University of Louisville

ThinkIR: The University of Louisville's Institutional Repository

Electronic Theses and Dissertations

$5-2021$

\title{
Genetic and functional analysis of rat mammary cancer susceptibility.
}

Emily L Duderstadt

University of Louisville

Follow this and additional works at: https://ir.library.louisville.edu/etd

Part of the Disease Modeling Commons

\section{Recommended Citation}

Duderstadt, Emily L, "Genetic and functional analysis of rat mammary cancer susceptibility." (2021). Electronic Theses and Dissertations. Paper 3628.

https://doi.org/10.18297/etd/3628

This Doctoral Dissertation is brought to you for free and open access by ThinkIR: The University of Louisville's Institutional Repository. It has been accepted for inclusion in Electronic Theses and Dissertations by an authorized administrator of ThinkIR: The University of Louisville's Institutional Repository. This title appears here courtesy of the author, who has retained all other copyrights. For more information, please contact thinkir@louisville.edu. 


\title{
GENETIC AND FUNCTIONAL ANALYSIS OF RAT MAMMARY CANCER
}

\section{SUSCEPTIBILITY}

\section{By}

Emily L. Duderstadt

B.S. Wittenberg University 2016

M.S. University of Louisville 2018

\author{
A Dissertation \\ Submitted to the Faculty of the \\ School of Medicine \\ of the University of Louisville \\ in Partial Fulfillment of the Requirements \\ for the Degree of
}

Doctor of Philosophy in Biochemistry and Molecular Genetics

Department of Biochemistry and Molecular Genetics

University of Louisville

Louisville, KY

May 2021 
Copyright 2021 by Emily Lauren Duderstadt

All rights reserved 

GENETIC AND FUNCTIONAL ANALYSIS OF RAT MAMMARY CANCER SUSCEPTIBILITY

\author{
By \\ Emily L. Duderstadt \\ B.S. Wittenberg University 2016 \\ M.S. University of Louisville 2018 \\ A dissertation approved on
}

April 21, 2021

By the following Dissertation Committee:

Dr. David J. Samuelson - Dissertation Director

Dr. Carolyn M. Klinge

Dr. Ronald G. Gregg

Dr. Timothy D. Cummins

Dr. Mary Ann Sanders 


\section{ACKNOWLEDGEMENTS}

I would like to thank my parents, Chuck and Jackie, for investing in my education since my birth. I would not be here without their willingness to sacrifice a comfortable life for themselves so I could have a better educational environment. They also instilled in me a strong work ethic and academic drive. I would like to thank my brother Nathan, who as my "twin" has been my companion and supporter through every shared milestone since pre-school. I would like to thank my best friend Lisa Green, who has been there for half of my life and supported me on our shared career path. Additionally, I would like to express an appreciation for my hound dog Lena, who has been a source of comfort to me through all of the lonely and terrible times in college and graduate school. 


\title{
ABSTRACT \\ GENETIC AND FUNCTIONAL ANALYSIS OF RAT MAMMARY CANCER SUSCEPTIBILITY
}

\author{
Emily L. Duderstadt
}

April 21, 2021

Breast cancer genetic susceptibility is modulated by high to low penetrance risk alleles. Population frequencies of high and moderate penetrant predisposing mutations are rare and responsible for a small percentage of population cases. Most genetic variation in breast cancer susceptibility is low penetrance common variants where causal genes and risk-associated mechanisms are largely unknown. Comparative genetics uses animal models to isolate and mechanistically evaluate genetic susceptibility to human disease. Rat strains have different susceptibility phenotypes to chemical-induced carcinogenesis. Selective breeding and genetic linkage analysis using a 7,12-dimethylbenz(a)anthracene (DMBA) rat mammary carcinogenesis model have identified Mammary carcinoma susceptibility quantitative trait loci (Mcs QTLs). Research completed for this dissertation focused on Mcs3 and Mcs $1 b$ QTLs. I found elevated mammary gland expression of Mcs3nominated and established breast cancer gene p21-activated kinase (Pakl) in response to DMBA administration. This highlighted a potential role for this gene in disease initiation. Mcs3 was previously mapped to a $29.4 \mathrm{Mb}$ region on rat chromosome 1 using WF.COP 
congenic lines. I developed my own congenic lines and fine mapped this locus to $7.2 \mathrm{Mb}$, discovered pleiotropic effects of $M c s 3$ on mammary tumor multiplicity and body mass, and identified candidate breast cancer and body mass genes/variants in orthologous human regions. Rat $M c s 1 b$ maps to $1.8 \mathrm{Mb}$ on rat chromosome 2 and is orthologous to human breast cancer risk locus 5q11.2. An Mcs1b and 5q11.2-nominated gene is MIER family member 3 (MIER3). I used an established rat Mcs $1 b$ congenic model to study in situ Mier3 mammary gland expression and found most expression in luminal epithelial cells. Expression of Mier3 doubled upon DMBA administration and increased along a precursorcarcinoma disease progression model. Mier3 expression was significantly enhanced in embryonic mammary buds, neonatal, and pubertal mammary glands, with significant dropoff at adulthood. This implicates MIER3 as a breast cancer gene that may modulate disease susceptibility during early periods of female breast development. I also provide MIER3 mechanistic insight by identifying interactions of MIER3 with nuclear proteins involved in transcriptional regulation and heterochromatin formation. Thus, MIER3 may function within a novel HDAC1/2 co-repressor complex like NuRD, Sin3A, CoREST, and family member MIER1. 
TABLE OF CONTENTS

PAGE

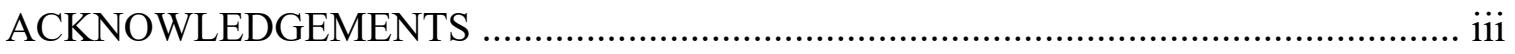

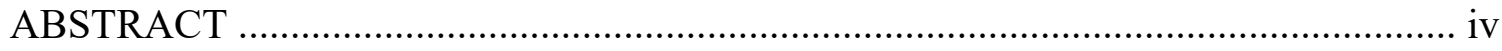

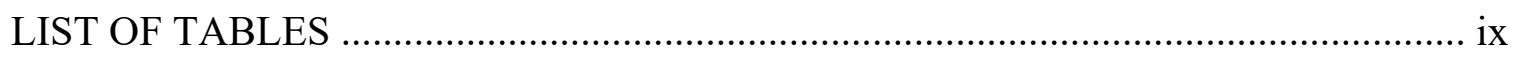

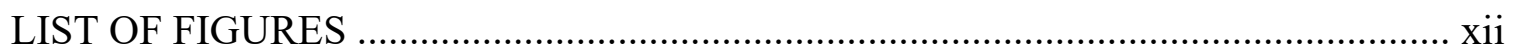

\section{CHAPTER}

I. BREAST CANCER INTRODUCTION …………………...................... 1

Breast Cancer Statistics ............................................................................... 1

Breast Cancer Risk Factors....................................................................... 1

Genetic Factors of Breast Cancer Risk ………………................................. 7

Timepoints of Altered Environmental and Genetic Susceptibility ................ 14

A Rat Model of Human Breast Cancer .............................................................. 19

Mammary Carcinoma Susceptibility Quantitative Trait Loci ........................ 30

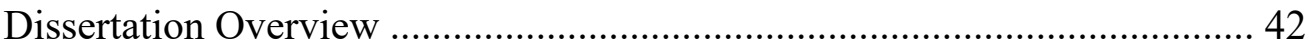

II. CHEMICAL CARCINOGEN INDUCED RAT MAMMARY

CARCINOGENESIS IS A POTENTIAL MODEL OF P21 ACTIVATED

KINASE POSITIVE FEMALE BREAST CANCER ………………............ 44

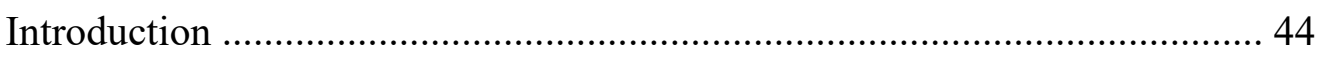




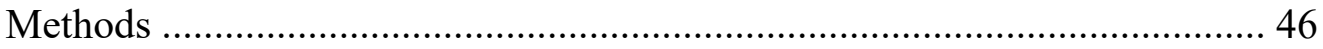

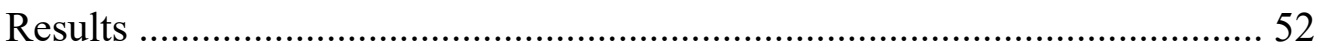

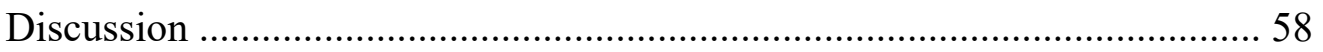

III. MAMMARY CARCINOMA SUSCEPTIBILITY LOCUS, MCS3: FINE

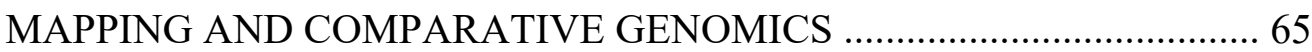

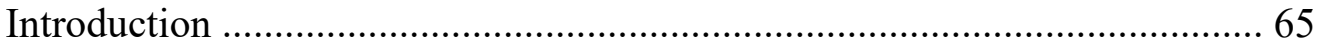

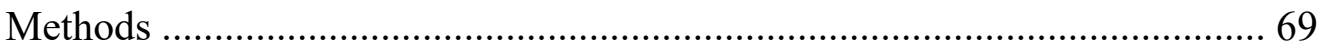

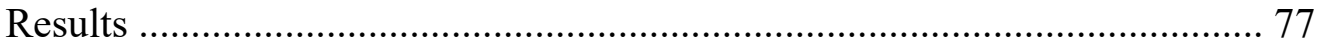

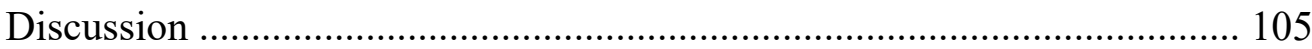

IV. A METHOD TO PRE-SCREEN RAT MAMMARY GLAND WHOLEMOUNTS PRIOR TO RNASCOPE ………………................................. 119

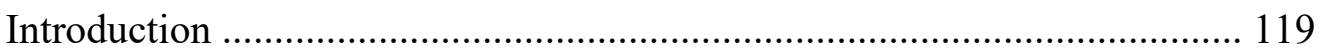

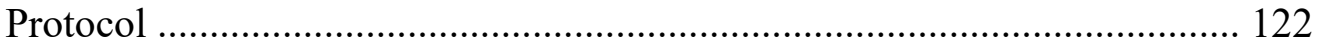

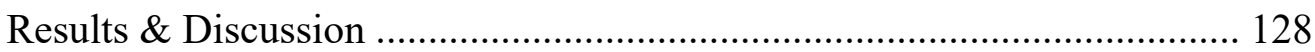

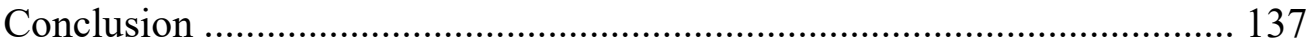

V. MIER3 EXPRESSION INCREASES THROUGHOUT RAT MAMMARY

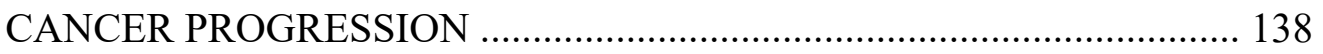

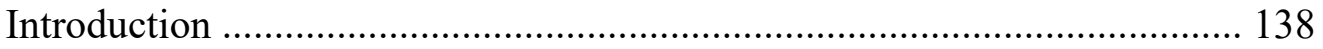

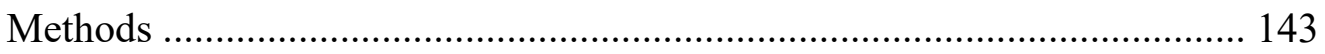

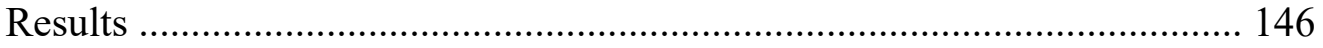

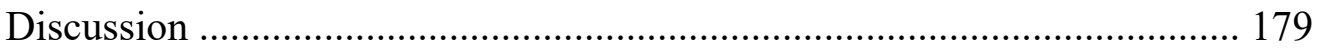

VI. MIER3 EXPRESSION DURING MAMMARY GLAND DEVELOPMENT AND MAMMARY CARCINOMA SUSCEPTIBILITY WINDOWS ....... 186 


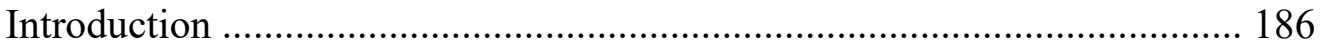

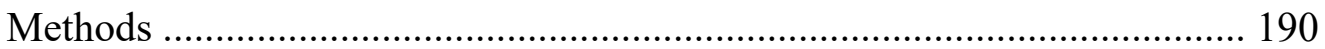

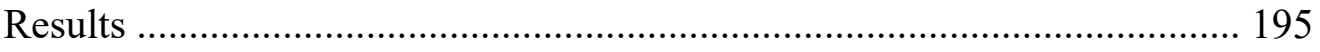

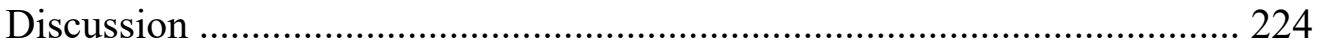

VII. MIER3 PROTEIN INTERACTIONS SUGGEST A FUNCTIONAL ROLE IN A TRANSCRIPTIONAL CO-REPRESSOR COMPLEX ...................... 231

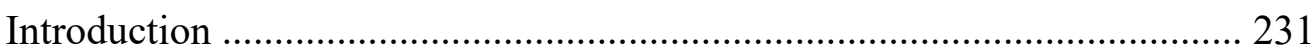

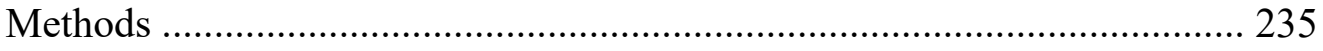

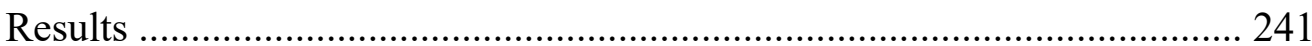

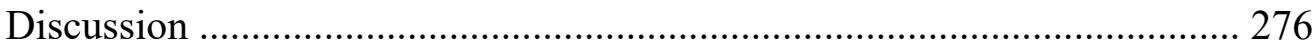

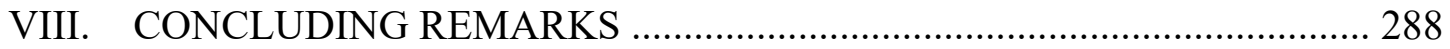

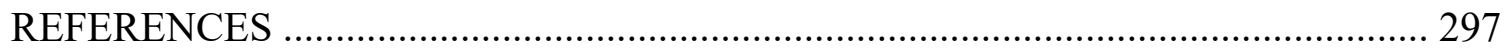

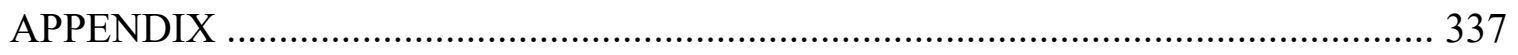

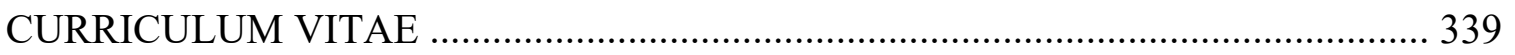




\section{LIST OF TABLES}

TABLE $\quad$ PAGE

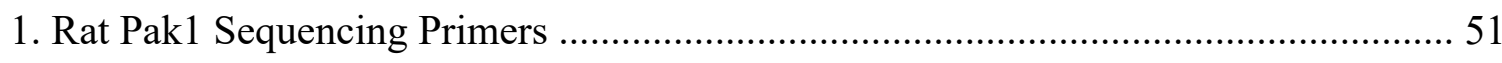

2. Rat Pak1 sequence variants between strains …………………………………….... 56

3. Rat mammary glands positive for Pak1 staining ……………………..................... 59

4. Female breast disease samples positive for PAK1 staining ................................................... 61

5. Primer Sequences for Mcs3 Microsatellite Markers in RNO1 used in Screening for

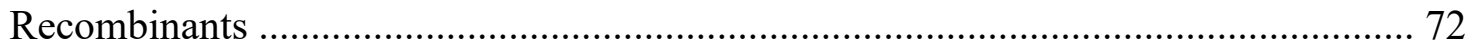

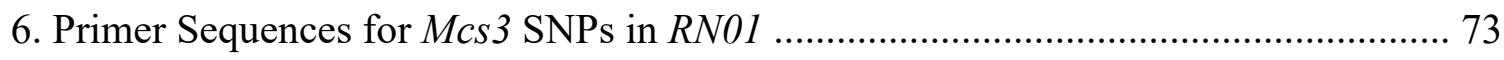

7. Positional markers and genomic locations defining WF.COP Mcs 3 congenic lines ... 79

8. Tumor Multiplicity Summary Data of Mcs3 WF.COP Congenic Lines ..................... 82

9. Primer Sequences Tested for New Mcs3 Informative Microsatellites ......................... 86

10. SNP Markers Tested in WF and COP Rats Based on Published Data in the Mcs3

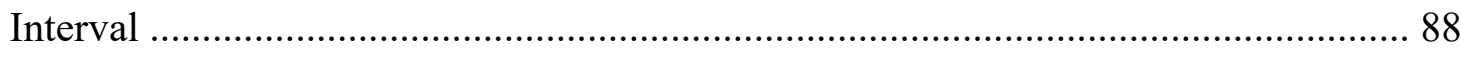

11. Body Mass Summary Data of Mcs3 WF.COP Congenic Females 15 weeks Post

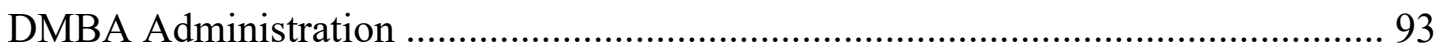

12. Body Mass Summary Data of WF/NHsd and WF.COP Line J Breeders .................. 95

13. Orthologous Human Loci Syntenic to Rat Mcs3 ………...................................... 101

14. List of Genes and Genomic Position in Rat Mcs 3 and the Orthologous Human

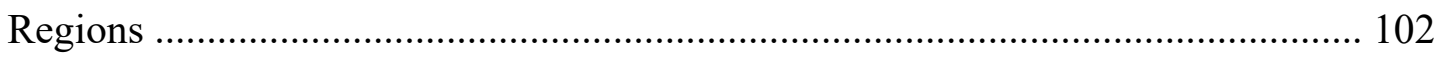

15. Rat Genome Positions of Mcs 3 Non-Coding DNA and Orthologous Human Genome 
Segments 104

16. Human Cancer-associated Genes Located in Rat Mcs3 106

17. Breast Cancer Risk Correlated Traits with Human GWAS Significant Variants in Genomic Segments Orthologous to Rat Mcs3 108

18. Spatial expression of Mier3 in 8-week-old mammary gland tissue from female WF/NHsd rats 152

19. Mammary Gland Expression of Mier3 in 12-week-old female WF/NHsd rats exposed or not exposed to DMBA 158

20. Mier3 mammary ductal expression in WF/NHsd and WF.COP Mcs $1 b$-N3

Females 4 weeks post-DMBA administration 162

21. Mier3 expression by mammary cancer progression morphologies in WF/NHsd and WF.COP $M c s 1 b$-N3 females 8 weeks post-DMBA administration 167

22. Mier 3 expression by mammary cancer progression morphologies in WF/NHsd and WF.COP $M c s 1 b$-N3 females 12 weeks post-DMBA administration

23. Mier 3 expression by mammary cancer progression morphologies in WF/NHsd and WF.COP Mcs $1 b$-N3 females 15 weeks post-DMBA administration 175

24. Mier3 expression in WF/NHsd and WF.COP Mcs $1 b$-N3 mammary epithelial structures throughout early development

25. Mier3 expression in WF/NHsd and WF.COP $M c s 1 b-\mathrm{N} 3$ mammary epithelial structures throughout reproductive development

26. Mier3 expression in combined WF/NHsd and WF.COP Mcs $1 b$-N3 mammary epithelial structures throughout the rat estrus cycle 223

27. Mass spectrometry peptide IDs from total elutions of GFP-trap pulldowns unique 
to MIER3 and MIER3-LXXAA

28. Additional mass spectrometry peptide IDs that appear from serum-starved and stimulated GFP-Trap pulldowns unique to MIER3 and MIER3-LXXAA

29. Total peptide IDs unique to MIER3 from mass spectrometry of individual bands in GFP-trap pulldown SDS-PAGE gels

30. Top $25 \%$ of mass spectometry peptide IDs meeting Contaminant Repository for affinity Purification (CRAPome) stringency criteria unique to MIER3 from individual band digestion in GFP-trap pulldown SDS-PAGE gels 265

31. Gene ontology of biological process of proteins meeting top stringency criteria ... 267

32. Gene ontology based on molecular function of proteins meeting top stringency criteria 268

33. Gene ontology based on biological processes of nuclear proteins meeting top stringency criteria 270

34. Gene ontology based on molecular function of nuclear proteins meeting top stringency criteria

35. Proteins meeting top stringency criteria associated with breast cancer and located in the nucleus 275 


\section{LIST OF FIGURES}

FIGURE

PAGE

1. Architecture of genetic susceptibility to breast cancer ......................................... 13

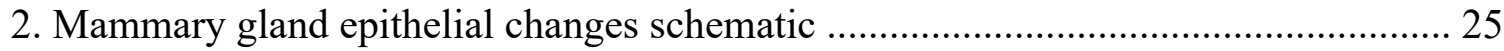

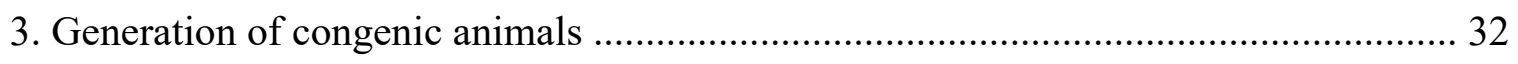

4. Rat $M c s 1 b$ and orthologous 5q11.2 breast cancer risk allele share two human/rat

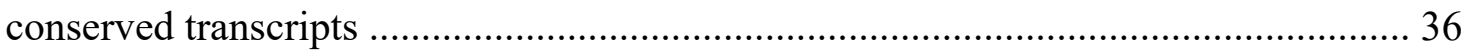

5. Protein domains of MIER family member 3 (MIER3) ............................................. 39

6. Increased Pak1 transcript levels in DMBA-induced rat mammary glands .................. 53

7. Correlation of Pakl and Ilk expression in rat mammary glands is lost with DMBA

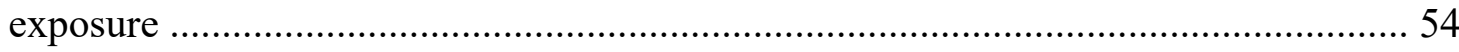

8. Representative Pak1 staining images of morphologically normal DMBA-induced

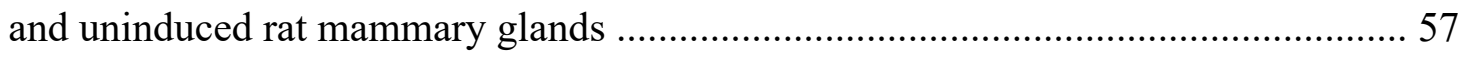

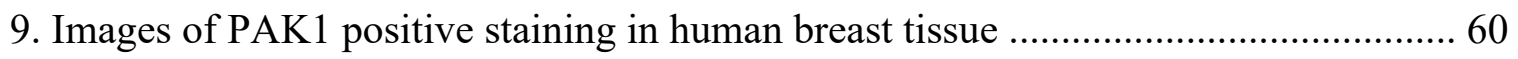

10. Timeline of Congenic Breeding and Strain Development ..................................... 71

11. WF.COP congenic map of $M c s 3$ recombinants used as congenic line founders ....... 78

12. Tumor Multiplicity of WF.COP Mcs3 Congenic Lines ...................................... 81

13. WF.COP Congenic Map Defining the Genomic Position of Mcs3 …..................... 83

14. WF.COP Mcs3 congenic lines develop invasive papillary carcinomas ................... 90

15. WF.COP Mcs 3 congenic lines develop invasive cribriform carcinomas ................ 91 
16. Body mass of $M c s 3$ experimental females at 15 weeks post-DMBA administration..92

17. Body mass of WF and Line J rats over development ............................................ 94

18. Body mass of WF and Line J females raised in an all-female environment .............. 97

19. Rat mammary gland histology in WF and line J females over development ............ 98

20. Diagram of overlapping GWAS breast cancer associated traits orthologous to rat $\operatorname{Mcs} 3$ 110

21. Mammary Gland Tissue Extraction 124

22. Flow chart outlining difference in tissue preparation methods 125

23. Premalignant and malignant lesions were visible in mammary gland tissue whole mounts following alum carmine staining

24. Representative RNAscope results from Mier3, Polr2a, and DapB probes in tissue sections obtained from rat mammary gland whole-mounts

25. Representative images of RNAscope assays using Mier3, Polr2a, and DapB probes in rat mammary gland and mammary carcinoma tissue

26. Quantitative analysis of RNAscope assays 135

27. Precursor-Carcinoma Sequence of Mammary Cancer Development 140

28 Disease progression mammary gland collection timeline

29. RNA in situ hybridization (RNAscope) control and Mier3 probes function properly in rat mammary tissue

30. Mammary ducts possess the greatest Mier 3 expression levels of all rat mammary gland tissue elements 150

31. The majority of ductal Mier3 expression is localized to luminal epithelial cells ... 154

32. Ductal Mier3 expression is significantly increased with DMBA-exposure 155 
33. Mier 3 expression in rat mammary glands 4 weeks post DMBA administration ..... 160

34. Mier 3 expression in rat mammary glands 8 weeks post DMBA administration ..... 164

35. Mier 3 expression in rat mammary glands 12 weeks post DMBA administration ... 168

36. Mier3 expression in rat mammary glands 15 weeks post DMBA administration ... 172

37. Rat mammary carcinoma cells express variable levels of Mier3 …........................ 177

38. Mier3 expression in the histopathological stages of the precursor-carcinoma sequence of rat mammary cancer progression ………………................................ 178

39. Mammary gland collection timeline of windows of susceptibility during

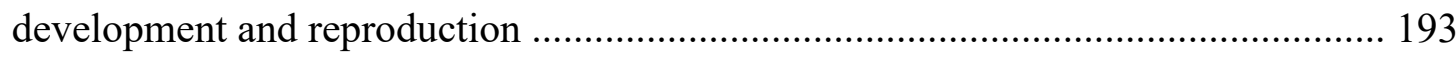

40. Mammary Gland Appearance and Histology during Development ......................... 197

41. Mammary Gland Appearance and Histology during the Reproduction Cycle ........ 199

42. RNAscope assay probes in rat mammary glands throughout development ............ 202

43. RNAscope assay probes in rat mammary glands during the reproductive cycle ..... 203

44. Mier3 expression in MECs of rat embryonic mammary buds ................................ 204

45. Mier3 expression in MECs of rat mammary glands at 9 days of age ..................... 205

46. Mier3 expression in MECs of rat mammary glands at 4 weeks of age .................. 207

47. Mier3 expression in MECs of rat mammary glands at 8 weeks of age .................. 208

48. Mier3 expression in MECs of rat mammary glands at 12 weeks of age ................ 209

49. Mier3 expression in MECs of rat mammary glands during pregnancy .................. 212

50. Mier3 expression in MECs of rat mammary glands during lactation ...................... 213

51. Mier3 expression in MECs of rat mammary glands during involution ................... 215

52. Mier3 expression in MECs of parous rat mammary glands .................................. 216

53. Ductal Expression of Mier3 In MECs of rat mammary gland during development. 219 
54. Mier3 expression in rat mammary glands based on stage of the rat estrus cycle .... 221

55. Cells transfected with pEGFP-MIER3-C1 display low levels of fluorescence ....... 243

56. pEGFP-MIER3-C1 transfected cells display increased fluorescence with

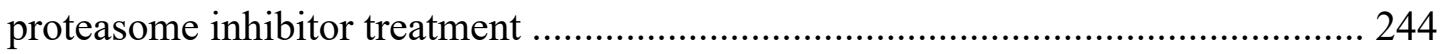

57. pEGFP-MIER3-C1 transfected cells display increased fluorescence over a 48 hour time course with proteasome inhibitor treatment 246

58. Screening of transformed colonies for LXXLL mutations of MIER3 248

59. The LXXLL sequence motifs of MIER3 were successfully mutated to LXXAA ... 249

60. MIER3 proteins appear at the correct molecular weight following affinity purification of GFP 251

61. Overlapping peptide numbers from affinity purification-mass spectroscopy experiments with pEGFP-C1, pEGFP-MIER3-C1, and pEGFP-MIER3-C1-LXXAA 255

62. GFP and GFP-MIER3 band excision following affinity purification 258

63. Model of the function of a novel HDAC1/2-containing MIER3 repressive complex in heterochromatin formation and gene repression in breast cancer 274 


\section{CHAPTER I}

\section{BREAST CANCER INTRODUCTION}

\section{$\underline{\text { Breast Cancer Statistics }}$}

Breast cancer is the most frequently diagnosed cancer among women, accounting for $30 \%$ of all diagnosed cancers. This is significant as the second most common cancer in women is lung cancer, with $13 \%$ of cancer incidences [1]. In the U.S. alone in 2021 there are projected to be 281,550 new cases and an estimated 43,600 deaths due to breast cancer [1]. Although the breast cancer death rate has dropped by $41 \%$ since 1989 , it is still the second leading cause of cancer-related deaths in women at $15 \%$ of cancer mortalities [1]. Over the last two decades, breast cancer incidence rates have increased by about $0.5 \%$ per year, which is partly attributable to lifestyle risk factors such as older age at first birth and increased body weight [2]. This highlights a pressing need to identify and investigate further genetic and molecular susceptibility factors to aid in early screening and prevention strategies.

\section{$\underline{\text { Breast Cancer Risk Factors }}$}

Breast cancer is a highly complex disease comprised of environmental and genetic components, as well as the interplay between them. A woman's individual disease risk varies depending on her possession of underlying factors that modulate overall susceptibility. Risk factors attributable to breast disease initiation can be divided into two 
groups, intrinsic and extrinsic factors. Intrinsic risk factors are inherent and cannot be changed by outside intervention. Extrinsic factors can be modified to a certain degree within individuals and include lifestyle choices and environmental influences. Some of the major risk factors are outlined below. This is not an exhaustive list, but serves to illustrate the broad range of risk factors that influence breast cancer susceptibility.

\section{Intrinsic risk factors:}

Sex:

Biological sex is a major risk factor for developing breast cancer, as women have 100 times greater disease incidence than men [3]. However, despite diagnosis predominantly in women, the number of male breast cancer cases is increasing, which is thought to be attributable to obesity and longer life expectancy $[4,5]$.

Age:

Breast cancer incidence is highly related to increasing age, with $80 \%$ of diagnosis coming from women aged 50 and over [6]. The number of breast cancer cases is very low in women younger than $30(<25$ cases per 100,000$)$, after which there is a steady linear increase and a plateau around the age of $80(\sim 500$ cases per 100,000) [7]. Hormone responsiveness is also stratified by age. Estrogen receptor negative (ER-) tumors occur more frequently until around 50 years of age, i.e., the approximate average age at menopause, followed by a greater incidence of ER + tumors in women after menopause [8]. 


\section{Endogenous estrogen levels:}

High levels of estrogen-related hormones (total estradiol, free estradiol, estrone, estrone sulfate) are a well-defined breast cancer risk factor. Increasing estrogen levels especially enhance breast cancer risk in post-menopausal women and is correlated with increased incidence of ER + breast tumors $[9,10]$. This phenomenon has been demonstrated using aromatase inhibitors (AI), which block the conversion of androgens to estrogens and lowers blood estrogen levels. Phase III trials have demonstrated a 50-65\% reduction in invasive breast cancer in post-menopausal women treated with AI (which ones) at high risk of breast cancer compared to placebo $[11,12]$. Although estrogens confer higher risk in post-menopausal women, there is still a critical role of estrogens in the etiology of premenopausal breast cancer $[13,14]$.

\section{Reproductive factors:}

Exposure to and changes in endogenous sex hormones can influence breast cancer risk. Early menarche (less than 12 years of age) is associated with higher risk as compared to menses onset after age 15 [15]. Every two-year delay of menarche reduces disease risk by $10 \%[15-17]$. At the opposite end of reproduction, breast cancer risk increases $3 \%$ for every one year increase in the age at menopause [18]. These higher risks of disease occurrence are thought to be due to the duration of menstrual cycles over a woman's lifetime, with prolonged estrogen exposure resulting from early menarche and delayed menopause. The risk of breast cancer is also increased in nulliparous women or women who have a fist-time birth after age $30[19,20]$. 


\section{Genetic susceptibility:}

Genetic predisposition to breast cancer can come from inherited familial susceptibility or de novo germline mutations. Cases of familial disease is a major risk factor as $12 \%$ of women diagnosed with breast cancer have at least one affected family member [21]. Women with one first-degree relative with breast cancer have a 1.75-fold higher risk of disease occurrence, which increases to 2.5-fold higher in women with two or more firstdegree relatives with breast cancer [22].

\section{Mammographic density:}

A mammography assesses the radiographic appearance of breast tissue, which varies depending on the ratio of fat, connective tissue, and epithelial tissue (radiographically most dense). Women with more than $75 \%$ of their breast tissue radiographically defined as dense have a 5-fold greater risk of breast cancer development than women with only $5 \%$ of tissue classified as dense [23]. Thinner and younger women, as well as both pre- and post-menopausal nulliparous women, generally have increased breast density and therefore may have increased risk of breast cancer [24].

\section{Extrinsic Risk Factors:}

Diet:

The modern human diet contains a variety of natural and chemical carcinogens which can lead to DNA damage and cancer initiating events $[25,26]$. Many of these come from the processing and preservation of food, especially the preparation of meat [27]. A diet low in bread and fruits, but high in meat, fish, butter, and other animal fats is associated 
with increased disease risk [28]. A major dietary risk factor is the excess intake of saturated fat, which is highly concerning for a western diet containing too much saturated fats [29, 30]. This risk may be attributable to an increase in circulating estradiol due to saturated fats [31]. It has been demonstrated that implementing a low-fat diet in post-menopausal women, after a primary breast cancer surgical procedure, significantly reduced the risk of relapse [26]. Additionally, a diet rich in fiber, omega-3 polyunsaturated fatty acids, fruits, and vegetables seems to have a protective effect against breast cancer [32-34].

\section{Alcohol consumption:}

There is a positive association between alcohol intake and breast cancer risk. Ingesting 35-44 grams of alcohol per day can cause a $32 \%$ increase in risk, with each additional 10 grams resulting in a $7.1 \%$ increase [35]. The main breast cancer specific risk is the effect of alcohol on estrogen metabolism, which results in elevated serum levels of estrogen-related hormones and induction of ER pathways in pre- and post-menopausal women [36-38]. Another critical pathogenic mechanism is through the action of the alcohol metabolite acetaldehyde, which is an established carcinogen [39].

\section{Exercise:}

Low levels of physical activity are associated with breast cancer risk at any age. There is a $25 \%$ average reduction in breast cancer risk across 73 studies that compared the most active to least physically active women [40]. Physical activity during adolescence and young adulthood significantly reduces breast cancer risk, as there is a $3 \%$ reduction in risk for each one-hour increase in recreational activity per week [41]. Associations for reduced 
risk are strongest for activity that is minimally moderate in intensity and is sustained over a lifetime or done after menopause [42]. Mechanisms of reduced risk are thought to stem from reduction in circulating sex hormones which lessons cumulative exposure and activation of hormonal pathways, as well as modulation of adipocytokines and inflammation [43].

\section{Obesity:}

The risk of breast cancer in association with obesity appears to be dependent on a woman's menopausal status. In post-menopausal women obesity positively associates with breast cancer risk, with an $8 \%$ risk increase for each $5 \mathrm{~kg}$ of weight gain since the lowest adult weight [44]. This is largely thought to be due to the greater amounts of adipose tissue in obese women which serves as an extragonadal source of estrogen, resulting in continued estrogen exposure despite loss of menstrual cycling [45, 46]. In contrast, obesity is associated with a decreased risk of breast cancer in pre-menopausal women, although the underlying mechanism is not understood $[47,48]$.

\section{Smoking:}

The relationship between smoking and breast cancer risk is not definitive, probably due to a genetic and environmental interplay on risk association that has been demonstrated and low carcinogen dose $[49,50]$. Some studies have indicated early age of smoking initiation and long duration of smoking years can increase breast cancer risk $15 \%$ to $40 \%$ [50]. Of the thousands of chemicals in cigarette smoke, 69 are carcinogenic and 20 of these

specific mammary carcinogens [51]. Mammary tissue can take up and bioactivate 
polycyclic aromatic hydrocarbons and $\mathrm{N}$-nitrosamines from cigarette smoke, eventually resulting in formation of DNA adducts $[49,52,53]$.

\section{Environmental pollutants:}

There are two main environmental pollutants that are associated with breast cancer risk, both of which are lipophilic and accumulate in the high adipose content of breast tissue - polycyclic aromatic hydrocarbons (PAHs) and polychlorinated biphenyls (PCBs). PAHs are a by-product of combustion and are prevalent in grilled foods, air pollution, vehicle exhaust, and cigarette smoke [51,54]. Several PAHs, including one of the more widely recognized PAHs benzo(a)pyrene, are mammary mutagens that form DNA adducts that induce DNA damage and repair responses [55]. PCBs were contained in lubricants, liquid sealants, flame retardants, and electrical insulators until banned in the U.S. in 1979. They remain pervasive in the environment, and the main route of PCB exposure is through ingestion of contaminated fish and animal fats. PCBs are found in most samples of human breast milk [56]. PCBs are highly relevant to breast cancer risk since metabolites serve as endocrine disrupting chemicals. PCBs also induce DNA damage, which can lead to carcinogenesis [56].

\section{Genetic Factors of Breast Cancer Risk}

Among women diagnosed with breast cancer, $10-20 \%$ already have a family history of disease [57, 58]. This clustering of breast cancer cases within families provides evidence there is a genetic predisposition underlying cancer development, though it is difficult to parse out genetic and environmental factors in disease epidemiology. The genetic 
contribution has been estimated using twin studies that compare disease occurrence in monozygotic (genetically identical) and dizygotic (share half of their segregating genes) twin pairs [59]. Monozygotic twins share a greater disease concordance than dizygotic twins, meaning a monozygotic twin has a higher risk of developing breast cancer if their twin is affected, demonstrating there are powerful hereditary factors contributing to breast cancer susceptibility [59-62]. Analysis of familial clustering has estimated the heritability of breast cancer to be $73 \%$ within families [63], while $10-30 \%$ of non-familial disease susceptibility has been attributed to genetic factors $[59,61,62,64]$. Since recognizing the large genetic component to the origin of breast disease, it became essential to identify inherited susceptibility factors and their biological mechanism.

Breast cancer risk genes can be divided into three classes based on allele frequency and conferred risk. The first class is comprised of high penetrance risk alleles such as BRCA1, BRCA2, TP53, STK11, and $C D H 1$ [64]. Risk variants in these genes are very rare in the population but each individual allele has a strong effect on breast cancer risk [65]. Early genetic studies using linkage analysis within families displaying aggregation of breast cancer risk first identified $B R C A 1$ and $B R C A 2$ as highly penetrant susceptibility genes $[66,67]$. These cause breast cancer incidences of $65 \%$ and $45 \%$ in carrier women of $B R C A 1$ and $B R C A 2$ mutations, respectively, by the age of 70 [68]. Further linkage studies and candidate gene approaches in breast cancer families that do not segregate $B R C A 1$ or $B R C A 2$ mutations have identified additional genes. These studies were successful in accounting for up to $25 \%$ of the heritability of familial breast cancer $[65,69]$; however, they do little in explaining genetic factors behind sporadic disease development, accounting for only up to $5 \%$ of heritability [65]. Since the search for new highly penetrant 
genes eventually proved exhaustive, the unexplained genetic risk provided evidence of existence of other undetected susceptibility genes.

Moderate risk alleles make up the second class of breast cancer heritability factors, which are variants with a rare population frequency and a moderate effect on disease risk. Genes in this class of breast cancer risk alleles include PTEN, CHECK2, PALB2, ATM, BRIP1, BARD1, ABRAXAS [64]. These predisposing genes have been identified through linkage analysis and candidate gene approaches, which used targeted sequencing of genes known to interact with BRCA1 and BRCA2 or DNA repair pathway genes, followed by further evaluation in case-control association studies [63]. Other moderate risk alleles have been identified using linkage analysis in families with rare syndromes that include breast malignancies [70]. Similar to the high penetrance mutations, these moderately penetrant alleles account for very little non-familial disease risk and contribute less then 3\% of the heritable familial risk [71]. High penetrance and moderate penetrance alleles together account for no more than $25 \%$ of familial breast cancer risk [69, 72], leaving the majority of genetic susceptibility unidentified.

The remaining fraction of breast cancer genetic risk is thought to be driven by lowpenetrance alleles, which make up the third class of breast cancer risk alleles [73, 74]. Variants in this class are very common, each affecting $>5 \%$ of the population, but individually have weak associations with risk [75]. It is estimated that the collective effects of these low penetrance risk alleles on population genetic risk exceeds that of highly penetrant genes [76, 77]. In addition to their combined effect on sporadic disease development, low-risk variants are thought to explain part of the residual genetic component of carriers of high-risk alleles by interacting multiplicatively on overall 
susceptibility $[78,79]$. Common low-penetrance alleles were initially identified by casecontrol genetic association studies targeting candidate genes known to be involved in carcinogenesis [80]. This approach is biased because it relies on prior knowledge of the genes and types of causative polymorphisms and it ignores uncharacterized genes [75]. The first genome-wide association study (GWAS) of breast cancer was published in 2007 and GWAS has since become the standard approach to identifying risk-associated variants and low-penetrance alleles [64]. In these studies, several hundred thousand SNPs are genotyped across very large study populations, allowing for association with disease risk to be conferred without assumption of biological function or location [70, 81]. As the number of GWAS have grown, meta-analysis combining multiple studies have become important in identifying additional susceptibility loci. To date, more than 180 breast cancer susceptibility loci have been identified [82]. There is now a great need to identify causal variants in these loci and biological mechanism behind disease susceptibility.

It is estimated that the collective common low penetrance risk variants identified by GWAS only account for $18 \%$ of familial breast cancer risk, and most of these reported associations have failed to be confirmed in validation studies [83, 84]. The remaining heritability from low penetrance alleles may remain unidentified due to limitations in GWAS. There were high levels of false-positives in initial reports because investigators failed to use stringent statistical evaluation to identify true risk loci and variants in which the probability of an association is small $[83,85]$. A Bonferroni corrected p-value of less than $10^{-7}$ has been recommended as necessary to reduce the rate of false-positives, while other studies utilize a very stringent criteria of $p<10^{-8}$ [85-87]. From this increased stringency has risen the idea that this increases the rate of false-negatives, meaning SNPs 
associated with disease risk are missed due to too conservative of statistical methods in evaluating GWAS [88]. Thus, parsing out true risk-associated alleles and variants from GWAS proves difficult without complementary studies of genetic risk. Additionally, GWAS use "tag-SNPS" to tag common variation across the genome, but these only serve as a marker of a disease-associated region [70]. This is a result of linkage disequilibrium (LD), where numerous SNPs in a region of low recombination are inherited together [63]. These LD blocks, or haplotype blocks, are bound by regions of high recombination [63]. This delimits the region and means the GWAS tag-SNP identifies that LD block as associated with a disease phenotype, but leaves the true causal variant unknown [70]. Fine mapping of these LD blocks is then necessary to obtain a complete list of variants in the region, followed by genotyping of all SNPs in LD with the tag-SNP across large casecontrol studies to identify candidate causal variants based on the strongest diseaseassociations $[63,70]$.

GWA studies by design are capable of identifying common variants, but it is estimated a portion of the unidentified genetic risk of breast cancer stems from its inability to interrogate rare variants conferring moderate risks since these SNPs are not tagged or well represented in population-based studies [89]. The increase in the scale of next generation sequencing (NGS) and decline in sequencing cost has elevated this platform as the newest route for discovery of both rare and common breast cancer-associated variants $[90,91]$. When investigating rare risk alleles there is reduced statistical power, requiring filtering of studies or sequencing of a large number of cases and controls. Some of these strategies include initially sequencing within cases of familial aggregation, selecting candidate genes to sequence based on pathways or disease implications, or filtering based 
on consequences of variation on translation and protein function [63, 92]. Following discovery, variants can be validated in larger cohorts that contain controls and patients with sporadic and familial breast cancer [93].

Although high and moderate penetrant alleles have deleterious effects for familial breast cancer patients, the majority of the heritability remains unexplained in these families and do not provide insight into the risk of sporadic disease. The major genetic component of breast cancer most likely follows a polygenic model, where the aggregation of low penetrant alleles alters disease susceptibility in sporadic cases and can further modulate familial disease-risk through interaction with higher penetrant alleles. The genetic architecture of breast cancer, comprised of the three classes of genetic susceptibility and their minor allele frequency, is depicted in Figure 1. High and moderate penetrant alleles are typically found in coding regions of genes, making the identification of the causative gene clear and usually resulting in protein truncation due to nonsense mutations, frameshift insertions or deletions, or variants interrupting splice sites [63]. However, low penetrance alleles are generally found in non-coding or intergenic regions, which makes identifying the causative gene more difficult since the effect is mainly on regulation of gene expression. These risk alleles may affect promoter function, modulate binding of transcription factors, or reside at distal regulatory elements such as enhancers and affect gene regulation through complex chromatin arrangements [70]. In continuing the discovery of unexplained genetic factors behind breast cancer susceptibility, there is a dual challenge in identifying causative variants within susceptibility loci, as well as parsing a biological mechanism behind the associated risk. 


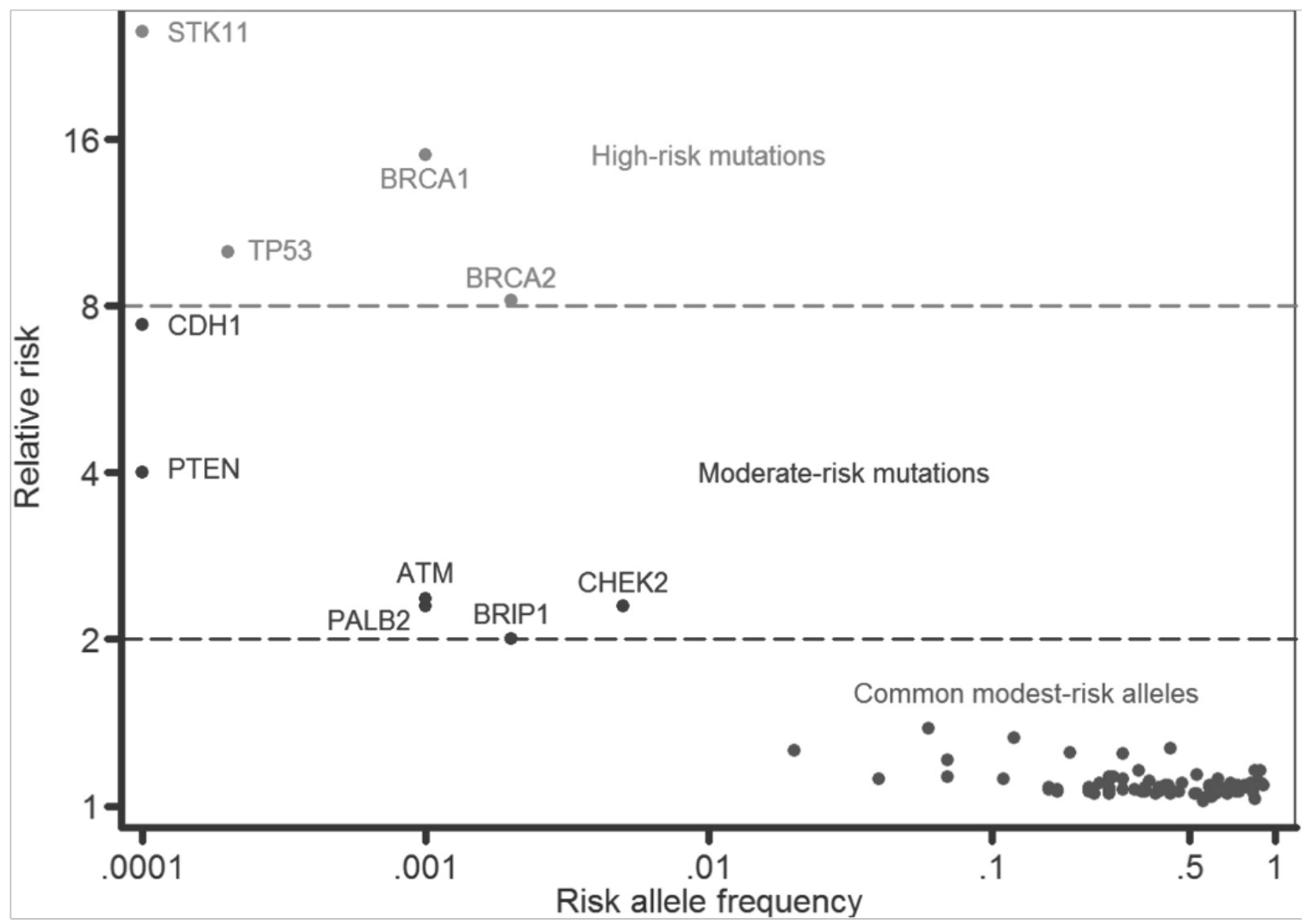

Figure 1: Architecture of genetic susceptibility to breast cancer. Adapted from Ghoussaini et al. (2013) [70]. Some of the major genetic loci identified for breast cancer risk are shown in this figure. Breast cancer risk genes are subdivided into classes by allele frequency and level of risk. 


\section{Timepoints of Altered Environmental and Genetic Susceptibility}

There are susceptibility timepoints when genetic risk factors and environmental perturbations overlap with morphological changes in breast tissue that result in increased lifetime risk of developing breast cancer [94-99]. These "windows of susceptibility" are timepoints characterized by an increased risk of cancer initiating events due to molecular alterations that drive tissue remodeling, which can have a role in breast cancer development later in life [94, 96, 98, 99]. Windows of susceptibility encompass developmental and reproductive time intervals that are each unique in their susceptibility response due to distinct transcriptomes, epigenetic state, tissue microenvironment, and signaling processes that comprise breast tissue and its biological function at that time $[94,96,97,100]$.

\section{Breast cancer susceptibility during puberty:}

The most susceptible structures in the female breast are terminal ductal lobular unit (TDLU) or Lobules type 1 (Lob 1), which become a milk-secreting population during lactation [100-102]. These structures contain undifferentiated epithelial cells with high proliferative activity that first appear during puberty and expand during this time period. It is notable that Lob 1 epithelial cells can be transformed to induce mammary cancer following in vitro exposure to the same chemical carcinogens administered in experimental mammary cancer models, such as N-methyl-nitrosourea and 9,10-dimethyl-1,2benzanthracene $[100,103]$. Lob 1 structures have also been shown to have the highest rate of DNA binding by carcinogen [104]. This suggests that chemical insults affect Lob 1 structures, and a mutagenic event is most likely to transform breast tissue during this susceptibility window that begins at puberty and extends until a first full-term pregnancy 
$[100,104]$. From studies like these, there arises a concern that inadvertent chemical exposure during this high risk window may drastically increase susceptibility to breast cancer since it is the terminal ductal structures that give rise to the majority of breast cancers [105-112]. Thus, it is crucial to understand the biological characteristics of Lob 1 as these are the major sites of cancer initiation events.

\section{Breast cancer protection timepoints due to tissue differentiation:}

Differentiation is an inhibitor of cancer development, which is clinically relevant as undifferentiated structures are more likely to develop aggressive carcinomas [113-115]. This has been demonstrated in the human breast, which attains maximum development and differentiation during pregnancy, resulting in parous women displaying the greatest proportion of differentiated lobules and lower sensitivity to carcinogens $[100,107,116$, 117]. As a result of differentiation, an early first full-term birth is the most effective method of breast cancer prevention, which can reduce a woman's lifetime risk up to $50 \%$, with additional pregnancies increasing this protective effect compared to nulliparous women $[116,118,119]$. Breast cancer protection due to pregnancy is inversely related to the length of time between menarche and the first reproductive cycle, as breast cancer risk increases with increased time of exposure of undifferentiated tissue $[119,120]$. This protective effect is extremely powerful as it persists in all subsequent ages and through menopause [119, 120]. Breast cancer protection due to tissue differentiation is also demonstrated in parous women who do develop breast cancer, as their lobular profiles match that of nulliparous women. This indicates these women harbor a defective response to differentiation signaling and do not achieve full differentiation during the reproductive cycle, and thus retain 
susceptible cell types [121-123]. Understanding the molecular mechanisms behind mammary gland differentiation would thus provide valuable knowledge to develop novel approaches of breast cancer prevention.

The protective effect of pregnancy has been recapitulated in a rat model of mammary cancer. Rats administered a chemical carcinogen to induce mammary tumors are similarly protected by pregnancy as compared to nulliparous females [124-126]. Interestingly, merely replicating the hormonal exposures of the mammary gland during pregnancy in virgin females can induce protection from carcinogen-induced mammary tumor multiplicity [127-130]. Combinatorial treatments with estradiol and progesterone shortly before or after chemical carcinogen administration have been shown to mimic pregnancy by inducing mammary gland lobuloalveolar formation and inhibiting mammary tumor formation $[128,131]$. It is assumed that the same molecular factors in the human breast and rat mammary gland are responsible for tissue differentiation that inhibits cancer initiation [103, 121-123]. These molecular mechanisms during pregnancy may remove or modify more highly susceptible cell types and structures, accounting for the decreased risk of breast cancer $[131,132]$.

During breast tissue remodeling throughout pregnancy, lobular structures progress to a more differentiated state that makes up the fully developed gland [100]. The undifferentiated Lobules type 1 (Lob 1) structures are most susceptible to carcinogen [133]. In nulliparous women, the breast has the highest portion of Lob 1 structures, with slightly more differentiated Lob 2 present in modest numbers [100, 121]. Throughout breast development during pregnancy, Lob 1 and 2 progress to Lob 3 in the early stages, then to the fully differentiated Lob 4 at the later stages of pregnancy $[100,121]$. During involution, 
Lob 4 regresses back to Lob 3 [100]. This remains the predominant structure in the once again quiescent gland, which are absent in nulliparous women [100, 121]. Even after full breast development during the reproductive cycle, the mammary epithelium remains responsive to proliferative signaling. However, these stimulated cells have undergone differentiation and can more efficiently metabolize carcinogens and direct a DNA repair response, making them less susceptible to carcinogenesis [100]. Although more differentiated lobular structures are still affected by carcinogen, the malignancy of initiated lesions is decreased with greater degrees of differentiation [133]. Lob 1 gives rise to ductal carcinomas which comprise the majority of breast cancer cases, Lob 2 develops into lobular carcinomas in situ, Lob 3 advances to benign breast lesions, such as hyperplastic lobules, cysts, fibroadenomas, and adenomas; and Lob 4 originates lactating adenomas [100, 101, 133]. Thus, differentiation during pregnancy gives rise to different types of lesions and altered susceptibility to breast cancer $[100,101,122]$.

\section{Breast cancer susceptibility timepoints due to tissue remodeling:}

Post-lactational involution is characterized by extensive tissue remodeling that eliminates a large portion of the mammary epithelium through apoptosis [100]. No other processes or pathological conditions compare to the rapid nature of involution [134]. This reconstruction process is similar to wound healing as the gland is filled with activated fibroblasts and immune infiltration, and the extracellular matrix becomes enriched with high levels of fibrillar collagen, laminin, fibronectin and matrix metalloproteinase activity [135-140]. In fact, the transcriptome of the mammary gland undergoing involution indicates that the same tissue-remodeling processes involved in wound healing and 
inflammation are activated during this period, though involution itself is controlled and developmentally programmed $[135,141,142]$. Inflammation is a pro-oncogenic process, such that cancers with an inflammation component have a poorer prognosis than those cancers that do not [143-145]. Due to a microenvironment that mimics wound healing and inflammation, it is believed that involution creates tumor-promoting activities that may support transformation of epithelial cells to cancer cells and allow for tumor cell dissemination [146-148]. This idea has been investigated using rat mammary gland extracellular matrix (ECM) isolated from quiescent nulliparous vs weaning-induced involuting mammary glands. Involution ECM increased breast cancer cell invasiveness and motility as compared to quiescent ECM in vitro [139, 145, 147, 149]. A xenograft model of human breast cancer found that rates of metastasis significantly increased when breast cancer cells were mixed with involution ECM compared with cells mixed with nulliparous ECM or Matrigel [147, 148]. Collectively, these studies indicate that involution indeed creates a microenvironment permissive to tumor initiation and represents a metastatic window of susceptibility $[139,147]$.

A transient increase in breast cancer risk following pregnancy has been established, referred to as pregnancy-associated breast cancer [148]. This cancer is defined as breast cancer occurring within 5 years of pregnancy, and is associated with poor prognosis, an increased rate of metastasis, and a high mortality rate [150-152]. This may appear contradictory to the established protective effect of pregnancy. It is true that an early first full-term pregnancy and multiple pregnancies are associated with a reduction in breast cancer risk for the lifetime of these women [153]. However, a first full-term pregnancy in older women is associated with a perpetual increase in breast cancer risk, possibly due to 
a longer period of exposures in undifferentiated breast tissue to estradiol and progesterone prior to pregnancy [153-155]. It is likely that there is a period of tumor-promoting activity during pregnancy due to the growth and invasion-promoting effects of gestational hormones and following pregnancy due to the tumorigenic microenvironment during involution $[148,156,157]$. These risk-associated timepoints would precede the eventual protective effect of pregnancy $[120,153]$. Thus, the reproductive cycle both suppresses and promotes breast cancer development [148].

\section{$\underline{\text { Rat Model of Human Breast Cancer }}$}

A comparative genomics approach using a rat model presents an unbiased method of genome-wide screening to identify novel homologous human candidate breast cancer genes that may have been unknown or unsuspected through population studies $[158,159]$. These rodent models have the power to identify low penetrance genes or loci that modulate breast cancer risk and translate these to human populations for comparative evaluation [159]. The rat model also allows for exploration of the mechanism through which genes and genetic variants modulate breast cancer susceptibility by studying the natural initiation and progression of mammary cancer. Rat mammary carcinogenesis is a good model of human breast cancer, as induced rat mammary carcinomas display the same histopathological stages and features of malignant breast carcinomas, including epithelial ductal cell origin, progression, and frequency of hormone responsive and nonresponsiveness [76, 101, 160-164]. 


\section{Structure of the rat mammary gland:}

The rat has six pairs of mammary glands that are situated along the ventrolateral sides of the body, extending from the cervical glands at the anterior, two pairs of thoracic glands, a pair of abdominal glands, and 2 pairs of inguinal glands at the posterior [165]. These mammary glands appear as subcutaneous sheets of tissue that lay between the skin and body cavity [114]. Mammary tissue is composed of adipocytes and fibroblasts that make up the mammary fat pad, with epithelial structures embedded throughout [166]. The mammary gland can thus be divided into two compartments; the epithelial and the stromal compartments [114]. Adipocytes make up much of the stroma, which is comprised of other cell types, including fibroblasts present within connective tissue and infiltrating vascular endothelial cells and lymphocytes [94, 114]. The epithelium consists of a primary collecting duct that extends from the nipple, with branching luminal ducts whose tertiary branching elongates into smaller ductules [114]. The ductules end as either proliferative terminal end buds, blunt end buds, alveolar buds, or alveoli, with these alveoli developing into milk secreting lobules during lactation $[114,166]$. These various epithelial structures all possess distinct morphologies and functionality.

The two main cell types of the mammary epithelium are luminal epithelial cells and myoepithelial cells, which together form bi-layered tubular branching ducts [166-168]. The luminal epithelial cells make up a single cuboidal inner cell layer of ductal structures and develop into the milk-secreting cells of lobuloalveolar structures during lactation $[114,166$, 168]. The outer layer is comprised of myoepithelial cells, which secrete a continuous basement membrane that separates the mammary epithelium from the surrounding stroma [114, 117]. Myoepithelial cells contain numerous fine filaments, similar to smooth muscle 
cells, which provide a contractile mechanism in response to oxytocin released during lactation [167]. These two cell types work in conjunction during lactation when the outer myoepithelial layer contracts, which squeezes and transports milk released from the inner luminal epithelial layer $[94,167]$. Luminal epithelial cells are most relevant to cancer as their cell phenotype is the most similar to breast cancer cells and are thought to give rise to the majority of female breast cancers $[114,117]$. Myoepithelial cells are also important in cancer, as their absence within a ductal lesion indicates development into an invasive breast carcinoma $[114,169]$.

\section{Rat mammary epithelium morphological changes throughout tissue development:}

The mammary gland is one of the few organs to undergo most development postnatally, where a large portion of the epithelial ductal structures appear during adolescent rather than fetal development $[167,170,171]$. During fetal growth, the mammary gland develops only as a rudimentary ductal system, comprised mainly of an embryonic mammary stalk that penetrates what is to become the mammary fat pad and with a limited number of branching ducts $[170,171]$. At birth, mammary glands grow isometrically in keeping up with normal body growth [170]. It is not until the onset of puberty when hormone-dependent expansive epithelial proliferation occurs and fills the fat pad [170]. This period of development is defined by enrichment of mammary stem cells, high levels of proliferation, epithelial invasion of the mammary fat pad, and ductal morphogenesis [114, 172-174] which is driven by terminal end buds (TEBs) [117]. TEBs are clusters of undifferentiated epithelial cells at the distal end of primary ductules, which form highly proliferative bulbous structures that penetrate the fat pad and result in ductal 
elongation and branching $[97,104,114,117,170,175]$. TEBs continuously expand with each estrous cycle, resulting in new primary ducts with secondary and tertiary branches sprouting laterally until this ductal tree has expanded to occupy up to $60 \%$ of the mammary fat pad $[114,117,167,170,176]$. Alveolar buds develop at the ends of tertiary branching, capable of developing into the lobuloalveolar structures responsible for milk secretion during lactation $[117,167,170,176]$. Overall, puberty is a critical period of epithelial growth that establishes the ductal tree and primitive lobular structures throughout the mammary fat pad that make up the inactive mammary gland.

The next developmental stage is during pregnancy, when the mammary gland attains maximum development. Pregnancy is comprised of an early phase of growth and a late phase of lobular differentiation [133]. During pregnancy, there is yet a further increase in ductal side branching, which provides connections for the greater number of alveolar buds that develop $[94,114,133]$. These alveolar buds grow and differentiate into functional alveoli, which cluster around terminal ducts to become milk-secreting lobules during lactation $[94,133,171]$. By day 14 of the 21 day gestation period in the rat, there is a distinct increase in both number and size of alveolar lobules [114]. As this intensive ductal side branching and lobuloalveolar formation takes place, there is a continuous increase in the mammary gland epithelium $[114,117,177,178]$. By late pregnancy, interductal adipose tissue reaches a minimum as the alveolar epithelium comprises most of the mammary fat pad $[94,117]$. The expansive proliferation of the alveolar epithelium causes the outer myoepithelial layer to become discontinuous so that the alveolar luminal epithelium comes into direct contact with the basement membrane $[114,168]$. This contact does not occur in the ductal epithelium which retains a continuous myoepithelial layer, 
accounting for the differences in secretory potential of the ducts vs lobuloalveolar structures [114]. As pregnancy approaches term and growth reaches a maximum, lobules can begin to show some secretory activity prior to their function in lactation [94].

The lactating mammary gland has a distinct appearance from the mammary gland during late pregnancy. Though the mammary gland epithelium continues to display a predominantly lobuloalveolar morphology with fewer ducts, there is a large increase in total cell number which reaches a peak in the first few days of lactation $[114,117,171$, 179]. The lobuloalveolar epithelium also swells osmotically due to increased intracellular lipid and lactose content, causing the outer myoepithelial cell layer to develop an even more discontinuous appearance $[114,168]$. During lactation, the luminal epithelium of lobuloalveolar structures function in milk production while luminal epithelium of ducts serves as a conduit for the movement of milk $[114,171]$. The myoepithelial cell layer contracts upon stimulation by oxytocin, causing the secretion of milk into the alveolar lumen and the subsequent emptying into the ductules $[114,117]$. This lactation cycle persists for about 21 days postpartum, with natural involution beginning in an asynchronous manner near the end of this cycle as pups begin eating other foods [94, 114, 180].

Involution is characterized by remodeling of the mammary gland back to a quiescent state, which is attained by apoptotic activity that decreases the size and number of alveolar lobules whose secretory function is no longer needed [117, 127, 170, 181-184]. Loss of suckling through weaning causes milk to stagnate in the mammary epithelium, initiating the process of involution $[94,183,185,186]$. There are two phases of involution that have been described. The first phase is a reversible period of involution, where a 
resumption in suckling can reestablish a lactation function [94]. During this stage, alveolar cells begin to detach from the basement membrane and undergo apoptosis, resulting in an accumulation of cell debris in the alveolar lumen and lobuloalveolar engorgement due to milk stagnation $[94,114,168,186]$. The mammary gland enters the second irreversible phase of involution after 2 days of sustained milk stasis and it is only in this stage that tissue remodeling takes place $[94,113,186]$. Proteinases disrupt epithelial junctions and degrade the epithelial basement membrane and extracellular matrix, causing alveoli to regress in size and the myoepithelial cells to attain a more continuous layer $[94,114,168$, 170, 186]. Continued apoptosis results in massive degeneration of the lobuloalveolar structures that once predominated the mammary gland $[113,133,170,186]$. By six days, most of the secretory lobuloalveolar epithelium is removed and replaced by adipocytes and connective tissue [94]. Ductal structures are regenerated by terminal tubules that continue to branch and reestablish the simple epithelial ductal tree characteristic of the quiescent gland [94, 133].

Figure 2 provides a schematic depicting the gross morphological mammary epithelial changes that occur throughout these key stages of mammary gland development.

\section{Rat mammary epithelial cell proliferation in relation to the estrus cycle:}

The onset of puberty in the female rat begins at approximately 30 days of age with the release of the pituitary luteinizing hormone, which stimulates ovarian maturation during this anestrus period [187, 188]. Estrus cycling begins 8-9 days later [189] and occurs every 4-5 days $[190,191]$. The human menstrual cycle has an estrogen-dominated follicular stage, a progesterone-dominated luteal stage in which mammary epithelial cell 


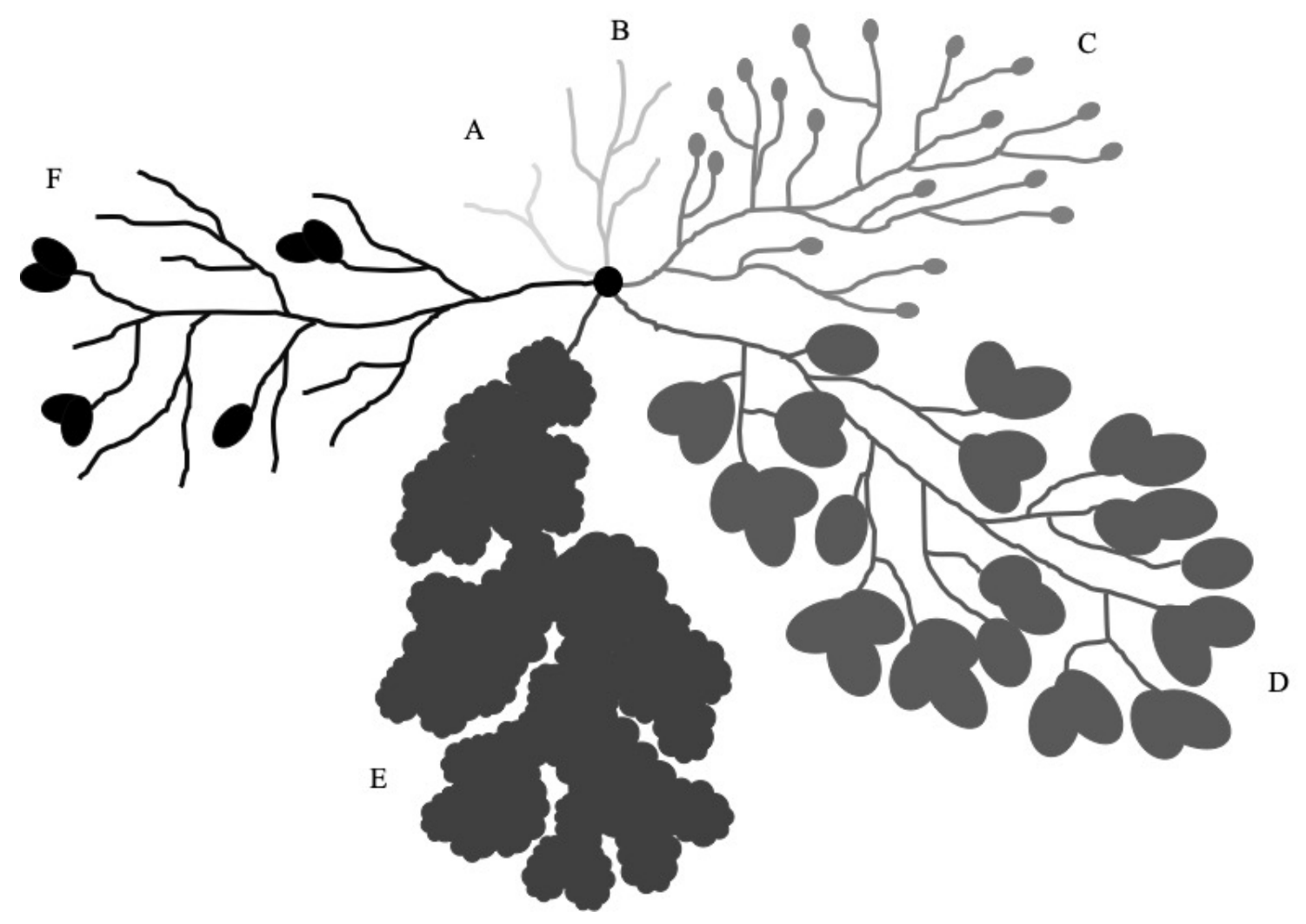

Figure 2: Mammary gland epithelial changes schematic: (A) Embryonic development

- the rudimentary mammary gland begins as a bud containing a limited number of branching ducts. (B) Neonatal development - allometric growth with some extensions to the ductal system. (C) Puberty - epithelial proliferation and invasion of the mammary fat pad establishes the ductal tree. Distal terminal end buds drive ductal elongation and branching. (D) Pregnancy - predominantly lobuloalveolar morphology due to extensive alveolar bud differentiation into milk-secreting lobules. (E) Lactation - the lobuloalveolar epithelium reaches a maximum. The remaining ducts serve as conduits for milk movement. (F) Involution - degeneration of the lobuloalveolar structures and re-establishment of the ductal tree, returning the mammary gland to a quiescent state and the epithelium again predominantly ductal. 
(MEC) proliferation reaches a maximum, and a menstrual stage that is characterized by low ovarian hormone levels and MEC apoptosis, balancing cyclical proliferation [192-196]. Similar events occur in the rat estrus cycle, but it is divided into 4 or 5 stages depending on individual classifications: proestrus, estrus, metestrus, which can be further divided into metestrus I and metestrus II, and diestrus [197, 198]. The proestrus phase is a period of high estrogen levels, follicular development, and endometrial proliferation, comparable to the human follicular phase [199, 200]. The estrus phase encompasses ovulation and is marked by increased levels of progesterone, similar to the luteal phase in human menstruation [199]. Early metestrus mimics the human luteal phase and is a progesterone-dominated phase with maximum development of the endometrium $[201,202]$. During late metestrus, ovarian hormone levels begin to fall and there is a regression of the endometrium, equivalent to entry into menstruation in humans [199]. Diestrus is characterized by minimal hormone levels, similar to the human menstrual phase, with a beginning rise in estrogen levels towards the end initiating a new round of the estrus cycle [200].

Of the few studies that have analyzed MEC proliferation in rat mammary glands based on estrus cycle staging, there have been conflicting reports, with some indicating peak MEC proliferation during proestrus and diestrus [196, 203], and another study reporting high proliferation during metestrus and early diestrus [199]. Thus, the impact of the estrus cycle on mammary gland biology is still not well characterized. This leaves a necessity to understand the factors involved in normal cyclical growth and regression in the mammary gland as they can be potentially dysregulated during carcinogenesis. 


\section{Chemical induction of rat mammary carcinogenesis:}

Mammary tumors develop in rats both spontaneously and by oncogene- and chemical-induction. Spontaneous mammary tumors occur frequently in some rat strains and are the most common tumor type in rats [204-206]. Benign mammary tumors appear more often than malignant tumors, and arise mostly from fibrous and connective mammary tissue [206]. Of malignant tumors, fibrosarcomas originating from proliferating stromal fibroblasts are the most common, with one report finding an incidence of $59 \%$ of all spontaneous malignant tumors [206]. Mammary tumors are also chemically induced in a reproducibly high percentage of rats, which allowed early researchers to investigate types of carcinogen, routes of administration, and age of recipient rats on the incidence of mammary tumors. Unlike spontaneous tumors, induced tumors are generally carcinomas [206]. Induced rat carcinomas are highly hormone responsive, have short latency, and rarely metastasize; and, since their discovery. have been utilized to simulate human breast carcinogenesis [207-209].

The chemical induction of cancer in rodents was first observed by Maisen and Coolen who applied the carcinogen 3-methylcholanthrene (3-MC) onto the skin of mice [210]. Later it was found that the feeding of polycyclic hydrocarbons induced mammary cancer preferentially in rats $[211,212]$. The first compound used in this way was 2acetylaminofluorene (2-AAF), which was incorporated into the food of rats over several months [213]. Building on these studies, it was discovered that up to three IV injections of polycyclic aromatic hydrocarbons such as 7,12-dimethylbenz(a)anthracene (DMBA) resulted in a high incidence of female mammary cancer in $80 \%$ of the Wistar and SpragueDawley strains of rats $[214,215]$. However, Huggins et. al was the first to show a single 
oral dose of DMBA administered to 50-65 day old female Sprague-Dawley rats resulted in $100 \%$ mammary carcinoma incidence in just 2 months, and was much more effective than similar administration of 3-MC and 2-AAF that required several doses [212]. A positive correlation was found between dose of carcinogen and incidence of mammary tumors. An optimal oral DMBA dose of $20 \mathrm{mg} / \mathrm{kg}$ body mass in sesame oil was published and is now a standard procedure $[212,216]$. The DMBA-induced rat mammary tumor model became known as the "Huggins tumor" and is the most widely utilized animal model of human breast cancer [217]. This discovery of a single dose of carcinogen was crucial as it allowed for the study of cancer initiation, carcinogenesis, and tumor biology, while providing the most adequate experimental model to study breast cancer development [217].

\section{DMBA model of rat mammary carcinogenesis:}

The DMBA-induced rat mammary tumor model is the most efficient method to study human breast cancer as tumors develop quickly and in the form of invasive ductal carcinomas, which account for $80 \%$ of diagnosed breast cancers worldwide [218]. DMBAinduced mammary tumors can be reproducibly induced at a high frequency and develop on average 5 weeks following administration [211,219]. These mammary tumors are multifocal and occur at different sizes within the same animal, eventually resulting in skin ulceration, necrosis, infection, and death [219]. DMBA-induced mammary cancer displays similar pathology to human breast cancer, including a common site of disease initiation and morphology throughout tumor progression [219-221]. The carcinogenic activity of DMBA is induced by metabolic activation [222]. The liver is the major site of metabolic activation for many chemical carcinogens, but the highly lipophilic nature of polycyclic 
aromatic hydrocarbons, including DMBA, results in accumulation in fatty tissue [222, 223]. DMBA concentrates in the significant amount of adipose tissue that comprises the mammary gland and local metabolic activation occurs [223].

Age is a critical factor in the DMBA mammary carcinogenesis model, as the incidence of tumors reaches a peak when administered to female rats at 50-55 days old, before rapidly declining $[175,224-226]$. Tumors will rarely be induced in rats older than 100 days [223]. This 50-55 day timepoint coincides with peak DNA synthesis, as shown through $3 \mathrm{H}$-thymidine incorporation in mammary gland DNA that reaches a maximum at 50 days of age $[225,226]$. The terminal ductal structures in mammary glands are believed to be the site of action by DMBA as they are highly proliferative and display an elevated rate of DNA synthesis at 50-55 days of age [217, 227]. DMBA is metabolically activated by CYP1A1 and CYP1B1 isoforms from the P450 family of metabolic enzymes, which creates unstable diol epoxides [228, 229]. These epoxides bind and form adducts onto the DNA of proliferative mammary structures [222, 230]. Notably, DMBA itself is capable of inducing expression of CYP1A1 and CYP1B1 in the mammary gland, which can further increase metabolic activation [222]. DNA adducts of DMBA metabolites hinder DNA repair enzymes and cause mutations, which contributes to malignant transformation of epithelial cells $[222,223,231]$. The intrinsic affinity of DMBA to collect in mammary tissue and the ability of mammary tissue to locally activate DMBA creates a clean, mammary-specific model of carcinogenesis. 


\section{Mammary Carcinoma Susceptibility Quantitative Trait Loci}

\section{Susceptibility alleles:}

Rat strains vary in their susceptibility to carcinogen-induced development of mammary cancer [232]. The inbred Copenhagen (COP) strain displays nearly complete resistance to mammary carcinogenesis, developing an average of 0.25 carcinomas per rat with a single dose of DMBA $[159,164,232,233]$. In contrast, the highly susceptible Wistar Furth (WF) strain displays $>90 \%$ tumor incidence after a single dose of DMBA $[159,160$, 164, 232, 233]. These divergent mammary cancer susceptibility phenotypes between strains allow for the ability to isolate genetic loci that contribute to mammary cancer susceptibility [159]. Inbred rat strains that differ in their susceptibility to DMBA-, Nmethyl-N-nitrosourea (NMU)-, or estrogen-induced mammary carcinogenesis have been used predict locations of quantitative trait loci (QTL) that control susceptibility to mammary carcinogenesis, termed the Mammary carcinoma susceptibility (Mcs) and Estrogen-induced mammary cancer (Emca) loci [164, 234-239]. Quantitative trait linkage analysis studies were conducted in the 1980s using segregating backcrosses between WF and either resistant rat strain COP or Wistar Kyoto. These studies identified eight QTLs that modified susceptibility to DMBA-induced mammary cancer, termed mammary carcinoma susceptibility loci 1-8 (Mcs1-Mcs8) [77, 158, 161, 164, 234, 240]. Mcs1-4 are present in the COP strain genome while Mcs5-8 stem from the Wistar Kyoto (WKy) genome $[164,241]$.

Congenic rat lines carrying a specific Mcs on a WF genetic background have been used to validate these QTLs [76, 161]. Congenic lines are generated by first mating two inbred rat strains to create an N1 heterozygote generation. In subsequent matings, one strain 
is considered the donor (for Mcs analysis this is COP or WKy) and the other is the recipient (WF). The N1 generation is backcrossed to the recipient strain for up to ten generations. Within each generation, animals are genotyped at the predicted region and selected for based on possession of the desired donor allele. This results in introgression of the donor allele at the region of interest into a recipient genetic background. The phenotypes of congenic lines are then compared to inbred parental strains to determine if the isolated region of interest modulates the overall phenotype. This breeding scheme is diagrammed in Figure 3. Of the COP loci, it was found that Mcs4 contributes to increased susceptibility while Mcsl-3 decrease susceptibility [164]. Even though resistance to mammary carcinomas is a multilocus trait in the COP genome, when rats are homozygous for Mcs I3 , this is enough the suppress tumor development regardless of Mcs4, indicating these three QTLs account for the majority of the phenotypic differences between WF and COP [164].

To limit the set of candidate genes that confer disease susceptibility, the QTL must be fine mapped to a higher resolution using genetic markers, such a single nucleotide polymorphisms (SNPs) and microsatellites, spaced at regular intervals across the QTL [161, 240]. Congenic lines harboring the Mcs chromosomal region are repeatedly backcrossed to the WF susceptible genetic background to produce lines that carry delimited QTL intervals due to homologous recombination [240]. These new lines are phenotyped for retained mammary cancer susceptibility or resistance following DMBA administration $[161,240]$. These fine mapping studies in congenic lines allow the smaller selection of potential candidate genes that can be extended to orthologous human studies of breast cancer risk $[158,161]$. The work presented in this dissertation will involve two different mammary cancer susceptibility loci, Mcs3 and Mcs $1 b$. 


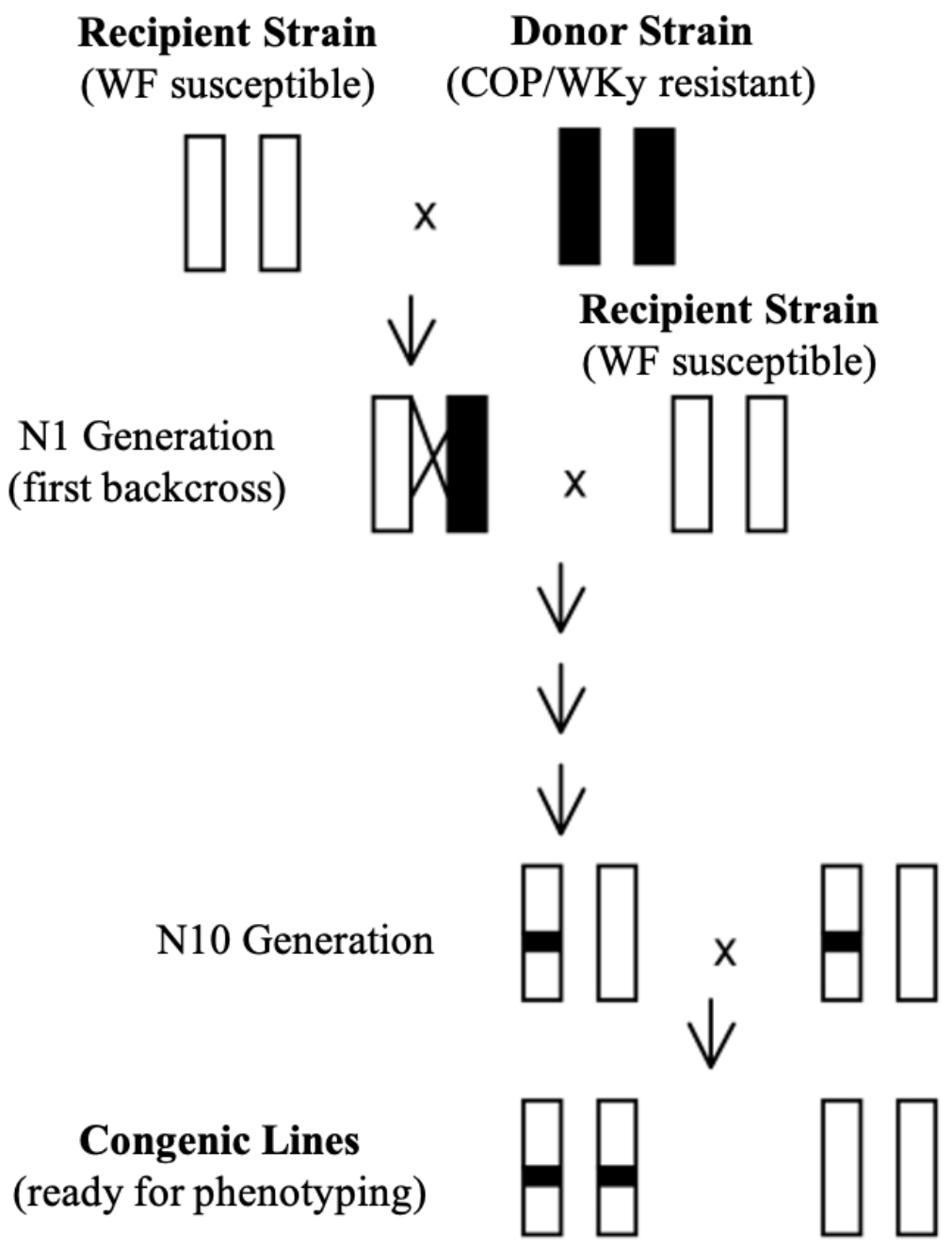

Figure 3. Generation of congenic animals. Adapted from Kim et al. (2010) [242]. Mating of two inbred rat strains generates a heterozygous N1 generation. These animals are backcrossed to the recipient strain and this cycle of backcrossing repeated for 10 generations. At each generation animals are genotyped to ensure the desired donor allele is present in the region of interest. Heterozygotes are crossed to yield offspring homozygous for the donor region of interest. This method introgresses the donor allele (black bar) into the recipient genome (white bars). 


\section{Mcs3:}

From linkage analysis using the susceptible WF and the resistant COP strain postDMBA treatment, $M c s 3$ was initially predicted to reside within a $30 \mathrm{cM}$ region of rat chromosome one that acted as a dominant resistance loci [164]. The COP resistant Mcs3 was physically confirmed and delimited to a $29.4 \mathrm{Mb}$ region on rat chromosome one in 2017 and results in around a 50\% reduction in mammary cancer susceptibility in homozygous rats [243]. This rat region aligns to four syntenic regions of human chromosomes 11 and 15 and four other regions of the human genome that contain a single gene, with 198 genes in common or orthologous between these species [243]. Most notable in the context of breast cancer, rat $M c s 3$ contains a sequence orthologous to human $11 \mathrm{q} 13 / 14$, which is amplified in a subset of female breast carcinomas with poor prognosis [243, 244]. Potential breast cancer risk associated variants at Mcs 3 orthologous loci 2p16.3, 11p15.4, 11q14.1, 11q14.2, and 15q25.2 have also been identified by population-based genetic association studies [243, 245-247]. The Mcs3 locus is currently too large for functional studies so fine mapping is needed to reduce the pool of candidate genes. Work with $M c s 3$ in this dissertation focuses on evaluation of expression of known breast cancer genes in this locus and further fine mapping to increase the resolution at this QTL.

\section{Mcs1b:}

Another independently acting Mcs QTL, Mcs 1, was first identified in 1998 as a susceptibility locus that modulates tumor multiplicity phenotype [164]. Genetic linkage analysis of (WF.COP)F $\mathrm{F}_{1} \mathrm{x}$ WF backcrosses with mammary carcinogenesis induction by DMBA predicted $M c s 1$ localized to a $30 \mathrm{cM}$ region at the centromeric end of rat 
chromosome 2 [164]. Gould and his group created an Mcs 1 congenic line by transferring the Mcs 1 COP allele onto a WF genetic background [159]. In delineating the large Mcs 1 locus, recombinant lines were created by backcrossing the Mcsl congenic line to the WF strain. These new congenic lines were phenotyped for their sensitivity to DMBA-induced mammary cancer. COP-homozygous rats from three nonoverlapping lines showed resistance to DMBA-induced mammary tumorigenesis. From these lines, Gould's group identified three Mcs 1 subloci independently capable of conferring mammary cancer resistance, termed Mcs Ia, Mcs 1b, and Mcs 1c. This positional mapping of Mcs 1 determined that this locus contains more than one gene that contributes to mammary cancer susceptibility.

$M c s 1 b$, was mapped to a region of $13 \mathrm{cM}$ by Gould's group, which resulted in a $58 \%$ reduction in DMBA-induced mammary carcinomas per rat as compared to the WF strain [159]. In 2012, Mcs $1 b$ was further fine mapped and delimited to a $1.8 \mathrm{Mb}$ region corresponding to rat Chr2:42364155-44195382 (Rnor_6.0) [248]. Females homozygous for this delimited COP allele displayed 56\% fewer mammary carcinomas compared to susceptible WF females [248]. The current Mcs $1 b$ is highly relevant to human breast cancer as it contains a genomic sequence orthologous to human 5q11.2, a breast cancer susceptibility locus identified in multiple GWA studies [245, 248-252]. Genetic variation at $5 \mathrm{q} 11.2$ susceptibility allele is associated with breast cancer invasive ductal carcinoma, invasive lobular breast carcinoma, ER+, ER-, and triple negative and breast cancer subtypes [253-255].

Mapping of Mcslb to a much shorter genomic interval allows for selection of candidate breast cancer susceptibility genes and functional studies of this QTL. Within the 
overlap of rat Mcslb and orthologous human 5q11.2 are two genes, mitogen-activated protein kinase kinase kinase 1 (MAP3K1/Map3k1) and MIER family member 3 (MIER3/Mier3). Present in Mcs $1 b$ but not 5q11.2 is GC-rich promoter binding protein 1 (Gpbp1) (Figure 4). Rat Mcs $1 b$ was found not to contain any protein-coding differences between COP and WF alleles, suggesting that differences in mammary cancer susceptibility may be due to variation in one or more nonprotein-coding regulatory elements that differentially control gene expression between Mcslb genotypes [248]. Mammary gland and mammary carcinoma transcript levels of $M c s 1 b$ genes were measured in both DMBA-exposed and age matched control WF and Mcs $1 b$ females. The effect of mammary cancer susceptible and $M c s 1 b$ resistant genotypes were statistically significant for Gpbp1, Mier3, Map3k1. However, only Mier3 showed significant expression differences when comparing Mcslb genotypes of females exposed to DMBA [248]. Additionally, there were no significant Mier3 transcript differences between Mcs $1 b$ resistant and susceptible genotypes in spleen, thymus, ovary, or brain tissues, indicating Mier3 differences between $M c s 1 b$ alleles may be specific to mammary gland tissue [248]. Work with $M c s 1 b$ in this dissertation focuses on further expression analysis of Mier3 within rat mammary glands through mammary cancer progression and time windows of susceptibility. Additionally, I investigate the molecular function of MIER3 in human breast cancer through identification of protein-protein interactions.

\section{The MIER family:}

The MIER gene family consists of three related genes that were named based on shared homology between their ELM2 and SANT domains. The prototypical member, 


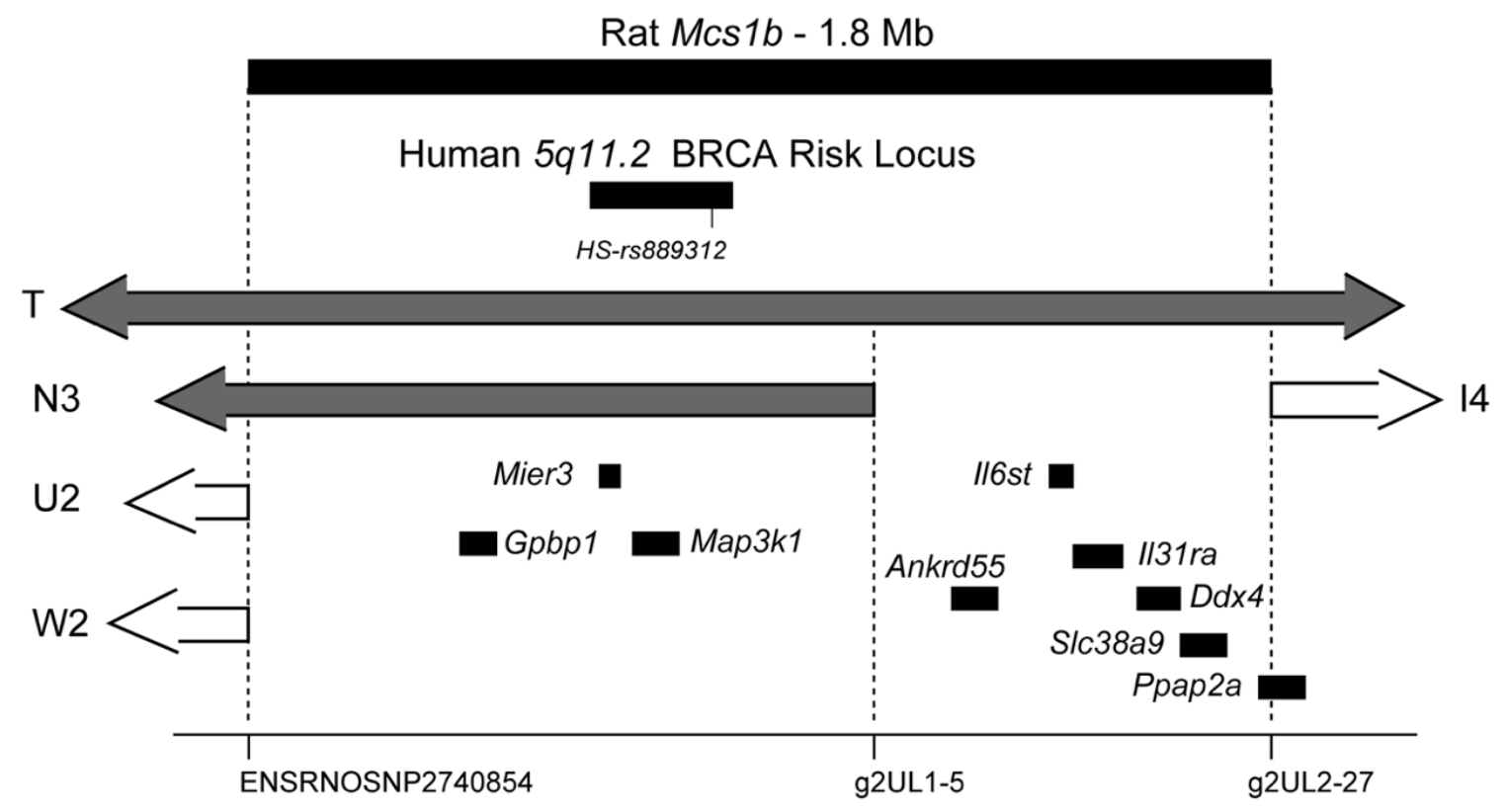

Figure 4. Rat Mcs1b and orthologous 5q11.2 breast cancer risk allele share two human/rat conserved transcripts. Adapted from denDekker et al. (2012) [256]. Genetic markers used to map $M c s 1 b$ to this region are marked on the X-axis. The ends of congenic lines used to delimit $M c s 1 b$ are included for orientation (white is susceptible, gray is resistant). Potential candidate breast cancer susceptibility genes Map3k1 and Mier3 overlapping Mcs $1 b$ and 5q11.2 are shown as filled black bars that represent exonic and intronic DNA. 
MIER1, was first discovered as a fibroblast growth factor early response gene [257]. Hence, the original nomenclature Mesoderm Induction Early Response (MIER) genes. Human MIER1 is a single copy gene on chromosome 1 that consists of 17 exons and gives rise to four major protein isoforms due to alternative splicing and promoter usage but retain a common internal region with functional domains $[258,259]$. There are two C-termini variants named MIER $1 \alpha$ and $\beta$, with $\beta$ arising from the inclusion of a facultative intron [260]. The $\alpha$ C-terminus contains 23 amino acids and includes an LXXLL motif, canonically responsible for interaction with nuclear hormone receptors [259, 261, 262]. The $\beta$ C-terminus contains 102 amino acids and possesses a functional nuclear localization signal $[259,263]$. To date, much of the research into MIER1 has focused on MIER1 $\alpha$ and $\beta$, both of which have been shown to function as transcriptional repressors [261]. There are also two major MIER1 isoforms that differ at their $\mathrm{N}$-termini as a consequence of alternate promoter usage, named P1 and P2 [259]. MIER1 is transcribed from P2, and MIER-3A is transcribed from P1 and includes an additional exon (3A) [261, 262]. This encodes 25 amino acids that contain a nuclear export signal $[259,261,263]$. MIER2 is a single copy gene on chromosome 19 consisting of 14 exons that is transcribed into a single transcript. It also possesses internal ELM2 and SANT domains, as well as a predicted nuclear localization signal. MIER 3 is a single copy gene on chromosome 5 consisting of 13 exons and giving rise to 5 isoforms due to alternative splicing. Isoform 1 is the canonical sequence and isoform 3 only differs by a single amino acid deletion in the ELM2 domain. There is a 5 amino acid insert near the N-terminus of isoform 2, and isoform 4 is missing the first 63 amino acids. These isoforms possess the ELM2 and SANT domains common to this gene family, as well as a predicted nuclear localization signal. MIER3 also has two LXXLL 
sequence motifs, one upstream of the ELM2-SANT domains and another within the ELM2 domain (Figure 5). Isoform 5 is a truncated variant of only 119 amino acids at the Nterminus and lacks the ELM2 and SANT domains [264]. Human MIER1 and MIER3 are similar, with BLAST analysis showing 54\% identical amino acid sequence [265]. However, MIER3 differs in its possession of two LXXLL sequence motifs as compared to one within MIER1. Co-immunoprecipitation analysis has also demonstrated that the MIER family members do not dimerize and act autonomously from each other [266]. Thus, although similar in sequence and protein domains, the MIER family may possess differing functional roles.

Within the MIER family, a functional role of MIER1 in transcriptional regulation has been well characterized, though there is little published research functionally annotating MIER2 and MIER3 [266]. Co-immunoprecipitation and mass spectrometryproteomic approaches have identified the ELM2 domain of MIER1 $\alpha$ and MIER1 $\beta$ as necessary to recruit histone deacetylase 1 and 2 (HDAC1/2) and repress transcription through histone remodifying complexes [263, 266-269]. In addition to HDAC recruitment, MIER1 has other mechanisms of transcriptional repression, including physical association with the histone acetyltransferase CREB-binding protein (CBP) and inhibition of its activity [270]. The SANT domain of MIER1 can also disrupt promoter binding by the transcription factor Sp1 and alter downstream transcription of target genes [263, 266].

Proteomic studies of Bromo-Adjacent-Homology domain-containing 1 (BAHD1) have reproducibly co-purified this protein with each of the MIER proteins and HDAC1/2 [271]. BAHD1 acts as a transcriptional co-repressor by interaction with other proteins to 


\section{MIER3 (NP_001284527.1)}

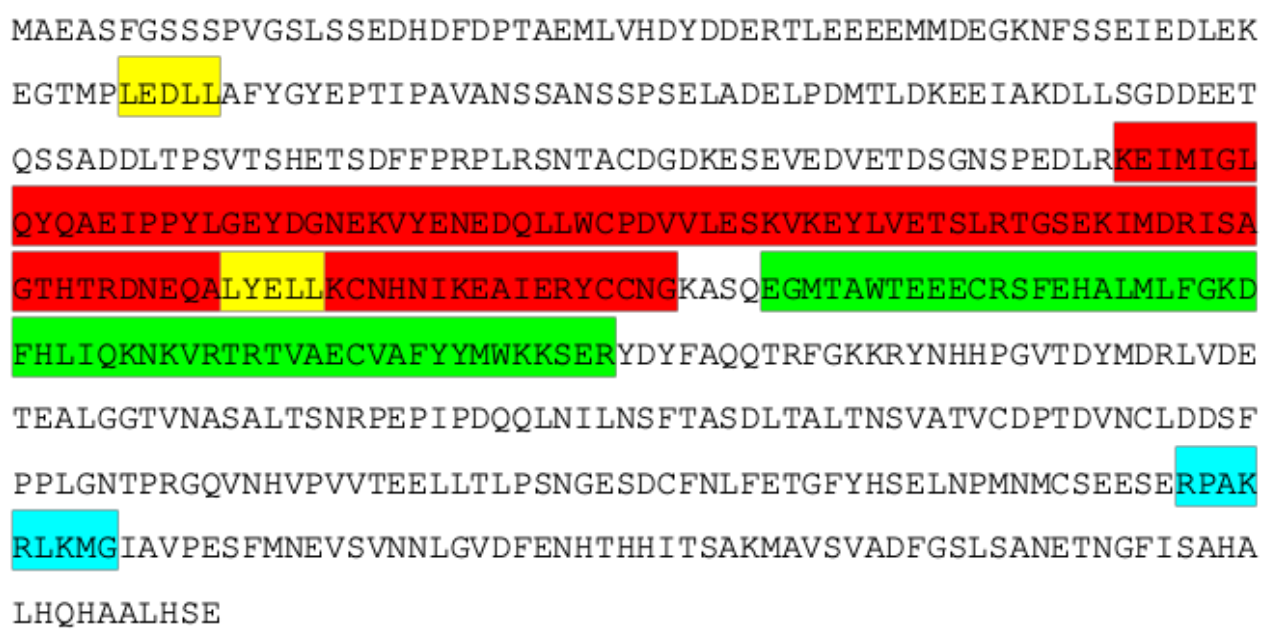

\section{LXXLL Motifs}

ELM2 Domain

SANT Domain

Nuclear localization signal

Figure 5: Protein domains of MIER family member 3 (MIER3). MIER3 possesses a juxtaposed ELM2 and SANT domain. One LXXLL sequence motif is near the N-terminus and the other is within the C-terminal region of the ELM2 domain. There is also a nuclear localization signal near the C-terminus of the protein. 
promote chromatin condensation [271]. As such, BAHD1 is reported to consistently interact with the chromatin writer G9a, readers HP1 $\alpha / \beta / \gamma$, CDYL1/2, MBD1, and remodelers CHD3, RUVBL1/2 [271]. These data provide evidence for the first time of the other MIER proteins as subunits of a multiprotein complex that may have a role in transcriptional repression.

\section{MIER1 function in breast cancer:}

An immunohistochemical study of the MIER1 $\alpha$ protein expression pattern in human tissues found expression in all endocrine and reproductive tissues except the thyroid gland, with the majority of non-endocrine tissues negative for MIER1 $\alpha$ [259]. The most intense staining was found in the ovaries, testis, breast, pancreas, and adrenal gland and pituitary. The subcellular localization of MIER1 $\alpha$ was cytoplasmic across all tissues examined except oocytes, germinal epithelial cells, and ductal epithelial cells of the breast which were exclusively nuclear [259]. This is concordant with findings in mice, in which there was no immunoreactivity of MIER $1 \alpha$ in most tissues, except for high levels in endocrine tissue, and localization was largely cytoplasmic [272]. These collectively suggest an endocrine role of MIER $1 \alpha$. In the breast specifically, another study found very low MIERI mRNA levels in non-diseased breast cell lines and tissue, but significantly higher expression in breast cancer cell lines and tumor tissue, highlighting an association with human breast cancer [273]. It has been reported that there is no difference in MIER1 $\alpha$

protein levels between non-diseased breast tissue and breast carcinoma tissue, but there is a drastic shift from nuclear to cytoplasmic subcellular localization with progression to invasive ductal carcinoma [274]. Nuclear MIER1 $\alpha$ was detectable in 75\% of normal tissue, 
$51 \%$ of DCIS and only $4 \%$ of IDC [274]. It has been demonstrated that interaction of HDAC1 and 2 are necessary for the nuclear translocation of MIER1 $\alpha$, and that insulin or insulin-like growth factor induces cytoplasmic localization in breast cancer cells [262, 275]. Additionally, inclusion of exon $3 \mathrm{~A}$ in the MIER1-3A $\alpha$ isoform results in cytoplasmic translocation due to inclusion of a nuclear export signal but has no effect on MIER1-3A $\beta$ localization [261]. MIER1 $\alpha$ can also physically bind estrogen receptor alpha (ER $\alpha)$ with and without the presence of estrogen, and it has been shown that overexpression of MIER1 $\alpha$ inhibits estrogen-stimulated growth of breast cancer cells [274]. These results indicate that a combination of alternative splicing and interacting partners effects the subcellular location of MIER1 $\alpha$ in the context of breast cancer, and the potential function of MIER1 $\alpha$ as an ER $\alpha$ corepressor could either indicate an additional non-genomic role of MIER1 or enhance ER $\alpha$ non-genomic activities in breast cancer development.

\section{MIER3 function in breast cancer:}

Human MIER3 localizes to the nucleus of breast cancer cells and MIER3 levels are elevated in invasive ductal and invasive lobular carcinoma samples compared to normal breast tissue [256]. However, much of what is known about MIER3 comes from studies of the rat ortholog. Human and rat MIER3/Mier3 (GenBank ref NP_689835.3 and NP_001161472.1) gene products share 93\% amino acid sequence identity. While 5 isoforms have been isolated in the human, there are 3 reported Mier3 isoforms in the rat (NCBI). One isoform differs from the full-length transcript via intra-exonal splicing of a $363 \mathrm{bp}$ segment in exon 1, resulting in an alternate translational start site at position 1013 and a 23 amino acid truncation of the $\mathrm{N}$-terminus. The other isoform utilizes an alternative 
transcription start site upstream of the normal start site, which results in usage of three short exons in place of a skipped exon 1 and slightly alters the N-terminus. These result in fulllength, spliced, and alternative 5'-transcript start site variants of Mier3. Rat Mier3 is contained within the mammary cancer susceptibility locus $M c s 1 b$. Using rat lines that are resistant or susceptible to mammary cancer based on inclusion of this locus, lower levels of the alternative start site variant (Mier3-alt5' $P$ ) has been found in the resistant strain. However, no differences were identified between the full-length and splice variant forms (Samuelson Lab, unpublished). This indicates that lower levels of Mier3 are genetically determined and transcript variant-specific, and that while loss of MIER1 may contribute to breast cancer progression, a reduction in Mier 3 expression appears to be protective against mammary carcinoma development. Additionally, no differences in Mier3 expression were found between rat strains in spleen, thymus, or ovary tissue (Samuelson Lab, unpublished), further indicating Mier3 expression differences and downstream effects on cancer development occurs in a mammary gland autonomous manner.

\section{$\underline{\text { Dissertation Overview }}$}

\section{Overall goal:}

This dissertation focuses on using WF.COP Mcs 3 congenic lines to fine map this locus with the goal to narrow the genetic interval and reduce the pool of candidate susceptibility genes for comparative analysis in human breast cancer risk. In addition, analyzing Mier3 expression and protein function in this dissertation begins to identify the role of MIER3 in disease progression and developmental timepoints of altered 
susceptibility. The goal here is to identify a MIER3-driven mechanism of breast cancer susceptibility for future preventative or screening strategies.

\section{Hypothesis and Aims:}

The hypothesis is that (1) the Mcs3 locus contains genetic elements that control mammary cancer susceptibility, and (2) MIER3/Mier3 has a functional role in human breast cancer/rat mammary cancer development.

Aim 1: Physically narrow mammary carcinoma susceptibility 3 (Mcs3) region on RNO1 using WF.COP congenic rat lines.

Aim 2: Associate Mier3 expression with mammary gland cell type, mammary cancer progression, and time windows of susceptibility

Aim 3: Identify protein interactions of MIER3 (specifically determine if MIER3 interacts with nuclear hormone receptors) 


\section{CHAPTER II}

CHEMICAL CARCINOGEN INDUCED RAT MAMMARY CARCINOGENESIS IS A POTENTIAL MODEL OF P21-ACTIVATED KINASE POSITIVE FEMALE BREAST

\section{CANCER}

\section{Introduction:}

Amplification of human chromosome 11q13/14 in breast cancer is associated with poor prognosis, with patient tumors displaying chemoresistance and a 10 year survival rate of only $50 \%[244,277-280]$. Amplification of $11 q 13 / 14$ contains several known and putative driver genes, but it remains unclear if a cancer phenotype is driven by a cassette of genes, or a single oncogene $[279,281]$. A recent study which profiled nearly 2500 primary breast tumors with copy number aberrations determined $11 q 13 / 14$ contains two gene cassettes, one centered around cyclin D1 (CCND1, 11q13.3), and the other around p21 activated kinase (PAK1, 11q14.1) [278].

Human breast epithelial cells normally have low PAKl expression; however, amplification of PAK1 is found in 33\% of breast tumors and cancer cell lines [282-285]. $P A K 1$ gene expression is associated with aggressive breast cancer type, histologic grade and proliferation level $[283,285,286]$. A meta-analysis of 15 independent studies that

Adapted from Duderstadt et al. (2021) [276]. See Appendix 
investigated association of PAK1 with disease prognosis, found high PAK1 expression in tumors is negatively associated with overall survival and disease-specific survival [287]. Expression of PAK1 is also relevant to breast cancer survival, as its expression inhibits antiestrogenic action of tamoxifen in tamoxifen-sensitive breast cancer cell lines. Furthermore, $P A K 1$ expression is linked to tamoxifen resistance and disease recurrence in primary breast carcinoma samples $[283,288]$.

The $P A K 1$ gene product is a serine/threonine kinase involved in regulating cell morphology and motility through mediation of actin organization [289]. In vitro expression of PAK1 is implicated in a broad array of signaling processes that correspond to breast cancer-related phenotypes, including cell migration, invasion, and anchorage-independent growth [290, 291]. Regulation of anchorage-independent growth signaling and development of invasive breast carcinomas is mediated by proteins that directly interact with PAK1, including cyclin-D1 (CCND1), mitogen-activated protein kinase-1 (MAPK1), and RAS-homolog-family-member-A (RHOA) [282, 292]. Overexpression of PAK1 has been shown to lead to focal adhesion complex disassembly, formation of lamellipodia and filopodia, as well as colocalization with actin filaments at the leading periphery of cells $[289,291,293]$.

The Mammary carcinoma susceptibility (Mcs3) QTL, a 27.8 Mb segment on rat chromosome 1 that controls susceptibility to developing mammary carcinomas, contains sequence orthologous to human 11q13/14 [243]. These orthologous sequences contain rat/human Pakl/PAK1 orthologs. Integrin-linked kinase (ILK), another Mcs3-nominated candidate gene, [243] is on a different human chromosome than PAK1; however, a physical interaction between ILK and PAK1 has been documented [294]. Similar to PAKl, 
expression of $I L K$ is associated with breast cancer and five-year survival [295]. PAKl and $I L K$ expression are both associated with female breast cancer [282, 290, 295], and rat orthologs of these genes are located at Mcs3. We sought to determine if expression of either rat Pakl or Ilk is associated with rat Mcs3 genotype and DMBA-induced rat mammary carcinogenesis, using our integrated model of breast cancer susceptibility and development $[163,296]$.

\section{Methods:}

Congenic Strains and Animal Breeding

Wistar Furth.Copenhagen (WF.COP) congenic rat strains D and E used in this study are described in [243]. Briefly, strain D contains a segment of Copenhagen (COP/NHsd) RNO1 from markers D1Rat277 (Chr1:134,120,332-134,120,480) to D1Rat321 (Chr1:171,716,519-171,716,714). Strain D is positive for the Mcs3 COP allele associated with decreased mammary cancer susceptibility. Strain E contains a segment of Copenhagen (COP/NHsd) RNO1 from markers D1Rat214 (Chr1:105,499,947-105,500,046) to D1Rat350 (Chr1:141,580,935-141,581,089). Strain E is negative for an independently acting Mcs 3 decreased susceptibility allele. Rat chromosome physical positions are from UCSC rat genome assembly Rnor_6.0 [297, 298]. Animals were housed in an Association for the Assessment and Accreditation of Laboratory Animal Care approved facility on a 12-hr light/dark cycle. LabDiet 5001 Rodent Diet (PMI Nutrition International) and acidified water was provided ad libitum. All protocols involving animals were approved by the University of Louisville Institutional Animal Care and Use Committee. 


\section{DMBA Administration and Tissue Collection}

At 50-55 days of age, 7,12-Dimethylbenz(a)anthracene (DMBA Acros Organics, CAS\#: 57-97-6, $20 \mathrm{mg} / \mathrm{mL}$ in sesame oil) was given by single oral gavage (65 $\mathrm{mg}$ DMBA/kg body mass) to 10 WF.COP-RN01 strain D and 11 strain E females to induce mammary carcinogenesis. Age matched females (9 strain D and 12 strain E) not exposed to DMBA were used as untreated controls. Four weeks following DMBA administration, the DMBA-exposed and age-matched non-exposed rats were euthanized by $\mathrm{CO}_{2}$ asphyxiation. Abdominal-inguinal mammary glands were flash frozen and stored at $-80^{\circ}$ prior to RNA extraction. Right thoracic mammary glands were formalin fixed and paraffin embedded (FFPE) for use in immunohistochemistry (IHC).

\section{Quantitative PCR}

Mammary tissue was homogenized in TRI-reagent (Molecular Research Center TR118) for total RNA extraction. Three molar sodium acetate $(1 / 10 \mathrm{v} / \mathrm{v})$ followed by a $100 \%$ ethanol $(2.5 \mathrm{x} \mathrm{v} / \mathrm{v})$ wash was used to remove potential solvent contaminants. Samples were then treated with Turbo DNAse (Life Technologies). Reverse transcription reactions, containing $1 \mu \mathrm{g}$ RNA, $0.5 \mathrm{x}$ RNAsecure, $5 \mu \mathrm{M}$ random hexamers (ThermoFisher N8080127), 25ng/ $\mu \mathrm{L}$ oligo dT18 (ThermoFisher 18418012), and $0.5 \mathrm{mM}$ dNTPs (ThermoFisher 10297018), were incubated for 5 minutes at $65^{\circ} \mathrm{C}$. First strand buffer (1x), 100mM DTT, and $1 \mu \mathrm{L}$ Superscript IV reverse transcriptase (ThermoFisher 18090010) were added and incubated $5 \mathrm{~m}$ at $25^{\circ} \mathrm{C}, 1 \mathrm{~h}$ at $50^{\circ} \mathrm{C}$, and $15 \mathrm{~m}$ at $70^{\circ} \mathrm{C}$. Quantitative PCR reactions containing 1x TaqMan buffer $\mathrm{A}$ (ThermoFisher), $3.5 \mathrm{mM} \mathrm{MgCl} 2,0.2 \mathrm{mM}$ dNTP, $500 \mathrm{nM}$ each forward and reverse primer, $200 \mathrm{nM}$ target TaqMan gene-expression assays 
(ThermoFisher) for Pakl (Rn00664986_m1), Ilk (Rn00591471_m1), or Rplp2 (Rn01479927_g1), 0.025 U/ $\mu 1$ Taq Gold DNA pol (ThermoFisher 18080240), and $50 \mathrm{ng}$ cDNA were run at $50^{\circ} \mathrm{C}$ for $2 \mathrm{~min}$ and $95^{\circ} \mathrm{C}$ for $10 \mathrm{~min}$, followed by 40 cycles of $95^{\circ} \mathrm{C}$ for $15 \mathrm{~s}$ and $60^{\circ} \mathrm{C}$ for $1 \mathrm{~min}$. TaqMan gene-expression assay probe and primer sequences were: Pakl FAM-ACTATCTGGACAGTTACCTTG with forward 5'TGGTCATGAGGGAAAACAAAAA and 5'TCCATGACAACCCATAGCTCATC; Ilk FAM-CGGCTCAGGATTTT with forward 5'GCAGGGACTTCAATGAGGAATG and reverse CGGAAGCACGTTAGGATGAGA; and Rplp2 VIC-CTGAATGGAAAGAATATTGAG with forward 5'-TGAACGACTCAACAAGGTCATCA and reverse 5'CAACACCCTGAGCGATGACA. The manufacturer indicates that amplification efficiency is $100 \% \pm 10 \%$ for TaqMan gene-expression assays [299]. Amplification efficiencies for TaqMan assays ranged from 95 to $99 \%$ in our experiments. Quantitative PCR was conducted using an ABI Prism 7900HT Sequence Detection System with SDS v2.3 software (Applied Biosystems).

\section{Immunohistochemistry}

Tissue was cut in $4 \mu \mathrm{m}$ sections from FFPE blocks onto superfrost plus microscope slides (VWR). Slides were deparafinized in xylene and ethanol, boiled in sodium citrate buffer (10mM sodium citrate, $0.05 \%$ tween, $\mathrm{pH} 6.0$ ) for 20 minutes, washed in $1 \mathrm{X}$ TBS with $0.025 \%$ Triton X-100 for 10 minutes, then blocked in $10 \%$ normal goat serum with $1 \%$ BSA in TBS for 2 hours. Primary antibodies were diluted 1:100 in TBS with 1\% BSA and kept with samples overnight at $4^{\circ} \mathrm{C}$ (Anti-PAK1 Abcam131522, rabbit IgG Abcam 
37415). Slides were visualized using an HRP/DAB detection IHC kit (Abcam ab64261), counterstained with hematoxylin, destained using xylene and ethanol, and coverslipped with ProLong Gold antifade mountant (Thermofisher P36930). Slides were imaged at 40x magnification on an Aperio Imagescope 12.4 Software (Leica Biosystems).

De-identified human breast disease clinical samples for IHC were from female patients seen at the UofL James Graham Brown Cancer Center (JG-BCC). Samples were obtained under an approved UofL IRB protocol to protect the privacy of research subjects.

The intensity of DAB staining of IHC samples was quantified using open-source ImageJ Fiji software [300]. Corresponding tissue sections were quantified for Pak1 and control IgG. Microscopic images were submitted to the ImageJ plug-in vector HDAB, which color deconvolutes and outputs separate images of DAB staining and hematoxylin counterstaining. Mammary ductal structures were outlined in the deconvoluted DAB image output and mean intensity measured. The intensity value range is from 0 , representing the darkest shade, to 255 , representing a pure white, 8-bit image. Intensity values of Pak1 and IgG images were converted to optical density (OD), using the equation:

$$
\mathrm{OD}=\log (\max \text { intensity/mean intensity })
$$

where maximum intensity is 255 . Ratios of Pak1 OD to IgG OD for each image were used to determine relative Pak1 staining. Ratios were binned and assigned to a histogram profile using GraphPad Prism version 7.00. A gaussian curve was fit to this plot. Samples with ratios that were greater than one standard deviation from the mean were counted as positive for Pak1 staining. 
Sanger Sequencing and Strain Comparison

A reference rat Pakl gene sequence was obtained from Ensembl ID ENSRNOG00000029784. Primer3 plus software was used to design PCR primers flanking Pak1 intron/exon splice sites and UTRs based on rat reference sequence RGSC 6.0/rn6. Primers and sequences are located in table 1, which is available at Mendeley Data (http://dx.doi.org/10.17632/s5ywd3525d.1) [301]. The UCSC genome browser in silico PCR tool was used to exclude off target primers. Genomic DNA was extracted from rat spleen tissue, and PCR amplified at cycling conditions of $94^{\circ} \mathrm{C}$ for 2 minutes, 18 cycles of $94^{\circ} \mathrm{C}$ for 15 seconds and $68^{\circ} \mathrm{C}$ for 2 minutes, 22 cycles of $94^{\circ} \mathrm{C}$ for 15 seconds and $59^{\circ} \mathrm{C}$ for 2 minutes, and $59^{\circ} \mathrm{C}$ for 10 minutes. PCR samples were Sanger sequenced using an ABI PRISM 3130xl Genetic Analyzer. Sequences were compared between WF, COP, and WF.COP-RN01 strain D rats. Identified variants were given dbSNP identifiers from NCBI and submitted to the European Variation Archive (EVA) project and Rat Genome Database ID. The database, UTRdb, contains curated 5' and 3' UTRs of eukaryotic mRNAs [302] and was used to annotate functional UTR elements. The miRNA database miRBase v22 was used to annotate miRNA binding sites within UTRs [303]. When comparing sequences at intron/exon junctions, the consensus 5' splice donor sequence GG(cut)GTRAGT and 3' splice acceptor site $\mathrm{CAG}(\mathrm{cut}) \mathrm{S}$ were used, where R denotes any purine and $\mathrm{S}$ represents $\mathrm{G}$ or C. Branch sites were searched between 18-40 nucleotides upstream from the $3^{\prime}$ end of an intron using the consensus sequence YYRAY, where $\mathrm{Y}$ indicates a pyrimidine and $\mathrm{R}$ denotes any purine. 


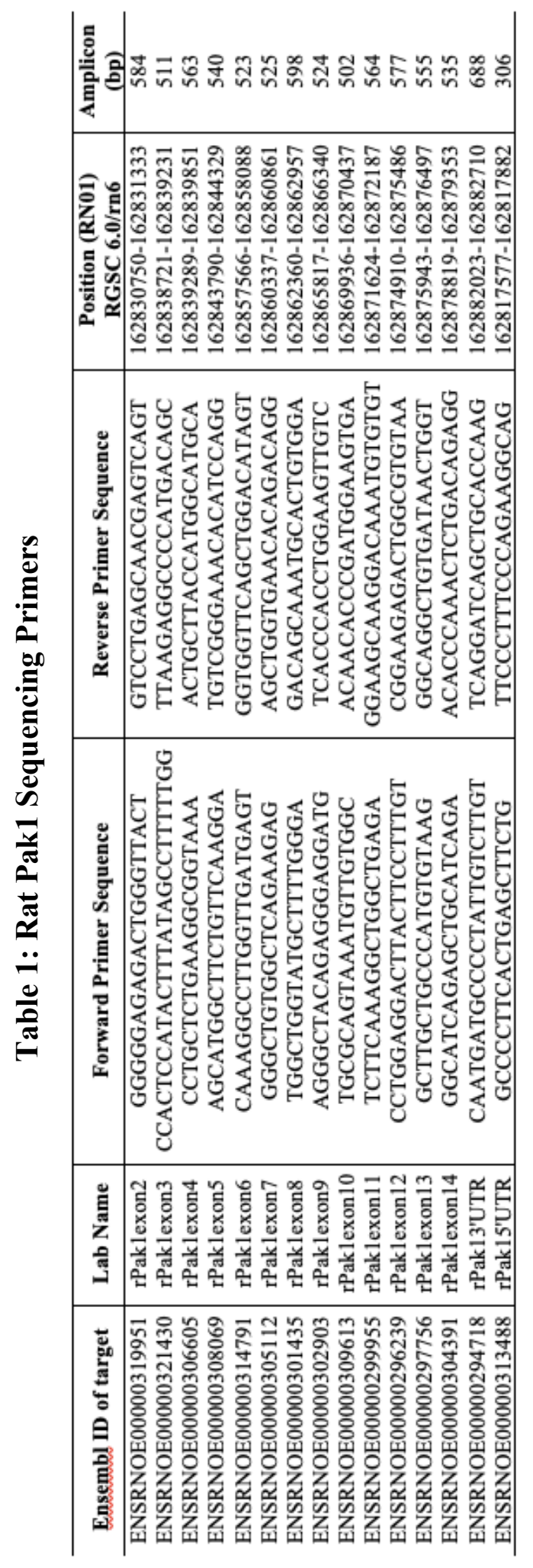




\section{Data Handling and Statistical Analysis}

Relative quantification, based on a standard curve, was used for QPCR. Mean quantities of triplicate QPCRs for each unknown sample were analyzed using Two-Way ANOVA with $\log 2$ (Target quantity/Rplp2 quantity) as the dependent variable. Independent variables for comparing mammary gland transcript levels were $M c s 3$ genotype and DMBA exposure. A two-tailed, unpaired T test was performed post hoc. Pearson's correlation coefficients were calculated for Pakl and Ilk transcript levels from $M c s 3$ genotypes. The independent variables were $M c s 3$ genotype and DMBA exposure. All statistical analysis was done using GraphPad Prism version 7.00 for Windows, GraphPad Software, La Jolla California USA.

\section{Results:}

Quantitative PCR (QPCR) was used to compare Ilk and Pakl mammary gland transcript levels between Mcs3-resistance associated WF.COP congenic strain D and mammary carcinoma susceptible WF.COP strain E females following DMBA-exposure. A time point of four weeks post-DMBA administration was used. This time represents a stage of early tumorigenesis before frank carcinomas are detectable in a DMBA-induced mammary carcinogenesis model. No effect of Mcs 3 haplotype on expression of Ilk or Pak1 was observed at this time point, which was 12 weeks of age $(\mathrm{p}>0.05)$. Expression of Pak1 was significantly higher in rat mammary glands exposed to DMBA compared to unexposed age matched controls ( $\mathrm{p}=0.02$, Figure 6A). There was no effect of either Mcs3 haplotype or DMBA-exposure on mammary gland $I l k$ transcript levels (Figure 6B). There was a correlation between Ilk and Pakl mammary gland transcript levels in the mammary cancer 
Transcript Levels of Pak1 and Ilk in DMBA-exposed Rat Mammary Glands
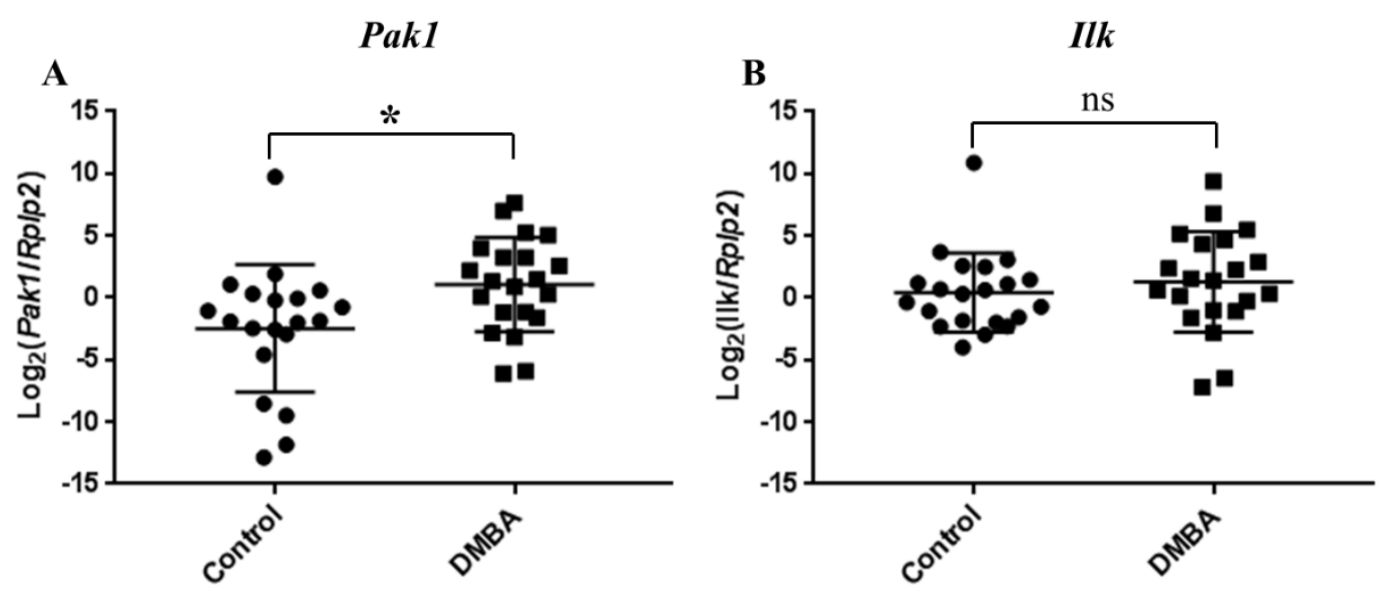

Figure 6: Increased Pak1 transcript levels in DMBA-induced rat mammary glands.

RT-qPCR of Pakl and Ilk levels in rat mammary gland tissue 4 weeks after DMBAinduction of mammary carcinogenesis compared to age-matched uninduced controls. QPCRs, run in triplicate for each sample, were used to measure target gene quantity standardized by Rplp2 quantity. QPCR Ct values were quantified using linear standard curves derived from known cDNA concentrations. P values are from two-way ANOVA Ftests including exposure and genotype. $M c s 3$ genotypes were pooled because genotype was not significant for either Pakl or Ilk. (A: $n=21$ DMBA rats, 21 control rats; $\mathrm{P}=0.02$. B: $\mathrm{n}=21$ DMBA rats, 21 control rats; $\mathrm{P}=0.47$ ) 


\section{Correlation of Pak1 and Ilk Transcript Levels in Rat Mammary Gland Tissue}

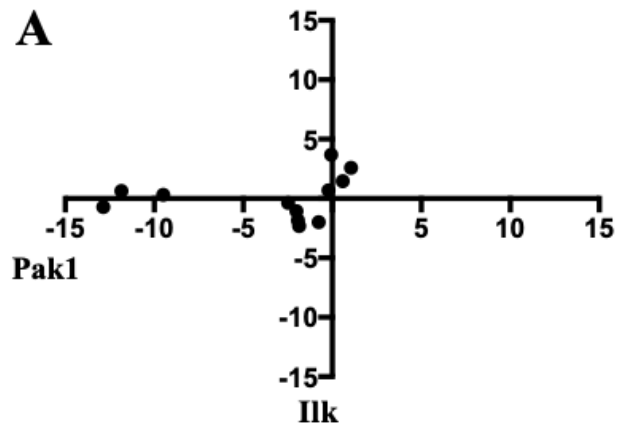

Strain E

Unexposed

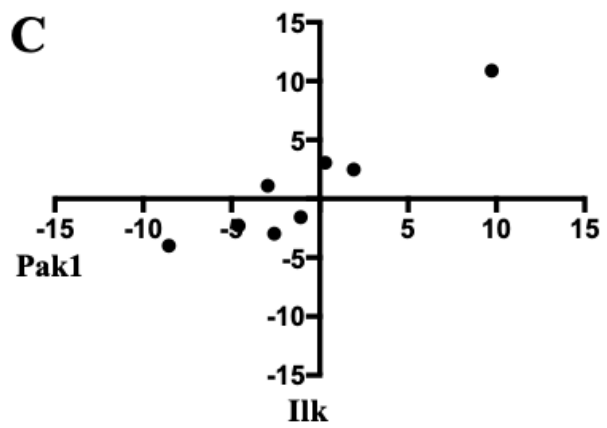

Strain D

Unexposed

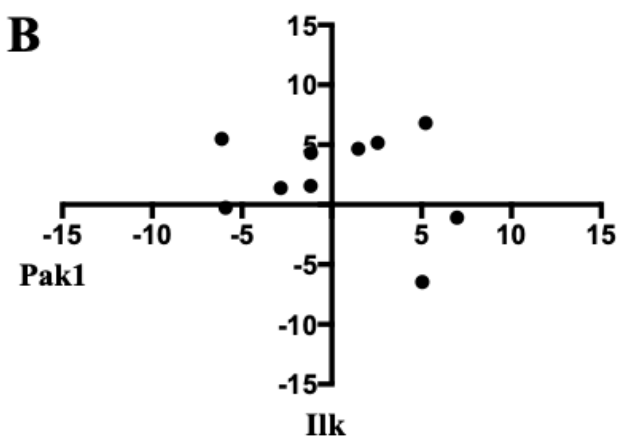

Strain E

DMBA-exposed

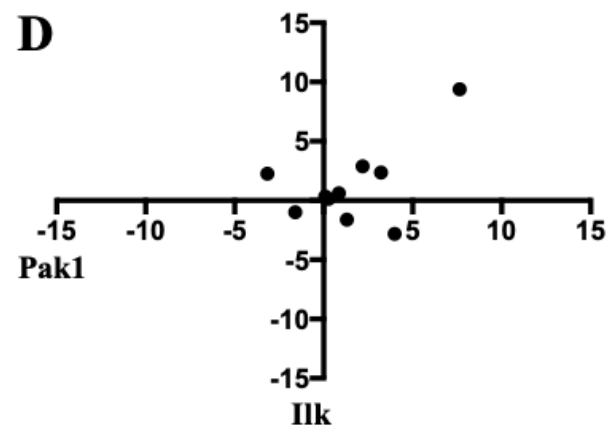

Strain D

DMBA-exposed

Figure 7: Correlation of Pakl and $I l k$ expression in rat mammary glands is lost with DMBA exposure. Standardized values of Pakl and $l l k$ expression from previous RTqPCR of rat mammary gland tissue (Fig 6) were analyzed by Pearson's correlation. A: Rat strain $\mathrm{E}$ (susceptible) mammary glands, not exposed to DMBA $\mathrm{n}=12$ control rats, $\mathrm{r}=0.1811$, $\mathrm{p}=0.5732$. B: Strain E age matched rat mammary glands 4 weeks after DMBA-induction of mammary carcinogenesis $n=10$ DMBA rats, $r=-0.2158, p=0.5494$. C: Strain D (resistant) rat mammary glands, not exposed to DMBA $n=8$ control rats, $r=0.9349$, $\mathrm{p}=0.0007$. D: Strain D age matched rat mammary glands, 4 weeks after DMBA-induction of mammary carcinogenesis $\mathrm{n}=10$ DMBA rats, $\mathrm{r}=0.5275, \mathrm{p}=0.1171$. 
resistant WF.COP strain D unexposed control rats $(r=0.9349, \mathrm{p}=0.0007$, Figure 7 C $)$, but this correlation was lost with DMBA exposure $(r=0.5275, \mathrm{p}=0.1171$, Figure 7D). There was no correlation between Pakl and Ilk in the mammary cancer susceptible strain E with and without DMBA-exposure.

To determine if genetic variants in Pakl protein-coding sequence existed between susceptible and Mcs3-resistance associated strains we sequenced WF/NHsd, COP/NHsd, and Mcs3- resistance associated WF.COP strain D. This revealed SNVs in exons 1, 2, 11, and 15 that differed between WF and COP or WF.COP strain D, but did not result in amino acid changes (Table 2). One variant was found in the 3 ' splice site junction of exon 7; however, based on consensus splice sequence, this variant was not predicted to disrupt the splice site. We used the UTRdb database to predict three different upstream open reading frames (uORFs) in the 5' UTR and a polyadenylation signal (PAS) within the 3' UTR of Pak1. Variants located in Pak1 UTRs were not located in these elements. The database miRBase v22 was used to annotate miRNA binding sites within rat Pakl UTRs. No sites were identified in the 5'UTR. The 3'UTR contains an rno-miR-217-5p site; however, there were no variants between WF and COP or WF.COP strain D at this site.

To determine if the DMBA-induced mammary carcinogenesis model is also a model of PAK1 expression during breast cancer development, IHC was performed on noncancerous rat mammary tissue exposed to DMBA. Figure 8 contains images of a rat mammary gland that displays positive Pak1 staining compared to an IgG background control. We quantitated relative Pak1 staining in mammary glands again at 4 weeks postDMBA administration, as this represents an early stage of carcinogenesis. Overall, four out of 17 (24\%) DMBA-exposed mammary glands were positive for Pak1 staining; whereas, 


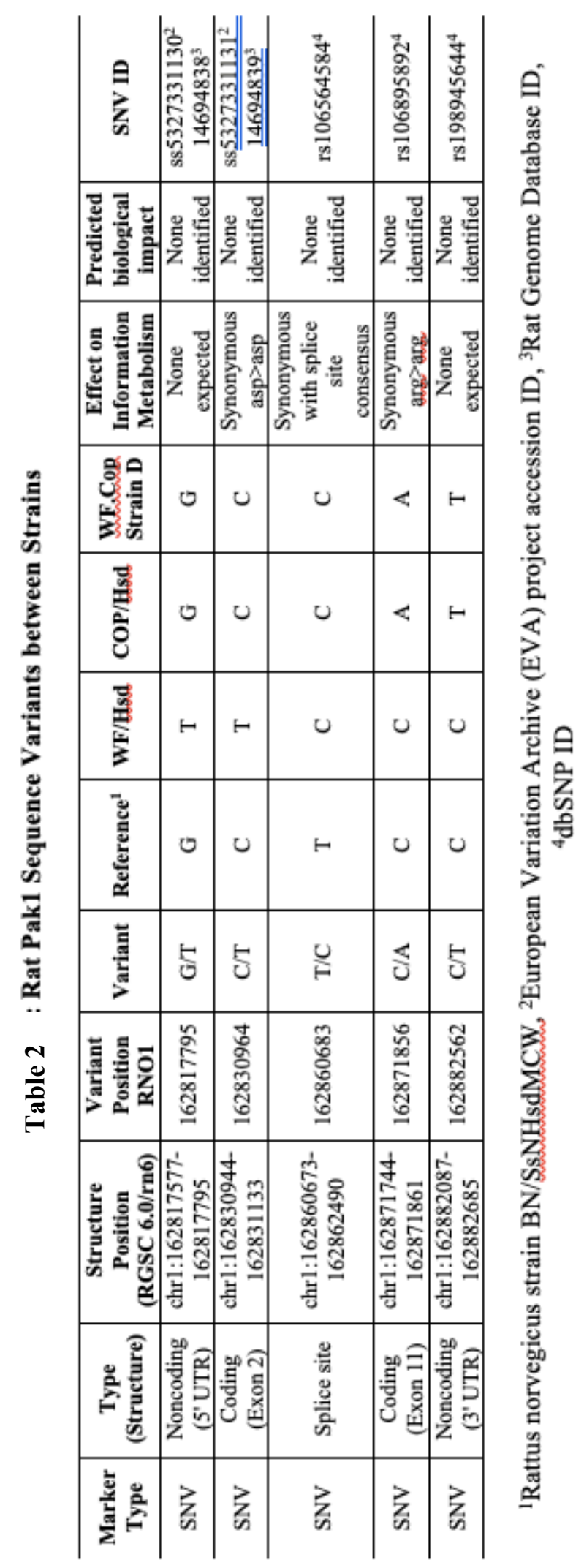



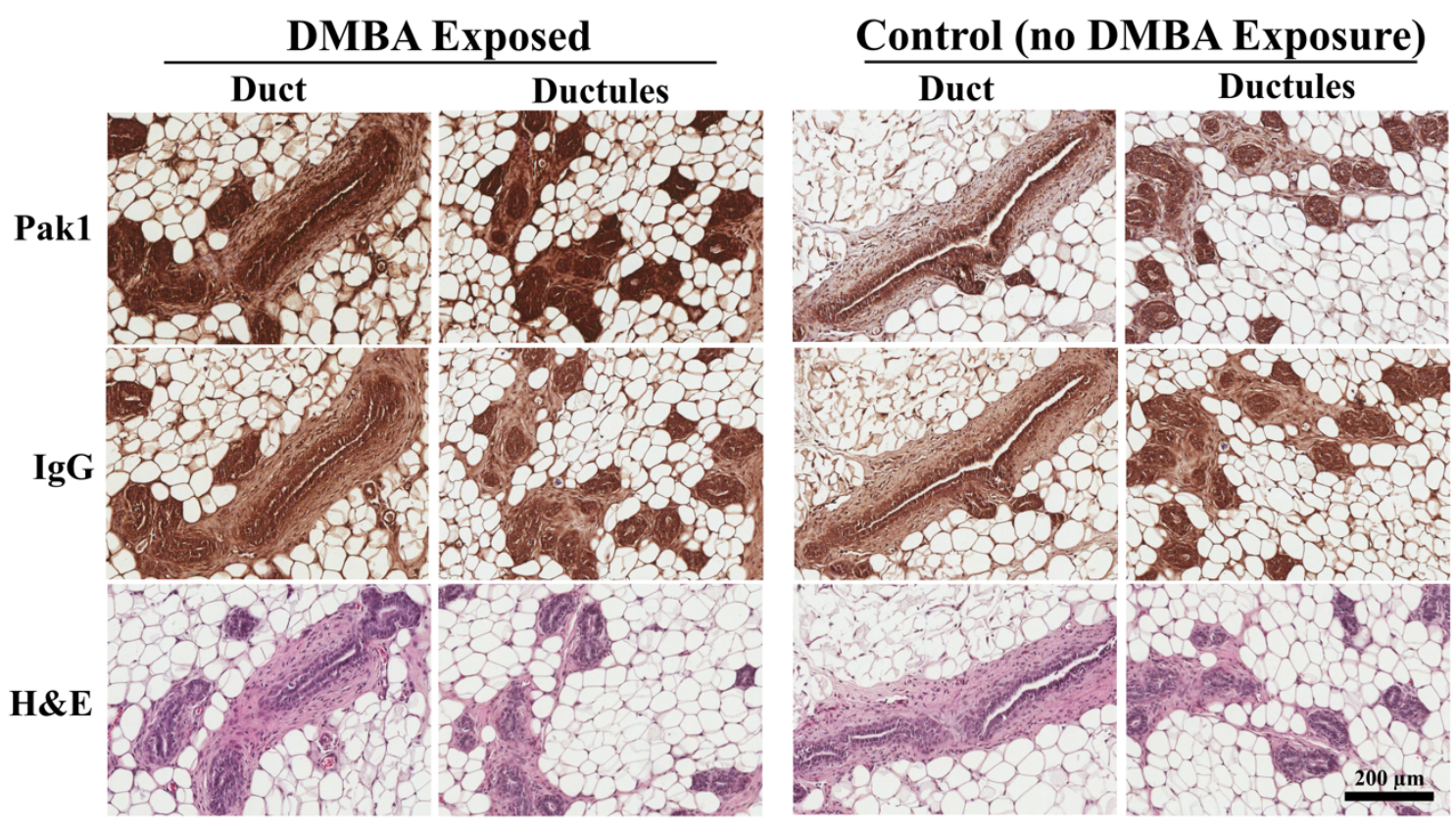

Figure 8: Representative Pak1 staining images of morphologically normal DMBAinduced and uninduced rat mammary glands. IHC images of mammary ducts and smaller ductules of a morphologically normal rat mammary gland, 4 weeks after DMBAinduction of mammary carcinogenesis (left two columns) compared to an age-matched rat, not exposed to DMBA (right two columns). Darker staining, relative to the IgG control, is evident in the DMBA-exposed mammary ducts, but not in the uninduced control mammary gland. H\&E staining is shown for a histological view of tissue sections. Images were taken at 40x magnification on an Aperio ImageScope CS2. 
zero mammary glands out of seven $(0 \%)$ taken from seven age-matched controls, not exposed to DMBA, stained positive for Pak1 (Table 3). Mammary gland tissue, positive for Pak1 staining at four weeks post-DMBA exposure, appeared histologically normal (Figure 8).

To compare our findings in rat mammary glands with reported results of PAK1 positivity in human breast disease, IHC was used to visualize PAK1 expression in ER+/HER2- ductal carcinoma in situ (DCIS) and triple-negative (TN), ER+/HER2-, and HER2+ invasive ductal breast carcinomas (IDC). Images of positive PAK1 staining, relative to IgG control, in TN, ER+/HER2-, HER2+ IDC, and ER+/HER2- DCIS are shown in Figure 9. This was performed as a comparative experiment, with anticipation that our results would recapitulate those reported for PAK1 positivity in human breast disease. One out of six samples stained positive for PAK1 in both TN and HER2+ IDC cases. One out four ER+/HER2- IDC samples displayed positive PAK1 staining, and one out of two ER+/HER2- DCIS samples were positive for PAK1. Overall, four breast disease tissue samples out of $18(22 \%)$ analyzed were positive for PAK1 staining (Table 4).

\section{Discussion:}

We report that a DMBA-induced rat mammary carcinogenesis model of human breast cancer has utility to investigate a potential role for PAK1 at early stages of breast cancer development. Human $P A K 1$ is located within the breast cancer-associated $11 q 13 / 14$ amplicon. Human chromosome $11 q 13 / 14$ amplification is reported to occur in $19.6 \%$ of breast tumors and is associated with poor prognosis [244, 277-280, 304]. Functional significance of $P A K 1$ expression, due to $11 q 13 / 14$ amplification, has been investigated. 
Table 3. Rat mammary glands positive for Pak1 staining

\begin{tabular}{c|c|c|c}
\hline Treatment & $\begin{array}{c}\text { Total } \\
\text { Samples }\end{array}$ & $\begin{array}{c}\text { Pak1 } \\
\text { Positive }\end{array}$ & $\begin{array}{c}\text { Percent } \\
\text { Positive }\end{array}$ \\
\hline Unexposed controls & 7 & 0 & 0 \\
DMBA & 17 & 4 & 24 \\
\hline
\end{tabular}




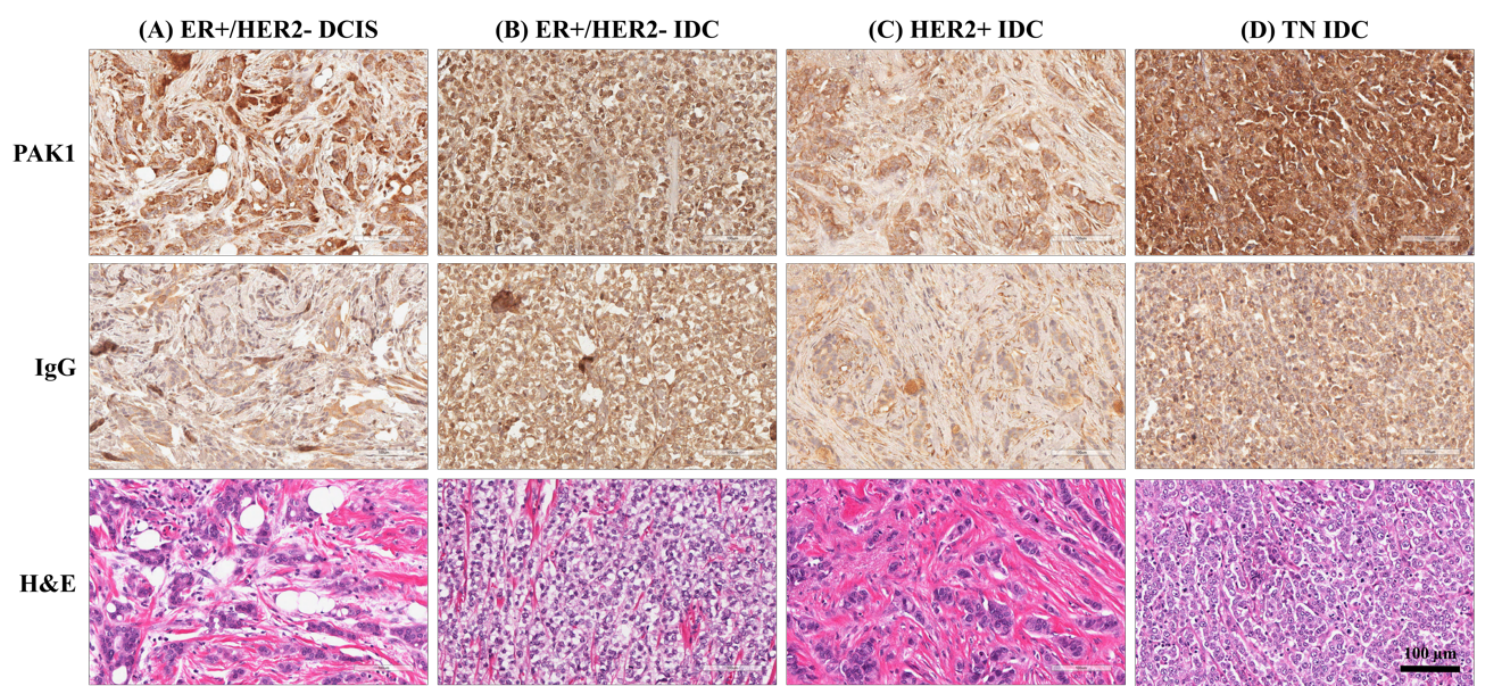

Figure 9: Images of PAK1 positive staining in human breast tissue. Representative IHC images from (A) ER+/HER2- ductal carcinoma in situ (DCIS) (B) ER+/HER2- invasive ductal carcinoma (IDC) (C) HER2+ IDC and (D) triple negative IDC. Darker PAK1 staining is evident compared to respective IgG controls. H\&E staining is shown to provide a detailed view of the tissue. Images were taken at 40x magnification on an Aperio ImageScope CS2. 
Table 4. Female breast disease samples positive for PAK1 staining

\begin{tabular}{cccc}
\hline Breast Disease Subtype & $\begin{array}{c}\text { Total } \\
\text { Samples }\end{array}$ & $\begin{array}{c}\text { PAK1 } \\
\text { Positive }\end{array}$ & $\begin{array}{c}\text { Percent } \\
\text { Positive }\end{array}$ \\
\hline IDC TN & 6 & 1 & 17 \\
IDC HER2+ & 6 & 1 & 17 \\
IDC ER+/HER2- & 4 & 1 & 25 \\
1DCIS ER+/HER2- & 2 & 1 & 50 \\
\hline
\end{tabular}

${ }^{1}$ Only a limited number of DCIS samples were available for study 
using breast cancer cell lines in tissue culture and xenograft tumors. [288, 289, 305-308]. These in vitro studies provide insight into PAK1-driven progression of breast cancer cell lines, but do not indicate if aberrant PAK1 expression possesses a functional role during early disease pathogenesis, as normal tissue transitions toward a carcinoma. We did not uncover evidence supporting a hypothesis that Pakl is an Mcs3 causal gene in this study. There are multiple Mcs3 nominated genes that will need to be studied in this context [243]. We did discover an independent effect of DMBA exposure on Pakl expression in mammary glands. Positive Pak1 expression was detected before pathological signs of disease developed in DMBA-exposed mammary glands. This suggests a potential early functional role of Pak1 in at least a subset of mammary glands suspected to be undergoing carcinogenesis.

We did not measure Pak1 protein and Pakl transcript levels in the same samples; therefore, we have neither determined this relationship nor the mechanism for Pak1 positivity in a subset of rat mammary glands exposed to DMBA. We suspect that the increase in Pakl transcript levels observed in mammary gland tissue following chemical exposure is due to either repopulation of the mammary gland with a high proportion of Pak1 expressing cells, or increased transcription and/or amplification of the Pakl locus in pre-existing cells. Increased mRNA and protein stability are additional mechanisms that will need to be considered to determine the mechanism of Pak1 positivity.

Frequencies of PAK1 positive female breast disease samples found in samples we tested were consistent with a published study of PAK1 staining [282]. We also detected positive PAK1 staining in a human DCIS sample. This matches previous findings of PAK1 positivity in human DCIS lesions using immunohistochemistry [309], and together suggest 
PAK1 expression may be involved in the progression of some female invasive breast carcinomas. This is concordant with a transcript profiling study of breast cancer progression that reported higher $P A K 1$ transcript levels in human DCIS compared to nondiseased breast epithelium [310]. A study of breast cancer cell lines serially passed through xenografts in mice also reported a positive correlation between $P A K 1$ expression and stage of premalignant progression [311]. Our finding of elevated Pak1 expression in chemical carcinogen-induced non-diseased rat mammary glands further suggests that PAK1 has a role during early tumorigenesis of female breast cancer, potentially prior to the development of premalignant lesions.

Rodent models have long been used to explore gene function during cancer progression [312]. The only published rodent study of PAK1 and breast disease to our knowledge used transgenic mice expressing an activated form of Pak1 [313]. These authors demonstrated a role for Pak1 in mammary lesion and tumor formation; however, the expression of an activated-Pakl transgene was dependent upon a pituitary gland isograft. Few mammary tumors developed in this model over a long latency period of 2 years. We propose that laboratory rats, well-known to serve as a female breast cancer relevant in vivo system, could be used to study Pak1-mediated mechanisms using a shorter time interval. Palpable mammary tumors typically develop in susceptible rat strains around six-weeks following a single DMBA exposure. A laboratory rat model recapitulates a natural course of breast cancer development, which provides an in vivo model to study Pak1-mediated carcinogenesis. The laboratory rat is an excellent model of human breast cancer, as induced rat mammary carcinomas display the same histopathological stages and features of malignant breast carcinomas, including epithelial ductal cell origin, progression, and an 
array of hormone responsive and non-responsiveness [76, 101, 160-163, 314]. The mammary carcinoma susceptible rat strains used in this study develop on average 7 mammary tumors per rat upon DMBA induction, which is about 0.58 tumors per mammary gland (MG). Our finding that $24 \%$ of DMBA-induced mammary glands were Pak1 positive is extrapolated to estimate Pak1 positivity in $14 \%$ of rat mammary tumors (MT) $(0.58$ MT/MG x $0.24 \mathrm{Pak}^{+}-\mathrm{MGs}$ ). This is in line with the $15-25 \%$ PAK1 positivity of human breast carcinoma samples in this study, and experimentally demonstrates similar Pak1/PAK1 expression patterns in mammary/breast disease. Therefore, a rat model allows for experimentation and pathway analyses of Pak1 in the context of natural disease initiation, development, and progression that would complement human breast cancer studies.

In this study, we discovered that DMBA-induction of mammary carcinogenesis increased Pakl transcript levels in mammary gland tissue appearing morphologically normal. We also reported that Pak1 protein expression in rat mammary glands mirrored what we and others have found in human breast DCIS and IDC samples. These findings of rat/human Pak1/PAK1 staining at early stages of rat mammary carcinogenesis and premalignant stages of female breast cancer warrant further functional and diagnostic studies of PAK1 expression during early stages of rat mammary and human breast cancer development. 


\section{CHAPTER III}

\section{MAMMARY CARCINOMA SUSCEPTIBILITY LOCUS, MCS3: FINE MAPPING AND COMPARATIVE GENOMICS}

\section{Introduction:}

Breast cancer is the most frequently diagnosed form of cancer among women and is the leading cause of premature mortality $[315,316]$. It is estimated that $5-10 \%$ of familial breast cancer incidences are due to inherited gene mutations such as BRCA1 and BRCA2 [317]. Many of these established breast cancer predisposition, or susceptibility genes, are highly penetrant yet occur at a low population frequency [318]. However, most of the unexplained familial risk and spontaneous disease occurrence is due to a polygenic disease model, where the additive effects of low penetrant variants contribute to increased breast cancer risk [83, 319]. Linkage analysis and candidate gene approaches have historically been utilized to identify high risk breast cancer loci that segregate with breast cancer incidences in families, but they are limited in their ability to identify novel susceptibility genes $[65,320]$. Linkage analysis cannot detect the low penetrance alleles that individually confer a small risk on disease development [320] and studies of breast cancer candidate genes are implicitly biased as they evaluate variants in genes with known biological function or involvement in carcinogenic pathways [83, 319]. Their use in identifying genetic factors behind sporadic disease development and the residual heritability of familial breast cancer risk have proved exhaustive. 
Genome-wide association studies (GWAS) of breast cancer risk in the general population allows for an objective approach as this method scans the genome for causal variants and does not rely on previous assumptions of biological function or genetic locations [85]. From these studies, a high density of single nucleotide polymorphisms (SNPs) across the genome are identified and mapped as common genetic susceptibility factors, which would have remained unsuspected through linkage analysis and candidate gene approaches [85]. Some of these identified regions contain known genes and indicate new potential pathways with a role in breast carcinogenesis, while others are located in intronic or distal regulatory elements and suggest risk is modulated through regulatory effects $[85,319,321,322]$. Following identification of variants in risk-associated loci, subsequent fine-mapping and functional studies aim to validate causal SNPs and understand their biological mechanisms [319]. Currently, the causal variants and biological mechanisms underlying these GWAS-identified loci are largely unknown and unannotated $[319,321,322]$.

Numerous association studies have been performed but only a small proportion of identified SNPs have true associations with breast cancer, leaving a high false positivity rate. Stringent $\mathrm{P}$ values are necessary to lower the false positivity rate, but this also lowers the detection limit and can exclude true risk alleles with very low effect sizes [80]. Furthermore, these studies have only explained a small percentage of breast cancer heritability, indicating there remains considerable breast cancer risk-associated variation to be identified [88]. A comparative genomics strategy employs a model organism in which to genetically identify disease risk loci and evaluate human orthologs of these loci [161]. Integrating combinatorial GWAS and comparative genomics approaches allows for the 
discovery of novel risk-associated variants, the identification of true-positive GWAS riskassociations due to overlap in genetic susceptibility, and the ability to mechanistically characterize overlapping breast cancer risk alleles in a model organism that may translate directly to human susceptibility $[88,161,323]$.

An extremely useful comparative genomics strategy in studying human breast cancer utilizes a rat model of mammary cancer susceptibility. This consists of mapping mammary cancer risk loci and evaluating human orthologous loci, which may have previously been nominated from independent GWA studies or may have an unidentified role in disease risk [161]. The utility of the rat genome in identifying true positive GWASnominated risk associations has been established [88]. One study found that significantly more SNPs from GWA studies mapped to human orthologous regions of rat mammary cancer loci than to human orthologous regions of randomly selected rat genomic regions not known to associate with mammary cancer susceptibility, regardless of the human population used for a GWA study [88]. This study also highlighted that conservative GWAS corrections are too stringent as a considerable number of GWAS-nominated SNPs not meeting genome-wide significance levels had genomic overlap with rat mammary cancer susceptibility loci [88]. This complementary method of risk analysis that integrates rat genomics facilitates validation of variants identified in GWA studies and further studies of these defined orthologous risk loci. Comparative genomics also aids in prioritizing which variants to study amidst the plethora of breast cancer risk associated alleles.

The laboratory rat presents one of the best in vivo human breast cancer models as the induced rat carcinoma displays the same histopathological stages and features of malignant breast carcinomas, including epithelial ductal cell origin, progression, and an 
array of hormone responsive and non-responsiveness [76, 101, 160-164]. An initial observation found a single oral dose of the chemical carcinogen dimethylbenzanthracene (DMBA) rapidly and reproducibly induces mammary cancer in female Sprague-Dawley rats [212]. Reports followed that outlined differences in carcinogen sensitivity amongst various rat strains. Certain strains displayed a resistance to tumor induction, requiring multiple doses of carcinogen to match the high tumor incidence found in the SpragueDawley strain [211]. This finding that inbred rat strains have natural genetic variation in their susceptibility to carcinogen-induced and spontaneous development of mammary cancer led to genetic analysis of mammary cancer susceptibility [232]. Inbred rat strains that differ in their susceptibility to DMBA-, N-methyl-N-nitrosourea (NMU)-, or estrogeninduced mammary carcinogenesis have been used predict locations of quantitative trait loci (QTL) that control susceptibility to mammary carcinogenesis, termed the Mammary carcinoma susceptibility (Mcs) and Estrogen-induced mammary cancer (Emca) loci [164, 234-239]. In the DMBA mammary cancer model specifically, linkage analysis of segregating populations from crosses between the DMBA-induced mammary carcinoma susceptible Wistar-Furth (WF) and resistant Copenhagen (COP) strains resulted in four predicted Mcs QTLs named Mcs1, Mcs2, Mcs3, and Mcs4 [164, 235].

The existence of Mcs 1, Mcs2, and Mcs3 QTLs were validated using congenic rat lines carrying the resistant $M c s$ of interest introgressed on a WF susceptible background $[159,243,324]$. These congenic substitutions encompassing the introgressed QTL are tens of megabases in size, so fine mapping of this congenic interval is required to increase the resolution at this locus. Physical mapping can determine if more than one independently acting QTL exists at this locus while also reducing the pool of candidate variants to 
functionally characterize [161]. The COP resistant Mcs3 was physically confirmed and delimited to a $29.4 \mathrm{Mb}$ region on rat chromosome one (RNO1) in 2017 [243]. Here, I report results from fine-physical mapping using WF.COP Mcs3 congenic strains with differing portions of this COP locus that further delimits $M c s 3$ to a $7.2 \mathrm{Mb}$ region on RNO1. I also report findings of breast cancer associated variants using comparative analysis of human orthologous syntenic regions to the narrowed rat Mcs3.

\section{Methods:}

Congenic breeding:

Congenic rat lines were established and maintained in an Association for the Assessment and Accreditation of Laboratory Animal Care (AAALAC)-approved facility on a 12-hr light/dark cycle and provided LabDiet 5001 Rodent Diet (PMI Nutrition International) and water ad libitum. All animal protocols were approved by the University of Louisville Animal Care and Use Committee. Mcs 3 congenics are defined as genetic lines developed on a WF genome and carrying the selected COP alleles at the Mcs3 locus. Mcs3associated resistant line D rats have a fixed COP genotype for RN01 Mcs3 segment from single nucleotide variant (SNV) rs8149408 to SNV rs105131702. Line D males were crossed with inbred Wistar Furth (WF/NHsd) female rats (Envigo). Resulting Mcs3 heterozygotes were backcrossed to WF females and offspring screened for unique recombination intervals between microsatellite markers near $r s 8149408$ and $r s 105131702$. Novel recombinant rats were backcrossed to WF animals to expand the genotype, and subsequent offspring were inbred by brother-sister mating to establish unique WF.COP- 
RNO1 congenic strains for phenotyping. A timeline of this congenic strain development is depicted in Figure 10.

Sequence information and locations of genetic markers defining the ends of COP alleles in the WF.COP Mcs3 congenics are available at the UCSC Genome Browser (www.genome.ucsc.edu), the Rat Genome Database (http://rgd.mcw.edu/), or Table 5 and 6.

\section{Genotyping:}

Animals were genotyped as described in Samuelson et al. (2003) [325]. Briefly, a small tail tip section from each rat was removed at 8-11 days of age, lysed, and DNA extracted. Microsatellite marker primers were used to PCR amplify DNA to determine genotypes at the congenic ends of Mcs 3 and at intervals between. Fast-PCR reactions underwent denaturation of $95^{\circ} \mathrm{C}$ for $10 \mathrm{sec}$, followed by 40 cycles of $94^{\circ} \mathrm{C}$ for $0 \mathrm{sec}$ and $63^{\circ} \mathrm{C}$ for $8 \mathrm{sec}$, and an extension at $72^{\circ} \mathrm{C}$ for $30 \mathrm{sec}$ on an Applied Biosystems Veriti Fast Thermal Cycler. PCR products were resolved on 3\% high resolution agarose gels, stained with ethidium bromide, scanned using a Typhoon Imager, and visualized with ImageQuant software (Amersham Biosciences, Piscataway, NJ). Visible gel-bands of the appropriate size were analyzed along with DNA from homozygous COP and WF, and (WF x COP) $)_{\mathrm{F} 1}$ (heterozygous) rats. Four Mcs $3 \mathrm{WF} / \mathrm{COP}$ informative SNPs were PCR amplified and samples sequenced by the University of Louisville, Center for Genetics and Molecular Medicine, sequencing core using an ABI PRISM 3130XL Sequence Detection System (Life Technologies). 


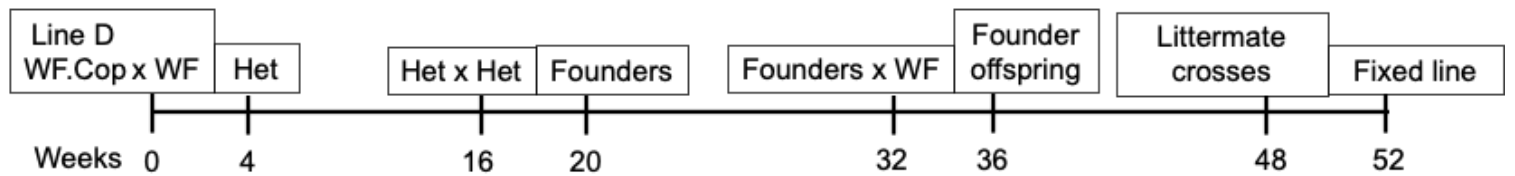

Figure 10: Timeline of Congenic Breeding and Strain Development. The top row indicates rat breedings and generations. The bottom row shows weeks between crosses and new generations. Line D containing the COP Mcs3 allele is crossed with the WF strain to generate a heterozygous generation. Heterozygotes are bred together to produce a generation of recombinant animals used as founders of new $M c s 3$ congenic lines. Founder genotypes are expanded by backcrossing to the WF strain. Brother-sister matings fix these genotypes and establish unique WF.COP Mcs3 congenic lines. In total it takes about 1 year to develop a new congenic line. 
Table 5. Primer Sequences for Mcs3 Microsatellite Markers in RN01 used in Screening for Recombinants

\begin{tabular}{|c|c|c|c|c|}
\hline $\begin{array}{c}\text { Samuelson } \\
\text { Lab ID }\end{array}$ & Forward Sequence & Reverse Sequence & $\begin{array}{l}\text { Amplicon } \\
\text { Position* }\end{array}$ & $\begin{array}{l}\text { Amplicon size } \\
\text { WF/COP (bp) }\end{array}$ \\
\hline D1Rat382 & $\begin{array}{c}\text { GGCCGAATGCTTT } \\
\text { CAATAGA }\end{array}$ & $\begin{array}{c}\text { GGCATACATGCTC } \\
\text { AAACTGC }\end{array}$ & $\begin{array}{l}137503081- \\
137503201\end{array}$ & $123 / 139$ \\
\hline D1Rat173 & $\begin{array}{l}\text { GATCCCTTGACAA } \\
\text { GCATGGT }\end{array}$ & $\begin{array}{c}\text { GATGGAGGCAGTT } \\
\text { TTTCCAA }\end{array}$ & $\begin{array}{l}152519382- \\
152519547\end{array}$ & $158 / 174$ \\
\hline D1Mit3 & $\begin{array}{c}\text { ACTTGGTGAAGAA } \\
\text { GAGTCAGGG }\end{array}$ & $\begin{array}{c}\text { GATTTACTGTGCC } \\
\text { TGTGGTTTT }\end{array}$ & $\begin{array}{c}156446196- \\
156446783\end{array}$ & $129 / 121$ \\
\hline D1Rat274 & $\begin{array}{c}\text { TTGGTACACACAC } \\
\text { GCACATG }\end{array}$ & $\begin{array}{c}\text { CTGGACATAAGGA } \\
\text { CAACTGGAA }\end{array}$ & $\begin{array}{l}161677683- \\
161677836\end{array}$ & $175 / 157$ \\
\hline D1Mgh8 & $\begin{array}{c}\text { CCTCTGGATTCTG } \\
\text { CCAGAAG }\end{array}$ & $\begin{array}{c}\text { TTTCAAATGTACA } \\
\text { GGCTGAAACA }\end{array}$ & $\begin{array}{l}163796316- \\
163796432\end{array}$ & $143 / 111$ \\
\hline D1Rat277 & $\begin{array}{c}\text { TCTGGTCTTTACAT } \\
\text { GTATGTGCA }\end{array}$ & $\begin{array}{c}\text { TTCACATCAGTTTT } \\
\text { GGCCAC }\end{array}$ & $\begin{array}{l}171716519- \\
171716714\end{array}$ & $194 / 210$ \\
\hline
\end{tabular}

$\mathrm{WF}=$ Wistar Furth, $\mathrm{COP}=$ Copenhagen

* Rattus norvegicus genome build version RGSC 6.0/rn6 


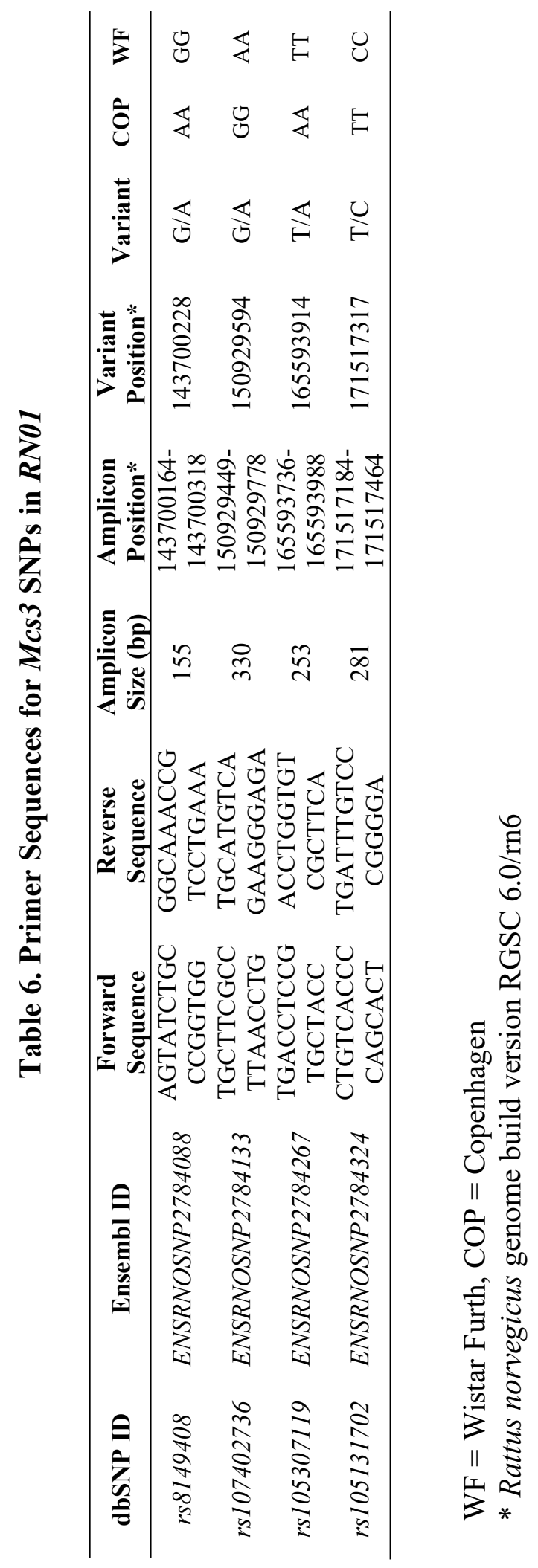




\section{Resequencing:}

Intergenic regions at the distal ends of the newly delimited Mcs3 and approximately the middle of the locus (Chr1:143700228-150929594) were selected to be amplified and sequenced in order to identify additional variants. This locus was searched using rat reference sequence RGSC 6.0/rn6using UCSC genome browser for regions containing diand tri-nucleotide repeats. Primers flanking these regions were designed using Primer3 plus oligonucleotide design software and the UCSC genome browser in silico PCR tool was used to exclude off target primers. Primers were obtained from Integrated DNA Technologies and used to PCR amplify genomic DNA as described in the previous heading. PCR reactions were run on high-resolution agarose gels to identify potential microsatellite size differences between WF and COP (Table 7).

The genome browser tool JBrowse database v6 as part of the Rat Genome Database was used to search for informative variants. The WF/NHsd genome is not sequenced, so the $\mathrm{COP} / \mathrm{CrCrl}$ genome was compared to the Lewis (Lew/Crl), Milan Hypertensive Strain (MHS/Gib), and inbred Wistar (WN/N) genomes. Lewis and MHS are derived from Wistar stock, and together with the Wistar strain have similar genetic backgrounds as the WF/NHsd strain. Primers were designed to amplify a region roughly $150-300 \mathrm{bp}$ in size that encompass the SNP. These regions were PCR amplified at cycling conditions of $94^{\circ} \mathrm{C}$ for 2 minutes, 18 cycles of $94^{\circ} \mathrm{C}$ for 15 seconds and $68^{\circ} \mathrm{C}$ for 2 minutes, 22 cycles of $94^{\circ} \mathrm{C}$ for 15 seconds and $59^{\circ} \mathrm{C}$ for 2 minutes, and $59^{\circ} \mathrm{C}$ for 10 minutes. PCR reactions were cleaned up using ExoSAP-IT (Thermofisher) at cycling conditions of $37^{\circ} \mathrm{C}$ for 15 minutes and $80^{\circ} \mathrm{C}$ for 15 minutes. Samples were then Sanger sequenced using an ABI PRISM 3130xl Genetic Analyzer (Table 9). 


\section{Phenotyping:}

Female WF.COP-RNO1 congenic rats, along with female WF/NHSd rats (Envigo), as positive (susceptible) controls, were administered a single oral gavage of DMBA (65 mg DMBA/kg body mass)(ACROS Organics; Fisher Scientific, Pittsburgh, PA) in sesame oil at 50-55 days of age. Mammary carcinomas $\geq 3 \times 3 \mathrm{~mm}^{2}$ were counted at 15 weeks posttreatment. $\mathrm{N}=28 \mathrm{WF}, 20$ line $\mathrm{H}, 26$ line $\mathrm{I}, 30$ line $\mathrm{J}$, and 25 line $\mathrm{K}$.

WF.COP Line J congenic rats and WF/NHsd rats (Envigo) were maintained in facilities described in congenic breeding. Rats were weighed at 4, 8, and 12 weeks of age ( $\mathrm{N}=31 \mathrm{WF}$ males, $\mathrm{N}=34 \mathrm{WF}$ females, $\mathrm{N}=30$ line $\mathrm{J}$ males, and $\mathrm{N}=29$ line $\mathrm{J}$ females). Separate female WF.COP Line J congenic rats and WF/NHsd rats (Envigo) were weighed at 4 weeks of age (weaning), then placed into a room of only female housing. Rats were again weighed at 8 and 12 weeks of age $(\mathrm{N}=15 \mathrm{WF}$ females, $\mathrm{N}=24$ line $\mathrm{J}$ females).

\section{Tumor Collection and Hematoxylin and Eosin Staining:}

Representative mammary tumors (invasive ductal carcinomas) were resected from females from each WF.COP-RNO1 congenic line and WF/NHSd rats (Envigo) at 15 weeks post-DMBA administration. Tumors were formalin fixed and paraffin embedded (FFPE). FFPE blocks were cut into $4 \mu \mathrm{m}$ sections and placed onto Superfrost Plus microscope slides using a rotary microtome (Thermo HM 355S). Slides were deparaffinized and rehydrated using 3 separate xylene washes and 4 washes in 100\% ethanol, 90\% ethanol, $80 \%$ ethanol, and DI water. Tissue was stained with hematoxylin (Fisher Scientific 469803) for 3 minutes, rinsed in running DI water, dipped in bluing reagent (Fisher Scientific 22-220106), and rinsed in running DI water. Tissue was stained with Eosin-Y (Fisher Scientific $22-220-104$ ) for $30-45$ seconds. Tissue was dehydrated in washes of $90 \%$ ethanol, $100 \%$ 
ethanol, and 3 separate xylene washes. Tissue was coverslipped with Permount Mounting Medium (Electron Microscopy Sciences 17986-05)

\section{Statistical analysis:}

Mammary carcinoma multiplicity data were analyzed by comparing congenic strain phenotypes to a susceptible WF/NHsd phenotype. Mammary carcinoma multiplicity phenotypes for all lines were compared using a one-way ANOVA. Following a significant one-way ANOVA ( $\mathrm{p}$ value $<0.05$ ), each congenic strain was compared to the WF/NHsd susceptible control group using Dunnett's multiple comparison test. P values $\leq 0.05$ were considered statistically significant. Body mass data were analyzed by a 2-way ANOVA using strain and age as the independent variables. Tukey's test for multiple comparisons was used post hoc.

\section{Comparative genomics:}

Human and mouse orthologous syntenic regions that mapped to the delimited rat Mcs3 were identified using the convert function of the UCSC Genome Browser. The Rattus norvegicus reference genome build version RGSC 6.0/rn6, Homo sapiens version GRCh38/hg38, and Mus musculus version GRCm38/mm10 were used. Rat Mcs3 and human and mouse orthologous syntenic regions were annotated for genes and non-coding DNA using the table browser function of the UCSC Genome Browser. The NHGRI-EBI Catalog of human genome-wide association studies was used to identify breast cancer disease and associated traits that had $\mathrm{p}$ values of $1 \times 10^{-3}$ or less in the human syntenic regions. PubMed was used to data mine Mcs3 and human orthologous genes associated with cancer. 


\section{Results:}

\section{I. $\quad$ Fine Mapping Mcs3 Using WF.COP Congenic Rat Lines}

\section{Backcrosses of WF.COP Mcs3 line D result in novel WF.COP Mcs3 congenic lines}

Mcs3 was previously predicted as a DMBA-induced mammary cancer susceptibility QTL by Michael Gould's group, and was physically confirmed and delimited to a 29.4 Mb region on rat chromosome 1 by Le et al in 2017 [164, 243]. I sought to further map the Mcs3 QTL to a shorter interval to reduce the pool of candidate genes and variants that could be modulating mammary cancer susceptibility. To accomplish this fine mapping, an additional four congenic lines were derived from the original Copenhagen (COP) locus in WF.COP Mcs3 Line D. These were termed lines H, I, J, and K and possessed a unique portion of the COP introgression on chromosome 1 from the previously defined Mcs 3 on a susceptible WF genetic background (Fig 11). The genomic location and positional markers defining the COP region of these lines are given in Table 7.

\section{Mammary carcinoma susceptibility phenotypes of WF.COP congenic lines delimit}

\section{Mcs3 to a 7.2 Mb region on rat chromosome 1 (Chr1:143700228-150929594)}

The new WF.COP Mcs3 congenic lines were tested for DMBA-induced mammary carcinoma susceptibility phenotypes in order to further delimit the Mcs3 QTL. Tumor multiplicity data for each WF.COP Mcs3 congenic lines depicted in Figure 11 is shown in Figure 12 and quantified in Table 9. Tumor multiplicity is counted 15 weeks following administration of DMBA and represents the DMBA-induced mammary carcinoma susceptibility phenotypes. WF females were administered DMBA alongside WF.COP 


\section{WF.COP Mcs3 Congenic Map}

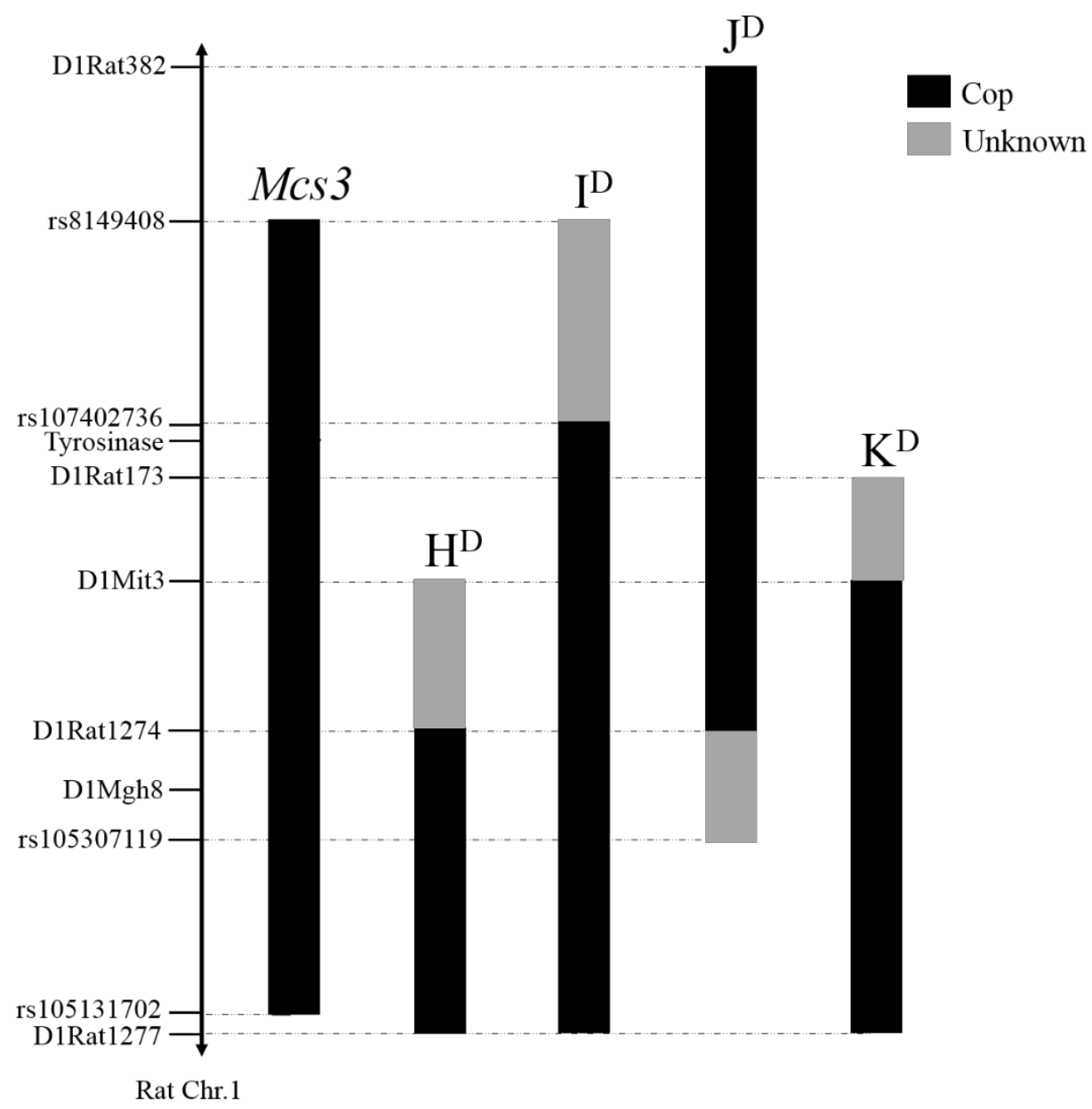

Figure 11. WF.COP congenic map of Mcs3 region recombinants used as congenic line founders. The leftward black bar represents the previously delimited Mcs3, defined by a COP segment (black) of RNO1 introgressed into a WF/NHsd strain genetic background. Horizontal lines along the axis represent informative markers used to distinguish WF and COP alleles along the Mcs3 locus. The remaining black bars represent the retained COP segments in the congenic lines used to narrow Mcs3. Gray bars at the ends of congenic segments mark regions of unknown genotype. 
Table 7. Positional markers and genomic locations defining WF.COP Mcs3 congenic lines

\begin{tabular}{ccccc}
\hline $\begin{array}{c}\text { WF.COP } \\
M c s 3 \text { line }\end{array}$ & $\begin{array}{c}\text { Congenic region } \\
\text { marker/marker }\end{array}$ & Genomic position* & $\begin{array}{c}\text { Size } \\
(\mathrm{Mb})\end{array}$ & $\begin{array}{c}\text { Percent of } \\
M c s 3 \text { span }\end{array}$ \\
\hline H & D1Rat274 to D1 Rat277 & Chr1:161677683-171716714 & 10 & 34 \\
I & rs107402736 to D1Rat277 & Chr1:150929594-171716714 & 20.8 & 71 \\
J & D1Rat382 to D1 Rat274 & Chr1:137503201-161677683 & 24.2 & 61 \\
K & D1Mit3 to D1Rat277 & Chr1:156446196-171716714 & 15.3 & 52 \\
\hline
\end{tabular}

* Rattus norvegicus genome build version RGSC 6.0/rn6 
Mcs3 congenic lines to serve as controls for a susceptible phenotype and developed an average of $7.9 \pm 2.9$ (mean \pm SD) tumors per rat. The susceptible WF phenotype in this study was similar to previously published DMBA-induced susceptibility phenotypes in WF females. Veillet et al. reported an average of $7.9 \pm 0.5$ tumors per WF rat, and denDekker et al. reported WF rats developed $6.3 \pm 3.3$ tumors per rat upon DMBA induction [243, 248, 326]. Line H, I, and K displayed average high mammary tumor numbers per rat similar to the WF strain of $6.7 \pm 3.4,5.9 \pm 3.8$, and $7.0 \pm 3.5$ respectively. Line J showed a lower average of $3.4 \pm 2.7$ tumors per rat. Tumor multiplicity data was analyzed by a statistically significant One-way ANOVA ( $<<0.0001)$, followed by Dunnett's nonparametric post hoc tests to compare each congenic line to the WF susceptible control. Line J was the only line to display a DMBA-resistant phenotype as it was significantly different from the WF strain. The resulting mammary carcinoma phenotypes and positional maps of the original founder lines and congenic lines in this study are depicted in Figure 13. The overlap of the susceptible lines do not indicate that there are independently acting Mcs sub-QTLs. Taken all together, the congenic lines delimit rat $M c s 3$ to a $7.2 \mathrm{Mb}$ region on rat chromosome 1 that spans from $r s 8149408$ to $r s 107402736$ (Chr1:143700228-150929594).

To further fine map the newly delimited $M c s 3$, the rat genome database (RGD) was queried for microsatellite markers in this region but reported markers did not differ in size between WF and COP alleles and were thus not informative. To uncover additional microsatellite markers within $M c s 3$, intergenic regions of the middle segment and distal ends of the newly delimited Mcs3 spanning from markers $r s 8149408$ to $r s 107402736$ were scanned using the UCSC genome browser to find short genomic regions rich in di- and trinucleotide repeats within this interval. A total of 33 of these potentially 


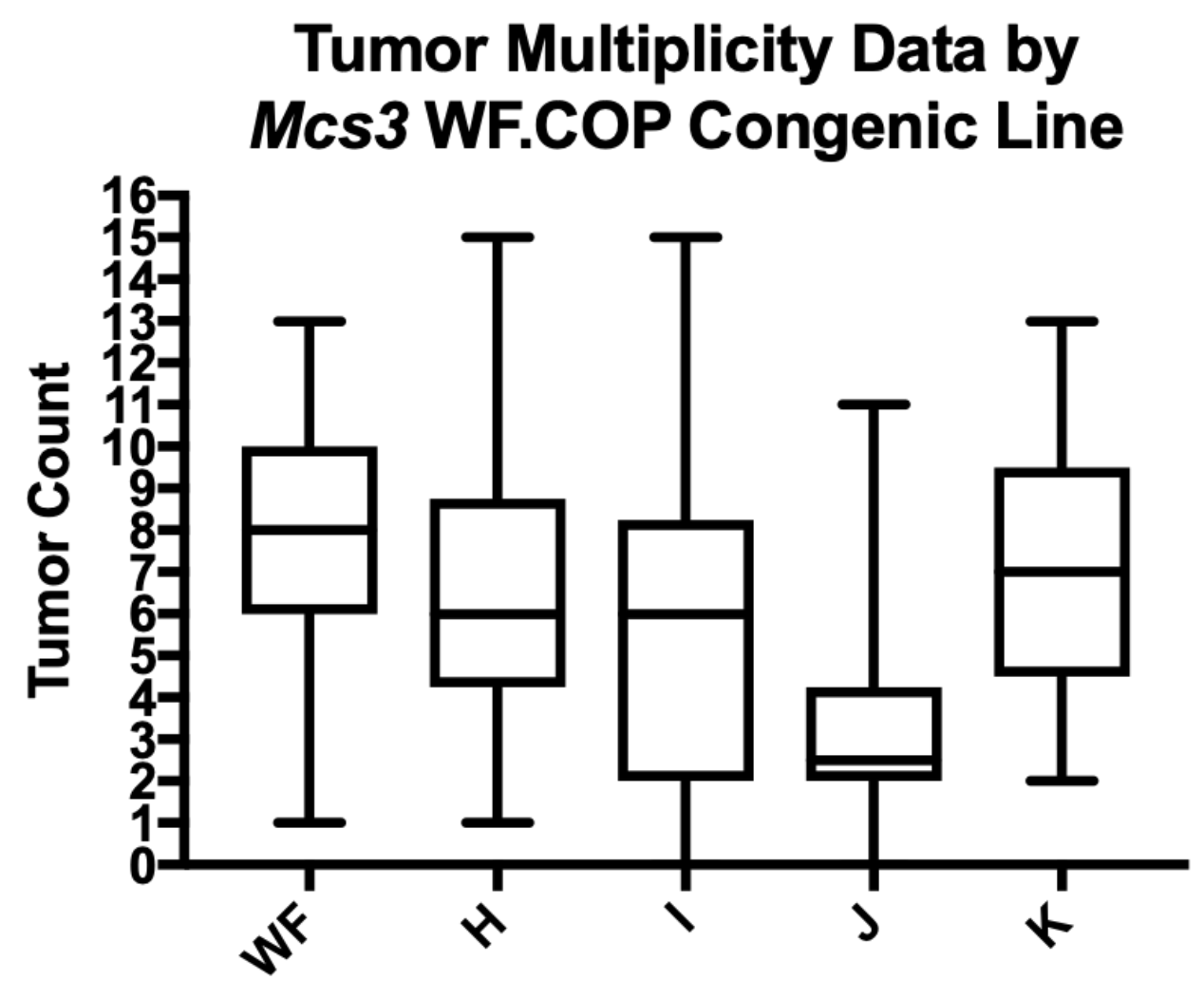

Figure 12: Tumor Multiplicity of WF.COP Mcs3 Congenic Lines. Shown are the distribution of tumor count data of congenic line $\mathrm{H}, \mathrm{I}, \mathrm{J}$, and $\mathrm{K}$ alongside the WF strain as a susceptible phenotype control. Tumors were counted at 15 weeks post-DMBA administration when rats were 23 weeks of age. A Kruskal-Wallis test was performed ( $\mathrm{p}<0.0001$ ), followed by Dunn's nonparametric post hoc tests comparing each line to the control WF strain. Line J was the only line that significantly differed from the WF strain in tumor multiplicity $(\mathrm{p}<0.0001)$. 
Table 8. Tumor Multiplicity Summary Data of Mcs3 WF.COP Congenic Lines

\begin{tabular}{ccccc}
\hline Line & $\begin{array}{c}\text { Congenic region } \\
\text { marker/marker }\end{array}$ & $\begin{array}{c}\text { Mean (SD) Mammary } \\
\text { Carcinomas per } \text { Rat }\end{array}$ & $\mathrm{N}$ & $\begin{array}{c}\text { p-value against } \\
\text { WF/NHsd }\end{array}$ \\
\hline D & D1Rat320/D1Rat65 & $2.8(2.3)$ & 19 & - \\
H & D1Rat274/D1Rat277 & $6.7(3.4)$ & 20 & 0.7666 \\
I & rs107402736/D1Rat277 & $5.9(3.8)$ & 26 & 0.0932 \\
J & D1Rat382/D1Rat274 & $3.4(2.7)$ & 30 & $<0.0001$ \\
K & D1Mit3/D1Rat277 & $7.0(3.5)$ & 25 & $>0.9999$ \\
WF/NHsd & - & $7.9(2.9)$ & 28 & - \\
\hline
\end{tabular}




\section{WF.COP Mcs3 Congenic Map}

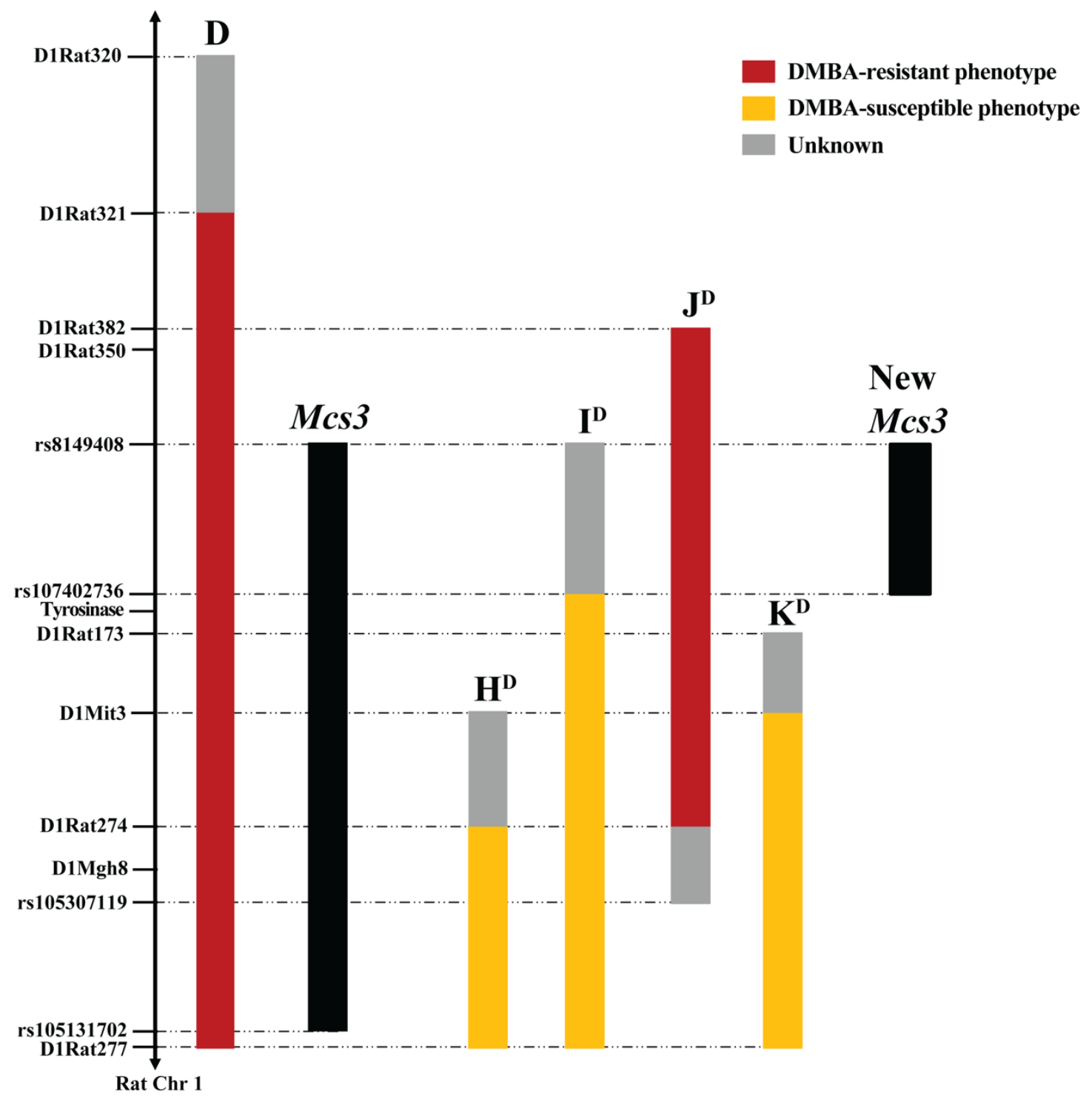


Figure 13. WF.COP Congenic Map Defining the Genomic Position of Mcs3. Vertical bars define the COP segment of RNO1 introgressed into a WF/NHsd inbred strain genetic background. Red colored bars represent congenic lines that display a resistant phenotype, yellow bars represent a susceptible phenotype, orange bars represent a mixed phenotype, and grey bars represent segments of unknown recombination where the genotype wasn't determined. The leftward lines D and E are from the previous physical mapping of Mcs3 and represent how this locus was delimited. Congenic lines on the right are derived from line D and further narrow this locus (rightward black bar). 
polymorphic microsatellite markers were tested by designing flanking primers, amplifying the region, and running PCR products on high-resolution agarose gels (Table 9). However, none of these were informative between WF and COP genotypes. After failing to discover new Mcs3 microsatellite markers, the RGD genome browser tool JBrowse database v6 was searched for differing variants between genotypes. Since the WF/NHsd genome is not available, the $\mathrm{COP} / \mathrm{CrCrl}$ genome was compared to the Lewis (Lew/Crl), Milan Hypertensive Strain (MHS/Gib), and inbred Wistar (WN/N) genomes. Lewis and MHS are derived from Wistar stock, and together with the Wistar strain have similar genetic backgrounds as the WF strain. Within Mcs3, 20 SNPs were identified that reportedly differed in the COP strain. After designing primers and sequencing across these variants, it was found that none of these SNPs differed between COP and WF (Table 10). This inability to find informative markers between WF and COP suggest that they share similar haplotypes at Mcs3. As a result, I stopped searching for markers in this tedious manner, so $M c s 3$ was unable to be mapped to a shorter genomic interval than what was found from the development and phenotyping of our congenic lines.

\section{WF.COP Mcs3 congenic lines have differing mammary tumor multiplicities but share similar tumor histopathology}

To determine if a difference in tumor histopathology was associated with the observed differences in tumor multiplicity, mammary tumor sections from WF and each WF.COP Mcs3 congenic line were stained by hematoxylin and eosin (H\&E). The DMBAinduced mammary tumors from each line were all of ductal origin, with the majority tumor histological subtypes being invasive papillary carcinoma (Fig 14) and invasive cribriform 
Table 9. Primer Sequences Tested for New Mcs3 Informative Microsatellites

\begin{tabular}{|c|c|c|c|}
\hline \multirow{2}{*}{$\begin{array}{l}\text { Samuelson Lab ID } \\
\text { A186_gULRN01_1 }\end{array}$} & Forward Sequence & Reverse Sequence & Chr 1 Position* \\
\hline & $\begin{array}{l}\text { AGGCTCTCCTTTC } \\
\text { TACCTCTGT }\end{array}$ & $\begin{array}{c}\text { GGTGGGCGTACACT } \\
\text { TTAGGT }\end{array}$ & $145501208-145501435$ \\
\hline A186_gULRN01_2 & $\begin{array}{l}\text { TGAGCTAAGGAT } \\
\text { GAGGGGCT }\end{array}$ & $\begin{array}{l}\text { TCCTGTCCTCAAAT } \\
\text { GCCCAC }\end{array}$ & $145506302-145506564$ \\
\hline A186_gULRN01_3 & $\begin{array}{l}\text { ATACAGGCACAT } \\
\text { GCTTCGTG }\end{array}$ & $\begin{array}{l}\text { ACATCAAATCAAA } \\
\text { GCAAAATCGATCA }\end{array}$ & $145509109-145509341$ \\
\hline A186_gULRN01_4 & $\begin{array}{l}\text { CCAGATCAGTGA } \\
\text { GAGGCTCC }\end{array}$ & $\begin{array}{c}\text { GAACATCCCACCAC } \\
\text { TGCCAG }\end{array}$ & $145510601-145510801$ \\
\hline A186_gULRN01_5 & $\begin{array}{l}\text { TGTGATACCCTCT } \\
\text { TCTGGTGTG }\end{array}$ & $\begin{array}{c}\text { CACCTAAGGTAGCG } \\
\text { AAAGAGCA }\end{array}$ & $145516753-145516980$ \\
\hline A186_gULRN01_6 & $\begin{array}{l}\text { AGACAGACAAAT } \\
\text { CTTAAGGGACCA }\end{array}$ & $\begin{array}{l}\text { TGCTTTAGTACCAA } \\
\text { GGATCTGAGT }\end{array}$ & $145532420-145532725$ \\
\hline A186_gULRN01_7 & $\begin{array}{l}\text { CGCATGTGACTG } \\
\text { GAGGAGAA }\end{array}$ & $\begin{array}{l}\text { GAGAGCATATAGG } \\
\text { TTCCACCCA }\end{array}$ & $145534867-145535141$ \\
\hline A186_gULRN01_8 & $\begin{array}{c}\text { CCTCTGATGCCCA } \\
\text { CATGTGT }\end{array}$ & $\begin{array}{c}\text { TGTGTGTATGTGCA } \\
\text { TGTAGGT }\end{array}$ & $145538047-145538198$ \\
\hline A186_gULRN01_9 & $\begin{array}{l}\text { TCAAGGAGGTTC } \\
\text { TCAGTTGTGA }\end{array}$ & $\begin{array}{c}\text { CTTGCAGCTCTGGG } \\
\text { GATCAA }\end{array}$ & $145545762-145545974$ \\
\hline A186_gULRN01_10 & $\begin{array}{l}\text { GCATGAGCTTTCT } \\
\text { AATTCACACTGA }\end{array}$ & $\begin{array}{l}\text { AGAGAAGATCCCG } \\
\text { CTAGGTCT }\end{array}$ & $145549788-145549991$ \\
\hline A186_gULRN01_11 & $\begin{array}{l}\text { TCTCCCCCATCCA } \\
\text { AATCACA }\end{array}$ & $\begin{array}{l}\text { GGGAGGAAGGGAG } \\
\text { GACAGAA }\end{array}$ & $145559506-145559729$ \\
\hline A186_gULRN01_12 & $\begin{array}{c}\text { ACCTTGACTCCAA } \\
\text { AGCCAAT }\end{array}$ & $\begin{array}{c}\text { GGCTCAAGCTTGCA } \\
\text { ACAGTT }\end{array}$ & $145575831-145575998$ \\
\hline A186_gULRN01_13 & $\begin{array}{l}\text { AAGTCCAGGCAG } \\
\text { AGTGAGGA }\end{array}$ & $\begin{array}{c}\text { AGTCACAGAAAGG } \\
\text { AGCATGGA }\end{array}$ & $145576351-145576497$ \\
\hline A186_gULRN01_14 & $\begin{array}{c}\text { CCTCCCTGGCCCA } \\
\text { GTAATTC }\end{array}$ & $\begin{array}{l}\text { GCAGGAGGGAGAA } \\
\text { GTGTTCC }\end{array}$ & $145581088-145581249$ \\
\hline A186_gULRN01_15 & $\begin{array}{l}\text { CTCACAGAGCTTC } \\
\text { CATCCCC }\end{array}$ & $\begin{array}{l}\text { GAGGGAAAAGGGA } \\
\text { CAGTGGG }\end{array}$ & $145588803-145588952$ \\
\hline A186_gULRN01_16 & $\begin{array}{l}\text { TGAGTCTGCATTG } \\
\text { TGGGTGC }\end{array}$ & $\begin{array}{l}\text { AGTTCAGTGAGAG } \\
\text { ACTCCGTATT }\end{array}$ & $145591580-145591793$ \\
\hline A186_gULRN01_17 & $\begin{array}{l}\text { AAGCCAAGCAAA } \\
\text { TAAATTTCATTCA }\end{array}$ & $\begin{array}{c}\text { GGCAGTAGCTACAC } \\
\text { CATGGA }\end{array}$ & $145595659-145595873$ \\
\hline A186_gULRN01_18 & $\begin{array}{l}\text { AGTCTGTGAGGA } \\
\text { TGGTCCCT }\end{array}$ & $\begin{array}{l}\text { TGCAGAGAACAAT } \\
\text { GAGATGGCT }\end{array}$ & $145605424-145605629$ \\
\hline A186_gULRN01_19 & $\begin{array}{l}\text { ATATTCCAGACTT } \\
\text { GCCTACAGG }\end{array}$ & $\begin{array}{c}\text { CTCCCCTCAGTCCC } \\
\text { TGTGTT }\end{array}$ & $145626825-145626998$ \\
\hline A186_gULRN01_20 & $\begin{array}{c}\text { CCTTCTGGCAACT } \\
\text { AAGGGCA }\end{array}$ & $\begin{array}{l}\text { TGGAATTCTTAACT } \\
\text { ATGAGATGAGATG }\end{array}$ & $145638005-145638182$ \\
\hline A186_gULRN01_21 & $\begin{array}{l}\text { GGGTCGTTACAG } \\
\text { AGCGGTG }\end{array}$ & $\begin{array}{l}\text { CCAGTGGGCAAGG } \\
\text { GAGGC }\end{array}$ & $147112809-147113012$ \\
\hline A186_gULRN01_22 & $\begin{array}{c}\text { CCTTTCTTGTGGC } \\
\text { TTTTGGGT }\end{array}$ & $\begin{array}{l}\text { TCCAAATGGGCAA } \\
\text { ATGGTTTGA }\end{array}$ & $146907551-146907671$ \\
\hline A186_gULRN01_23 & $\begin{array}{l}\text { TGGTACACATAT } \\
\text { ATGCCACCCA }\end{array}$ & $\begin{array}{l}\text { TCTGGATAACCAAC } \\
\text { TTAATGGCA }\end{array}$ & $146908208-146908398$ \\
\hline A186_gULRN01_24 & $\begin{array}{l}\text { TGTTTAGTATGCA } \\
\text { TGGAGCCCT }\end{array}$ & $\begin{array}{c}\text { TCATGTTCTTTATTT } \\
\text { ACAAGCCTAACA }\end{array}$ & $146854985-146855162$ \\
\hline A186_gULRN01_25 & $\begin{array}{c}\text { GGTATGGGCTTGT } \\
\text { TGGAGGT }\end{array}$ & $\begin{array}{c}\text { CTGGAGGCATAGCT } \\
\text { GGGAG }\end{array}$ & $146856680-146856848$ \\
\hline A186_gULRN01_26 & $\begin{array}{l}\text { GACCCAATGGCC } \\
\text { ATTTTCTCTG }\end{array}$ & $\begin{array}{c}\text { GGGAGGCTCTGCTC } \\
\text { AATGTT }\end{array}$ & $146867455-146867635$ \\
\hline
\end{tabular}




\begin{tabular}{|c|c|c|c|}
\hline A186_gULRN01_27 & $\begin{array}{l}\text { GCTGGACGGTTG } \\
\text { TGTTAGCT }\end{array}$ & $\begin{array}{c}\text { GTTGTACACTGGCC } \\
\text { TCCACA }\end{array}$ & $146872229-146872360$ \\
\hline A186_gULRN01_28 & $\begin{array}{l}\text { TGGGGCAGAGTT } \\
\text { AACACAGAC }\end{array}$ & $\begin{array}{c}\text { TCCTTCAAGACTGT } \\
\text { ACCCTGT }\end{array}$ & $148319017-148319217$ \\
\hline A186_gULRN01_29 & $\begin{array}{l}\text { AGCCATAGAGGT } \\
\text { CAGGAAGA }\end{array}$ & $\begin{array}{c}\text { CCTGAGATTTCACC } \\
\text { TCACAGCA }\end{array}$ & $148330186-148330327$ \\
\hline A186_gULRN01_30 & $\begin{array}{l}\text { ACAAGCTTGTTTT } \\
\text { CAGAATTTCTGT }\end{array}$ & $\begin{array}{c}\text { TGCATTCCCAAGAT } \\
\text { TACATGCT }\end{array}$ & $148363769-148363904$ \\
\hline A186_gULRN01_31 & $\begin{array}{l}\text { CTCGCAAGCACA } \\
\text { CCAAAATCT }\end{array}$ & $\begin{array}{c}\text { TCAGGTCCAGTAGT } \\
\text { TTTTCAGGT }\end{array}$ & $148387678-148387840$ \\
\hline A186_gULRN01_32 & $\begin{array}{l}\text { TCCCCTCAGAAG } \\
\text { AAACCCCT }\end{array}$ & $\begin{array}{c}\text { ACCCTAGAGTATAG } \\
\text { AGTAGAGGAGA }\end{array}$ & $148267287-148267422$ \\
\hline A186_gULRN01_33 & $\begin{array}{l}\text { TGTTTTCTAAACT } \\
\text { GCTTTCACCTGT }\end{array}$ & $\begin{array}{c}\text { TGTGACCATAGGTA } \\
\text { TGTGGGT }\end{array}$ & $148287640-148287840$ \\
\hline
\end{tabular}

* Rattus norvegicus genome build version RGSC 6.0/rn6 


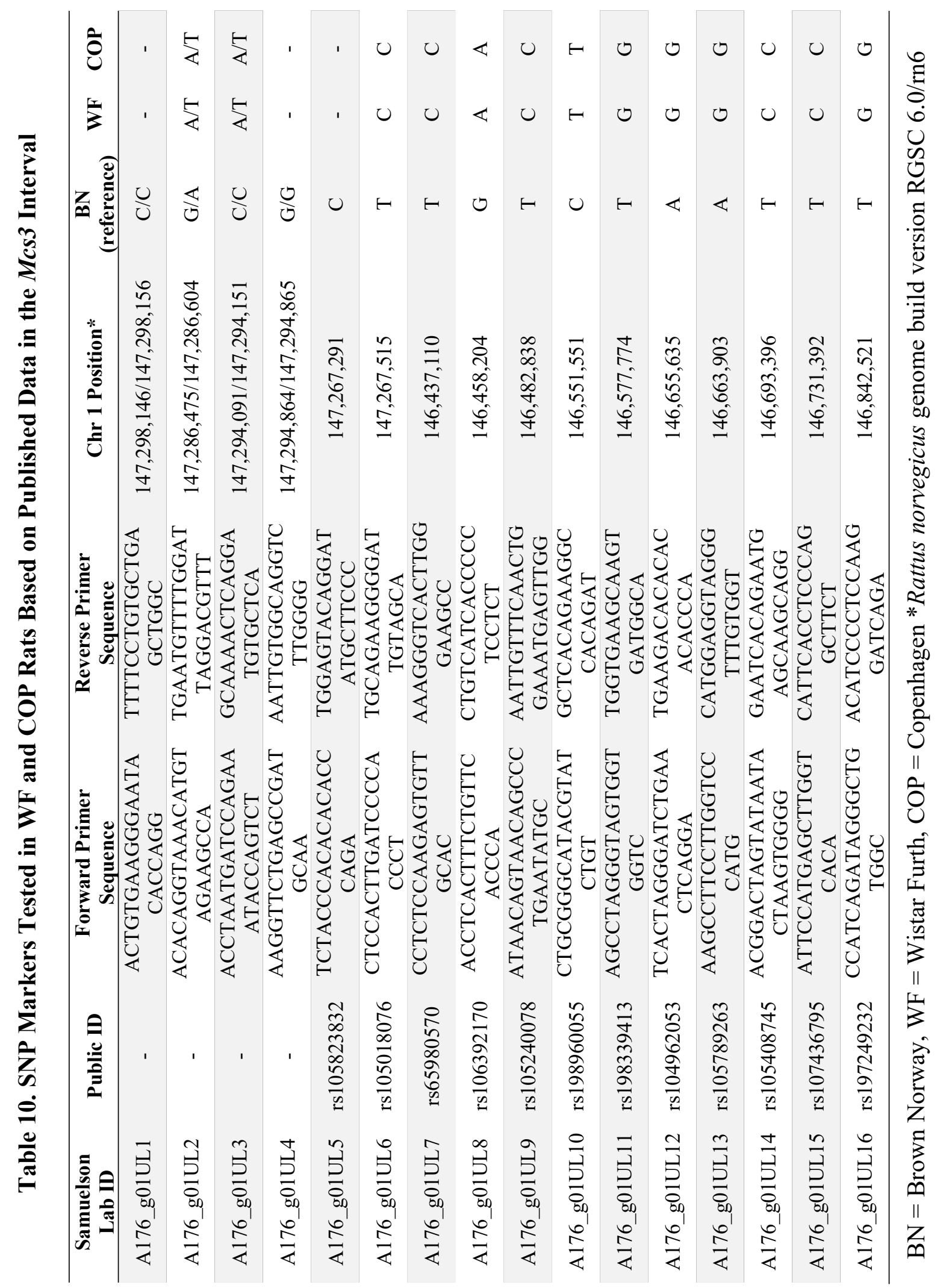


carcinoma (Fig 15). Thus, each line appeared to develop invasive ductal carcinomas with no discernable differences in tumor histopathologies between lines.

\section{Mcs3 has a pleiotropic effect on mammary tumor susceptibility and body mass which is not due to a delay in mammary gland development}

In addition to mammary tumor susceptibility, there was a pleiotropic effect of Mcs 3 on body mass. At 15 weeks post-DMBA administration there was a significant difference in body mass in line $\mathrm{J}$ females as compared to the other Mcs 3 congenic lines and WF strain (Fig 16). Lines $\mathrm{K}$ and $\mathrm{H}$ displayed an average body mass of $210 \mathrm{~g}$, WF showed an average of $201 \mathrm{~g}$, and line I showed an average of $202 \mathrm{~g}$. Line J was significantly lower with an average body mass of $179 \mathrm{~g}$ (one-way ANOVA followed by Dunnett's post hoc test $\mathrm{p}<0.0001)$ (Table 11). Body mass data collection was extended to the regular breeding colonies of WF and line $\mathrm{J}$ to determine if this lower body mass phenotype was solely attributable to the effects of DMBA. Weights were recorded at timepoints of 4, 8, and 12 weeks of age in order to capture stages of development (weaning age, puberty, and breeding age) (Fig 17) (Table 12). There was no significant difference between lines or sex at 4 weeks of age. There was a significant difference between WF males and line J males at 8 weeks of age, with WF weighing an average of $186 \mathrm{~g}$ and line J weighing $150 \mathrm{~g}$ (2-way ANOVA followed by Tukeys post hoc test $\mathrm{p}<0.0001)$. There was also a significant difference between WF and line J males at 12 weeks of age, with WF displaying an average body mass of $274 \mathrm{~g}$ and line $\mathrm{J}$ displaying $244 \mathrm{~g}$ (2-way ANOVA followed by Tukeys post hoc test $\mathrm{p}<0.0001$ ). Contrasting with the body mass data from DMBA females, line $\mathrm{J}$ 


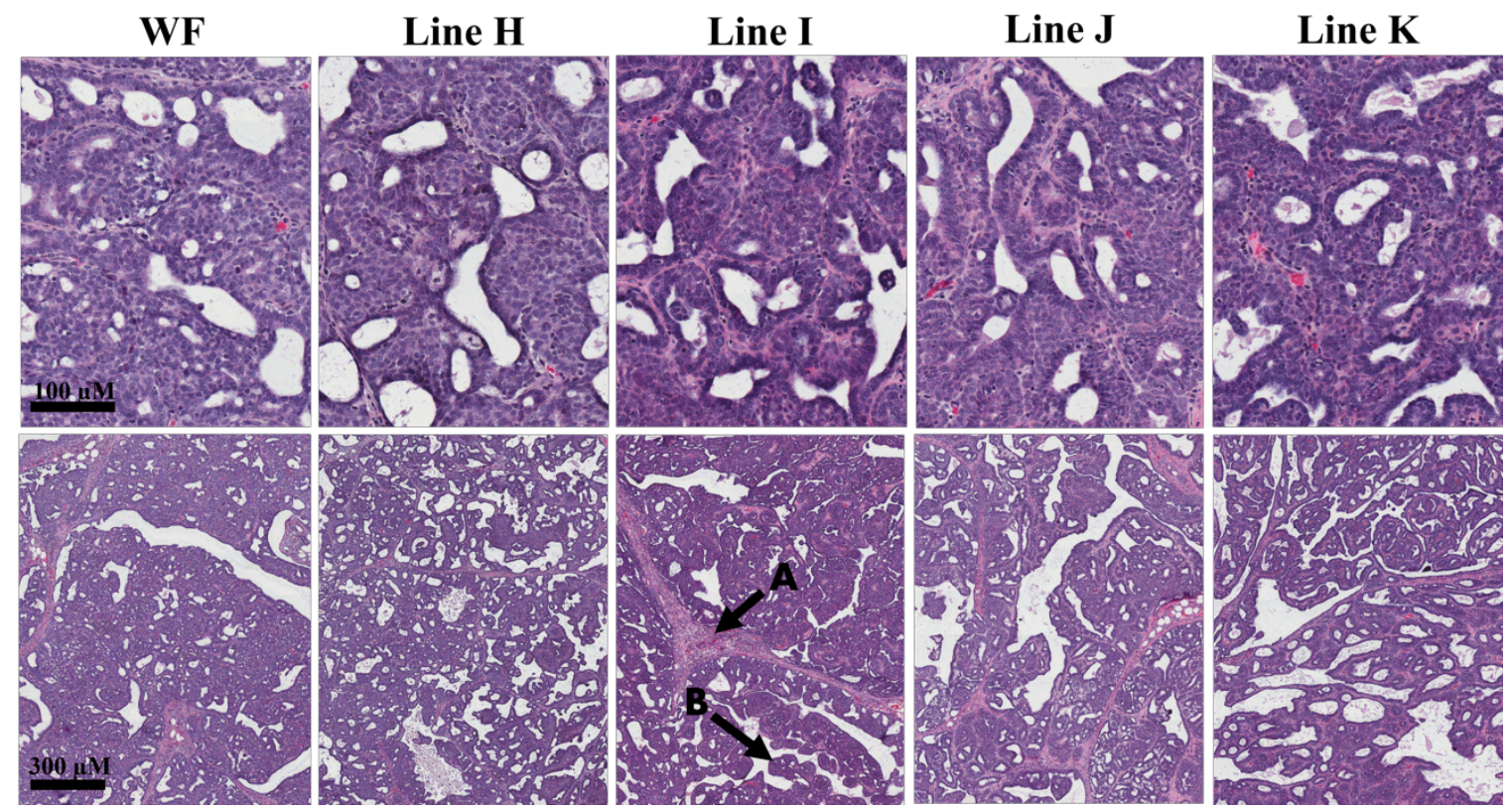

Figure 14: WF.COP Mcs3 congenic lines develop invasive papillary carcinomas. H\&E

staining of DMBA-induced mammary tumors from WF strain and derived congenic lines (columns). The top and bottom rows show differing magnifications. The papillary pattern is defined by fibrovascular cores that form a network throughout the tumor (arrow A), with neoplastic epithelial cells that grow and expand the lesion via papillary projections (arrow B). Images were taken at 40x magnification on an Aperio ImageScope CS2. 


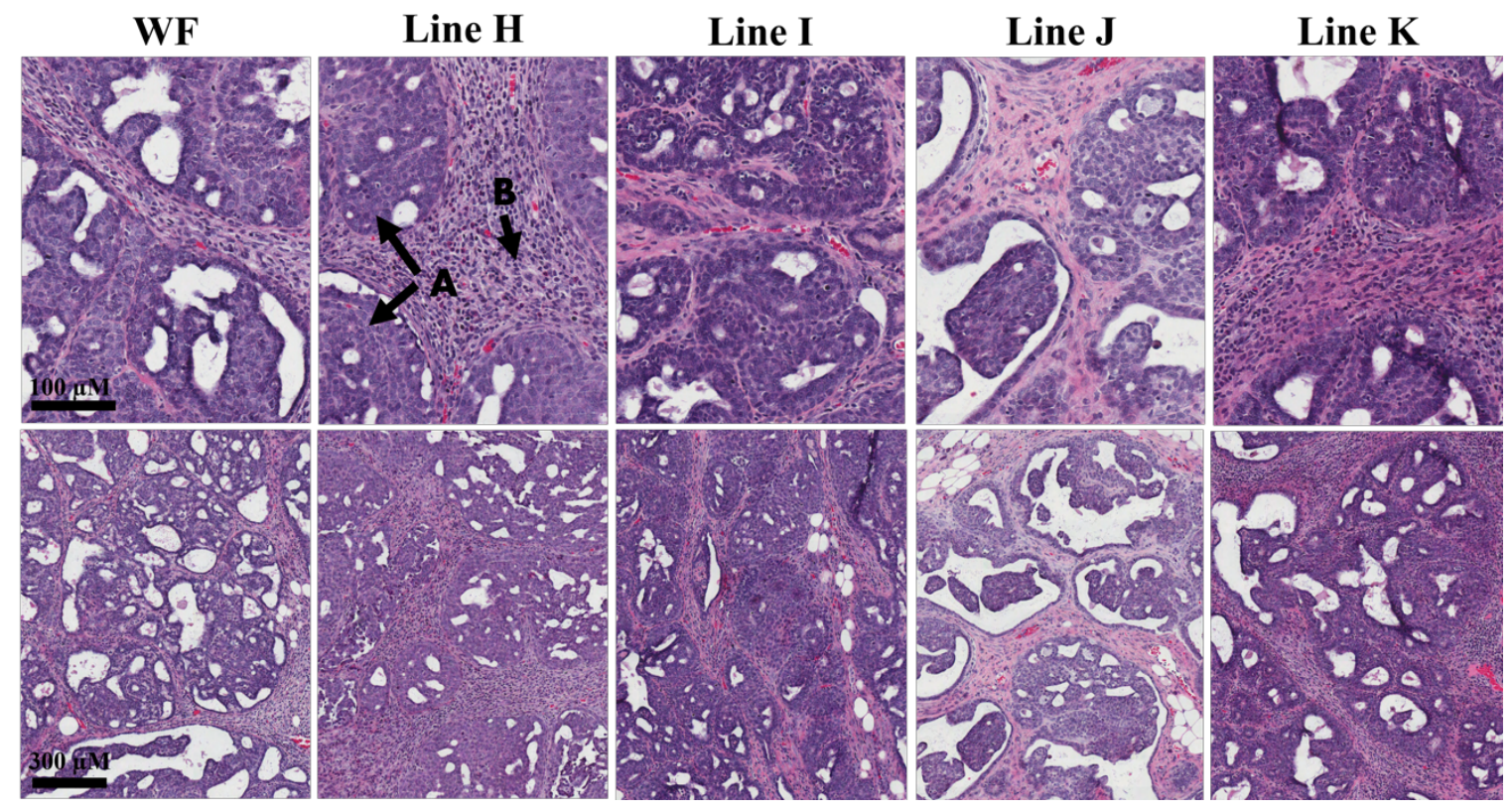

Figure 15: WF.COP Mcs3 congenic lines develop invasive cribriform carcinomas.

H\&E staining of DMBA-induced mammary tumors from WF strain and derived congenic lines (columns). The top and bottom rows show differing magnifications. The cribriform pattern is defined by neoplastic epithelial cell tumor nests (arrows A) within a surrounding desmoplastic stroma (arrow B). Images were taken at 40x magnification on an Aperio ImageScope CS2. 


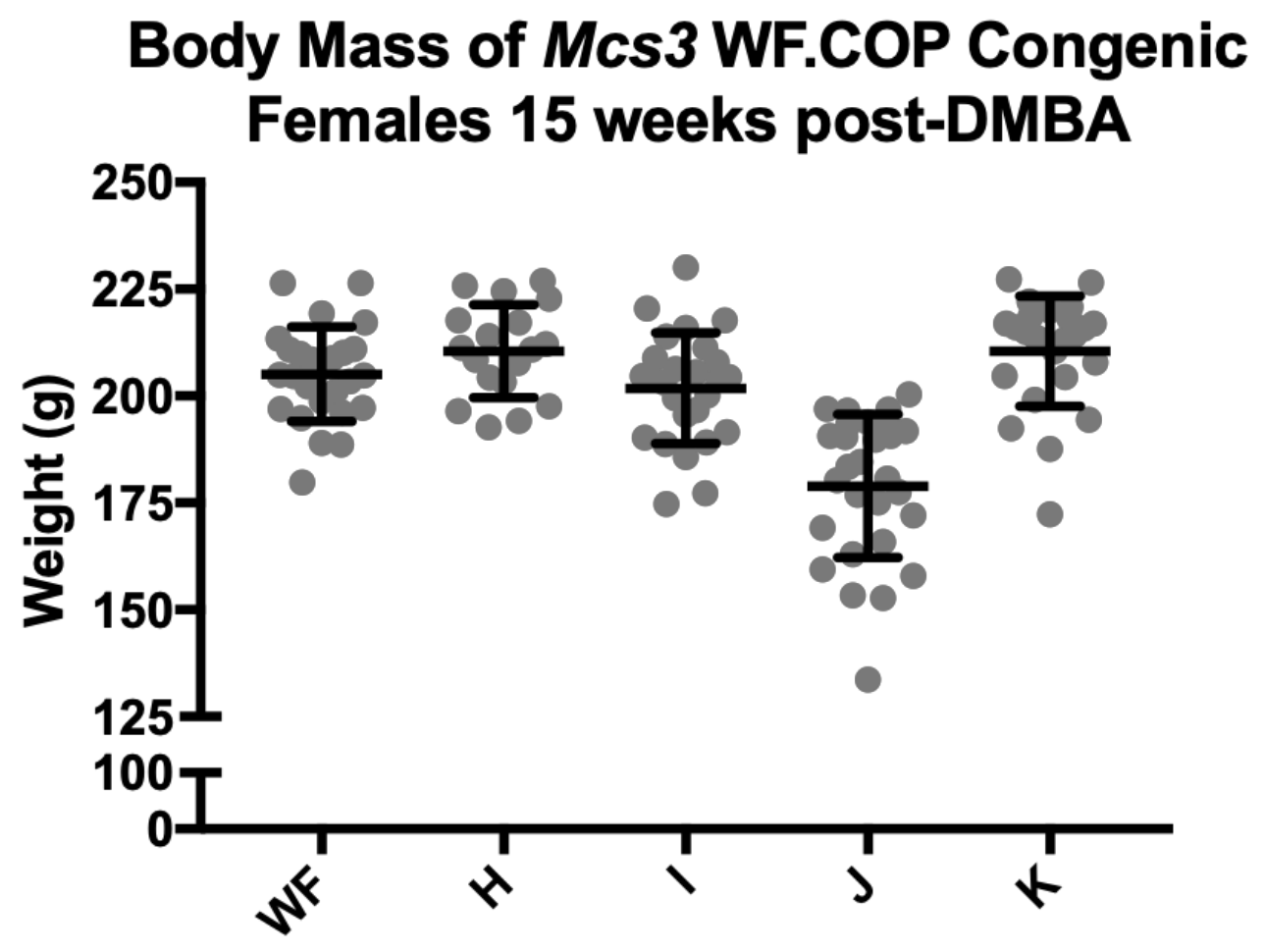

Figure 16: Body mass of Mcs3 experimental females at 15 weeks post-DMBA administration. The WF strain and $M c s 3$ congenic lines were weighed at 23 weeks of age at the time of tumor multiplicity counts. A one-way ANOVA was performed $(\mathrm{p}<0.0001)$, followed by Dunnetts's post hoc tests comparing each line to the control WF strain. Line J was significantly different from WF $(\mathrm{p}<0.0001)$. 
Table 11. Body Mass of Mcs3 WF.COP Congenic Females 15 weeks Post-DMBA Administration

\begin{tabular}{cccc}
\hline Mcs3 WF.COP Line & Mean (SD) weight (g) & N & $\begin{array}{c}\text { p-value against } \\
\text { WF/NHsd }\end{array}$ \\
\hline H & $210(11)$ & 18 & 0.9560 \\
I & $202(13)$ & 26 & $>0.9999$ \\
J & $179(17)$ & 28 & $<0.0001$ \\
K & $210(13)$ & 25 & 0.4788 \\
WF/NHsd & $201(21)$ & 26 & - \\
\hline
\end{tabular}




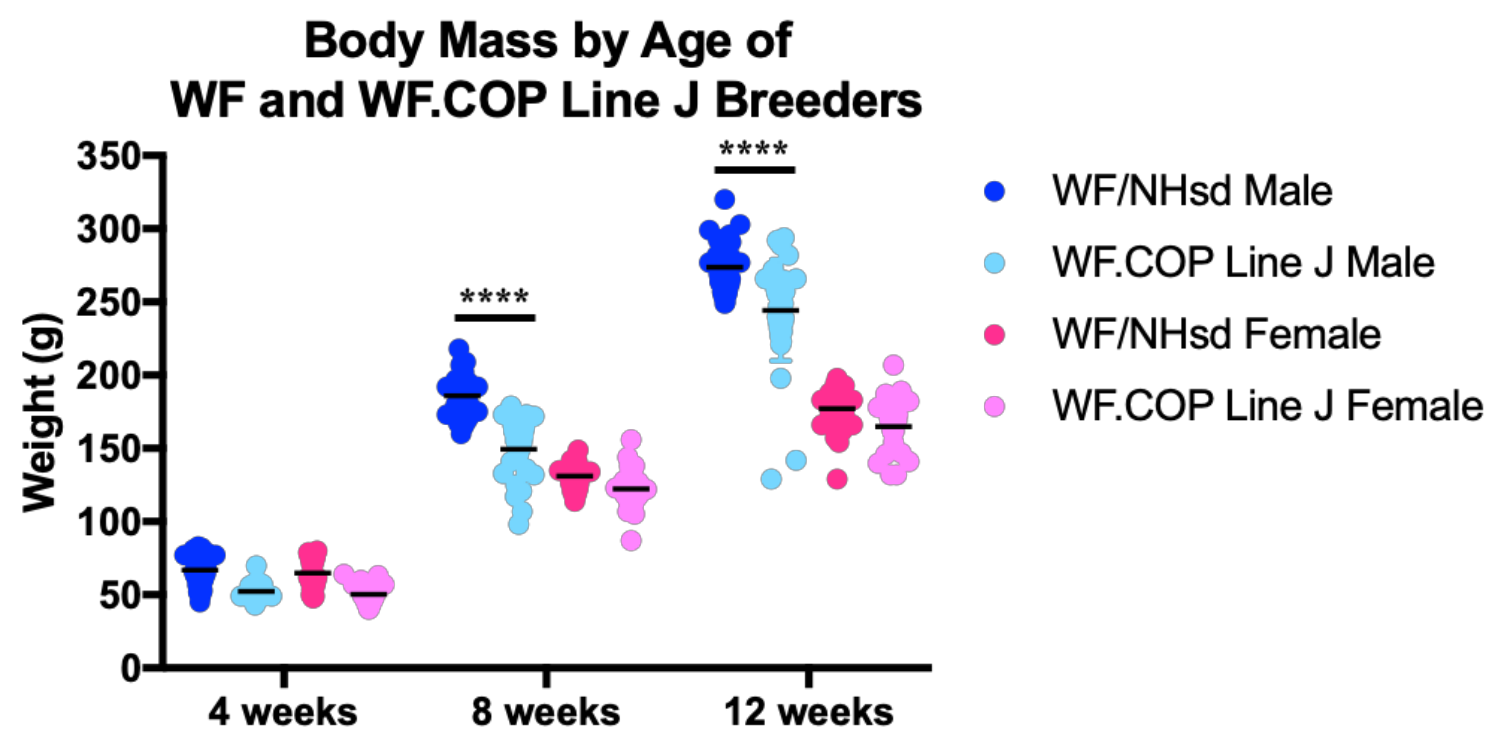

Figure 17: Body mass of WF and Line $\mathbf{J}$ rats over development. Male and female rats of each strain were weighed at weaning age ( 4 weeks), puberty ( 8 weeks), and breeding age (12 weeks). There was no significant difference in body mass at 4 weeks of age or in any age comparing WF or line $\mathrm{J}$ females. WF and line $\mathrm{J}$ males had significantly different weights at 8 and 12 weeks of age (2-way ANOVA followed by Tukeys multiple comparisons post hoc test $\mathrm{p}<0.0001)$. 
Table 12. Body Mass Summary Data of WF/NHsd and WF.COP Line J Breeders

\begin{tabular}{cllccc}
\hline Strain & Sex & Housing & $\begin{array}{c}\text { Age } \\
(\text { weeks })\end{array}$ & Mean (SD) weight (g) & N \\
\hline WF/NHsd & Male & Regular & 4 & $67(11)$ & 31 \\
WF/NHsd & Male & Regular & 8 & $186(15)$ & 27 \\
WF/NHsd & Male & Regular & 12 & $267(29)$ & 37 \\
\hline WF.COP Line J & Male & Regular & 4 & $52(6)$ & 17 \\
WF.COP Line J & Male & Regular & 8 & $149(21)$ & 28 \\
WF.COP Line J & Male & Regular & 12 & $244(37)$ & 29 \\
\hline WF/NHsd & Female & Regular & 4 & $65(11)$ & 22 \\
WF/NHsd & Female & Regular & 8 & $130(8)$ & 31 \\
WF/NHsd & Female & Regular & 12 & $175(14)$ & 38 \\
\hline WF.COP Line J & Female & Regular & 4 & $51(8)$ & 20 \\
WF.COP Line J & Female & Regular & 8 & $122(13)$ & 29 \\
WF.COP Line J & Female & Regular & 12 & $168(18)$ & 23 \\
\hline WF/NHsd & Female & Female Only & 8 & $134(5)$ & 15 \\
WF/NHsd & Female & Female Only & 12 & $178(7)$ & 15 \\
\hline WF.COP Line J & Female & Female Only & 8 & $114(9)$ & 24 \\
WF.COP Line J & Female & Female Only & 12 & $152(13)$ & 24 \\
\hline
\end{tabular}


females in general housing displayed no significant difference in weight from the WF strain. Since DMBA females are kept in a separate room that only houses female rats, WF and line $\mathrm{J}$ females (not exposed to DMBA) were moved to this room and body mass data re-collected (Fig 18). This time there was a significant difference between WF and line J females at 4 weeks of age ( $65 \mathrm{~g}$ vs $50 \mathrm{~g}), 8$ weeks of age (134g vs $113 \mathrm{~g})$ and 12 weeks of age (178g and 152g) (2-way ANOVA followed by Tukeys post hoc test $\mathrm{p}<0.0001)$.

To explore if reduced body mass was reflected as a delay in mammary gland growth and development, mammary glands were collected and H\&E stained from WF and line $\mathrm{J}$ females at 4, 8 and 12 weeks of age (Fig 19). Line J and WF females displayed similar morphologies of mixed terminal end buds (TEBs) and ductal structures at 4 weeks of age. At 8 weeks of age, both rat lines displayed a mammary gland histology of mostly TEB structures. Adult line J and WF rats at 12 weeks of age exhibited a largely ductal histology. There were no discernable differences in gross mammary gland histology or morphological structure between rat lines at these developmental timepoints.

\section{Human Orthologs to the Newly Delimited Rat Mcs3}

\section{Comparative genomics identifies human syntenic regions to rat Mcs3 with orthologous genes}

Using the convert genomes function in the UCSC Genome Browser, the newly delimited rat $M c s 3(7.2 \mathrm{Mb})$ was found to align to three orthologous human syntenic regions on chromosomes 11 and 15 (Table 13). The July 2014 rat assembly (build 6.0/rn6) and December 2013 human assembly (build GRCh38/hg38) at the UCSC Genome Browser 


\section{Body Mass by Age of WF and WF.COP Line $\mathbf{J}$ Females Raised in all Female Enviroment}

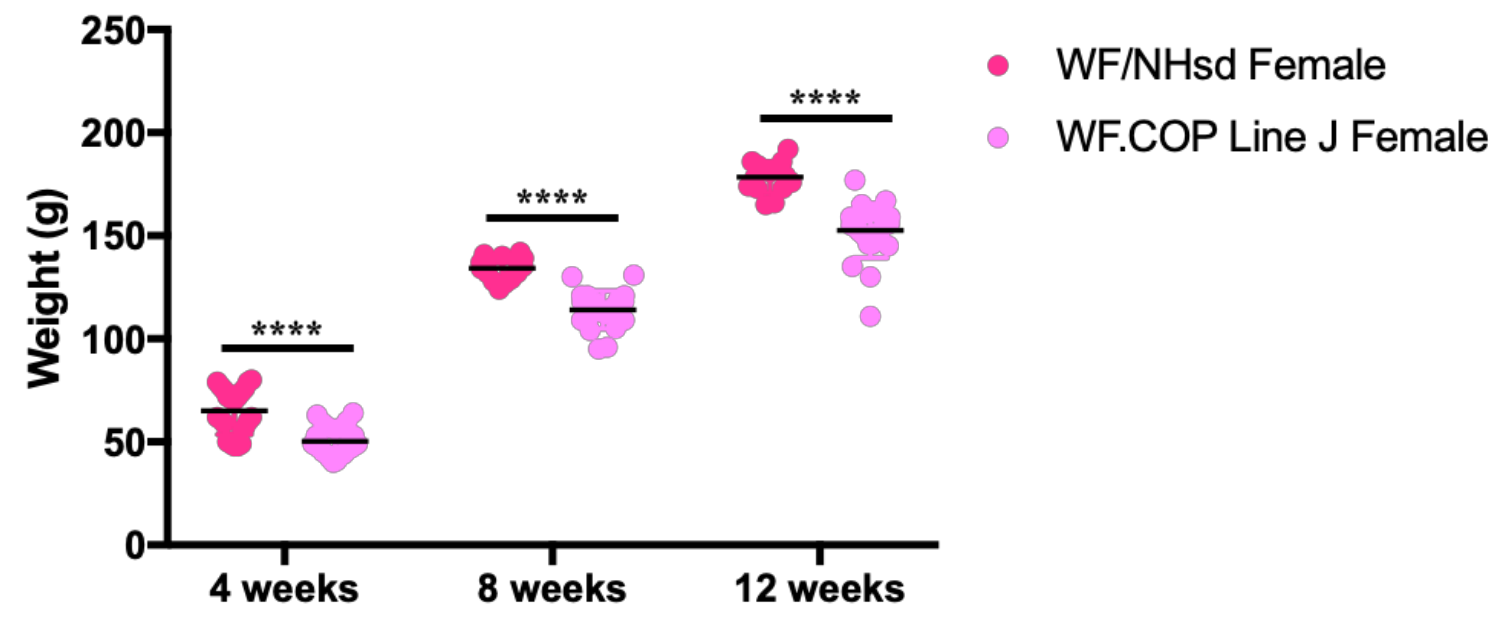

Figure 18: Body mass of $W F$ and Line $J$ females raised in an all-female environment.

Female rats of each strain were kept in all female housing and weighed at 4, 8, and 12 weeks of age. There was a significant effect of strain on body mass at all stages of development (2-way ANOVA p<0.0001). 
A

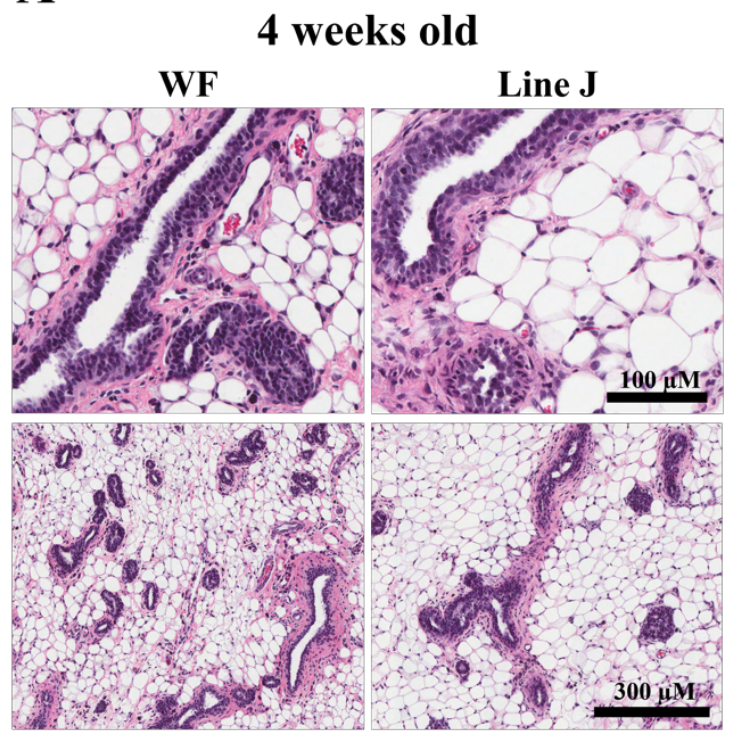

B

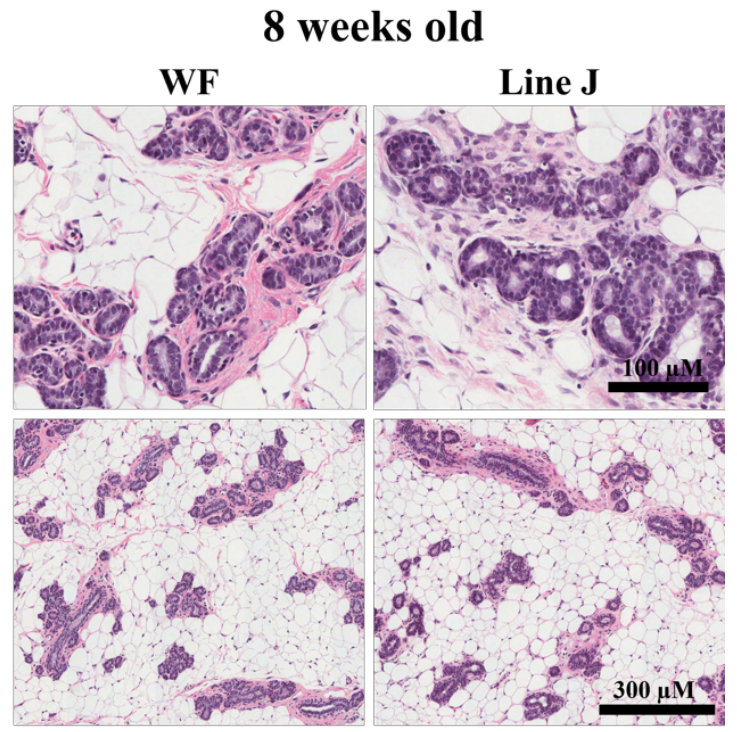

C

12 weeks old

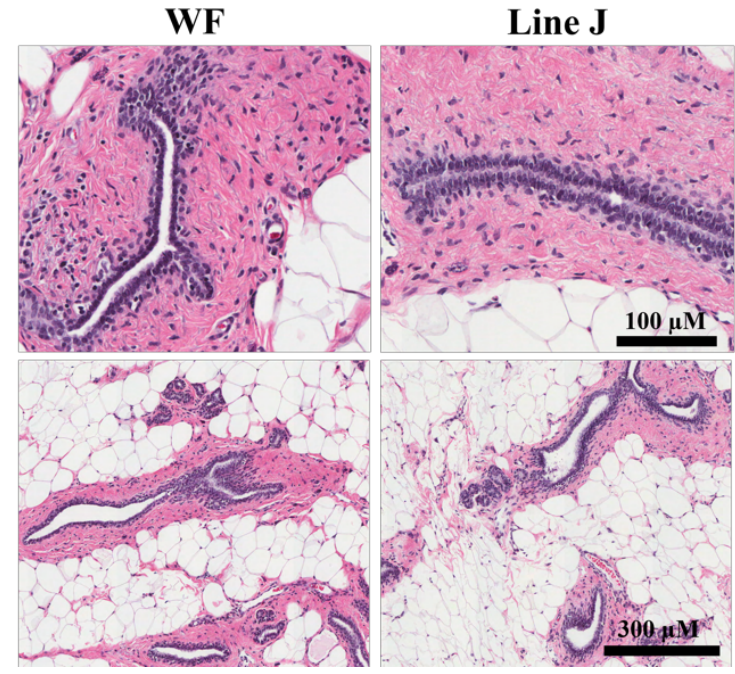


Figure 19: Rat mammary gland histology in WF and line $J$ females over development.

H\&E staining of mammary glands, top and bottom rows display different magnification. Images were taken at 40x magnification on an Aperio ImageScope CS2. A: mammary gland morphology is a mix of ductal and terminal end bud (TEB) epithelial structures embedded throughout the adipocyte matrix at the start of puberty in both WF and line J. B: TEB structures have reached maximal numbers during puberty and appear as the predominant morphology in WF and line J females. C: TEB structures are no longer readily apparent in the adult mammary gland. Greater developed ductal structures within a more pronounced fibrous stroma appear as the major epithelial morphology in WF and line $\mathrm{J}$ females. 
table browser function were used to annotate rat $M c s 3$ and human syntenic regions. A list of genes encompassed in these regions are given in table 13, and non-coding DNA given in Table 15. Orthologous syntenic regions to rat Mcs3 were also annotated in the mouse genome (December 2011 assembly, build GRCm38/mm10). This was done to potentially place human syntenic genes that were not annotated in rat $M c s 3$ since the human and rat genomes are more divergent than human and mouse. However, no genes or non-coding DNA was shared exclusively between the mouse and human syntenic regions. Overall, 23 orthologous genes were found in common between rat $M c s 3$ and human syntenic regions, with 12 genes annotated in the human only and 20 in the rat only. A majority of these ratonly genes encode olfactory receptors not found in the human. There are also 30 lncRNAs encoded in rat Mcs 3 and 16 lncRNAs and 4 miRNAs in the human syntenic regions.

\section{Human orthologous genes and variants have associations with cancer and body mass traits}

PubMed was used to data mine $M c s 3$ and human orthologous genes associated with cancer, which are listed in Table 16. The human orthologous syntenic regions were also analyzed for breast cancer disease and associated traits using the NHGRI-EBI Catalog of human genome-wide association studies. Two of the human syntenic regions at chr15:80005820-82285404 and chr15:83134545-84130720 possessed 72 relevant variants. Table 16 lists overlapping GWAS SNPs and associated traits within these regions, which include breast cancer specific mortality in ER+ breast cancer, age to menarche, body mass index, waist-hip ratio, fat body mass, body height, lean body mass, BMI-adjusted hip circumference, BMI-adjusted waist circumference, body fat distribution, body fat 
Table 13. Orthologous Human Loci Syntenic to Rat Mcs3*

\begin{tabular}{ccc}
\hline $\begin{array}{c}\text { Human chromosome position } \\
\text { (GRCh38/hg38) }\end{array}$ & Percent of bases & Percent of span \\
\hline chr15:80005820-82285404 & 12 & 31.6 \\
chr15:83134545-84130720 & 4.4 & 13.2 \\
chr11:89365341-89617253 & 1.1 & 4.2 \\
\hline
\end{tabular}

*Mcs3 position Chr1:143700228-150929594 (assembly RGSC 6.0/Rn6) 
Table 14. List of Genes and Genomic Position in Rat Mcs3 and the Orthologous Human Regions

\begin{tabular}{|c|c|c|c|c|c|}
\hline \multicolumn{3}{|c|}{ Rattus norvegicus (RGSC 6.0/Rn6) } & \multicolumn{3}{|c|}{ Homo sapiens (GRCh38/hg38) } \\
\hline Gene & $\begin{array}{c}\text { Accession } \\
\text { number }\end{array}$ & $\begin{array}{l}\text { Genomic } \\
\text { Position }\end{array}$ & Gene & $\begin{array}{c}\text { Accession } \\
\text { number }\end{array}$ & $\begin{array}{l}\text { Genomic } \\
\text { Position }\end{array}$ \\
\hline Tm6sf1 & NM_001108490.1 & $\begin{array}{l}\text { Chr1: } 143657606- \\
\quad 143703759\end{array}$ & TM6SF1 & NM_023003.5 & $\begin{array}{l}\text { Chr15: } 83107644- \\
\quad 83137408\end{array}$ \\
\hline Hdgfrp3 & NM_145785.1 & $\begin{array}{l}\text { Chr1: } 143702863- \\
143751789\end{array}$ & HDGFL3 & NM_016073.4 & $\begin{array}{l}\text { Chr15: } 83127752- \\
\quad 83207823\end{array}$ \\
\hline Bnc1 & NM_001108916.1 & $\begin{array}{l}\text { Chr1:143869439- } \\
143878077\end{array}$ & $\mathrm{BNC1}$ & NM_001717.4 & $\begin{array}{l}\text { Chr15:83255883- } \\
\quad 83284664\end{array}$ \\
\hline Sh3gl3 & NM_031238.2 & $\begin{array}{l}\text { Chr1:144069640- } \\
144200030\end{array}$ & SH3GL3 & NM_003027.5 & $\begin{array}{l}\text { Chr15:83447340- } \\
\quad 83618743\end{array}$ \\
\hline Adamtsl3 & NM_001107533.2 & $\begin{array}{l}\text { Chr1:144238957- } \\
\quad 144561065\end{array}$ & ADAMTSL3 & NM_207517.3 & $\begin{array}{l}\text { Chr15:83654122- } \\
\quad 84039842\end{array}$ \\
\hline Saxo2 & NM_001106277.1 & $\begin{array}{l}\text { Chr1:144586259- } \\
\quad 144601327\end{array}$ & $\mathrm{SAXO} 2$ & NM_001348699.2 & $\begin{array}{l}\text { Chr15:82262817- } \\
\quad 82284927\end{array}$ \\
\hline Efl1 & NM_001107534.1 & $\begin{array}{l}\text { Chr1:144601424- } \\
144740634\end{array}$ & EFL1 & NM_024580.6 & $\begin{array}{l}\text { Chr15:82130232- } \\
82262734\end{array}$ \\
\hline Mex3b & NM_001191626.1 & $\begin{array}{l}\text { Chr1:144831522- } \\
\quad 144835758\end{array}$ & MEX3B & NM_032246.6 & $\begin{array}{l}\text { Chr15:82041777- } \\
\quad 82046018\end{array}$ \\
\hline Tmc3 & NM_001170432.1 & $\begin{array}{l}\text { Chr1:145715968- } \\
145760623\end{array}$ & TMC3 & NM_001080532.3 & $\begin{array}{l}\text { Chr15:81331087- } \\
\quad 81374213\end{array}$ \\
\hline Stard5 & NM_001192011.1 & $\begin{array}{l}\text { Chr1:145770134- } \\
145780001\end{array}$ & STARD5 & NM_181900.3 & $\begin{array}{l}\text { Chr15: } 81309052- \\
\quad 81324141\end{array}$ \\
\hline Il16 & NM_001105749.1 & $\begin{array}{c}\text { Chr1:145781927- } \\
145870912\end{array}$ & IL16 & NM_004513.6 & $\begin{array}{l}\text { Chr15:81296969- } \\
81314058\end{array}$ \\
\hline Cfap161 & NM_001100581.1 & $\begin{array}{l}\text { Chr1:145907092- } \\
\quad 145931583\end{array}$ & CFAP161 & NM_173528.4 & $\begin{array}{l}\text { Chr15:81134269- } \\
\quad 81149179\end{array}$ \\
\hline Mesdc1 & NM_001013149.1 & $\begin{array}{c}\text { Chr1:146027820- } \\
\quad 146029840\end{array}$ & TLNRD1 & NM_022566.3 & $\begin{array}{l}\text { Chr15:81000922- } \\
\quad 81005788\end{array}$ \\
\hline Mesdc2 & NM_001008345.1 & $\begin{array}{l}\text { Chr1:146037425- } \\
146043097\end{array}$ & MESD & NM_015154.3 & $\begin{array}{l}\text { Chr15:80975751- } \\
\quad 80989819\end{array}$ \\
\hline Abhd17c & NM_001100736.1 & $\begin{array}{l}\text { Chr1:146248504- } \\
146289465\end{array}$ & ABHD17C & NM_021214.2 & $\begin{array}{l}\text { Chr15:80695309- } \\
\quad 80755621\end{array}$ \\
\hline Arnt2 & NM_012781.3 & $\begin{array}{l}\text { Chr1:146399216- } \\
146556437\end{array}$ & ARNT2 & NM_014862.4 & $\begin{array}{l}\text { Chr15:80404381- } \\
\quad 80597933\end{array}$ \\
\hline Fah & NM_017181.2 & $\begin{array}{c}\text { Chr1:146713662- } \\
146736339\end{array}$ & FAH & NM_000137.3 & $\begin{array}{l}\text { Chr15:80152998- } \\
\quad 80186949\end{array}$ \\
\hline Zfand6 & NM_001007630.1 & $\begin{array}{l}\text { Chr1:146745849- } \\
\quad 146823762\end{array}$ & ZFAND6 & NM_019006.4 & $\begin{array}{l}\text { Chr15:80059675- } \\
\quad 80138393\end{array}$ \\
\hline F8a1 & NM_001109323.1 & $\begin{array}{l}\text { Chr1:147021435- } \\
147022950\end{array}$ & F8A3 & NM_001007524.1 & $\begin{array}{c}\text { ChrX: } 15545748415 \\
5458600\end{array}$ \\
\hline Cyp2c6v1 & NM_001013904.1 & $\begin{array}{l}\text { Chr1:147713900- } \\
147814395\end{array}$ & CYP2C19 & NM_000769.4 & $\begin{array}{l}\text { Chr10:94762680- } \\
\quad 94855547\end{array}$ \\
\hline Сур2c7 & NM_017158.2 & $\begin{array}{l}\text { Chr1:147422998- } \\
148119857\end{array}$ & \multicolumn{3}{|c|}{ No human ortholog } \\
\hline Mpp1 & NM_001037659.1 & $\begin{array}{l}\text { Chrl:148450212- } \\
148458945\end{array}$ & \multicolumn{3}{|c|}{ No human ortholog } \\
\hline Folh1 & NM_057185.2 & $\begin{array}{l}\text { Chr1:150323767- } \\
150395415\end{array}$ & FOLH1 & NM_004476.3 & $\begin{array}{l}\text { Chr11:49145091- } \\
\quad 49208602\end{array}$ \\
\hline Nox4 & NM_053524.1 & $\begin{array}{l}\text { Chr1:150797201- } \\
\quad 150974737\end{array}$ & NOX4 & NM_016931.5 & $\begin{array}{l}\text { Chr1 1:89324352- } \\
\quad 89491375\end{array}$ \\
\hline Cemip & XM_218855.9 & $\begin{array}{l}\text { Chr1:146069773- } \\
\quad 146226320\end{array}$ & CEMIP & NM_018689.3 & $\begin{array}{l}\text { Chr15:80779369- } \\
\quad 80951771\end{array}$ \\
\hline Vbp1 & XM_006229645.3 & $\begin{array}{l}\text { Chr1:148425045- } \\
\quad 148443505\end{array}$ & \multicolumn{3}{|c|}{ No human ortholog } \\
\hline
\end{tabular}




\begin{tabular}{|c|c|c|}
\hline Vom $2 \mathrm{r} 40$ & NM_173317.1 & $\begin{array}{c}\text { Chr1:148927531- } \\
148974509\end{array}$ \\
\hline Vom $2 \mathrm{r} 42$ & NM_001099511.1 & $\begin{array}{c}\text { Chr1:149132319- } \\
149175214\end{array}$ \\
\hline Vom 2 r43 & NM_001099512.1 & $\begin{array}{c}\text { Chr1:149506821- } \\
149529350\end{array}$ \\
\hline Olr12 & NM_001000540.1 & $\begin{array}{c}\text { Chr1:149578356- } \\
149579280\end{array}$ \\
\hline Olr16 & NM_001000694.1 & $\begin{array}{c}\text { Chr1:149674492- } \\
149675443\end{array}$ \\
\hline Olr17 & NM_001000937.1 & $\begin{array}{c}\text { Chr1:149682400- } \\
149683357\end{array}$ \\
\hline Olr13 & NM_001000116.1 & $\begin{array}{c}\text { Chr1:149786381- } \\
149787308\end{array}$ \\
\hline Olr14 & NM_001000695.1 & $\begin{array}{c}\text { Chr1:149816123- } \\
149817065\end{array}$ \\
\hline Olr19 & NM_001000117.1 & $\begin{array}{c}\text { Chr1:149913646- } \\
\text { 149914606 }\end{array}$ \\
\hline Olr20 & NM_001000118.1 & $\begin{array}{c}\text { Chr1:149963546- } \\
149964539\end{array}$ \\
\hline Olr23 & NM_001000119.1 & $\begin{array}{c}\text { Chr1:150067241- } \\
150068237\end{array}$ \\
\hline Olr24 & NM_001000693.1 & $\begin{array}{c}\text { Chrl:150100235- } \\
150101177\end{array}$ \\
\hline Olr25 & NM_001000692.1 & $\begin{array}{c}\text { Chrl:150130083- } \\
150131076\end{array}$ \\
\hline Olr27 & NM_001000936.1 & $\begin{array}{c}\text { Chrl:150172374- } \\
150173316\end{array}$ \\
\hline Olr29 & NM_001000691.1 & $\begin{array}{c}\text { Chr1:150209395- } \\
150210403\end{array}$ \\
\hline Olr30 & NM_001000120.1 & $\begin{array}{c}\text { Chrl:150225372- } \\
150226302\end{array}$ \\
\hline Olr32 & NM_001000690.1 & $\begin{array}{c}\text { Chr1:150261205- } \\
150262213\end{array}$ \\
\hline Olr34 & NM_001000689.1 & $\begin{array}{c}\text { Chrl:150310318- } \\
150311257\end{array}$ \\
\hline \multicolumn{3}{|c|}{ No rat ortholog } \\
\hline \multicolumn{3}{|c|}{ No rat ortholog } \\
\hline \multicolumn{3}{|c|}{ No rat ortholog } \\
\hline \multicolumn{3}{|c|}{ No rat ortholog } \\
\hline \multicolumn{3}{|c|}{ No rat ortholog } \\
\hline \multicolumn{3}{|c|}{ No rat ortholog } \\
\hline \multicolumn{3}{|c|}{ No rat ortholog } \\
\hline \multicolumn{3}{|c|}{ No rat ortholog } \\
\hline \multicolumn{3}{|c|}{ No rat ortholog } \\
\hline \multicolumn{3}{|c|}{ No rat ortholog } \\
\hline \multicolumn{3}{|c|}{ No rat ortholog } \\
\hline & No rat ortholog & \\
\hline
\end{tabular}

\begin{tabular}{|c|c|c|}
\hline \multicolumn{3}{|c|}{ No human ortholog } \\
\hline \multicolumn{3}{|c|}{ No human ortholog } \\
\hline \multicolumn{3}{|c|}{ No human ortholog } \\
\hline OR13G1 & NM_001005487.1 & $\begin{array}{c}\text { Chr1:247672117- } \\
247673041\end{array}$ \\
\hline \multicolumn{3}{|c|}{ No human ortholog } \\
\hline \multicolumn{3}{|c|}{ No human ortholog } \\
\hline \multicolumn{3}{|c|}{ No human ortholog } \\
\hline \multicolumn{3}{|c|}{ No human ortholog } \\
\hline \multicolumn{3}{|c|}{ No human ortholog } \\
\hline \multicolumn{3}{|c|}{ No human ortholog } \\
\hline \multicolumn{3}{|c|}{ No human ortholog } \\
\hline \multicolumn{3}{|c|}{ No human ortholog } \\
\hline \multicolumn{3}{|c|}{ No human ortholog } \\
\hline \multicolumn{3}{|c|}{ No human ortholog } \\
\hline \multicolumn{3}{|c|}{ No human ortholog } \\
\hline \multicolumn{3}{|c|}{ No human ortholog } \\
\hline \multicolumn{3}{|c|}{ No human ortholog } \\
\hline \multicolumn{3}{|c|}{ No human ortholog } \\
\hline CTXND1 & NM_001352888.2 & $\begin{array}{c}\text { Chr15:80195480- } \\
80252213\end{array}$ \\
\hline OR6A2 & NM_003696.2 & $\begin{array}{c}\text { Chr11:6794524- } \\
6795908\end{array}$ \\
\hline TWNK & NM_021830.5 & $\begin{array}{c}\text { Chr10:100987542- } \\
100994403\end{array}$ \\
\hline OR6F1 & NM_001005286.1 & $\begin{array}{c}\text { Chr1:247711828- } \\
247712755\end{array}$ \\
\hline OR10A5 & NM_178168.1 & $\begin{array}{c}\text { Chr11:684568- } \\
6846636\end{array}$ \\
\hline H2AB3 & NM_080720.1 & $\begin{array}{c}\text { ChrX:155459418- } \\
155459935\end{array}$ \\
\hline OR14A2 & NM_001355292.1 & $\begin{array}{c}\text { Chr1:247722956- } \\
247748158\end{array}$ \\
\hline CYP2C9 & NM_000771.4 & $\begin{array}{c}\text { Chr10:94938657- } \\
94990091\end{array}$ \\
\hline CYP2C8 & NM_000770.3 & $\begin{array}{c}\text { Chr10:95036771- } \\
95069497\end{array}$ \\
\hline CYP4A11 & NM_000778.4 & $\begin{array}{c}\text { Chr1:46929187- } \\
46941476\end{array}$ \\
\hline BCL2A1 & NM_004049.4 & $\begin{array}{c}\text { Chr15:79960891- } \\
79971196\end{array}$ \\
\hline ZSCAN5A & NM_001322072.1 & $\begin{array}{c}\text { Chr19:56221302- } \\
56368383\end{array}$ \\
\hline
\end{tabular}


Table 15. Genome Positions of Rat Mcs3 Non-Coding DNA and Orthologous Human Genome Segments

\begin{tabular}{|c|c|c|c|}
\hline IncRNA/miRNA & Accession number & Genomic Position & Species* \\
\hline LOC102556513 & XR 590364.1 & Chr1:143846546-143861632 & Rat \\
\hline LOC103691175 & XR_590365.1 & Chr1:143930927-143936603 & Rat \\
\hline LOC 108348871 & XR_001835889.1 & Chr1:144778613-144798063 & Rat \\
\hline LOC103691179 & XR_590366.2 & Chr1:145146668-145149394 & Rat \\
\hline LOC103691180 & $\mathrm{XR}^{-} 590367.2$ & Chr1:145360463-145363189 & Rat \\
\hline LOC102552825 & XR_350537.1 & Chr1:145448880-145450795 & Rat \\
\hline LOC103691181 & XR_590368.1 & Chr1:145505885-145521853 & Rat \\
\hline LOC108349601 & XR 001835891.1 & Chr1:145525669-145537559 & Rat \\
\hline LOC108349171 & $\mathrm{XR}^{-} 001835892.1$ & Chr1:146289822-146320389 & Rat \\
\hline LOC108349102 & XR_001835893.1 & Chr1:146346478-146376238 & Rat \\
\hline LOC 102553298 & XR_590369.2 & Chr1:146682691-146706084 & Rat \\
\hline LOC108349602 & XR_001835899.1 & Chr1:146898565-146906850 & Rat \\
\hline LOC690082 & XM_-017590171.1 & Chr1:146966353-146972459 & Rat \\
\hline LOC100911002 & XM_003748903.4 & Chr1:146977349-146989856 & Rat \\
\hline LOC100911047 & XM_006229628.3 & Chr1:147000036-147012436 & Rat \\
\hline LOC100366231 & XM-003748905.4 & Chr1:147060790-147072122 & Rat \\
\hline LOC100911148 & XM_017590168.1 & Chr1:147077010-147083118 & Rat \\
\hline LOC100361434 & XM_017590456.1 & Chr1:147524188-147596924 & Rat \\
\hline LOC102550489 & X⿳亠口冋_590372.2 & Chr1:147641561-147642719 & Rat \\
\hline LOC100911718 & XM_0̄03748910.4 & Chr1:147883945-147887671 & Rat \\
\hline LOC103691183 & X⿳亠口冋 590373.2 & Chr1:148193185-148196949 & Rat \\
\hline LOC100361547 & XM_0̄08759635.1 & Chr1:148232437-148287458 & Rat \\
\hline LOC100911879 & X⿳亠口冋_590374.2 & Chr1:148363574-148380117 & Rat \\
\hline LOC102546990 & XM 006229649.3 & Chr1:148445119-148446339 & Rat \\
\hline LOC100912097 & XM_003748913.1 & Chr1:148862492-148863446 & Rat \\
\hline LOC679574 & XM_008759645.1 & Chr1:149437495-149476747 & Rat \\
\hline LOC100909966 & XM_003748916.2 & Chr1:149602345-149603284 & Rat \\
\hline LOC100910049 & XM- 017590559.1 & Chr1:149632087-149633029 & Rat \\
\hline LOC100909935 & XM_003748915.3 & Chr1:149777725-149778838 & Rat \\
\hline LOC100910009 & XM_003748917.2 & Chr1:149858459-149859555 & Rat \\
\hline LINC00927 & NR_033833.1 & Chr15:80263067-80341805 & Human \\
\hline LOC101929560 & XR_932523.2 & Chr15:80344848-80362464 & Human \\
\hline LOC101929586 & NR_-120363.1 & Chr15:80554608-80562944 & Human \\
\hline LOC107984749 & XR_001751630.2 & Chr15:80896190-80911991 & Human \\
\hline LOC102723985 & XR_001751634.1 & Chr15:81614941-81664130 & Human \\
\hline LOC105370921 & XR_-932526.2 & Chr15:81665145-81690642 & Human \\
\hline LOC102724001 & XR_932527.2 & Chr15:81912868-81967182 & Human \\
\hline LOC105370922 & XR_932529.2 & Chr15:81977933-82013733 & Human \\
\hline LOC 105370923 & XR_932531.2 & Chr15:82026116-82032619 & Human \\
\hline LINC01583 & NR_120367.1 & Chr15:82088593-82097694 & Human \\
\hline LOC105370924 & XR_932532.1 & Chr15:82096581-82099672 & Human \\
\hline LOC105370932 & XR_932541.2 & Chr15:83283474-83318663 & Human \\
\hline LOC105370933 & XR_932544.1 & Chr15:83345641-83350586 & Human \\
\hline LOC105370934 & XR_932545.2 & Chr15:83365867-83368716 & Human \\
\hline LOC105370935 & XR_001751646.1 & Chr15:83944102-83949251 & Human \\
\hline LOC112268243 & XR_002958412.1 & Chr19:56314620-56341105 & Human \\
\hline MIR5572 & NR 049837.1 & Chr15:80581102-80581239 & Human \\
\hline MIR549A & NR_030393.1 & Chr15:80841977-80842073 & Human \\
\hline MIR4514 & NR_039739.1 & Chr15:80997416-80997473 & Human \\
\hline MIR1184-3 & NR 036260.1 & ChrX:155457516-155457615 & Human \\
\hline
\end{tabular}


percentage, and fat body mass. A lollipop diagram of GWAS variants contained within these two human syntenic regions is shown in Figure 20. Only one of these variants has a direct association with breast cancer. The remaining variants stem from breast cancer associated traits, namely body mass.

\section{Discussion:}

The focus of this study was to enhance our understanding of genetic risk and predisposition of human breast cancer using a rat mammary cancer model. Specifically, I sought to further narrow $M c s 3$ to reduce the pool of potential candidate breast cancer susceptibility genes. In this way, a subset of genomic variants and genes can be identified for orthologous risk analysis in human disease, and this rat model further utilized to investigate these genes and their underlying molecular mechanisms in breast/mammary carcinogenesis.

Mcs3 was first identified as a putative QTL associated with a decrease in the number of mammary tumors in female rats carrying the COP allele by Michael Gould's group. These studies conducted genome scans for loci that modulate induction of mammary carcinomas in (WFxCOP)F $\mathrm{F}_{1} \mathrm{x}$ WF backcross rats [314]. Peak logarithm of odds (LOD) scores estimated an $M c s 3$ interval of around $30 \mathrm{cM}$ and predicted this loci contained potential mammary cancer resistance genes [314]. Mcs3 was physically confirmed in 2017 using WF.COP congenic rat strains and delimited to a $29.4 \mathrm{Mb}$ region on rat chromosome 1:143700228-171517317 (build 6.0/rn6) [243].

My congenic rat lines further delimit $M c s 3$ to a 7.2 Mb region of rat chromosome 1 that spans from $r s 8149408$ to $r s 107402736$ (Chr1:143700228-150929594). I was 
Table 16. Human Cancer-associated Genes Located in Rat Mcs3

\begin{tabular}{|c|c|c|}
\hline Gene & Cancer association & Basis for Association \\
\hline MPP1* & $\begin{array}{c}\text { Lung adenocarcinomas and } \\
\text { neoplasms [327] }\end{array}$ & Meta-analysis of microarray datasets \\
\hline IL16 & $\begin{array}{l}\text { cutaneous T cell lymphoma } \\
\text { [328], multiple myeloma } \\
\text { [329], breast [330, 331], } \\
\text { gastrointestinal [331, 332], } \\
\text { colorectal [332], renal cell } \\
\text { carcinoma [333], lung } \\
\text { [334], prostate [335, 336] }\end{array}$ & $\begin{array}{c}\text { Flow cytometry [328, 331]. ELISA of } \\
\text { patient serum samples [329, 331-333], } \\
\text { Cell culture tumor spheroids and tumor } \\
\text { xenografts [330], RT-qPCR based on } \\
\text { patient genotypes [334], GWAS [335, } \\
336]\end{array}$ \\
\hline $\begin{array}{l}\text { FOLH1 } \\
\text { (PSMA) }\end{array}$ & $\begin{array}{c}\text { Prostate }[337,338] \text {, breast } \\
{[339,340]}\end{array}$ & $\begin{array}{c}\text { RT-PCR and PDX models from patient } \\
\text { samples [337], IHC of patient tissue } \\
\text { microarrays [338], IHC and IF of patient } \\
\text { samples }[339,340]\end{array}$ \\
\hline NOX4 & $\begin{array}{l}\text { Glioblastoma [341], thyroid } \\
\text { [342], melanoma [343], } \\
\text { gastric [344], colorectal } \\
\text { [345], prostate [346], lung } \\
\text { [347], endometrial [348] }\end{array}$ & $\begin{array}{c}\text { RT-PCR and IHC of patient samples } \\
\text { [341-347], tumorigenicity in nude mice } \\
\text { [343], expression microarray [344-347], } \\
\text { xenografts [347], in situ hybridization } \\
\text { [348] }\end{array}$ \\
\hline BCL2A $1 * *$ & Breast $[349,350]$ & $\begin{array}{c}\text { IHC of patient samples [349], differential } \\
\text { expression analysis [350] }\end{array}$ \\
\hline $\begin{array}{l}\text { CYP2C19/ } \\
\text { Cyp2c6v1 }\end{array}$ & Breast $[351,352]$ & $\begin{array}{c}\text { Global screening array of patient } \\
\text { genotypes [351], genotyping of patient } \\
\text { samples [352] }\end{array}$ \\
\hline EFL1 & Glioma [353], breast [354] & $\begin{array}{c}\text { WB, IHC of patient samples, xenograft } \\
\text { tumor model [353], qRT-PCR [353, } \\
\text { 354], RNA-seq [354] }\end{array}$ \\
\hline $\begin{array}{l}\text { CYP2C8/ } \\
\text { Cyp2c79 }\end{array}$ & $\begin{array}{l}\text { Breast [355], hepatocellular } \\
\text { carcinoma [356] }\end{array}$ & $\begin{array}{c}\text { genotyping of patient samples [355], RT- } \\
\text { PCR and IHC of patient samples [356] }\end{array}$ \\
\hline CYP4A11 & $\begin{array}{l}\text { Hepatocellular carcinoma } \\
{[357,358], \text { renal cell }} \\
\text { carcinoma [359] }\end{array}$ & $\begin{array}{l}\text { co-expression network from clinical } \\
\text { data, RNA-seq [357], IHC, WB, RT- } \\
\text { PCR of patient samples [358, 359] }\end{array}$ \\
\hline ARNT2 & $\begin{array}{c}\text { Hepatocellular carcinoma } \\
\text { [360], oral squamous cell } \\
\text { carcinoma [361], breast } \\
\text { [362], lung [363], gastric } \\
\text { [364] } \\
\end{array}$ & $\begin{array}{c}\text { IHC and tissue microarray of patient } \\
\text { samples [360], qRT-PCR, WB, and IHC } \\
\text { of patient samples [361], whole-genome } \\
\text { microarray [362], xenograft tumors } \\
\text { [363], IHC of patient samples }[363,364]\end{array}$ \\
\hline CEMIP & $\begin{array}{c}\text { Pancreatic [365], colorectal } \\
\text { [366, 367], gastric [368, } \\
\text { 369], breast [370], ovarian } \\
\text { [371], prostate [372] }\end{array}$ & $\begin{array}{c}\text { ELISA of patient serum [365], } \\
\text { microarray analysis }[366,372], \text { transcript } \\
\text { profiling [367], IHC of patient samples } \\
{[367,368,371,372], \text { RT-PCR }[368,}\end{array}$ \\
\hline
\end{tabular}




\begin{tabular}{|c|c|c|}
\hline & & $\begin{array}{l}\text { 369], cDNA microarray [369], IHC of } \\
\text { tissue microarray [370] }\end{array}$ \\
\hline ABHD17C & Breast [373] & gene expression profiles from PDX \\
\hline $\begin{array}{l}\text { TLNRD1 } \\
\text { (MESDC1) }\end{array}$ & $\begin{array}{l}\text { Hepatocellular carcinoma } \\
{[374]}\end{array}$ & $\begin{array}{l}\text { RT-PCR of patient samples, tumor } \\
\text { xenografts }\end{array}$ \\
\hline MEX3B & Melanoma [375] & $\begin{array}{c}\text { RNA-seq and RT-qPCR of patient } \\
\text { samples }\end{array}$ \\
\hline TM6SF1 & $\begin{array}{l}\text { Breast [376], hepatocellular } \\
\text { carcinoma [377] }\end{array}$ & $\begin{array}{l}\text { Methylation analysis in patient samples } \\
{[376,377]}\end{array}$ \\
\hline BNC1 & $\begin{array}{c}\text { Pancreatic [378], breast } \\
\text { [379], hepatocellular } \\
\text { carcinoma [380], squamous } \\
\text { cell carcinoma [381, 382] }\end{array}$ & $\begin{array}{l}\text { Methylation analysis in patient samples } \\
\text { [378-380], RT-PCR of patient samples } \\
\text { [380-382], expression array and IHC } \\
\text { from patient samples [382] }\end{array}$ \\
\hline SH3GL3 & $\begin{array}{c}\text { Glioblastoma [383], } \\
\text { colorectal [384], oral } \\
\text { squamous cell carcinoma } \\
{[385]}\end{array}$ & $\begin{array}{l}\text { Expression microarray [383, 385], IHC } \\
\text { from patient samples [383], methylation } \\
\text { analysis in patient samples [384], qRT- } \\
\text { PCR [385] }\end{array}$ \\
\hline ADAMTSL3 & $\begin{array}{c}\text { Colorectal [386], } \\
\text { hepatocellular carcinoma } \\
{[387]}\end{array}$ & $\begin{array}{c}\text { in situ hybridization and qRT-PCR of } \\
\text { patient samples [386], IHC of patient } \\
\text { samples [386, 387], in vivo screening } \\
\text { [387] }\end{array}$ \\
\hline LINC01583 & Colorectal [388] & Expression profile screening \\
\hline
\end{tabular}

*Not orthologous to rat Mcs3

**Rat segment not located at Mcs 3 
Table 17. Breast Cancer Risk Correlated Traits with Human GWAS Significant Variants in Genomic Segments Orthologous to Rat Mcs3

\begin{tabular}{|c|c|c|c|c|c|}
\hline $\begin{array}{c}\text { Variant } \\
\text { dbSNP ID }\end{array}$ & $\begin{array}{c}\text { P- } \\
\text { Value* }\end{array}$ & Trait & $\begin{array}{l}\text { Genomic } \\
\text { position }\end{array}$ & Gene/locus & $\begin{array}{c}\text { Accession } \\
\text { number }\end{array}$ \\
\hline rs 7165759 & $1 \times 10^{-10}$ & $\begin{array}{c}\text { visceral adipose tissue } \\
\text { measurement }\end{array}$ & Chr15:80696259 & ABHD17C & NM_021214.2 \\
\hline rs12914623 & $2 \times 10-16$ & body mass index & Chr15:80701229 & ABHD17C & NM_021214.2 \\
\hline rs57783949 & $4 \times 10-8$ & body mass index & Chr15:80710515 & ABHD17C & NM_021214.2 \\
\hline rs34769775 & $9 \times 10-14$ & body mass index & Chr15:80696831 & ABHD17C & NM_021214.2 \\
\hline rs2759315 & $5 \times 10-11$ & body mass index & Chr15:80717305 & ABHD17C & NM_-021214.2 \\
\hline rs12593036 & $2 \times 10-15$ & body mass index & Chr15:80766311 & ABHD17C & NM_-021214.2 \\
\hline rs12593088 & $5 \times 10-12$ & Waist-hip ratio & Chr15:80766299 & ABHD17C & NM_021214.2 \\
\hline rs149479659 & $2 \times 10-6$ & $\begin{array}{l}\text { Breast cancer specific } \\
\text { mortality in estrogen } \\
\text { receptor positive } \\
\text { breast cancer }\end{array}$ & Chr15:80763524 & ABHD17C & NM_021214.2 \\
\hline rs530826342 & $3 \times 10-6$ & Fat body mass & Chr15:81398425 & TMC3 & NM_001080532.3 \\
\hline rs12324805 & $7 \times 10-6$ & body mass index & Chr15:82059859 & MEX3B & NM̄_032246.6 \\
\hline rs111491984 & $1 \times 10-9$ & age at menarche & Chr15:82195898 & EFL1 & NM 024580.6 \\
\hline rs 11325 & $5 \times 10-9$ & Body height & Chr15:81308999 & IL16 & NM 004513.6 \\
\hline rs4448892 & $2 \times 10-12$ & Body height & Chr15:81196722 & IL16 & NM_-004513.6 \\
\hline rs17875533 & $1 \times 10-18$ & Body height & Chr15:81305884 & IL16 & NM_004513.6 \\
\hline rs11857976 & $2 \times 10-30$ & Body height & Chr15:81531197 & AC060809 & AC0 60809.1 \\
\hline rs 28463783 & $1 \times 10-10$ & Body height & Chr15:81954237 & AC104041 & AC104041.1 \\
\hline rs2654210 & $6 \times 10-8$ & Waist-hip ratio & Chr15:82113300 & LINC01583 & NR_120367.1 \\
\hline rs35056343 & $2 \times 10-13$ & Body height & Chr15:81543120 & AC060809 & AC060809.1 \\
\hline rs 2554380 & $9 \times 10-7$ & Body height & Chr15:83647132 & $\begin{array}{l}\text { ADAMTSL3/ } \\
\text { SH3GL3 }\end{array}$ & $\begin{array}{l}\text { NM_207517.3/ } \\
\text { NM_003027.5 }\end{array}$ \\
\hline rs10906982 & $2 \times 10-8$ & Body height & Chr15:83899406 & ADAMTSL3 & NM 207517.3 \\
\hline rs11259933 & $1 \times 10-19$ & Body height & Chr15:83911404 & ADAMTSL3 & NM_207517.3 \\
\hline rs7183263 & $4 \times 10-7$ & Body height & Chr15:83904289 & ADAMTSL3 & NM_207517.3 \\
\hline rs 1383484 & $9 \times 10-7$ & Body height & Chr15:83854003 & ADAMTSL3 & NM 207517.3 \\
\hline rs11259936 & $2 \times 10-35$ & Body height & Chr15:83911830 & ADAMTSL3 & NM 207517.3 \\
\hline rs2401171 & $4 \times 10-21$ & Body height & Chr15:83888924 & ADAMTSL3 & NM_-207517.3 \\
\hline rs 2257011 & $1 \times 10-47$ & Body height & Chr15:83597393 & SH3GL3 & NM_003027.5 \\
\hline rs7162542 & $8 \times 10-55$ & $\begin{array}{l}\text { body height, BMI- } \\
\text { adjusted waist } \\
\text { circumference, BMI- } \\
\text { adjusted hip } \\
\text { circumference }\end{array}$ & Chr15:83845538 & ADAMTSL3 & NM_207517.3 \\
\hline rs4842924 & $7 \times 10-10$ & Lean body mass & Chr15:83918855 & ADAMTSL3 & NM 207517.3 \\
\hline rs2585061 & $5 \times 10-13$ & Body height & Chr15:83678999 & ADAMTSL3 & NM_-207517.3 \\
\hline rs1896796 & $4 \times 10-6$ & Body height & Chr15:83606228 & SH3GL3 & NM_003027.5 \\
\hline rs8024628 & $5 \times 10-19$ & $\begin{array}{l}\text { body height, BMI- } \\
\text { adjusted waist } \\
\text { circumference, BMI- } \\
\text { adjusted hip } \\
\text { circumference }\end{array}$ & Chr15:83917711 & ADAMTSL3 & NM_207517.3 \\
\hline rs950169 & $2 \times 10-7$ & Body height & Chr15:84037709 & ADAMTSL3 & NM_207517.3 \\
\hline rs2562784 & $2 \times 10-6$ & Body height & Chr15:83617740 & SH3GL3 & NM 003027.5 \\
\hline rs28728297 & $7 \times 10-6$ & Body height & Chr15:83877660 & ADAMTSL3 & NM 207517.3 \\
\hline rs 2562781 & $2 \times 10-9$ & $\begin{array}{l}\text { BMI-adjusted hip } \\
\text { circumference }\end{array}$ & Chr15:83643816 & $\begin{array}{l}\text { ADAMTSL3/ } \\
\text { SH3GL3 }\end{array}$ & $\begin{array}{l}\text { NM_207517.3/ } \\
\text { NM_003027.5 }\end{array}$ \\
\hline rs 4842838 & $6 \times 10-27$ & $\begin{array}{c}\text { BMI-adjusted waist } \\
\text { circumference, body } \\
\text { height }\end{array}$ & Chr15:83913372 & ADAMTSL3 & NM_207517.3 \\
\hline rs8030379 & $9 \times 10-27$ & $\begin{array}{l}\text { BMI-adjusted waist } \\
\text { circumference }\end{array}$ & Chr15:83922158 & ADAMTSL3 & NM_207517.3 \\
\hline rs2030839 & $1 \times 10-15$ & $\begin{array}{l}\text { BMI-adjusted waist } \\
\text { circumference }\end{array}$ & Chr15:83915207 & ADAMTSL3 & NM_207517.3 \\
\hline
\end{tabular}




\begin{tabular}{|c|c|c|c|c|c|}
\hline rs111470917 & $7 \times 10-37$ & Body height & Chr15:83916787 & ADAMTSL3 & NM_207517.3 \\
\hline rs8038454 & $4 \times 10-11$ & Body height & Chr15:83670684 & ADAMTSL3 & NM_207517.3 \\
\hline rs11856122 & $2 \times 10-31$ & Body fat distribution & Chr15:83907596 & ADAMTSL3 & NM_207517.3 \\
\hline rs8031704 & $1 \times 10-15$ & Body fat percentage & Chr15:83982433 & ADAMTSL3 & NM_207517.3 \\
\hline rs62025778 & $1 \times 10-20$ & $\begin{array}{l}\text { BMI-adjusted waist } \\
\text { circumference }\end{array}$ & Chr15:83776366 & ADAMTSL3 & NM_207517.3 \\
\hline rs75821416 & $2 \times 10-13$ & $\begin{array}{l}\text { BMI-adjusted waist } \\
\text { circumference }\end{array}$ & Chr15:83657008 & ADAMTSL3 & NM_207517.3 \\
\hline rs75666428 & $8 \times 10-11$ & $\begin{array}{l}\text { BMI-adjusted waist } \\
\text { circumference }\end{array}$ & Chr15:84012650 & ADAMTSL3 & NM_207517.3 \\
\hline rs55685171 & $6 \times 10-10$ & $\begin{array}{l}\text { BMI-adjusted waist } \\
\text { circumference }\end{array}$ & Chr15:83757828 & ADAMTSL3 & NM_207517.3 \\
\hline rs7169595 & $9 \times 10-9$ & $\begin{array}{l}\text { BMI-adjusted waist } \\
\text { circumference }\end{array}$ & Chr15:83736822 & ADAMTSL3 & NM_207517.3 \\
\hline rs 140574883 & $1 \times 10-13$ & $\begin{array}{l}\text { BMI-adjusted waist } \\
\text { circumference }\end{array}$ & Chr15:84074328 & AC027807 & AC027807.1 \\
\hline rs 141724470 & $6 \times 10-9$ & $\begin{array}{l}\text { BMI-adjusted waist } \\
\text { circumference }\end{array}$ & Chr15:84044702 & ADAMTSL3 & NM_207517.3 \\
\hline rs11259926 & $7 \times 10-21$ & $\begin{array}{l}\text { BMI-adjusted waist } \\
\text { circumference }\end{array}$ & Chr15:83897496 & ADAMTSL3 & NM_207517.3 \\
\hline rs4842918 & $1 \times 10-62$ & $\begin{array}{l}\text { BMI-adjusted waist } \\
\text { circumference }\end{array}$ & Chr15:83868247 & ADAMTSL3 & NM_207517.3 \\
\hline rs4583201 & $5 \times 10-11$ & $\begin{array}{l}\text { BMI-adjusted waist } \\
\text { circumference }\end{array}$ & Chr15:83820297 & ADAMTSL3 & NM_207517.3 \\
\hline rs113817743 & $4 \times 10-9$ & $\begin{array}{l}\text { BMI-adjusted waist } \\
\text { circumference }\end{array}$ & Chr15:83817917 & ADAMTSL3 & NM_207517.3 \\
\hline rs4842926 & $1 \times 10-44$ & $\begin{array}{l}\text { BMI-adjusted waist } \\
\text { circumference }\end{array}$ & Chr15:83939736 & ADAMTSL3 & NM_207517.3 \\
\hline rs8035886 & $2 \times 10-34$ & $\begin{array}{l}\text { BMI-adjusted waist } \\
\text { circumference }\end{array}$ & Chr15:83790608 & ADAMTSL3 & NM_207517.3 \\
\hline rs 77378263 & $3 \times 10-14$ & $\begin{array}{l}\text { BMI-adjusted waist } \\
\text { circumference }\end{array}$ & Chr15:83571369 & SH3GL3 & NM_003027.5 \\
\hline rs2730075 & $6 \times 10-12$ & $\begin{array}{l}\text { BMI-adjusted waist } \\
\text { circumference }\end{array}$ & Chr15:83645689 & $\begin{array}{l}\text { ADAMTSL3/ } \\
\text { SH3GL3 }\end{array}$ & $\begin{array}{l}\text { NM_207517.3/ } \\
\text { NM_003027.5 }\end{array}$ \\
\hline rs 75340848 & $2 \times 10-10$ & $\begin{array}{l}\text { BMI-adjusted waist } \\
\text { circumference }\end{array}$ & Chr15:83420223 & AC103876 & AC103876.1 \\
\hline rs2220856 & $5 \times 10-22$ & $\begin{array}{l}\text { BMI-adjusted waist } \\
\text { circumference }\end{array}$ & Chr15:83526475 & SH3GL3 & NM_003027.5 \\
\hline rs 10152514 & $5 \times 10-14$ & $\begin{array}{l}\text { BMI-adjusted waist } \\
\text { circumference }\end{array}$ & Chr15:83583309 & SH3GL3 & NM_003027.5 \\
\hline rs2732151 & $8 \times 10-10$ & $\begin{array}{l}\text { BMI-adjusted waist } \\
\text { circumference }\end{array}$ & Chr15:83534933 & SH3GL3 & NM_003027.5 \\
\hline rs4843136 & $2 \times 10-8$ & $\begin{array}{l}\text { BMI-adjusted waist } \\
\text { circumference }\end{array}$ & Chr15:83403098 & AC103876 & AC103876.1 \\
\hline rs4842921 & $8 \times 10-11$ & $\begin{array}{c}\text { BMI-adjusted wait to } \\
\text { hip ratio }\end{array}$ & Chr15:83887871 & ADAMTSL3 & NM_207517.3 \\
\hline rs185392832 & $2 \times 10-8$ & Body height & Chr15:83162219 & HDGFL3 & NM_016073.4 \\
\hline rs6602982 & $3 \times 10-42$ & Body height & Chr15:83588992 & SH3GL3 & NM_003027.5 \\
\hline rs118136305 & $2 \times 10-16$ & Body height & Chr15:83769541 & ADAMTSL3 & NM_207517.3 \\
\hline rs2135880 & $\begin{array}{c}3 \times 10- \\
222\end{array}$ & Body height & Chr15:83920861 & ADAMTSL3 & NM_207517.3 \\
\hline rs11630762 & $5 \times 10-10$ & Waist to hip ratio & Chr15:83946227 & ADAMTSL3 & NM_207517.3 \\
\hline rs 2289125 & $2 \times 10-16$ & Body height & Chr11:89491285 & NOX4 & NM_016931.5 \\
\hline rs317162 & $5 \times 10-9$ & body mass index & Chr11:89371405 & NOX4 & NM_016931.5 \\
\hline rs61886764 & $3 \times 10-8$ & $\begin{array}{l}\text { BMI-adjusted waist } \\
\text { circumference }\end{array}$ & Chr10:94912317 & CYP2C9 & NM_000771.4 \\
\hline rs530826342 & $3 \times 10-6$ & fat body mass & Chr15:81398425 & TMC3-AS1 & NR_120365.1 \\
\hline
\end{tabular}

*P-value for association

** Homo sapiens assembly GRCh38/hg38 

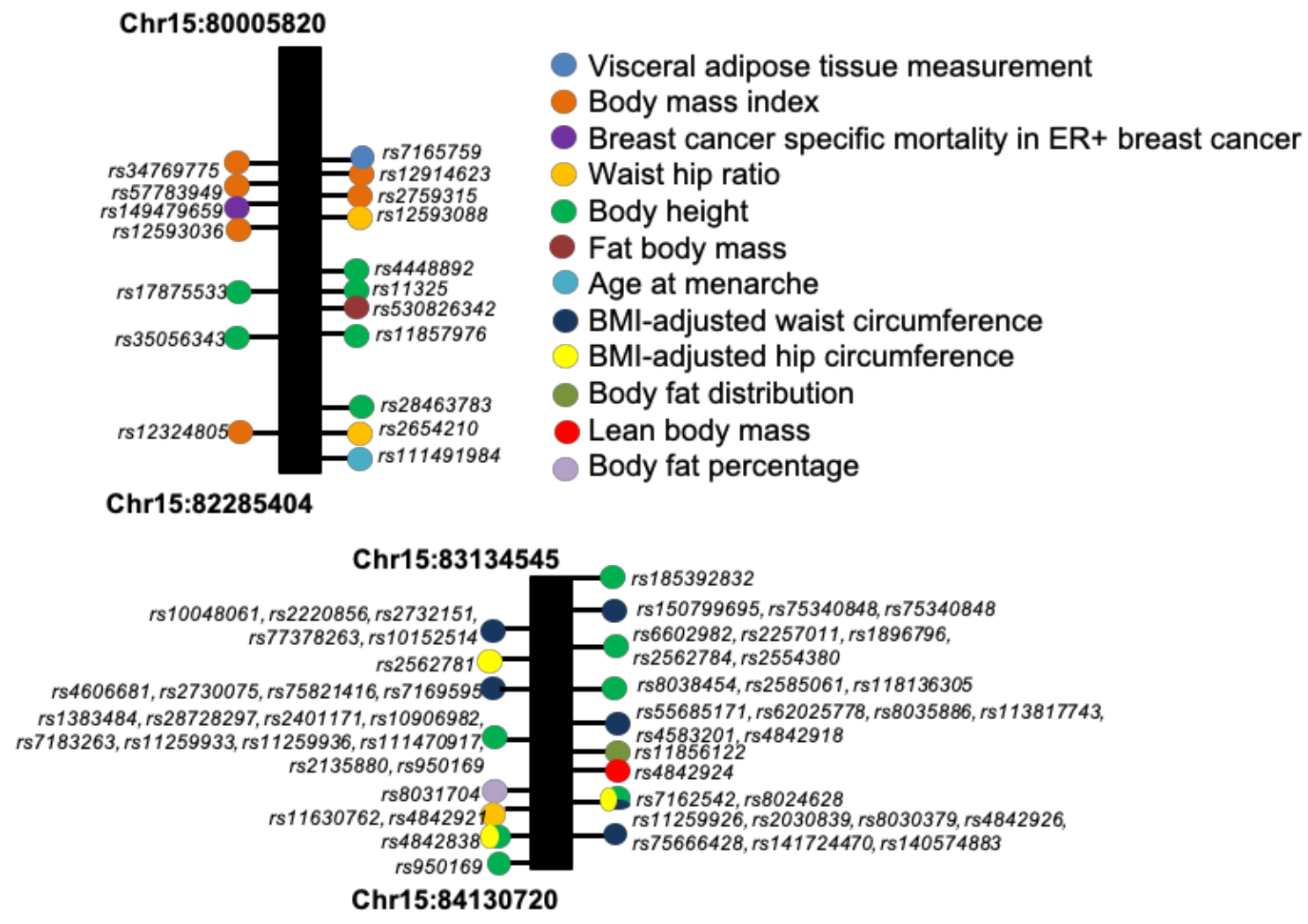

Figure 20: Diagram of overlapping GWAS breast cancer associated traits orthologous

to rat Mcs3. Shown are human syntenic regions at chr15:80005820-82285404 and chr15:83134545-84130720. Each color represents a different trait as indicated by the legend. Multiple nearby variants associated with the same trait share a circle. 
unable to uncover additional informative markers between WF and COP strains to further fine map $M c s 3$, largely due to the published genomes on the Rat Genome Database. The uploaded COP genome has a high depth of coverage and number of reads, but the other rat strain genomes searched possessed a low depth of sequencing across reported SNPs. My inability to find informative SNPs was likely due to the uploaded sequencing data that were not high quality enough to identify true variants differing from the COP genome. This genome possessed a high frequency of variants because it is highly dissimilar from the Brown Norway rat reference genome, but likely is more similar to the Wistar genomes than the low-quality genome sequencing makes it appear. This difficulty in finding informative markers at higher resolution in fine mapping Mcs3 suggests WF and COP share similar haplotypes at Mcs3. Thus, more work in finding genetic markers will need to be conducted if future work in deriving more congenic lines is pursued. Mcs3 is also at a shortened enough interval now that it could also possibly be sequenced between WF and COP strains to identify and evaluate causal genetic elements or transcriptional differences that contribute to mammary cancer susceptibility.

A single COP allele was predicted to reduce mammary carcinoma susceptibility by $42 \%$ in female rats heterozygous at the original Mcs3 locus [164]. This study found that female rats homozygous for the COP allele at the delimited Mcs3 locus had a 57\% reduction in mammary cancer susceptibility. The difference in the susceptibility between the two studies might be explained by either a possible degree of dominance of the COP allele at the Mcs3 locus, or by background COP alleles potentially present in the females included in the original linkage analysis.

Breast carcinomas are usually classified based on histological appearance, with up to 21 histological types identified via differing cytoarchitectural features and 
morphological variation [389]. Invasive ductal carcinomas (IDC), specifically, account for $80 \%$ of diagnosed female breast cancers [390]. The majority of IDCs do not exhibit enough histological features, other than proliferative invasion through the ductal basement membrane, to be classified as a specific tumor subtype [389, 391]. These IDCs of no special type, or not otherwise specified account for $75 \%$ of invasive carcinomas [391]. It is also well established that chemically-induced rat mammary tumors share similar histopathology to human breast carcinomas $[221,392,393]$. In pubescent nulliparous rats, carcinogenic initiation using 7,12-dimethylbenz(a)anthracene (DMBA) primarily occurs in the terminal end buds and terminal ducts within mammary tissue, giving rise to adenocarcinomas of ductal cell origin [394]. These are intraductal and invasive ductal carcinomas, similar to what is observed in the majority of human breast malignancies of no specific type [394]. The majority of DMBA-induced rat mammary carcinomas are classified as invasive papillary carcinomas, comprising $32 \%$ of tumor histology [394]. The invasive cribriform carcinoma is the second most common histologically-defined rat mammary carcinoma at $27 \%$ of DMBA-rat mammary cancers [394]. These mammary IDC subtypes were the most frequently observed tumor histologies in the WF strain and each Mcs 3 WF.COP congenic lines $\mathrm{H}$, I, J, and $\mathrm{K}$.

The papillary carcinoma is defined by branching fibrovascular cores throughout the lesion that contain variable degrees of stromal fibrosis (Fig 14, Arrow A) [395-397]. These cores support neoplastic epithelial proliferations, which form papillary projections that comprise this invasive growth pattern [394-397] (Fig 14, Arrow B). The invasive cribriform carcinoma is defined by invasion of the stroma by neoplastic epithelial cells that form nestlike tumor structures $[398,399]$. These tumor nests are characterized by glandular 
spaces with no solid component that create a sieve-like appearance (Fig 15, Arrow A) [394, 398, 399]. The cribriform architecture also displays a surrounding desmoplastic stroma with lymphocyte infiltration (Fig 15, Arrow B) [394, 399]. Since these two most common DMBA-mammary tumor subtypes were the majority histologies observed in each of the Mcs3 WF.COP congenic lines, it was concluded that these rat lines share the same histopathological tumor development pathways in response to DMBA-initiation. As a result, it wasn't the tumor subtype or pathology that accounted for the differences in tumor multiplicity between congenic lines. This also indicates that the mammary tumor resistant phenotype observed in line $\mathrm{J}$, as opposed to the susceptible phenotypes of lines H, I, and $\mathrm{K}$, do not result in differences in tumor development and morphology.

In addition to mammary cancer resistance, a secondary phenotype of lower body mass was documented in line $\mathrm{J}$ rats. This indicates a developmental delay in line $\mathrm{J}$ growth; however, it doesn't appear to affect mammary gland development. H\&E staining showed the observed morphologies match known rat mammary gland developmental structures, such as terminal end bud appearances during puberty, and appears consistent between line $\mathrm{J}$ and WF females across all developmental timepoints. Lower body mass also doesn't seem to be associated with a developmental delay in body growth as it persists over the lifetime of line $\mathrm{J}$ rats.

It is possible that these pleiotropic traits of mammary cancer resistance and low body mass phenotypes are connected, as obesity, characterized by excess body fat, is associated with greater breast cancer incidence [400-402]. Specifically, obesity is associated with larger tumor size, positive lymph node status, shorter time to disease recurrence, and mortality [401, 403-405]. There exists a positive association between 
obesity and breast cancer risk in postmenopausal women [406-409], likely due to adipose tissue being the main site of estrogen synthesis in postmenopausal women [410] and higher concentrations of estrogen in the excess fat reserves of obese women [411]. These greater estrogen levels could be expected to drive estrogen-dependent tumors, which is supported by the fact that most postmenopausal breast cancer cases in women with obesity are ERpositive [412-416]. Inversely, there is a negative relationship between obesity and breast cancer risk in premenopausal women [411, 417-419]. It has been speculated this may be due to less estrogen in circulation during premenopausal cycling [409], and suggested that this effect is reversed after adjusting for breast density [420-422]. Extra adipose tissue in general mimics an inflammatory transcriptome and produces inflammatory cytokines, creating a microenvironment conducive to cancer initiation, invasion, and metastasis [423425]. The higher body mass in the WF strain as compared to line $\mathrm{J}$ could result in subclinical inflammation of mammary tissue that contributes to the differences in mammary tumor incidence. Conversely, the lower body mass observed in line J could lend a protective effect to disease development, similar to what is observed in human epidemiological studies.

The lower body mass phenotype in line $\mathrm{J}$ rats appears to be a sex-dependent trait. In general housing, only line $\mathrm{J}$ males had a significantly lower body mass compared to WF. Although the mammary tumor phenotype is only studied in females, this body mass phenotype indicates that the Mcs3 genotype also has an effect in males. In female rats, it wasn't until placed in a female-only environment that female line $\mathrm{J}$ rats displayed a lower body mass compared to WF. It appears that the presence of male rats somehow masks the effect of $M c s 3$ genotype on female body mass. The only apparent environmental difference 
is the lack of male pheromones. In rodents, male pheromones can induce a physiological change in the female endocrine and reproductive system [426]. In rats, this includes induction of puberty, a reduction in the duration of the estrus cycle, induced diestrus staging, and restoration of estrus cycling in aging females [426-428]. These endocrine disruptions are due to changes in hormonal signaling. For example, male urinary pheromones have been demonstrated to increase plasma progesterone levels in females [429]. The endocrine system also has a role in body weight regulation, disruptions to which could be theorized to have an effect on overall body mass. The genomic actions of ER $\alpha$ specifically has a known downstream effect on body weight [430, 431]. Estrogen is demonstrated to reduce food intake and body weight in ovariectomized rats, with restoration of eating behavior and normal body weight restored with estradiol treatment [431, 432]. Targeted deletion of an ER $\alpha$ subunit was also reported to increase body mass and adiposity [433]. Additionally, estrogen modulates leptin synthesis and secretion, and so regulates energy homeostasis and body weight in an indirect manner [434-436]. Thus, the differences in female line $\mathrm{J}$ body mass with and without the presence of male pheromones could be due to disruptions in endocrine signaling. The lower body mass as compared to WF females is presumed to be an effect of the genomic interplay of the Mcs 3 reduced mammary cancer susceptibility allele.

Rat $M c s 3$ was found to align to three human syntenic loci on chromosomes 11 and 15. These rat and human orthologous regions contain 16 annotated genes with cancer associations, as well as an additional rat only gene, and human only gene and lncRNA, not annotated in either orthologous regions. Of these genes, 11 have reported associations with breast cancer. There is reported significant increases in the cytokine interleukin-16 (IL16) 
in serum levels of stage III and IV breast cancer patients without or after chemo/radiotherapy compared to healthy controls [331]. Elevated IL16 levels in tumor spheroids and xenografts also result in greater migration and invasion of blood monocytes and macrophages in the tumor microenvironment, potentially serving as a chemoattractant in tumorigenesis [330]. Folate hydrolase 1 (FOLH1), also known by its protein product prostate-specific membrane antigen (PSMA), has been shown to localize to tumor cells of primary breast tumors and neovasculature of breast tumors and distant metastasis, serving as a potential therapeutic vascular target [339]. Additionally, the highest levels of PSMA are observed in the aggressive triple negative breast cancer molecular subtype [340]. The pro-survival/apoptotic-inhibitor B-cell lymphoma 2-related protein A1 (BCL2A1) is upregulated in breast tumor samples as compared to non-diseased, where it potentially has a role in protecting pro-inflammatory cells from apoptosis [350, 437]. Increased expression is positively associated with advanced TNM stage and poor prognosis in triple negative breast cancer [349]. Overexpression of $B C L 2 A 1$ has also been demonstrated to prevent mammary gland involution via apoptosis, indicating a physiological role in mammary gland function [438]. Inherited mutations of cytochrome P450 2C19 (CYP2C19), which has a major function in estrogen catabolism, have poor associations in breast cancer patients, including triple negative breast cancer [351, 352]. CYP2C19 may then serve as a potential prognostic biomarker. Additionally, gene variants of cytochrome P450 2 C8 (CYP2C8) are associated with increased risk of breast cancer [355]. Elongation factor like GTPase 1 (EFL1) is a domain of elongation factor-1 alpha (EF1 alpha), which has a role in proliferation, metastasis, and apoptosis [353, 439, 440]. Vitamin D3 has been shown to directly upregulate expression of EFL1 in ex vivo explants of human breast tissue where it 
may serve in a potential tumor suppressor role [354]. Aryl hydrocarbon receptor nuclear translocator 2 (ARNT2) is significantly overexpressed in breast tumors as compared to normal breast tissue, and positively correlates with patient relapse-free survival and overall survival [362]. As a result, it may be useful as a prognostic biomarker of breast cancer. Cell migration-inducing and hyaluronan-binding protein (CEMIP), formerly known as KIAA1199, has significantly increased expression in invasive breast carcinomas as compared to non-diseased breast tissue, and expression is inversely associated with patient survival [370]. CEMIP overexpression in vitro has also been demonstrated to increase migration of breast cancer cells and markers of epithelial-to-mesenchymal transition, as well as mammary gland tumor metastasis in vivo, serving a critical role in producing a metastatic cancer phenotype [370]. High expression of alpha/beta hydrolase domaincontaining protein $17 C(A B H D 17 C)$ has been reported in breast cancer patient samples and patient-derived tumor xenografts, although no studies into the molecular role in breast cancer have been done to date [373]. The promoter of transmembrane 6 superfamily member 1 (TM6SF1) was found to be significantly differentially methylated in normal breast tissue as compared malignant breast tissue. Though the function of TM6SF1 remains unknown, it may serve as a potential methylation biomarker for early breast cancer detection [376]. The transcription factor zinc finger protein basonuclin-1(BNC1) is reported to be methylated and silenced during breast to brain metastasis, but not in primary breast carcinomas, suggesting a role in tumor progression and metastasis [379].

The majority of GWAS-nominated variants identified in human orthologous syntenic regions to rat Mcs3 reside in SH3 Domain Containing GRB2 Like 3, Endophilin A3 (SH3GL3) and ADAMTS-like protein 3 (ADAMTSL3). These variants are associated 
with body mass and ratio traits, including body mass index, waist-hip ratio, fat body mass, body height, lean body mass, BMI-adjusted hip circumference, BMI-adjusted waist circumference, body fat distribution, body fat percentage, BMI-adjusted waist circumference, and fat body mass. These regions contain no direct association with breast cancer; however, are relevant to our congenic studies as line $\mathrm{J}$ was positive for the Mcs 3 reduced susceptibility locus and displayed a significantly lower body mass as compared to the other congenic lines. This suggests that $M c s 3$ is indeed modulating both body mass and mammary cancer susceptibility, and that these orthologous genic regions in the human that are associated with body mass may have a novel, secondary role in cancer development. Additionally, there was one variant, $r s 149479659$, with a direct breast cancer association, which indicates that this genetic region may warrant ultra-fine mapping to further investigate underlying susceptibility mechanisms at this locus. Commonly in these susceptibility loci, it is gene overexpression that has a mechanistic role in driving a cancer phenotype. However, genetic variants, especially in non-coding regions, modulate gene expression levels by altering the function of regulatory DNA. Thus, unannotated variants within rat Mcs3 and human orthologous regions may alter the expression patterns of nominated candidate cancer genes contained in these loci. Future studies aimed at further physical mapping of $M c s 3$ would reduce the pool of candidate genes and create a smaller orthologous region in the human genome in which to fine map and identify causal disease variants. Since WF and COP seem to share similar haplotypes at $M c s 3$, it may also be more feasible to directly sequence this locus in both strains and isolate potential causal differences leading to altered susceptibility. 


\section{CHAPTER IV}

\section{A METHOD TO PRE-SCREEN RAT MAMMARY GLAND WHOLE-MOUNTS PRIOR TO RNASCOPE}

\section{Introduction:}

Protein, DNA, and RNA biomarkers are important clinical diagnostic and prognostic tools $[442,443]$. Gene expression profiling has highlighted an abundance of novel RNA biomarkers associated with cancer [443-445]. Expression profiling of specific RNAs using whole tissue homogenates is problematic because of signal interference from multiple cell types. Furthermore, spatial and cell-specific information are lost in approaches using disrupted tissue [443, 446]. New technologies, including single-cell sequencing and RNAscope, have made single-cell RNA transcript profiling a reality. Gall and Pardue introduced molecular hybridization using radiolabeled nucleic acid sequences in situ $[447,448]$. Since their work, many additional techniques have been established, including RNAscope, a proprietary RNA in situ hybridization (RNA ISH) method that provides quantitative gene expression analysis within intact tissue [446].

Commercially available RNAscope technology utilizes a novel target probe design, along with a series of hybridization-based signal amplification steps, to provide single molecule visualization within specific cell populations of intact tissue. Oligo "Z probes"

Adapted from Duderstadt et al. (2021). [441] See Appendix 
for RNAscope contain $18-25$ base pair complementarity to a target transcript, a spacer sequence, and a 14 base pair tail sequence. These assays require adjacent target probes (a double $\mathrm{Z}$ pair) to hybridize contiguously to target RNA. The combined tail sequences of a double $\mathrm{Z}$ pair provide for hybridization of a preamplification scaffold, which contains multiple binding sites for subsequent amplifier molecules. Next, fluorescent-dye or chromogenic labeled probes bind to the amplifier molecules, which contain multiple binding sites for the labeled probes. Double $\mathrm{Z}$ probe pairing results in target-specific amplification and a reduction in background signal because binding of a single probe is not sufficient for preamplification scaffold hybridization [446].

Rodent models have been used extensively to study morphological changes during mammary gland development and disease progression [449-451]. Gross morphology of the mammary gland can be examined by whole-mounting tissue to a microscope slide and staining of intact mammary gland tissue $[450,451]$. Alum carmine is a biological nuclear stain derived from dried female cochineal insects [452]. Mammary ductal structures are easily visualized when fat-cleared mammary gland tissue is stained with carmine [449]. Whole-mounts are extremely useful to observe epithelial changes that occur during mammary gland development, function, and disease. Tissue morphologies can be excised, processed, and sectioned for further evaluation. This approach provides efficiency in capturing disease morphologies, as standard procedures would require extensive serial sectioning to find morphologies of interest [451]. For example, rat mammary carcinogenesis serves to model the natural histopathological lesions and malignancies found in human breast disease. Visualization of rat mammary gland ductal structures in situ is useful because, similar to a majority of human breast cancers, neoplasms arise in 
mammary ducts [391, 453, 454]. Screening tissue whole-mounts for morphologies of interest is a valuable technique to visualize and study changes occurring during carcinogenesis $[453,455]$.

RNAscope assays are designed and recommended by the manufacturer to be used on recently processed formalin fixed and paraffin embedded (FFPE) or cryopreserved tissue samples. Morphological features are retained in FFPE tissues, making it a preservation method of choice for decades. Integrity of RNA is preserved for long periods of time in FFPE tissues $[456,457]$. This is important because nucleic acid degradation is a pervasive problem that must be avoided in samples used for any RNA ISH method, including RNAscope. Loss of nucleic acid quality has a negative impact on the reproducibility and quantitative accuracy of subsequent assays. The duration of fixation and pre-treatment conditions can negatively impact nucleic acid integrity and compromise RNAscope assays $[458,459]$; thus, care must be taken to limit exposure time to harsh conditions prior to paraffin embedding tissue. We report that RNAscope can be effectively utilized following screening of mammary gland whole-mounts for lesions of interest. This is useful as it allows for the identification and study of diseased tissue morphologies without apparent loss of RNA integrity. We show, using probes targeting genes with known expression level differences, that the quantitative capacity of RNAscope is retained in tissue excised from mammary gland whole-mounts. This is applicable to mammary gland biology studies requiring pre-screening of mammary gland whole-mounts. 


\section{Protocol:}

Animal husbandry

Inbred Wistar Furth (WF/Hsd; Envigo) female rats were maintained in an AAALAC-approved facility on a $12 \mathrm{~h}$ light/dark cycle and provided LabDiet 5001 Rodent Diet (PMI® Nutrition International) and water ad libitum. The University of Louisville Institutional Animal Care and Use Committee approved all animal protocols.

\section{DMBA Administration and Mammary Gland Tissue Resection}

At 50-55 days of age, 7,12-dimethylbenz(a)anthracene (DMBA Acros Organics, CAS\#: 57-97-6, $20 \mathrm{mg} / \mathrm{mL}$ in sesame oil) was given by single oral gavage (65 mg DMBA/kg body mass) to induce mammary carcinogenesis. Rats were euthanized by $\mathrm{CO}_{2}$ asphyxiation at four or twelve weeks following DMBA administration and mammary glands excised. Briefly, one skin incision was made at the lower abdomen and extended forward, along the midline, to the neck (Fig 21). Four additional skin incisions, extending down each limb from the midline, were made and skin was separated from the body cavity to reveal mammary glands. Abdominal $4^{\text {th }}$ mammary glands (Fig 21) were used in this study. Mammary gland tissue was carefully trimmed away from skin by moving dorsally. Figure 22 contains a flowchart of tissue processing procedures used in this study. One mammary gland from each rat was immediately processed by FFPE, while the other was processed as a whole-mount. Tissues were fixed overnight in $10 \%$ neutral buffered formalin. 


\section{Whole-Mount Preparation:}

Following resection, mammary glands were immediately stretched across an electrostatically charged slide and fixed by submerging in $10 \%$ neutral buffered formalin at room temperature overnight. This whole-mount procedure minimized solvent exposure and staining time [455]. Slides were removed from formalin and placed into an acetone bath at room temperature overnight to de-fat. Overnight in acetone did not always completely clear the whole-mount of fat; however, it was sufficient time for successful carmine staining to visualize mammary gland structures and lesions.

Following defatting of mammary glands, slides were submerged in an alum carmine staining solution, prepared by boiling $1 \mathrm{~g}$ of carmine and $2.5 \mathrm{~g}$ of potassium alum in $500 \mathrm{~mL}$ of RNase-free DI water for 20 minutes. Carmine solution was stored at $4^{\circ} \mathrm{C}$ and reused after filtering to remove oil. Carmine staining took 1-2 days depending on mammary gland thickness. Glands were considered fully stained when white tissue was no longer visible through the microscope slide. Whole-mounts, not used for RNAscope analysis, were dehydrated in ethanol gradients, cleared overnight in xylene, and submerged in room temperature mineral oil for long-term storage.

Mammary gland tissue processing, embedding, and sectioning:

Morphological structures and lesions of interest, such as hyperplasia and ductal carcinoma in situ (DCIS) (Fig 23), within carmine stained whole-mounts were excised using a surgical scalpel. Excised tissue was placed in histology cassettes and processed using a standard tissue processor. Tissues were oriented with the flat side, originally against 

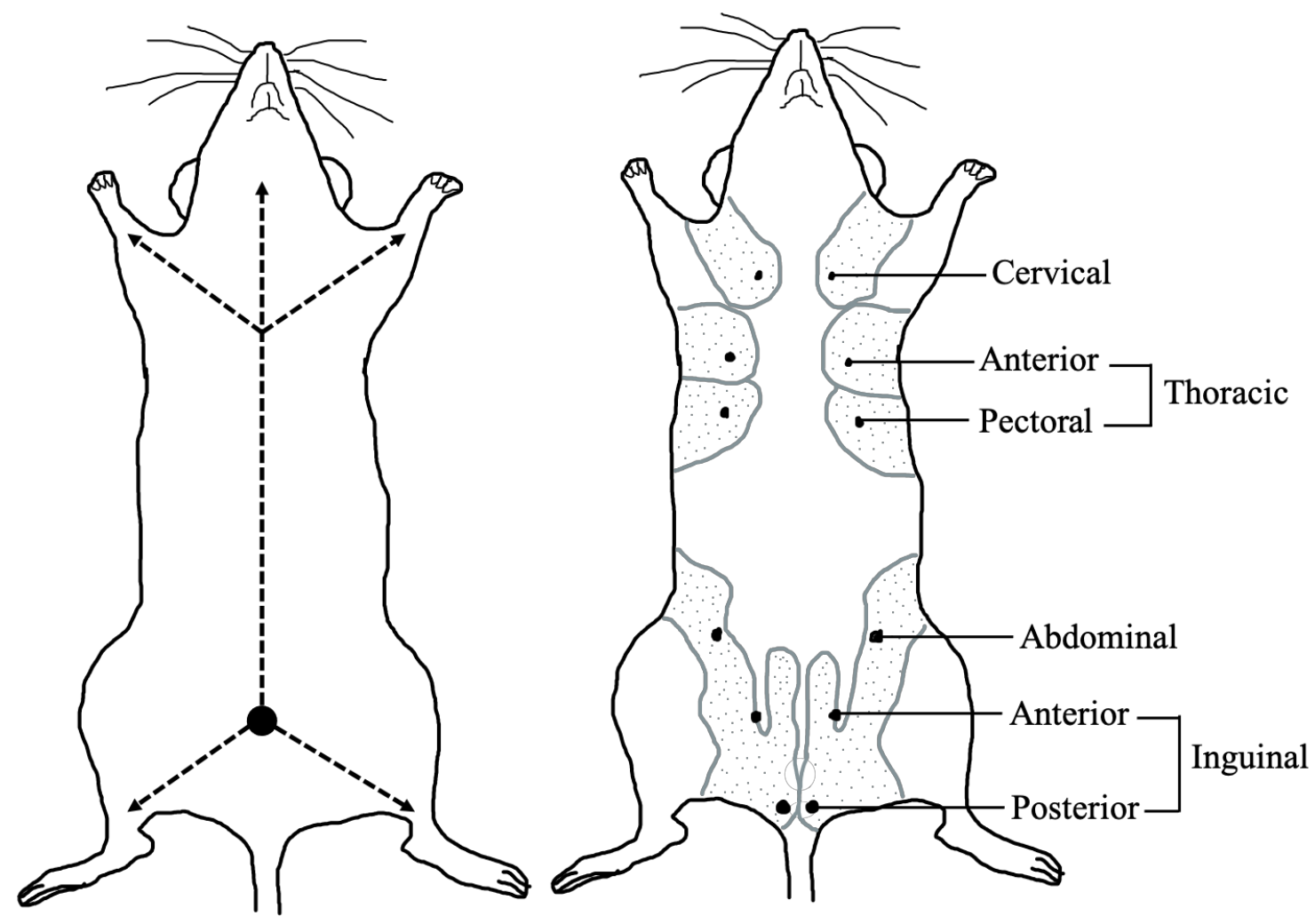

Figure 21: Mammary gland tissue extraction. Left panel: to reveal and expose mammary gland tissue, an incision was made in the skin at the lower abdomen (black circle) and extended along the midline. Four additional incisions were made extending from the midline down each limb. Skin was separated from the body cavity to expose mammary glands. Right panel: Rats have six ventral mammary gland pairs, which are located in cervical, thoracic, abdominal, and inguinal regions. 


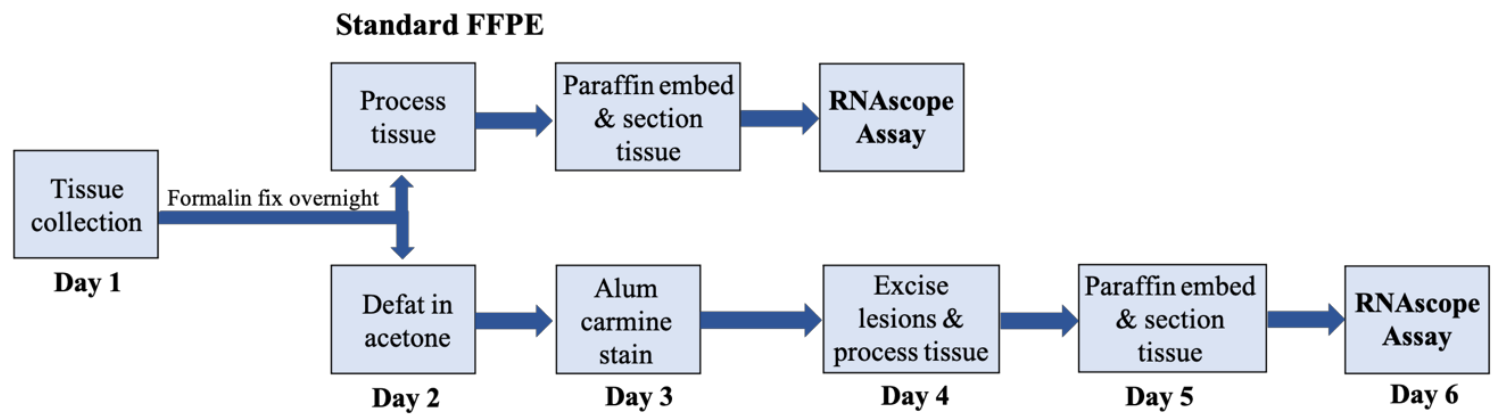

Whole mount followed by FFPE

Figure 22: Flow chart outlining difference in tissue preparation methods. Mammary glands were collected, processed, and used in RNAscope assays within 4 days (top). Alternatively, whole-mounts were processed and excised tissue used in RNAscope assays within 6 days of mammary gland collection (bottom). These whole-mounts had been processed and stained with alum carmine to pre-screen mammary glands for disease lesions. 
the microscope slide, facing the bottom of the paraffin mold. Paraffin embedded tissues were stored at $4{ }^{\circ} \mathrm{C}$ until sectioning. Tissues in paraffin blocks were sectioned one day prior to an RNAscope assay. This was important because RNA is more stable while the tissue is in paraffin blocks than after tissue sectioning [460]. Prior to sectioning, paraffin blocks were placed at $-20^{\circ} \mathrm{C}$ for a minimum of 30 minutes to improve sectioning and reduce wrinkling of tissue sections. Tissues were cut into $4 \mu \mathrm{m}$ sections and placed onto Superfrost Plus microscope slides (VWR). Other slide types, suitable for RNA ISH, may also be used. Up to three tissue sections, depending on tissue size, were placed on each slide. Slides were kept at room temperature overnight to completely dry.

Hematoxylin and Eosin (H\&E) staining of sectioned mammary glands:

Slides were deparaffinized and rehydrated using three separate xylene washes, followed by single washes in $100 \%$ ethanol, $90 \%$ ethanol, $80 \%$ ethanol, and DI water. Tissues were stained with hematoxylin for three minutes, rinsed under running DI water for three minutes, soaked in bluing reagent for less than one minute, and rinsed with DI water. Slides were dipped a at least three times in $100 \%$ ethanol to eliminate water before staining in eosin-Y for 30-45 seconds. Tissue samples were then dehydrated using washes of $90 \%$ ethanol, $100 \%$ ethanol, and 3 separate xylene washes, air dried, and coverslipped using permount or a similar mounting media.

RNAscope Assay

In this report, RNAscope assays were performed on abdominal $4^{\text {th }}$ mammary gland pairs, one immediately processed by FFPE and the other whole-mounted, defatted, carmine 
stained, and processed and paraffin embedded later. The RNAscope 2.5 HD Detection Reagent-RED kit (Advanced Cell Diagnostics \#322360) was used according to manufacturer's protocol, except for the following modifications that served to minimize harsh pre-hybridization treatment conditions that could compromise fragile mammary tissue [461]. Slides were not rocked or agitated during any step of the hybridization procedure. Slides were deparaffinized in two xylene, followed by two $100 \%$ ethanol washes. A hydrophobic barrier was drawn around tissue sections with an ImmEdge pen (Vector Laboratories). It was important that tissue sections not dry out from this point. Sections were blocked with $\mathrm{H}_{2} \mathrm{O}_{2}$ for 10 minutes at room temperature. Slides were submerged in target retrieval solution at a temperature just below boiling for 10 minutes, rather than the manufacturer recommended boiling for 15 minutes. Protease plus solution was added to slides and incubated in $\mathrm{HybEZ}$ oven (ACD Bio) at $40^{\circ} \mathrm{C}$ for 10 minutes, rather than the manufacturer recommended 30 minutes. Target probe was added and allowed to hybridize for two hours at $40^{\circ} \mathrm{C}$ in the HybEZ oven. Serial steps to amplify signal involved the following solutions and conditions: Amp1 for 30 minutes at $40^{\circ} \mathrm{C}$, Amp2 for 15 minutes at $40^{\circ} \mathrm{C}$, Amp3 for 30 minutes at $40^{\circ} \mathrm{C}$, Amp 4 for 15 minutes at $40^{\circ} \mathrm{C}$, Amp5 for 30 minutes at room temperature, Amp6 for 15 minutes at room temperature, and detection reagent for 10 minutes at room temperature. Slides were washed in wash buffer between each probe hybridization and amplification step. Slides were counterstained with 1:1 Gill's hematoxylin and water, allowed to air dry, and coverslipped with mounting media. Slides were imaged on an Aperio ImageScope CS2. 


\section{Expression Quantification and Statistics:}

Representative images were selected from $n=6$ mammary carcinomas and $n=6$ nondiseased mammary tissues that were either immediately FFPE processed or wholemounted and carmine stained prior to standard processing. Mean probe per epithelial/neoplastic cell was measured within outlined mammary ducts and cancerous lesions for DapB, Mier3, and Polr2a expression using the HALO-ISH quantification software (Indica Labs). Mean \pm standard deviation of each RNAscope probe were used for quantitative comparisons. Two independent DMBA groups were sampled for mammary gland or mammary carcinoma tissue. Each tissue type was analyzed separately by TwoWay ANOVA. Independent variables were processing method (2 levels) and RNAscope probe (3 levels). Dependent variable was gene expression level measured as RNAscope target probe per cell. Tukey's post hoc test was used for group comparisons. Statistical analysis was performed using GraphPad Prism, version 7.00 for Windows (GraphPad Software).

\section{Results \& Discussion:}

Alum carmine stained mammary gland whole-mount screening was used to detect various lesions known to develop during mammary cancer progression, including premalignant hyperplasia and pre-invasive ductal carcinoma in situ (DCIS) not detectable by palpation. Mammary ductal structures, interspersed within stromal tissue, were visible upon staining with alum carmine. Early hyperplastic proliferative lesions were detected, as expected, in alum carmine stained mammary gland whole-mounts (Fig 23A) [449, 450, 462]. The gross morphology of hyperplasia was extended, thickened, and amplified ductal branches (Fig 23B), that appeared as ductal proliferation of more than two cell layers (Fig 
23C). Ductal carcinoma in situ (DCIS), an intraductal proliferation of epithelial cells, [453] was evident in whole-mounts as darkened structures (Fig 23A). These tumor lesions appeared as darkened structures due a dense concentration of neoplastic epithelial cells inhabiting these diseased morphologies (Fig 23D). Staining of tissue sections with H\&E was used to confirm morphological classifications made in mammary gland whole-mounts. Tissue sections of lesions were also used to identify specific histopathological subtypes of excised lesions. Mammary tumor lesions appeared as a population of cells with characteristics of a papillary growth pattern (Fig 23E). Invasive ductal carcinoma (IDC) presented in tissue sections as migratory epithelial cells penetrating ductal walls and invading stroma (Fig 24) [391, 453]. These IDCs appeared as palpable mammary tumors that were visible without alum carmine staining.

Whole-mount processing and screening for lesions of interest, followed by RNAscope, was successfully used in this study to produce RNAscope results similar to results obtained using samples directly processed by FFPE, which is the recommended protocol. Exposure to additional reagents prior to FFPE processing, required for wholemount preparation, did not have a negative effect on mRNA quality or abundance. No positive staining for the bacterial gene four-hydroxy-tetrahydrodipicolinate reductase $(D a p B)$ was observed (Fig 24). This indicated our negative control functioned properly as there was no signal for DapB RNA, which was not expected to be present. To test the sensitivity of RNAscope following whole-mount preparation and screening, we targeted the Mier family member 3 (Mier3) gene, a relatively low to moderately expressed gene transcript in rat mammary and female breast tissue, with average expression of 11 transcripts per million [463]. For comparison, we probed for RNA Polymerase II Subunit 





Figure 23: Premalignant and malignant lesions were visible in mammary gland tissue whole-mounts following alum carmine staining. A: A representative mammary gland from a Wistar Furth (WF/Hsd) virgin female eight weeks following 7,12dimethylbenz(a)anthracene (DMBA) induction of mammary carcinogenesis. Hyperplasia appeared as dark thickened branching (black box). DCIS appeared as a dark mass within mammary fat pads (black arrow). B: Thickened hyperplastic ductal structures in a mammary fat pad. C: H\&E staining of representative hyperplasia, indicated by proliferative ductal structures of more than two cell layers thick. D: A representative DCIS, indicated by a darkly stained tumor mass within the mammary fat pad. E: H\&E staining of DCIS, indicated by a monoclonal expansion of cells in a papillary growth pattern. 


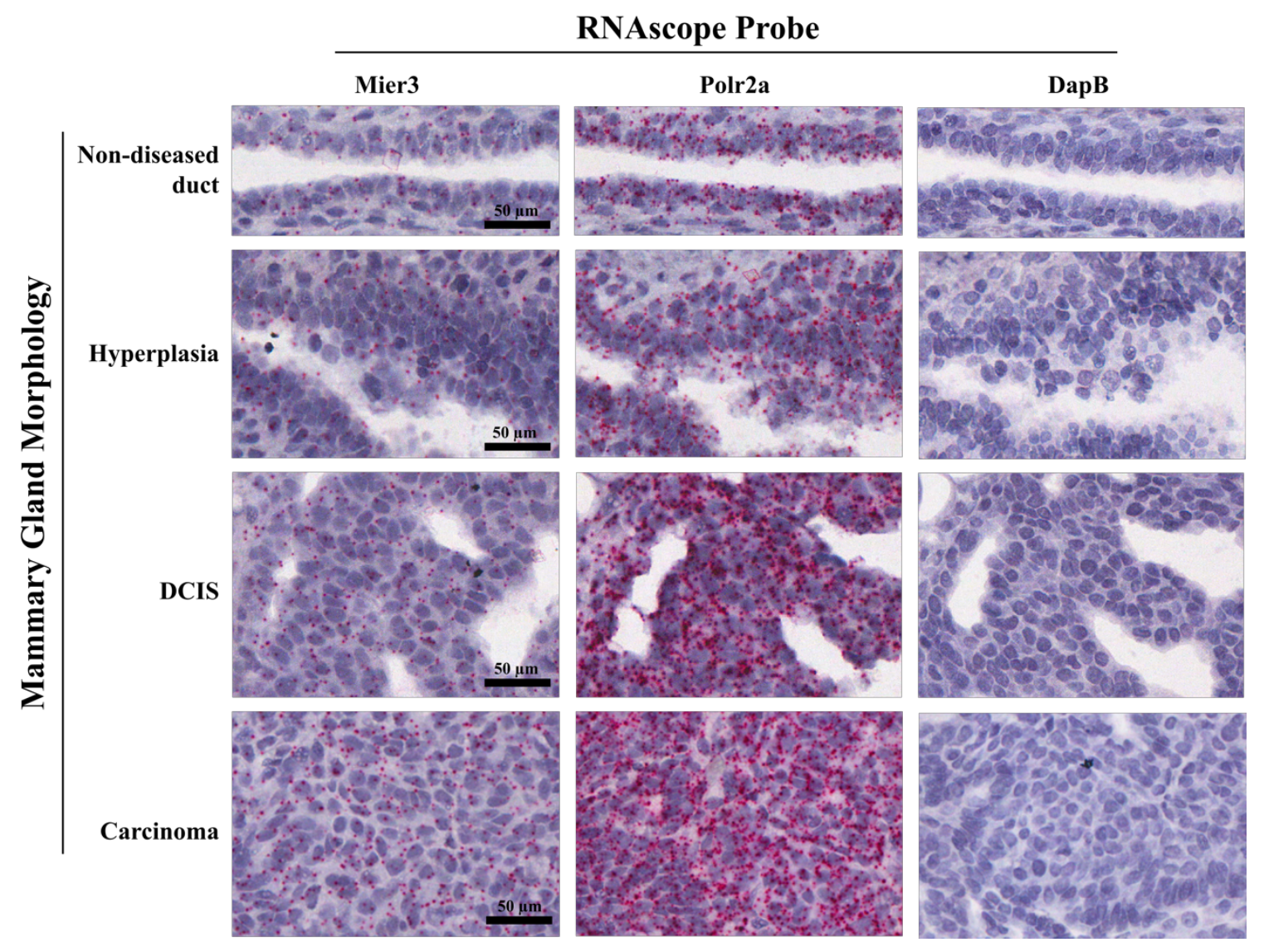

Figure 24: Representative RNAscope results from Mier3, Polr2a, and DapB probes in tissue sections from rat mammary gland whole-mounts. Rat mammary gland ductal hyperplasia, ductal carcinoma in situ (DCIS) and invasive ductal carcinoma (IDC) were identified in formalin-fixed alum-carmine stained whole-mounts. These lesions were excised and processed for RNAscope assays targeting Mier family member 3 (Mier3), a moderately expressed gene (left column), RNA Polymerase II Subunit A (Polr2a), a highly expressed gene (middle column), and bacterial 4-hydroxy-tetrahydrodipicolinate reductase $(D a p B)$, a negative control gene (right column). Each red puncta represents a single mRNA transcript [446]. Tissues were counterstained with hematoxylin. Images were taken at 40x magnification on an Aperio ImageScope CS2. 
$A($ Polr $2 A)$, a relatively highly expressed gene with an average of 79 transcripts per million in human breast tissue [463]. RNAscope assays probing for Polr2a and Mier3 reflected these expected expression differences (Fig 24). Results for Polr2a were densely packed red puncta within mammary ducts, while a probe targeting Mier 3 resulted in more scattered red puncta. There was no discernable difference in puncta abundance between RNAscope assays performed on samples excised from carmine stained whole-mounts (Fig 24) and mammary glands processed by FFPE immediately following tissue resection (Fig 25). This lack of difference between tissue treatment methods, along with an expected difference between independent gene target probes, indicated that the pre-screening process used did not negatively impact mRNA stability or abundance.

To determine if similar quantitative results of puncta density were obtained from different tissue processing methods, RNAscope images were quantified using HALO-ISH software (Indica Labs) and compared. Non-diseased mammary ducts had levels of DapB probe per mammary epithelial cell of $0.28 \pm 0.26$ by immediate FFPE and $0.35 \pm 0.19$ in tissue from whole-mounts (Fig 26). An RNAscope probe for Mier3 gave results of $2.34 \pm$ 0.46 in immediate FFPE tissue and $2.32 \pm 0.14$ in tissue processed from whole-mounts (Fig 26). The Polr $2 a$ probe per mammary epithelial cell were $7.55 \pm 1.22$ in immediately processed FFPE tissue and $7.21 \pm 1.22$ in tissue from whole-mounts (Fig 26). There was no statistically significant difference in quantities between tissues that were immediately FFPE processed and tissues excised from mammary gland whole-mounts. There was, as expected, a statistically significant difference between gene probes $(\mathrm{p}<0.0001)$. This confirmed anticipated results of no DapB expression, moderate Mier3 expression in both 


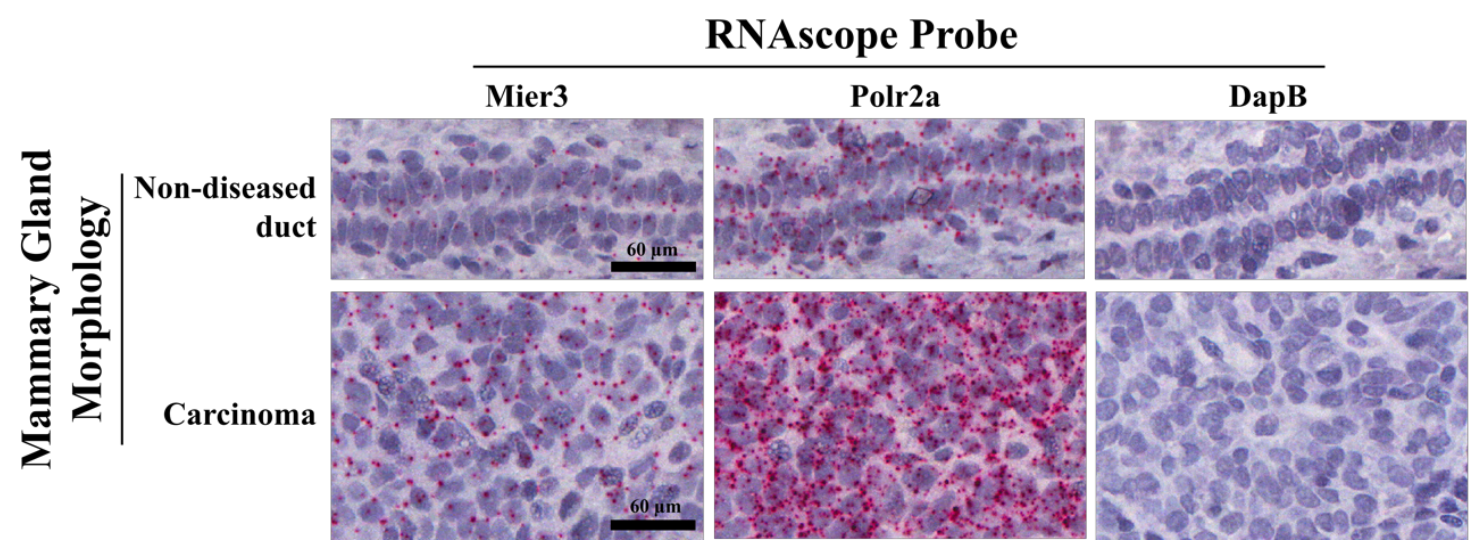

Figure 25: Representative images of RNAscope assays using Mier3, Polr2a, and DapB probes in rat mammary gland and mammary carcinoma tissue. Immediately processed FFPE tissue samples from non-diseased mammary glands or DMBA-induced mammary carcinomas were used. RNAscope assay probes for Mier family member 3 (Mier3), a moderately expressed gene (left column), RNA Polymerase II Subunit A (Polr2a), a highly expressed gene (middle column), and bacterial 4-hydroxy-tetrahydrodipicolinate reductase $(D a p B)$, a negative control gene (right column), were targeted. Each red puncta represents a single mRNA transcript [446]. Tissues were counterstained with hematoxylin. Images were taken at 40x magnification on an Aperio ImageScope CS. 
A

Non-diseased Mammary Ducts

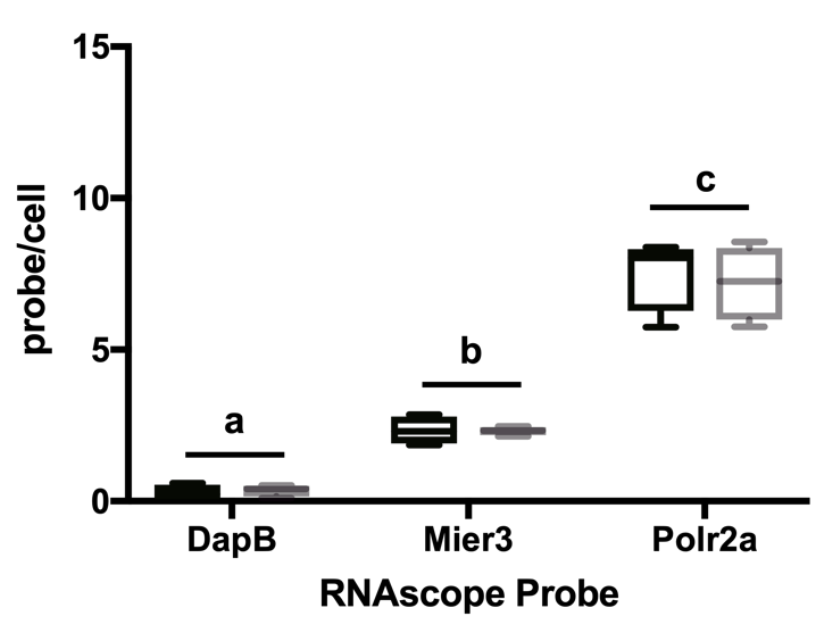

$\square$ FFPE

$\square$ Whole Mount

followed by FFPE

B

Mammary Carcinomas

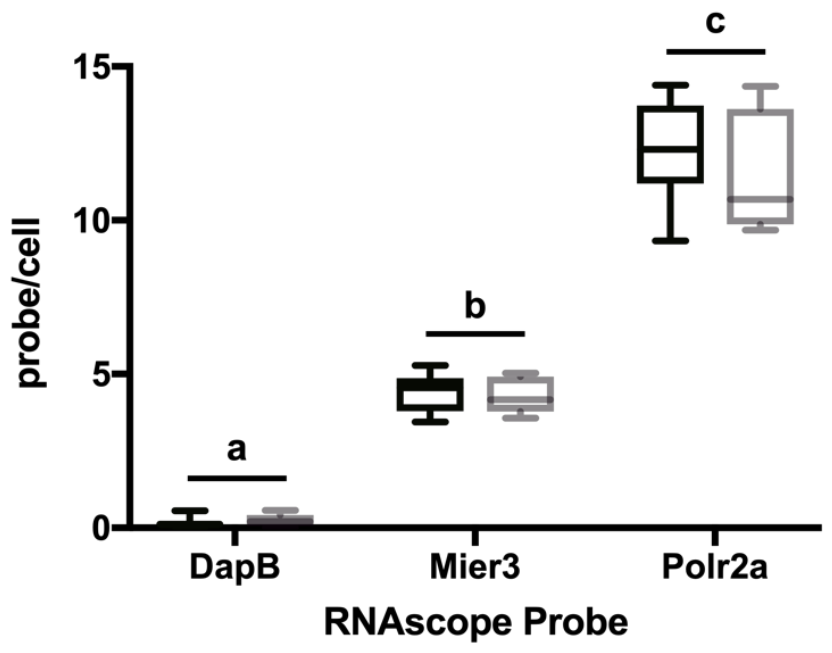


Figure 26: Quantitative analysis of RNAscope assays. RNAscope assays were performed on non-diseased mammary gland $(n=6$; panel A) and mammary carcinoma $(n=6$, panel B) tissue from the same rats. Tissues were either immediately processed by FFPE (manufacturer recommended) or excised from whole-mounts prior RNAscope assays. Tissue sections were probed for Mier family member 3 (Mier3), a moderately expressed gene, RNA Polymerase II Subunit A (Polr2a), a highly expressed gene, and bacterial 4hydroxy-tetrahydrodipicolinate reductase (DapB), a negative control gene that is not present in mammalian tissues. RNAscope probes per cell in non-diseased mammary ducts and mammary carcinomas were quantified using HALO-ISH quantification software (Indica Labs). There was no significant effect of processing method within either tissue type (Panel A- $\mathrm{p}=0.5057$, Panel B- $\mathrm{p}=0.6086$ ). There was a significant effect of RNAscope probe in both tissue types (Panels A and B p values $<0.0001$ ). RNAscope probe gene targets with different lowercase letters were significantly different $(\mathrm{p}<0.05)$. 
tissue processing methods, and high Polr $2 a$ expression, which indicated the quantitative sensitivity of RNAscope assays were retained in samples taken from whole-mounts.

Similar quantitative results between tissue processing methods were obtained when RNAscope assays from mammary carcinomas were analyzed (Fig 26). The DapB probe per carcinoma cell results were $0.15 \pm 0.17$ in immediate FFPE tumors and $0.23 \pm 0.20$ in mammary tumors taken from whole-mounts. The Mier 3 probe per cell results were $4.45 \pm$ 0.64 in immediate FFPE tumor tissue and $4.30 \pm 0.59$ in tumor tissue from whole-mounts. The Polr $2 a$ probe resulted per cell quantities of $12.27 \pm 1.65$ in immediate FFPE processed carcinomas and $11.52 \pm 1.90$ in carcinoma tissue from whole-mounts. There was no statistically significant effect of tissue processing method; however, there was a significant effect of RNAscope target probe $(\mathrm{p}<0.0001)$. These results validated that RNAscope was a robust and appropriate procedure for quantitative analysis of gene expression in mammary carcinoma tissue excised from mammary gland whole-mounts.

\section{Conclusion:}

RNAscope was used successfully on tissue collected from mammary gland wholemounts that had been processed for carmine staining. There was no loss in qualitative or quantitative gene expression measurements using RNAscope on tissue excised from whole-mounts. This method allowed for in situ pre-screening of mammary tissue to identify lesions or morphological epithelial changes that were visually detectable after whole tissue carmine staining. Methods outlined in this report provide a procedure to identify and quantitatively evaluate gene expression in various mammary gland pathologies associated with disease progression. 


\section{CHAPTER V \\ MIER3 EXPRESSION INCREASES THROUGHOUT RAT MAMMARY CANCER PROGRESSION}

\section{Introduction:}

The human breast contains small alveolar buds at the distal ends of ducts that are collectively referred to as terminal ductal lobular units. In the rat mammary gland these distal ductal buds are referred to a terminal end buds. It is commonly hypothesized that these structures are the initiation sites of invasive ductal carcinomas as they are the most rapidly proliferative components of the mammary gland, and thus contain vulnerable precursor cells $[102,464]$. Ductal carcinomas, which comprise the majority of breast cancers, are believed to develop along a precursor-carcinoma sequence $[218,465,466]$ beginning with normal ducts which can initiate proliferative disease. Disease progression of ductal origin can develop in sequential stages characterized by increasing cellular abnormalities and proliferation $[465,466]$. This simplified model is also applicable to DMBA-induced rat mammary cancer. In fact, the earliest observable change in rat mammary tissue following DMBA administration is atypical hyperplasia of cells surrounding ducts and ductules, which is the earliest disease morphology of the progression model [219].

Normally ducts are lined by two cell layers, the inner luminal cell layer and the 
outer myoepithelial layer. Proliferative cells can increase the cell number of this bi-cell layer, referred to as hyperplasia [467]. Usual ductal hyperplasia comprises a still heterogeneous population of cells that retains the ductal structure, and while it carries a 1.5 relative risk for development of invasive breast cancer, it does not significantly increase the risk of breast cancer development [467-469]. Atypical ductal hyperplasia (ADH) is a premalignant stage of the precursor-carcinoma disease sequence characterized by excessive proliferation of the mammary epithelium. In this stage, an outgrowth of the luminal epithelial cell layer is morphologically distinct from usual hyperplasia through clonal expansion of a now uniform population of cells, which can sometimes encompass the entire luminal space and distort the ductal architecture $[465,467,470]$. ADH can progress to ductal carcinoma in situ (DCIS), which is a preinvasive stage characterized by what is now considered neoplastic proliferation of epithelial cells that are still retained within the ductal-lobular structures [465]. ADH and low-grade DCIS share the same low grade monoclonal cell population, but ADH partially fills duct spaces whereas DCIS completely fills the duct spaces. Distinguishing between ADH and low-grade DCIS is based on quantitative criteria, with classification of a lesion as low-grade DCIS requiring complete involvement of at least two duct spaces or completely filled duct spaces measuring $>2 \mathrm{~mm}[465,470,471]$. Histologically, DCIS is considered to progress to invasive ductal carcinoma (IDC) when this lesion has extended through the basement membrane and invaded into the surrounding stroma [465]. This stage is characterized by a fragmented myoepithelial layer and basement membrane, if present at all [472]. IDC is a dangerous form of cancer as it can lead to metastasis and the formation of secondary tumors $[472,473]$. In summary, this precursor-carcinoma sequence of breast cancer development 

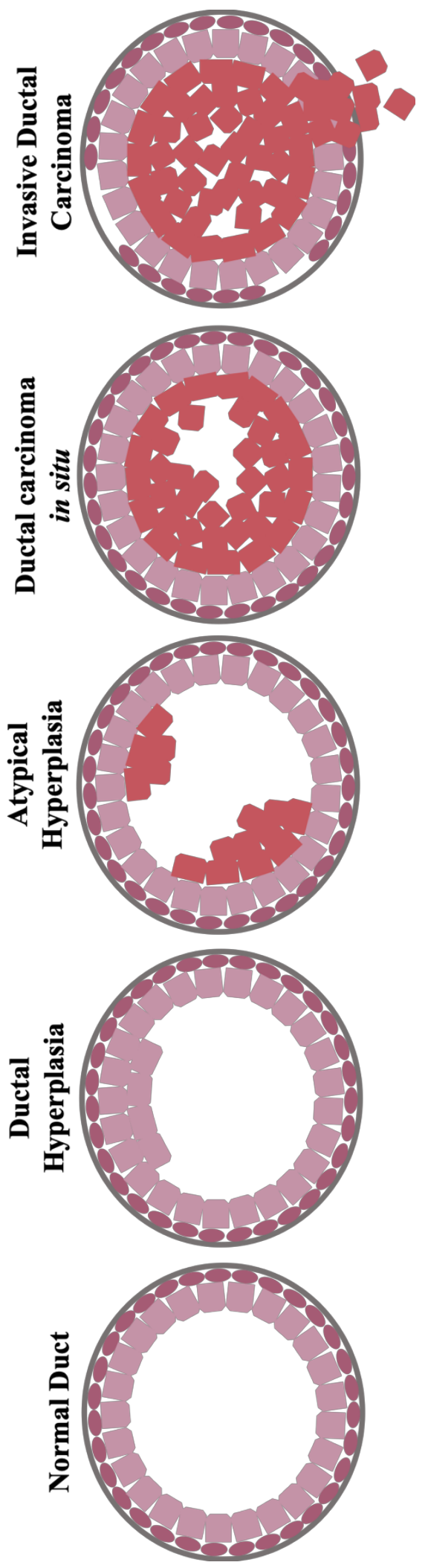
Figure 27: Precursor-Carcinoma Sequence of Mammary Cancer Development: A normal mammary duct is bilayered, with an inner luminal cell layer (light pink) and an outer myoepithelial cell layer (dark pink), and a basement membrane (gray circle) that separates the epithelial components from the outside stroma and adipose tissue. Noncancerous usual ductal hyperplasia occurs when luminal cells proliferate and increase the cell numbers from a bilayer. Atypical ductal hyperplasia (ADH) is a premalignant stage that is a result of clonal expansion of the luminal epithelial cell layer (bright pink) and outgrowth into the luminal space. ADH can progress to the preinvasive stage ductal carcinoma in situ (DCIS). This is a further expanded lesion from neoplastic cells but is still retained by the myoepithelial layer and basement membrane. DCIS is classified as an invasive ductal carcinoma (IDC) when the myoepithelial cell layer begins to disintegrate and the malignancy has extended through the basement membrane and invaded into the surrounding stroma. 
is comprised of definitive pathological stages, initiating as a premalignant stage of $\mathrm{ADH}$, progressing to a preinvasive stage of DCIS, and culminating in the potentially lethal stage of IDC (Fig 27) [218, 464, 466].

Cell proliferation drives a cancer phenotype. The average proliferation rate of terminal ductal lobular units, the most proliferative structure in the non-diseased breast is $2 \%$, which rises to $5 \%$ in $\mathrm{ADH}$ and $15 \%$ in DCIS [471]. It is widely held that cells acquire proliferative and malignant phenotypes through the accumulation of genetic activations/inactivations and mutations throughout cancer progression [474]. Transcriptional analysis of normal breast epithelium as compared to ADH, DCIS, and IDC has revealed distinct global alterations in gene expression shared amongst premalignant and malignant disease $[464,466,470,475]$. The finding that gene expression profiles are highly similar at each of these stages of breast cancer progression suggests a common clonal origin and that genes conferring proliferative and invasive potential are active at premalignant and preinvasive stages and maintained through later disease stages $[464,470$, 476]. As a result, the transcriptome of an early disease stage could be a useful indicator of the invasive potential of a later pathological lesion [464].

Previous work identified Mier 3 as a candidate mammary cancer susceptibility gene through physical mapping of the rat mammary cancer susceptibility quantitative trait locus, Mcs $1 b$. This locus was narrowed to a $1.8 \mathrm{Mb}$ region on rat chromosome 2 and results in around a $50 \%$ reduction in DMBA-induced mammary tumor multiplicity in rats homozygous for the Copenhagen strain Mcs $1 b$ resistance allele [248]. There are two genes encompassed in the overlap between rat $M c s 1 b$ and established human breast cancer risk allele 5q11.2, MIER3 and Mitogen-Activated Protein Kinase Kinase Kinase 1 (MAP3K1). 
MAP3K1 is an established breast cancer gene [477]. In this study, I used a DMBA-induced rat mammary cancer model of female breast cancer to investigate Mier3 expression throughout a precursor-carcinoma sequence of ductal origin. I report the predominant Mier3-expressing mammary gland cell type as ductal luminal epithelial cells, significant increases in Mier3 expression with increasing malignancy of each histopathological stage of progression, and the highest Mier 3 levels in invasive ductal carcinomas. These findings suggest a potential role in disease progression and elevate MIER3 as a possible biomarker or therapeutic target in breast disease development.

\section{Methods:}

Animal husbandry

Rats were maintained in an AAALAC-approved facility on a $12 \mathrm{~h}$ light/dark cycle and provided LabDiet 5001 Rodent Diet (PMI ${ }^{\circledR}$ Nutrition International) and water ad libitum. Inbred Wistar Furth (WF/Hsd) females were obtained from Envigo. All animal protocols were approved by the University of Louisville Animal Care and Use Committee.

\section{Congenic Strains and Animal Breeding}

The Wistar Furth (WF/NHsd) inbred rat strain was used as a mammary cancer susceptible line. The Wistar Furth.Copenhagen (WF.COP) congenic rat strain N3 contains a segment of Copenhagen (COP/NHsd) on $\mathrm{RNO} 2$ from markers D2Mgh2 to g2UL1-5 (Chr2:40171863 - 43756834, build RGSC 6.0/rn6) and is positive for the Mcs $1 b$ decreased susceptibility allele. The Mcs $1 b$ N3 strain at generation N16F40 was used as a mammary cancer resistance line and compared to the susceptible WF strain. 


\section{DMBA Administration and Mammary Gland Tissue Collection}

At 50-55 days of age 7,12-Dimethylbenz(a)anthracene (DMBA Acros Organics, CAS\#: $57-97-6,20 \mathrm{mg} / \mathrm{mL}$ in sesame oil) was given by single oral gavage (65 $\mathrm{mg}$ DMBA/kg body mass) to WF and WF.COP-Mcs $1 b$ strain N3 females to induce mammary carcinogenesis. At time points of 4, 8, 12, and 15 weeks following DMBA administration, six rats of each strain were euthanized and mammary glands formalin fixed and paraffin embedded (FFPE) (Fig 28).

Tissue selection and Classification

Sectioning of FFPE blocks was done one day prior to a planned RNAscope assay, as mRNA remains more stable within paraffin blocks than tissue sections [460]. Mammary gland FFPE blocks were cut in 4 um sections onto Superfrost Plus microscope slides using a rotary microtome (Thermo HM 355S). Representative slides from mammary glands exposed to DMBA were stained by hematoxylin and eosin to identify histopathological features of mammary cancer progression. Minimally 6 slides from each Mcs $1 b$ genotype that displayed normal mammary duct morphology and usual hyperplasia were selected to assay within the timepoint of 4 weeks post-DMBA administration. At least 6 slides per genotype within each of the remaining carcinogenesis timepoints of 8,12 , and 15 weeks post-DMBA administration were selected to assay that displayed stages of progression normal morphology, usual hyperplasia, ADH, DCIS, and IDC. 
Hematoxylin and Eosin (H\&E) staining of sectioned mammary glands:

An H\&E stain on sectioned tissues was used to assess tissue sample quality and to confirm the lesion of interest was present in the section. Slides were deparaffinized and rehydrated using 3 separate xylene washes followed by single washes each in $100 \%$ ethanol, $90 \%$ ethanol, $80 \%$ ethanol, and DI water. Tissues were stained with hematoxylin for 3 minutes, rinsed under running DI water for 3 minutes, soaked in bluing reagent for less than a minute, and again rinsed with DI water. Slides were dipped a few times in $100 \%$ ethanol to eliminate water before staining in eosin-Y for 30-45 seconds. Tissue was then dehydrated using washes of $90 \%$ ethanol, $100 \%$ ethanol, and 3 separate xylene washes. Samples were air dried and coverslipped using permount or similar mounting media.

RNA in situ hybridization

RNAscope was used to identify the spatiotemporal cell-specific expression of Mier3 in rat mammary tissue according to manufacturer's directions (ACD Bio). Briefly, slides were deparaffinized in xylene and $100 \%$ ethanol, pretreated with an $\mathrm{H}_{2} \mathrm{O}_{2}$ block for 10 minutes, placed in target retrieval solution that was just under boiling (ACD Bio 322000 ) for 10 minutes, and treated with protease plus solution for 10 minutes (ACD Bio 322330). The target probe was added and slides were incubated in HybEZ oven (ACD Bio) at $40^{\circ} \mathrm{C}$ for 2 hours. Amplification steps were as follows using an RNAscope $2.5 \mathrm{HD}$ red detection kit (ACD Bio 322360): Amp1 for 30 minutes at $40^{\circ} \mathrm{C}$, Amp2 for 15 minutes at $40^{\circ} \mathrm{C}$, Amp3 for 30 minutes at $40^{\circ} \mathrm{C}$, Amp4 for 15 minutes at $40^{\circ} \mathrm{C}$, Amp5 for 30 minutes at room temp, Amp6 for 15 minutes at room temp, and detection reagent for 10 minutes at room temp. Slides were counterstained with 1:1 Gill's hematoxylin and water. Vectashield 
mounting media (Vector Laboratories H-1000) was added to protect staining. Slides were imaged at 40x on an Aperio ImageScope CS2.

Expression Quantification, Data Handling, and Statistical Analysis

Within images of tissue slides following the RNAscope assay, occurrences of normal mammary ducts, usual hyperplasia, ADH, DCIS, and IDC were outlined and selected for analysis using the HALO-ISH quantification software from Indica Labs. Mean probe per mammary epithelial cell and percent of Mier3 positive mammary epithelial cell data was collected. Average Mier 3 transcript quantification values were transformed by taking the natural log. Percent positivity values were transformed by taking the arcsin of the square root of the decimal percent values. Transformed data were analyzed via oneway or two-way ANOVA depending on the number of variables. Independent variables included timepoint, genotype, and morphology. All statistical analysis was done using GraphPad Prism version 7.00 for Windows, GraphPad Software, La Jolla California USA.

\section{Results:}

\section{The RNAscope assay functions properly in rat mammary gland and disease tissue}

To study Mier3 expression throughout mammary cancer progression, WF susceptible and WF.COP Mcslb-resistant N3 females were administered DMBA and mammary glands analyzed at various timepoints following this exposure. A mammary gland collection timeline of all experimental stages used in this study is outlined in Figure 28. To begin, the utility of the RNAscope assay in rat mammary tissue was tested using the experimental Mier3 probe and manufacturer recommended (ACD Bio) control probes. A 


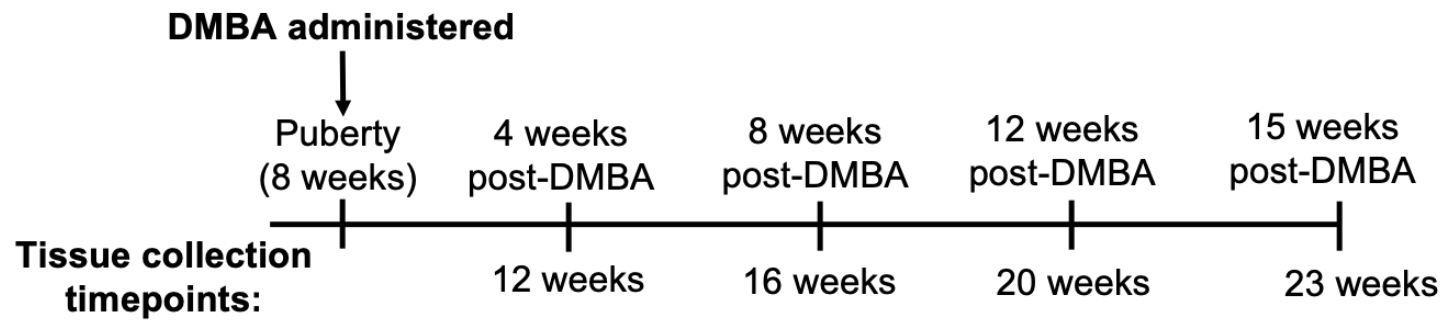

Figure 28: Disease progression mammary gland collection timeline: Female rats from the WF and WF.COP Mcs $1 b$ N3 strain were administered the chemical carcinogen DMBA at 50-55 days of age. Mammary glands were collected at 4, 8, 12, and 15 weeks post administration (top row), corresponding to 12, 16, 20, and 23 weeks of age (bottom row). 
probe for RNA Polymerase II Subunit A (Polr2a) was used as a positive control due to its ubiquitous expression and the probe for the bacterial gene 4-hydroxytetrahydrodipicolinate reductase (DapB) used as a negative control. Mammary tissue has a high adipose content so is not as robust as other tissue types. As a result, the manufacturers protocol had to be experimentally modified to reduce the harsh pretreatment conditions, namely lower temperature of the target retrieval buffer, and less exposure time to the buffer and protease treatment. Following optimization of the protocol, all three probes functioned as expected in each progression stage using the RNAscope assay, with indistinct stains due to high Polr2a expression, median Mier3 expression appearing as distinct puncta, and no DapB expression. This demonstrates the specificity of the Mier3 probe in rat mammary tissue (Fig 29).

\section{Mammary epithelial cells are the highest Mier3-expressing cells in the rat mammary gland}

To determine if there is cell-specific expression of Mier 3 in rat mammary glands, RNAscope assays probing for Mier3 followed by quantification with HALO-ISH module software from Indica Labs were performed on mammary glands from 8-week-old WF strain females. Regions of assayed mammary glands were classified according to major tissue elements (Fig 30A) and collectively quantified (Fig 30B) (Table 18). Mammary ducts displayed the greatest average Mier3 probe per mammary epithelial cell (MEC) followed by lymph nodes. Blood vessels, stroma, and adipose tissue showed negligible Mier3 expression. One-way ANOVA followed by Tukey's multiple comparisons test was performed. No difference was found between mammary ducts and lymph nodes, as well as 


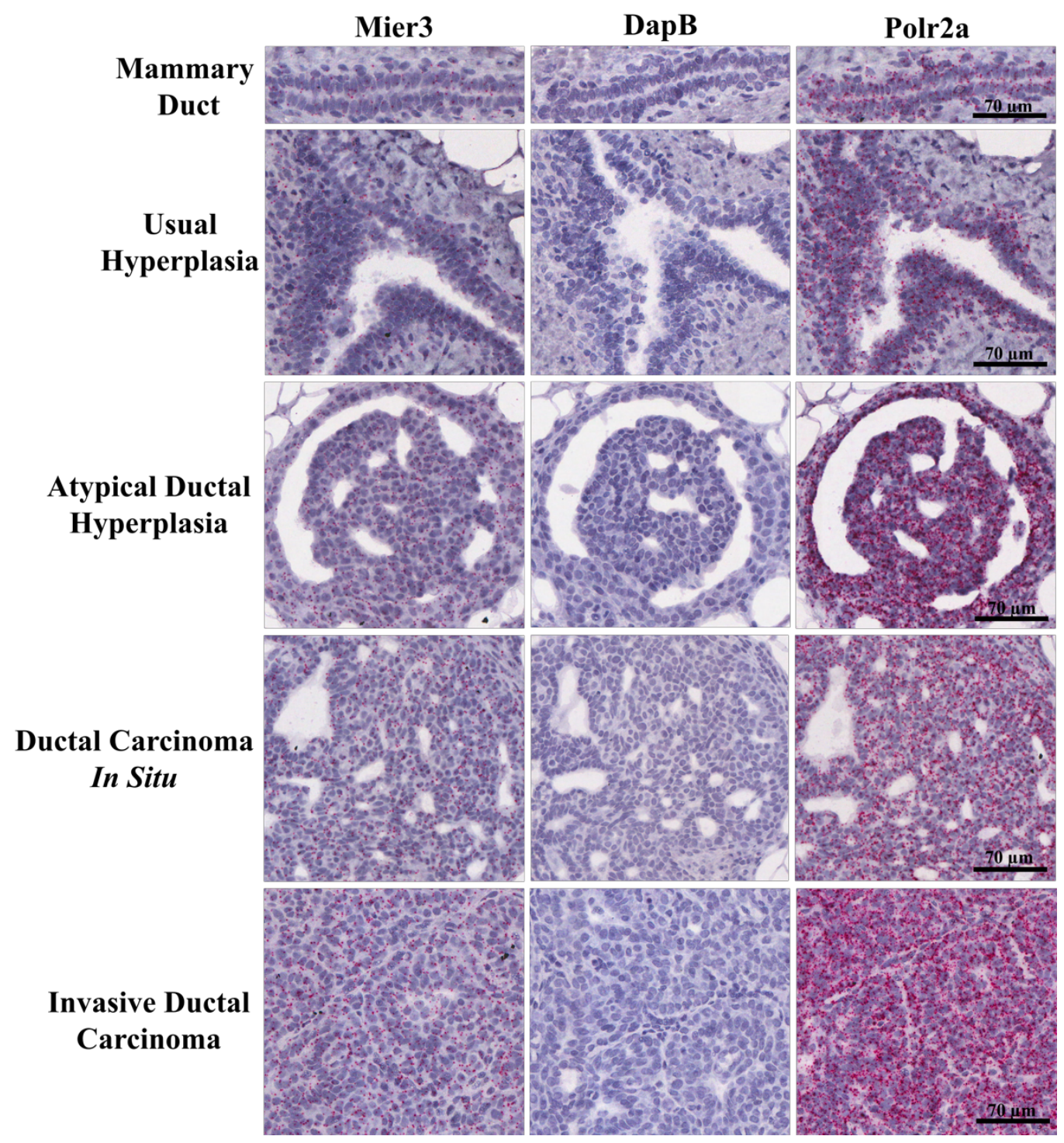

Figure 29: RNA in situ hybridization (RNAscope) control and Mier3 probes function

properly in rat mammary tissue: Shown are representative images of the ductal histopathologies along the precursor-carcinoma sequence of mammary cancer progression following RNAscope assays (top to bottom). Columns display assays that probed for Mier3, 4-hydroxy-tetrahydrodipicolinate reductase probe (DapB, negative control), and RNA Polymerase II Subunit A probe (Polr2A, positive control). Images were taken at 40x magnification on an Aperio ImageScope CS2. 


\section{A}
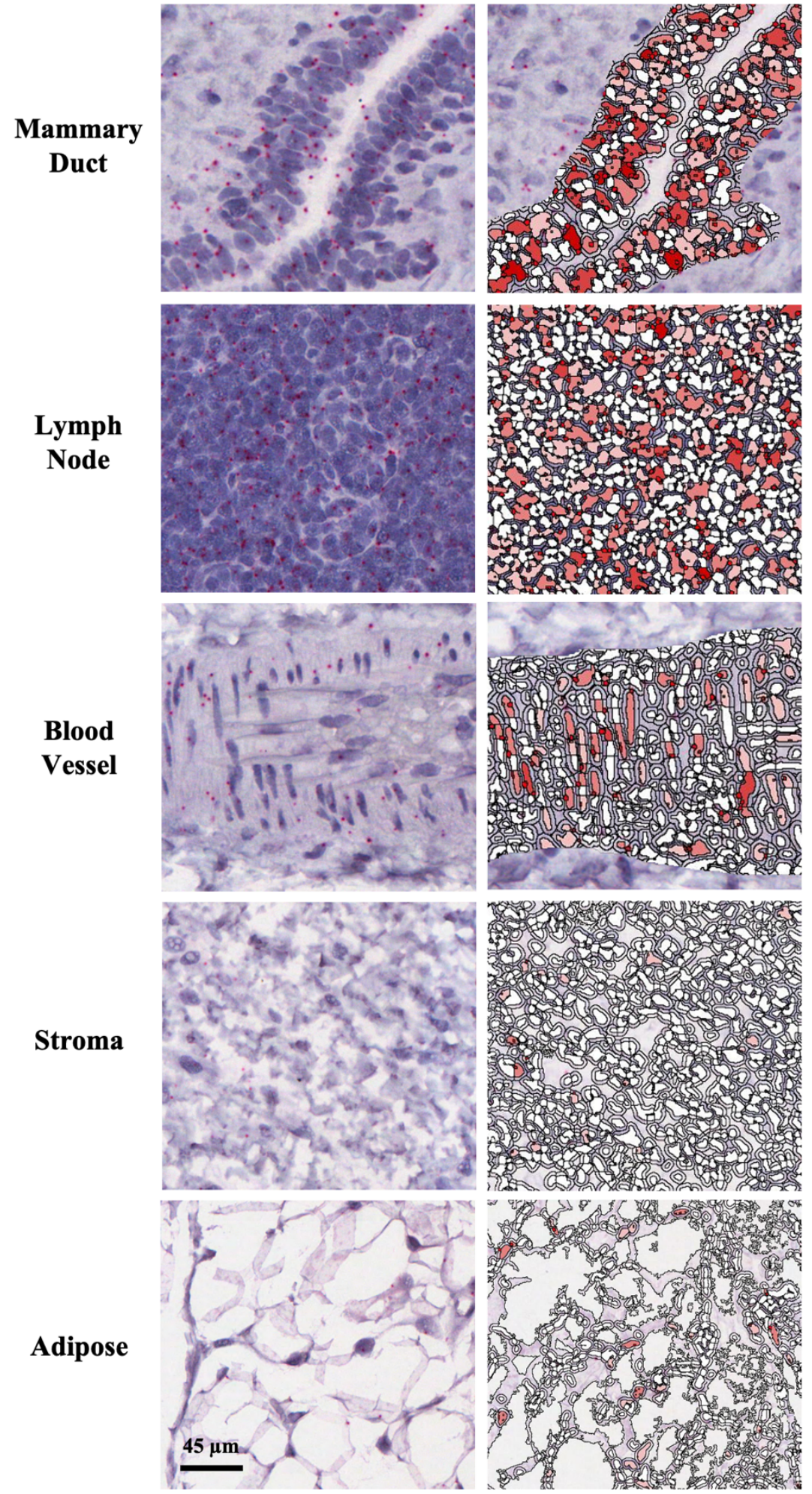


\section{Mier3 expression in rat mammary gland tissue elements}

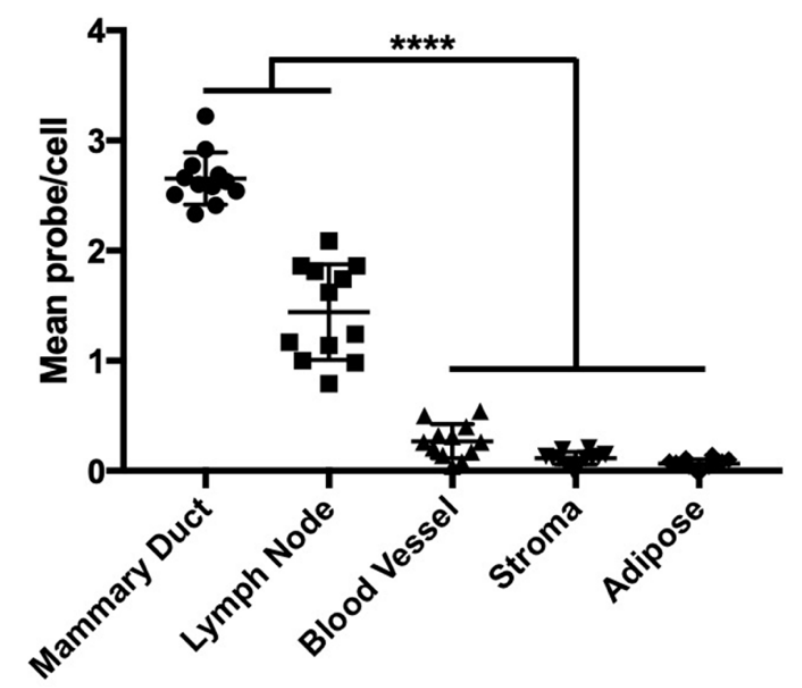

Figure 30: Mammary ducts possess the greatest Mier3 expression levels of all rat mammary gland tissue elements: RNAscope assays probing for Mier3 were done on 8week-old WF female mammary glands.

A- 40x mammary gland histologic images show red puncta (Mier3 transcripts) counterstained with hematoxylin. Rows display a representative mammary duct, lymph node, blood vessel, stroma, and adipose tissue. The right column is the red probe and nuclei markup from the HALO-ISH module software from Indica Labs. Negative cells are white. Cells positive for Mier3 are shades of red, with light pink corresponding to 1-3 puncta and dark red displaying $>14$ puncta.

B- Quantification of mean Mier3 expression per cell analyzed in each mammary tissue element. One-way ANOVA followed by Tukey’s multiple comparisons test. Connected morphology groups indicate Tukey's post hoc test $\mathrm{p}<0.0001$. $\mathrm{n}=6$ individual rat mammary glands, $\mathrm{n}=2$ viewpoints per sample, for $\mathrm{N}=12$ per tissue element. 
Table 18. Spatial expression of Mier3 in 8-week-old mammary gland tissue from female WF/NHsd rats

\begin{tabular}{cccc}
\hline Tissue Element & Cell Types & $\begin{array}{c}\text { Mean probe per } \\
\text { cell (SD) }\end{array}$ & $\begin{array}{c}\text { Percent of } \\
\text { positive cells (SD) }\end{array}$ \\
\hline Mammary Ducts & $\begin{array}{c}\text { Myoepithelial, luminal } \\
\text { epithelial cells }\end{array}$ & $2.66(0.24)$ & $58.2(8.1)$ \\
Lymph Nodes & Immune cells & $1.44(0.43)$ & $32.4(8.5)$ \\
Blood Vessels & Vascular endothelial cells & $0.27(0.16)$ & $10.2(5.3)$ \\
Stroma & Fibroblasts & $0.12(0.06)$ & $5.3(2.8)$ \\
Adipose & Adipocytes & $0.07(0.04)$ & $4.1(2.0)$ \\
\hline
\end{tabular}

$\mathrm{n}=6$ mammary glands, $\mathrm{n}=2$ viewpoints per tissue element, $\mathrm{N}=12$ 
between blood vessels, stroma, or adipose. However, there was a significant difference between these two groups $(\mathrm{p}<0.0001)$. In the mammary ducts specifically, the inner luminal epithelial cell layer visibly possesses the majority of Mier3 expression, with the outer myoepithelial cell layer displaying nominal expression (Fig 31).

\section{DMBA induces Mier3 expression in non-diseased rat mammary glands}

Following the finding that MECs in mammary ducts are the major Mier3expressing cell type in rat mammary glands, the effect of administration of the mammary carcinogen DMBA on Mier3 expression was explored. Mammary glands were collected from 12-week-old age-matched WF strain females either unexposed (control) or 4 weeks post-DMBA administration. This age was selected for tissue collection because it follows the acute phase for DMBA-toxicity but is also prior to the development of mammary lesions and carcinomas in susceptible rat strains. RNAscope assays probing for Mier3 and downstream HALO-ISH module software quantification was performed on mammary ducts (Fig 32A) (Table 19). DMBA-exposed mammary ducts had significantly greater Mier3 expression levels (unpaired 2-tailed t test $\mathrm{p}=0.0001$ ) (Fig 32B). DMBA-exposed MECs also had greater percent positivity for overall Mier3 expression (unpaired 2-tailed $\mathrm{t}$ test $\mathrm{p}=0.0001)($ Fig $32 \mathrm{C})$.

\section{There is no difference in Mier3 expression between bi-layered and hyperplastic mammary ducts}

After determining an effect of DMBA administration of Mier3 expression, Mier3 expression differences were explored throughout mammary cancer progression in 


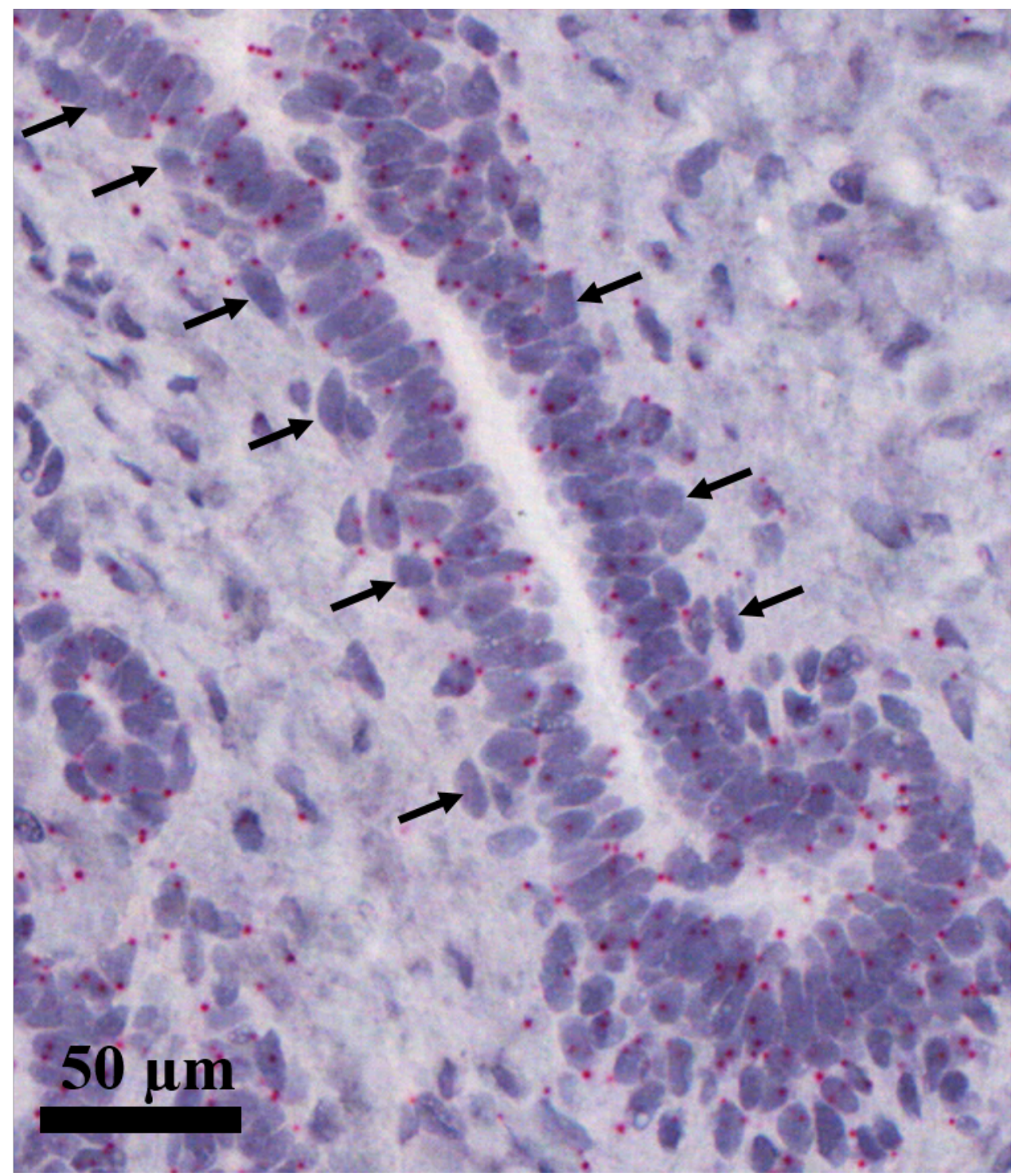

Figure 31: The majority of ductal Mier3 expression is localized to luminal epithelial cells. This representative mammary duct histologic image shows red puncta (Mier3 transcripts) counterstained with hematoxylin. Image was taken at 40x magnification on an Aperio ImageScope CS2. Black arrows point to outer myoepithelial cells that show nominal Mier3 expression. Inner columnar luminal epithelial cells display the greatest Mier3 expression levels. 
A

12 weeks Control
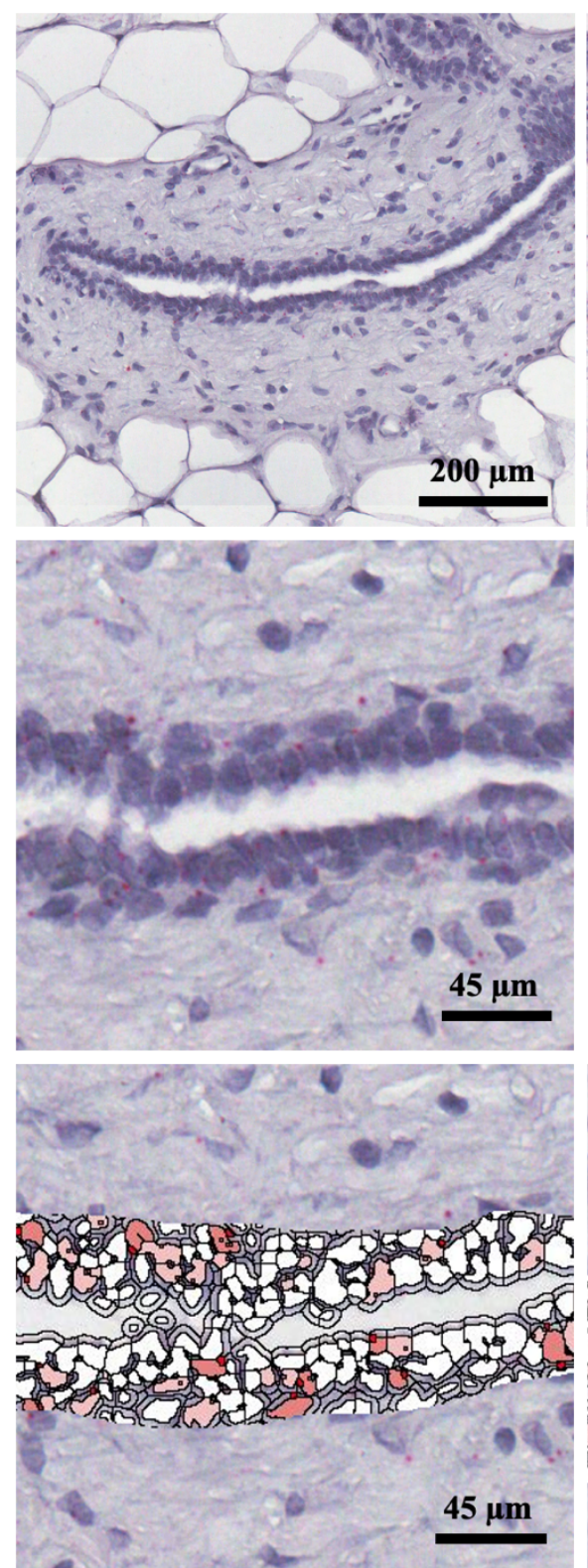

12 weeks +DMBA
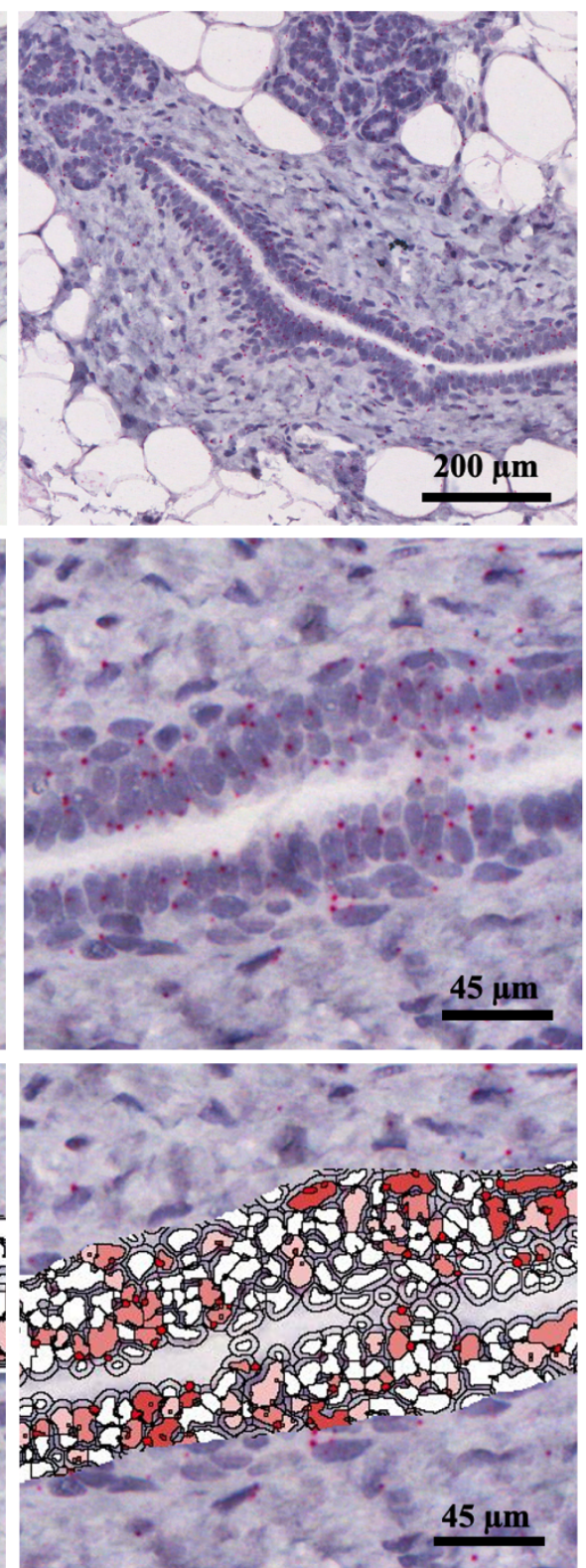
B

Ductal Mier3 expression in rat mammary glands at 12 weeks of age with and without DMBA exposure

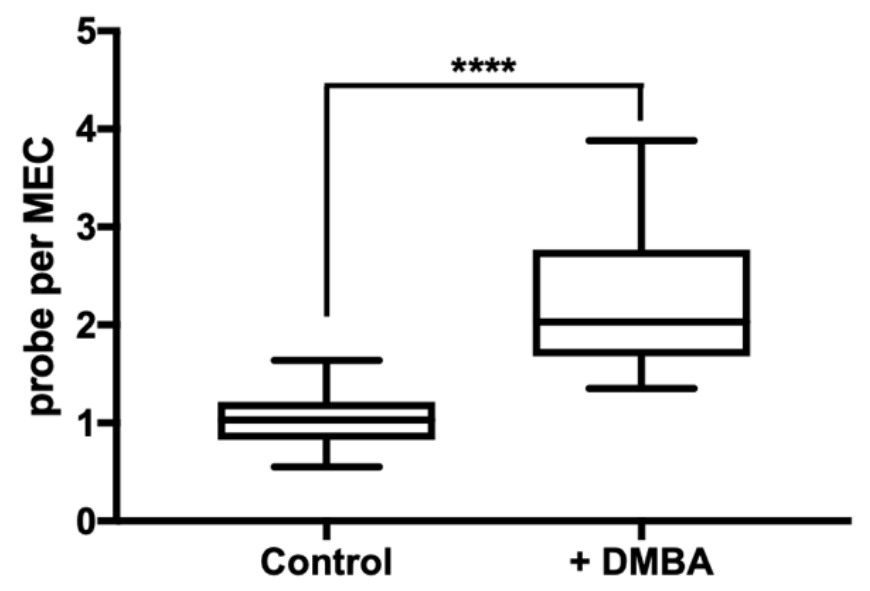

C

Mier3 positive MECs in rat mammary glands at 12 weeks of age with and without DMBA exposure

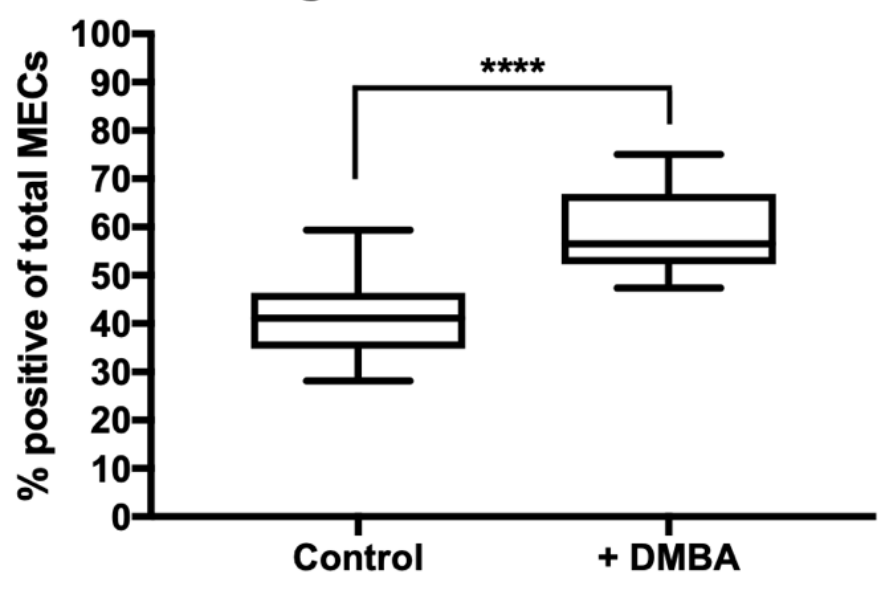


Figure 32: Ductal Mier3 expression is significantly increased with DMBA-exposure: RNAscope assays probing for Mier3 were done on age-matched 12-week-old WF female rat mammary glands that were either unexposed to DMBA (control) or administered DMBA 4 weeks prior. (A) 40x mammary gland histologic images show red puncta (Mier3 transcripts) counterstained with hematoxylin. Columns display an unexposed mammary duct (left) and a DMBA-exposed mammary duct (right). The bottom row is the red probe and nuclei markup from the HALO-ISH module software. (B) Quantification of mean Mier3 expression per MEC. There was a significant difference in expression with DMBA exposure using a one-way ANOVA followed by Tukey's post hoc test $(\mathrm{p}=0.0001)$. (C) Quantification of MECs positive for Mier3 expression. There was a significant difference in Mier3 positive cells with DMBA exposure using an unpaired 2-tailed t test $(\mathrm{p}=0.0001)$. $\mathrm{N}=12$ mammary glands per group. 
Table 19. Mier3 expression in 12-week-old female WF/NHsd rat mammary glands with and without DMBA exposure

\begin{tabular}{ccc}
\hline Treatment & Mean probe per cell (SD) & Percent of positive cells (SD) \\
\hline None & $1.03(0.28)$ & $41.3(8.2)$ \\
DMBA & $2.24(0.68)$ & $59.4(8.5)$ \\
\hline
\end{tabular}

$\mathrm{N}=12$ mammary glands per group 
mammary cancer resistant vs susceptible strains of rat. Mammary glands from the WF and WF.COP Mcs $1 b$-N3 strains of rats were first collected at an early timepoint of 4 weeks following administration of DMBA. RNAscope assays probing for Mier3 transcripts was performed and resulting images quantified using the HALO-ISH module software from Indica Labs (Fig 33A) (Table 20). Mammary ducts were classified as normal based on a typical bi-cell layer or usual ductal hyperplasia if there was an increase in cell number across the duct. There was no significant difference in Mier3 expression based on genotype or morphology ( $p=0.3604$ genotype, $p=0.3661$ morphology, two-way ANOVA) (Fig 33B). There was also no difference when quantifying based on MEC Mier3 percent positivity ( $p=0.4048$ genotype, $p=0.1793$ morphology, two-way ANOVA) (Fig 33C).

\section{Mier3 expression significantly increases with each histopathological stage along a precursor-carcinoma sequence of mammary cancer development}

To continue Mier3 expression analysis throughout disease progression, the process of mammary gland tissue collection, RNAscope assays, and quantification using HALOISH module software was performed at 8-, 12-, and 15-weeks post-DMBA administration in WF and WF.COP Mcs $1 b$-N3 females. At these timepoints, IDC and the morphologies of the precursor-carcinoma sequence that can lead to IDC are present, including ADH and DCIS. These progression morphologies, as well as normal mammary ducts and hyperplastic ducts, were assessed for Mier3 expression (Fig 34A, 35A, 36A). 
A

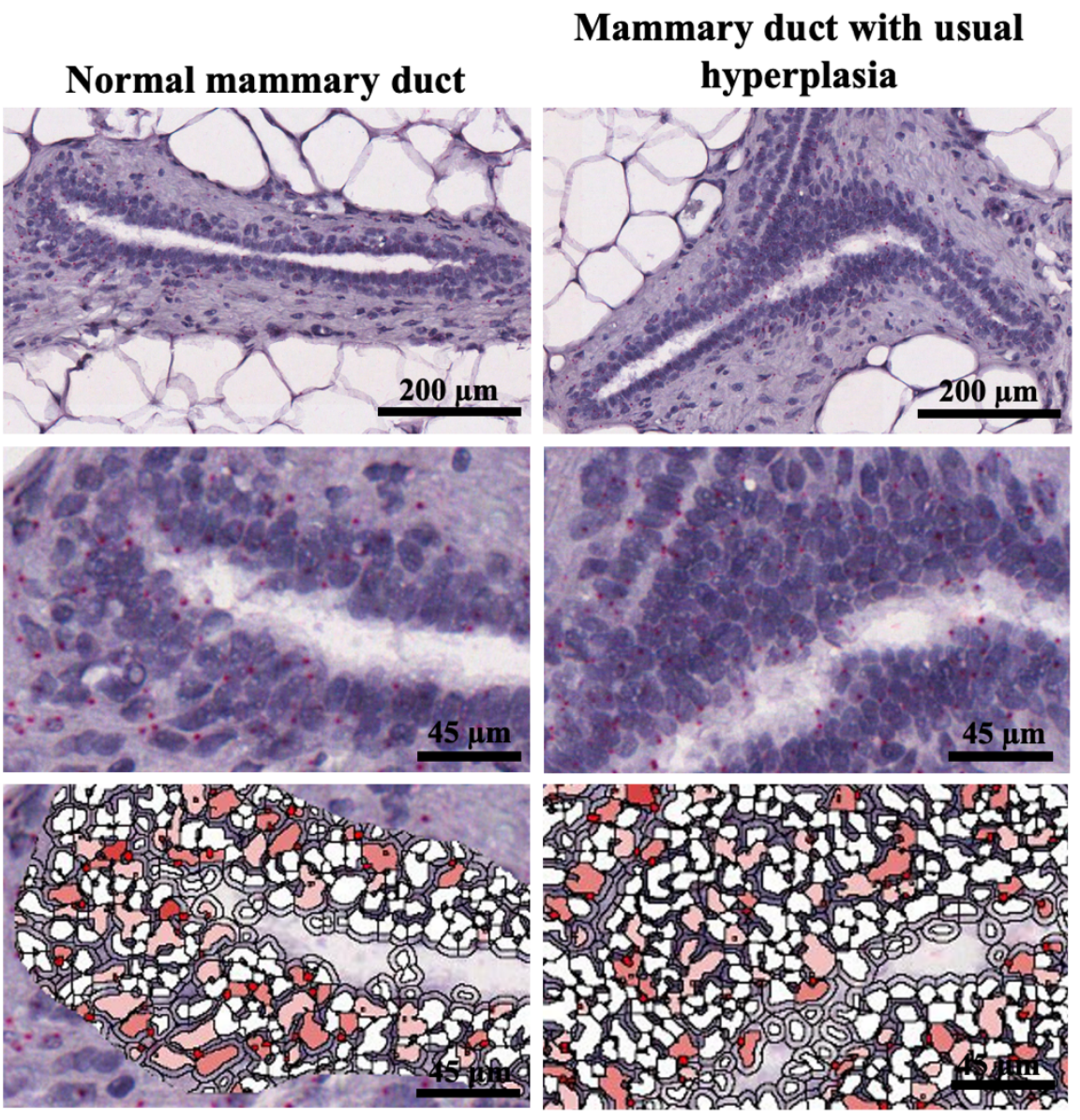

B

Mier3 expression in rat mammary glands 4 weeks post DMBA administration

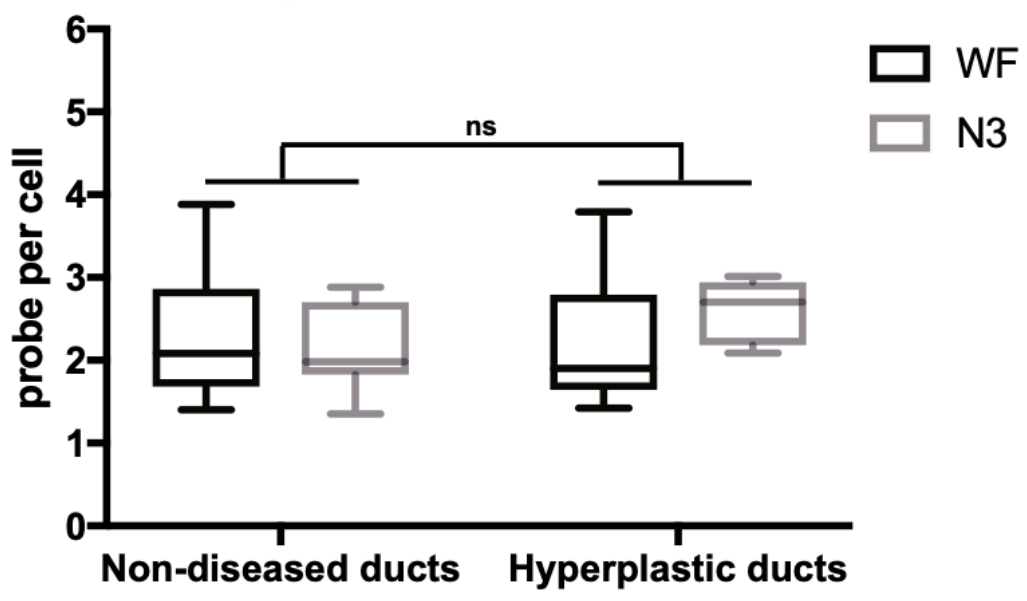




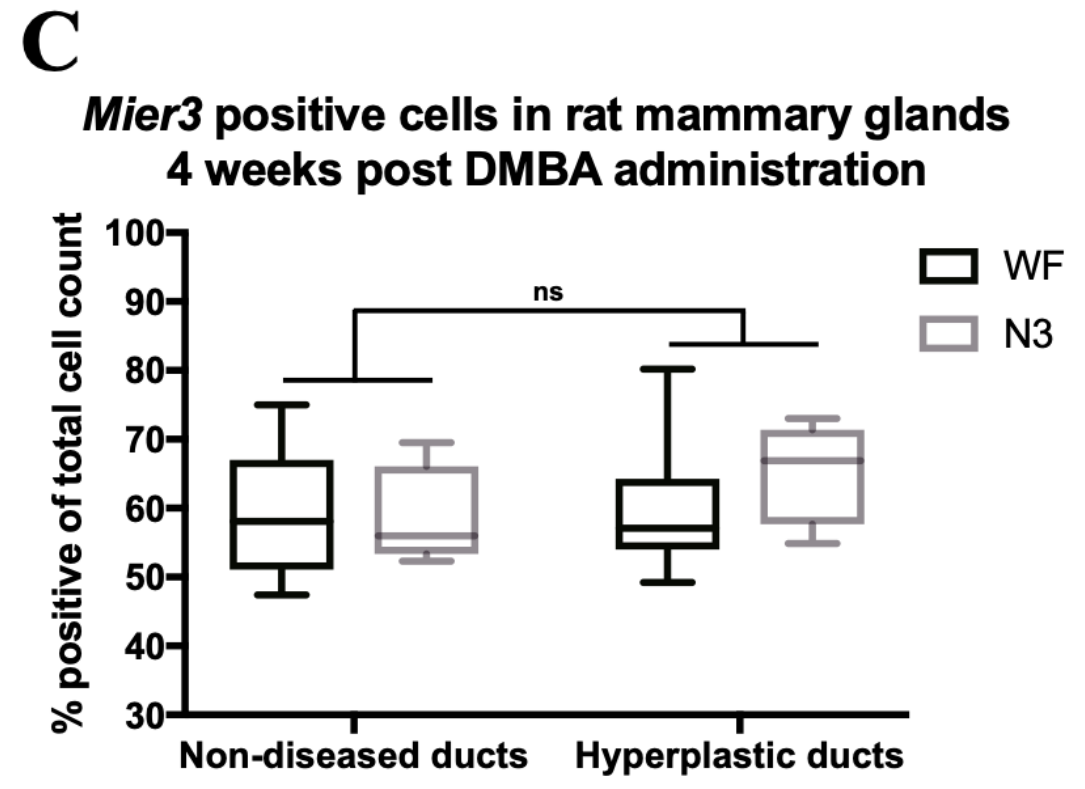

Figure 33: Mier3 expression in rat mammary glands 4 weeks post DMBA administration: (A) 40x mammary gland histologic images from RNAscope assays probing for Mier3 show red puncta (Mier3 transcripts) counterstained with hematoxylin. Columns display a representative non-diseased mammary duct (left) and a mammary duct with usual hyperplasia (right). The middle row provides a closer view to observe red puncta. The bottom row is the red probe and nuclei markup from the HALO-ISH module software. (B) Quantification of mean Mier3 expression per MEC in normal mammary ducts and ducts with usual hyperplasia in the WF vs N3 strain of rat. Two-way ANOVA followed by Tukey's multiple comparisons test found no significant difference based on genotype or morphology. (C) Quantification of MECs positive for Mier3 expression. Two-way ANOVA followed by Tukey's multiple comparisons test found no significant difference based on genotype or morphology. $N=6$ mammary glands per genotype, minimally $n=8$ viewpoints per morphology per genotype. 
Table 20. Mier3 mammary ductal expression in WF/NHsd and WF.COP Mcs1b-N3 females 4 weeks post-DMBA administration

\begin{tabular}{cccc}
\hline Genotype & Morphology & $\begin{array}{c}\text { Mean probe per } \\
\text { cell (SD) }\end{array}$ & $\begin{array}{c}\text { Percent of positive } \\
\text { cells (SD) }\end{array}$ \\
\hline WF/NHsd & Bi-layered ducts & $2.27(0.73)$ & $59.6(9.1)$ \\
WF.COP Mcs $1 b$-N3 & Bi-layered ducts & $2.15(0.53)$ & $59.0(6.7)$ \\
WF/NHsd & Hyperplastic ducts & $2.18(0.67)$ & $60.3(8.5)$ \\
WF.COP Mcs $1 b$-N3 & Hyperplastic ducts & $2.56(0.38)$ & $65.2(6.7)$ \\
\hline N=6 mammary glands per genotype &
\end{tabular}


Quantification of mean Mier3 probe per MEC/carcinoma cell at 8 weeks postDMBA administration found no significant difference based on genotype, but there was for morphology ( $\mathrm{p}=0.4096$ genotype, $\mathrm{p}<0.0001$ morphology, two-way ANOVA) (Fig 34B) (Table 21). Normal/hyperplastic ducts were significantly different from ADH $(\mathrm{p}=0.0001$, Tukeys post hoc) and DCIS and IDC ( $<<0.0001)$. ADH significantly differed from IDC $(\mathrm{p}=0.01)$. Overall cells positive for Mier 3 was also assessed $(\mathrm{p}=0.0552$ genotype, $\mathrm{p}<0.0001$ morphology, two-way ANOVA) (Fig 34C). Normal/hyperplastic ducts differed from ADH $(\mathrm{p}=0.0001)$, DCIS $(\mathrm{P}<0.0001)$, and IDC $(\mathrm{p}=0.001)$. ADH differed from IDC $(\mathrm{p}=0.01)$.

At 12 weeks post-DMBA administration, there was again only a difference based on mammary gland disease morphology when analyzing by mean Mier3 probe per cell $(\mathrm{p}=0.6544$ genotype, $\mathrm{p}<0.0001$ morphology, two-way ANOVA) and overall positivity $(\mathrm{p}=0.2624$ genotype, $\mathrm{p}<0.0001$ morphology, two-way ANOVA) (Fig 35B\&C) (Table 22). With both quantification methods, there was a significant difference between normal/hyperplastic ducts and ADH, DCIS, and IDC ( $\mathrm{p}<0.0001$, Tukeys post hoc).

In the final mammary cancer progression timepoint of 15 weeks post-DMBA administration, quantification once again only yielded a difference based on disease morphology by mean probe per cell analysis $(\mathrm{p}=0.5837$ genotype, $\mathrm{p}<0.0001$ morphology, two-way ANOVA) and overall Mier3 positive cells ( $\mathrm{p}=0.5470$ genotype, $\mathrm{p}<0.0001$ morphology, two-way ANOVA) (Fig 36B\&C) (Table 23). With both quantification methods, there was a significant difference between normal/hyperplastic ducts and $\mathrm{ADH}$, DCIS, and IDC ( $\mathrm{p}<0.0001$, Tukeys post hoc). When analyzing mean probe per cell there was also a difference between $\mathrm{ADH}$ and $\mathrm{IDC}(\mathrm{p}=0.001)$, but this was lost when assessing overall positivity. 

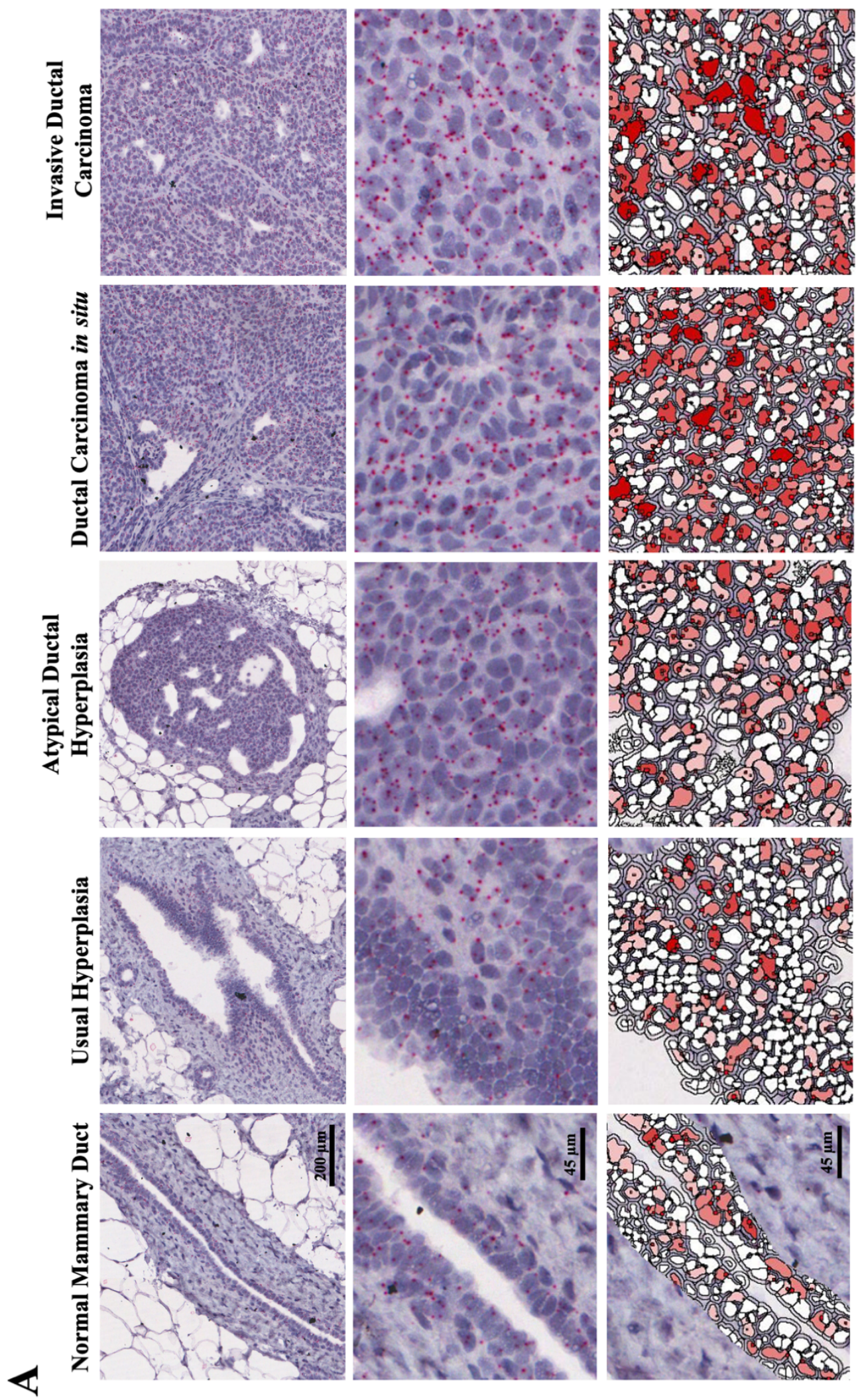
B Mier3 expression in rat mammary glands 8 weeks post DMBA administration

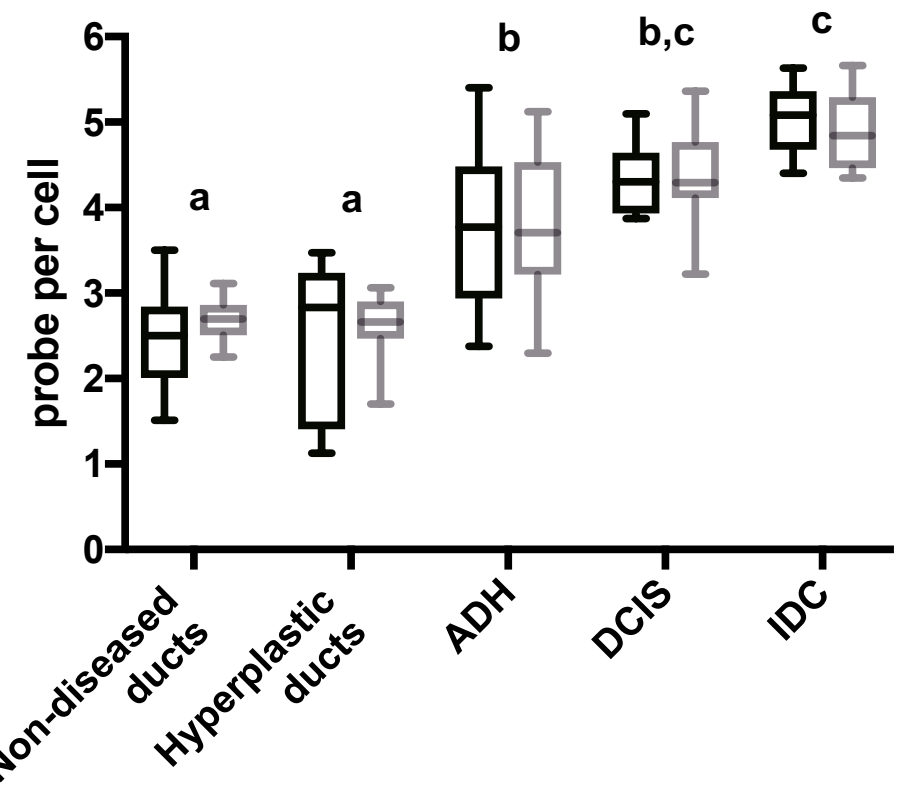

$\square$ WF

$\square$ N3

C Mier3 positive cells in rat mammary glands

8 weeks post DMBA administration

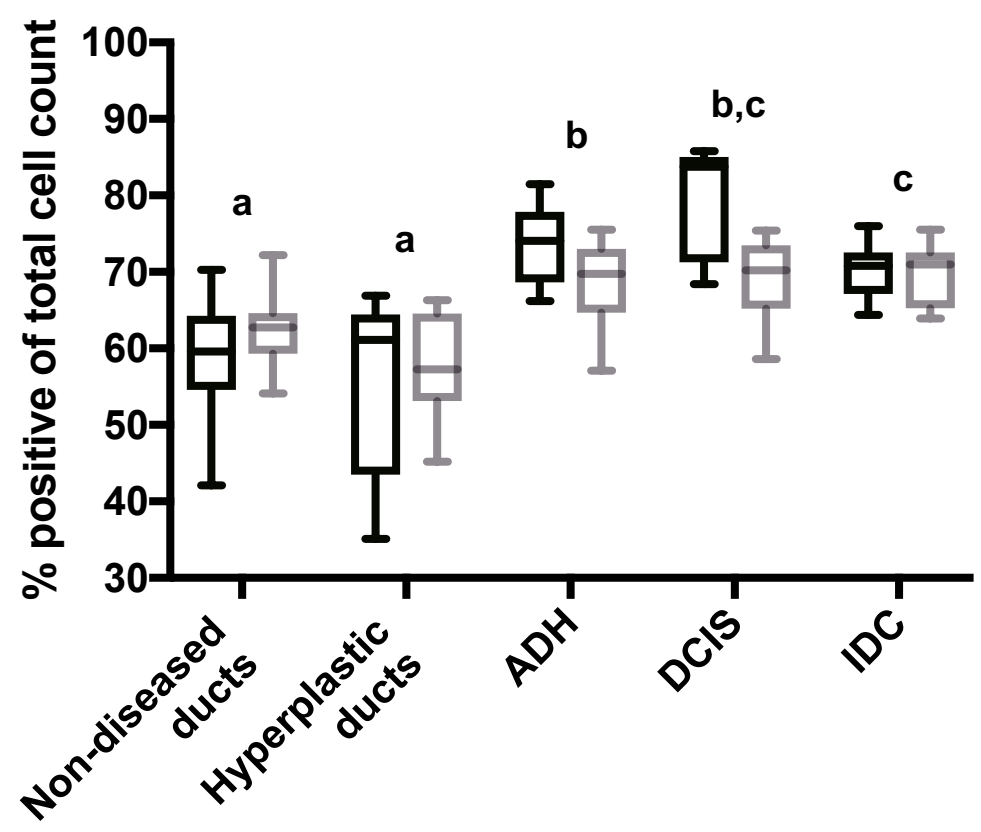


Figure 34: Mier3 expression in rat mammary glands 8 weeks post DMBA

administration. (A) 40x mammary gland histologic images from RNAscope assays probing for Mier3 show red puncta (Mier3 transcripts) counterstained with hematoxylin. Columns display (from left to right) representative non-diseased duct, hyperplastic duct, ADH, DCIS, and IDC. The bottom row is the red probe and nuclei markup from the HALO-ISH module software. (B) Quantification of mean Mier3 expression per MEC or cancer cell in the WF vs N3 strain. (C) Quantification of Mier3-positive cells in a set field of view of MECs/cancer cells. In both graphs morphology groups with different lowercase letters are significantly different at Tukey's post hoc test $\mathrm{p}<0.01$. N=6 mammary glands per genotype, minimally $n=6$ viewpoints per morphology per genotype 
Table 21. Mier3 expression by mammary cancer progression morphologies in WF/NHsd and WF.COP Mcs $1 b$-N3 females 8 weeks post-DMBA administration

\begin{tabular}{cccc}
\hline Genotype & Morphology & $\begin{array}{c}\text { Mean probe } \\
\text { per cell (SD) }\end{array}$ & $\begin{array}{c}\% \text { of positive } \\
\text { cells (SD) }\end{array}$ \\
\hline WF/NHsd & Non-diseased ducts & $2.48(0.56)$ & $59.2(7.0)$ \\
WF.COP $M c s 1 b$-N3 & Non-diseased ducts & $2.69(0.23)$ & $62.1(4.4)$ \\
\hline WF/NHsd & Hyperplastic ducts & $2.48(0.92)$ & $55.6(11.7)$ \\
WF.COP $M c s 1 b$-N3 & Hyperplastic ducts & $2.60(0.42)$ & $57.8(7.0)$ \\
\hline WF/NHsd & ADH & $3.74(0.97)$ & $73.3(5.4)$ \\
WF.COP $M c s 1 b$-N3 & ADH & $3.77(0.88)$ & $68.6(5.9)$ \\
\hline WF/NHsd & DCIS & $4.34(0.44)$ & $79.8(7.5)$ \\
WF.COP $M c s 1 b-\mathrm{N} 3$ & DCIS & $4.30(0.61)$ & $68.7(5.8)$ \\
\hline WF/NHsd & IDC & $5.04(0.39)$ & $70.0(3.5)$ \\
WF.COP $M c s 1 b-\mathrm{N} 3$ & IDC & $4.89(0.46)$ & $69.8(4.1)$ \\
\hline
\end{tabular}

$\mathrm{N}=6$ mammary glands per genotype 

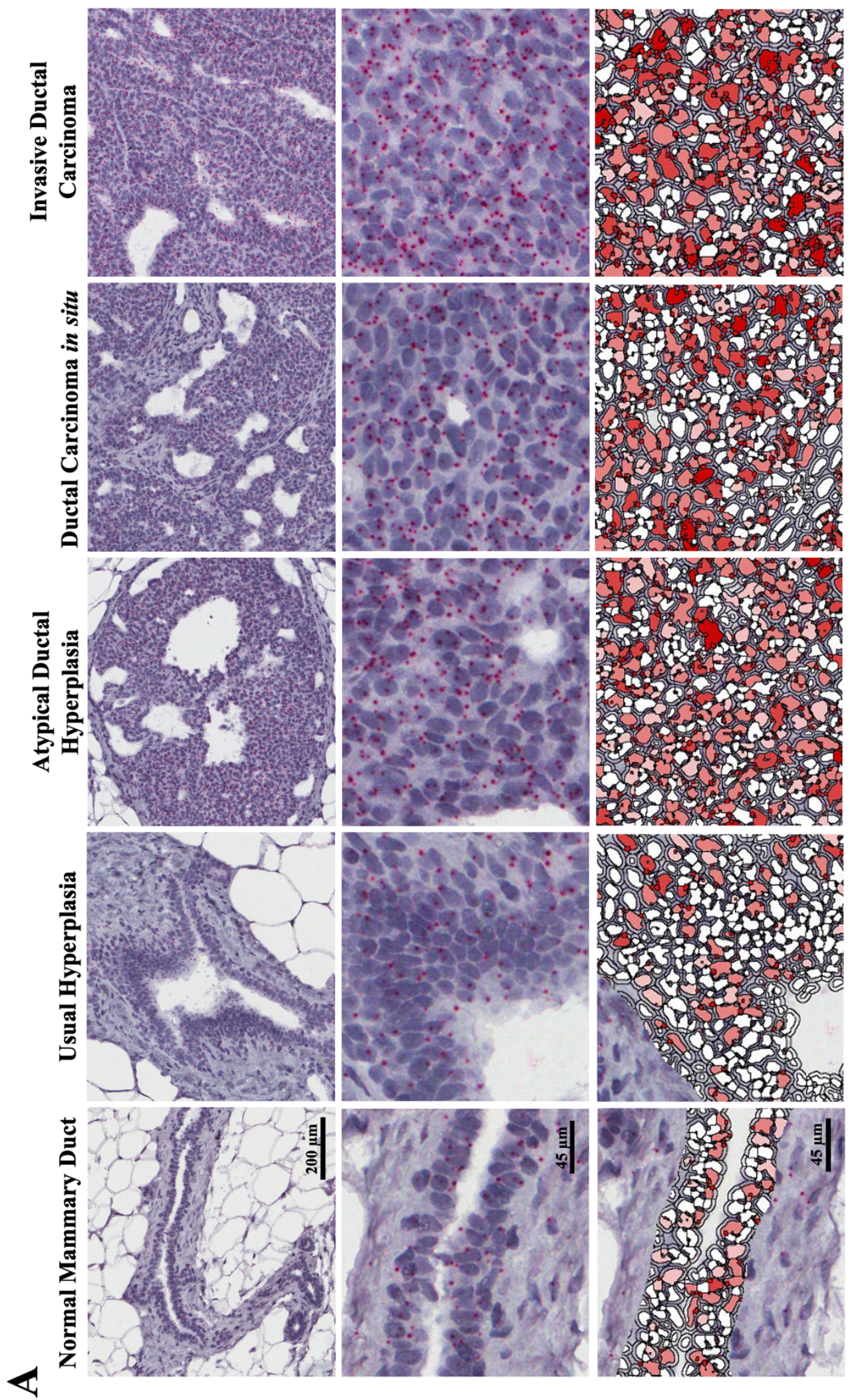
B Mier3 expression in rat mammary glands

12 weeks post DMBA administration

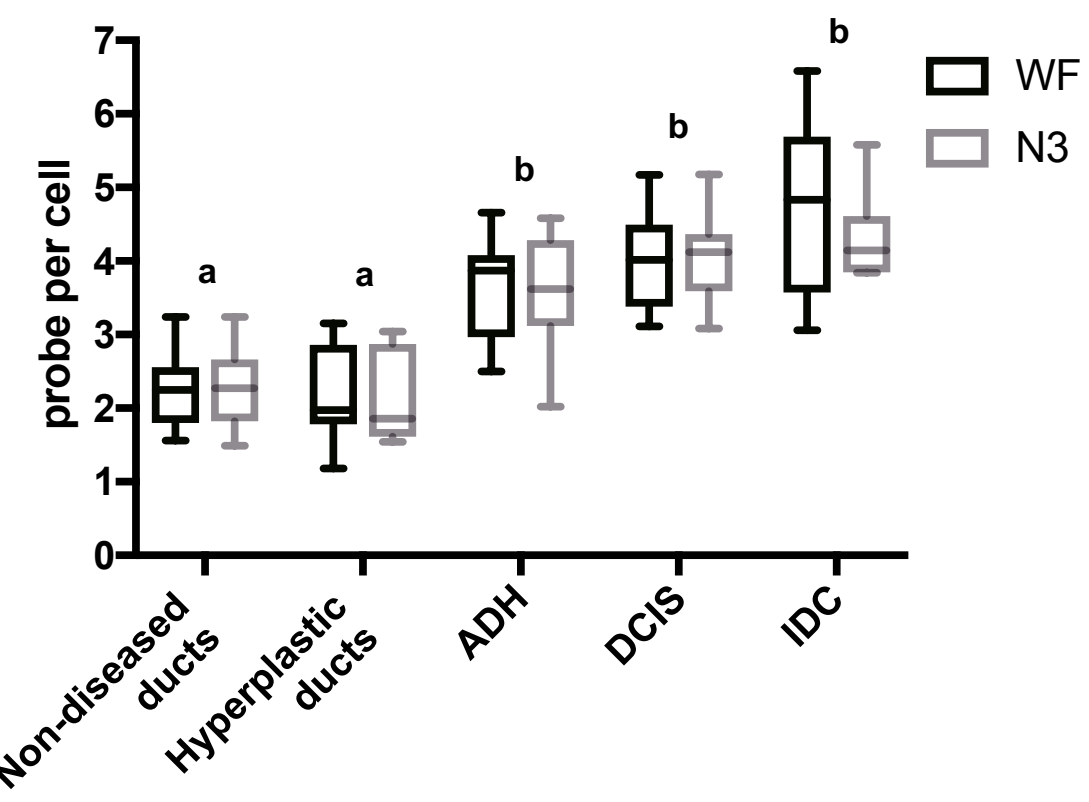

C Mier3 positive cells in rat mammary glands

12 weeks post DMBA administration

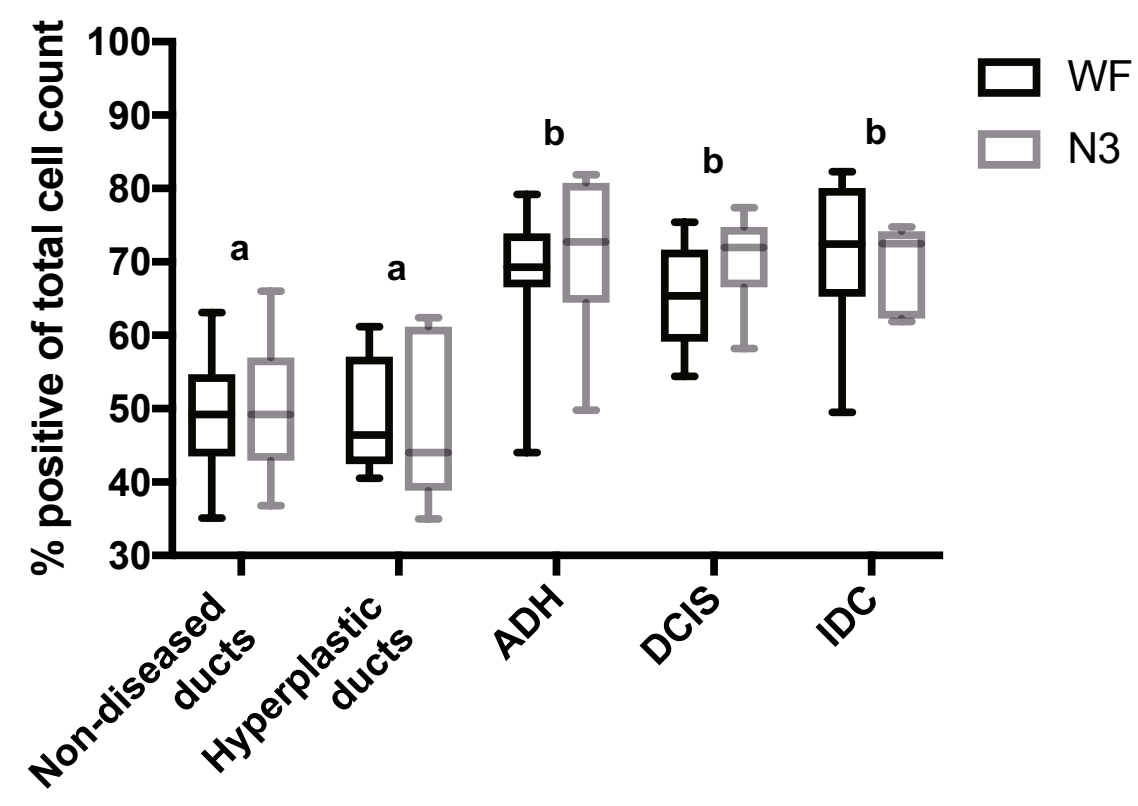


Figure 35: Mier3 expression in rat mammary glands 12 weeks post DMBA

administration. (A) 40x mammary gland histologic images from RNAscope assays probing for Mier3 show red puncta (Mier3 transcripts) counterstained with hematoxylin. Columns display (from left to right) representative non-diseased duct, hyperplastic duct, ADH, DCIS, and IDC. The bottom row is the red probe and nuclei markup from the HALO-ISH module software. (B) Quantification of mean Mier3 expression per MEC or cancer cell in the WF vs N3 strain. (C) Quantification of Mier3-positive cells in a set field of view of MECs/cancer cells. For both graphs morphology groups with different lowercase letters indicate Tukey's post hoc test $\mathrm{p}<0.0001$. $\mathrm{N}=6$ mammary glands per genotype, minimally $n=6$ viewpoints per morphology per genotype. 
Table 22. Mier3 expression by mammary cancer progression morphologies in WF/NHsd and WF.COP Mcs1b-N3 females 12 weeks post-DMBA administration

\begin{tabular}{cccc}
\hline Genotype & Morphology & $\begin{array}{c}\text { Mean probe } \\
\text { per cell (SD) }\end{array}$ & $\begin{array}{c}\% \text { of positive } \\
\text { cells (SD) }\end{array}$ \\
\hline WF/NHsd & Non-diseased ducts & $2.22(0.44)$ & $48.5(7.7)$ \\
WF.COP $M c s 1 b$-N3 & Non-diseased ducts & $2.26(0.53)$ & $50.3(8.6)$ \\
\hline WF/NHsd & Hyperplastic ducts & $2.23(0.65)$ & $49.3(7.6)$ \\
WF.COP $M c s 1 b$-N3 & Hyperplastic ducts & $2.20(0.65)$ & $48.6(11.3)$ \\
\hline WF/NHsd & ADH & $3.64(0.68)$ & $68.1(9.5)$ \\
WF.COP $M c s 1 b$-N3 & ADH & $3.56(0.74)$ & $71.2(10.0)$ \\
\hline WF/NHsd & DCIS & $3.98(0.68)$ & $65.2(6.8)$ \\
WF.COP $M c s 1 b-\mathrm{N} 3$ & DCIS & $4.04(0.49)$ & $70.3(5.5)$ \\
\hline WF/NHsd & IDC & $4.76(1.12)$ & $70.2(10.4)$ \\
WF.COP $M c s 1 b$-N3 & IDC & $4.31(0.65)$ & $69.7(5.9)$ \\
\hline N=6 mammary glands per genotype & &
\end{tabular}



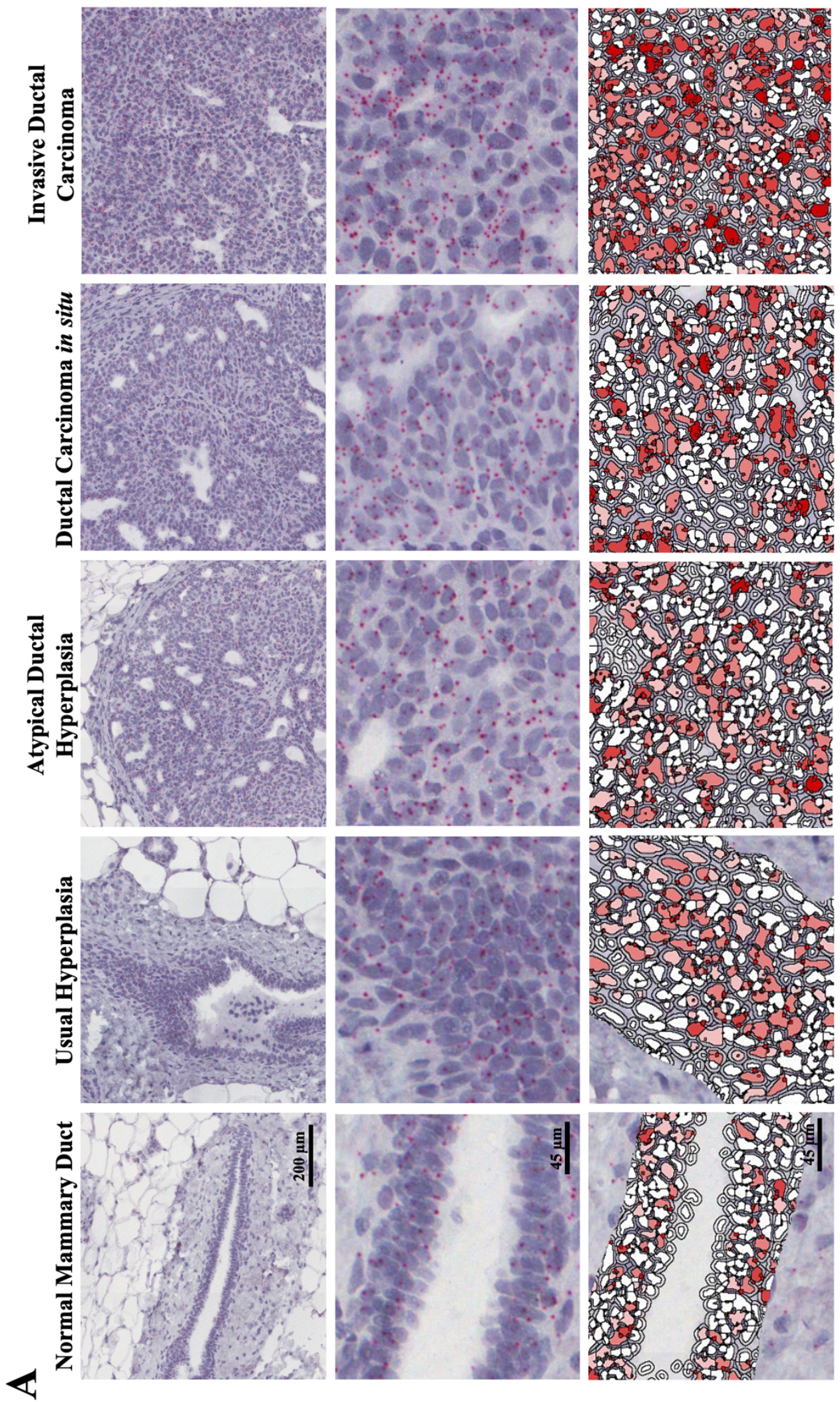
B Mier3 expression in rat mammary glands 15 weeks post DMBA administration

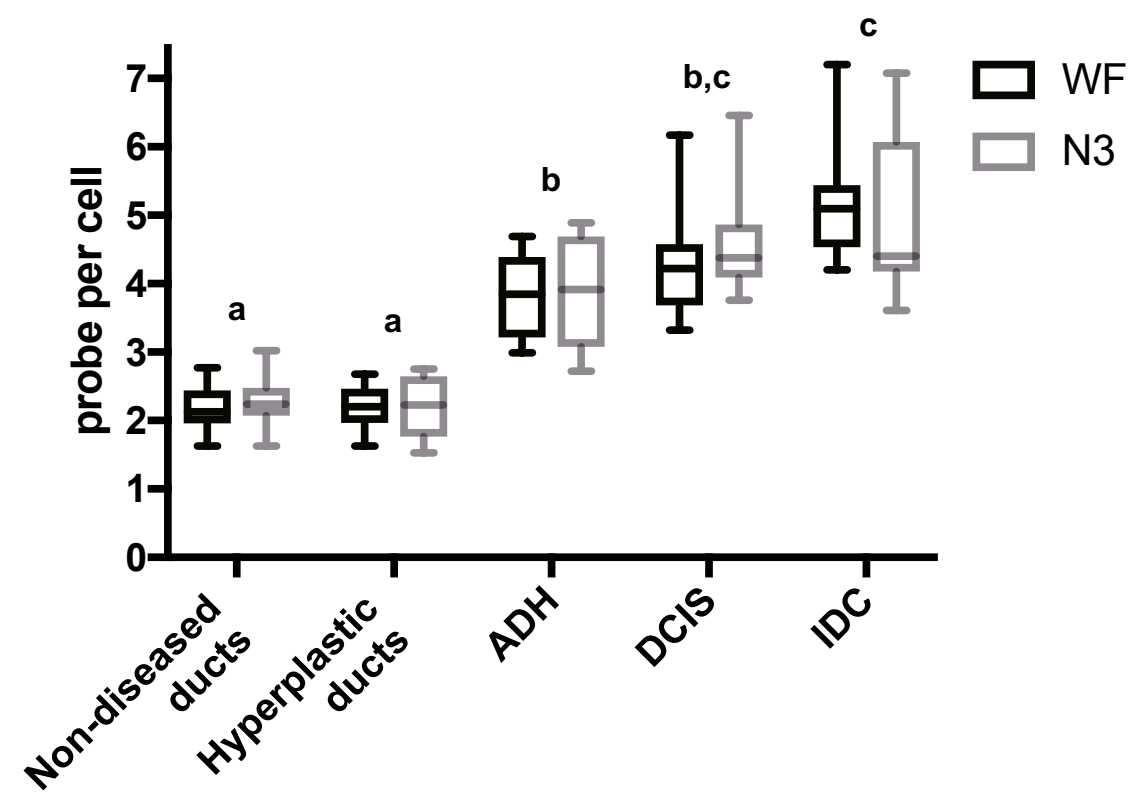

C Mier3 positive cells in rat mammary glands

15 weeks post DMBA administration

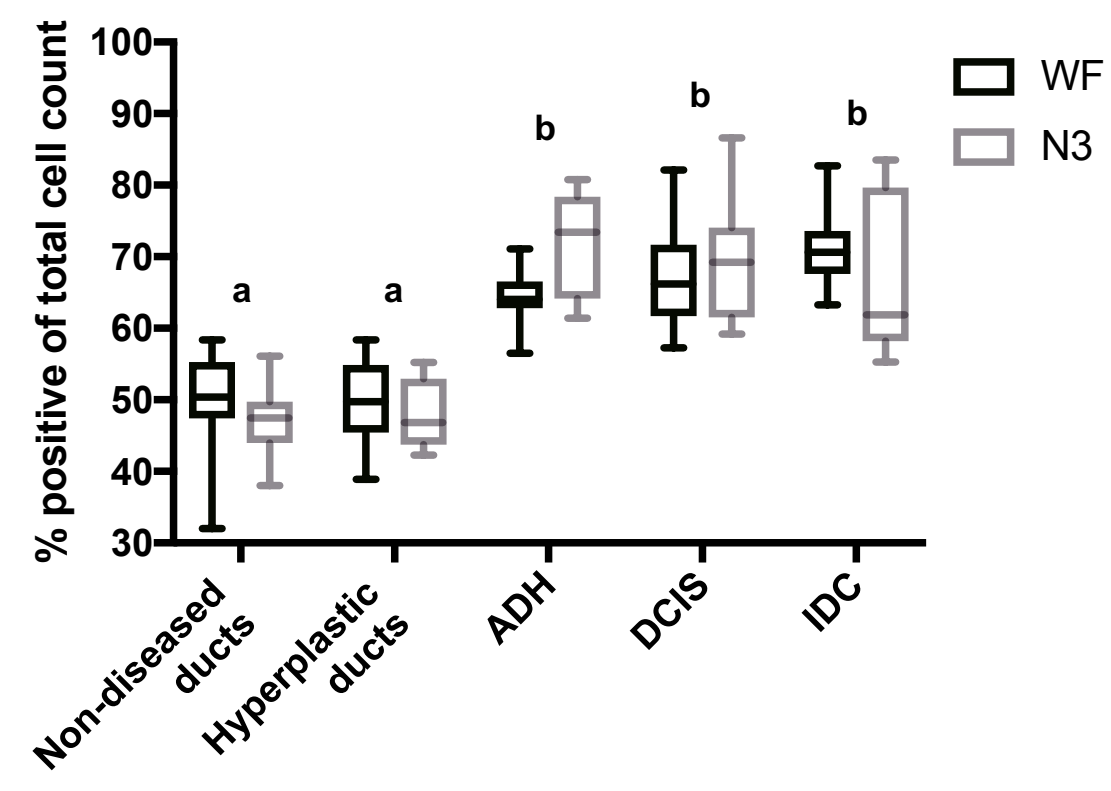


Figure 36: Mier3 expression in rat mammary glands 15 weeks post DMBA

administration. (A) 40x mammary gland histologic images from RNAscope assays probing for Mier3 show red puncta (Mier3 transcripts) counterstained with hematoxylin. Columns display (from left to right) representative non-diseased duct, hyperplastic duct, ADH, DCIS, and IDC. The bottom row is the red probe and nuclei markup from the HALO-ISH module software. (B) Quantification of mean Mier3 expression per MEC or cancer cell in the WF vs N3 strain. (C) Quantification of Mier3-positive cells in a set field of view of MECs/cancer cells. For both graphs morphology groups with different lowercase letters indicate Tukey's post hoc test $\mathrm{p}<0.0001$. $\mathrm{N}=6$ mammary glands per genotype, minimally 8 viewpoints per morphology per genotype. 
Table 23. Mier3 expression by mammary cancer progression morphologies in WF/NHsd and WF.COP Mcs1b-N3 females 15 weeks post-DMBA administration

\begin{tabular}{cccc}
\hline Genotype & Morphology & $\begin{array}{c}\text { Mean probe } \\
\text { per cell (SD) }\end{array}$ & $\begin{array}{c}\% \text { of positive } \\
\text { cells (SD) }\end{array}$ \\
\hline WF/NHsd & Non-diseased ducts & $2.19(0.30)$ & $50.2(6.1)$ \\
WF.COP $M c s 1 b$-N3 & Non-diseased ducts & $2.27(0.34)$ & $47.0(4.8)$ \\
\hline WF/NHsd & Hyperplastic ducts & $2.21(0.31)$ & $49.6(6.5)$ \\
WF.COP $M c s 1 b$-N3 & Hyperplastic ducts & $2.21(0.43)$ & $48.1(4.8)$ \\
\hline WF/NHsd & ADH & $3.78(0.60)$ & $64.0(3.61)$ \\
WF.COP $M c s 1 b-\mathrm{N} 3$ & ADH & $3.88(0.81)$ & $71.9(7.2)$ \\
\hline WF/NHsd & DCIS & $4.25(0.71)$ & $67.4(7.0)$ \\
WF.COP $M c s 1 b-\mathrm{N} 3$ & DCIS & $4.60(0.82)$ & $69.3(8.5)$ \\
\hline WF/NHsd & IDC & $5.13(0.70)$ & $71.0(5.1)$ \\
WF.COP $M c s 1 b-\mathrm{N} 3$ & IDC & $4.97(1.07)$ & $68.3(11.1)$ \\
\hline
\end{tabular}

$\mathrm{N}=6$ mammary glands per genotype 


\section{Carcinoma cells display heterogenous Mier3 expression in mammary tumors}

In analyzing each progression stage based on histology as opposed to absolute quantification, there was an interesting appearance of IDCs, in which certain carcinoma cells express markedly increased levels of Mier3 as compared to neighboring cells (Fig 37A). This was consistent across tumors taken at 8-, 12-, and 15-weeks post-DMBA administration. This heterogeneity in expression is observed throughout tumor sections (Fig 37B) and is seen in neoplastic cells but is not observed in healthy morphologies.

\section{Summary: Mier3 expression increases with greater malignancy of mammary lesions, but the overall number of cells expressing Mier3 does not change}

In the summation of the expression data, there were no statistically significant differences between pathology stages based on Mcs $1 \mathrm{~b}$ genotype and timepoint collected following DMBA administration. Data was pooled to provide a final quantification of mean Mier3 expression (Fig 38A) and percent of Mier3 positive cells (Fig 38B) within each histopathological stage of the precursor-carcinoma sequence of rat mammary cancer development. There was a significant increase in mean Mier3 expression between nondiseased mammary ducts and cancerous lesions, as well as between each pathology stage $(\mathrm{p}<0.001)$. There was also a significant increase in cells positive for Mier3 expression between non-diseased mammary ducts and cancerous lesions $(\mathrm{p}<0.001)$. However, there were no differences between pathology stages. In summary, there were no differences in expression based on time post-DMBA administration or Mcs $1 b$ genotype. However, there was an increase in Mier 3 expression with each increase in malignancy of histopathological 


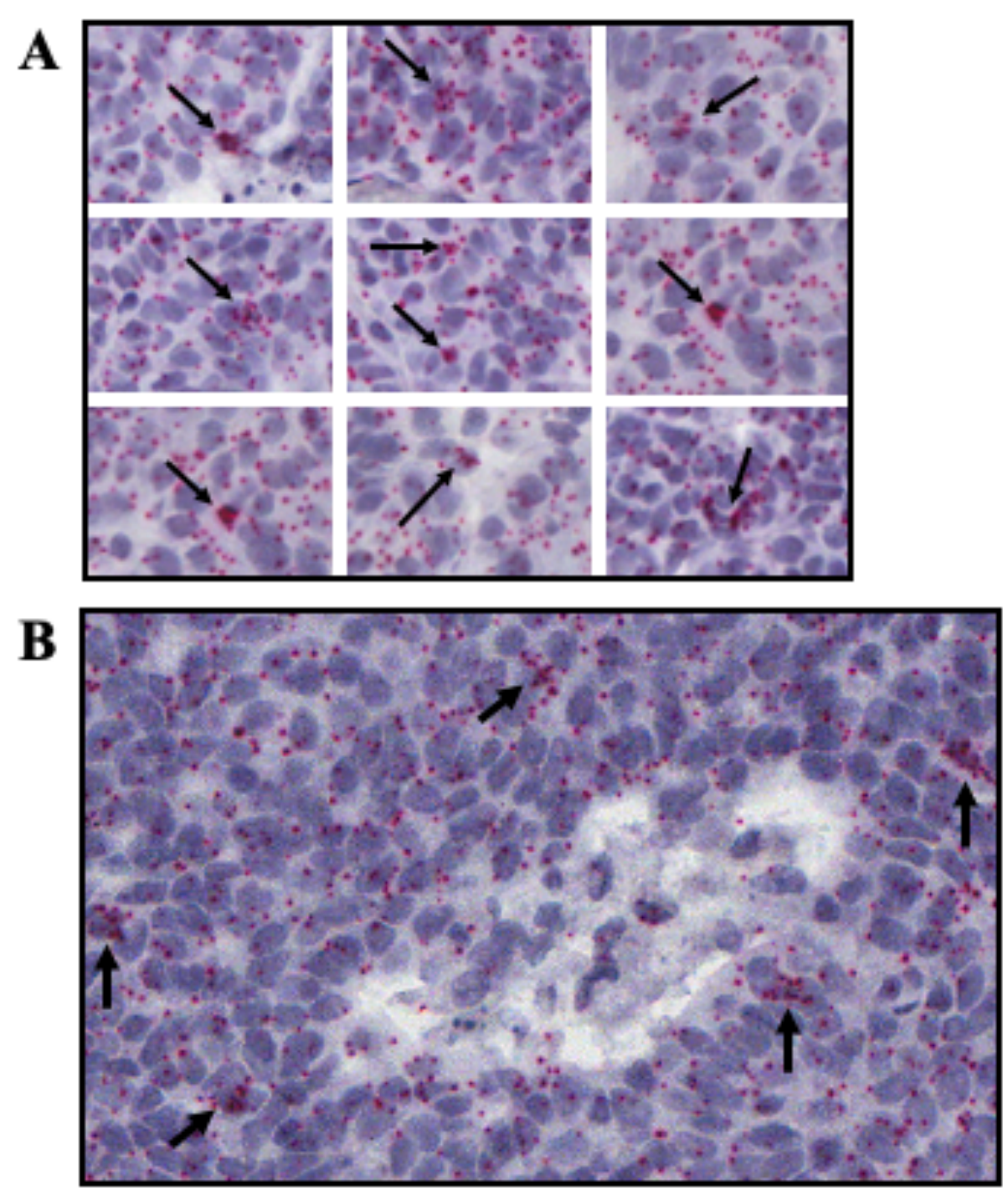

Figure 37: Rat mammary carcinoma cells express variable levels of Mier3:

Mammary carcinoma histologic images from RNAscope assays probing for Mier3 show red puncta (Mier 3 transcripts) counterstained with hematoxylin. Images were taken at 40x magnification on an Aperio ImageScope CS2. (A) Within various mammary IDCs, certain carcinoma cells express very high levels of Mier3 (black arrows) as compared to neighboring cells. (B) This single IDC field of view provides an uncropped example of the intra-tumoral heterogeneity of Mier3 expression. 


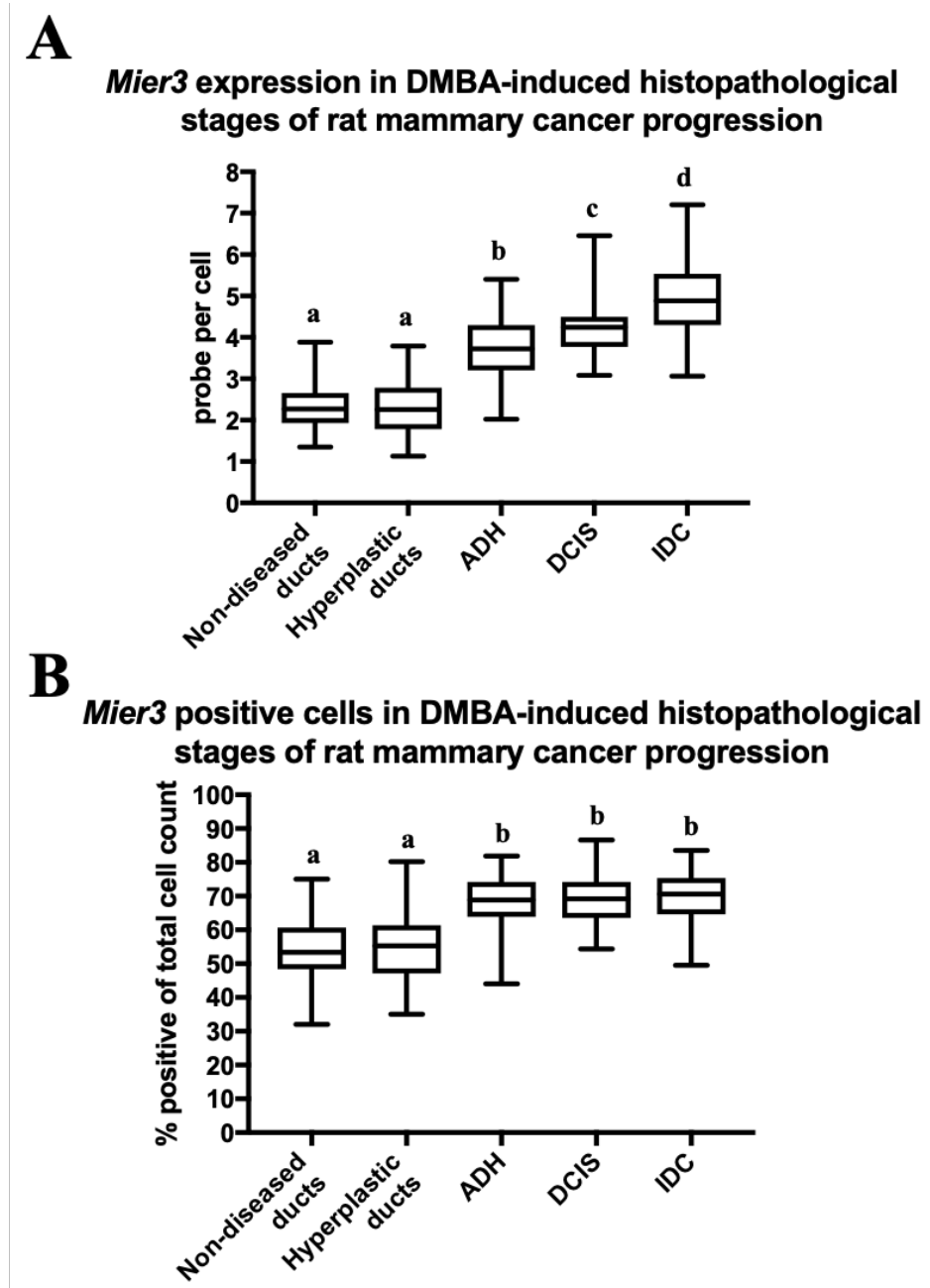

Figure 38: Mier3 expression in the histopathological stages of the precursorcarcinoma sequence of rat mammary cancer progression: Quantification by stage of disease progression for (A) mean Mier3 probe per MEC/cancer cell and (B) Quantification of Mier3-positive cells in a set field of view of MECs/cancer cells. Data was pooled from $4,8,12$, and 15 weeks post DMBA-administration and combined genotypes as there was no difference between morphologies based on time or genotype. Following a significant one-way ANOVA $(\mathrm{p}<0.0001)$ different lowercase letters indicate significant Tukey's multiple comparisons tests $(\mathrm{p}<0.001)$. Minimally $\mathrm{N}=64$ quantified fields of view per morphology. 
progression stage, with no differences in total Mier3 positive cells between cancerous lesions.

\section{Discussion:}

When first analyzing the rat mammary gland for in situ, cell-specific expression of Mier3, I found nominal, if any expression, in vascular endothelial cells, fibroblasts, and adipocytes, indicating Mier3 does not have a role in the stromal compartment of the mammary gland. I found the highest levels in mammary epithelial cells (MECs) that make up the mammary ducts. This is relevant to mammary cancer as the epithelial compartment is the most active component of the mammary gland and ductal structures are most susceptible to chemical insult $[114,117]$. Terminal ductal lobular units are also the site of origin of invasive ductal carcinomas (IDC), which account for $80 \%$ of diagnosed breast cancers worldwide [218, 478]. When evaluating MECs specifically, luminal epithelial cells were found to express greater levels of Mier3 as compared to myoepithelial cells. This is important to breast cancer etiology as the majority of breast cancers have luminal epithelial cell characteristics, making these cells the likely precursors for neoplastic transformation $[117,479]$. This analysis provided crucial cell-specific expression information adding to the potential role of Mier3 in mammary cancer by identifying its most prominent expression levels as stemming from the cell population that originates the majority of breast carcinomas.

I next examined the effect of carcinogen exposure on Mier3 expression in rat mammary glands. There was two-fold greater Mier 3 expression in non-diseased mammary ducts of rats exposed to DMBA as compared to age-matched, non-exposed control 
mammary glands. Changes in gene expression in DMBA-induced lesions has been analyzed for decades [480, 481], and minimally nominates these identified genes as markers of mammary carcinogenesis. However, our analysis was conducted at an early induction time point prior to the formation of pathological disease lesions, indicating a potential role of Mier3 in disease initiation. Luminal epithelial cells are described as being on a path of continuous differentiation, with the population displaying variable levels of progenitor and differentiation markers [482], making them as a whole susceptible to carcinogen initiation. As a result, the higher Mier3 levels found after carcinogen-exposure could be a reflection of early carcinogenesis, and possible reprogramming of the transcriptome of MECs preceding full cellular transformation.

I chose to evaluate Mier3 expression at progressive pathological stages, as opposed to endpoint analysis in mammary tumors, as it is crucial to understand how the transcriptome changes during early carcinogenesis and disease progression to uncover prognostic or targetable molecular markers. There is a higher priority to identify a wide array of prognostic factors in an early disease state and prevent the development to a potentially lethal IDC altogether. Tumorigenesis in mammary glands is a multistep process that stems from the acquisition of genetic alterations that drive progressive transformation of MECs into malignant cells [474]. This can be studied within intermediate pathological stages, which in DMBA-induced mammary cancer progression is development of nondiseased mammary ducts to premalignant $\mathrm{ADH}$, preinvasive DCIS, and to a full invasive carcinoma [483]. I evaluated Mier3 expression based on (1) progression with time following carcinogen administration, and (2) tissue disease progression stages within each timepoint. I found significant increases in Mier3 expression with increasing malignancy of 
each disease morphology stage along the precursor-carcinoma sequence of ADH, DCIS, and IDC. This was found at all timepoints, with no differences in expression based on pathology when comparing across tissue collection timepoints. This indicates that it is not the time to progression that Mier3 has a role in, for example a carcinoma that develops at 8 weeks post DMBA administration as compared to 15 weeks. Rather, Mier3 expression increases between progressive tissue changes, indicating Mier3 has a role in or is a product of the cellular transformations that occur throughout carcinogenic development to IDC.

In my progression analysis, I found the non-cancerous morphologies of nondiseased mammary ducts and ducts with usual hyperplasia possessed no differences in Mier3 expression levels. Although usual hyperplasia encompasses a proliferation of MECs, this is not driven by carcinogenic transformation and does not contribute to breast cancer risk [467, 468], so the finding of similar Mier3 levels to normal mammary ducts is not surprising. This indicates a shared role of Mier3 in mammary ducts as in non-cancerous hyperplasias. In the early disease stage of $\mathrm{ADH}$, I found a 1.6-fold increase in expression on top of the 2.2-fold increase in expression induced by DMBA-exposure. Following this large increase in Mier3 expression, there was a progressive increase in expression within each of the final stages of DCIS and IDC. This suggests Mier3 may have a role in tumor initiation and development. The initial increase in Mier3 expression at the first pathological stage of ADH is noteworthy. These lesions are the earliest detectable changes to the breast epithelium and it is suggested that the molecular profile displays underlying susceptibility factors that drive further disease progression [484]. The increased Mier3 expression between ADH and DCIS is possibly explained by the fact that DCIS typically displays much more frequent genetic alterations that drive tumorigenesis, even though these are 
both preinvasive disease stages [471]. When evaluating disease pathologies based on overall percent of cells positive for Mier 3 expression rather than absolute quantification, I found a similar increase in Mier3-positive cells between non-diseased mammary ducts and ADH. This again suggests Mier3 may have a role in pre-malignant cell transformation. However, there was no significant difference in the percent of cells positive for Mier3 expression between ADH, DCIS, and IDC, even though the average transcripts per cell are increasing between these stages. This highlights that all cells in which Mier3 has a potential tumorigenic role are present at the earliest disease stage. This complements what is known about global alterations in gene expression throughout mammary cancer progression, where significant transcriptional changes are observed during $\mathrm{ADH}$ and are maintained in DCIS and IDC [485]. This is thought to be due to the clonal relationship between these progression stages and suggests that the genes involved in carcinogenic growth and progressive potential are active at the earliest disease stage $[464,486]$. Thus, increased Mier3 expression in ADH may be due to neoplastic transformation and reflect a possible carcinogenic role of Mier3 in early disease stages, which may be maintained and expanded along pathological progression to DCIS and IDC.

In my evaluation throughout rat mammary cancer progression, I found Mier3 expression was most enhanced in mammary tumors. The correlation of increased Mier3 expression with increasing malignancy of pathological stage, along with the greatest levels in IDC, suggests Mier3 could have a role in tumor expansion or transition from preinvasive to invasive growth. I also found variable expression levels of Mier3 in IDCs, with some cells displaying such elevated levels that they are not discernable as distinct puncta, but rather appeared as a red smear across the cell nuclei. This may be a reflection of the 
intra-tumoral heterogeneity that is common in DMBA-induced tumors and intrinsic to breast cancer [487, 488]. DMBA-induced mammary carcinomas consist of pleomorphic cells [487], with individual tumor cells differing in phenotypes and function, such as proliferation rates, angiogenic and metastatic potential, migration, induction of senescence, activation of signaling pathways, and response to anticancer drugs [488]. In addition to the neoplastic cells that constitute the tumor mass, there are networks of soluble factors, immune cells, vasculature cells, stromal cells, and extra-cellular matrix that together comprise the tumor microenvironment $[489,490]$. These components can interact with the inherent genome instability of cancer cells and create diverse subpopulations within a tumor [490]. As a result, the extensive intra-tumoral variation in Mier3 expression may have been a response to certain heterogeneous selective pressures that necessitate a role of Mier3, potentially in tumor growth, transition, invasion, or adaptation.

The overlap of the Mcs $1 b$ mammary cancer susceptibility gene locus and human breast cancer risk allele 5q11.2 nominates Mier3/MIER3 as a breast cancer risk gene. In this study, I used a DMBA model of rat mammary cancer to investigate Mier3 expression throughout a precursor-carcinoma sequence of disease development of ductal origin. This study shared similar goals as gene expression profiling; namely, to identify gene(s) that have the potential to be a molecular target in drug development or a molecular marker of disease diagnosis or prognosis $[491,492]$. Importantly, this candidate gene approach was based on published experimental rationale that had established the phenotype of mammary cancer susceptibility was encompassed within this genetic region, as opposed to an uninformed, forward genetics approach. 
A null result in this study, worthy of discussion, was the lack of $M c s 1 b$ genotypic differences in Mier3 expression between my mammary cancer susceptible WF strain and my WF.COP Mcs $1 b$ resistant N3 strain. This could be a result of three Mier3 transcript isoforms that arise from alternative splicing of exon 1 and an alternate 5' start site, generating transcripts with differing 5' ends and truncated N-termini of the translated protein products [256, 493]. However, these Mier3 isoforms are not differentiated between using the Mier3 RNAscope probe, which is designed to bind within nucleotides 2357-3614 of the 4758 base pair transcript, corresponding to the 3'UTR of Mier3. Previous analysis in our lab found lower expression of alternative isoforms in the resistant strain compared to the susceptible strain, but no differences with other Mier3 transcripts (Samuelson lab, unpublished). Thus, the two genotypes may have variability in Mier3 expression at the level of isoform differences specific to the mammary gland which could account for the difference in susceptibility. Additionally, this study only provides correlative information of Mier3 expression quantification based on pathology stage. The purpose of this study was to identify mammary gland cell-specific expression of Mier 3 and changes with disease pathology in order to determine if this candidate breast cancer gene had a potential role in cancer risk and progression. The intent was to establish rationale to warrant in depth analysis of the function of MIER3 in breast cancer. Further mechanistic studies would need to be done to understand a molecular function of MIER3 in the context of breast cancer.

Genetic analysis has unveiled the complexity of breast disease, but there remains a lot to be uncovered about the molecular factors and changes that drive development and progression of premalignant breast lesions. Standard histopathology is limited in the ability to predict risk of disease progression, making it crucial to investigate the underlying 
molecular biology. In this study, I detected a significant increase in Mier3 expression in susceptible MECs with carcinogen exposure prior to development of any disease morphology, as well as increasing expression concurrent with increasing malignancy of pathological disease stage. This suggests a functional role of Mier3 in disease initiation and tumorigenesis and elevates it as a potential biomarker or therapeutic target in early disease development. The highest Mier 3 levels were discovered in invasive carcinomas, which suggests an additional role in tumor growth. It is established that variation in transcriptional programs account for much of tumoral diversity and function [489]. Given the putative role of Mier3 as a transcriptional repressor [494], elevated Mier3 levels may reflect a potential role in MECs as a driver of breast carcinogenesis through transcriptional remodeling during disease initiation up through progression to invasive carcinomas. In summary, Mier3 has a potential role in breast carcinogenesis and tumorigenesis, and may serve as a clinically meaningful target in disease progression. 


\section{CHAPTER VI \\ MIER3 EXPRESSION DURING MAMMARY GLAND DEVELOPMENT AND MAMMARY CARCINOMA SUSCEPTIBILITY WINDOWS}

\section{Introduction:}

The developmental stages and morphology of the rat mammary gland closely matches that of the human breast $[95,96,101,117]$, and the DMBA-induced rat mammary cancer model induces similar pathology to human breast cancer [96, 219-221], making the rat a useful model in studying both human breast development and carcinogenesis. When studying the morphological and gene expression changes associated with mammary cancer progression, it is critical to examine these same changes that occur during mammary tissue development as many of the same factors that modify the developmental profile can contribute to carcinogenesis susceptibility later in life $[95,114,117,495,496]$. There are obvious similarities between the morphologies and growth pathways utilized during both development and carcinogenesis. The pubertal period displays rapid epithelial proliferation and expansion that is also necessary for tumor outgrowth, and the reproductive cycle undergoes controlled angiogenesis during pregnancy and lactation, as well as vascular regression and inflammation during involution that is also required to sustain tumor growth $[114,117,132,174,495,497]$. Thus, the same processes involved in growth, proliferation, migration, invasion, and apoptosis to create a developed mammary gland are unfortunately 
employed throughout tumorigenesis [117, 495, 498]. Unlike during carcinogenesis, the onset of these processes are tightly controlled and regulated during mammary gland development through interactions of hormones, growth factors, and signaling molecules with components of the extracellular matrix in the basement membrane as well as fibroblasts and adipocytes in the stroma [184, 495, 497, 499-501]. However, these same hormones and signaling factors in development can influence susceptibility to carcinogens at these time points and become dysregulated during breast cancer [95, 96, 502-504]. Overall, it is established that with any organ, it is crucial to obtain thorough knowledge of normal cellular and molecular mechanisms that guide development in order to understand initiation and progression of cancer [96, 133].

In addition to gross morphological changes that occur during mammary gland development, there exist cyclical changes to the human breast and rat mammary epithelium in response to ovarian hormone levels during the human menstrual cycle/rat estrus cycle $[192,199,200]$. Although tissue-specific changes are primarily confined to the ovaries and uterus, the mammary gland also undergoes cyclic stages of mammary epithelial cell (MEC) proliferation and regression due to ovarian hormone fluctuations, namely estrogen and progesterone [196, 199, 505-508]. Estrogen, specifically, has been associated with ductal elongation, and progesterone with ductal branching and lobulo-alveolar development [192, 196, 200, 509]. These morphological changes are more limited and minute, yet still mimic the broad tissue remodeling that occurs during pregnancy, lactation, and involution [200]. Epidemiological evidence indicates that the menstrual cycle increases the risk of female breast cancer, which may be attributable to endocrine control over susceptible MECs [510512]. Rat estrus cycle-dependent stages of MEC proliferation and regression closely 
matches changes in the human breast tissue during the menstrual cycle $[193,199,513$, 514], in which dysregulation of growth by ovarian hormone signaling could induce transformative events and contribute to mammary carcinogenesis [199].

The key stages of morphological changes in the mammary gland/breast in both the rat and human have been termed "windows of susceptibility" and represent timepoints when genetic risk factors and environmental perturbations result in increased lifetime risk of developing breast cancer [94-99]. Though the interaction of genetic and environmental factors can contribute to breast cancer risk at any age, these windows of susceptibility are time points in which a woman is more susceptible to cancer initiating events due to the rapid structural and molecular alterations taking place, which can play a role in breast cancer development later in life $[94,96,98,99]$. Windows of susceptibility are comprised of developmental and reproductive time intervals in humans and rats, which include prenatal and pubertal development, pregnancy, lactation, involution, menopause, and premenopausal cyclical hormonal changes [97, 99]. Each time point has a distinct transcriptome, epigenetic changes, microenvironments, and signaling mechanisms unique to the biological function of the mammary gland/breast at that time, which can alter responses to environmental insults and heighten susceptibility to carcinogenesis [94, 96, 97, 100]. Clinical data has modeled this window of susceptibility phenomenon, where environmental exposures at these critical periods of susceptibility influence risk of future breast cancer development [97, 123, 515-518]. For example, exposure to ionizing radiation during childhood and adolescence increases the number of breast cancer cases but does not confer increased risk at older ages or after pregnancy [108, 519, 520]. This occurrence has also been demonstrated in rats using exposure to endocrine-disrupting carcinogens during 
gestation, puberty, and pregnancy, which alters mammary gland development and results in increased tumor incidence following carcinogen administration [95, 112, 521-523]. Many of these environmental chemicals given to rats result in histological abnormalities and activate hormonal pathways that are postulated to enhance mammary gland susceptibility to carcinogenesis $[95,112,517,524,525]$, but the molecular mechanisms responsible for effects on breast cancer risk remain largely unknown $[99,526]$. To date, there appears to be only one report outlining mechanistically distinct susceptibility windows, where a noncoding locus in the rat was found to regulate downstream gene expression mediated by age-dependent chromatin looping and genotype-dependent DNA methylation [99]. There are over 180 genetic loci affecting breast cancer susceptibility that have been identified in human genome-wide association studies (GWAS) [99, 527], but their function throughout critical windows of susceptibility has not been assessed.

The rat model of the Mcslb genetic susceptibility locus has nominated Mier3/MIER3 as a breast cancer risk gene, which I confirmed as a breast cancer gene in my expression analysis throughout mammary cancer progression. Here, I sought to further our understanding of Mier 3 expression and ascertain a potential functional role during specific windows of susceptibility in the rat. This study was performed with the idea that a knowledge of Mier3 expression at these time points could elucidate at what stages Mier3 activity is potentially enhanced and responsible for increased risk of female breast cancer development. Analysis of Mier3 expression during the stages of the estrus cycle was included to examine expression in relation to modulation of normal cyclical growth and regression of the mammary epithelium, which could contribute to carcinogenesis susceptibility. I report the highest Mier3 levels in early mammary gland development 
stages that are accompanied by extensive morphological changes, as well as fluctuations in expression correlating with the estrus cycle. These findings suggest Mier3 may be hormonally regulated and possess a functional role in growth of MECs during embryonic through pubertal development. Combined with my previous finding of increased Mier3 expression throughout mammary cancer progression, this indicates Mier3 may serve a role in developmental mammary gland growth as well as a driver of mammary carcinogenesis at these crucial early windows of susceptibility.

\section{Methods:}

Animal husbandry

Rats were maintained in an AAALAC-approved facility on a $12 \mathrm{~h}$ light/dark cycle and provided LabDiet 5001 Rodent Diet (PMI ${ }^{\circ}$ Nutrition International) and water ad libitum. Inbred Wistar Furth (WF/Hsd) females were obtained from Envigo. All animal protocols were approved by the University of Louisville Animal Care and Use Committee.

\section{Congenic Strains}

The Wistar Furth (WF/NHsd) rat strain was used as a mammary cancer susceptible line. The Wistar Furth.Copenhagen (WF.COP) congenic rat strain N3 contains a segment of Copenhagen (COP/NHsd) on RNO2 from markers D2Mgh2 to g2UL1-5 (Chr2:40171863-43756834, build RGSC 6.0/rn6) and is positive for the Mcs $1 b$ decreased susceptibility allele. The Mcs $1 b$ N3 strain at generation N16F41 - N16F42 was used as a mammary cancer resistance line and compared to the susceptible WF strain. 


\section{Mammary Gland Tissue Collection}

Rats were euthanized by $\mathrm{CO}_{2}$ asphyxiation. Abdominal-inguinal mammary glands from six females of both WF and WF.COP Mcs $1 b$ genotypes were resected and formalinfixed and paraffin embedded (FFPE) at each post-natal developmental stage. Embryos were FFPE processed following head and limb removal. Limbs were kept for DNA extraction to sex embryos. Early development stages were taken at embryonic day 19, neonatal day 9 , an early pubescent timepoint at 4 weeks of age, a pubescent timepoint at 8 weeks of age, and a nulliparous timepoint at 12 weeks of age. Reproductive cycle stages were taken at gestation day 19, lactation at 11 days post-parturition, involution 2 days following pup removal, and a parous timepoint at 24 weeks of age (4 weeks following litter weaning). Mammary glands used in analysis of the estrus cycle were also collected from parous animals at 24 weeks of age (Fig 39). Three animals were used per stage of the cycle.

\section{Rat Embryo Sexing}

Limbs removed from embryos were digested and DNA extracted. Gene specific oligonucleotide primers were used to PCR amplify portions of $K d m 5 c$ on the Xchromosome and $K d m 5 d$ on the Y-chromosome according to [528]. Primers for $K d m 5 c$ were F: 5'TTTGTACGACTAGGCCCCAC and R: 5'CCGCTGCCAAATTCTTTGG. Primers for $K d m 5 d$ were F: 5'TTGGGTGAGATGGCTGATTCC and R: 5'CCGCTGCCAAATTCTTTGG. Cycling conditions were as follows: initial denaturation $95^{\circ} \mathrm{C}$ for $2 \mathrm{~min}$, followed by 35 cycles of denaturation at $95^{\circ} \mathrm{C}$ for $30 \mathrm{~s}$, annealing at $55^{\circ} \mathrm{C}$ for $30 \mathrm{~s}$, and extension at $72^{\circ} \mathrm{C}$ for $42 \mathrm{~s}$, followed by a final extension for $7 \mathrm{~min}$ at $72^{\circ} \mathrm{C}$ 
using an Applied Biosystems Veriti Fast Thermal Cycler. PCR products were resolved on a $1 \%$ LE agarose gel.

\section{Estrus Cycle Staging}

Vaginal cytology was used to classify combined WF and WF.COP Mcs $1 b$-N3 females based on their stage in the estrus cycle. Vaginal cells were collected in PBS from rats using a plastic transfer pipet. Proestrus was classified by the appearance of small, rounded nucleated cells. Estrus appears as a majority cornified cells. Metestrus I appears as a late cornified stage with the presence of caseous fluid. At metestrus II there are leukocytes present with cornified cells. Diestrus is characterized by the presence of epithelial cells and leukocytes [529, 530].

\section{Tissue Sectioning and Selection}

Sectioning of FFPE blocks was done one day prior to a planned RNAscope assay, as mRNA remains more stable within paraffin blocks than tissue sections [460]. Mammary gland FFPE blocks were cut in 4um sections onto superfrost plus microscope slides (VWR) using a rotary microtome (Thermo HM 355S). Sections were assessed for integrity of mammary epithelial structures by H\&E staining. Embryonic mammary buds from female embryos were captured by serial sectioning across the FFPE embryo body cavity and identifying the presence of mammary tissue by H\&E staining. 


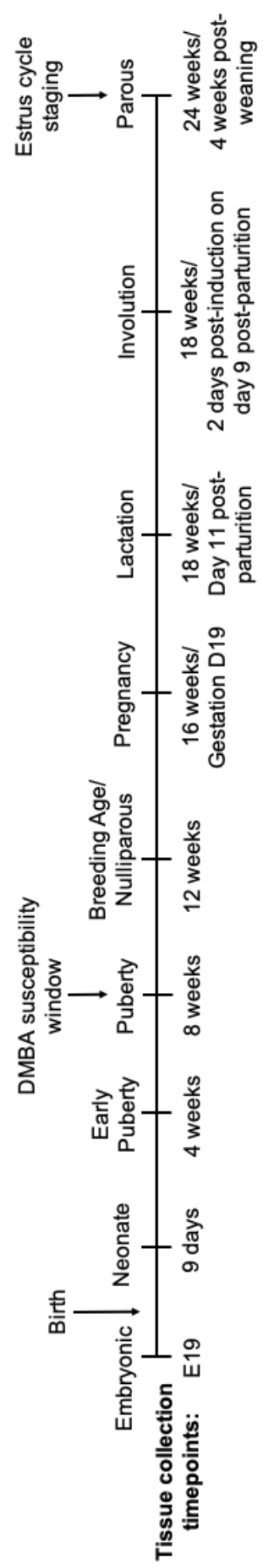

Figure 39: Mammary gland collection timeline of windows of susceptibility during development and reproduction: Experimental stages of development, the reproductive cycle, and estrus cycle (top row) and corresponding timepoints (bottom row). 
Hematoxylin and Eosin (H\&E) staining of sectioned mammary glands:

Microscope slides were deparaffinized and rehydrated using 3 separate xylene washes followed by single washes each in $100 \%$ ethanol, $90 \%$ ethanol, $80 \%$ ethanol, and DI water. Tissues were stained with hematoxylin for 3 minutes, rinsed under running DI water for 3 minutes, soaked in bluing reagent for less than a minute, and again rinsed with DI water. Slides were dipped a few times in $100 \%$ ethanol to eliminate water before staining in eosin-Y for 30-45 seconds. Tissue was then dehydrated using washes of $90 \%$ ethanol, $100 \%$ ethanol, and 3 separate xylene washes. Samples were air dried and coverslipped using permount or similar mounting media.

\section{RNA in situ hybridization}

RNAscope was used to identify the spatiotemporal cell-specific expression of Mier3 in rat mammary tissue according to manufacturer's directions (ACD Bio). Briefly, slides were deparaffinized in xylene and $100 \%$ ethanol, pretreated with an $\mathrm{H}_{2} \mathrm{O}_{2}$ block for 10 minutes, placed in target retrieval solution that was just under boiling (ACD Bio 322000 ) for 10 minutes, and treated with protease plus solution for 10 minutes (ACD Bio 322330). The target probe was added and slides were incubated in HybEZ oven (ACD Bio) at $40^{\circ} \mathrm{C}$ for 2 hours. Amplification steps were as follows using an RNAscope $2.5 \mathrm{HD}$ red detection kit (ACD Bio 322360): Amp1 for 30 minutes at $40^{\circ} \mathrm{C}$, Amp2 for 15 minutes at $40^{\circ} \mathrm{C}$, Amp3 for 30 minutes at $40^{\circ} \mathrm{C}$, Amp 4 for 15 minutes at $40^{\circ} \mathrm{C}$, Amp5 for 30 minutes at room temp, Amp6 for 15 minutes at room temp, and detection reagent for 10 minutes at room temp. Slides were counterstained with 1:1 Gill's hematoxylin and water. Vectashield 
mounting media (Vector Laboratories H-1000) was added to protect staining. Slides were imaged at 40x on an Aperio ImageScope CS2.

Expression Quantification, Data Handling, and Statistical Analysis

Within images of tissue slides following the RNAscope assay, mammary duct, TEB, or lobular morphologies were outlined and selected for analysis using the HALOISH quantification software from Indica Labs. Mean probe per mammary epithelial cell and percent of Mier3 positive mammary epithelial cells data were collected. Average Mier3 transcript quantification values were transformed by taking the natural log. Percent positivity values were transformed by taking the arcsin of the square root of the percent values in decimal form. Transformed data were analyzed via one-way or two-way ANOVAs depending on the number of variables. Independent variables included timepoint, genotype, and morphology. All statistical analysis was done using GraphPad Prism version 7.00 for Windows, GraphPad Software, La Jolla California USA.

\section{Results:}

To study mammary gland Mier 3 expression throughout mammary cancer windows of susceptibility, rat lifecycle was evaluated in three parts: (1) early mammary gland development, (2) the reproductive cycle, and (3) the estrus cycle. A mammary gland collection timeline of all experimental stages used in this study is outlined in Figure 39. 


\section{WF and WF.COP Mcs1b-N3 females have normal mammary gland morphology and express Mier3 at each developmental timepoint}

Early mammary gland development was analyzed in embryonic mammary buds at gestation day 19, and mammary glands at neonatal day 9, 4 weeks of age, 8 weeks of age, and 12 weeks of age. Figure 40 shows representative images of mammary gland gross morphologies and H\&E stains at these developmental timepoints. Embryonic and neonatal timepoints were necessary to elucidate Mier3 involvement at these critical structural periods of the primitive gland, while 4,8 , and 12 weeks of age were important to evaluate Mier3 expression changes with onset of the estrous cycle and pubertal changes into an inactive adult mammary gland by 12 weeks of age. To study Mier3 expression throughout the reproductive cycle, mammary glands were collected from pregnant, lactating, involuting, and parous rats. The previous 12 week timepoint dually functioned as a nulliparous counterpart to the parous stage. Figure 41 shows representative images of the mammary gland gross morphologies and $H \& E$ stains at these reproductive timepoints. Nulliparous and parous timepoints were selected to determine baseline Mier3 expression in inactive mammary glands, whether virgin or fully differentiated. The other timepoints were critical to evaluate Mier 3 expression changes with tissue remodeling and the different functional roles of active, secretory mammary glands during pregnancy, lactation, and involution. Figures 40 and 41 also display that mammary glands collected at each developmental stage appear morphologically as expected, indicating our rat strains used have normal mammary gland development and are appropriate to assay with RNAscope.

The sensitivity of the RNAscope assay was tested using Mier3 as a moderately expressing gene, and control probes RNA Polymerase II Subunit A (Polr2a) 

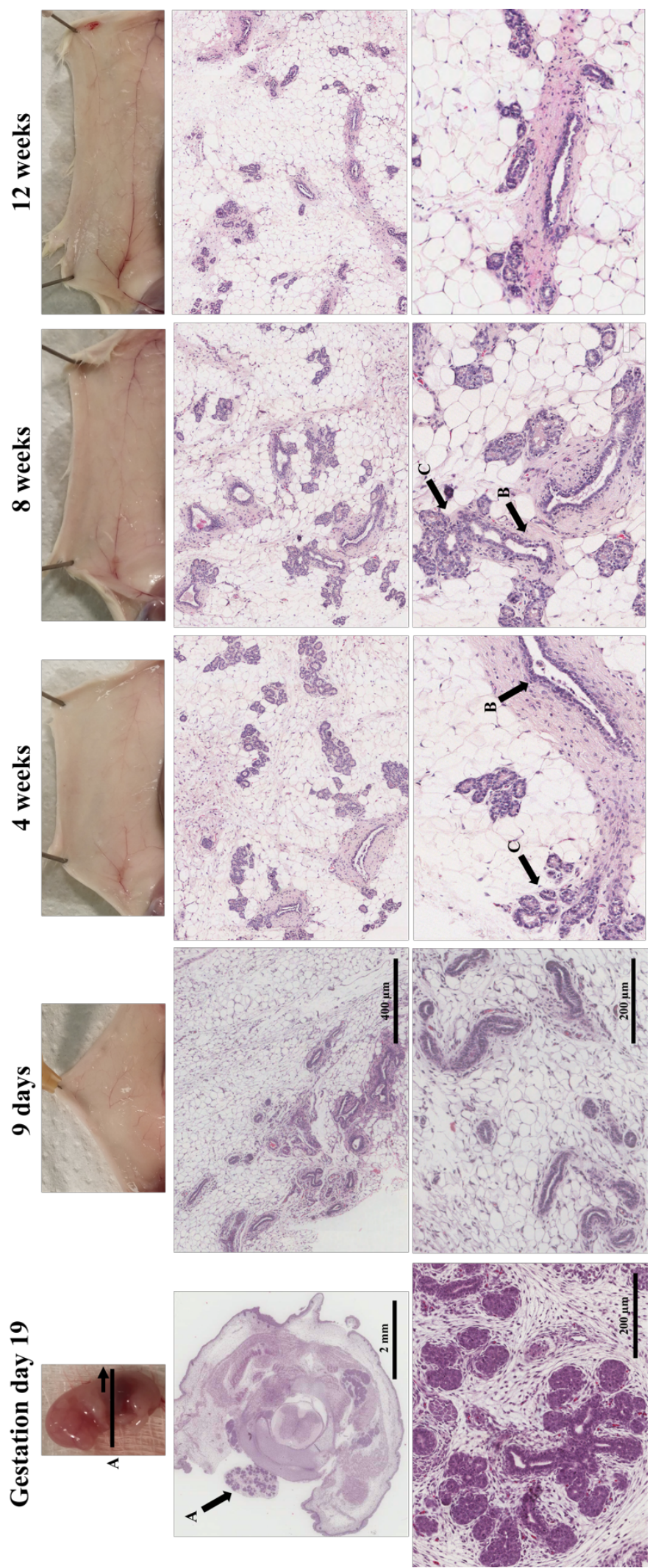
Figure 40: Mammary Gland Appearance and Histology during Development: Top row - gross morphology of the intact mammary gland at each time point, except for a full embryo at E19 shown. Middle row - Low power view of H\&E stained mammary glands to show overall histology. Bottom row - High power view of H\&E stained mammary glands to show morphological features.

The embryonic mammary bud extends from the body cavity and is captured by sectioning across (A). Primitive epithelial structures can be observed at late embryonic development. The postnatal mammary fat pad grows ventro-laterally along the skin. During neonatal development (9 days) a clustering of epithelial ducts can be detected within a mostly stromal mammary gland component. At puberty (4 and 8 weeks) fully developed bi-layered epithelial ducts (B) are apparent throughout the stromal matrix. At the ends of ducts are proliferative terminal end buds (TEBs) (C) that drive ductal extension and branching. At 12 weeks of age the epithelial tree has fully expanded and developed within the mammary fat pad, and is marked by a loss of the majority of TEB structures. 


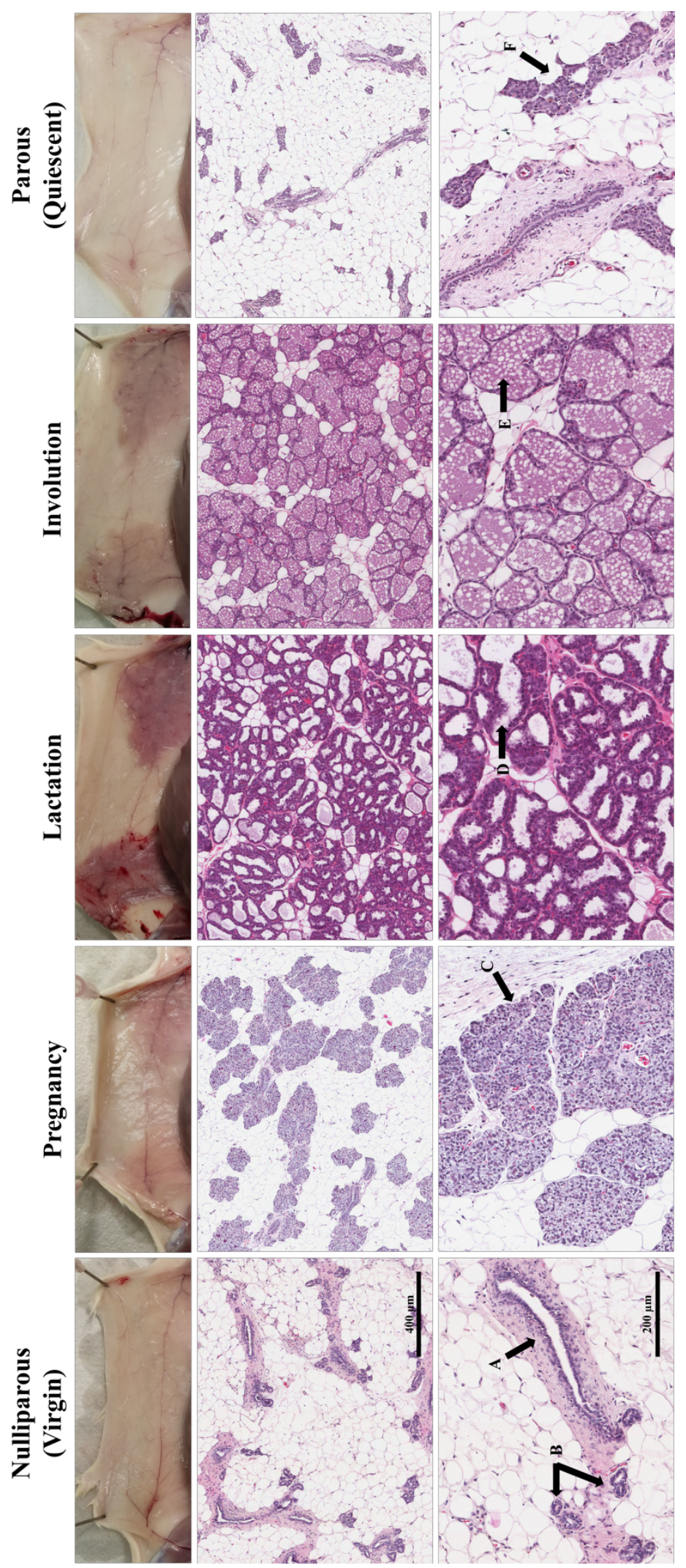




\section{Figure 41: Mammary Gland Appearance and Histology during the Reproduction}

Cycle: Top row - gross morphology of the intact mammary gland at each time point. Middle row - Low power view of H\&E stained mammary glands to show overall histology. Bottom row - High power view of H\&E stained mammary glands to show morphological features.

The fully developed virgin (nulliparous) mammary gland is mainly comprised of branching epithelial ducts (A) interspersed within connective tissue stroma and surrounded by adipose tissue. Smaller ductules (B) and alveolar buds (B) are also apparent as tubular clusters. During pregnancy, alveolar buds differentiate into milk-secreting lobular structures (C) that encompass the majority of the fat pad. The ductal tree regresses and becomes a minimal structure at this time. The lactating mammary gland consists of actively secreting lobular structures (D) that have attained maximal volume in the mammary fat pad. Loss of suckling results in retention of milk and fat globules within lobules (E) that initiates involution. Tissue remodeling, mainly controlled apoptosis of lobules and re-establishment of the ductal tree, brings the mammary gland back to a quiescent state. The parous gland resembles the nulliparous gland except for some retained differentiated lobular structures (F). 
as a highly expressed gene and bacterial 4-hydroxy-tetrahydrodipicolinate reductase $(D a p B)$ as a negative control. Each probe functioned as expected based on differences in density of puncta between Mier3 and Polr2a, and lack of puncta with DapB. Probes were assayed in tissue used to study early development (Fig 42) and the reproductive cycle (Fig 43).

\section{Mier3 expression is greatly enhanced in mammary ducts and terminal end buds during embryonic through pubertal development, but significantly drops off at adulthood}

Mier3 expression was first analyzed in mammary epithelial structures during the early stages of mammary gland development in WF and WF.COP Mcs $1 b$-N3 females. Primitive embryonic mammary tissue was unable to be excised in the same manner as mammary glands post-birth, so embryonic body cavities were serial sectioned across and stained to find tissue. Since this is an extremely laborious process, tissue was analyzed from combined genotypes. This late stage of embryonic development possessed the greatest levels of Mier3 (Fig 44). There was significantly greater Mier3 expression in WF mammary ducts at the neonatal timepoint of 9 days old, quantified as either mean probe per MEC ( $\mathrm{p}=0.0380$ unpaired two-tailed t test) or percent of MECs positive for Mier3 $(\mathrm{p}=0.0025)($ Fig 45).

During puberty, terminal end bud (TEB) morphologies first appear as a prominent, proliferative epithelial structure of the pubescent mammary gland. As such, there was separate analysis of ductal and TEB morphologies in early stage puberty (4 weeks old) and standard puberty ( 8 weeks old). There was no significant genotypic difference during early 


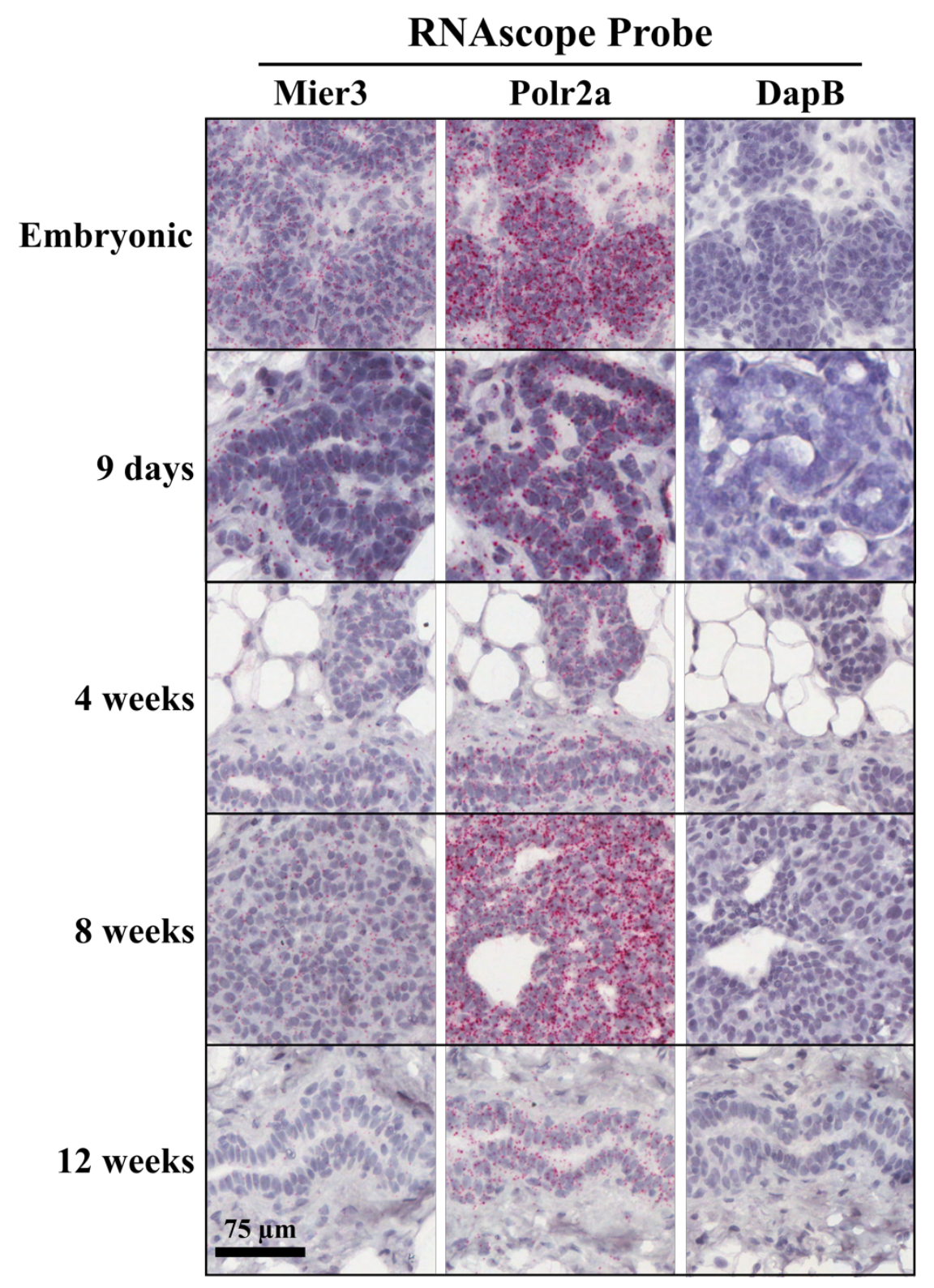

Figure 42: RNAscope assay probes in rat mammary glands throughout development.

From top to bottom are representative 40x images of embryonic mammary buds, primitive ducts at 9 days, a mix of ductule and terminal end bud (TEB) morphology at 4 weeks, TEBs at 8 weeks, and ducts at 12 weeks. The RNAscope assay probed for Mier3 as a moderately expressed gene (left column), Polr $2 a$ as a highly expressed gene (middle column) and bacterial $D a p B$ as a negative control (right column). 


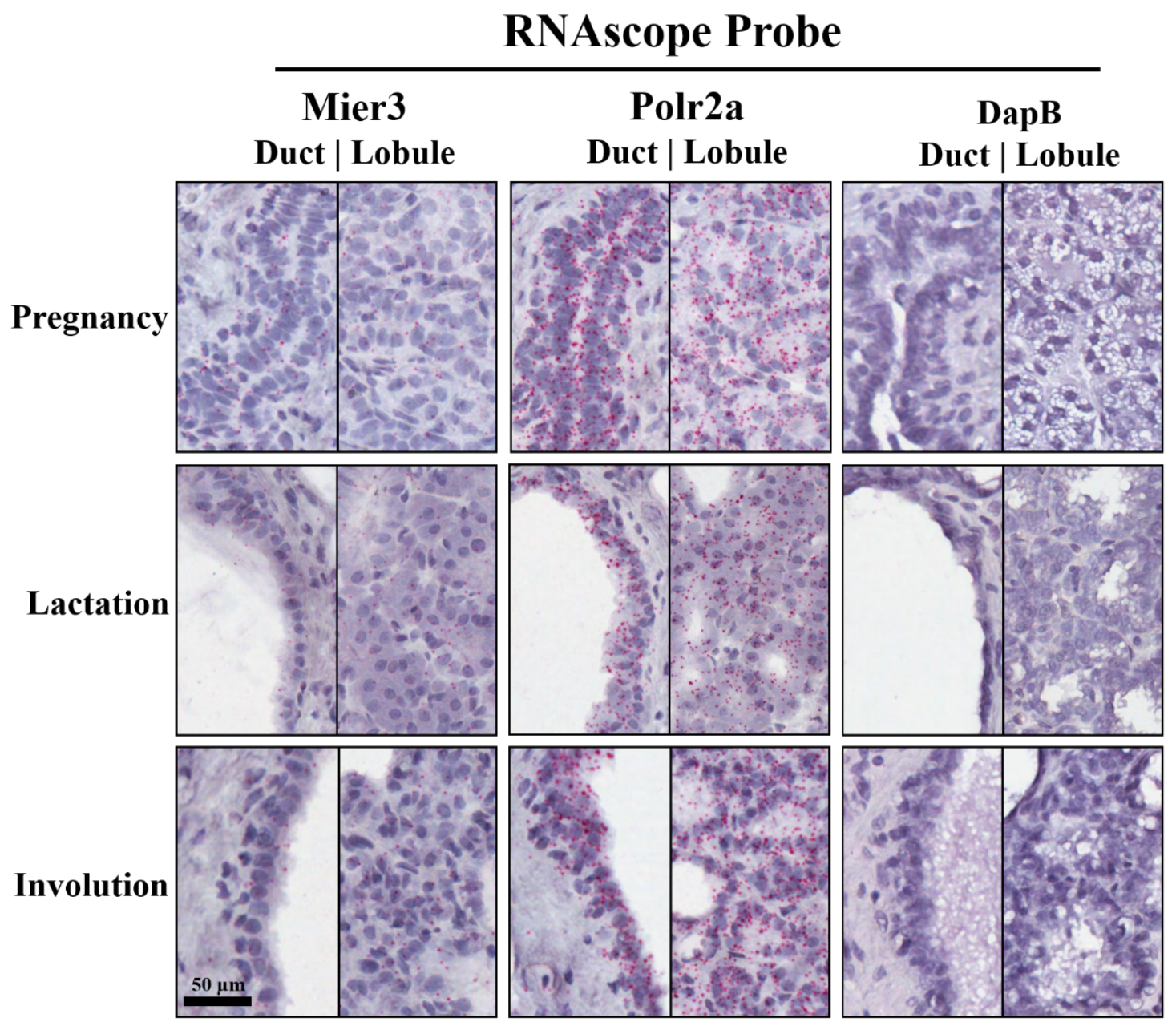

Figure 43: RNAscope assay probes in rat mammary glands during the reproductive

cycle. From top to bottom are $40 \mathrm{x}$ representative images of mammary glands during pregnancy, lactation, and involution. At each timepoint ductal (left panel) and lobular (right panel) morphologies are shown. The RNAscope assay probed for Mier3 as a moderately expressed gene (left column), Polr2a as a highly expressed gene (middle column) and bacterial $D a p B$ as a negative control (right column). 
A

Embryonic Mammary Bud

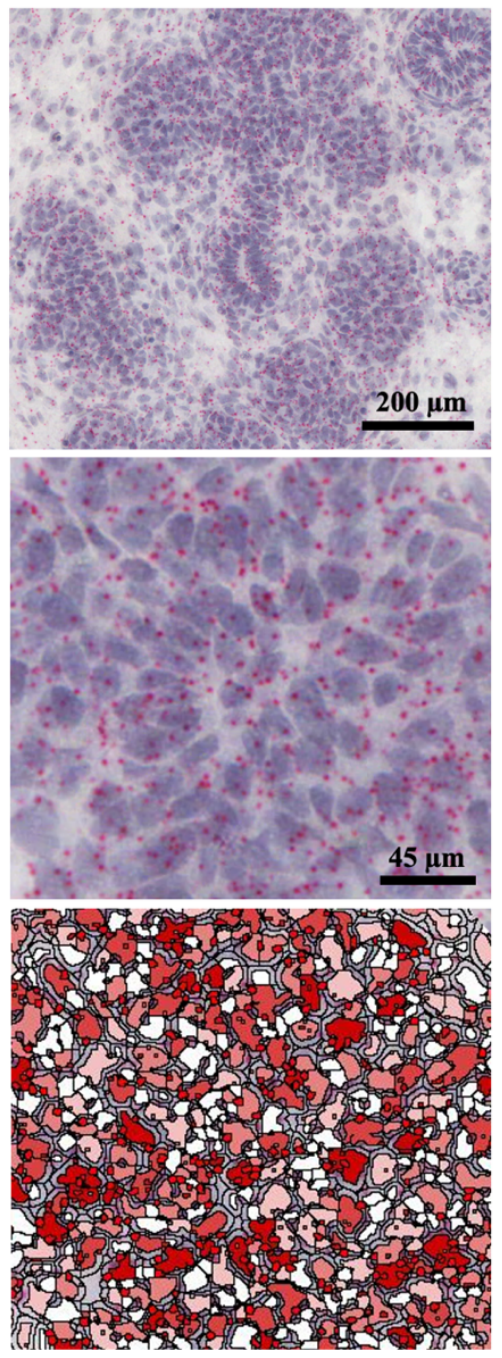

B

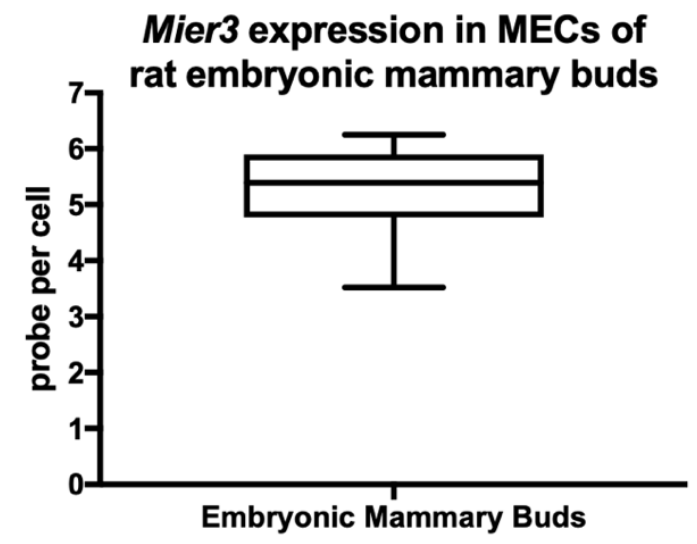

C

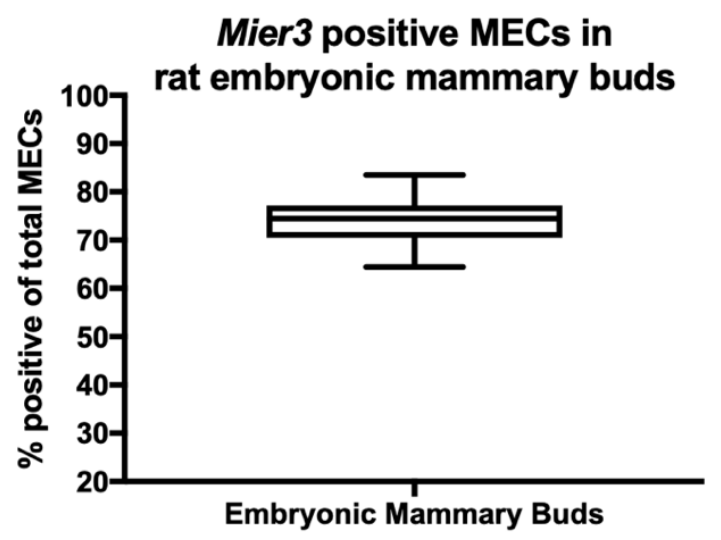

Figure 44: Mier3 expression in MECs of rat embryonic mammary buds: (A) 40x mammary bud histologic images from RNAscope assays probing for Mier3 show red puncta (Mier3 transcripts) counterstained with hematoxylin. The bottom row is the red probe and nuclei markup from the HALO-ISH module software. (B) Quantification of mean Mier3 expression per MEC in mammary buds. (C) Quantification of MECs positive for Mier3 expression. $\mathrm{N}=6$ embryonic mammary buds, $\mathrm{N}=26$ total fields of view. 
A

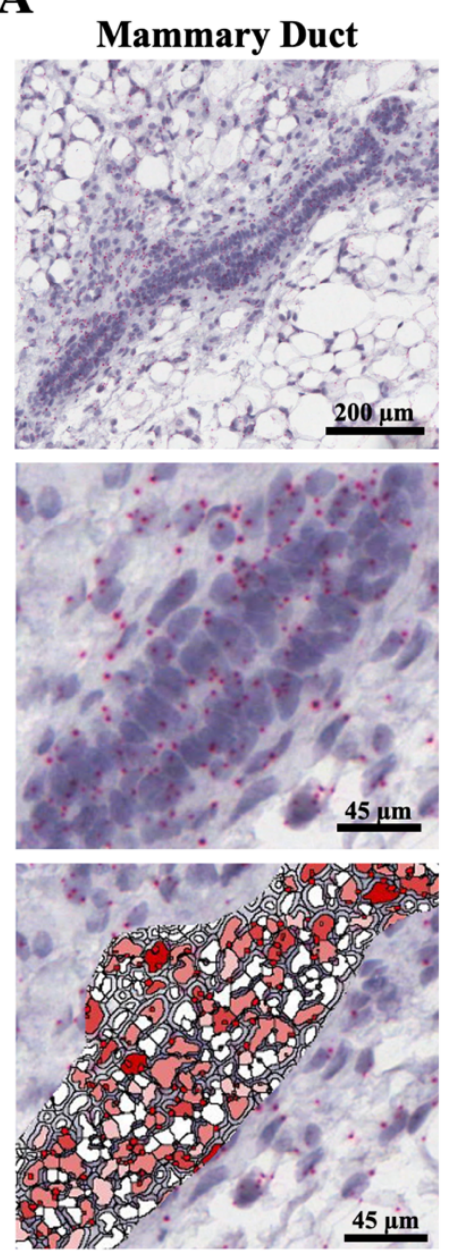

B

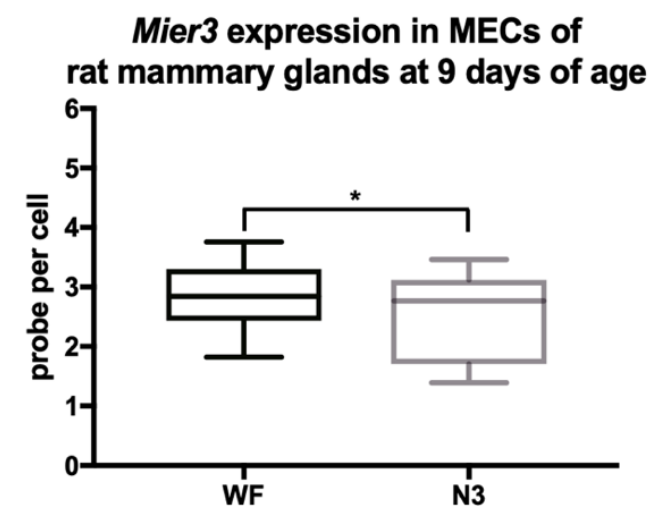

C

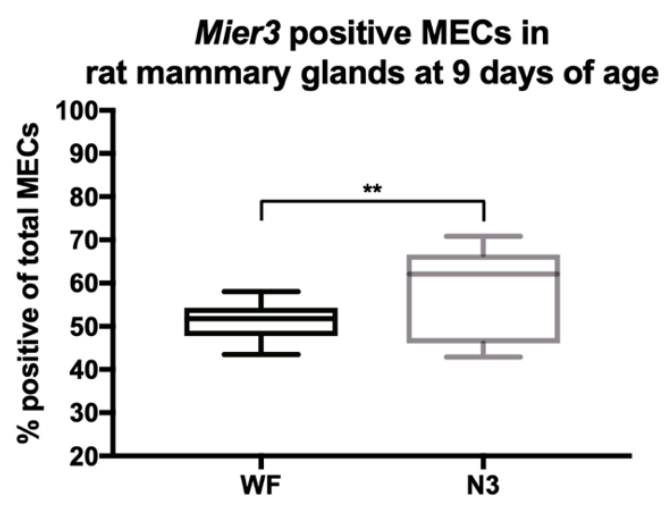

Figure 45: Mier3 expression in MECs of rat mammary glands at 9 days of age: (A) 40x mammary duct histologic images from RNAscope assays probing for Mier3 show red puncta (Mier3 transcripts) counterstained with hematoxylin. The bottom row is the red probe and nuclei markup from the HALO-ISH module software. (B) Quantification of mean Mier3 expression per MEC in mammary ducts in the WF vs N3 strain. There was fa significant difference based on genotype using an unpaired two-tailed $t$ test $(p<0.05)$. (C) Quantification of MECs positive for Mier3 expression. There was a significant difference based on genotype using an unpaired two-tailed t test $(\mathrm{p}<0.01) . \mathrm{N}=6$ mammary glands per genotype, minimally $n=14$ fields of view per genotype. 
puberty at 4 weeks of age, but there was a morphology difference when quantifying by mean Mier3 probe per MEC ( $\mathrm{p}=0.9892$ genotype, $\mathrm{p}<0.0001$ morphology, two-way ANOVA) and percent of MECs positive for Mier3 expression ( $\mathrm{p}=0.8399$ genotype, $\mathrm{p}<0.0001$ morphology, two-way ANOVA). In both strains, TEBs displayed greater Mier3 levels than mammary ducts by mean Mier3 probe quantification per MEC $(\mathrm{p}<0.0001$ Tukeys post hoc) and overall MECs positive for Mier3 ( $\mathrm{p}=0.0003$ ) (Fig 46). The main effect at this timepoint was morphology as there was initially a genotypic difference during the standard pubertal timepoint when quantifying by mean probe per MEC, but this was lost in post hoc analysis ( $\mathrm{p}=0.0095$ genotype, $\mathrm{p}=0.0020$ morphology, two-way ANOVA). When quantifying by percent of Mier3 positive MECs, there were significant differences during puberty at 8 weeks of age $(p=0.0154$ genotype, $p<0.0001$ morphology, two-way ANOVA). There was a significant genotypic difference in Mier3 expression within ductal morphologies ( $\mathrm{p}=0.03$ Tukeys post hoc), but none in comparing TEBs. There was also significantly greater expression in TEBs in the WF strain $(\mathrm{p}=0.03)$ and WF.COP Mcs $1 b$ $\mathrm{N} 3$ strain $(\mathrm{p}=0.0002)$ (Fig 47).

A final developmental timepoint of 12 weeks of age was next evaluated, as this is breeding age in the rat and is representative of fully developed, adult mammary glands. Pubescent TEB structures are minimal at the timepoint, so only mammary ducts were evaluated. Mier 3 expression was greatly reduced at this stage compared to previous timepoints. There was no significant difference between genotypes based on mean Mier3 probe analysis ( $\mathrm{p}=0.4089$ unpaired two-tailed $\mathrm{t}$ test) but there was a greater percent of Mier3-positive MECs in the WF strain ( $\mathrm{p}=0.0179)$ (Fig 48). Total quantification of Mier3 expression at all developmental timepoints is given in Table 24 . 
A

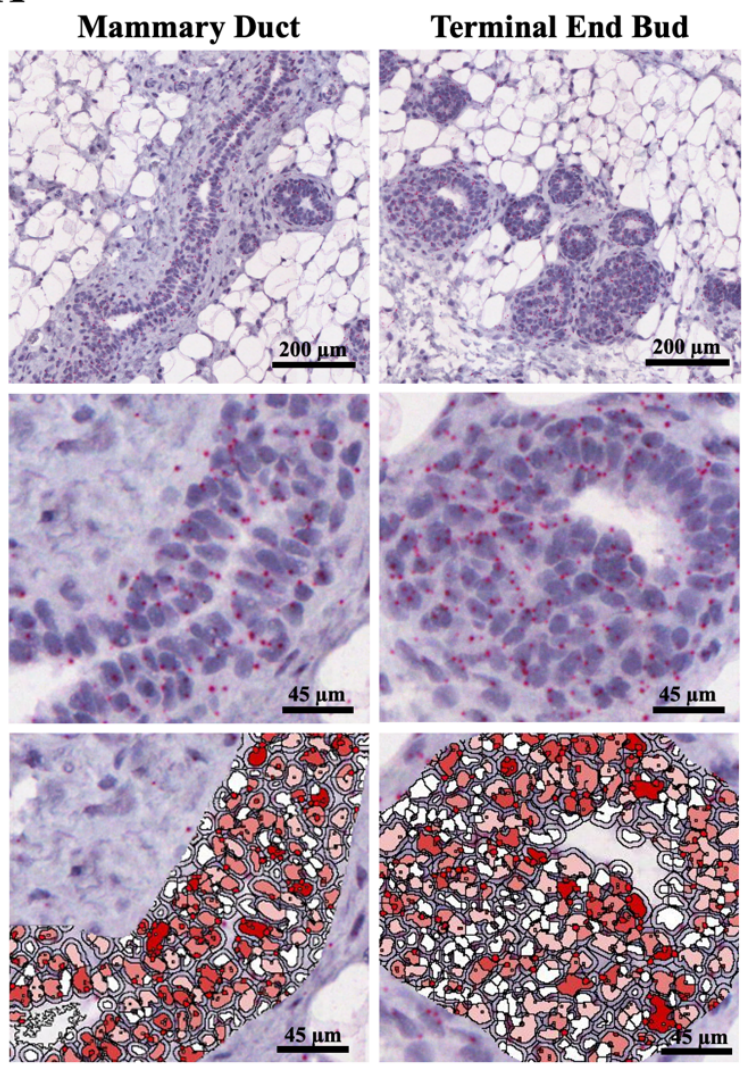

B

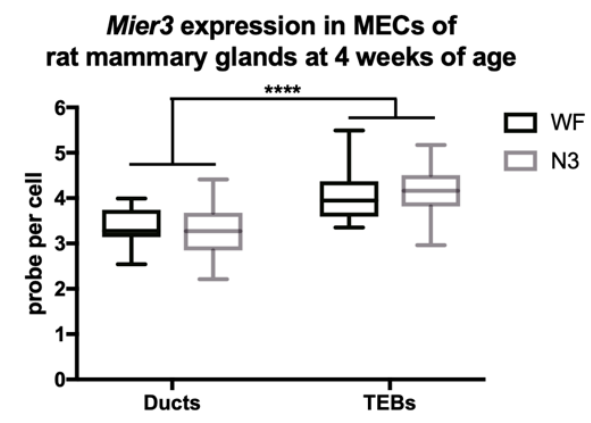

C

Mier3 positive MECs in rat mammary glands

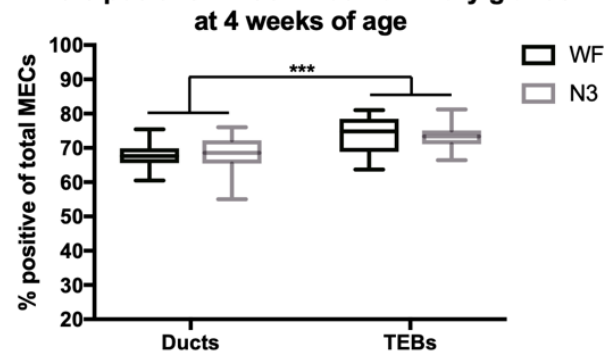

Figure 46: Mier3 expression in MECs of rat mammary glands at 4 weeks of age:

(A) 40x images of a mammary duct (left) and TEB (right) from RNAscope assays probing for Mier3 show red puncta (Mier3 transcripts) counterstained with hematoxylin. The bottom row is the red probe and nuclei markup from the HALO-ISH module software. (B) Quantification of mean Mier3 expression per MEC in mammary ducts and TEBs in the WF vs N3 strain. There was no difference based on genotype, but a significant difference based on morphology (2-way ANOVA, Tukeys post hoc $\mathrm{p}<0.0001)$. (C) Quantification of MECs positive for Mier3 expression in mammary ducts and TEBs. There was no difference based on genotype, but a significant difference based on morphology (2-way ANOVA, Tukeys post hoc $\mathrm{p}=0.0003$ ). $\mathrm{N}=6$ mammary glands per genotype, minimally $\mathrm{n}=20$ fields of view per genotype per morphology. 
A

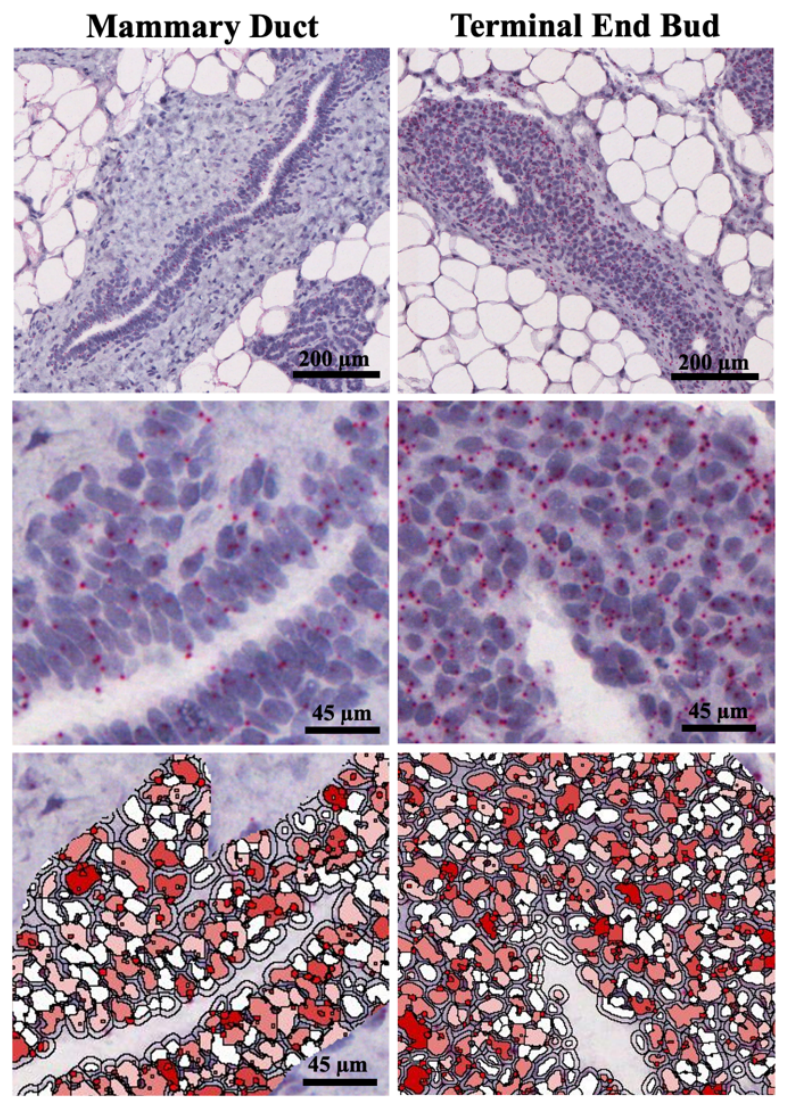

B

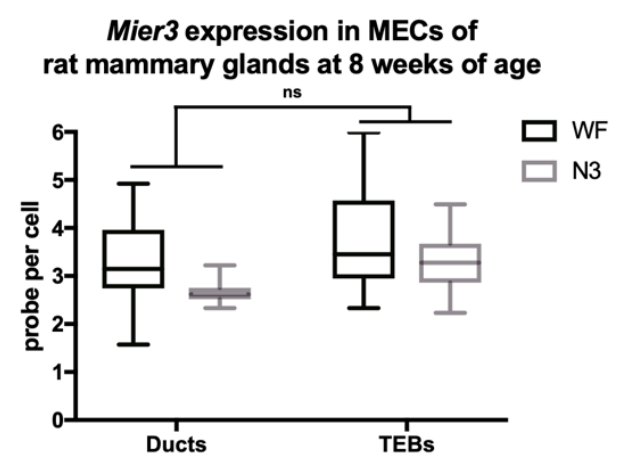

$\mathbf{C}$

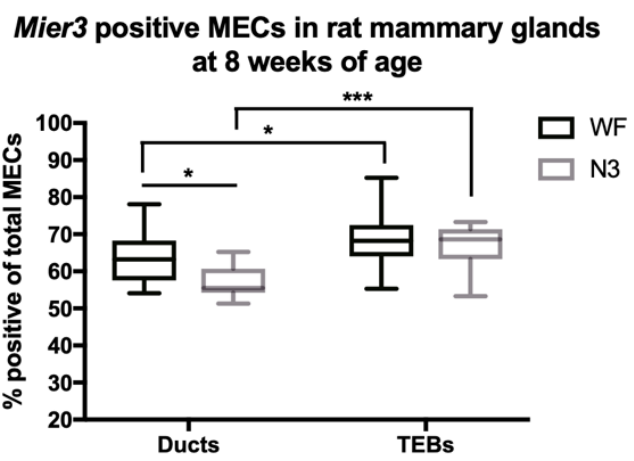

Figure 47: Mier3 expression in MECs of rat mammary glands at 8 weeks of age:

(A) 40x images of a mammary duct (left) and TEB (right) from RNAscope assays probing for Mier3 show red puncta (Mier3 transcripts) counterstained with hematoxylin. The bottom row is the red probe and nuclei markup from the HALO-ISH module software. (B) Quantification of mean Mier 3 expression per MEC in mammary ducts and TEBs in the WF vs N3 strain. There was no significant difference based on genotype or morphology. N=6 per genotype. (C) Quantification of MECs positive for Mier3 expression in mammary ducts and TEBs. There was a significant difference based on genotype in ducts (2-way ANOVA, Tukeys post hoc $\mathrm{p}=0.01)$ and between morphologies $(\mathrm{p}<0.0001)$. $\mathrm{N}=6$ mammary glands per genotype, minimally $n=12$ fields of view per genotype per morphology. 
A

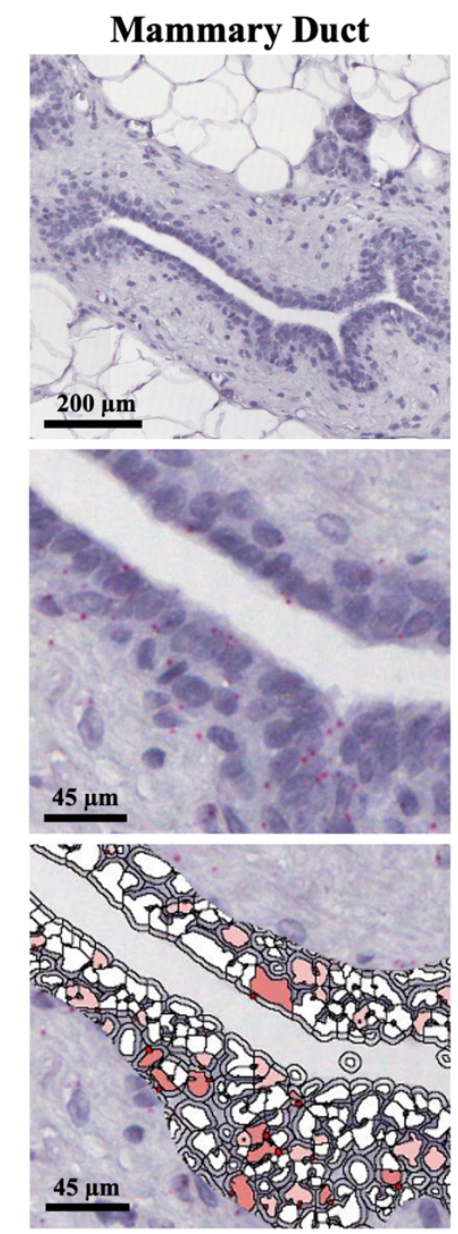

B

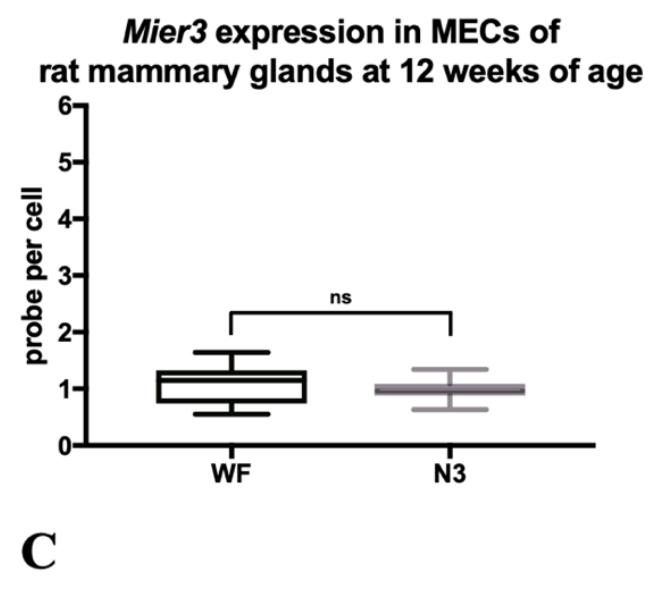

Mier3 positive MECs in rat mammary glands

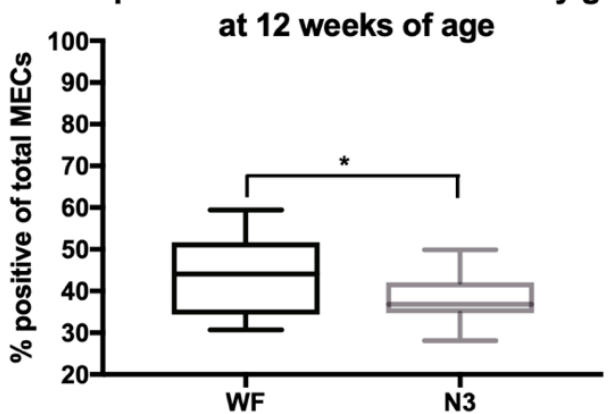

Figure 48: Mier3 expression in MECs of rat mammary glands at 12 weeks of age:

(A) 40x mammary duct histologic images from RNAscope assays probing for Mier3 show red puncta (Mier3 transcripts) counterstained with hematoxylin. The bottom row is the red probe and nuclei markup from the HALO-ISH module software. (B) Quantification of mean Mier3 expression per MEC in mammary ducts in the WF vs N3 strain. There was no significant difference based on genotype. (C) Quantification of MECs positive for Mier3 expression in mammary ducts. There was a significant difference based on genotype (unpaired two-tailed t test $\mathrm{p}=0.02$ ). $\mathrm{N}=6$ per mammary glands genotype, minimally $\mathrm{n}=20$ fields of view per genotype. 
Table 24. Mier3 expression in WF/NHsd and WF.COP Mcs1b-N3 mammary epithelial structures throughout early development

\begin{tabular}{|c|c|c|c|c|c|}
\hline Genotype & Age & Stage & Morphology & $\begin{array}{c}\text { Mean } \\
\text { probe per } \\
\text { cell (SD) }\end{array}$ & $\begin{array}{c}\% \text { of } \\
\text { positive } \\
\text { cells (SD) }\end{array}$ \\
\hline $\begin{array}{c}\text { WF/NHsd and } \\
\text { WF.COP } M c s 1 b-\mathrm{N} 3\end{array}$ & E19 & Embryonic & Mammary bud & $5.27(0.75)$ & $74.0(4.6)$ \\
\hline WF/NHsd & P9 & Neonatal & Duct & $2.83(0.54)$ & $51.2(3.8)$ \\
\hline WF.COP Mcs $1 b-\mathrm{N} 3$ & P9 & Neonatal & Duct & $2.48(0.74)$ & $57.5(10.2)$ \\
\hline WF/NHsd & 4wks & Early puberty & Duct & $3.35(0.39)$ & $67.9(3.6)$ \\
\hline WF/NHsd & $4 w k s$ & Early puberty & Terminal end bud & $4.08(0.58)$ & $73.7(5.2)$ \\
\hline WF.COP $M c s 1 b-\mathrm{N} 3$ & 4wks & Early puberty & Duct & $3.28(0.53)$ & $68.5(5.0)$ \\
\hline WF.COP $M c s 1 b-\mathrm{N} 3$ & $4 \mathrm{wks}$ & Early puberty & Terminal end bud & $4.17(0.50)$ & $73.6(3.7)$ \\
\hline WF/NHsd & $8 w k s$ & Puberty & Duct & $3.25(0.82)$ & $63.4(6.6)$ \\
\hline WF/NHsd & $8 w k s$ & Puberty & Terminal end bud & $3.75(1.06)$ & $68.1(6.5)$ \\
\hline WF.COP $M c s 1 b-\mathrm{N} 3$ & $8 w k s$ & Puberty & Duct & $2.66(0.24)$ & $57.3(4.4)$ \\
\hline WF.COP $M c s 1 b-\mathrm{N} 3$ & $8 w k s$ & Puberty & Terminal end bud & $3.28(0.56)$ & $67.2(5.4)$ \\
\hline WF/NHsd & $12 \mathrm{wks}$ & Adult & Duct & $1.08(0.33)$ & $43.9(9.4)$ \\
\hline WF.COP $M c s 1 b-\mathrm{N} 3$ & $12 \mathrm{wks}$ & Adult & Duct & $0.97(0.18)$ & $38.0(5.0)$ \\
\hline
\end{tabular}

$\mathrm{N}=6$ mammary glands per genotype per timepoint, E19=6 mammary buds total 


\section{Mier3 expression significantly increases during growth phases of the reproductive cycle and has variation between ductal vs lobular morphologies}

The next phase of Mier 3 expression analysis throughout windows of susceptibility in the rat mammary gland was broken up into reproductive development. The adult timepoint of 12 weeks of age in the previous early development phase was also included as the start to the reproductive cycle, since this nulliparous stage provides baseline Mier3 expression information in undifferentiated and inactive mammary glands. During late pregnancy, lobuloalveolar structures appear as the predominant morphology in meeting the demands of lactation and persist throughout the cycle. As such, Mier3 probe quantification was performed separately in mammary duct and lobular morphologies at each stage analyzed.

At 19 days gestation, there was significantly greater $M i e r 3$ expression compared to the nulliparous stage. In evaluating this pregnancy timepoint, there were significant differences based on genotype and morphology when quantifying mean Mier3 probe per MEC ( $\mathrm{p}=0.0107$ genotype, $\mathrm{p}<0.0001$ morphology, two-way ANOVA) and overall Mier3positive MECs ( $\mathrm{p}=0.00006$ genotype, $\mathrm{p}<0.0001$ morphology, two-way ANOVA). However, the effect of genotype was lost in post hoc analysis in mean quantification and only persisted in lobular morphologies in percent positivity analysis ( $\mathrm{p}=0.04$ Tukeys post hoc). Mammary ducts possessed significantly greater Mier 3 levels than lobules $(\mathrm{p}=0.02)$, as well as greater percent of Mier3-positive MECs ( $\mathrm{p}=0.002 \mathrm{WF}, \mathrm{p}=0.003$ N3) (Fig 49).

The next stage of the reproductive cycle selected was 11 days post-parturition, which represents a timepoint of peak mammary gland lactation. Quantification by mean Mier3 probe per MEC and Mier3-positive MECs gave significant differences based on 
$\mathbf{A}$

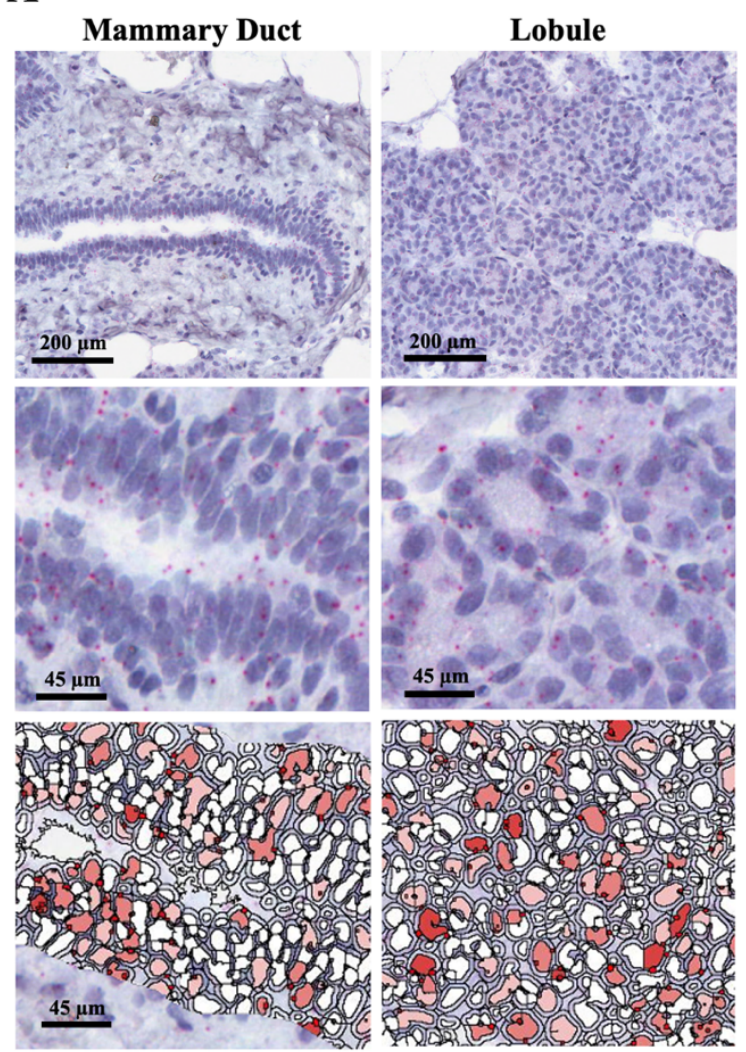

B

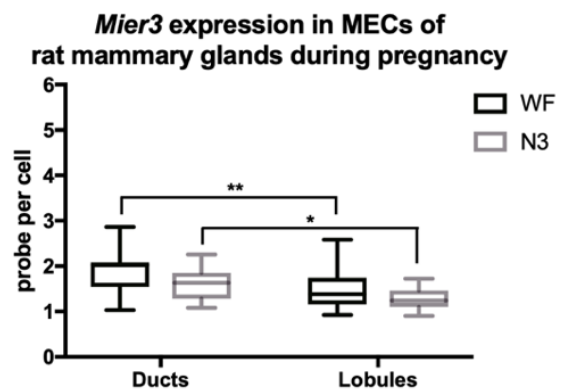

C

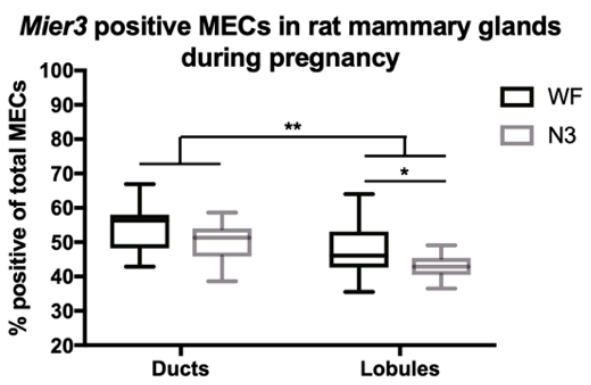

Figure 49: Mier3 expression in MECs of rat mammary glands during pregnancy:

(A) 40x images of a mammary duct (left) and lobule (right) from RNAscope assays probing for Mier3 show red puncta (Mier3 transcripts) counterstained with hematoxylin. The bottom row is the red probe and nuclei markup from the HALO-ISH module software.

(B) Quantification of mean Mier3 expression per MEC in mammary ducts and lobules in the WF vs N3 strain. There was no difference based on genotype, but a significant difference between morphologies (2-way ANOVA, Tukeys post hoc $\mathrm{p}<0.0001)$. (C) Quantification of MECs positive for Mier3 expression in mammary ducts and lobules. There was a significant difference based on genotype in lobules (2-way ANOVA, Tukeys post hoc $\mathrm{p}=0.0006)$ and between morphologies $(\mathrm{p}<0.0001) . \mathrm{N}=6$ mammary glands per genotype, minimally $n=20$ fields of view per genotype per morphology. 
A

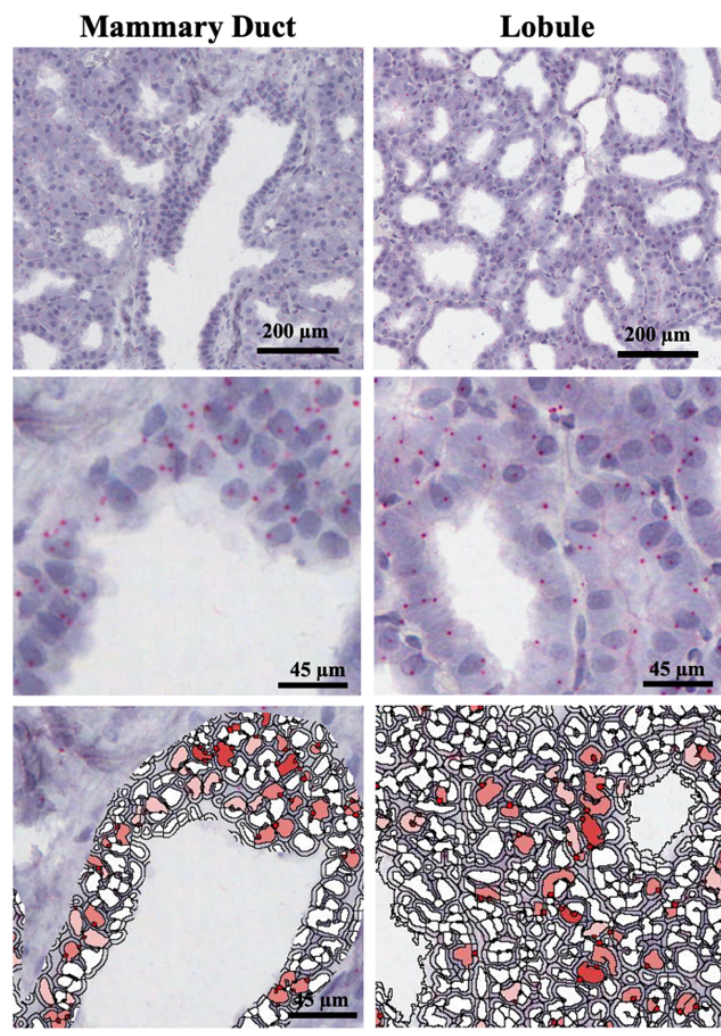

B

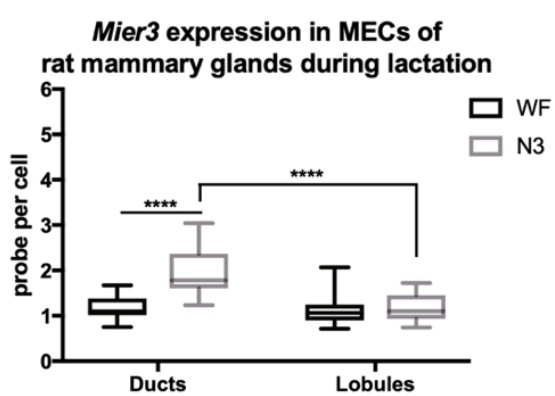

C

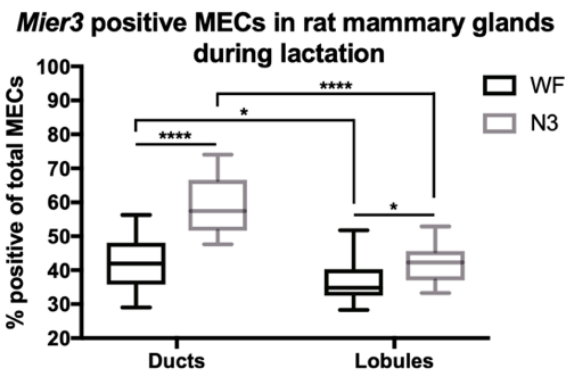

Figure 50: Mier3 expression in MECs of rat mammary glands during lactation:

(A) 40x images of a mammary duct (left) and lobule (right) from RNAscope assays probing for Mier3 show red puncta (Mier3 transcripts) counterstained with hematoxylin. The bottom row is the red probe and nuclei markup from the HALO-ISH module software. (B) Quantification of mean Mier3 expression per MEC in mammary ducts and lobules in the WF vs N3 strain. There was a significant difference between genotype in ducts (2-way ANOVA, Tukeys post hoc $\mathrm{p}<0.0001)$ and between morphologies $(\mathrm{p}<0.0001)$. (C) Quantification of MECs positive for Mier3 expression in mammary ducts and lobules. There was a significant difference based on genotype (2-way ANOVA, Tukeys post hoc $\mathrm{p}<0.0001)$ and morphology $(\mathrm{p}<0.0001)$. $\mathrm{N}=6$ mammary glands per genotype, minimally $\mathrm{n}=17$ fields of view per genotype per morphology. 
genotype and morphology ( $<<0.0001$ two-way ANOVA). Analyzing by mean Mier3 probe quantification, there were greater levels in ductal structures in the N3 strain $(p<0.0001$ Tukeys). Post hoc analysis of percent of Mier3-positive MECs yielded significantly greater levels in the $\mathrm{N} 3$ strain $(\mathrm{p}<0.0001$ duct, $\mathrm{p}=0.03$ lobule) as well as greater ductal positivity in both strains ( $\mathrm{p}=0.04 \mathrm{WF}, \mathrm{p}<0.0001 \mathrm{~N} 3$ ) (Fig 50).

To evaluate the process of involution, the timepoint of 2 days following cessation of lactation via pup removal was selected, as this represents entry into the irreversible phase of involution. Quantification via mean Mier3 probe per MEC was significant ( $\mathrm{p}=0.0007$ genotype, $\mathrm{p}<0.0001$ morphology, two-way ANOVA), but post hoc analysis showed the genotypic significance only in lobular structures ( $\mathrm{p}=0.02$ Tukey's post hoc). Analysis by percents of Mier3-positive MECs yielded only a morphological difference $(\mathrm{p}=0.1391$ genotype, $\mathrm{p}<0.0001$ morphology, two-way ANOVA). Differing from the phases of active growth in pregnancy and lactation, involution displayed greater Mier3 levels in lobular structures as opposed to ductal (Fig 51).

A final parous timepoint was selected for studying Mier3 mammary gland expression at 4 weeks following weaning of pups. This represents a time where involution has remodeled the mammary gland back into an inactive, yet now fully differentiated, gland. Although mammary ducts are once again the predominant structures, quantification was separated based on morphology since some lobules remain present. Mier3 levels fell back down and were similar to that found in nulliparous mammary glands. Analysis by mean Mier3 probe quantification and percent of Mier3-postivie MECs gave no significant differences based on genotype or morphology (two-way ANOVA) (Fig 52). Total quantification of Mier3 expression at all reproductive timepoints is given in Table 25. 
A

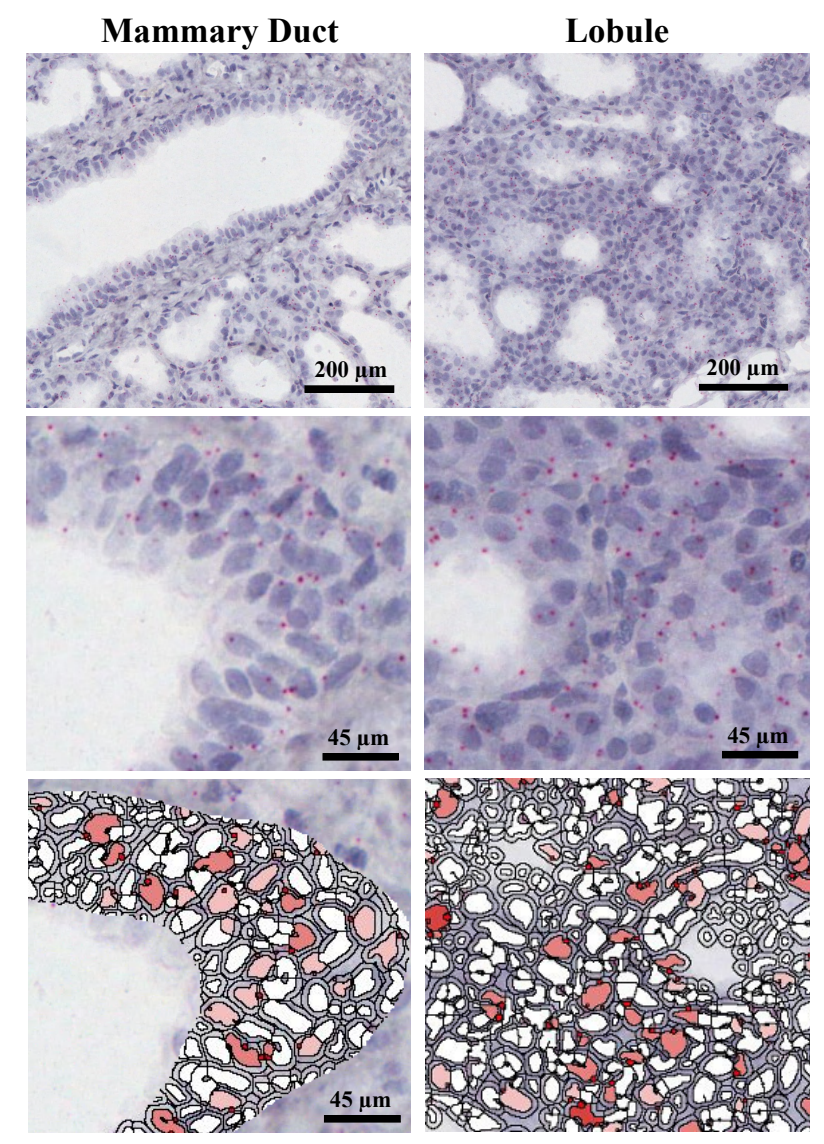

B

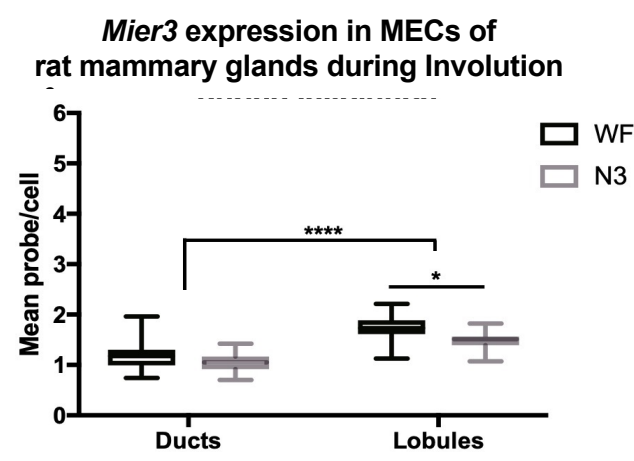

C

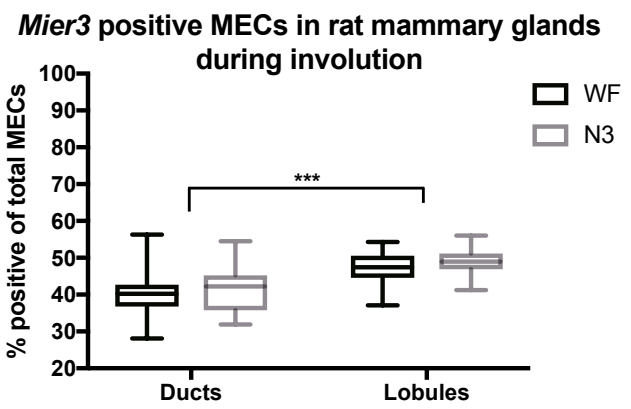

Figure 51: Mier3 expression in MECs of rat mammary glands during involution:

(A) 40x images of a mammary duct (left) and lobule (right) from RNAscope assays probing for Mier3 show red puncta (Mier3 transcripts) counterstained with hematoxylin. The bottom row is the red probe and nuclei markup from the HALO-ISH module software. (B) Quantification of mean Mier3 expression per MEC in mammary ducts and lobules in the WF vs N3 strain. There was a significant difference between genotype (2-way ANOVA, Tukeys post hoc $\mathrm{p}=0.0007)$ and morphologies $(\mathrm{p}<0.0001)$. (C) Quantification of MECs positive for Mier3 expression in mammary ducts and lobules. There was a significant difference in morphologies (2-way ANOVA, Tukeys post hoc $\mathrm{p}<0.0001$ ). N=6 mammary glands per genotype, minimally $n=17$ fields of view per genotype per morphology. 
$\mathbf{A}$

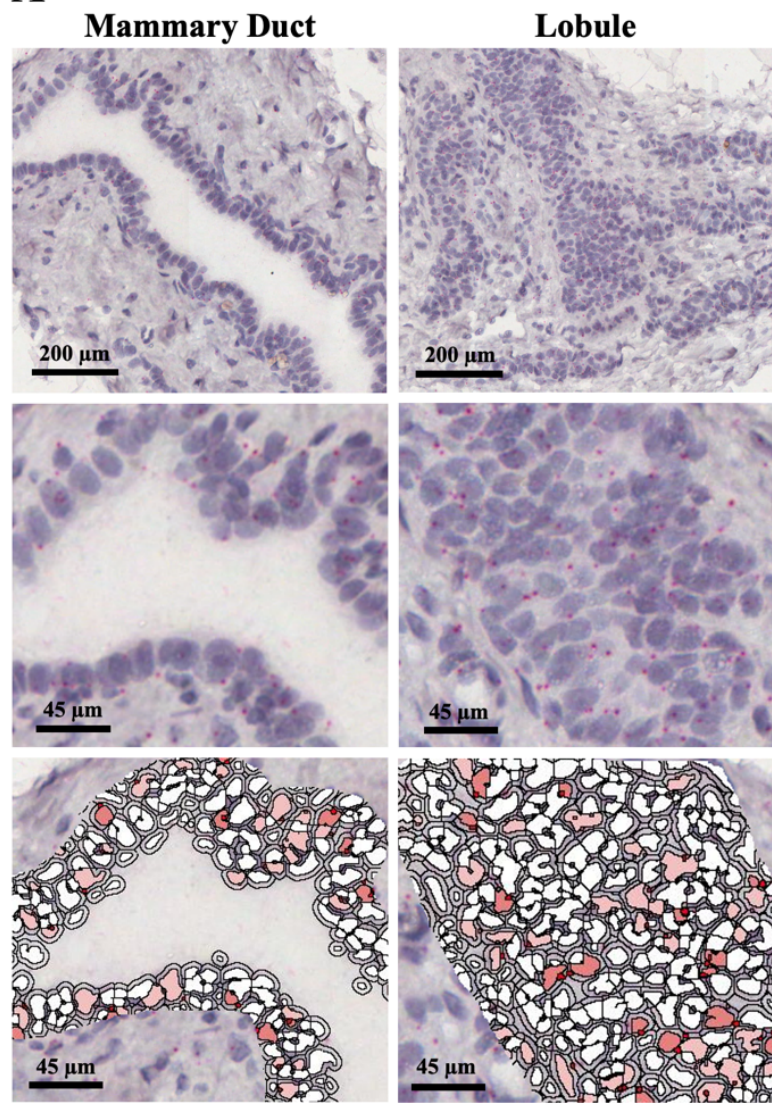

B

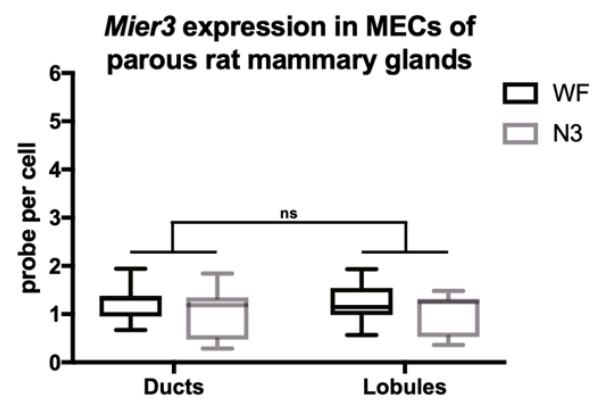

C

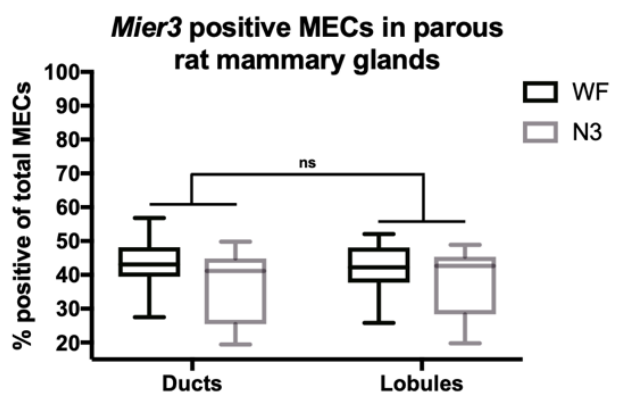

Figure 52: Mier3 expression in MECs of parous rat mammary glands: (A) 40x images of a mammary duct (left) and lobule (right) from RNAscope assays probing for Mier 3 show red puncta (Mier3 transcripts) counterstained with hematoxylin. The bottom row is the red probe and nuclei markup from the HALO-ISH module software. (B) Quantification of mean Mier3 expression per MEC in mammary ducts and lobules in the WF vs N3 strain. (C) Quantification of MECs positive for Mier3 expression in mammary ducts and lobules. For both graphs there was no significant difference based on genotype or morphology. $N=6$ mammary glands per genotype, minimally $n=18$ fields of view per genotype per morphology. 
Table 25. Mier3 expression in WF/NHsd and WF.COP Mcs1b-N3 mammary epithelial structures throughout reproductive development

\begin{tabular}{ccccc}
\hline Genotype & Stage & Morphology & $\begin{array}{c}\text { Mean probe } \\
\text { per cell (SD) }\end{array}$ & $\begin{array}{c}\text { \%oof positive } \\
\text { cells (SD) }\end{array}$ \\
\hline WF/NHsd & Nulliparous* & Duct & $1.08(0.33)$ & $43.9(9.4)$ \\
WF.COP $M c s 1 b$-N3 & Nulliparous* & Duct & $0.97(0.18)$ & $38.0(5.0)$ \\
\hline WF/NHsd & Pregnancy & Duct & $1.99(0.48)$ & $54.2(6.4)$ \\
WF/NHsd & Pregnancy & Lobule & $1.50(0.48)$ & $47.9(7.8)$ \\
WF.COP $M c s 1 b-\mathrm{N} 3$ & Pregnancy & Duct & $1.61(0.32)$ & $49.9(5.8)$ \\
WF.COP $M c s 1 b-\mathrm{N} 3$ & Pregnancy & Lobule & $1.27(0.21)$ & $43.0(3.3)$ \\
\hline WF/NHsd & Lactation & Duct & $1.18(0.26)$ & $41.8(7.2)$ \\
WF/NHsd & Lactation & Lobule & $1.13(0.35)$ & $36.1(5.8)$ \\
WF.COP $M c s 1 b-\mathrm{N} 3$ & Lactation & Duct & $1.95(0.50)$ & $58.7(8.5)$ \\
WF.COP $M c s 1 b-\mathrm{N} 3$ & Lactation & Lobule & $1.17(0.29)$ & $42.0(5.6)$ \\
\hline WF/NHsd & Involution & Duct & $1.18(0.27)$ & $40.3(5.7)$ \\
WF/NHsd & Involution & Lobule & $1.73(0.27)$ & $46.8(4.1)$ \\
WF.COP $M c s 1 b-\mathrm{N} 3$ & Involution & Duct & $1.04(0.18)$ & $41.6(6.7)$ \\
WF.COP $M c s 1 b-\mathrm{N} 3$ & Involution & Lobule & $1.48(0.17)$ & $48.9(3.6)$ \\
\hline WF/NHsd & Parous & Duct & $1.22(0.34)$ & $43.1(7.2)$ \\
WF/NHsd & Parous & Lobule & $1.23(0.34)$ & $42.7(6.6)$ \\
WF.COP $M c s 1 b-\mathrm{N} 3$ & Parous & Duct & $1.06(0.48)$ & $37.3(10.2)$ \\
WF.COP $M c s 1 b-\mathrm{N} 3$ & Parous & Lobule & $1.03(0.41)$ & $38.4(10.0)$ \\
\hline
\end{tabular}

* Same values as adult stages in Table 23

$\mathrm{N}=6$ mammary glands per genotype per timepoint 


\section{Summary: Mier3 expression is highest at embryonic through pubertal stages within developmental susceptibility windows in the rat mammary gland}

Comparing across stages of development in this study, there was a significant difference based on timepoint but no effect of genotype $(p=0.0647$ genotype, $p<0.0001$ timepoint, two-way ANOVA). WF and WF.COP Mcs $1 b$-N3 genotype data were combined for ductal morphologies at each stage, since TEB and lobular morphologies are not present across all developmental timepoints. There were significant expression differences across mammary gland development stages evaluated ( $\mathrm{p}<0.0001$ one-way ANOVA) (Fig 53). Mier3 expression was highest during the embryonic stage, and significantly differed from every other stage $(\mathrm{p}<0.0001$ Tukey's post hoc). The next highest timepoints of 9 days of age and 4 weeks of age significantly different from each other ( $\mathrm{p}=0.0033$ Tukey's post hoc), but not from the timepoint of 8 weeks of age. Expression dropped to the lowest levels at 12 weeks of age/nulliparous glands, during involution, and in parous glands. These timepoints did not significantly differ from each other. There was a significant increase in

Mier3 expression during pregnancy and lactation as compared to the other stages of the reproductive cycle ( $<<0.0001$ Tukey's post hoc), but this was also significantly lower than the early development stages.

The rat mammary epithelium displays cyclical changes in Mier3 expression, with the greatest levels correlated with the hormonal stages of the estrus cycle

In the concluding analysis of windows of susceptibility timepoints in rat mammary glands, Mier3 expression was evaluated at each stage of the rat estrus cycle since cyclical 


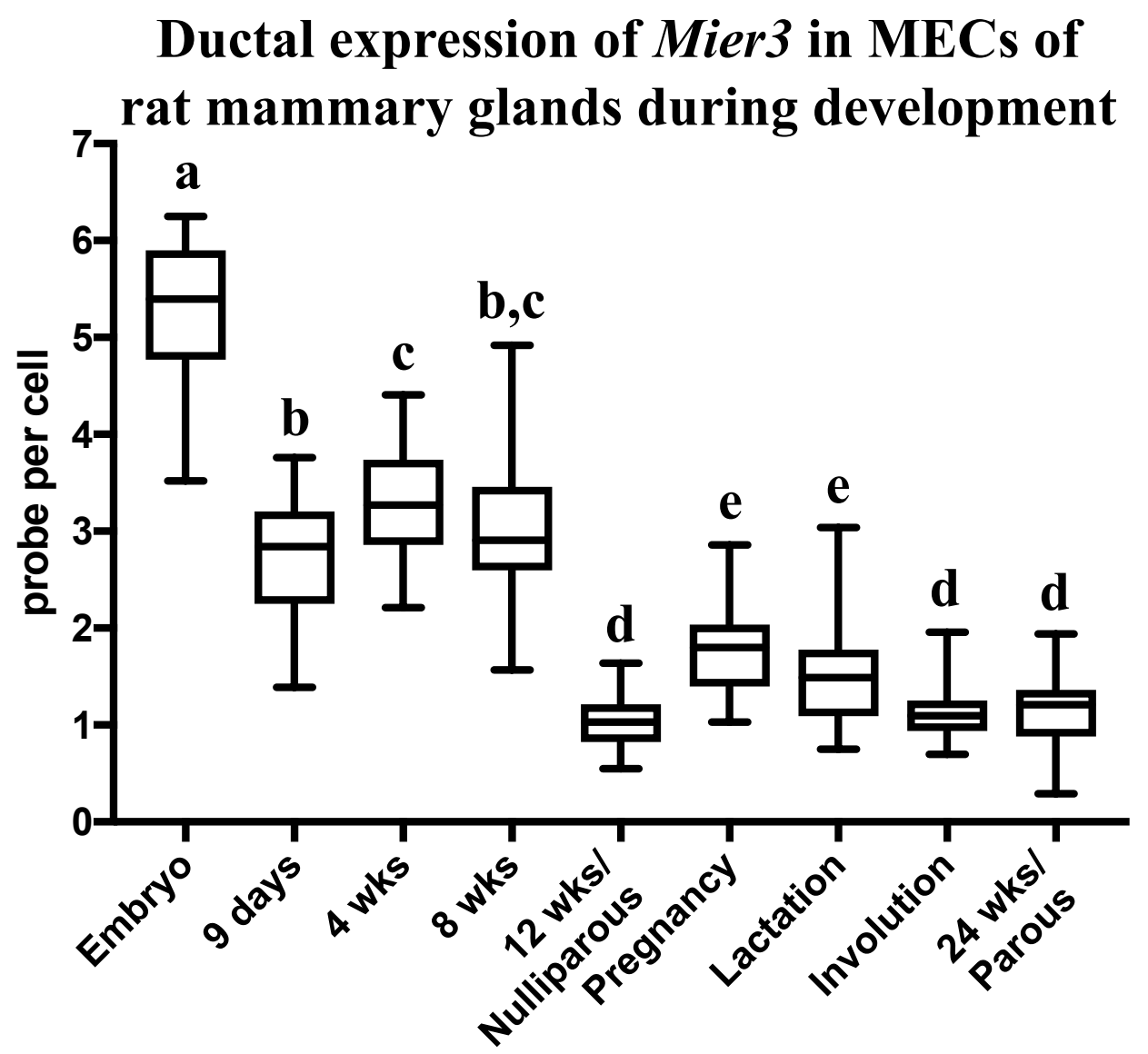

Figure 53: Ductal Expression of Mier3 in MECs of rat mammary glands during development: Quantification of mean Mier3 probe per MEC in combined WF and WF.COP Mcs 1b-N3 mammary duct morphology at each mammary gland development stage. Different lowercase letters indicate timepoints with significantly different average transcript levels $(p<0.001)$. A significant one-way ANOVA $(p<0.0001)$ followed by Tukey's multiple comparisons test was performed using GraphPad Prism version 7.00 Software. Minimally N=26 total fields of view per timepoint. 
changes to the mammary gland can alter susceptibility. Staging was determined based on vaginal cytology, which is informative of the cyclical growth and regression of the mammary epithelium. Combined WF and WF.COP Mcs $1 b$-N3 genotypes were used since these tissues were collected at the same age as the parous timepoint which showed no genotypic differences. Quantification was based on stage and morphology, with ductal and lobular morphology separated at each timepoint (Fig 54) (Table 26). Quantification based on mean Mier3 probe per MEC yielded no difference between ductal and lobular morphologies, but showed a significant difference between stages of the cycle in ( $\mathrm{p}=0.1095$ morphology, $\mathrm{p}<0.0001$ stage, two-way ANOVA). Using Tukey's post hoc analysis, the stages of proestrus, estrus, and metestrus I were grouped as significantly higher than the other stages, but not different from each other. Expression at metestrus II was significantly lower than this collective group ( $\mathrm{p}=0.001$ Tukey's post hoc), followed by diestrus which possessed the lowest levels ( $\mathrm{p}=0.0001$ Tukey's post hoc). Quantification by overall MECs positive for Mier 3 gave a significant difference based on morphology and stage $(\mathrm{p}=0.0268$ morphology, $\mathrm{p}<0.0001$ stage, two-way ANOVA). In the post hoc analysis, proestrus and estrus were found to significantly differ from each other ( $\mathrm{p}=0.0004$ Tukey's post hoc) but neither differed from metestrus I. Similar to mean probe quantification, metestrus II was significantly lower than these stages ( $\mathrm{p}=0.001$ Tukey's post hoc) and diestrus was lowest overall ( $\mathrm{p}=0.0001$ Tukey's post hoc). Using both quantification methods, the greatest Mier3 levels corresponded with the most hormonally-active stages of the estrus cycle. 
A
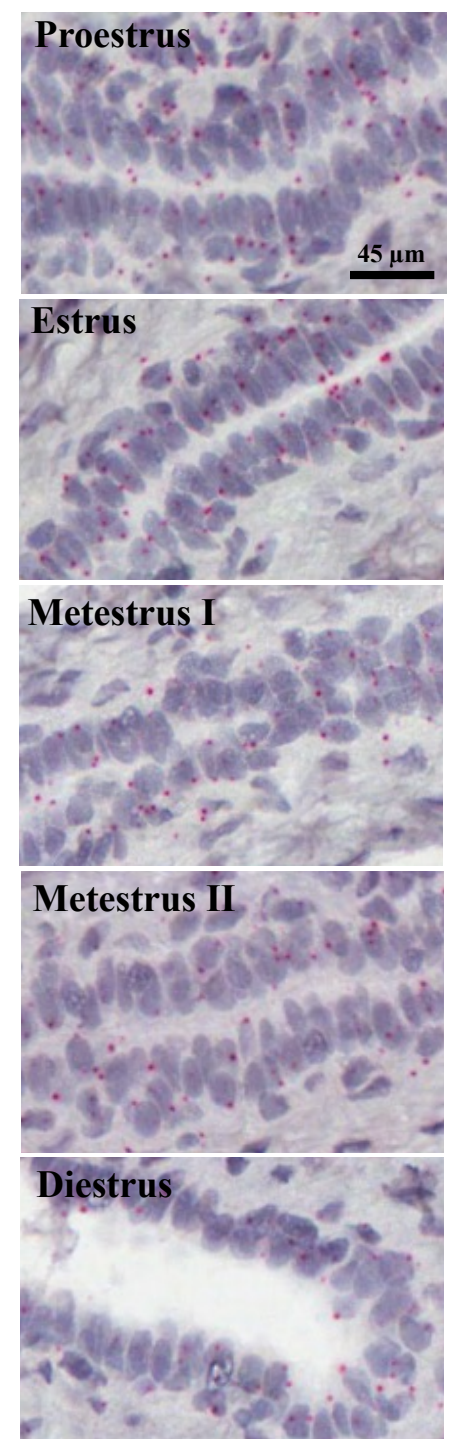

B

Mier3 expression in MECs of rat mammary glands by stages of the estrus cycle

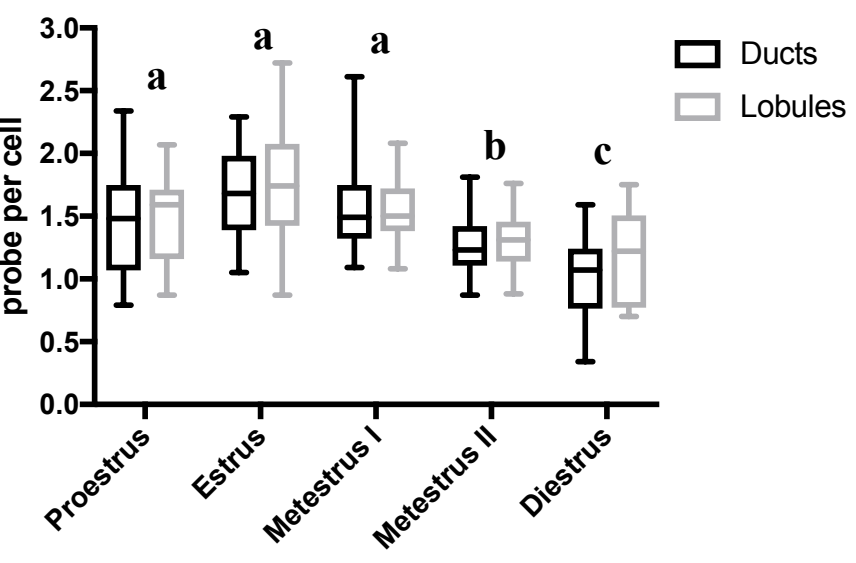

C

Mier3 positive MECs in rat mammary glands at stages of the estrus cycle

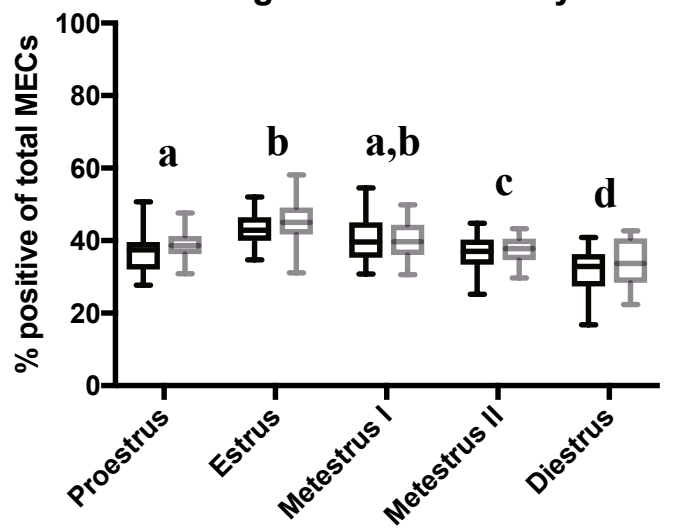


Figure 54: Mier3 expression in rat mammary glands based on stage of the rat estrus cycle: (A) 40x mammary gland histologic images from RNAscope assays probing for Mier3 show red puncta (Mier3 transcripts) counterstained with hematoxylin. From top to bottom shows a representative mammary duct at each consecutive stage of the estrus cycle - proestrus, estrus, metestrus I, metestrus II, and diestrus. (B) Quantification of mean Mier3 expression per MEC in mammary ducts and lobules in the WF strain. There was no significant difference based on morphology, but a significant difference based on stage of the cycle (2-way ANOVA, Tukeys post hoc $\mathrm{p}<0.0001)$. (C) Quantification of MECs positive for Mier3 expression in mammary ducts and lobules. There was a significant difference based on morphology (2-way ANOVA, Tukeys post hoc $\mathrm{p}=0.03$ ) and stage of the cycle (Tukeys post hoc $\mathrm{p}<0.0001$ ). Different lowercase letters indicate significant Tukey's multiple comparisons tests $(\mathrm{p}<0.001)$. N=3 mammary glands per estrus cycle stage, minimally $n=20$ fields of view per genotype per morphology. 
Table 26. Mier3 expression in combined WF/NHsd and WF.COP Mcs1b-N3 mammary epithelial structures throughout the rat estrus cycle

\begin{tabular}{cccc}
\hline Estrus Cycle Stage & Morphology & $\begin{array}{c}\text { Mean probe per } \\
\text { cell (SD) }\end{array}$ & $\begin{array}{c}\% \text { of positive } \\
\text { cells (SD) }\end{array}$ \\
\hline Proestrus & Duct & $1.44(0.44)$ & $36.8(5.2)$ \\
Proestrus & Lobule & $1.44(0.35)$ & $38.9(4.4)$ \\
\hline Estrus & Duct & $1.66(0.37)$ & $43.2(4.3)$ \\
Estrus & Lobule & $1.77(0.47)$ & $45.2(6.3)$ \\
\hline Metestrus I & Duct & $1.56(0.35)$ & $40.4(5.9)$ \\
Metestrus I & Lobule & $1.55(0.27)$ & $40.3(5.3)$ \\
\hline Metestrus II & Duct & $1.28(0.23)$ & $36.8(4.7)$ \\
Metestrus II & Lobule & $1.30(0.20)$ & $37.3(3.8)$ \\
\hline Diestrus & Duct & $1.03(0.32)$ & $31.6(6.2)$ \\
Diestrus & Lobule & $1.19(0.36)$ & $34.2(6.2)$ \\
\hline
\end{tabular}

$\mathrm{N}=3$ mammary glands per stage 


\section{Discussion:}

In my analysis of mammary gland Mier3 expression during windows of susceptibility, I quantified expression in mammary epithelial cells (MECs) based on average transcripts per cell, as well as overall percent of MECs positive for Mier3 expression. At each stage assayed, a change in average probe number per cell positively correlated with a change in percent positivity. This indicates that Mier3 expression is modulated not within a set subpopulation of MECs, but within the MEC population as a whole. As a result, Mier3 may serve a universal function in MECs at each developmental stage.

I found a positive correlation between Mier3 mammary gland expression and known ovarian hormone signaling levels based on stages of the rat estrus cycle. Mier3 expression was highest during proestrus, estrus, and metestrus I, which encompass a window of peak estrogen surging and ovulation, followed by a rise in progesterone levels, and a peak in progesterone during metestrus I [199]. Mier3 levels began to decline at metestrus II, corresponding to when ovarian hormone signaling significantly drops off $[199,202]$. I found the lowest levels of Mier3 during diestrus, which is characterized as a non-hormonal phase of the estrus cycle [200]. These findings are significant because even though these are inactive mammary glands, Mier3 expression still seems to be modulated in relation to hormonal cycling. It is established that the mammary epithelium undergoes cyclical proliferation and regression due to endocrine control over MECs, namely estrogen and progesterone $[199,505,506]$. However, there are differing publications of MEC changes based on the rat estrus cycle stage, with proestrus, metestrus, and diestrus all reportedly possessing peak MEC proliferation levels [196, 199, 203]. There remains a lot 
to be uncovered regarding how the estrus cycle effects the mammary epithelium, but the high expression of Mier3 in MECs and cyclical changes in Mier3 expression suggests Mier3 may have a role in this process. The correlation of highest Mier3 expression during the hormonally-dominated stages of the estrus cycle also suggests Mier3 may be hormonally regulated.

I found the highest Mier3 expression levels ranging from embryonic development through pubertal development, with a drastic decrease in expression in the adult mammary gland. This loss in expression in the adult gland indicates a diminished role of Mier3 once the mammary gland has achieved pubertal development and entered into an inactive stage. High Mier3 expression during embryonic and pubertal development corresponds with significant periods of allometric growth in the mammary gland, where in rats, the gland grows at 3.5-5 times the rate of the rest of the body [531]. There was a small dip in expression during neonatal development which corresponds to a period of isometric growth, where the rate of mammary gland growth is kept in rate with regular body growth [532]. Allometric mammary gland growth during this developmental period is largely in response to estrogen signaling, which is responsible for the expansive ductal growth that occurs, especially during puberty $[531,533]$. In fact, deletion of ER $\alpha$ or its pharmacological inhibition hinders ductal growth, which is restored with exogenous estrogen treatment $[507,531,534,535]$. There are other endocrine cues driving morphological development, including progesterone and prolactin, whose mammogenic effects include induction of ductal side branching and alveolar bud formation [536]. Additionally, growth hormone and insulin-like growth factor-I influence ductal elongation [535]. Thus, different levels of mammogenic hormones establish numerous conditions for 
stages of morphogenesis of the mammary gland $[535,536]$, but the exact roles and levels of hormones and growth factors in rat mammary gland development is unclear. Estrogen is known to bind to its receptor and stimulate production of other growth factors [536], which may include Mier3 given its association with cancerous and developmental growth. However, ER does not appear in MECs in either rodents or humans during fetal development [536-538]. I determined from this study of the estrus cycle that Mier3 appears to act in concert with ovarian hormone fluctuations and is possibly hormonally regulated. Yet, given its highest involvement during fetal development, which lacks ER, Mier3 may be stimulated by alternative hormonal pathways, in addition to or independent of estrogen. Increased expression of Mier 3 was found during the pubertal phase of mammary gland development, including early initiation of puberty at 4 weeks of age. At each pubescent time point analyzed, terminal end bud (TEB) morphology had greater Mier3 levels as compared to ductal structures. This finding correlates with my discovery of increasing Mier 3 expression along a ductal mammary cancer progression model, as these proliferative structures contain vulnerable precursor cells that are commonly held to be the initiation sites of ductal carcinogenesis [102, 209, 464]. TEBs are clusters of undifferentiated epithelial cells at the distal ends of primary ducts, which continuously expand with each round of estrus cycling and drive proliferation of the mammary epithelium $[104,114,167]$. The finding that the most proliferative mammary gland structure possesses the highest Mier3 expression levels adds further support that Mier3 has an important functional role in MEC growth.

I report significantly higher Mier3 expression in MECs during late gestation and lactation stages as compared to nulliparous glands. However, the overall average Mier3 
transcripts per MEC at these time points do not match the high expression levels found during embryonic, neonatal, and pubertal development. This is likely due to the drastic architectural and hormonal differences that exist in the mammary gland between pubertal and reproductive development. During pregnancy, there is a great increase in alveolar bud formation and differentiation into alveoli, which cluster and form functional secretory lobules. This lobuloalveolar growth continues post-parturition [94, 133]. Lobuloalveolar formation relies on differing signaling mechanisms than ductal morphogenesis. For example, progesterone and prolactin are largely responsible for driving alveologenesis and differentiation [94]. As a result, the differences in Mier3 expression found between prepubescent/pubescent growth and reproductive growth may be a result of the different hormonal milieu, which creates a reduced role for Mier3 or differentially modulates Mier3 expression. There is also a known decrease in proliferative activity corresponding with increasing differentiation of mammary structures, so reduced Mier3 expression may be attributable to the state of differentiation of MECs. I believe the increased levels of Mier3 I found during pregnancy and lactation is correlated with its presumed role in MEC growth; however, the substantially lower levels as compared to early development is likely due to the differences in types of MEC growth and differentiation that occur during reproductive development.

In addition to analyzing overall Mier3 expression during pregnancy and lactation, I quantified expression based on lobular or ductal morphology, and found greater expression in MECs of ducts as compared to lobules. This may be due to the differing functions of luminal epithelial cells based on morphology; serving a secretory role in lobules as opposed to solely a conduit for the movement of milk in ducts. As a result, these 
cells may respond to growth stimuli or signaling molecules differently. Mier3 is expressed at lower levels in lobules, and late gestation and lactation are mostly lobular growth, which could indicate why this growth period doesn't recapitulate the high levels recorded in early developmental growth. Conversely, during involution we found greater lobular expression of Mier3 as compared to ductal. I took mammary glands at the start of the irreversible phase of involution, which is marked by massive rates of apoptosis and removal of the secretory epithelium [94]. Overall Mier3 expression during the period of involution was significantly lower than pregnancy and lactation, and no different than the quiescent nulliparous and parous stages. Thus, the greater lobular expression may not necessarily indicate a role of Mier3, but instead point to a lack of a role of Mier3 in the inactive ductal structures at this stage.

I found no significant differences in Mier3 expression between nulliparous mammary glands at 12 weeks of age and parous glands at 24 weeks. In the parous mammary gland there still remains some alveoli, but overall it is morphologically similar to the virgin gland [94]. The major difference between these stages of mammary gland development is at the molecular level, as parity results in the achievement of complete differentiation and a distinct transcriptional profile from the undifferentiated, nulliparous counterpart $[539,540]$. Pregnancy also drives terminal differentiation of the proliferative MECs most vulnerable to carcinogenesis, resulting in a protective effect from breast cancer risk [541]. My results indicate that Mier3 expression, although specific to MECs, is not contingent upon or driven by the state of differentiation of these cells. Additionally, since Mier3 expression is unchanged going to a differentiated stage, it is not part of the transcriptional reprogramming of MECs and likely doesn't have a mechanistic role in 
altered disease susceptibility post-parturition. The nulliparous and parous stages displayed the lowest Mier3 levels in our study of windows of susceptibility, so the lack of difference in expression may also be attributable to the quiescent state of the mammary glands, not the degree of differentiation.

A null result in this study was no genotype differences for the most part between my mammary cancer susceptible WF strain and my WF.COP Mcs $1 b$ resistant N3 strain. It is possible that this is due to the existence of three Mier3 alternative splice variants [256, 493]. Populations of MECs from the opposing genotypes may exhibit variable expression and functionality of the differing splice variants but have no differences in total Mier3 expression overall. The Mier3 RNAscope probe mix possesses 20 probe pairs that are designed to bind within nucleotides $2357-3614$ of the 4758 base pair transcript, so no variation in alternative splice forms would be captured in this assay. There was statistical indication of genotypic differences at 9 days of age and during lactation; however, the lack of a difference in genotype at any other stage puts this finding under scrutiny. I only used 6 animals per genotype at each stage of development so we could be statistically underpowered in our analysis. The purpose of this study was to determine what stage of development Mier3 potentially has a role in, with the intent of further evaluation at interesting stages. I found that early developmental stages encompassing embryonic, neonatal and pubertal development displayed the highest Mier3 expression, making these stages the best candidates for re-evaluation and further analysis into the role of Mier3.

In summary, I found high Mier3 expression levels at stages of mammary gland development that are characterized by extreme morphological changes that make the gland lactation-competent. Morphological re-structuring of the mammary gland is driven by 
transcriptional reprogramming, as well as hormonal signaling and growth factor stimulation. The high expression levels I report at these stages complement the putative transcriptional repressive function of MIER3 [494], that could have a mechanistic role in promoting tissue evolution. Based on my results, I propose a functional role of Mier3 in the growth or differentiation of MECs during embryonic through pubertal mammary gland development. I also suggest that Mier3 is hormonally regulated. Given its identified role throughout mammary cancer progression, it is especially relevant that Mier3 is highly expressed in the exact cell type and stages most vulnerable to cancer initiating events, in what is most often a hormonally-driven disease. Thus, I conclude that in addition to a developmental growth function, MIER3 could serve as a potential driver of breast carcinogenesis at these developmental time windows of susceptibility. 


\section{CHAPTER VII}

\section{MIER3 PROTEIN INTERACTIONS SUGGEST A FUNCTIONAL ROLE IN A TRANSCRIPTIONAL CO-REPRESSOR COMPLEX}

\section{Introduction:}

The MIER family members share structural features in common with known transcriptional co-repressors, including the ELM2 and SANT domains [261, 262]. The ELM2 (Egl-27 and MTA1 homology 2) domain was initially identified in the MTA1 protein that is a component of the NuRD chromatin regulatory complex [542] and has since been shown to recruit trichostatin A-sensitive (class I) histone deacetylase (HDAC) proteins that lead to changes in chromatin structure and transcriptional repression [543, 544]. It has been demonstrated that the ELM2 domain of MIER $1 \alpha$ and $\beta$ is responsible for recruiting and binding HDAC 1 and 2, providing one mechanism of transcriptional repression [543]. The ELM2 domain of MIER1 is also necessary for binding the histone acetyltransferase (HAT) Creb-binding protein (CBP), which provides another mechanism of transcriptional repression as this interaction inhibits the histone acetyltransferase activity of CBP [270]. The vast majority of ELM2 containing proteins also possess a SANT domain immediately downstream, indicating a functional or structural relationship between these motifs. SANT domains are highly conserved sequences of around 50 amino acids that were first identified in the transcriptional factors SWI3, ADA2, N-CoR, and TFIIIB, generating 
the acronym "SANT" [545]. They have since been found in the subunits of many chromatin-remodeling complexes [546]. The SANT domain is characterized by tandem repeats of three $\alpha$-helices that are arranged in a helix-turn-helix motif, similar to the DNAbinding domain of Myb proteins; however, the SANT domain does not bind DNA or contribute to transcriptional repression in the same manner [547, 548]. The SANT domain is thought to instead serve a chromatin remodeling function by facilitating interactions with histones [545]. In MIER1, the SANT domain has been demonstrated to bind transcription factor specificity protein $1(\mathrm{Sp} 1)$ at the MIER1 promoter, displacing it from its cognate sites, and repressing its own transcription as well as other Sp-1 regulated genes [263]. This represents an HDAC-independent manner of transcriptional repression by MIER1. The protein binding domains of the other MIER family members may also facilitate interactions with chromatin modifying proteins, similar to established co-repressors.

MIER2 and MIER3 are hypothesized to possess transcriptional repressive roles similar to MIER1 due to sequence homology and shared ELM2/SANT domains, but investigations into their individual mechanisms are still in their infancy. It has been shown that all MIER family members associate in HDAC complexes, but never with each other or as dimers [264]. They also do not appear in complexes with other corepressor complexes such as CoREST (co-repressor of RE1-silencing transcription factor), NuRD (nucleosome remodeling deacetylase), Sin3, or NCoR (nuclear receptor corepressor), suggesting each MIER forms independent HDAC-containing complexes that are functionally distinct from each other [264]. HDAC recruitment also appears to be cell-line dependent. For example, in HEK293 cells, MIER1 and 2 associates with HDAC1 and 2, but not MIER3. In MCF-7 and HeLa cells, MIER1 associates with both HDACs, MIER2 only with HDAC1, and 
MIER3 with neither [264]. In CEM-T cells all MIER family members were found in HDAC1 and 2-containing complexes [267]. Finally, in K562 cells MIER2 and 3 were found to only associate with HDAC2 [549]. This variability in MIER-HDAC complexes highlights not only the differences between family members, and likely underlying functionality, but also the cell-line dependent manner of the composition of the complexes, indicating environment and tissue type create alternative roles for the MIER proteins. Despite shared similarities with respect to their domains and regions of high homology, these proteins are also divergent, especially at their C-termini, suggesting distinct molecular mechanisms among them in transcriptional regulation. MIER1 and 2 also share more similarities between them than with MIER3, indicating MIER3 especially has diverse functional roles. For example, the presence of MIER1 and 2 have been reported in chromodomain Y like (CDYL) chromatin repressive complexes, and HDAC1/2 association with this complex is improved by MIER1/2 interaction [550-552]. However, MIER3 is not found within this complex. MIER1/2, but not MIER3, are also enriched at RE1-silencing transcription factor (REST) target genes [552]. Even the shared domains of the MIER family may be functionally distinct. MIER1/2 are predicted to contain coiled-coiled motifs in their C-terminal regions of the ELM2 domain while MIER3 possess an LXXLL motif at this region instead [552]. MIER3 also contains an additional LXXLL motif near its Nterminus, while MIER $1 \alpha$ only contains one at the C-terminus, and MIER2 does not possess any. Thus, the MIER family members appear to participate in unique complexes from each other, which may serve overlapping functions as transcriptional repressors, but there are likely distinct effects on downstream gene regulation. 
The established and predicted transcriptional repressive roles of the MIER family as members of co-repressor complexes also indicates dysregulation of these genes could have an important impact on cancer biology and disease development. Epigenetic regulation of gene expression allows for cell-specific transcriptional programs that impart differing cell functions despite having the same genetic information [553]. However, perturbations to these processes can lead to aberrant gene expression which contribute to disease initiation and tumor progression $[554,555]$. There exist multiple layers of transcriptional regulation, which include direct methylation of DNA, covalent modifications to histone proteins which contribute to chromatin structure, and nucleosome remodeling which represents higher order chromatin organization [556]. Co-repressor complexes are often recruited and bound to DNA by transcription factors, where the enzymatic activities of chromatin-modifying protein subunits work in a combinatorial fashion to recognize, remove, and place epigenetic marks that result in chromatin condensation [557, 558]. Differences in subunit composition create unique specificities for certain genomic loci [559]. Altered levels of chromatin co-repressor proteins disrupts the balance of transcriptional activation and repression that results in oncogenesis $[560,561]$. In breast cancer specifically, there is global hypomethylation accompanied by formation of repressive chromatin domains and gene silencing early in disease development [562]. MIER1 has an established role in breast cancer as member of a co-repressor complex, which may contribute to these disease-specific repressive chromatin domains. Although it is unknown whether MIER3 exists in a co-repressor complex, it has an association with human breast cancer as a candidate rat mammary cancer susceptibility gene [256]. In this study, I sought to identify the protein-protein interactors of MIER3 within a hormone 
receptor positive breast cancer cell line in order to determine if MIER3 exists in a corepressor complex and what the subunit composition is in a breast disease state. I also approached this study with a specific hypothesis that MIER3 interacts with a nuclear hormone receptor via its LXXLL sequence motifs that directs certain chromatin interactions, since the possession of two motifs as compared the single motif in MIER1 $\alpha$ could elucidate an impotent functional difference between these MIER proteins.

\section{Methods:}

Plasmid Construction and Site Directed Mutagenesis

Homo sapiens MIER3 ORF (NCBI/GenBank ref NM_152622.3) from pooled human breast total RNA (\#AM6952, Life Technologies) was cloned into pEGFP-C1 vector at EcoRI and KpnI sites. Primer sequences for cDNA amplification were 5'CGGAATTCTATGGCGGAGGCTTCTTTTGGAAGT-3' ${ }^{\prime}$ and CGGGGTACCCTCAGAGTGTAGGGCCGCGTGC-3'. MIER3 LXXLL sequence motifs were mutated to LXXAA using a QuikChange Multi Site-Directed Mutagenesis kit (Agilent 200515). Primer sequences for LEDLL mutagenesis was 5'GGAAGGAACCATGCCTCTAGAAGATGCAGCGGCATTCTATGGCTATGAAC

and LYELL mutagenesis was AAGGGACAATGAACAGGCATTATATGAAGCTGCCAAGTGTAACCACAATATA AAGGAAGC-3'. Mutations were verified using primer sequences for cDNA amplification, and Sanger sequenced with an ABI PRISM 7700 Sequence Detection System using the LEDLL sequencing primer 5'-CCCACTGCTGAGATGTTGGT-3' and LYELL sequencing primer 5'-ACTTTGGTGTCCTGATGTGGT-3'. 


\section{Cell culture and Transfection (standard conditions)}

T47D (\#HTB-133) cell line was purchased from American Type Culture Collection (ATCC) and cultured in RPMI 1640 with 10\% FBS, 1\% antibiotic-antimycotic, and 0.2 $\mathrm{U} / \mathrm{mL}$ bovine insulin. Cell authentication was guaranteed by ATCC and morphology was confirmed under a phase-contrast light microscope. T47D cells were plated at $5 \times 10^{6}$ cells per 10cm dish. Using Lipfectamine 3000 transfection reagent (ThermoFisher L3000015) and following the manufacturers protocol, 20 plates were transfected with $28 \mu \mathrm{g}$ plasmid in opti-MEM with $1 \%$ antibiotic-antimycotic for each plasmid- pEGFP-C1, pEGFP-C1MIER3, and pEGFP-C1-MIER3-LXXAA.

\section{Cell culture and Transfection (serum starvation and stimulation)}

Following adherence, cells were serum starved in RPMI 1640 without L-glutamine and phenol red with $1 \%$ charcoal/dextran treated FBS and $1 \%$ antibiotic-antimycotic. Using Lipfectamine 3000 transfection reagent (ThermoFisher L3000015) and following the manufacturers protocol, 20 plates were transfected with $28 \mu \mathrm{g}$ plasmid in opti-MEM with $1 \%$ anti-anti for each plasmid- pEGFP-C1, pEGFP-C1-MIER3, and pEGFP-C1MIER3-LXXAA. Cells were re-stimulated 6 hours post transfection with the addition of RPMI 1640 with $40 \%$ FBS and $1 \%$ anti-anti to the transfection mixture, for a total of $20 \%$ serum.

\section{Evaluation of transfection efficiency}

At 24 hours post transfection, efficiency was estimated in 3 plates each of cells transfected with pEGP-C1 or pEGFP-C1-MIER3. This was done using T47D and HEK293 
cell lines. Cells positive for GFP fluorescence were identified using an EVOS digital inverted fluorescent microscope. Representative images were taken at 10x.

Transfection of pEGP-C1 and pEGFP-C1-MIER3 was repeated in 6 plates of T47D cells each. At 8 hours post transfection, 3 plates per plasmid were treated with $10 \mu \mathrm{M}$ MG132 [563]. At 24 hours post transfection, the number of GFP-positive cells were counted in minimally seven separate 10x fields of view per plasmid using an EVOS digital inverted fluorescent microscope. Representative images were taken at 10x. This experiment was repeated and positive cells counted every 2 hours up to 24 hours post transfection, with a final assessment at 48 hours.

\section{Cell Lysis and Affinity Purification of GFP-interacting proteins}

At 24 hours following transfection, cells were harvested on ice in lysis buffer with $2.5 \mathrm{mg} / \mathrm{mL}$ DSP (40 mM HEPES pH 7.4, 120 mM NaCl, 1 mM EDTA, 1\% Triton X-100, 1x protease/phosphatase cocktail inhibitors, and dithiobis succinimidyl propionate (DSP, Pierce) $200 \mathrm{mg} / \mathrm{ml}$ in DMSO). Total cell lysate was collected into a single tube per transfected plasmid- pEGFP-C1, pEGFP-C1-MIER3, and pEGFP-C1-MIER3-LXXAA. Cell lysates were incubated on ice for 30 minutes, cross linking quenched with 1M Tris$\mathrm{HCl} \mathrm{pH} 7.4$ in a ration of $1: 4$, and incubated for a further 30 minutes on ice. Lysates were clarified by centrifugation at $4500 \mathrm{xg}$ for $40 \mathrm{~min}$ at $4{ }^{\circ} \mathrm{C}$ and filtered through SpinX columns (Corning). Lysates were pre-cleared by incubating with $50 \mu \mathrm{L}$ packed sepharose beads (Sigma Aldrich) for 1 hour at $4^{\circ} \mathrm{C}$ on a rotating wheel. GFP-TRAP beads (ChromoTek) were equilibrated in lysis buffer and $30 \mu \mathrm{L}$ of pelleted beads added to each lysate. Lysates were spun on a rotating wheel at $4^{\circ} \mathrm{C}$ for 4 hours. Beads were washed $4 \mathrm{x}$ for 1 hour each 
at $4^{\circ} \mathrm{C}$ in lysis buffer supplemented with $0.5 \mathrm{M} \mathrm{NaCl}$, and washed $1 \mathrm{x}$ in $50 \mathrm{mM}$ Tris- $\mathrm{HCl}$, pH 7.5, 0.1mM EGTA. Beads were reconstituted in $80 \mu \mathrm{L} 1 \mathrm{x}$ LDS sample buffer with 0.1M DTT, incubated 1 hour at $37^{\circ} \mathrm{C}$, and boiled for 5 minutes at $95^{\circ} \mathrm{C}$. Denatured proteins were eluted through SpinX columns.

\section{In-Gel Protein Digestion and Extraction:}

Protein elution was run on an $4-20 \%$ gradient polyacrylamide gel (BioRad 4561094) at 100V for 1 hour then stained with Coomassie blue to visualize bands. Bands were cut into roughly $1 \mathrm{~mm}^{3}$ plugs, place in $1.5 \mathrm{~mL}$ tubes, $10 \mu \mathrm{L}$ of $100 \mathrm{mM}$ triethylammonium bicarbonate (TEA-BC) added, and tubes incubate at room temperature for 15 minutes. Tubes were incubated for another 15 minutes following addition of $15 \mu \mathrm{L}$ acetonitrile (ACN). Solvent was removed by SpeedVac for about five minutes. To the dry tubes, $20 \mu \mathrm{L}$ of $20 \mathrm{mM}$ DTT/100mM TEA-BC was added and tubes incubated at $56^{\circ} \mathrm{C}$ for 45 minutes. DTT solution was removed, $20 \mu \mathrm{L}$ of $55 \mathrm{mM}$ iodoacetamide/100mM TEA$\mathrm{BC}$ was added, and tubes incubated at room temperature for 30 minutes in the dark. Iodoacetamide solution was removed, $200 \mu \mathrm{L}$ of $50 \mathrm{mM}$ TEA-BC was added, and tubes incubated at room temperature for 15 minutes. Tubes were incubated for another 15 minutes following addition of $200 \mu \mathrm{L} \mathrm{ACN}$. Solvent was removed and $3 \mu \mathrm{L}$ of digestion buffer ( $20 \mathrm{ng} / \mu \mathrm{L}$ modified trypsin in $50 \mathrm{mM}$ TEA-BC) was added to gel pieces. After about 10 minutes gel pieces swelled and $50 \mathrm{mM}$ TEA-BC was added to a final volume of $25 \mu \mathrm{L}$. Tubes were incubated at $37^{\circ} \mathrm{C}$ overnight in a shaker.

To extract peptides, $25 \mu \mathrm{L}$ of LC-MS grade water was added to the tubes, followed by $100 \mu \mathrm{L}$ of $1: 25 \% \mathrm{v} / \mathrm{v}$ formic acid in water:acetonitrile, and tubes incubated at room 
temperature for 15 minutes in a shaker. Liquid surrounding the gel pieces was transferred to a clean tube and solvent removed in a SpeedVac. Protein was re-dissolved in $20 \mu \mathrm{L} 2 \%$ $\mathrm{v} / \mathrm{v}$ acetonitrile / $0.1 \% \mathrm{v} / \mathrm{v}$ formic acid. Sample was filtered through a $0.45 \mu \mathrm{m}$ regenerated cellulose syringe filter (Thermo \#F2504-7) to remove any gel material, and 2-20 $\mu \mathrm{L}$ injected for LC-MS analysis

1-Dimensional Reverse Phase Liquid Chromatography (LC)-MS/MS:

Approximately $400 \mathrm{ng}$ of fractionated peptides were analyzed by 1-dimensional nano-LC on a Thermo Ultimate 3000 HPLC system directly eluting into a Thermo QExactive-HF mass spectrometer. Peptides were separated on a $15 \mathrm{~cm}$ in-house packed tip reverse phase 3.6 $\mu \mathrm{m}$ XB-C-18 resin (Phenomenex, Aeris C18) column and direct eluted at $200 \mathrm{nl} / \mathrm{min}$ into the MS. Briefly, a reverse phase gradient from 5 minutes to 90 minutes was applied with $0 \%$ solvent B ( $80 \%$ acetonitrile, $0.1 \%$ formic acid) to $45 \%$ solvent B for each of the 18 fractions in sequence. Peptides were eluted directly into a Thermo QExactive-HF at a spray voltage of $1.65 \mathrm{kV}, 250^{\circ} \mathrm{C}$ and spectra were acquired in data dependent top 15 ion mode.

\section{Computational analysis:}

Spectra were deconvoluted by Proteome Discoverer v2.2 and the built-in SEQUEST analysis program used to map peptides to the in silico Uniprot human reviewed database. Peptide false discovery rate was held at $<1 \%$. LC-MS/MS analysis performed following band excision gave a more comprehensive list of peptides than analysis of direct protein elution, so identifications of less than 1 unique peptide were not considered in 
downstream analysis and were removed from the dataset of these experiments. SEQUEST compares projected theoretical spectra to the observed tandem mass spectrum using cross correlation analysis [564]. Resulting SEQUEST HT scores were used to evaluate the strongest candidate peptides. Proteins in the MIER3-GFP interaction list were compared to the GFP trap interaction list and removed if deemed non-specific. Protein lists were submitted to the CRAPome (a Contaminant Repository for Affinity Purification Mass Spectrometry Data) (www.crapome.org), which was used to further identify background contaminants and score interacting proteins ranked by confidence [565]. Protein IDs were filtered out for highly non-specific interactions as predicted from literature reports aggregated in the CRAPome database. CRAPome performs a fold change (FC) calculation on the ratio of average normalized spectral counts in bait purifications versus negative controls. The more stringent secondary FC-B score was accepted as the final stringency criteria in parsing out the strongest protein interactors.

A final list of the strongest protein interactors in which to focus further analysis on was created by accepting the top $25 \%$ of protein IDs across the three stringency criteria of SEQUEST HT score, number of peptide fragments detected, and CRAPome FC-B score. Proteins that did not appear in at least 2 of these 3 lists were excluded.

This list was subjected to gene ontology analysis through the Gene Ontology Consortium (www.geneontology.org) to analyze interacting proteins based on shared biological processes and molecular function [566]. A Fishers exact test with stringent Bonferroni correction was used and analysis type was a PANTHER Overrepresentation Test (GO Ontology database DOI: 10.5281/zenodo.4081749 Released 2020-10-09). The 
output with each topic is the number of proteins from the input list, the expected number of proteins, the resulting fold enrichment, and the associated Bonferroni P-value.

Toppgene, a portal for gene list enrichment analysis and candidate gene prioritization, was used to further functionally analyze the list of strongest nuclear protein interactors (www.toppgene.cchmc.org) [567]. Specifically in this portal, ToppFun was used for functional enrichment of the input protein list based on gene expression, protein domains and interactions, and ontologies. Co-expression analysis within ToppFun found breast cancer microarrays as the top hits $[444,568,569]$ which output gene lists and Bonferroni P-values.

\section{Results:}

\section{MIER3 overexpression is not permissible and the protein product is likely rapidly}

\section{degraded}

The overall goal of this analysis was to identify MIER3 protein-protein interactions, with the specific intention of isolating a nuclear hormone receptor, to determine if MIER3 is participating in a chromatin repressive complex similar to MIER1. To first test the transfection efficiency of the MIER3 plasmid, T47D breast cancer cells were transfected with either pEGFP-C1 empty vector or pEGFP-MIER3-C1and resulting GFP fluorescence analyzed 24 hours later. Cells transfected with empty vector displayed high levels of fluorescence while pEGFP-MIER3-C1 transfected cells showed very low levels of fluorescence at only 0-2 cells fluorescing in 10x fields of view (Figure 55). To determine if this low transfection efficiency resulted from the MIER3 insert, this same transfection procedure was repeated in HEK293 cells. This cell line has an established high transfection 
efficiency and dependable protein translation and processing [570, 571]. HEK293 cells displayed even greater GFP fluorescence intensity with the empty vector control than T47D cells, which was expected from this highly transfectable cell line. There again were only a few fluorescent cells observed in each 10x field of view with cells transfected with pEGFPMIER3-C1 (Figure 55). This same disparity in fluorescence between plasmids indicates that transfection is successful in both cell lines, but MIER3 overexpression may be signaling rapid degradation of its protein product.

\section{Proteasome inhibitor treatment increases MIER3 expression but does not recapitulate levels of the empty vector control}

To superficially test if the hypothesis of rapid MIER3 protein degradation might hold some validity, T47D cells were treated with the proteasome inhibitor MG132 at 8 hours following transfection to halt possible MIER3 protein degradation. A dose of $10 \mu \mathrm{M}$ MG132 was selected as this has been shown to effectively inhibit protein degradation in vitro in carcinoma cell lines, including breast carcinoma lines MCF-7 and T47D [572-574]. Fluorescence was then analyzed at 24 hours post-transfection. As before, T47D cells transfected with eGFP-C1 displayed significantly greater numbers of fluorescent cells as compared to pEGFP-MIER3-C1, but there was no effect of MG132 treatment. Cells transfected with pEGFP-MIER3-C1 displayed significantly greater numbers of fluorescent cells when treated with MG132 as compared to untreated (Figure 56). This experiment was repeated, and fluorescence monitoring extended to every 2 hours following MG132 treatment. Results were consistent between experiments. Cells transfected with pEGFP-C1 displayed no difference in fluorescence when treated with MG132. Cells transfected with 
(A) eGFP-C1 in T47D

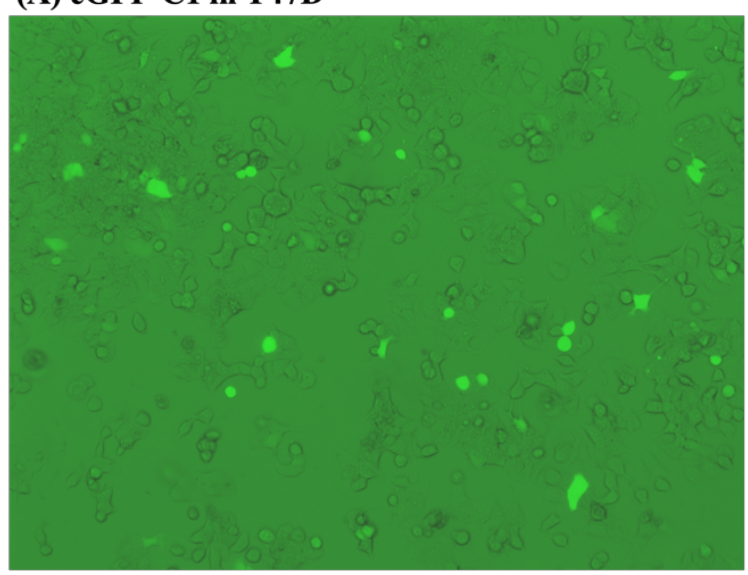

(C) eGFP-C1 in HEK293

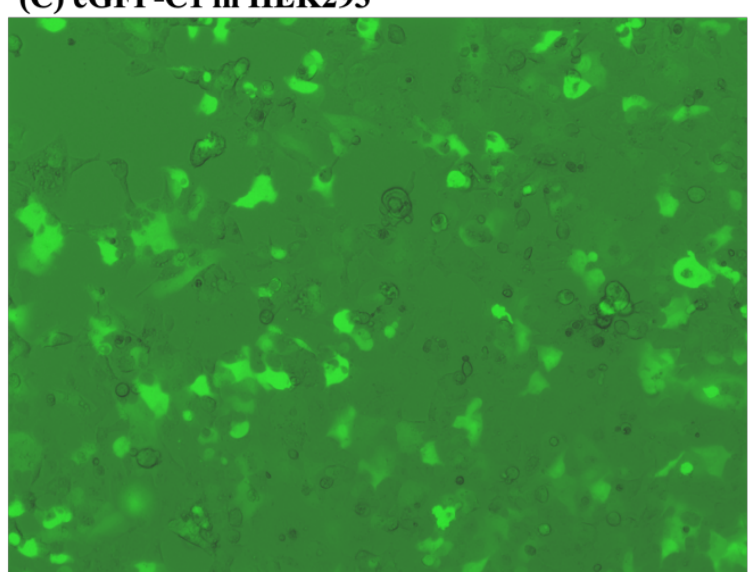

(B) eGFP-MIER3-C1 in T47D

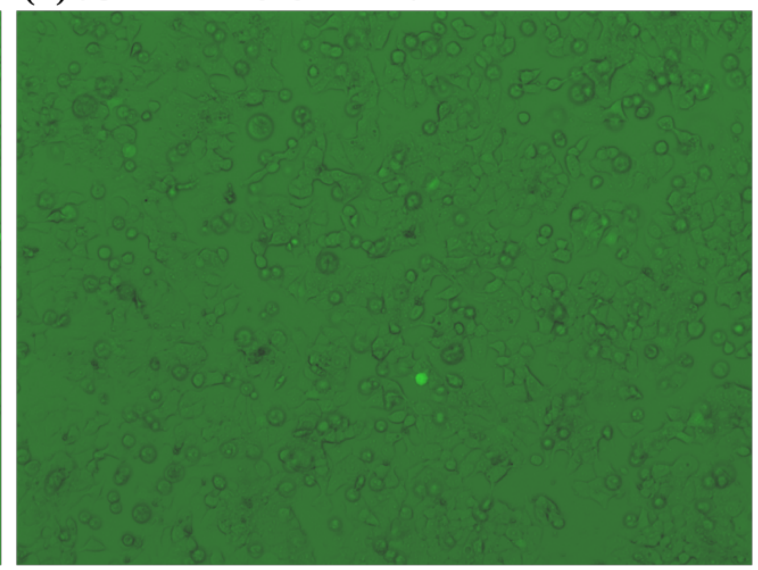

(D) eGFP-MIER3-C1 in HEK293

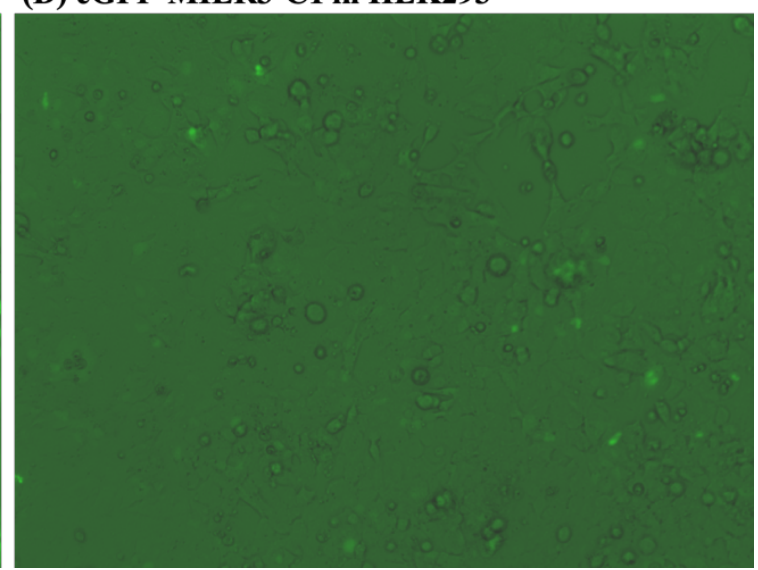

Figure 55: Cells transfected with pEGFP-MIER3-C1 display low levels of

fluorescence. Shown are representative images of cells at 10x magnification on an EVOS digital inverted fluorescent microscope at 24 hours post-transfection. A, C: T47D cells (top row) and HEK293 cells (bottom row) transfected with pEGFP-C1 empty vector display high levels of florescence. B, D: Florescence levels drop substantially in both cell lines transfected with pEGFP-MIER3-C1. 
A

T47D cells transfected with pEGFP-C1-MIER3

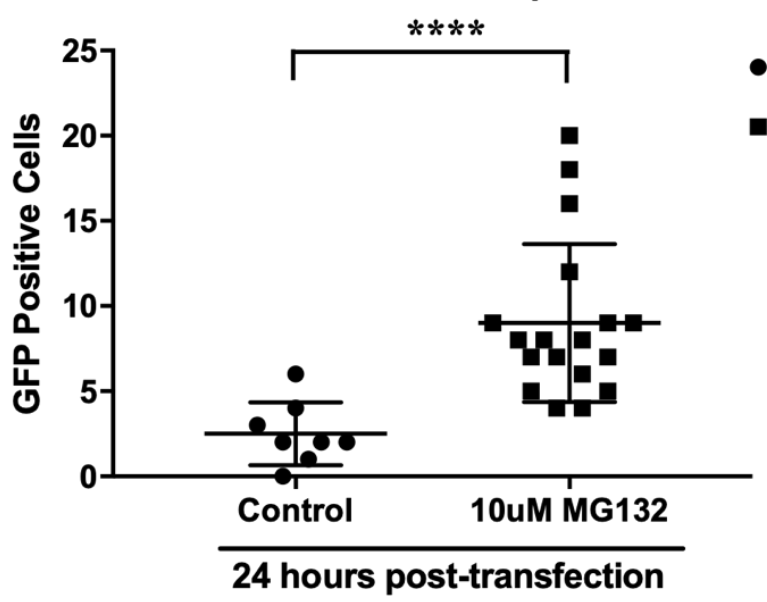

- Control

- 10uM MG132

B

T47D cells transfected with pEGFP-C1

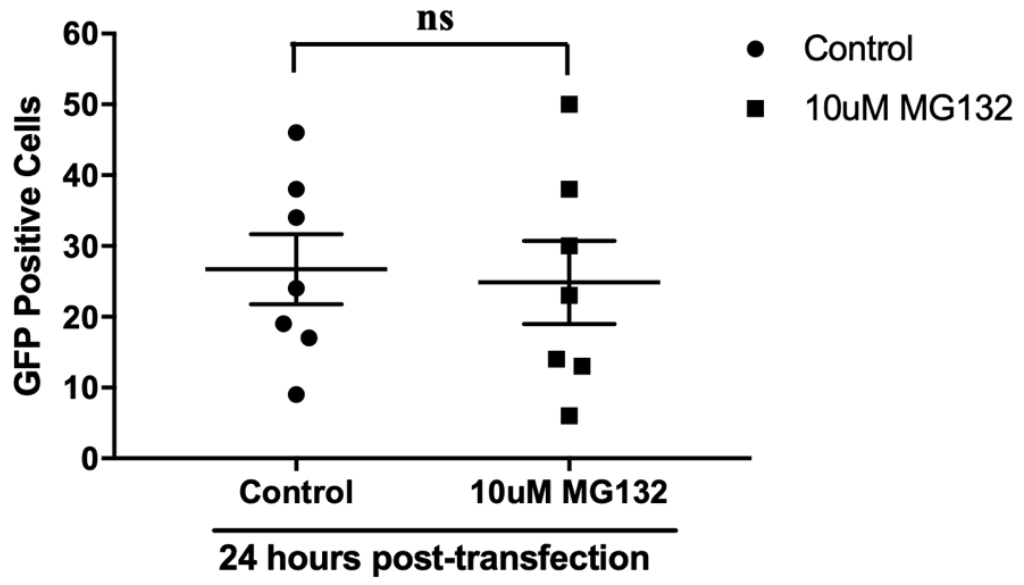


C T47D cells transfected with pEGFP-C1-MIER3 (24 hours):

Control

10uM MG132

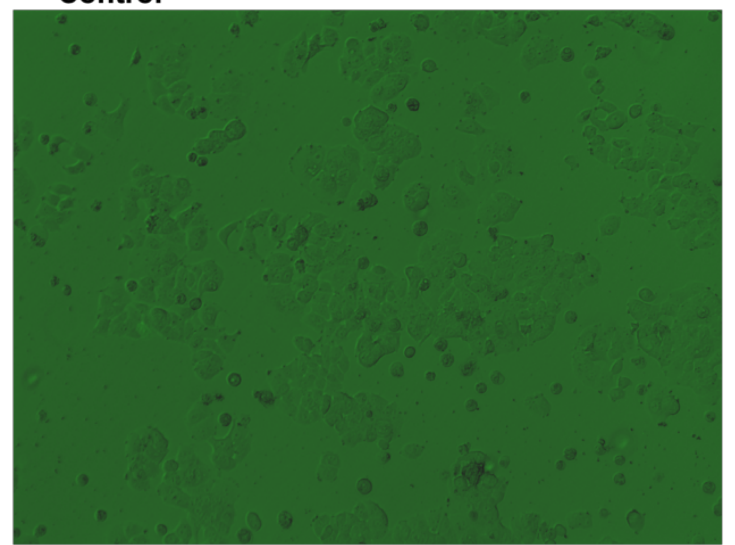

D T47D cells transfected with pEGFP-C1 (24 hours): Control
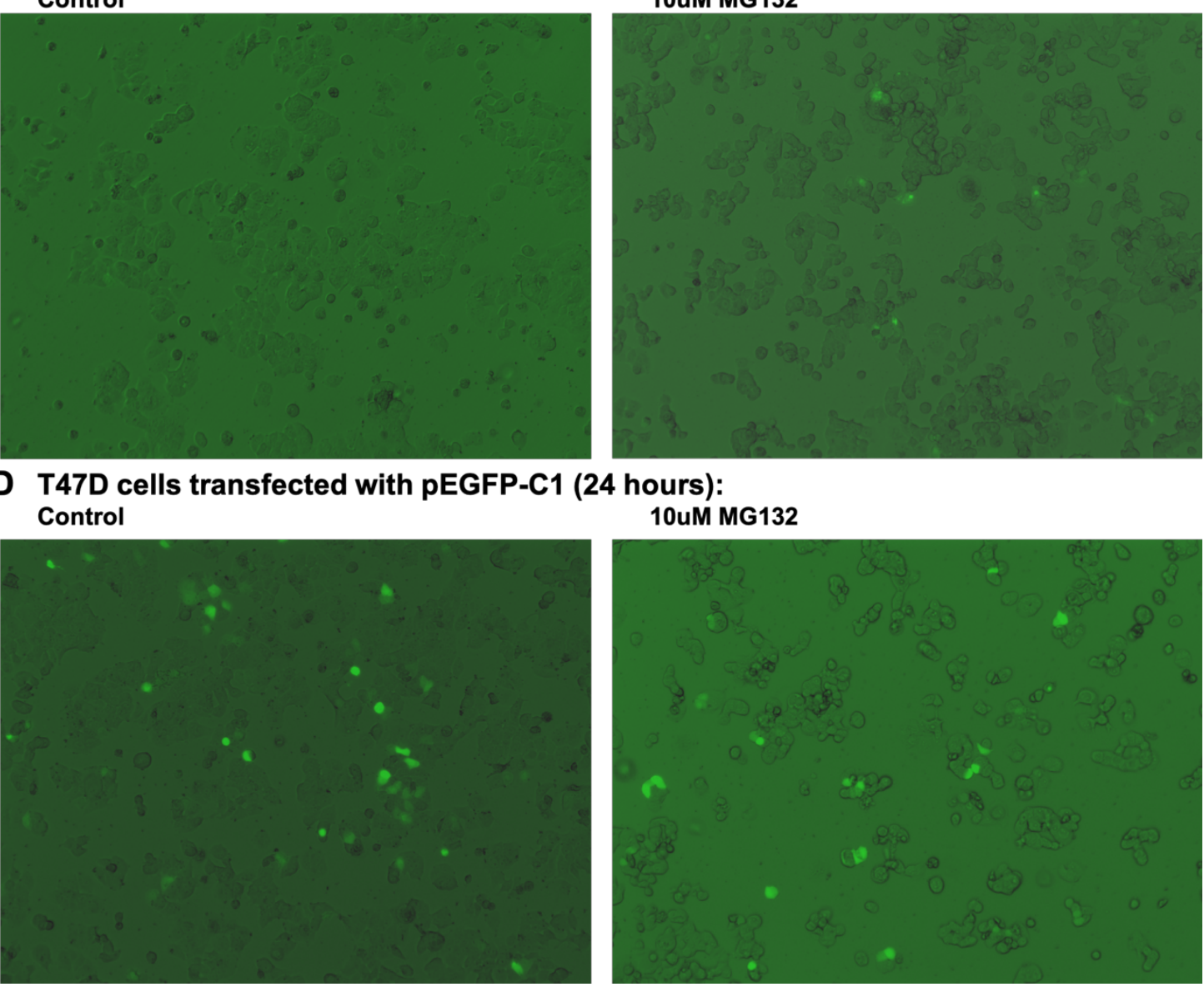

Figure 56: pEGFP-MIER3-C1 transfected cells display increased fluorescence with proteasome inhibitor treatment. Shown are representative images of T47D cells at 10x magnification on an EVOS digital inverted fluorescent microscope at 24 hours posttransfection alongside the quantification of GFP positive cells from differing fields of view. A, C: Cells transfected with pEGFP-MIER3-C1 display greater numbers of GFP positive cells with $10 \mu \mathrm{M}$ MG132 treatment as compared to control. B, D: 16 hours of MG132 treatment does not have an effect in pEGFP-C1 transfected cells. Data were analyzed by a 2-tailed Mann-Whitney test, $* * * * \mathrm{p}<0.0001$ 
A

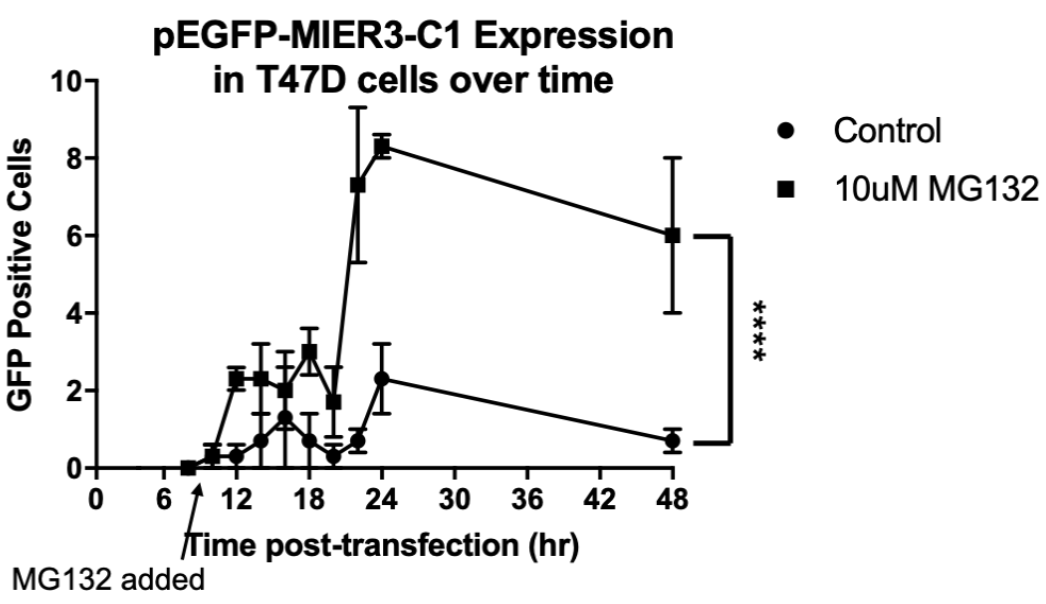

B

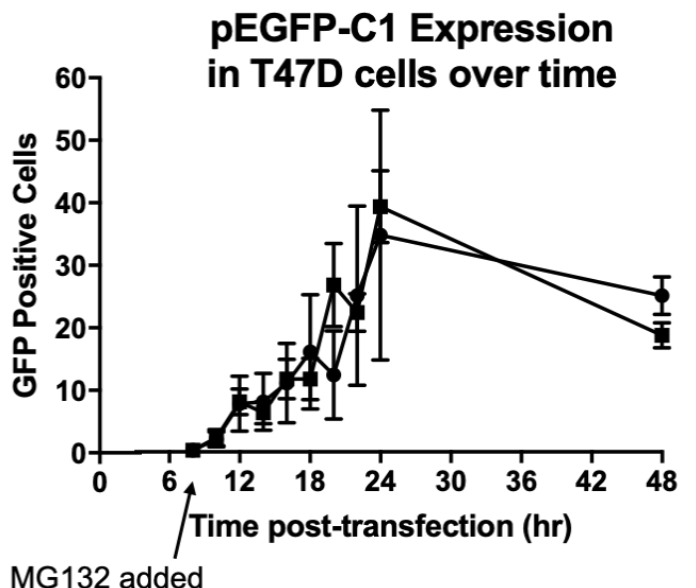

- Control

- 10uM MG132

Figure 57: pEGFP-MIER3-C1 transfected cells display increased fluorescence over a 48 hour time course with proteasome inhibitor treatment. Shown is the quantification of GFP positive T47D cells at 10x magnification and differing fields of view at every 2 hours following MG132 treatment. A: pEGFP-MIER3-C1 transfected cells display greater numbers of GFP positive cells with MG132 treatment as compared to control. This is greatly enhanced by 14 hours post-treatment. B: MG132 treatment does not have an effect in pEGFP-C1 transfected cells over time. Fluorescence data for each plasmid transfection with and without MG132 treatment were separately analyzed by 2-tailed Mann-Whitney tests, $* * * * \mathrm{p}<0.0001$. 
pEGFP-MIER3-C1 and treated with MG132 displayed significantly greater numbers of GFP positive cells beginning at 20 hours post-transfection (Figure 57). In all groups, levels drop off at the final timepoint of 48 hours as cell death begins to take place. Although GFP positive cells as a result of pEGFP-MIER3-C1 transfection don't recapitulate the numbers from the GFP empty vector, the significant increase in numbers from proteosome inhibition lends support to the hypothesis that the MIER3 protein may be signaled for degradation as a result of overexpression. These experiments determined that pEGFP-MIER3-C1 transfection was occurring, albeit resulting in lower GFP-MIER3 protein levels than desired.

\section{Affinity purification of GFP-MIER3 followed by LC-MS/MS recapitulates reported HDAC1/2 and BAHD1 interactions, with BAHD1 specific to the LXXLL motifs}

Towards the goal of isolating and confirming an interaction with a nuclear hormone receptor, site-directed mutagenesis was performed on the pEGFP-MIER3-C1 plasmid to mutate the LXXLL sequence motifs of MIER3 to LXXAA. This particular mutation has been established as sufficient to disrupt protein-protein binding at LXXLL motifs [575577]. This plasmid contains isoform 1 of human MIER3, which possess an LEDLL sequence at amino acids 66-70 and a downstream LYELL sequence within the ELM2 domain at amino acids 251-255. The resulting plasmid population from site-directed mutagenesis was used to transform competent cells. Colonies were selected, the MIER3 insert PCR amplified, and PCR products digested by the restriction enzyme TseI to screen for LXXLL mutations (Figure 58). Plasmid was purified from colonies indicating single and double mutations from TseI digestion and were Sanger sequenced to verify LXXLL to 


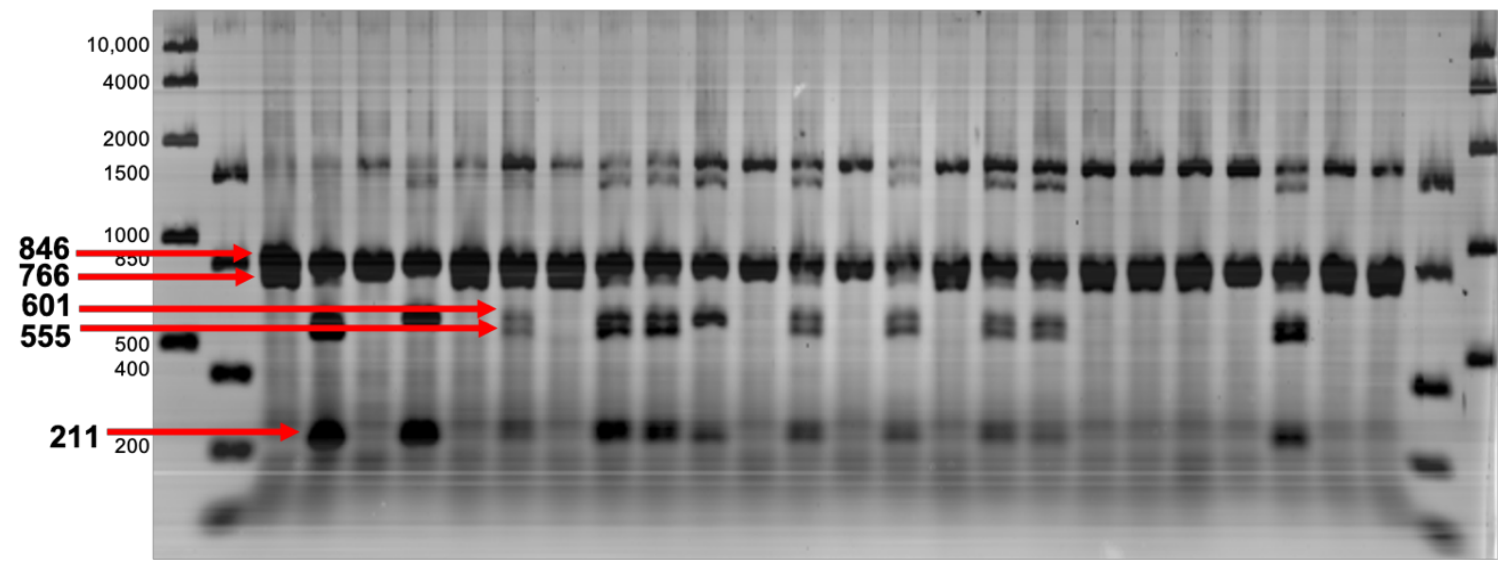

Figure 58: Screening of transformed colonies for LXXLL mutations of MIER3.

MIER3 contains two LXXLL sequence motifs, LEDLL and LYELL. Shown is a $1.4 \%$ agarose gel of the MIER3 PCR product from E. Coli colonies transformed with sitedirected mutagenesis treated pEGFP-MIER3-C1 that was digested with TseI. Expected bands from digestion of non-mutated MIER3 is 812 and $846 \mathrm{bp}$; single LEDAA mutant is 211, 601, and 846 bp; single LYEAA mutant is 46, 766, and 845 bp; and double LEDAA/LYEAA mutant is 46, 211, 555, and 846 bp. 


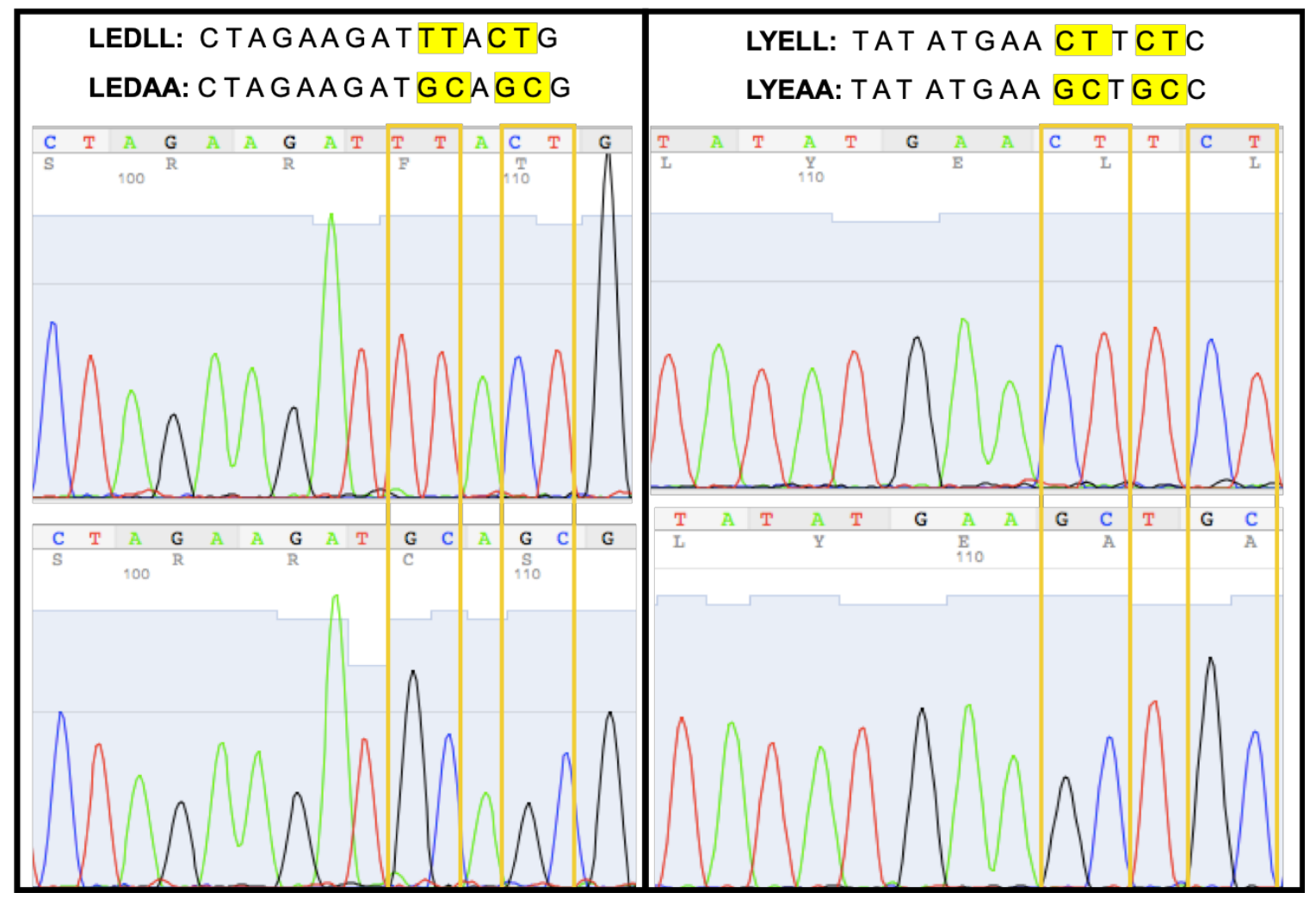

Figure 59: The LXXLL sequence motifs of MIER3 were successfully mutated to LXXAA. Shown are electropherograms from Sanger sequencing of pEGFP-MIER3-C1 plasmids that contain non-mutated sequences for LEDLL and LYELL (top row) or mutated sequences for LEDAA and LYEAA (bottom row). 
LXXAA mutations (Figure 59). This yielded MIER3 plasmid with LXXAA mutations to compare protein interactions specific to the LXXLL motifs of the non-mutated pEGFPMIER3-C1 plasmid.

The previous analysis of the transfection efficiency of pEGFP-MIER3-C1 showed a low amount of GFP-MIER3 protein being translated. To overcome this low protein concentration, a total of 60 dishes $(10 \mathrm{~cm})$ of T47D cells were plated, with 20 dishes per plasmid - GFP empty vector, GFP-MIER3, and GFP-MIER3-LXXAA. Whole cell lysate was collected 24 hours post transfection and GFP-trap beads used to affinity purify GFP tags following protein crosslinking. GFP, GFP-MIER3, and GFP-MIER3-LXXAA ran on an SDS-PAGE gel at their expected molecular weights of $27 \mathrm{kD}$ for GFP and a combined $88 \mathrm{kD}$ for GFP-MIER3 (Figure 60). The total elution obtained from affinity purification was applied to LC-MS/MS analysis. This experiment was repeated once more, and the peptide IDs identified by mass spectroscopy appearing in both pull downs were accepted as "real" interactors. Table 27 provides a list of these IDs unique to MIER3 and MIER3LXXAA following removal of peptides also appearing with the GFP empty vector and common GFP contaminants identified by CRAPome analysis. Figure 61 illustrates the number of overlapping peptides identified. Most notable in this list are HDAC1, HDAC2, and BAHD1, all which have been previously reported [264, 578]. HDAC1/2 have been demonstrated to bind to the ELM2 domain of the MIER family of proteins, and as a result also appear with the MIER3-LXXAA. However, the BAHD1 interaction disappears with the mutant, indicating this interaction is likely specific to the LXXLL sequence motifs. From these experiments we did not detect the hypothesized interaction with a nuclear hormone receptor. 


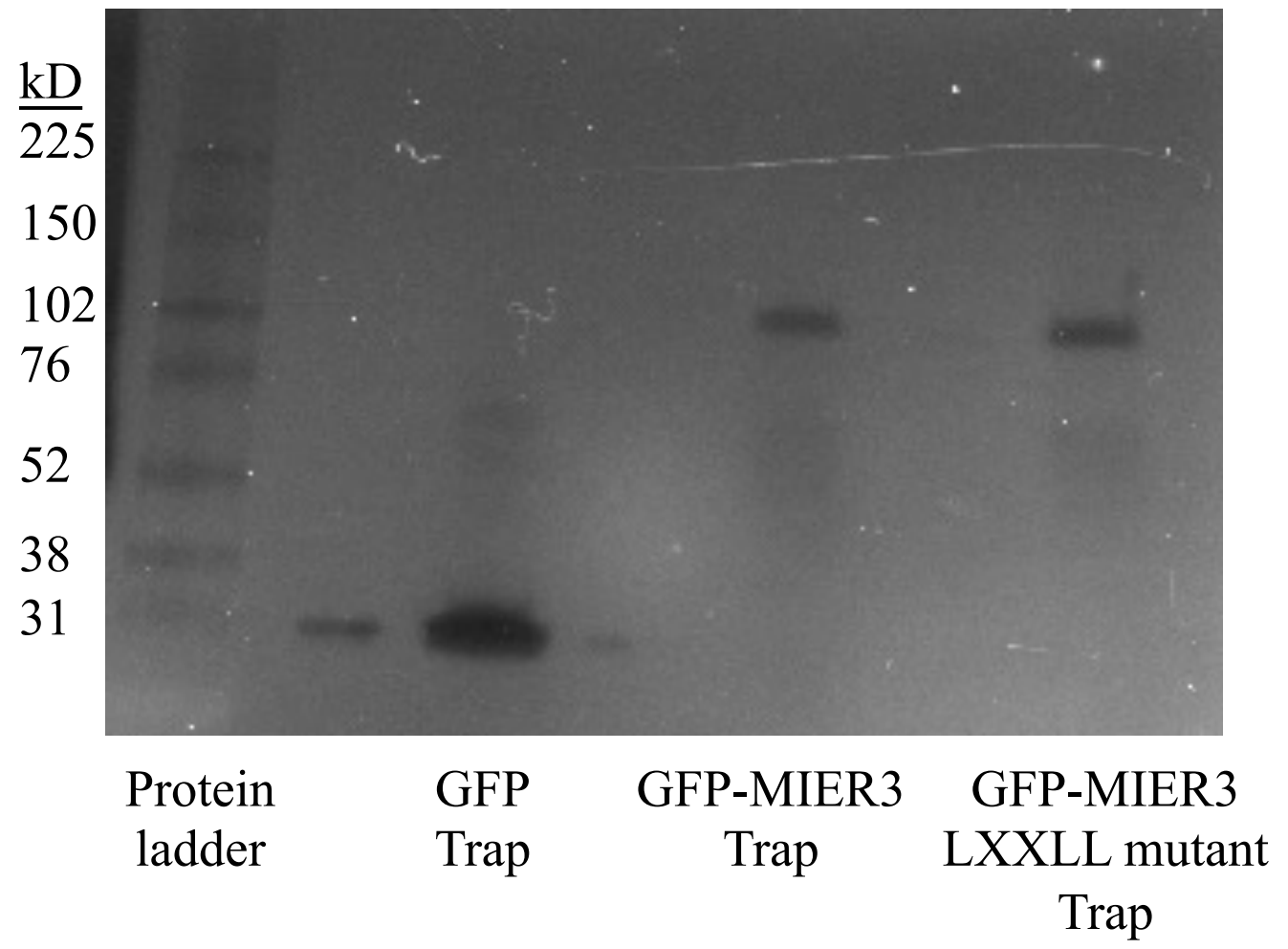

Figure 60: MIER3 proteins appear at the correct molecular weight following affinity purification of GFP. Coomassie blue staining of an SDS-PAGE gel run after a GFP-trap experiment using pEGFP-C1, pEGFP-MIER3-C1, and pEGFP-MIER3-C1-LXXAA. GFP runs at the expected molecular weight of $27 \mathrm{kD}$, while GFP-MIER3 with and without mutations runs at the expected weight of $88 \mathrm{kD}$. 
Table 27. Mass spectrosmetry peptide IDs from total elutions of GFP-trap pulldowns unique to MIER3 and MIER3-LXXAA

\begin{tabular}{|c|c|c|c|c|c|}
\hline \multicolumn{3}{|c|}{ MIER3 } & \multicolumn{3}{|c|}{ MIER3-LXXLL Mutant } \\
\hline Gene & Description & $\begin{array}{l}\text { Sequest } \\
\text { HT }\end{array}$ & Gene & Description & $\begin{array}{c}\text { Sequest } \\
\text { HT }\end{array}$ \\
\hline MIER3 & $\begin{array}{l}\text { Mesoderm induction early } \\
\text { response protein } 3\end{array}$ & 536.21 & FASN & Fatty acid synthase & 12.93 \\
\hline LMNB1 & Lamin-B1 & 43.58 & MIER3 & $\begin{array}{l}\text { Mesoderm induction early } \\
\text { response protein } 3\end{array}$ & 13.98 \\
\hline ATP1A1 & $\begin{array}{l}\text { Sodium/potassium-transporting } \\
\text { ATPase subunit alpha-1 }\end{array}$ & 39.78 & PPIA & $\begin{array}{c}\text { Peptidyl-prolyl cis-trans } \\
\text { isomerase A }\end{array}$ & 28.79 \\
\hline STAT1 & $\begin{array}{l}\text { Signal transducer and activator } \\
\text { of transcription 1-alpha/beta }\end{array}$ & 44.25 & CLTC & Clathrin heavy chain 1 & 6.91 \\
\hline ATAD3 & $\begin{array}{l}\text { ATPase family AAA domain- } \\
\text { containing protein } 3 \mathrm{~A}\end{array}$ & 40.16 & GSR & Glutathione reductase & 2.28 \\
\hline SAMHD1 & $\begin{array}{l}\text { Deoxynucleoside triphosphate } \\
\text { triphosphohydrolase SAMHD1 }\end{array}$ & 29.99 & PRDX2 & Peroxiredoxin-2 & 6.66 \\
\hline BAHD1 & $\begin{array}{l}\text { Bromo adjacent homology } \\
\text { domain-containing } 1 \text { protein }\end{array}$ & 75.8 & FLNB & Isoform 8 of Filamin-B & 3.61 \\
\hline KPNA2 & Importin subunit alpha-1 & 39.96 & ARF3 & ADP-ribosylation factor 3 & 2.41 \\
\hline IDH2 & $\begin{array}{l}\text { Isocitrate dehydrogenase, } \\
\text { mitochondrial }\end{array}$ & 51.32 & RAB1C & $\begin{array}{l}\text { Putative Ras-related protein } \\
\text { Rab-1C }\end{array}$ & 2.37 \\
\hline PHGDH & $\begin{array}{c}\text { D-3-phosphoglycerate } \\
\text { dehydrogenase }\end{array}$ & 36.06 & CFL1 & Cofilin-1 & 7.32 \\
\hline MAGED2 & $\begin{array}{c}\text { Melanoma-associated antigen } \\
\text { D2 }\end{array}$ & 38.65 & PDIA6 & $\begin{array}{c}\text { Isoform } 2 \text { of Protein disulfide- } \\
\text { isomerase A6 }\end{array}$ & 4.65 \\
\hline TCP1 & $\begin{array}{l}\text { T-complex protein } 1 \text { subunit } \\
\text { alpha }\end{array}$ & 29.7 & HSD17B10 & $\begin{array}{c}\text { 3-hydroxyacyl-CoA } \\
\text { dehydrogenase type-2 }\end{array}$ & 4.24 \\
\hline AIFM1 & Apoptosis-inducing factor 1 & 21.26 & ALYREF & THO complex subunit 4 & 2.11 \\
\hline OAS2 & 2'-5'-oligoadenylate synthase 2 & 32.12 & IQGAP1 & $\begin{array}{l}\text { Ras GTPase-activating-like } \\
\text { protein IQGAP1 }\end{array}$ & 2.06 \\
\hline SQOR & Sulfide:quinone oxidoreductase & 45.65 & AHNAK & $\begin{array}{l}\text { Neuroblast differentiation- } \\
\text { associated protein AHNAK }\end{array}$ & 1.86 \\
\hline ATP5F1B & ATP synthase subunit beta & 46.66 & CAPZA1 & $\begin{array}{c}\text { F-actin-capping protein } \\
\text { subunit alpha-1 }\end{array}$ & 0 \\
\hline HDAC2 & Histone deacetylase 2 & $\underline{90.93}$ & RAB5C & Ras-related protein $\mathrm{Rab}-5 \mathrm{C}$ & 0 \\
\hline TRIP13 & $\begin{array}{c}\text { Pachytene checkpoint protein } 2 \\
\text { homolog }\end{array}$ & 26.15 & STIP1 & $\begin{array}{l}\text { Isoform } 2 \text { of Stress-induced- } \\
\text { phosphoprotein } 1\end{array}$ & 0 \\
\hline GALK1 & Galactokinase & 36.4 & CRKL & Crk-like protein & 0 \\
\hline PRKDC & $\begin{array}{l}\text { DNA-dependent protein kinase } \\
\text { catalytic subunit }\end{array}$ & 31.17 & VCP & $\begin{array}{l}\text { Transitional endoplasmic } \\
\text { reticulum ATPase }\end{array}$ & 0 \\
\hline FAR1 & Fatty acyl-CoA reductase 1 & 31.17 & EPPK1 & Epiplakin & 0 \\
\hline HNRNPC & $\begin{array}{l}\text { Heterogeneous nuclear } \\
\text { ribonucleoproteins } \mathrm{C} 1 / \mathrm{C} 2\end{array}$ & 19.84 & PFKP & $\begin{array}{l}\text { ATP-dependent 6- } \\
\text { phosphofructokinase }\end{array}$ & 0 \\
\hline UNC45A & Protein unc-45 homolog A & 16.56 & HDAC1 & Histone deacetylase 1 & 3.67 \\
\hline DDX21 & Nucleolar RNA helicase 2 & 16.27 & HDAC2 & Histone deacetylase 2 & 1.66 \\
\hline $\mathrm{CNP}$ & $\begin{array}{l}\text { 2',3'-cyclic-nucleotide 3'- } \\
\text { phosphodiesterase }\end{array}$ & 19.94 & STAT1 & $\begin{array}{c}\text { Signal transducer and } \\
\text { activator of transcription 1- } \\
\text { alpha/beta }\end{array}$ & 2.14 \\
\hline SLC25A6 & ADP/ATP translocase 3 & 43.51 & KPNB1 & Importin subunit beta-1 & 0 \\
\hline RARS & Arginine--tRNA ligase & 16.43 & FN1 & Isoform 15 of Fibronectin & 2.96 \\
\hline CNP & $\begin{array}{l}\text { 2',3'-cyclic-nucleotide } 3^{\prime}- \\
\text { phosphodiesterase }\end{array}$ & 19.94 & NPEPPS & $\begin{array}{l}\text { Puromycin-sensitive } \\
\text { aminopeptidase }\end{array}$ & 2.85 \\
\hline NSF & Vesicle-fusing ATPase & 32.45 & PARK7 & $\begin{array}{c}\text { Protein/nucleic acid deglycase } \\
\text { DJ-1 }\end{array}$ & 2.76 \\
\hline BAG3 & $\begin{array}{l}\text { BAG family molecular } \\
\text { chaperone regulator } 3\end{array}$ & 30.2 & FARSA & $\begin{array}{c}\text { Phenylalanine--tRNA ligase } \\
\text { alpha subunit }\end{array}$ & 2.69 \\
\hline HLA-B & $\begin{array}{l}\text { HLA class I histocompatibility } \\
\text { antigen, B-37 }\end{array}$ & 25.37 & CRIP2 & Cysteine-rich protein 2 & 2.59 \\
\hline
\end{tabular}




\begin{tabular}{|c|c|c|c|c|c|}
\hline EHHADH & $\begin{array}{l}\text { Peroxisomal bifunctional } \\
\text { enzyme }\end{array}$ & 25.37 & CALML5 & Calmodulin-like protein 5 & 2.5 \\
\hline FOXK1 & Forkhead box protein K1 & 19.55 & LYZ & Lysozyme C & 2.45 \\
\hline ARAF & $\begin{array}{c}\text { Serine/threonine-protein kinase } \\
\text { A-Raf }\end{array}$ & 19.46 & PPA1 & Inorganic pyrophosphatase & 2.39 \\
\hline IFIT3 & $\begin{array}{l}\text { Interferon-induced protein with } \\
\text { tetratricopeptide repeats } 3\end{array}$ & 18.41 & ABHD14B & Protein ABHD14B & 2.34 \\
\hline EHD4 & $\begin{array}{c}\text { EH domain-containing protein } \\
4\end{array}$ & 16.52 & TGM1 & $\begin{array}{l}\text { Protein-glutamine gamma- } \\
\text { glutamyltransferase K }\end{array}$ & 2.26 \\
\hline ACSL3 & $\begin{array}{c}\text { Long-chain-fatty-acid--CoA } \\
\text { ligase } 3\end{array}$ & 13.31 & PSMA8 & $\begin{array}{l}\text { Proteasome subunit alpha- } \\
\text { type } 8\end{array}$ & 2.22 \\
\hline FARSA & $\begin{array}{c}\text { Phenylalanine--tRNA ligase } \\
\text { alpha subunit }\end{array}$ & 27.74 & SFXN2 & Sideroflexin-2 & 2.21 \\
\hline SLC25A3 & Phosphate carrier protein & 30.7 & LGALS1 & Galectin-1 & 1.88 \\
\hline MCCC2 & $\begin{array}{l}\text { Methylcrotonoyl-CoA } \\
\text { carboxylase beta chain }\end{array}$ & 16.94 & CDK1 & Cyclin-dependent kinase 1 & 1.87 \\
\hline SLC25A4 & ADP/ATP translocase 1 & 40.82 & TBR1 & T-box brain protein 1 & 1.82 \\
\hline CKAP4 & $\begin{array}{l}\text { Cytoskeleton-associated } \\
\text { protein } 4\end{array}$ & 16.16 & CORO1B & Coronin-1B & 1.75 \\
\hline $\mathrm{ABCF} 3$ & $\begin{array}{l}\text { ATP-binding cassette sub- } \\
\text { family F member } 3\end{array}$ & 19.81 & ECM1 & $\begin{array}{l}\text { Isoform } 4 \text { of Extracellular } \\
\text { matrix protein } 1\end{array}$ & 1.73 \\
\hline FXR1 & $\begin{array}{l}\text { Fragile } \mathrm{X} \text { mental retardation } \\
\text { syndrome-related protein } 1\end{array}$ & 25.21 & ATP5F1B & ATP synthase subunit beta & 1.71 \\
\hline CCT4 & $\begin{array}{l}\text { T-complex protein } 1 \text { subunit } \\
\text { delta }\end{array}$ & 18.12 & EPRS & $\begin{array}{c}\text { Bifunctional } \\
\text { glutamate/proline--tRNA } \\
\text { ligase }\end{array}$ & 1.7 \\
\hline DARS & Aspartate--tRNA ligase & 17.35 & PTBP1 & $\begin{array}{l}\text { Isoform } 3 \text { of Polypyrimidine } \\
\text { tract-binding protein } 1\end{array}$ & 1.7 \\
\hline ACSL1 & $\begin{array}{c}\text { Long-chain-fatty-acid--CoA } \\
\text { ligase } 1\end{array}$ & 13.27 & ATP5PO & ATP synthase subunit $O$ & 1.63 \\
\hline HADHB & $\begin{array}{c}\text { Trifunctional enzyme subunit } \\
\text { beta }\end{array}$ & 15.19 & IDH2 & Isocitrate dehydrogenase & 0 \\
\hline IMPDH2 & $\begin{array}{c}\text { Inosine-5'-monophosphate } \\
\text { dehydrogenase } 2\end{array}$ & 14.75 & ZNF354B & Zinc finger protein $354 \mathrm{~B}$ & 0 \\
\hline ESRP2 & $\begin{array}{l}\text { Epithelial splicing regulatory } \\
\text { protein } 2\end{array}$ & 15.43 & FARSB & $\begin{array}{c}\text { Phenylalanine--tRNA ligase } \\
\text { beta subunit }\end{array}$ & 0 \\
\hline ACAD11 & $\begin{array}{l}\text { Acyl-CoA dehydrogenase } \\
\text { family member } 11\end{array}$ & 25.92 & PRKDC & $\begin{array}{l}\text { DNA-dependent protein } \\
\text { kinase catalytic subunit }\end{array}$ & 0 \\
\hline SUCLA2 & $\begin{array}{c}\text { Succinate--CoA ligase subunit } \\
\text { beta, }\end{array}$ & 13.15 & IMPDH2 & $\begin{array}{c}\text { Inosine-5'-monophosphate } \\
\text { dehydrogenase } 2\end{array}$ & 0 \\
\hline ERLIN1 & Erlin-1 & 16.72 & MCM3 & $\begin{array}{l}\text { Isoform } 2 \text { of DNA replication } \\
\text { licensing factor MCM3 }\end{array}$ & 0 \\
\hline DTX3L & $\begin{array}{c}\text { E3 ubiquitin-protein ligase } \\
\text { DTX3L }\end{array}$ & 13.04 & TRIM28 & $\begin{array}{c}\text { Transcription intermediary } \\
\text { factor 1-beta }\end{array}$ & 0 \\
\hline MARK2 & $\begin{array}{c}\text { Serine/threonine-protein kinase } \\
\text { MARK2 }\end{array}$ & 16.2 & EMSY & $\begin{array}{c}\text { BRCA2-interacting } \\
\text { transcriptional repressor } \\
\text { EMSY }\end{array}$ & 0 \\
\hline GARS & Glycine--tRNA ligase & 13.56 & SLC25A13 & $\begin{array}{c}\text { Calcium-binding } \\
\text { mitochondrial carrier protein } \\
\text { Aralar2 }\end{array}$ & 0 \\
\hline DNAJA2 & $\begin{array}{c}\text { DnaJ homolog subfamily A } \\
\text { member } 2\end{array}$ & 11.92 & PCK2 & $\begin{array}{c}\text { Phosphoenolpyruvate } \\
\text { carboxykinase }\end{array}$ & 0 \\
\hline QARS & Glutamine--tRNA ligase & 12.7 & PPP2CB & $\begin{array}{l}\text { Serine/threonine-protein } \\
\text { phosphatase } 2 \mathrm{~A} \text { catalytic } \\
\text { subunit }\end{array}$ & 0 \\
\hline $\begin{array}{l}\mathrm{ALDH} 3 \mathrm{~A} \\
2\end{array}$ & $\begin{array}{c}\text { Isoform } 2 \text { of Fatty aldehyde } \\
\text { dehydrogenase }\end{array}$ & 10.27 & $\mathrm{P} 3 \mathrm{H} 4$ & $\begin{array}{c}\text { Endoplasmic reticulum } \\
\text { protein SC65 }\end{array}$ & 0 \\
\hline SDHA & $\begin{array}{l}\text { Succinate dehydrogenase } \\
\text { flavoprotein subunit }\end{array}$ & 11.4 & SRF3 & $\begin{array}{l}\text { Serine/arginine-rich splicing } \\
\text { factor } 3\end{array}$ & 0 \\
\hline CLTC & Clathrin heavy chain 1 & 9.14 & PFKL & $\begin{array}{l}\text { ATP-dependent 6- } \\
\text { phosphofructokinase }\end{array}$ & 0 \\
\hline
\end{tabular}




\begin{tabular}{|c|c|c|c|c|c|}
\hline KHSRP & $\begin{array}{l}\text { Far upstream element-binding } \\
\text { protein } 2\end{array}$ & 8.05 & GFPT1 & $\begin{array}{c}\text { Glutamine--fructose-6- } \\
\text { phosphate aminotransferase } 1\end{array}$ & 0 \\
\hline DDOST & $\begin{array}{l}\text { Dolichyl- } \\
\text { diphosphooligosaccharide-- } \\
\text { protein glycosyltransferase } 48 \\
\text { kDa subunit }\end{array}$ & 8.36 & OGA & $\begin{array}{l}\text { Isoform } 3 \text { of Protein O- } \\
\text { GlcNAcase }\end{array}$ & 0 \\
\hline MTA2 & $\begin{array}{c}\text { Metastasis-associated protein } \\
\text { MTA2 }\end{array}$ & 7.89 & ARL3 & $\begin{array}{l}\text { ADP-ribosylation factor-like } \\
\text { protein } 3\end{array}$ & 0 \\
\hline ATP5PO & ATP synthase subunit $\mathrm{O}$ & 7.53 & THBS1 & Thrombospondin-1 & 0 \\
\hline SEC23B & $\begin{array}{l}\text { Protein transport protein } \\
\text { Sec23B }\end{array}$ & 7.08 & CTSD & Cathepsin D & 0 \\
\hline NSUN2 & $\begin{array}{l}\text { tRNA (cytosine }(34)-C(5))- \\
\text { methyltransferase }\end{array}$ & 8.01 & DDX6 & $\begin{array}{l}\text { Probable ATP-dependent } \\
\text { RNA helicase DDX6 }\end{array}$ & 0 \\
\hline PRDX4 & Peroxiredoxin- 4 & 19.35 & CDSN & Corneodesmosin & 0 \\
\hline $\mathrm{P} 4 \mathrm{HB}$ & Protein disulfide-isomerase & 8.17 & CAPN1 & Calpain-1 catalytic subunit & 0 \\
\hline HDAC1 & Histone deacetylase 1 & 10.41 & AGR2 & $\begin{array}{l}\text { Anterior gradient protein } 2 \\
\text { homolog }\end{array}$ & 0 \\
\hline MAPK1 & $\begin{array}{c}\text { Mitogen-activated protein } \\
\text { kinase } 1\end{array}$ & 4.53 & RET & $\begin{array}{l}\text { Proto-oncogene tyrosine- } \\
\text { protein kinase receptor Ret }\end{array}$ & 0 \\
\hline MCM5 & $\begin{array}{l}\text { DNA replication licensing } \\
\text { factor MCM5 }\end{array}$ & 4.18 & GHX9 & $\begin{array}{c}\text { ATP-dependent RNA helicase } \\
\text { A }\end{array}$ & 0 \\
\hline MCM3 & $\begin{array}{l}\text { Isoform } 2 \text { of DNA replication } \\
\text { licensing factor MCM3 }\end{array}$ & 4.4 & DHTKD1 & $\begin{array}{c}\text { Probable 2-oxoglutarate } \\
\text { dehydrogenase E1 component } \\
\text { DHKTD1 }\end{array}$ & 0 \\
\hline MCM6 & $\begin{array}{l}\text { DNA replication licensing } \\
\text { factor MCM6 }\end{array}$ & 5.35 & RAB14 & Ras-related protein Rab-14 & 0 \\
\hline HSD17B4 & $\begin{array}{c}\text { Isoform } 2 \text { of Peroxisomal } \\
\text { multifunctional enzyme type } 2\end{array}$ & 5.75 & FUS & RNA-binding protein FUS & 0 \\
\hline PHB & Prohibitin & 4.37 & GGCT & $\begin{array}{l}\text { Gamma- } \\
\text { glutamylcyclotransferase }\end{array}$ & 0 \\
\hline LYZ & Lysozyme $\mathrm{C}$ & 17.32 & BLVRA & Biliverdin reductase $\mathrm{A}$ & 0 \\
\hline ANXA1 & Annexin A1 & 4.57 & COL6A3 & Collagen alpha-3(VI) chain & 0 \\
\hline \multirow[t]{18}{*}{ YWHAE } & 14-3-3 protein epsilon & 4.53 & GSN & Gelsolin & 0 \\
\hline & & & LARS & Leucine--tRNA ligase & 0 \\
\hline & & & HSD17B4 & $\begin{array}{l}\text { Peroxisomal multifunctional } \\
\text { enzyme type } 2\end{array}$ & 0 \\
\hline & & & ADAM33 & $\begin{array}{l}\text { Disintegrin and } \\
\text { metalloproteinase domain- } \\
\text { containing protein } 33\end{array}$ & 0 \\
\hline & & & DYNC1H1 & dynein 1 heavy chain 1 & 0 \\
\hline & & & PGK1 & Phosphoglycerate kinase 1 & 0 \\
\hline & & & PARG & $\begin{array}{l}\text { Poly(ADP-ribose) } \\
\text { glycohydrolase }\end{array}$ & 0 \\
\hline & & & SLC9A3R2 & $\begin{array}{l}\mathrm{Na}(+) / \mathrm{H}(+) \text { exchange } \\
\text { regulatory cofactor NHE-RF2 }\end{array}$ & 0 \\
\hline & & & TKT & Transketolase & 0 \\
\hline & & & CKMT1A & Creatine kinase U-type & 0 \\
\hline & & & NSF & Vesicle-fusing ATPase & 0 \\
\hline & & & BTBD11 & $\begin{array}{c}\text { Ankyrin repeat and } \mathrm{BTB} / \mathrm{POZ} \\
\text { domain-containing protein } \\
\text { BTBD11 }\end{array}$ & 0 \\
\hline & & & AHSA1 & $\begin{array}{c}\text { Activator of } 90 \mathrm{kDa} \text { heat } \\
\text { shock protein ATPase } \\
\text { homolog } 1\end{array}$ & 0 \\
\hline & & & NARS & Asparagine--tRNA ligase & 0 \\
\hline & & & RARS & Arginine--tRNA ligase & 0 \\
\hline & & & RAB13 & Ras-related protein Rab-13 & 0 \\
\hline & & & PDIA3 & $\begin{array}{c}\text { Protein disulfide-isomerase } \\
\text { A3 }\end{array}$ & 0 \\
\hline & & & MVK & Mevalonate kinase & 0 \\
\hline
\end{tabular}

\section{Red: Recapitulates findings reported in literature}




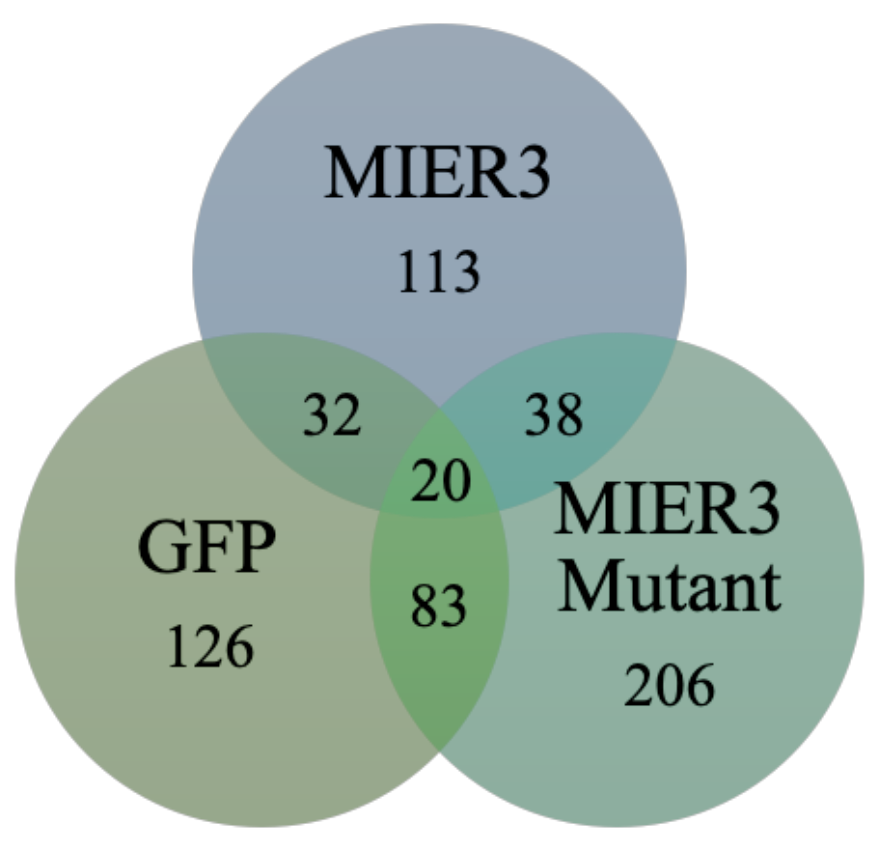

Figure 61: Overlapping peptide numbers from affinity purification-mass spectroscopy experiments with pEGFP-C1, pEGFP-MIER3-C1, and pEGFPMIER3-C1-LXXAA. 


\section{No evidence MIER3 interacts with a nuclear hormone receptor under conditions tested}

To see if I could induce expression of nuclear hormone receptors and identify an interaction with MIER3, the previous experiment was repeated using serum starvation prior to transfection, followed by serum stimulation with $20 \%$ FBS following transfection. A list of additional peptide IDs identified by mass spectrometry unique to MIER3 and MIER3LXXAA, but not present in the pulldowns using traditional media conditions, are listed in Table 28. A nuclear hormone receptor was not identified. However, this experiment recapitulated the previous results of $\mathrm{HDAC} 1 / 2$ and BAHD1 interactions, with the disappearance of BAHD1 in the LXXLL mutant pulldown.

\section{MIER3 interacts with a wide array of signaling, transport, and metabolic proteins}

The protein-protein interactions of MIER3, but not of the LXXLL mutant, were explored in a further affinity purification and mass spectroscopy experiment. The total elution from GFP empty vector and GFP-MIER3 pulldowns were run on an SDS-PAGE gel and bands individually excised and analyzed by LC-MS/MS (Figure 62). This resulted in detection of a greater number of peptide fragments per protein identifier and generated a more comprehensive list of interacting peptides. The greater number of peptide fragments that appeared with this method allowed for removal of IDs in which only one peptide was identified. Table 29 provides a list of these protein IDs following removal of peptides overlapping with the empty vector pull down and CRAPome-identified contaminants. SEQUEST software application assigned the tandem mass spectra to peptide sequences from databases of known protein sequences. From this, SEQUEST HT scores 
Table 28. Additional mass spectrometry peptide IDs* that appear from serumstarved and stimulated GFP-Trap pulldowns unique to MIER3 and MIER3-

\section{LXXAA}

\begin{tabular}{|c|c|c|c|c|c|}
\hline \multicolumn{3}{|c|}{ MIER3 } & \multicolumn{3}{|c|}{ MIER3-LXXLL Mutant } \\
\hline Gene & Description & $\begin{array}{l}\text { Sequest } \\
\text { HT }\end{array}$ & Gene & Description & $\begin{array}{l}\text { Sequest } \\
\text { HT }\end{array}$ \\
\hline MIER3 & $\begin{array}{l}\text { Mesoderm induction early } \\
\text { response protein } 3\end{array}$ & 83.62 & MIER3 & $\begin{array}{l}\text { Mesoderm induction early } \\
\text { response protein } 3\end{array}$ & 79.58 \\
\hline HDAC1 & Histone deacetylase 1 & 21.81 & HDAC1 & Histone deacetylase 1 & 13.27 \\
\hline HDAC2 & Histone deacetylase 2 & 17.5 & HDAC2 & Histone deacetylase 2 & 10.66 \\
\hline BAHD1 & $\begin{array}{l}\text { Bromo adjacent homology } \\
\text { domain-containing } 1 \text { protein }\end{array}$ & 1.68 & HRNR & Hornerin & 11.54 \\
\hline NOC4L & $\begin{array}{c}\text { Nucleolar complex protein } 4 \\
\text { homolog }\end{array}$ & 5.81 & NOC4L & $\begin{array}{l}\text { Nucleolar complex protein } 4 \\
\text { homolog }\end{array}$ & 9.09 \\
\hline PFKM & $\begin{array}{c}\text { Isoform } 3 \text { of ATP-dependent 6- } \\
\text { phosphofructokinase }\end{array}$ & 1.89 & MESP1 & Mesoderm posterior protein 1 & 0 \\
\hline HRNR & Hornerin & 0 & NEO1 & Neogenin & 0 \\
\hline ABCA9 & $\begin{array}{l}\text { ATP-binding cassette sub- } \\
\text { family A member } 9\end{array}$ & 0 & NCBP3 & $\begin{array}{c}\text { Nuclear cap-binding protein } \\
\text { subunit } 3\end{array}$ & 0 \\
\hline LIPE & Hormone-sensitive lipase & 0 & PLSCR5 & $\begin{array}{l}\text { Phospholipid scramblase family } \\
\text { member } 5\end{array}$ & 0 \\
\hline \multirow[t]{6}{*}{ SLC9A9 } & Sodium/hydrogen exchanger & 0 & FAM81B & Protein FAM81B & 0 \\
\hline & & & SHROOM3 & Protein Shroom3 & 0 \\
\hline & & & CAPN2 & Calpain-2 catalytic subunit & 0 \\
\hline & & & RIT2 & GTP-binding protein Rit2 & 0 \\
\hline & & & HIVEP3 & Transcription factor HIVEP3 & 0 \\
\hline & & & AMY1A & Alpha-amylase 1 & 0 \\
\hline
\end{tabular}

*IDs not present in previous mass spectrometry of total elution or gel extraction Red: Not unique to stimulation, but recapitulates previous findings 


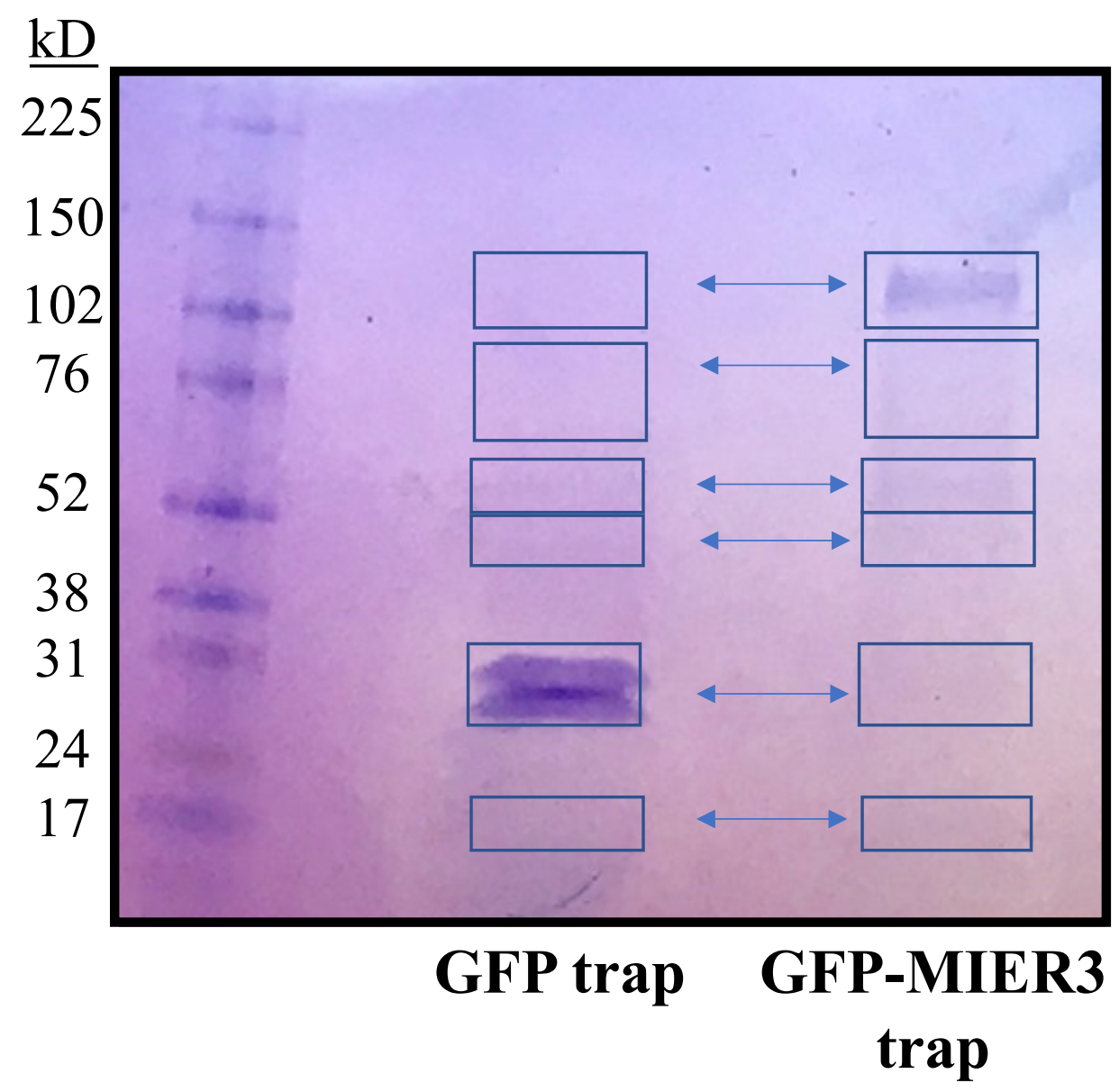

Figure 62: GFP and GFP-MIER3 band excision following affinity purification. Coomassie blue staining of an SDS-PAGE gel run after a GFP-trap experiment using pEGFP-C1 and pEGFP-MIER3-C1. Wherever a band appeared in either the GFP-trap or GFP-MIER3-trap column, a corresponding region was excised in the other. 
Table 29. Total peptide IDs unique to MIER3 from mass spectrometry of individual bands in GFP-trap pulldown SDS-PAGE gels

\begin{tabular}{|c|c|c|c|}
\hline Gene & Description & $\begin{array}{c}\# \\
\text { Peptides }\end{array}$ & $\begin{array}{c}\text { Sequest } \\
\text { HT }\end{array}$ \\
\hline LMNA & Prelamin-A/C & 27 & 140.31 \\
\hline MIER3 & Mesoderm induction early response protein 3 & 15 & 536.21 \\
\hline ATP2A2 & Sarcoplasmic/endoplasmic reticulum calcium ATPase 2 & 15 & 58.57 \\
\hline SLC25A13 & $\begin{array}{l}\text { Isoform } 2 \text { of Calcium-binding mitochondrial carrier protein } \\
\text { Aralar2 }\end{array}$ & 14 & 59.72 \\
\hline HDAC2 & Histone deacetylase 2 & 13 & 90.93 \\
\hline LMNB1 & Lamin-B1 & 13 & 43.58 \\
\hline ATP1A1 & Sodium/potassium-transporting ATPase subunit alpha-1 & 13 & 39.78 \\
\hline HSPA1L & Heat shock $70 \mathrm{kDa}$ protein 1-like & 13 & 189.44 \\
\hline HDAC1 & Histone deacetylase 1 & 13 & 90.36 \\
\hline ATAD3A & Isoform 2 of ATPase family AAA domain-containing protein $3 \mathrm{~A}$ & 12 & 40.16 \\
\hline STAT1 & Signal transducer and activator of transcription 1-alpha/beta & 12 & 44.25 \\
\hline SAMHD1 & Deoxynucleoside triphosphate triphosphohydrolase SAMHD1 & 11 & 29.99 \\
\hline SLC25A12 & Calcium-binding mitochondrial carrier protein Aralar1 & 10 & 32.03 \\
\hline KPNA2 & Importin subunit alpha-1 & 10 & 39.96 \\
\hline BAHD1 & Bromo adjacent homology domain-containing 1 protein & 10 & 75.8 \\
\hline PSMD2 & $26 \mathrm{~S}$ proteasome non-ATPase regulatory subunit 2 & 9 & 39.52 \\
\hline MAGED2 & Melanoma-associated antigen D2 & 9 & 38.65 \\
\hline ACTA1 & Actin, aortic smooth muscle & 9 & 65.3 \\
\hline AIFM1 & Apoptosis-inducing factor 1 , mitochondrial & 9 & 21.26 \\
\hline OAS2 & 2'-5'-oligoadenylate synthase 2 & 9 & 32.12 \\
\hline TRIP13 & Pachytene checkpoint protein 2 homolog & 8 & 26.15 \\
\hline GALK1 & Isoform 2 of Galactokinase & 8 & 36.4 \\
\hline PRKDC & DNA-dependent protein kinase catalytic subunit & 8 & 21.72 \\
\hline FAR1 & Fatty acyl-CoA reductase 1 & 8 & 31.17 \\
\hline HNRNPC & Heterogeneous nuclear ribonucleoproteins $\mathrm{C} 1 / \mathrm{C} 2$ & 7 & 19.84 \\
\hline UNC45A & Protein unc-45 homolog A & 7 & 16.56 \\
\hline SLC25A6 & ADP/ATP translocase 3 & 7 & 43.51 \\
\hline HLA-B & HLA class I histocompatibility antigen, B-37 alpha chain & 6 & 24.96 \\
\hline EHHADH & Peroxisomal bifunctional enzyme & 6 & 25.37 \\
\hline FOXK1 & Forkhead box protein K1 & 6 & 19.55 \\
\hline ARAF & Serine/threonine-protein kinase A-Raf & 6 & 19.46 \\
\hline IFIT3 & Interferon-induced protein with tetratricopeptide repeats 3 & 6 & 18.41 \\
\hline EHD4 & EH domain-containing protein 4 & 6 & 16.52 \\
\hline ACSL3 & Long-chain-fatty-acid--CoA ligase 3 & 6 & 13.31 \\
\hline FARSA & Phenylalanine--tRNA ligase alpha subunit & 6 & 27.74 \\
\hline ESYT2 & Isoform 2 of Extended synaptotagmin-2 & 6 & 19.58 \\
\hline MCCC2 & Methylcrotonoyl-CoA carboxylase beta chain, mitochondrial & 6 & 16.94 \\
\hline SLC25A4 & ADP/ATP translocase 1 & 6 & 40.82 \\
\hline CKAP4 & Cytoskeleton-associated protein 4 & 6 & 16.16 \\
\hline ABCF3 & ATP-binding cassette sub-family F member 3 & 6 & 19.81 \\
\hline FXR1 & Fragile $\mathrm{X}$ mental retardation syndrome-related protein 1 & 6 & 25.21 \\
\hline IMPDH2 & Inosine-5'-monophosphate dehydrogenase 2 & 5 & 14.75 \\
\hline GFPT1 & Glutamine--fructose-6-phosphate aminotransferase 1 & 5 & 13.75 \\
\hline NOP56 & Nucleolar protein 56 & 5 & 13.39 \\
\hline SLC25A10 & Isoform 2 of Mitochondrial dicarboxylate carrier & 5 & 15.29 \\
\hline ACAD11 & Acyl-CoA dehydrogenase family member 11 & 5 & 25.92 \\
\hline SUCLA2 & Succinate--CoA ligase subunit beta, mitochondrial & 5 & 13.15 \\
\hline ERLIN1 & Erlin-1 & 5 & 16.72 \\
\hline DTX3L & E3 ubiquitin-protein ligase DTX3L & 5 & 13.04 \\
\hline
\end{tabular}




\begin{tabular}{|c|c|c|c|}
\hline MARK2 & Serine/threonine-protein kinase MARK2 & 5 & 16.2 \\
\hline LARP4B & La-related protein $4 \mathrm{~B}$ & 5 & 12.25 \\
\hline YME1L1 & ATP-dependent zinc metalloprotease YME1L1 & 5 & 18.94 \\
\hline EHD1 & EH domain-containing protein 1 & 5 & 11.54 \\
\hline FASN & Fatty acid synthase & 5 & 12.63 \\
\hline SPTLC1 & Serine palmitoyltransferase 1 & 4 & 9.67 \\
\hline IMMT & MICOS complex subunit MIC60 & 4 & 10.4 \\
\hline PARP9 & Protein mono-ADP-ribosyltransferase PARP9 & 4 & 9.84 \\
\hline DNAJA1 & DnaJ homolog subfamily A member 1 & 4 & 16.29 \\
\hline ZC3HAV1 & Zinc finger CCCH-type antiviral protein 1 & 4 & 16.05 \\
\hline DYNC1LI1 & Cytoplasmic dynein 1 light intermediate chain 1 & 4 & 11.64 \\
\hline RAF1 & Isoform 2 of RAF proto-oncogene serine/threonine-protein kinase & 4 & 13.62 \\
\hline DHCR24 & Delta(24)-sterol reductase & 4 & 14.48 \\
\hline PSMC6 & $26 \mathrm{~S}$ proteasome regulatory subunit 10B & 4 & 9.94 \\
\hline MRPS27 & Isoform 2 of $28 \mathrm{~S}$ ribosomal protein $\mathrm{S} 27$, mitochondrial & 4 & 9.03 \\
\hline RFC2 & Replication factor $\mathrm{C}$ subunit 2 & 4 & 11.05 \\
\hline GNL3L & Guanine nucleotide-binding protein-like 3-like protein & 4 & 10.22 \\
\hline SSH3 & Protein phosphatase Slingshot homolog 3 & 4 & 8.34 \\
\hline LGALS3BP & Galectin-3-binding protein & 4 & 14.86 \\
\hline CSDE1 & Isoform 4 of Cold shock domain-containing protein E1 & 4 & 9.07 \\
\hline UGDH & UDP-glucose 6-dehydrogenase & 4 & 10 \\
\hline SLC25A11 & Mitochondrial 2-oxoglutarate/malate carrier protein & 4 & 16.47 \\
\hline ZNF385A & Zinc finger protein $385 \mathrm{~A}$ & 4 & 9.2 \\
\hline TAP2 & Antigen peptide transporter 2 & 4 & 9.59 \\
\hline KPNA1 & Importin subunit alpha-5 & 4 & 17.33 \\
\hline CCNK & Isoform 4 of Cyclin-K & 4 & 10.54 \\
\hline GTPBP4 & Nucleolar GTP-binding protein 1 & 4 & 9.41 \\
\hline NDUFA10 & $\begin{array}{l}\text { NADH dehydrogenase } 1 \text { alpha subcomplex subunit } 10, \\
\text { mitochondrial }\end{array}$ & 4 & 12.12 \\
\hline SLC3A2 & Isoform 4 of 4F2 cell-surface antigen heavy chain & 4 & 12.83 \\
\hline CSE1L & Exportin-2 & 4 & 9.14 \\
\hline VPS51 & Vacuolar protein sorting-associated protein 51 homolog & 4 & 10.45 \\
\hline CDK1 & Cyclin-dependent kinase 1 & 4 & 15.15 \\
\hline PDIA3 & Protein disulfide-isomerase A3 & 3 & 6.6 \\
\hline PRPF6 & Pre-mRNA-processing factor 6 & 3 & 7.54 \\
\hline RAB5C & Isoform 2 of Ras-related protein Rab-5C & 3 & 7.99 \\
\hline AHSA1 & Activator of $90 \mathrm{kDa}$ heat shock protein ATPase homolog 1 & 3 & 9.76 \\
\hline COG4 & Conserved oligomeric Golgi complex subunit 4 & 3 & 6.42 \\
\hline TPX2 & Isoform 2 of Targeting protein for Xklp2 & 3 & 7.04 \\
\hline MCM4 & DNA replication licensing factor MCM4 & 3 & 7.17 \\
\hline PURB & Transcriptional activator protein Pur-beta & 3 & 7.27 \\
\hline WDR18 & WD repeat-containing protein 18 & 3 & 6.39 \\
\hline ERAL1 & GTPase Era, mitochondrial & 3 & 7.56 \\
\hline IKBKB & Inhibitor of nuclear factor kappa-B kinase subunit beta & 3 & 7.37 \\
\hline IFIT1 & Interferon-induced protein with tetratricopeptide repeats 1 & 3 & 11.58 \\
\hline TXNDC5 & Thioredoxin domain-containing protein 5 & 3 & 7.08 \\
\hline RBM39 & RNA-binding protein 39 & 3 & 10.58 \\
\hline YWHAG & 14-3-3 protein gamma & 3 & 11.53 \\
\hline PES1 & Pescadillo homolog & 3 & 6.93 \\
\hline PDXDC1 & Pyridoxal-dependent decarboxylase domain-containing protein 1 & 3 & 6.98 \\
\hline FKBP4 & Peptidyl-prolyl cis-trans isomerase FKBP4 & 3 & 6.78 \\
\hline VPS53 & $\begin{array}{c}\text { Isoform } 4 \text { of Vacuolar protein sorting-associated protein } 53 \\
\text { homolog }\end{array}$ & 3 & 7.25 \\
\hline UFL1 & E3 UFM1-protein ligase 1 & 3 & 6.28 \\
\hline SLFN5 & Schlafen family member 5 & 3 & 7.69 \\
\hline RBM4 & RNA-binding protein 4 & 3 & 10.15 \\
\hline
\end{tabular}




\begin{tabular}{|c|c|c|c|}
\hline MLH1 & DNA mismatch repair protein Mlh1 & 3 & 6.33 \\
\hline PADI3 & Protein-arginine deiminase type- 3 & 3 & 7.21 \\
\hline DNAJC7 & DnaJ homolog subfamily $\mathrm{C}$ member 7 & 3 & 7.3 \\
\hline CTNND1 & Catenin delta-1 & 3 & 6.74 \\
\hline HLA-C & HLA class I histocompatibility antigen, $\mathrm{Cw}-17$ alpha chain & 3 & 12.92 \\
\hline PTPN1 & Tyrosine-protein phosphatase non-receptor type 1 & 3 & 7.48 \\
\hline HGH1 & Protein HGH1 homolog & 3 & 6.29 \\
\hline EPM2AIP1 & EPM2A-interacting protein 1 & 3 & 6.72 \\
\hline GTF3C5 & Isoform 3 of General transcription factor $3 \mathrm{C}$ polypeptide 5 & 3 & 6.49 \\
\hline SFXN2 & Sideroflexin-2 & 3 & 10.76 \\
\hline PGM3 & Isoform 3 of Phosphoacetylglucosamine mutase & 3 & 10.17 \\
\hline FASTKD5 & FAST kinase domain-containing protein 5 , mitochondrial & 3 & 6.58 \\
\hline PSMA7 & Proteasome subunit alpha type- 7 & 3 & 6.52 \\
\hline MYEF2 & Myelin expression factor 2 & 3 & 6.31 \\
\hline SPTLC2 & Serine palmitoyltransferase 2 & 3 & 8.21 \\
\hline TRAF4 & TNF receptor-associated factor 4 & 3 & 9.52 \\
\hline OAS3 & 2'-5'-oligoadenylate synthase 3 & 3 & 7.07 \\
\hline PRDX2 & Peroxiredoxin-2 & 3 & 19.35 \\
\hline TBL2 & Transducin beta-like protein 2 & 3 & 8.45 \\
\hline ZNF281 & Zinc finger protein 281 & 3 & 8.71 \\
\hline PKP3 & Isoform PKP3b of Plakophilin-3 & 3 & 11.36 \\
\hline GLT8D1 & Glycosyltransferase 8 domain-containing protein 1 & 3 & 7.76 \\
\hline HLA-E & HLA class I histocompatibility antigen, alpha chain E & 3 & 8.24 \\
\hline TARDBP & TAR DNA-binding protein 43 & 3 & 9.61 \\
\hline POLD3 & DNA polymerase delta subunit 3 & 3 & 9.18 \\
\hline YY1 & Transcriptional repressor protein YY1 & 3 & 6.45 \\
\hline LPCAT1 & Lysophosphatidylcholine acyltransferase 1 & 3 & 11.81 \\
\hline DNAJA3 & DnaJ homolog subfamily A member 3 , mitochondrial & 3 & 20.3 \\
\hline EBNA1BP2 & Probable rRNA-processing protein EBP2 & 3 & 7.32 \\
\hline SLC25A1 & Tricarboxylate transport protein, mitochondrial & 3 & 14.04 \\
\hline RAB25 & Ras-related protein Rab- 25 & 2 & 6.89 \\
\hline MCRS1 & Isoform 2 of Microspherule protein 1 & 2 & 5.87 \\
\hline DHRS13 & Dehydrogenase/reductase SDR family member 13 & 2 & 5.53 \\
\hline FAM83H & Protein FAM83H & 2 & 5.22 \\
\hline NTPCR & Cancer-related nucleoside-triphosphatase & 2 & 5.07 \\
\hline DNAJA4 & Isoform 2 of DnaJ homolog subfamily A member 4 & 2 & 4.56 \\
\hline DUSP23 & Dual specificity protein phosphatase 23 & 2 & 4.78 \\
\hline STAT3 & Signal transducer and activator of transcription 3 & 2 & 7.27 \\
\hline TOR1AIP1 & Torsin-1A-interacting protein 1 & 2 & 4.76 \\
\hline FIP1L1 & Pre-mRNA 3'-end-processing factor FIP1 & 2 & 5.13 \\
\hline $\mathrm{RCN} 1$ & Reticulocalbin-1 & 2 & 5.42 \\
\hline ECT2 & Isoform 3 of Protein ECT2 & 2 & 4.88 \\
\hline TMPO & Lamina-associated polypeptide 2 , isoforms beta/gamma & 2 & 4.45 \\
\hline LMF2 & Lipase maturation factor 2 & 2 & 4.61 \\
\hline EXOC3 & Exocyst complex component 3 & 2 & 4.85 \\
\hline RFC3 & Replication factor $\mathrm{C}$ subunit 3 & 2 & 6.22 \\
\hline CTSD & Cathepsin D & 2 & 8.64 \\
\hline STOML2 & Stomatin-like protein 2, mitochondrial & 2 & 6.17 \\
\hline HADHA & Trifunctional enzyme subunit alpha, mitochondrial & 2 & 5.95 \\
\hline MRE11 & Isoform 3 of Double-strand break repair protein MRE11 & 2 & 6.16 \\
\hline SFXN3 & Sideroflexin-3 & 2 & 4.81 \\
\hline SNRPA1 & U2 small nuclear ribonucleoprotein $\mathrm{A}^{\prime}$ & 2 & 4.6 \\
\hline SFXN1 & Sideroflexin-1 & 2 & 5.84 \\
\hline ZNHIT2 & Zinc finger HIT domain-containing protein 2 & 2 & 4.45 \\
\hline SLIRP & SRA stem-loop-interacting RNA-binding protein, mitochondrial & 2 & 8.67 \\
\hline ACAD9 & Acyl-CoA dehydrogenase family member 9, mitochondrial & 2 & 5.21 \\
\hline
\end{tabular}




\begin{tabular}{|c|c|c|c|}
\hline EXOC2 & Exocyst complex component 2 & 2 & 4.2 \\
\hline ATP2C1 & Isoform 7 of Calcium-transporting ATPase type $2 \mathrm{C}$ member 1 & 2 & 5.39 \\
\hline KIFC1 & Kinesin-like protein KIFC1 & 2 & 3.89 \\
\hline GATA3 & $\begin{array}{l}\text { Isoform } 2 \text { of Trans-acting T-cell-specific transcription factor } \\
\text { GATA-3 }\end{array}$ & 2 & 5.06 \\
\hline RAB5B & Ras-related protein Rab-5B & 2 & 5.64 \\
\hline RANBP9 & Ran-binding protein 9 & 2 & 4.41 \\
\hline CNDP2 & Cytosolic non-specific dipeptidase & 2 & 10.04 \\
\hline UQCRC1 & Cytochrome b-c 1 complex subunit 1 , mitochondrial & 2 & 5.7 \\
\hline TRIM26 & Tripartite motif-containing protein 26 & 2 & 4.38 \\
\hline MYL6 & Myosin light polypeptide 6 & 2 & 4.78 \\
\hline GANAB & Isoform 2 of Neutral alpha-glucosidase $\mathrm{AB}$ & 2 & 4.86 \\
\hline YARS & Tyrosine--tRNA ligase, cytoplasmic & 2 & 4.06 \\
\hline RIOX1 & Ribosomal oxygenase 1 & 2 & 6.35 \\
\hline TMEM165 & Transmembrane protein 165 & 2 & 7.87 \\
\hline TFRC & Transferrin receptor protein 1 & 2 & 4.48 \\
\hline ELAVL1 & Isoform 2 of ELAV-like protein 1 & 2 & 5.98 \\
\hline DPM1 & Dolichol-phosphate mannosyltransferase subunit 1 & 2 & 10.2 \\
\hline PRPSAP1 & $\begin{array}{c}\text { Isoform } 2 \text { of Phosphoribosyl pyrophosphate synthase-associated } \\
\text { protein } 1\end{array}$ & 2 & 5.99 \\
\hline SRSF6 & Serine/arginine-rich splicing factor 6 & 2 & 4.77 \\
\hline IKBKG & Isoform 2 of NF-kappa-B essential modulator & 2 & 5.36 \\
\hline SQSTM1 & Sequestosome-1 & 2 & 6.15 \\
\hline ITPK1 & Inositol-tetrakisphosphate 1-kinase & 2 & 5.4 \\
\hline ELAC2 & Zinc phosphodiesterase ELAC protein 2 & 2 & 4.5 \\
\hline AKAP8L & A-kinase anchor protein 8-like & 2 & 5.02 \\
\hline ADAM15 & Disintegrin and metalloproteinase domain-containing protein 15 & 2 & 5.27 \\
\hline DNAJC10 & DnaJ homolog subfamily C member 10 & 2 & 4.65 \\
\hline TBK1 & Serine/threonine-protein kinase TBK1 & 2 & 6.2 \\
\hline SNX1 & Isoform 3 of Sorting nexin-1 & 2 & 4.33 \\
\hline DHCR7 & 7-dehydrocholesterol reductase & 2 & 5 \\
\hline TIMM50 & $\begin{array}{c}\text { Isoform } 2 \text { of Mitochondrial import inner membrane translocase } \\
\text { subunit TIM50 }\end{array}$ & 2 & 4.47 \\
\hline PKP2 & Plakophilin-2 & 2 & 5.88 \\
\hline RALY & RNA-binding protein Raly & 2 & 4.19 \\
\hline NDUFS3 & NADH dehydrogenase iron-sulfur protein 3, mitochondrial & 2 & 5.83 \\
\hline SMARCB1 & $\begin{array}{c}\text { SWI/SNF-related matrix-associated actin-dependent regulator of } \\
\text { chromatin subfamily B member } 1\end{array}$ & 2 & 5.09 \\
\hline GART & Trifunctional purine biosynthetic protein adenosine-3 & 2 & 11.62 \\
\hline EMD & Emerin & 2 & 4.17 \\
\hline SLFN11 & Schlafen family member 11 & 2 & 4.62 \\
\hline GGCX & Vitamin K-dependent gamma-carboxylase & 2 & 4.18 \\
\hline CDK9 & Isoform 2 of Cyclin-dependent kinase 9 & 2 & 10.8 \\
\hline AP2M1 & AP-2 complex subunit mu & 2 & 4.09 \\
\hline SLC27A4 & Long-chain fatty acid transport protein 4 & 2 & 4.08 \\
\hline HOOK2 & Protein Hook homolog 2 & 2 & 5.64 \\
\hline FARSB & Phenylalanine--tRNA ligase beta subunit & 2 & 4.33 \\
\hline TBC1D5 & Isoform 2 of $\mathrm{TBC} 1$ domain family member 5 & 2 & 4.5 \\
\hline NCAPH2 & Isoform 4 of Condensin- 2 complex subunit $\mathrm{H} 2$ & 2 & 4.38 \\
\hline RBFA & Putative ribosome-binding factor $\mathrm{A}$, mitochondrial & 2 & 3.92 \\
\hline TRAFD1 & TRAF-type zinc finger domain-containing protein 1 & 2 & 5.42 \\
\hline ZW10 & Centromere/kinetochore protein zw10 homolog & 2 & 4.72 \\
\hline RHOT2 & Mitochondrial Rho GTPase 2 & 2 & 4.75 \\
\hline $\mathrm{CCDC} 47$ & Coiled-coil domain-containing protein 47 & 2 & 4.93 \\
\hline NLRP2 & NACHT, LRR and PYD domains-containing protein 2 & 2 & 4.73 \\
\hline NEU1 & Sialidase-1 & 2 & 4.13 \\
\hline
\end{tabular}




$\begin{array}{cccc}\text { RCOR1 } & \text { REST corepressor 1 } & 2 & 4.47 \\ \text { SMARCD2 } & \text { SWI/SNF-related matrix-associated actin-dependent regulator of } & 2 & 4.82 \\ \text { POLDIP3 } & \text { chromatin subfamily D member 2 } & 2 & 4.53 \\ \text { DNAJB12 } & \text { Polymerase delta-interacting protein 3 } & 2 & 4.55 \\ \text { TRIM28 } & \text { Isoform 2 of DnaJ homolog subfamily B member 12 } & 2 & 3.96 \\ \text { PSMB6 } & \text { Transcription intermediary factor 1-beta } & 2 & 5.03 \\ \text { DNAJB2 } & \text { Proteasome subunit beta type-6 } & 2 & 4.73 \\ \text { REPIN1 } & \text { DnaJ homolog subfamily B member 2 } & 2 & 5.43 \\ \text { AKAP10 } & \text { Isoform 2 of Replication initiator 1 } & 2 & 5.15 \\ \text { GCDH } & \text { A-kinase anchor protein 10, mitochondrial } & 2 & 4.97 \\ \text { CDC73 } & \text { Glutaryl-CoA dehydrogenase, mitochondrial } & 2 & 4.36 \\ \text { RPN2 } & \text { Parafibromin } & 2 & 4.14 \\ \text { ABCD3 } & \text { Dolichyl-diphosphooligosaccharide--protein glycosyltransferase } & 2 & 7.75 \\ \text { MSH2 } & \text { subunit 2 } & 2 & 5.11 \\ \text { TAP1 } & \text { ATP-binding cassette sub-family D member 3 } & 2 & 6 \\ \text { EXOC4 } & \text { DNA mismatch repair protein Msh2 } & 2 & 5.46 \\ \text { EXOC5 } & \text { Antigen peptide transporter 1 } & 2 & 4.77 \\ \text { SSBP1 } & \text { Exocyst complex component 4 } & 2 & 4.51 \\ \text { BUB3 } & \text { Exocyst complex component 5 } & 2 & 4.54 \\ \text { PPP2R1A } & \text { Serine/threonine-protein phosphatase 2A 65 kDa regulatory } & 2 & 4.01 \\ \text { DECR2 } & \text { subunit A alpha isoform } & 2 & 4.89 \\ \text { TELO2 } & \text { Peroxisomal 2,4-dienoyl-CoA reductase } & 2 & 4.06 \\ \text { STAU1 } & \text { Telomere length regulation protein TEL2 homolog } & 2 & 6.16 \\ \text { OXSR1 } & \text { Double-stranded RNA-binding protein Staufen homolog 1 } & 2 & 4.07 \\ \text { RAB5A } & \text { Serine/threonine-protein kinase OSR1 } & 2 & 5.09 \\ \text { STIP1 } & \text { Ras-related protein Rab-5A } & 2 & 4.33 \\ \text { UMPS } & \text { Isoform 2 of Stress-induced-phosphoprotein 1 } & 2 & 4.33 \\ \text { PRPF4 } & \text { Uridine 5'-monophosphate synthase } & 2 & 4.42 \\ \text { FKBP5 } & \text { U4/U6 small nuclear ribonucleoprotein Prp4 } & 6.68 \\ \text { ESRP1 } & \text { Peptidyl-prolyl cis-trans isomerase FKBP5 } & 9.57\end{array}$


were used to evaluate the strongest candidate peptides, stemming from a cross correlation of experimental and theoretical spectra. CRAPome was used to calculate stringent fold change calculations (FC-B) based on spectral peptide counts and negative controls. The top $25 \%$ of peptides within each stringency criteria were compared, this being number of peptide fragments detected, SEQUEST HT score, and FC-B score. Protein IDs belonging to at least 2 of 3 of these stringencies were accepted into a final list of "real" interactors (Table 30). This list again shows HDAC1/2 and BAHD1 as some of the strongest interactors. Broadly it includes proteins involved in signaling, apoptosis, antiviral response, chaperones, chromatin interaction, nuclear transport, transcription factors, mitochondrial transport and metabolism, steroid and fat transport and metabolism, translation, membrane dynamics, and cell proliferation.

The list of top protein interactors based on our stringency criteria were used for all subsequent analysis into the function of MIER3. The Gene Ontology Consortium was used to analyze this list based on common biological processes (Table 31) and shared molecular functions (Table 32). The independent ontologies provide a fold enrichment for each process or function based on the number of proteins from the input compared to what would be expected of all proteins. P values are derived from stringent Bonferroni corrections. In evaluating biological processes, the largest fold enrichment stems from C4-dicarboxylate transport, due to 4 members of the mitochondrial solute carrier family. Other processes with large fold enrichments but encompassing a larger number of proteins from the input list are ribose phosphate metabolism, purine ribonucleotide metabolism, and purinecontaining compound metabolic process, which include proteins prevalent in acyl-CoA 
Table 30. Top 25\% of mass spectrometry peptide IDs meeting Contaminant

Repository for Affinity Purification (CRAPome) stringency criteria unique to

MIER3 from digestion of individual bands in GFP-trap pulldown SDS-PAGE gels

\begin{tabular}{|c|c|c|c|c|}
\hline Gene & Description & Sequest HT & FC_B & \# Peptides \\
\hline MIER3 & Mesoderm induction early response protein 3 & 536.21 & & 15 \\
\hline LMNA & Prelamin-A/C & 140.31 & 26.86 & 27 \\
\hline HDAC2 & Histone deacetylase 2 & 90.93 & 6.73 & 13 \\
\hline HDAC1 & Histone deacetylase 1 & 90.36 & 6.73 & 13 \\
\hline BAHD1 & Bromo adjacent homology domain-containing 1 protein & 75.8 & 10.58 & 10 \\
\hline SLC25A13 & $\begin{array}{c}\text { Isoform } 2 \text { of Calcium-binding mitochondrial carrier } \\
\text { protein Aralar2 }\end{array}$ & 59.72 & 14.41 & 14 \\
\hline ATP2A2 & Sarcoplasmic/endoplasmic reticulum calcium ATPase 2 & 58.57 & 15.37 & 15 \\
\hline IDH2 & Isocitrate dehydrogenase, mitochondrial & 51.32 & 10.58 & \\
\hline STAT1 & $\begin{array}{l}\text { Signal transducer and activator of transcription 1- } \\
\text { alpha/beta }\end{array}$ & 44.25 & 12.49 & 12 \\
\hline LMNB1 & Lamin-B1 & 43.58 & 13.45 & 13 \\
\hline SLC25A6 & ADP/ATP translocase 3 & 43.51 & & 7 \\
\hline SLC25A4 & ADP/ATP translocase 1 & 40.82 & & 6 \\
\hline ATAD3A & $\begin{array}{c}\text { Isoform } 2 \text { of ATPase family AAA domain-containing } \\
\text { protein } 3 \mathrm{~A}\end{array}$ & 40.16 & 6.25 & 12 \\
\hline KPNA2 & Importin subunit alpha-1 & 39.96 & 10.58 & 10 \\
\hline ATP1A1 & Sodium/potassium-transporting ATPase subunit alpha-1 & 39.78 & 13.45 & 13 \\
\hline MAGED2 & Melanoma-associated antigen D2 & 38.65 & 9.62 & 9 \\
\hline GALK1 & Isoform 2 of Galactokinase & 36.4 & 8.66 & 8 \\
\hline OAS2 & 2'-5'-oligoadenylate synthase 2 & 32.12 & 9.62 & 9 \\
\hline SLC25A12 & Calcium-binding mitochondrial carrier protein Aralar1 & 32.03 & 10.58 & 10 \\
\hline FAR1 & Fatty acyl-CoA reductase 1 & 31.17 & 8.66 & 8 \\
\hline BAG3 & BAG family molecular chaperone regulator 3 & 30.2 & 6.75 & \\
\hline SAMHD1 & $\begin{array}{l}\text { Deoxynucleoside triphosphate triphosphohydrolase } \\
\text { SAMHD1 }\end{array}$ & 29.99 & 11.54 & 11 \\
\hline FARSA & Phenylalanine--tRNA ligase alpha subunit & 27.74 & 6.75 & 6 \\
\hline TRIP13 & Pachytene checkpoint protein 2 homolog & 26.15 & 8.66 & 8 \\
\hline ACAD11 & Acyl-CoA dehydrogenase family member 11 & 25.92 & 5.79 & 5 \\
\hline EHHADH & Peroxisomal bifunctional enzyme & 25.37 & 6.75 & 6 \\
\hline FXR1 & Fragile $\mathrm{X}$ mental retardation syndrome-related protein 1 & 25.21 & 6.75 & 6 \\
\hline HLA-B & $\begin{array}{l}\text { HLA class I histocompatibility antigen, B-37 alpha } \\
\text { chain }\end{array}$ & 24.96 & 6.75 & 6 \\
\hline PRKDC & DNA-dependent protein kinase catalytic subunit & 21.72 & 8.66 & 8 \\
\hline AIFM1 & Apoptosis-inducing factor 1, mitochondrial & 21.26 & 9.62 & 9 \\
\hline CNP & 2',3'-cyclic-nucleotide 3'-phosphodiesterase & 19.94 & 7.7 & \\
\hline HNRNPC & Heterogeneous nuclear ribonucleoproteins $\mathrm{C} 1 / \mathrm{C} 2$ & 19.84 & & 7 \\
\hline ABCF3 & ATP-binding cassette sub-family F member 3 & 19.81 & 6.75 & 6 \\
\hline ESYT2 & Isoform 2 of Extended synaptotagmin-2 & 19.58 & 6.75 & 6 \\
\hline FOXK1 & Forkhead box protein K1 & 19.55 & 6.75 & 6 \\
\hline ARAF & Serine/threonine-protein kinase A-Raf & 19.46 & 6.75 & 6 \\
\hline YME1L1 & ATP-dependent zinc metalloprotease YME1L1 & 18.94 & 5.79 & 5 \\
\hline IFIT3 & Interferon-induced protein with tetratricopeptide repeats & 18.41 & 6.75 & 6 \\
\hline KPNA1 & Importin subunit alpha-5 & 17.33 & 4.83 & 4 \\
\hline MCCC2 & $\begin{array}{l}\text { Methylcrotonoyl-CoA carboxylase beta chain, } \\
\text { mitochondrial }\end{array}$ & 16.94 & 6.75 & 6 \\
\hline ERLIN1 & Erlin-1 & 16.72 & 5.79 & 5 \\
\hline UNC45A & Protein unc-45 homolog A & 16.56 & 7.7 & 7 \\
\hline EHD4 & EH domain-containing protein 4 & 16.52 & 6.75 & 6 \\
\hline SLC25A11 & Mitochondrial 2-oxoglutarate/malate carrier protein & 16.47 & 4.83 & 4 \\
\hline DNAJA1 & DnaJ homolog subfamily A member 1 & 16.29 & 4.83 & 4 \\
\hline
\end{tabular}




\begin{tabular}{|c|c|c|c|c|}
\hline MARK2 & Serine/threonine-protein kinase MARK2 & 16.2 & 5.79 & 5 \\
\hline CKAP4 & Cytoskeleton-associated protein 4 & 16.16 & 6.75 & 6 \\
\hline ZC3HAV1 & Zinc finger $\mathrm{CCCH}$-type antiviral protein 1 & 16.05 & 4.83 & 4 \\
\hline ESRP2 & Epithelial splicing regulatory protein 2 & 15.43 & 5.79 & \\
\hline SLC25A10 & Isoform 2 of Mitochondrial dicarboxylate carrier & 15.29 & 5.79 & 5 \\
\hline HADHB & Trifunctional enzyme subunit beta, mitochondrial & 15.19 & 5.79 & \\
\hline LGALS3BP & Galectin-3-binding protein & 14.86 & & 4 \\
\hline IMPDH2 & Inosine-5'-monophosphate dehydrogenase 2 & 14.75 & 5.79 & 5 \\
\hline DHCR24 & Delta(24)-sterol reductase & 14.48 & & 4 \\
\hline GFPT1 & Glutamine--fructose-6-phosphate aminotransferase 1 & 13.75 & 5.79 & 5 \\
\hline RAF1 & $\begin{array}{c}\text { Isoform } 2 \text { of RAF proto-oncogene serine/threonine- } \\
\text { protein kinase }\end{array}$ & 13.62 & 4.83 & 4 \\
\hline ACSL3 & Long-chain-fatty-acid--CoA ligase 3 & 13.31 & 6.75 & 6 \\
\hline ACSL1 & Long-chain-fatty-acid--CoA ligase 1 & 13.27 & 5.79 & \\
\hline SUCLA2 & Succinate--CoA ligase subunit beta, mitochondrial & 13.15 & 5.79 & 5 \\
\hline DTX3L & E3 ubiquitin-protein ligase DTX3L & 13.04 & 5.79 & 5 \\
\hline FASN & Fatty acid synthase & 12.63 & 5.79 & 5 \\
\hline LARP4B & La-related protein $4 \mathrm{~B}$ & 12.25 & 5.79 & 5 \\
\hline EHD1 & EH domain-containing protein 1 & 11.54 & 5.79 & 5 \\
\hline SSH3 & Protein phosphatase Slingshot homolog 3 & & 4.83 & 4 \\
\hline RFC2 & Replication factor $\mathrm{C}$ subunit 2 & & 4.83 & 4 \\
\hline PARP9 & Protein mono-ADP-ribosyltransferase PARP9 & & 4.83 & 4 \\
\hline DYNC1LI1 & Cytoplasmic dynein 1 light intermediate chain 1 & 11.64 & 4.83 & 4 \\
\hline GNL3L & Guanine nucleotide-binding protein-like 3-like protein & & 4.83 & 4 \\
\hline UGDH & UDP-glucose 6-dehydrogenase & & 4.83 & 4 \\
\hline SPTLC1 & Serine palmitoyltransferase 2 & & 4.83 & 4 \\
\hline
\end{tabular}

Bold: Top 25\% in all 3 selection criteria

Red: Recapitulates findings reported in literature 
Table 31. Gene ontology based on biological process of proteins meeting top stringency criteria

\begin{tabular}{|c|c|c|c|c|}
\hline GO Biological Process & \# Proteins & $\begin{array}{c}\# \\
\text { Expected }\end{array}$ & $\begin{array}{c}\text { Fold } \\
\text { Enrichment }\end{array}$ & P value \\
\hline metabolic process & 49 & 28.93 & 1.69 & $1.88 \mathrm{E}-02$ \\
\hline cellular metabolic process & 46 & 26.23 & 1.75 & $2.34 \mathrm{E}-02$ \\
\hline primary metabolic process & 45 & 25.55 & 1.76 & $2.46 \mathrm{E}-02$ \\
\hline organic cyclic compound metabolic process & 29 & 10.84 & 2.67 & $1.71 \mathrm{E}-03$ \\
\hline cellular nitrogen compound metabolic process & 28 & 11.46 & 2.44 & $2.02 \mathrm{E}-02$ \\
\hline response to organic substance & 27 & 10.22 & 2.64 & $7.28 \mathrm{E}-03$ \\
\hline cellular aromatic compound metabolic process & 27 & 10.05 & 2.69 & $5.20 \mathrm{E}-03$ \\
\hline nucleobase-containing compound metabolic process & 27 & 9.22 & 2.93 & $9.06 \mathrm{E}-04$ \\
\hline heterocycle metabolic process & 27 & 9.88 & 2.73 & $3.69 \mathrm{E}-03$ \\
\hline small molecule metabolic process & 26 & 5.87 & 4.43 & $3.01 \mathrm{E}-07$ \\
\hline phosphorus metabolic process & 24 & 7.41 & 3.24 & $1.01 \mathrm{E}-03$ \\
\hline phosphate-containing compound metabolic process & 23 & 7.32 & 3.14 & $3.52 \mathrm{E}-03$ \\
\hline interspecies interaction between organisms & 21 & 7.22 & 2.91 & $4.60 \mathrm{E}-02$ \\
\hline organophosphate metabolic process & 17 & 3.04 & 5.59 & $6.13 \mathrm{E}-05$ \\
\hline organic acid metabolic process & 16 & 3.42 & 4.68 & $2.07 \mathrm{E}-03$ \\
\hline organic cyclic compound biosynthetic process & 16 & 4.15 & 3.86 & $2.55 \mathrm{E}-02$ \\
\hline oxoacid metabolic process & 16 & 3.35 & 4.78 & $1.56 \mathrm{E}-03$ \\
\hline oxidation-reduction process & 16 & 3.25 & 4.92 & $1.06 \mathrm{E}-03$ \\
\hline $\begin{array}{l}\text { nucleobase-containing small molecule metabolic } \\
\text { process }\end{array}$ & 16 & 1.76 & 9.07 & 2.03E-07 \\
\hline carboxylic acid metabolic process & 16 & 3.04 & 5.27 & 4.17E-04 \\
\hline aromatic compound biosynthetic process & 15 & 3.68 & 4.07 & $2.89 \mathrm{E}-02$ \\
\hline heterocycle biosynthetic process & 15 & 3.64 & 4.12 & $2.55 \mathrm{E}-02$ \\
\hline nucleoside phosphate metabolic process & 14 & 1.49 & 9.38 & $2.78 \mathrm{E}-06$ \\
\hline nucleotide metabolic process & 14 & 1.46 & 9.6 & $2.08 \mathrm{E}-06$ \\
\hline small molecule biosynthetic process & 13 & 1.98 & 6.55 & $8.18 \mathrm{E}-04$ \\
\hline purine nucleotide metabolic process & 11 & 1.14 & 9.63 & $2.08 \mathrm{E}-04$ \\
\hline purine-containing compound metabolic process & 11 & 1.26 & 8.75 & $5.39 \mathrm{E}-04$ \\
\hline ribonucleotide metabolic process & 10 & 1.11 & 8.97 & $1.82 \mathrm{E}-03$ \\
\hline purine ribonucleotide metabolic process & 10 & 1.06 & 9.46 & $1.13 \mathrm{E}-03$ \\
\hline ribose phosphate metabolic process & 10 & 1.15 & 8.7 & $2.38 \mathrm{E}-03$ \\
\hline defense response to virus & 9 & 0.73 & 12.37 & $5.65 \mathrm{E}-04$ \\
\hline response to virus & 9 & 1.03 & 8.77 & $9.34 \mathrm{E}-03$ \\
\hline type I interferon signaling pathway & 5 & 0.23 & 21.63 & $4.41 \mathrm{E}-02$ \\
\hline cellular response to type I interferon & 5 & 0.23 & 21.63 & $4.41 \mathrm{E}-02$ \\
\hline C4-dicarboxylate transport & 4 & 0.08 & 51.17 & $1.82 \mathrm{E}-02$ \\
\hline
\end{tabular}


Table 32. Gene ontology based on molecular function of proteins meeting top stringency criteria

\begin{tabular}{ccccc}
\hline GO Molecular Function & $\begin{array}{c}\# \\
\text { Proteins }\end{array}$ & $\begin{array}{c}\# \\
\text { Expected }\end{array}$ & $\begin{array}{c}\text { Fold } \\
\text { Enrichment }\end{array}$ & P value \\
\hline protein binding & 65 & 48.8 & 1.33 & $1.45 \mathrm{E}-02$ \\
organic cyclic compound binding & 51 & 20.45 & 2.49 & $1.87 \mathrm{E}-10$ \\
heterocyclic compound binding & 50 & 20.15 & 2.48 & $6.27 \mathrm{E}-10$ \\
catalytic activity & 40 & 19.6 & 2.04 & $1.04 \mathrm{E}-03$ \\
small molecule binding & 35 & 8.73 & 4.01 & $9.47 \mathrm{E}-11$ \\
nucleoside phosphate binding & 33 & 7.36 & 4.49 & $3.31 \mathrm{E}-11$ \\
nucleotide binding & 33 & 7.35 & 4.49 & $3.27 \mathrm{E}-11$ \\
anion binding & 31 & 9.77 & 3.17 & $2.45 \mathrm{E}-06$ \\
carbohydrate derivative binding & 26 & 7.72 & 3.37 & $3.28 \mathrm{E}-05$ \\
purine nucleotide binding & 25 & 6.55 & 3.82 & $5.64 \mathrm{E}-06$ \\
purine ribonucleoside triphosphate binding & 24 & 6.26 & 3.83 & $1.25 \mathrm{E}-05$ \\
purine ribonucleotide binding & 24 & 6.5 & 3.69 & $2.58 \mathrm{E}-05$ \\
ribonucleotide binding & 24 & 6.56 & 3.66 & $3.07 \mathrm{E}-05$ \\
adenyl nucleotide binding & 23 & 5.34 & 4.3 & $3.30 \mathrm{E}-06$ \\
adenyl ribonucleotide binding & 22 & 5.3 & 4.15 & $1.66 \mathrm{E}-05$ \\
ATP binding & 22 & 5.09 & 4.32 & $7.86 \mathrm{E}-06$ \\
RNA binding & 21 & 5.72 & 3.67 & $3.27 \mathrm{E}-04$ \\
cadherin binding & 10 & 1.1 & 9.11 & $4.99 \mathrm{E}-04$ \\
cell adhesion molecule binding & 10 & 1.84 & 5.44 & $4.54 \mathrm{E}-02$ \\
donors & 6 & 0.45 & 13.27 & $2.20 \mathrm{E}-02$ \\
activity, acting on CH-OH group of & & & & \\
activity & 4 & 0.11 & 35.66 & $2.11 \mathrm{E}-02$ \\
oxidoreductase & 4 & 0.05 & 73.55 & $1.61 \mathrm{E}-03$ \\
adenine nucleotide transmembrane transporter & 4 & 0.12 & 34.61 & $2.35 \mathrm{E}-02$ \\
C4-dicarboxylate transmembrane transporter activity & 4.52 & 3.97 & $3.52 \mathrm{E}-02$ \\
purine nucleotide transmembrane transporter activity & 4 & 0.13 & 32.03 & $1.16 \mathrm{E}-02$ \\
nucleotide transmembrane transporter activity & 4 & 0.1 & 42.03 \\
ATP transmembrane transporter activity & 4 & 0.11 & 35.66 & $2.11 \mathrm{E}-02$ \\
purine ribonucleotide transmembrane transporter & 4 & & & \\
activity & 4 & 0.13 & 30.18 & $3.87 \mathrm{E}-02$ \\
\hline dicarboxylic acid transmembrane transporter activity & 4 & & &
\end{tabular}


synthesis, mitochondrial metabolism, and fatty acid metabolism. Additionally, the viral response proteins rank highly. The largest fold enrichments in analyzing molecular function of our input proteins largely include transmembrane transporter activity, necessary for the previous mitochondrial and fatty acid metabolic proteins identified. Ribonucleotide and nucleotide binding functions do not have as large of fold enrichments but encompass a much greater proportion of the input proteins.

\section{MIER3 interacts strongly with nuclear proteins involved in DNA metabolism and chromosome organization}

Within my list of top interactors, $25 / 69$ proteins are located at the mitochondrion while 28/69 serve a nuclear function. Nuclear localization of MIER3 has been established [256], making the appearance of a large number of cytosolic and mitochondrial proteins perplexing. MIER3 has protein domains specific for tight protein-protein interactions, so MIER3 overexpression may promote a large non-nuclear presence and facilitate protein interactions that may not otherwise occur. As a result, I chose to further narrow my top interactors list by solely focusing on nuclear proteins to parse out the most biologically relevant interactions and provide greater insight into the nuclear function of MIER3. The nuclear proteins from the list of top interactors (Table 30) were input into ToppGene annotations, which provided more detailed gene ontology lists than the Gene Ontology Consortium. Table 33 lists the shared biological processes and Table 34 gives the molecular functions. The greatest number of input proteins participate in regulation of DNA metabolism and chromosome organization. The major function of these proteins is in chromatin and transcription factor binding, deacetylase activity, and catalytic activity on 
Table 33. Gene ontology based on biological process of nuclear proteins meeting top stringency criteria

\begin{tabular}{ccccc}
\hline GO Biological Process & $\begin{array}{c}\text { Genes from } \\
\text { Input* }\end{array}$ & $\begin{array}{c}\text { Genes in } \\
\text { Annotation }\end{array}$ & P value & Bonferroni \\
\hline DNA metabolic process & 13 & 1080 & $8.537 \mathrm{E}-10$ & $1.49 \mathrm{E}-06$ \\
chromosome organization & 12 & 1287 & $8.222 \mathrm{E}-08$ & $1.44 \mathrm{E}-04$ \\
regulation of response to stress & 11 & 1671 & 0.00001049 & $1.83 \mathrm{E}-02$ \\
regulation of DNA metabolic process & 10 & 389 & $9.266 \mathrm{E}-11$ & $1.62 \mathrm{E}-07$ \\
negative regulation of cellular component & 8 & 819 & 0.00001445 & $2.52 \mathrm{E}-02$ \\
organization & 8 & 669 & $3.277 \mathrm{E}-06$ & $5.73 \mathrm{E}-03$ \\
apoptotic signaling pathway & 7 & 556 & 0.0000108 & $1.89 \mathrm{E}-02$ \\
regulation of response to biotic stimulus & 6 & 300 & 0.00000369 & $6.45 \mathrm{E}-03$ \\
protein localization to nucleus & 5 & 167 & $3.725 \mathrm{E}-06$ & $6.51 \mathrm{E}-03$ \\
telomere maintenance & 5 & 180 & $5.372 \mathrm{E}-06$ & $9.39 \mathrm{E}-03$ \\
telomere organization & 5 & 251 & 0.00002681 & $4.68 \mathrm{E}-02$ \\
double-strand break repair & 4 & 84 & $6.166 \mathrm{E}-06$ & $1.08 \mathrm{E}-02$ \\
regulation of defense response to virus & 3 & 15 & $1.206 \mathrm{E}-06$ & $2.11 \mathrm{E}-03$ \\
positive regulation of double-strand break & & & & \\
repair via nonhomologous end joining & & & &
\end{tabular}

* 29 total genes were input 
Table 34. Gene ontology based on molecular function of nuclear proteins meeting top stringency criteria

\begin{tabular}{ccccc}
\hline GO Molecular Function & $\begin{array}{c}\text { Genes from } \\
\text { Input* }\end{array}$ & $\begin{array}{c}\text { Genes in } \\
\text { Annotation }\end{array}$ & P value & Bonferroni \\
\hline chromatin binding & 7 & 629 & $3.21 \mathrm{E}-05$ & $8.41 \mathrm{E}-03$ \\
transcription factor binding & 7 & 750 & $9.77 \mathrm{E}-05$ & $2.56 \mathrm{E}-02$ \\
DNA-binding transcription factor binding & 6 & 396 & $2.32 \mathrm{E}-05$ & $6.09 \mathrm{E}-03$ \\
catalytic activity, acting on DNA & 6 & 364 & $1.44 \mathrm{E}-05$ & $3.78 \mathrm{E}-03$ \\
repressing transcription factor binding & 5 & 75 & $8.66 \mathrm{E}-08$ & $2.27 \mathrm{E}-05$ \\
RNA polymerase II-specific DNA-binding & 5 & 311 & $9.21 \mathrm{E}-05$ & $2.41 \mathrm{E}-02$ \\
transcription factor binding & 4 & 128 & $3.90 \mathrm{E}-05$ & $1.02 \mathrm{E}-02$ \\
histone deacetylase binding & 3 & 45 & $4.18 \mathrm{E}-05$ & $1.10 \mathrm{E}-02$ \\
histone deacetylase activity & 3 & 47 & $4.77 \mathrm{E}-05$ & $1.25 \mathrm{E}-02$ \\
protein deacetylase activity & 3 & 54 & $7.24 \mathrm{E}-05$ & $1.90 \mathrm{E}-02$ \\
promoter-specific chromatin binding & 3 & 55 & $7.65 \mathrm{E}-05$ & $2.01 \mathrm{E}-02$ \\
nucleosomal DNA binding & 3 & 30 & $1.22 \mathrm{E}-05$ & $3.18 \mathrm{E}-03$ \\
NAD+ ADP-ribosyltransferase activity & 3 & 35 & $1.95 \mathrm{E}-05$ & $5.11 \mathrm{E}-03$ \\
RNA polymerase II repressing transcription & & & & \\
factor binding & 3 & 56 & $8.08 \mathrm{E}-05$ & $2.12 \mathrm{E}-02$ \\
transferase activity, transferring pentosyl & & & & \\
groups & 2 & 11 & $1.20 \mathrm{E}-04$ & $3.13 \mathrm{E}-02$ \\
NAD-dependent histone deacetylase activity & 2 & 11 & $1.20 \mathrm{E}-04$ & $3.13 \mathrm{E}-02$ \\
$\begin{array}{c}\text { (H3-K14 specific) } \\
\text { histone deacetylase activity (H3-K14 specific) }\end{array}$ & 2 & 9 & $7.84 \mathrm{E}-05$ & $2.05 \mathrm{E}-02$ \\
STAT family protein binding & 2 & 3 & $6.57 \mathrm{E}-06$ & $1.72 \mathrm{E}-03$ \\
\hline Krueppel-associated box domain binding & 2 & & & \\
\hline
\end{tabular}

* 29 total genes were input 
DNA. Relevant to the potential role of MIER3 in a chromatin repressive complex, there are 7 proteins that directly bind chromatin: protein mono-ADP-ribosyltransferase PARP9, E3 ubiquitin-protein ligase DTX3L, bromo adjacent homology domain-containing 1 (BAHD1), heterogeneous nuclear ribonucleoproteins $\mathrm{C} 1 / \mathrm{C} 2$ (HNRNPC), signal transducer and activator of transcription 1 (STAT1), and histone deacetylase 1/2 (HDAC1/2). There are 11 proteins that participate in chromosome organization: DTX3L, BAHD1, HNRNPC, HDAC1/2, DNA-dependent protein kinase catalytic subunit (PRKDC), guanine nucleotide-binding protein-like 3-like protein (GNL3L), replication factor C subunit 2 (RFC2), lamin A/C (LMNA), pachytene checkpoint protein 2 homolog (TRIP13), and apoptosis-inducing factor 1 (AIFM1). There are an additional 4 proteins that participate in repressing transcription factor binding: STAT1, $\mathrm{HDAC} 1 / 2$, and importin subunit alpha-1 (KPNA2).

\section{MIER3 interacts with co-repressor proteins that potentially facilitate}

\section{heterochromatin formation and transcriptional regulation as a multi-protein}

\section{repressive complex}

Of the nuclear proteins, BAHD1, HDAC1/2, LMNA/LMNB1, STAT1, and KPNA2 are the strongest interactors. The binding of nuclear import adaptor protein KPNA2, and also the weaker presence of KPNA1, indicates this is the mechanism of MIER3 nuclear transport via its nuclear localization symbol. The interaction of MIER3 with HDAC1/2 and BAHD1 has been established [264, 578], and provide evidence of MIER3 existing in a repressive complex. HDAC1/2 are critical catalytic components to many corepressor complexes through their histone deacetylase activity, including Sin3A, 
NuRD, and CoREST complexes [579]. BAHD1 has been shown to recruit proteins involved in heterochromatin formation and gene silencing [580]. The novel interaction with the transcription factor STAT1 may indicate the gene regions a MIER3-containing repressive complex is recruited to and downstream pathways that are modulated in hormone-responsive breast cancer. Additionally, interaction with nuclear lamins may indicate a role in positioning this complex, structural organization of chromatin, and further promotion of heterochromatin formation [581]. This model is depicted in Figure 63, whereby MIER3 is imported into the nucleus via KPNA1/2, docked at the nuclear lamina, and positioned as an adaptor protein in binding STAT1, HDAC1/2 via the ELM2 domain, and BAHD1 via an LXXLL motif. The lamins-MIER3 scaffold position chromatin and HDAC1/2 in such a way that chromatin is deacetylated, and BAHD1 recognizes H3K27me3 marks and facilitates further chromatin condensation. STAT1 may direct this repressive complex to specific gene regions and promote transcriptional silencing.

\section{The top MIER3-interacting nuclear proteins have associations with breast cancer}

I input the nuclear proteins from the top MIER3 protein interactors list into the coexpression analysis function within Toppgene, and the top hits were from three breast cancer microarray expression studies. This is interesting since I purposefully selected the breast cancer cell line T47D for analysis due to the potential role of MIER3 in breast cancer development. Nuclear proteins coinciding with a breast cancer association are listed in Table 35 [444, 568, 569]. Within this list are STAT1, HDAC1/2, KPNA2, and LMNA. This supports a functional role of this potential repressive complex in gene silencing and heterochromatin formation not only in normal gene regulation but in breast cancer. 


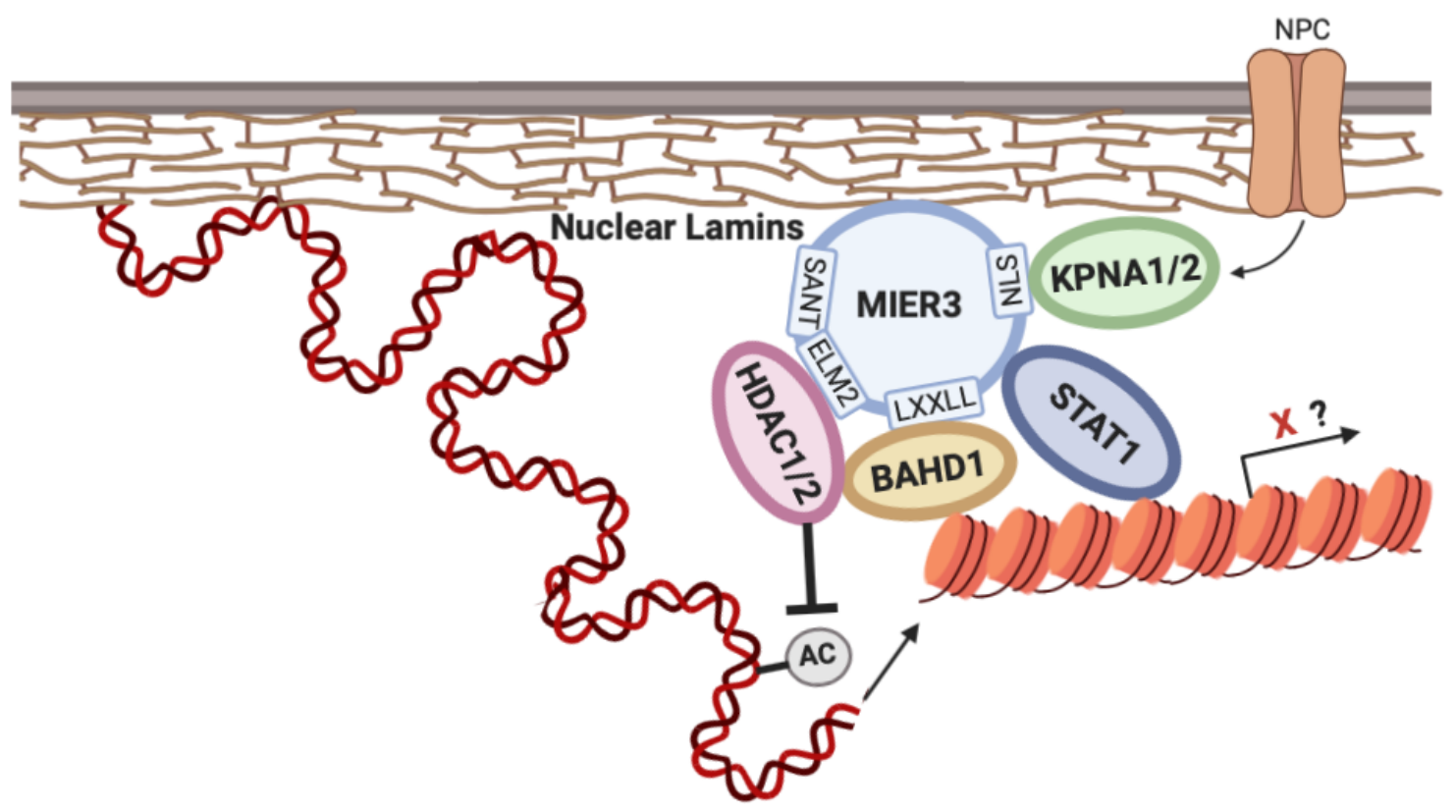

Figure 63: Model of the function of a novel HDAC1/2-containing MIER3 repressive complex in heterochromatin formation and gene repression in breast cancer. KPNA1/2 facilitates nuclear import of MIER3 where it is docked at the nuclear lamina. MIER3 serves an adaptor function in binding STAT1, HDAC1/2 via the ELM2 domain, and BAHD1 via an LXXLL motif. The lamins-MIER3 create a scaffold that positions chromatin and proteins for histone deacetylation by $\mathrm{HDAC} 1 / 2$ and recognition of H3K27me3 marks by BAHD1 that facilitates further chromatin condensation. STAT1 may direct this repressive complex to specific gene regions and promote transcriptional silencing. Created in BioRender (biorender.com). 
Table 35. Proteins meeting top stringency criteria associated with breast cancer and located in the nucleus

\begin{tabular}{|c|c|c|c|c|}
\hline Gene & Description & $\begin{array}{l}\text { Bertucci et } \\
\text { al. 2006* }\end{array}$ & $\begin{array}{l}\text { Van't Veer } \\
\text { et al. } 2002 * *\end{array}$ & $\begin{array}{l}\text { Mutarelli et } \\
\text { al. 2008*** }\end{array}$ \\
\hline IMPDH2 & Inosine-5'-monophosphate dehydrogenase & $\checkmark$ & $\checkmark$ & \\
\hline LMNA & Prelamin-A/C & $\checkmark$ & $\checkmark$ & \\
\hline MAGED2 & Melanoma-associated antigen D2 & $\checkmark$ & $\checkmark$ & \\
\hline SSH3 & Protein phosphatase Slingshot homolog 3 & $\checkmark$ & $\checkmark$ & $\checkmark$ \\
\hline SAMHD1 & $\begin{array}{l}\text { Deoxynucleoside triphosphate } \\
\text { triphosphohydrolase SAMHD1 }\end{array}$ & $\checkmark$ & $\checkmark$ & \\
\hline TRIP13 & Pachytene checkpoint protein 2 homolog & $\checkmark$ & $\checkmark$ & $\checkmark$ \\
\hline STAT1 & $\begin{array}{c}\text { Signal transducer and activator of } \\
\text { transcription } 1\end{array}$ & $\checkmark$ & $\checkmark$ & \\
\hline KPNA2 & Importin subunit alpha-1 & $\checkmark$ & $\checkmark$ & $\checkmark$ \\
\hline PARP9 & $\begin{array}{c}\text { Protein mono-ADP-ribosyltransferase } \\
\text { PARP9 }\end{array}$ & $\checkmark$ & & \\
\hline DTX3L & E3 ubiquitin-protein ligase DTX3L & $\checkmark$ & & \\
\hline PRKDC & $\begin{array}{l}\text { DNA-dependent protein kinase catalytic } \\
\text { subunit }\end{array}$ & $\checkmark$ & & $\checkmark$ \\
\hline DNAJA1 & DnaJ homolog subfamily A member 1 & $\checkmark$ & & $\checkmark$ \\
\hline HNRNPC & $\begin{array}{l}\text { Heterogeneous nuclear ribonucleoproteins } \\
\qquad \mathrm{C} 1 / \mathrm{C} 2\end{array}$ & $\checkmark$ & & \\
\hline ZC3HAV1 & Zinc finger $\mathrm{CCCH}$-type antiviral protein 1 & $\checkmark$ & & \\
\hline BAG3 & $\begin{array}{l}\text { BAG family molecular chaperone regulator } \\
3\end{array}$ & & $\checkmark$ & \\
\hline UGDH & UDP-glucose 6-dehydrogenase & & $\checkmark$ & $\checkmark$ \\
\hline HDAC2 & Histone deacetylase 2 & & $\checkmark$ & \\
\hline GNL3L & $\begin{array}{l}\text { Guanine nucleotide-binding protein-like 3- } \\
\text { like protein }\end{array}$ & & & $\checkmark$ \\
\hline RFC2 & Replication factor $\mathrm{C}$ subunit 2 & & & $\checkmark$ \\
\hline LMNB1 & Lamin-B1 & & & $\checkmark$ \\
\hline
\end{tabular}

Top hits were from breast cancer microarray expression data using co-expression analysis from Toppgene

* $\mathrm{P}$ value $1.038 \mathrm{E}-7$, Bonferroni 6.056E-4

** P value 1.382E-6, Bonferroni 8.062E-3

*** P value 9.039E-6, Bonferroni 3.100E-2 


\section{Discussion:}

Previous work in our lab and data in this dissertation has associated Mier3 with DMBA-induced rat mammary cancer susceptibility [256]. MIER3 is also increased in female breast tumors [582] and associates with lower overall survival and reduced relapsefree survival [583]. Here, I identified MIER3-interacting proteins in T47D luminal B breast cancer cells, which highlights a potential functional role of MIER3 in a complex that may promote heterochromatin formation and gene repression in breast cancer.

Although I utilized multiple cell lines to account for transfection issues, I repeatedly found MIER3 protein overexpression was only evident in a low number of cells, even with proteasome inhibitor treatment. This may be due to a stoichiometric imbalance, where overexpression of one member of a protein complex disrupts the molecular ratio that is otherwise normally kept in balance and leads to improper pathway modulation, abnormal complex formation, and cellular defects $[584,585]$. Since native MIER3 levels are low, it could be that overexpressed MIER3, not in complex with other proteins, leads to toxic effects and is targeted for rapid degradation. This could be particularly true for BAHD1 which also has intrinsically low protein levels, suggesting that MIER3-BAHD1 containing complexes must be tightly regulated and overexpression could be deleterious. The lack of MIER3 protein expression could also be due to resource overload, whereby overexpression requires too large of cellular resources for translation, folding, or localization [584]. MIER3 is likely imported into the nucleus via the karyopherin subunit beta 1 (KPNB1) pathway of nucleocytoplasmic transport based on strong interactions with adaptor proteins KPNA1/2. The strong demand for this pathway could exhaust its components, leading to unnatural accumulation in the cytoplasm. Build-up of cytosolic MIER3 could lead to 
promiscuous protein-protein interactions that are otherwise not biologically relevant [584]. It is important to note that intrinsically disordered proteins (IDPs), proteins lacking stable structures, enable non-physiological interactions [586, 587] and MIER3 is $>50 \%$ intrinsically disordered according to the Protein Disorder Prediction System (PrDOS) [588]. IDPs also tend to have low native expression levels [589], such as MIER3, of which overexpression and promiscuous interactions could modulate improper pathways or lead to degradation. This may explain why a major portion of MIER3 interacting proteins found following affinity purification and mass spectroscopy are involved in mitochondrial and fatty acid metabolism and transport. The structured regions of MIER3 - a SANT domain, ELM2 domain, and two LXXLL motifs - facilitate strong protein interactions. Compounded with the known promiscuous interactions of IDP regions, these pathways may be a result of forced interactions that could lead to MIER3 degradation. There would need to be additional experiments to determine if MIER3 does naturally exist in the cytoplasm and if these non-nuclear proteins are biologically relevant and indicate a cytosolic function of MIER3.

In addition to the overall goal of identifying the protein-protein interactions of MIER3 as an insight into its biological function, the specific hypothesis was that the LXXLL sequence motifs of MIER3 would interact with NRs. This hypothesis stemmed from the classical definition of the LXXLL motif as originally identified in coactivator proteins that bind directly to NRs in their ligand binding domains (LBD) and is referred to as the NR box [590]. In this mechanism, a ligand binds the LBD of a class II NR which releases a corepressor and allows the alpha-helical LXXLL motif of the coactivator to bind the NR and participate in transcriptional regulation of target genes [590, 591]. Corepressor 
proteins have extended LXXLL motifs while coactivators harbor the LXXLL [592]. MIER3 lacks the corepressor motif but possesses two LXXLLs. However, since no NR was identified in my mass spectrometry analysis, even following serum starvation and restimulation to induce NR expression, the function of the LXXLL motifs of MIER3 likely does not involve the canonical NR binding and transcriptional repression. A noncanonical role of the LXXLL motif was later identified, which showed the motif participates in protein-protein interactions with other regulatory proteins, including transcription factors and cofactors [593]. Thus, the LXXLL sequence is a protein recognition motif involved in transcriptional regulation in both NR-mediated and independent pathways. In my mass spectrometry analysis of MIER3 interactions, I found an interaction with BAHD1 which is reproducibly lost with the LXXAA double mutant that interrupts binding specificity. The specificity of the LXXLL motif for certain binding partners is complex, arising from the spacing and number of motifs which varies greatly between regulatory proteins, as well as the composition of the residues adjacent to the central motif [593, 594]. Thus, the double LXXLL motifs of MIER3 may modulate interaction with auxiliary transcriptional regulators given alternative tissue types, developmental, or disease states. However, BAHD1 appears to be a core interactor with MIER3 given reported interactions in human embryonic kidney (HEK293) and human proximal tubule (HPT) cells [578]. The MIER3BAHD1 interaction in breast cancer T47D cells in this study further displays BAHD1 appears to be a critical binding partner specific to the LXXLL motif and may possess a functional role in MIER3-associated susceptibility to breast cancer.

MIER3 is known to localize to the nucleus [256], so I chose to focus this study of MIER3-interacting proteins on those with nuclear functions, with BAHD1, HDAC1/2, 
LMNA/LMNB1, STAT1, and KPNA2 appearing as the strongest interactors. BAHD1 and HDAC1/2 have previously been reported to interact with MIER3, as well as a BAHD1 interaction with $\mathrm{HDAC} 1 / 2$, in other non-diseased cell lines [266, 578]. These proteins collectively may exist as core components of a nuclear complex with other more transient protein interactions. The bromo adjacent homology $(\mathrm{BAH})$ domain of BAHD1 is present in a number of nuclear proteins and has been demonstrated to facilitate chromatin processes such as protein-protein interactions, recognition of methylated histones, and nucleosome binding [595-597]. In addition to MIER3 and HDAC1/2, BAHD1 has been demonstrated to interact with KRAB-associated protein 1 (KAP1) and heterochromatin protein 1 (HP1 $\alpha$, $\beta, \gamma)$ which induce heterochromatin formation, the methyl-CpG-binding protein MBD1, and histone-lysine N-methyltransferases SETDB1 and SUV39-H1 [580, 598-600]. This supports a hypothesis that BAHD1 exists in a multiprotein repressive complex. HDAC1/2 are established core catalytic components of three co-repressor complexes that modulate transcription - NuRD (nucleosome remodeling deacetylase) [601, 602], Sin3A [603], and CoREST (co-repressor of RE1-silencing transcription factor) [604]. The components of these HDAC1/2-containing complexes possess DNA/chromatin recognition motifs and interact with transcription factors to target the HDACs to chromatin $[559,605]$. They incorporate different protein subunits which gives underlying specificity for genomic loci with context-dependent biological functions [560]. BAHD1 and MIER3 share domains found in other proteins within $\mathrm{HDAC} 1 / 2$ co-repressor complexes. For example, the metastasis-associated protein (MTA) subunits [606, 607] and RERE/atrophin-2 [608, 609] possess BAH domains and juxtaposed ELM2 and SANT domains. Thus, MIER3 and BAHD1 could serve similar co-repressive functions by creating a core scaffold that recruit 
HDACs via the established ELM2 domain [266], as well as combinatorial assembly of DNA binding transcription factors and chromatin modifying proteins to create a novel HDAC1/2 co-repressor complex.

Based on the established role of MIER1 in breast cancer development as part of a chromatin repressive complex, as well as the sequence homology to MIER3 and its association with breast cancer, MIER3 is projected to share a similar repressive role, though differing protein interactions may target a MIER3-complex to alternative loci and downstream pathways than MIER1. The function of HDAC1/2 and BAHD1 on histone modifications and recognition of repressive marks further indicates a MIER3-containing complex may have a role in facultative heterochromatin formation. This condensed higher order chromatin structure reduces access by transcriptional machinery and is achieved by the combination of functionally distinct proteins in complex that coordinate epigenetic modifications such as methylation of DNA and histone modifications [555, 558]. Maintenance of various regions of facultative heterochromatin is crucial between differing cell types, which give rise to alternative cellular functions. Therefore, deregulation of chromatin structure or the co-repressive proteins that promote it can have a role in various diseases, including breast cancer [610-612].

My analysis identified the nuclear lamins as strong interactors with MIER3 which further supports a chromatin co-repressor function of a MIER3-complex based on nuclear lamins' established role in chromosome positioning, epigenetic regulation of chromatin, and promotion of heterochromatin formation [613]. The nuclear lamins classically participate in the nucleoskeleton that supports the nuclear membrane, but also serve as docking sites for chromatin, transcription factors, and protein complexes involved in 
chromatin regulation $[581,614,615]$. Lamins participate in epigenetic regulation of DNA by binding histones and directly to DNA at scaffold/matrix attachment regions (S/MARs) [616-618]. The function of lamins in heterochromatin formation is observed in cells with LMNA mutations that results in a loss of heterochromatin from the periphery of nuclei $[581,613]$. These lamin-bound heterochromatin regions are referred to as lamin-associated domains (LADs), which are characterized by low gene density, minimal transcriptional activity, and repressive chromatin marks [619-621]. Hundreds of these LADs are further classified as facultative LADs, as they are cell type- and differentiation state-specific [619], generating an additional layer of context-dependent specificity of a bound co-repressor such as MIER3.

In addition to the nuclear lamins, BAHD1 also possesses an established role in facultative heterochromatin formation. In fact, BAHD1 overexpression induces large-scale chromatin condensation and DNA hypermethylation [580, 599]. Lamins and BAHD1 are both strong interactors with MIER3 and both may work in conjunction in heterochromatin formation as either a component of or in positioning a MIER3-containing complex. Of the BAHD1-induced heterochromatin domains, or BAHD1-associated differentially methylated domains (BADs), $60 \%$ overlap with LADs at the nuclear periphery [599]. It is also reported that the size distribution $(0.1-10 \mathrm{Mb})$ and the median sequence length $(0.5-$ $0.8 \mathrm{Mb}$ ) of LADs are very similar to that of BADs (0.3-6.5 Mb and median $0.5 \mathrm{Mb})$ [599]. BAHD1 has a further role in heterochromatin formation as the BAH domain is a H3K27me3 reader, a mark of facultative heterochromatin, and discriminates against H3K9me3, a mark of constitutive heterochromatin $[622,623]$. The BAD regions largely appear at satellites, interspersed repeats, and intergenic regions [599], though BAHD1 is 
also capable of directly binding to gene promoter regions, possibly by recognition of H3K27me3 marks, and recruiting repressive factors, thereby coordinating heterochromatin formation and gene silencing [580]. BAHD1 is known to bind HP1, MBD1, and HDAC5 [580], none of which appear as a MIER3 interactor. Thus, BAHD1 may exist in alternative repressive complexes that are context dependent, as these experiments were performed in HEK293 cells. Additionally, the MIER3-containing co-repressive complex may be larger and possess more heterochromatin factors than anticipated. For example, I did not identify any DNA methylation proteins in the MIER3 pulldowns in T47D cells, although methylation is an important factor in the formation of heterochromatin. However, MBD1 interacts with BAHD1 so may be present within a MIER3-containing complex despite no physical interaction with MIER3. It will be important to further investigate components of this repressive complex based on cell type, development, or disease state in order to marry the DNA methylation patterns, histone modifications, and transcription factor recruitment that drives the specificity and functionality of this complex.

The other core MIER3 interactors, HDAC1/2, are well characterized and possess an established role in facultative heterochromatin formation. They share $\sim 86 \%$ amino-acid sequence identity, often heterodimerize, and have high levels of functional redundancy [624-626]. HDAC1/2 do not have a DNA-binding domain, so exert their deacetylase function by interacting with transcription factors or as members of multi-component complexes, in which DNA-binding factors tether them to certain genomic loci $[556,561$, 627]. MIER3 may be an important tethering mechanism for this potentially novel complex, as it appears to bind the nuclear scaffold and the ELM2 domain has been established as crucial for HDAC1/2 binding in the MIER family [266]. The activity of HDAC1/2 in 
transcriptional silencing requires specific complex assembly, and the exact composition and recruitment proteins defines the repression of particular genomic regions and target genes [555, 628]. Through histone deacetylation, HDAC1/2 makes chromatin available for subsequent modifications by other complex components [628]. Within the MIER3 complex, deacetylation may allow BAHD1 and other histone-modifying factors associated with it to add additional repressive marks. In this way, layers of repression are created which all contribute to heterochromatin formation.

Of the MIER3-nuclear interactors, signal transducer and activator of transcription protein1 (STAT1) appears as the strongest transcription factor interactor. The STAT family of transcription factors are cytokine-activated proteins that have a role in the hematopoietic and immune systems [629]. In the canonical pathway of STAT1 function, STAT1 is activated by IFN type $1(\operatorname{IFN}-\alpha / \beta)$ or IFN type II (IFN $\gamma$ ) binding at membrane receptors [630], driving both downstream gene activation and repression [630, 631]. The type I pathway results in STAT1 and STAT2 trimerization with p48 to form the ISGF3 complex, which translocates into the nucleus and binds to interferon-stimulated response elements (ISREs) in the promoters of IFN $\alpha / \beta$-inducible genes [631]. The type II pathway results in STAT1 homodimerization that translocates into the nucleus and binds gamma activated sequence (GAS) in the promoters of IFN $\gamma$-responsive genes [632-634]. STAT1 also participates in constitutive activation of genes even without IFN activation [630]. This duality in activation and repression is likely due to cross talk between the numerous pathways STAT1 coordinates and the different interactions with transcriptional modulators $[630,635,636]$. 
The interaction of chromatin-remodeling complexes with transcription factors is thought to provide the biological specificity for these complexes by targeting them to certain genomic regions (39). Conversely, it is theorized that complexes are targeted to these regions by recognition of specific histone/DNA modifications, in which transcription factors bind after and exert their function [637]. Despite not understanding the order of appearance, transcription factors are crucial to co-repressor complexes in providing specificity of function that differs between cell types, as well as establishing and maintaining transcriptional repression $[558,560]$. The interaction of STAT1 with MIER3 seems similar to a reported interaction of STAT1 with protein inhibitor of activated STAT family (PIASy), in which the LXXLL motif of PIASy was found to be critical for repression of STAT1-mediated gene activation, although this motif does not facilitate the interaction with STAT1 or affect its DNA binding ability [591]. The authors proposed that PIASy may serve as an adaptor protein with the LXXLL motif facilitating the assembly of other components of a co-repressor complex with a functional role in repression of STAT1mediated gene activation [591]. MIER3 may participate with STAT1 in a similar manner, with a MIER3-repressive complex modulating STAT1 downstream pathways. However, the complexity of STAT1 signaling makes it difficult to hypothesize which genomic locations are bound and what are the possible downstream effects.

STAT1 and BAHD1 appear to function in a shared pathway, although no physical interaction between them has been demonstrated. BAHD1, along with HDAC1/2 and the co-repressor protein KAP1, are recruited at the promoters of certain interferon-stimulated genes (ISGs) and promote gene silencing [638-640]. The virulence factor LntA from Listeria monocytogenes infection displaces this BAHD1-HDAC1/2 complex from 
ISG promoters, de-repressing transcription [640, 641]. In this context, a BAHD1$\mathrm{HDACl} / 2$ complex is repressing transcription of ISGs that are normally expressed via STAT1-mediated transcription. Even though this was demonstrated in the context of listeriosis infection, it adds credibility to the premise that a MIER3-BAHD1-HDAC1/2 complex is participating in repression of STAT1 pathways. HDAC1 has also been shown to physically interact with STAT1 and modulate IFN- $\alpha$-induced transcription [638]. There is a general requirement for deacetylation in STAT1-dependent transcriptional regulation in response to IFN- $\alpha / \beta$ and IFN- $\gamma$ [638]. Although there are many regulatory complexes that contain HDAC1, a requirement for HDAC activity in STAT1-mediated pathways adds to a further potential functional role of a MIER3-containing co-repressive complex.

Since my T47D cells were not stimulated with IFNs, the contribution of STAT1 within a MIER3-containing complex likely diverges from its canonical role in interferon responses. There has been an increasing number of studies indicating that there are alternative mechanisms of STAT1 function compared to this standard model of IFNinduced STAT1 activation, including a role of unphosphorylated STAT1 (uSTAT1). In resting cells not stimulated with cytokines, STAT1 has been shown to be constitutively nuclear, accounting for up to $40 \%$ of total STAT1 [642]. In the nucleus, uSTAT1 can form stable homodimers [643] and uSTAT1 monomers are also capable of binding DNA [644]. uSTAT1 can form complexes with other transcription factors and accessory transcriptional regulators on DNA target sites, with specificity generated by the composition of the uSTAT1-complex [644]. Thus, uSTAT1 acts as a constitutive transcription factor and generates gene expression patterns entirely distinct from activated pSTAT1-homodimers or -heterotrimers [644]. Additionally, nuclear-localized uSTATs can promote 
heterochromatin formation, acting not only as transcriptional regulators at the expression level but also at the epigenetic level. This pathway was initially investigated in drosophila, in which the only encoded Stat protein, Stat92E, was found to bind Hp1 in its unphosphorylated form and localize to heterochromatin [645]. Stat activation lead to disruption of this complex and chromatin de-condensation, while increasing levels of uStat led to higher levels of heterochromatin [645]. This non-canonical function was found to be conserved in mammals and humans, in which uSTAT5A and USTAT3 have been shown to colocalize with HP1 1 , stabilize this interaction at heterochromatin, and promote heterochromatin formation [646, 647]. Although this non-canonical uSTAT function has not been investigated for uSTAT1, the association of STAT1 with BAHD1 and HDAC1/2, along with their established interaction with HP1 and role in heterochromatin formation, elevate this as a likely candidate function of STAT1 within a MIER3-repressive complex. Additionally, the established role of STAT1 as a constitutive transcription factor independent of IFN signaling indicates STAT1 is mediating non-canonical gene targeting and regulation within a MIER3 complex.

The ability of the identified MIER3-interacting proteins to modulate heterochromatin formation, and thus transcriptional repression, has potentially important ramifications on cancer biology, and our specific interest, the etiology of breast cancer. The epigenetic landscape found in breast cancer is largely characterized by hypomethylation of DNA accompanied by formation of repressive chromatin domains that leads to gene silencing [562]. These chromatin changes occur early in disease initiation and are thought to promote tumorigenesis by silencing tumor suppressor genes [562, 648]. Thus, chromatin repressive complexes serve a clear role in breast cancer development. I 
present my strongest experimental protein interactors with rational nuclear functions in my final model of MIER3 within a novel HDAC1/2-chromatin repressive complex in breast cancer. KPNA1/2 serve as adaptor proteins in a KPNB1 manner of MIER3 nuclear import. MIER3 is tethered to the nuclear lamin, and itself serves as an adaptor protein in binding BAHD1 via the LXXLL motifs, HDAC1/2 via the ELM2 domain, and STAT1 through an undetermined region. The lamins aid in positioning chromatin and organizing DNAprotein interactions. The order of DNA recognition remains unclear. Unphosphorylated STAT1 may lead targeted recognition of specific gene regions and promote transcriptional silencing. Additionally, BAHD1 may recognize and bind H3K27me3 marks, positioning HDAC1/2 for deacetylase activity and promoting facultative heterochromatin formation. The activity of this MIER3 co-repressive complex is potentially enhanced in breast cancer, which could contribute to greater levels of facultative heterochromatin and silencing of tumor suppressor genes. 


\section{CHAPTER VIII}

\section{CONCLUDING REMARKS}

Breast cancer is a complex disease modulated by interactions between genetic and environmental factors. The laboratory rat serves as a highly relevant model of human breast cancer for its use in studying the genetic components of breast cancer and evaluating molecular factors driving natural disease initiation and progression. There are eight identified quantitative trait loci in rats that associate with mammary cancer susceptibility (Mcs). This dissertation focuses on two of these genomic regions, Mcs3 and Mcs $1 b$, through genetic mapping and preliminary studies of $M c s 3$ and mammary gland expression analysis and mechanistic insight into Mcslb-nominated breast cancer gene MIER family member 3 (MIER3).

The Mcs3 locus was initially mapped to a $29.4 \mathrm{Mb}$ region on rat chromosome one which was too large to perform functional studies. As a precursory study, I selected candidate genes within $M c s 3$ that had known associations with human breast cancer and evaluated their expression in Mcs3-resistant and susceptible rat strains. I identified p21activated kinase (Pak1) as upregulated at the mRNA and protein level in a subset of carcinogen-exposed rat mammary glands prior to the development of any histopathological disease morphologies. These matched levels observed in human breast carcinomas and demonstrated a novel role of PAK1 in disease initiation. Although there was no effect of $M c s 3$ genotype on Pak1 expression (and the gene region was later excluded from the Mcs3 
locus by further mapping and narrowing), I elevated the rat as a valuable model to study the role of PAK1 in early human breast disease development.

Simultaneous to this study, congenic rat lines harboring differing portions of the Mcs3 locus were developed and evaluated for retained mammary cancer susceptibility or resistance phenotypes. From this, I concluded that Mcs 3 is located at a $7.2 \mathrm{Mb}$ region on rat chromosome 1: 143700228-150929594, which reduced the size of the locus by $75 \%$. I also discovered a novel pleiotropic effect of $M c s 3$ which confers reduced body mass in addition to mammary cancer resistance in those rats positive for this genetic region. In females, reduced body mass is dependent on separation from males, indicating an influence of pheromones and an environmental interplay with this genetic locus. Human GWASidentified variants that map to this region are also associated with body mass; however, none are associated with breast cancer. Further studies into this QTL are relevant. The shared genetic associations with body mass in the human and rat and the modulation of mammary cancer susceptibility in the rat, suggesting that there may also be unidentified breast cancer susceptibility genes in the orthologous human regions.

Another Mcs QTL, Mcs 1b, has been narrowed to only $1.2 \mathrm{Mb}$ and overlaps with human breast cancer risk allele 5q11.2. Encompassed in this overlap of the rat and human are two genes, Map $3 k 1$ and Mier3. Map $3 k 1$ is an established breast cancer gene so I chose to further our understanding of Mier 3 in rat mammary cancer by evaluating its expression at sequential pathological stages along a DMBA-induced model of mammary cancer development. I selected RNAscope to analyze the cell-specific expression and spatiotemporal context of Mier3 transcript abundance in preserved mammary tissue. Evaluating mammary glands for various disease lesions typically requires fixing whole mounted 
mammary glands, defatting, staining for epithelial structures, and excising and separately processing disease morphologies. However, I was unsure if this pre-screening process would be deleterious to mRNA integrity and the downstream quantifiability of the RNAscope assay. I developed a combinatorial method to pre-screen mammary glands for disease lesions followed by RNAscope assays. I proved that extended time and solvent exposures during whole mounting did not disrupt RNA integrity as the expression quantification of three separate genes was not different between mammary glands or carcinomas that were either processed immediately or whole mounted first. This is an important finding because in situ expression analysis is much more information rich than within homogenized tissue, but it is often critical to first observe or select for epithelial changes during normal mammary gland development, function, or disease progression.

Using RNAscope, I found that the luminal epithelial cells expressed the greatest levels of Mier3. This is critical given our knowledge of mammary cancer biology since these are the most proliferative cell types vulnerable to chemical insult and the majority of invasive ductal carcinomas, which account for $80 \%$ of diagnosed female breast cancers worldwide, demonstrate a luminal phenotype [114, 117, 218, 478]. Additionally, I found exposure to DMBA induces $>2 \mathrm{x}$ greater Mier3 expression in otherwise normal-appearing mammary ducts, indicating a potential role of Mier3 early in disease initiation. Overall, I found Mier3 expression significantly increases with each histopathological stage of a precursor-carcinoma disease sequence. This is a key finding that highlights a potential role of Mier3 not only in disease initiation but in tumorigenesis and warrants further study into the role of MIER3 in breast cancer development. Since I identified significant changes in Mier3 expression throughout disease progression, suggesting a functional role in tumor 
progression, I also evaluated Mier 3 expression in the normal (non-chemically-exposed) rat mammary gland during time windows of susceptibility during development and reproduction. These are critical windows in which the same factors that modify the developmental or growth profile of the tissue can alter or be utilized in carcinogenesis susceptibility later in life. Mier 3 abundance was highest in mammary epithelial cells during embryonic, neonatal, and pubertal development. Mier3 expression levels in embryonic mammary buds even surpassed that found in mammary tumors. Expression substantially dropped by adulthood and did not rise to the levels found in early development during the reproductive cycle. This study showed for the first time that in addition to a role in cancer, Mier3 potenitally has a temporal role during normal embryonic and post-natal development of the mammary gland. Understanding the function of Mier3 during this pivotal period then could elucidate its underlying function in cancer.

My studies coupling affinity purification of GFP-tagged human MIER3 with mass spectroscopy indicates MIER3 likely functions within a novel HDAC1 and 2-containing chromatin co-repressor complex. I identified a previously unknown function of the LXXLL sequence motifs of MIER3 in binding the heterochromatin-promoting protein BAHD1. I also show for the first time an interaction of STAT1 with MIER3. I hypothesize that this multi-protein complex serves to facilitate heterochromatin formation and downstream gene repression. This is important considering I found the most enhanced Mier 3 expression in embryonic mammary buds and mammary tumors. Transcriptional repression is critical in embryonic development for maintaining the proper transcriptional programming that orchestrate sequential events necessary for differentiation, and dysregulation in cancer can lead to silencing of tumor suppressors. Within this repressive complex, MIER3 appears to 
function as an adaptor/scaffold protein. MIER3 binds the nuclear lamin, which serves to dock protein complexes and chromatin, as well as facilitates binding of regulatory proteins HDAC1/2, BAHD1, and STAT1 through strong protein-protein interacting domains. Adaptor proteins can have prominent roles in disease development since small changes in their expression can have substantial effects on protein complex formation and the biological outcomes they mediate. This may explain the higher Mier 3 expression found in Mcs 1 -mammary cancer susceptible lines as compared to resistant [248]. Since endogenous levels of Mier3 are normally low, greater Mier3 levels in the susceptible strain could promote more co-repressive complex formation that drastically modify heterochromatin levels and global gene expression, accounting for the observed differences in cancer phenotypes. Additionally, since these genotypic differences in Mier3 expression have thus far only been observed in mammary tissue, the role of Mier3 in cancer development appears to function in a mammary gland autonomous manner. This merits further work to elucidate the function of MIER3 specifically in human breast cancer as well as in mammary gland development, where the high expression levels I found indicate modulation of Mier3-induced disease susceptibility may potentially occur during this time period.

The subunit composition of complexes that modulate chromatin structure and transcription varies between different cell types and developmental stages, which provides functional specificity. Since gene expression patterns during embryogenesis are entirely different from a developed adult or a disease state, the components of the MIER3 complex I identified in a breast cancer cell line may be drastically different from those that exist during fetal or pubescent development. For example, I identified the transcription factor 
STAT1 as one of the strongest MIER3 interacting proteins in hormone responsive breast cancer, but none of the STAT family members appear to have a role in mammary gland development during embryogenesis or pubertal epithelial expansion $[649,650]$. There may be other development-specific transcription factors that interact with MIER3 lead to targeting of alternate genomic sites and downstream pathways. Studies with the protein interactors of the MIER family and BAHD1 utilized numerous cell lines, which indicate HDAC1/2 and BAHD1 are core components of a MIER3 complex $[266,578]$. The interaction of $\mathrm{HDAC} 1 / 2$ with lineage-specific transcription factors suggests a role in modulating different transcriptional programs, and indeed $\mathrm{HDAC} 1 / 2$ is downregulated in certain cells during differentiation when the repression of lineage-specific genes is no longer needed $[627,628]$. The mammary gland does not become differentiated until the first reproductive cycle, so HDAC1/2, possibly within a MIER3-containing co-repressor complex, may be crucial in maintaining an undifferentiated epithelium during embryonic through pubertal development. The other core protein interactor of MIER3, BAHD1, has been demonstrated to modulate expression of estrogen receptor (ESRI) and progesterone receptor $(P G R)$ in combination with MIER1/MIER3 [578]. Since estrogen and progesterone are critical in signaling morphological tissue changes and differentiation of the mammary epithelium, epigenetic silencing by way of a MIER3-BAHD1 complex may contribute to regulation of correct temporal development. Future work investigating the mechanism and function of MIER3 would need to evaluate the combinatorial assembly of MIER3-HDAC1/2-BAHD1 with different transcription factors and chromatin-regulatory proteins in mammary epithelial cells from embryonic, pubescent, and developed mammary glands. 
I found the strongest MIER3-interacting proteins have a role in transcriptional repression via heterochromatin formation, dysregulation of which can have deleterious repercussions on breast cancer initiation and disease progression. Chromatin structure presents an additional layer of regulation of gene expression, but loss of epigenetic control by way of histone modifications or DNA methylation can lead to alterations in expression that result in cellular transformation and proliferation [562, 651]. Epigenetic deregulation is complex, as perturbations at the level of establishment, recognition, maintenance, and removal of chromatin marks can all contribute to oncogenesis [652]. The role of chromatinrepressive complexes in disease initiation can stem from either deregulation of protein subunits within the complex, which alters the balance of transcriptional activation and repression and promotes oncogenic transformation, or downstream epigenetic silencing of tumor suppressor genes, which is a central hallmark in the development of cancer [554, 556, 561]. The epigenetic landscape of breast cancer cells is comprised of global hypomethylation of DNA alongside repressive chromatin domains that leads to gene silencing [562]. These changes to chromatin structure occur early in breast cancer development and are thought to promote disease progression by silencing tumor suppressor genes $[562,648]$. Thus, chromatin repressive complexes have a clear function in breast cancer development. The increase in Mier3 expression I found throughout mammary cancer progression, coinciding with our mass spectroscopy analysis that indicates its presence in a chromatin co-repressor complex, strongly suggests dysregulation of MIER3 may contribute to the repressive chromatin domains characteristic in breast cancer.

Organismal development requires precise control of transcriptional programs in a spatially and temporally regulated manner, allowing for establishment and maintenance of 
various cell identities despite a single underlying genome $[556,560]$. This requires proper chromatin structure that is achieved by chromatin modifying enzymes [579, 653]. During the process of differentiation, chromatin architecture transitions towards a heterochromatin configuration, highlighting the instructional role of chromatin repressive complexes in orchestrating differentiation and preventing adverse transcriptional events [654-656]. The mammary gland is unique in that the majority of its development occurs postnatally. As a result, proper mammary gland transcriptional programming must be tightly regulated and maintained throughout the lifetime of the mammal. For example, luminal epithelial cells have critical repressive marks in the virgin gland, but these are decreased throughout pregnancy which allow these cells to differentiate into a milk-secreting population and create a lactation-competent mammary gland [653]. Changes in repressive histone modifications during the reproductive cycle may indicate a reduced role of Mier3, which coincides with the substantially lower Mier3 levels I found in mammary epithelial cells during the reproductive cycle as compared to early development. I found the highest Mier3 levels in embryonic through pubertal development. Alterations in chromatin remodeling and transcriptional programs during these early stages of mammary gland development can disrupt the function of progenitor cells that drive ductal branching and proliferation, which predispose the mammary gland to later disease susceptibility [657]. Thus, gaining insight into the function of MIER3 on chromatin structure and transcriptional regulation during mammary gland development will be critical to our understanding of its role in the etiology of breast cancer.

Separate from temporal chromatin changes, the mammary gland also possesses chromatin structural differences between cell populations. Stemming from their functional 
differences, the mammary epithelial subpopulations possess chromatin states that are as different as those between mammary epithelial and stromal cells [653, 658]. Luminal progenitor cells, which give rise to luminal epithelial cells of ductal or alveolar fate, are the adult mammary cell type that most closely resembles the chromatin architecture of fetal mammary stem cells [659]. This link between adult luminal epithelial cells and embryonic mammary cells is noteworthy since I found luminal epithelial cells possess the highest abundance of Mier3 transcripts and Mier3 abundance was highest during embryonic development. Because this cell population gives rise to the majority of female breast cancers, it will be important to identify the role of a MIER3 co-repressor complex in this cell type to define how chromatin remodeling drives tumorigenesis.

In conclusion, the laboratory rat will continue to be an important model to investigate genetically determined mechanisms of mammary cancer susceptibility, especially in studying $M c s 3$ and Mcs $1 b$ loci, which may translate directly to human breast cancer susceptibility. I propose a role of MIER3 within a novel HDAC1/2-containing corepressor complex and report an association with mammary gland development and disease progression. Continued evaluation of its regulatory function throughout development, possibly by differing combinatorial assembly of protein subunits, will be necessary in understanding its role in a neoplastic state. 


\section{REFERENCES}

1. Siegel, R.L., et al., Cancer Statistics, 2021. CA: A Cancer Journal for Clinicians, 2021. 71(1): p. 7-33.

2. Pfeiffer, R.M., et al., Proportion of U.S. Trends in Breast Cancer Incidence Attributable to Long-term Changes in Risk Factor Distributions. Cancer Epidemiol Biomarkers Prev, 2018. 27(10): p. 1214-1222.

3. Gnerlich, J.L., et al., Poorer survival outcomes for male breast cancer compared with female breast cancer may be attributable to in-stage migration. Ann Surg Oncol, 2011. 18(7): p. 1837-44.

4. Speirs, V. and A.M. Shaaban, The rising incidence of male breast cancer. Breast Cancer Res Treat, 2009. 115(2): p. 429-30.

5. Stang, A. and C. Thomssen, Decline in breast cancer incidence in the United States: what about male breast cancer? Breast Cancer Res Treat, 2008. 112(3): p. 595-6.

6. Kamińska, M., et al., Breast cancer risk factors. Prz Menopauzalny, 2015. 14(3): p. 196-202.

7. Singletary, S.E., Rating the risk factors for breast cancer. Ann Surg, 2003. 237(4): p. 474-82.

8. Ban, K.A. and C.V. Godellas, Epidemiology of breast cancer. Surg Oncol Clin N Am, 2014. 23(3): p. 409-22.

9. Key, T., et al., Endogenous sex hormones and breast cancer in postmenopausal women: reanalysis of nine prospective studies. J Natl Cancer Inst, 2002. 94(8): p. 606-16.

10. Farhat, G.N., et al., Sex hormone levels and risks of estrogen receptor-negative and estrogen receptor-positive breast cancers. J Natl Cancer Inst, 2011. 103(7): p. 562-70.

11. Goss, P.E., et al., Exemestane for breast-cancer prevention in postmenopausal women. N Engl J Med, 2011. 364(25): p. 2381-91.

12. Cuzick, J., et al., Anastrozole for prevention of breast cancer in high-risk postmenopausal women (IBIS-II): an international, double-blind, randomised placebo-controlled trial. Lancet, 2014. 383(9922): p. 1041-8.

13. Kaaks, R., et al., Serum sex steroids in premenopausal women and breast cancer risk within the European Prospective Investigation into Cancer and Nutrition (EPIC). J Natl Cancer Inst, 2005. 97(10): p. 755-65.

14. Eliassen, A.H., et al., Endogenous steroid hormone concentrations and risk of breast cancer among premenopausal women. J Natl Cancer Inst, 2006. 98(19): p. 1406-15 
15. Titus-Ernstoff, L., et al., Menstrual factors in relation to breast cancer risk. Cancer Epidemiol Biomarkers Prev, 1998. 7(9): p. 783-9.

16. Berkey, C.S., et al., Adolescence and breast carcinoma risk. Cancer, 1999. 85(11): p. 2400-9.

17. Hsieh, C.C., et al., Age at menarche, age at menopause, height and obesity as risk factors for breast cancer: associations and interactions in an international casecontrol study. Int J Cancer, 1990. 46(5): p. 796-800.

18. Dall, G.V. and K.L. Britt, Estrogen Effects on the Mammary Gland in Early and Late Life and Breast Cancer Risk. Front Oncol, 2017. 7: p. 110.

19. Brinton, L.A., R. Hoover, and J.F. Fraumeni, Jr., Reproductive factors in the aetiology of breast cancer. Br J Cancer, 1983. 47(6): p. 757-62.

20. Brinton, L.A., R. Hoover, and J.F. Fraumeni, Jr., Epidemiology of minimal breast cancer. Jama, 1983. 249(4): p. 483-7.

21. Familial breast cancer: collaborative reanalysis of individual data from 52 epidemiological studies including 58,209 women with breast cancer and 101,986 women without the disease. Lancet, 2001. 358(9291): p. 1389-99.

22. Brewer, H.R., et al., Family history and risk of breast cancer: an analysis accounting for family structure. Breast Cancer Res Treat, 2017. 165(1): p. 193200.

23. Boyd, N.F., et al., Quantitative classification of mammographic densities and breast cancer risk: results from the Canadian National Breast Screening Study. J Natl Cancer Inst, 1995. 87(9): p. 670-5.

24. Biglia, N., et al., Management of risk of breast carcinoma in postmenopausal women. Endocr Relat Cancer, 2004. 11(1): p. 69-83.

25. Sugimura, T., Nutrition and dietary carcinogens. Carcinogenesis, 2000. 21(3): p. 387-95.

26. Saxe, G.A., et al., Diet and risk for breast cancer recurrence and survival. Breast Cancer Res Treat, 1999. 53(3): p. 241-53.

27. Zheng, W., et al., Well-done meat intake and the risk of breast cancer. J Natl Cancer Inst, 1998. 90(22): p. 1724-9.

28. Schulz, M., et al., Identification of a dietary pattern characterized by high-fat food choices associated with increased risk of breast cancer: the European Prospective Investigation into Cancer and Nutrition (EPIC)-Potsdam Study. Br J Nutr, 2008. 100(5): p. 942-6.

29. Makarem, N., et al., Dietary fat in breast cancer survival. Annu Rev Nutr, 2013. 33: p. 319-48.

30. Velie, E., et al., Dietary fat, fat subtypes, and breast cancer in postmenopausal women: a prospective cohort study. J Natl Cancer Inst, 2000. 92(10): p. 833-9.

31. Young, L.R., et al., Effect of dietary fat and omega-3 fatty acids on urinary eicosanoids and sex hormone concentrations in postmenopausal women: a randomized controlled feeding trial. Nutr Cancer, 2011. 63(6): p. 930-9.

32. Dong, J.Y., et al., Dietary fiber intake and risk of breast cancer: a meta-analysis of prospective cohort studies. Am J Clin Nutr, 2011. 94(3): p. 900-5. 
33. Bartsch, H., J. Nair, and R.W. Owen, Dietary polyunsaturated fatty acids and cancers of the breast and colorectum: emerging evidence for their role as risk modifiers. Carcinogenesis, 1999. 20(12): p. 2209-18.

34. Lee, I.M., Antioxidant vitamins in the prevention of cancer. Proc Assoc Am Physicians, 1999. 111(1): p. 10-5.

35. Hamajima, N., et al., Alcohol, tobacco and breast cancer--collaborative reanalysis of individual data from 53 epidemiological studies, including 58,515 women with breast cancer and 95,067 women without the disease. Br J Cancer, 2002. 87(11): p. 1234-45.

36. Reichman, M.E., et al., Effects of alcohol consumption on plasma and urinary hormone concentrations in premenopausal women. J Natl Cancer Inst, 1993. 85(9): p. 722-7.

37. Onland-Moret, N.C., et al., Alcohol and endogenous sex steroid levels in postmenopausal women: a cross-sectional study. J Clin Endocrinol Metab, 2005. 90(3): p. 1414-9.

38. Ginsburg, E.S., et al., The effect of acute ethanol ingestion on estrogen levels in postmenopausal women using transdermal estradiol. J Soc Gynecol Investig, 1995. 2(1): p. 26-9.

39. Seitz, H.K. and P. Becker, Alcohol metabolism and cancer risk. Alcohol Res Health, 2007. 30(1): p. 38-41, 44-7.

40. Friedenreich, C.M., The role of physical activity in breast cancer etiology. Semin Oncol, 2010. 37(3): p. 297-302.

41. Lagerros, Y.T., S.F. Hsieh, and C.C. Hsieh, Physical activity in adolescence and young adulthood and breast cancer risk: a quantitative review. Eur J Cancer Prev, 2004. 13(1): p. 5-12.

42. Lynch, B.M., H.K. Neilson, and C.M. Friedenreich, Physical activity and breast cancer prevention. Recent Results Cancer Res, 2011. 186: p. 13-42.

43. Friedenreich, C.M. and A.E. Cust, Physical activity and breast cancer risk: impact of timing, type and dose of activity and population subgroup effects. Br J Sports Med, 2008. 42(8): p. 636-47.

44. Trentham-Dietz, A., et al., Weight change and risk of postmenopausal breast cancer (United States). Cancer Causes \& Control, 2000. 11(6): p. 533-542.

45. Clemons, M. and P. Goss, Estrogen and the risk of breast cancer. N Engl J Med, 2001. 344(4): p. 276-85.

46. McTiernan, A., et al., Adiposity and sex hormones in postmenopausal breast cancer survivors. J Clin Oncol, 2003. 21(10): p. 1961-6.

47. Huang, Z., et al., Dual effects of weight and weight gain on breast cancer risk. Jama, 1997. 278(17): p. 1407-11.

48. Lahmann, P.H., et al., Body size and breast cancer risk: findings from the European Prospective Investigation into Cancer And Nutrition (EPIC). Int J Cancer, 2004. 111(5): p. 762-71.

49. Hecht, S.S., Tobacco smoke carcinogens and breast cancer. Environ Mol Mutagen, 2002. 39(2-3): p. 119-26. 
50. Johnson, K.C., et al., Active smoking and secondhand smoke increase breast cancer risk: the report of the Canadian Expert Panel on Tobacco Smoke and Breast Cancer Risk (2009). Tob Control, 2011. 20(1): p. e2.

51. Reynolds, P., Smoking and breast cancer. J Mammary Gland Biol Neoplasia, 2013. 18(1): p. 15-23.

52. Li, D., et al., Characterization of a major aromatic DNA adduct detected in human breast tissues. Environ Mol Mutagen, 2002. 39(2-3): p. 193-200.

53. MacNicoll, A.D., et al., Metabolism and activation of carcinogenic polycyclic hydrocarbons by human mammary cells. Biochemical and Biophysical Research Communications, 1980. 95(4): p. 1599-1606.

54. Brody, J.G. and R.A. Rudel, Environmental pollutants and breast cancer. Environ Health Perspect, 2003. 111(8): p. 1007-19.

55. Rudel, R.A., et al., Chemicals causing mammary gland tumors in animals signal new directions for epidemiology, chemicals testing, and risk assessment for breast cancer prevention. Cancer, 2007. 109(12 Suppl): p. 2635-66.

56. Lauby-Secretan, B., et al., Carcinogenicity of polychlorinated biphenyls and polybrominated biphenyls. Lancet Oncol, 2013. 14(4): p. 287-8.

57. Pharoah, P.D.P., et al., Family history and the risk of breast cancer: A systematic review and meta-analysis. International Journal of Cancer, 1997. 71(5): p. 800809.

58. Rahman, N. and M.R. Stratton, The genetics of breast cancer susceptibility. Annu Rev Genet, 1998. 32: p. 95-121.

59. Ahlbom, A., et al., Cancer in twins: genetic and nongenetic familial risk factors. J Natl Cancer Inst, 1997. 89(4): p. 287-93.

60. Risch, N., The genetic epidemiology of cancer: interpreting family and twin studies and their implications for molecular genetic approaches. Cancer Epidemiol Biomarkers Prev, 2001. 10(7): p. 733-41.

61. Verkasalo, P.K., et al., Genetic predisposition, environment and cancer incidence: a nationwide twin study in Finland, 1976-1995. Int J Cancer, 1999. 83(6): p. 7439.

62. Lichtenstein, P., et al., Environmental and heritable factors in the causation of cancer--analyses of cohorts of twins from Sweden, Denmark, and Finland. N Engl J Med, 2000. 343(2): p. 78-85.

63. Wendt, C. and S. Margolin, Identifying breast cancer susceptibility genes - $a$ review of the genetic background in familial breast cancer. Acta Oncol, 2019. 58(2): p. 135-146.

64. Apostolou, P. and F. Fostira, Hereditary breast cancer: the era of new susceptibility genes. Biomed Res Int, 2013. 2013: p. 747318.

65. Mavaddat, N., et al., Genetic susceptibility to breast cancer. Molecular Oncology, 2010. 4(3): p. 174-191.

66. Wooster, R., et al., Identification of the breast cancer susceptibility gene BRCA2. Nature, 1995. 378(6559): p. 789-92.

67. Miki, Y., et al., A strong candidate for the breast and ovarian cancer susceptibility gene BRCA1. Science, 1994. 266(5182): p. 66-71. 
68. Antoniou, A., et al., Average risks of breast and ovarian cancer associated with BRCA1 or BRCA2 mutations detected in case Series unselected for family history: a combined analysis of 22 studies. Am J Hum Genet, 2003. 72(5): p. 1117-30.

69. Thompson, D. and D. Easton, The genetic epidemiology of breast cancer genes. J Mammary Gland Biol Neoplasia, 2004. 9(3): p. 221-36.

70. Ghoussaini, M., P.D.P. Pharoah, and D.F. Easton, Inherited Genetic Susceptibility to Breast Cancer: The Beginning of the End or the End of the Beginning? The American Journal of Pathology, 2013. 183(4): p. 1038-1051.

71. Rahman, N., et al., PALB2, which encodes a BRCA2-interacting protein, is a breast cancer susceptibility gene. Nat Genet, 2007. 39(2): p. 165-7.

72. Stratton, M.R. and N. Rahman, The emerging landscape of breast cancer susceptibility. Nat Genet, 2008. 40(1): p. 17-22.

73. Antoniou, A.C., et al., A comprehensive model for familial breast cancer incorporating BRCA1, BRCA2 and other genes. Br J Cancer, 2002. 86(1): p. 76-83.

74. Mack, T.M., et al., Heritable breast cancer in twins. Br J Cancer, 2002. 87(3): p. 294-300.

75. Easton, D.F., How many more breast cancer predisposition genes are there? Breast Cancer Res, 1999. 1(1): p. 14-7.

76. Samuelson, D.J., et al., Rat Mcs5a is a compound quantitative trait locus with orthologous human loci that associate with breast cancer risk. Proceedings of the National Academy of Sciences of the United States of America, 2007. 104(15): p. 6299-6304.

77. Samuelson, D.J., et al., Fine mapping reveals multiple loci and a possible epistatic interaction within the mammary carcinoma susceptibility quantitative trait locus, Mcs5. Cancer Res, 2005. 65(21): p. 9637-42.

78. Antoniou, A.C., et al., Common alleles at $6 q 25.1$ and $1 p 11.2$ are associated with breast cancer risk for BRCA1 and BRCA2 mutation carriers. Hum Mol Genet, 2011. 20(16): p. 3304-21.

79. Antoniou, A.C., et al., Common breast cancer-predisposition alleles are associated with breast cancer risk in BRCA1 and BRCA2 mutation carriers. Am J Hum Genet, 2008. 82(4): p. 937-48.

80. Antoniou, A.C. and D.F. Easton, Models of genetic susceptibility to breast cancer. Oncogene, 2006. 25(43): p. 5898-905.

81. Pe'er, I., et al., Evaluating and improving power in whole-genome association studies using fixed marker sets. Nature Genetics, 2006. 38(6): p. 663-667.

82. Shu, X., et al., Identification of novel breast cancer susceptibility loci in metaanalyses conducted among Asian and European descendants. Nat Commun, 2020. 11(1): p. 1217.

83. Commonly studied single-nucleotide polymorphisms and breast cancer: results from the Breast Cancer Association Consortium. J Natl Cancer Inst, 2006. 98(19): p. 1382-96.

84. Michailidou, K., et al., Association analysis identifies 65 new breast cancer risk loci. Nature, 2017. 551(7678): p. 92-94. 
85. Ghoussaini, M. and P.D. Pharoah, Polygenic susceptibility to breast cancer: current state-of-the-art. Future Oncol, 2009. 5(5): p. 689-701.

86. Pearson, T.A. and T.A. Manolio, How to interpret a genome-wide association study. Jama, 2008. 299(11): p. 1335-44.

87. Yang, Q., et al., Power and type I error rate of false discovery rate approaches in genome-wide association studies. BMC Genet, 2005. 6 Suppl 1: p. S134.

88. Sanders, J. and D.J. Samuelson, Significant overlap between human genome-wide association-study nominated breast cancer risk alleles and rat mammary cancer susceptibility loci. Breast cancer research : BCR, 2014. 16(1): p. R14-R14.

89. Zuk, O., et al., Searching for missing heritability: designing rare variant association studies. Proc Natl Acad Sci U S A, 2014. 111(4): p. E455-64.

90. Ruark, E., et al., Mosaic PPM1D mutations are associated with predisposition to breast and ovarian cancer. Nature, 2013. 493(7432): p. 406-10.

91. Shahi, R.B., et al., Identification of candidate cancer predisposing variants by performing whole-exome sequencing on index patients from BRCA1 and BRCA2negative breast cancer families. BMC Cancer, 2019. 19(1): p. 313.

92. Skol, A.D., M.M. Sasaki, and K. Onel, The genetics of breast cancer risk in the post-genome era: thoughts on study design to move past BRCA and towards clinical relevance. Breast Cancer Res, 2016. 18(1): p. 99.

93. Cybulski, C., et al., Germline RECQL mutations are associated with breast cancer susceptibility. Nat Genet, 2015. 47(6): p. 643-6.

94. Macias, H. and L. Hinck, Mammary gland development. Wiley Interdiscip Rev Dev Biol, 2012. 1(4): p. 533-57.

95. Rudel, R.A., et al., Environmental exposures and mammary gland development: state of the science, public health implications, and research recommendations. Environmental health perspectives, 2011. 119(8): p. 1053-1061.

96. Gopalakrishnan, K., et al., Histology and Transcriptome Profiles of the Mammary Gland across Critical Windows of Development in Sprague Dawley Rats. J Mammary Gland Biol Neoplasia, 2018. 23(3): p. 149-163.

97. Terry, M.B., et al., Environmental exposures during windows of susceptibility for breast cancer: a framework for prevention research. Breast Cancer Research, 2019. 21(1): p. 96.

98. Biro, F.M. and J. Deardorff, Identifying opportunities for cancer prevention during preadolescence and adolescence: puberty as a window of susceptibility. J Adolesc Health, 2013. 52(5 Suppl): p. S15-20.

99. Henning, A.N., et al., The Non-coding Mammary Carcinoma Susceptibility Locus, Mcs5c, Regulates Pappa Expression via Age-Specific Chromatin Folding and Allele-Dependent DNA Methylation. PLoS Genet, 2016. 12(8): p. e1006261.

100. Russo, J., et al., The protective role of pregnancy in breast cancer. Breast cancer research : BCR, 2005. 7(3): p. 131-142.

101. Russo, J., et al., Comparative study of human and rat mammary tumorigenesis. Lab Invest, 1990. 62(3): p. 244-78. 
102. Wellings, S.R., H.M. Jensen, and R.G. Marcum, An atlas of subgross pathology of the human breast with special reference to possible precancerous lesions. J Natl Cancer Inst, 1975. 55(2): p. 231-73.

103. Russo, J., et al., Expression of phenotypical changes by human breast epithelial cells treated with carcinogens in vitro. Cancer Res, 1988. 48(10): p. 2837-57.

104. Russo, J., L.K. Tay, and I.H. Russo, Differentiation of the mammary gland and susceptibility to carcinogenesis. Breast Cancer Res Treat, 1982. 2(1): p. 5-73.

105. Kleinberg, D.L., et al., Growth hormone and insulin-like growth factor-l in the transition from normal mammary development to preneoplastic mammary lesions. Endocr Rev, 2009. 30(1): p. 51-74.

106. Medina, D., Chemical carcinogenesis of rat and mouse mammary glands. Breast Dis, 2007. 28: p. 63-8.

107. Russo, J. and I.H. Russo, Development of the human breast. Maturitas, 2004. 49(1): p. 2-15.

108. Henderson, T.O., et al., Systematic review: surveillance for breast cancer in women treated with chest radiation for childhood, adolescent, or young adult cancer. Ann Intern Med, 2010. 152(7): p. 444-55; w144-54.

109. Imaoka, T., et al., Radiation-induced mammary carcinogenesis in rodent models: what's different from chemical carcinogenesis? J Radiat Res, 2009. 50(4): p. 28193.

110. Land, C.E., Studies of cancer and radiation dose among atomic bomb survivors. The example of breast cancer. Jama, 1995. 274(5): p. 402-7.

111. Birnbaum, L.S. and S.E. Fenton, Cancer and developmental exposure to endocrine disruptors. Environ Health Perspect, 2003. 111(4): p. 389-94.

112. Fenton, S.E., Endocrine-disrupting compounds and mammary gland development: early exposure and later life consequences. Endocrinology, 2006. 147(6 Suppl): p. S18-24.

113. Lund, L.R., et al., Two distinct phases of apoptosis in mammary gland involution: proteinase-independent and-dependent pathways. Development (Cambridge, England), 1996. 122(1): p. 181-193.

114. Masso-Welch, P.A., et al., A developmental atlas of rat mammary gland histology. J Mammary Gland Biol Neoplasia, 2000. 5(2): p. 165-85.

115. Russo, I.H. and J. Russo, Hormonal approach to breast cancer prevention. J Cell Biochem Suppl, 2000. 34: p. 1-6.

116. Katz, T.A., Potential Mechanisms underlying the Protective Effect of Pregnancy against Breast Cancer: A Focus on the IGF Pathway. Front Oncol, 2016. 6: p. 228.

117. Líška, J., et al., Relationship between histology, development and tumorigenesis of mammary gland in female rat. Experimental animals, 2016. 65(1): p. 1-9.

118. Lambe, M., et al., Parity, age at first and last birth, and risk of breast cancer: a population-based study in Sweden. Breast Cancer Res Treat, 1996. 38(3): p. 30511.

119. MacMahon, B., et al., Age at first birth and breast cancer risk. Bull World Health Organ, 1970. 43(2): p. 209-21. 
120. Rosner, B., G.A. Colditz, and W.C. Willett, Reproductive risk factors in a prospective study of breast cancer: the Nurses' Health Study. Am J Epidemiol, 1994. 139(8): p. 819-35.

121. Russo, J., R. Rivera, and I.H. Russo, Influence of age and parity on the development of the human breast. Breast Cancer Res Treat, 1992. 23(3): p. 2118.

122. Russo, J., A.L. Romero, and I.H. Russo, Architectural pattern of the normal and cancerous breast under the influence of parity. Cancer Epidemiol Biomarkers Prev, 1994. 3(3): p. 219-24.

123. Russo, J. and I.H. Russo, Toward a physiological approach to breast cancer prevention. Cancer Epidemiol Biomarkers Prev, 1994. 3(4): p. 353-64.

124. Sinha, D.K., J.E. Pazik, and T.L. Dao, Prevention of mammary carcinogenesis in rats by pregnancy: effect of full-term and interrupted pregnancy. Br J Cancer, 1988. 57(4): p. 390-4.

125. Russo, J. and I.H. Russo, Influence of differentiation and cell kinetics on the susceptibility of the rat mammary gland to carcinogenesis. Cancer Res, 1980. 40(8 Pt 1): p. 2677-87.

126. Yang, J., et al., Protective effects of pregnancy and lactation against $\mathrm{N}$-methyl-Nnitrosourea-induced mammary carcinomas in female Lewis rats. Carcinogenesis, 1999. 20(4): p. 623-8.

127. Guzman, R.C., et al., Hormonal prevention of breast cancer: mimicking the protective effect of pregnancy. Proc Natl Acad Sci U S A, 1999. 96(5): p. 2520-5.

128. Sivaraman, L., et al., Hormone-induced refractoriness to mammary carcinogenesis in Wistar-Furth rats. Carcinogenesis, 1998. 19(9): p. 1573-81.

129. Rajkumar, L., et al., Short-term exposure to pregnancy levels of estrogen prevents mammary carcinogenesis. Proc Natl Acad Sci U S A, 2001. 98(20): p. 11755-9.

130. Swanson, S.M., et al., Hormone levels and mammary epithelial cell proliferation in rats treated with a regimen of estradiol and progesterone that mimics the preventive effect of pregnancy against mammary cancer. Anticancer Res, 1997. 17(6d): p. 4639-45.

131. Grubbs, C.J., M.M. Juliana, and L.M. Whitaker, Short-term hormone treatment as a chemopreventive method against mammary cancer initiation in rats. Anticancer Res, 1988. 8(1): p. 113-7.

132. Russo, I.H. and J. Russo, Mammary gland neoplasia in long-term rodent studies. Environ Health Perspect, 1996. 104(9): p. 938-67.

133. Russo, J., et al., Cancer risk related to mammary gland structure and development. Microsc Res Tech, 2001. 52(2): p. 204-23.

134. Dickson, S.R. and M.J. Warburton, Enhanced synthesis of gelatinase and stromelysin by myoepithelial cells during involution of the rat mammary gland. J Histochem Cytochem, 1992. 40(5): p. 697-703.

135. Schedin, P., et al., Fibronectin fragments induce MMP activity in mouse mammary epithelial cells: evidence for a role in mammary tissue remodeling. J Cell Sci, 2000. 113 ( Pt 5): p. 795-806. 
136. Talhouk, R.S., M.J. Bissell, and Z. Werb, Coordinated expression of extracellular matrix-degrading proteinases and their inhibitors regulates mammary epithelial function during involution. J Cell Biol, 1992. 118(5): p. 1271-82.

137. Giannelli, G., et al., Expression of matrix metalloprotease-2-cleaved laminin-5 in breast remodeling stimulated by sex steroids. Am J Pathol, 1999. 154(4): p. 1193201.

138. Schedin, P., et al., Mammary ECM composition and function are altered by reproductive state. Mol Carcinog, 2004. 41(4): p. 207-20.

139. Bemis, L.T. and P. Schedin, Reproductive state of rat mammary gland stroma modulates human breast cancer cell migration and invasion. Cancer Res, 2000. 60(13): p. 3414-8.

140. Zhao, W., et al., Parity is associated with an expanded macrophage population in the mammary gland. Int J Oncol, 2010. 37(5): p. 1195-202.

141. Stein, T., et al., Involution of the mouse mammary gland is associated with an immune cascade and an acute-phase response, involving LBP, CD14 and STAT3. Breast Cancer Res, 2004. 6(2): p. R75-91.

142. Clarkson, R.W., et al., Gene expression profiling of mammary gland development reveals putative roles for death receptors and immune mediators in postlactational regression. Breast Cancer Res, 2004. 6(2): p. R92-109.

143. Mueller, M.M. and N.E. Fusenig, Friends or foes - bipolar effects of the tumour stroma in cancer. Nat Rev Cancer, 2004. 4(11): p. 839-49.

144. Coussens, L.M. and Z. Werb, Inflammation and cancer. Nature, 2002. 420(6917): p. 860-7.

145. Park, C.C., M.J. Bissell, and M.H. Barcellos-Hoff, The influence of the microenvironment on the malignant phenotype. Mol Med Today, 2000. 6(8): p. 324-9.

146. Clarkson, R.W. and C.J. Watson, Microarray analysis of the involution switch. J Mammary Gland Biol Neoplasia, 2003. 8(3): p. 309-19.

147. McDaniel, S.M., et al., Remodeling of the mammary microenvironment after lactation promotes breast tumor cell metastasis. The American journal of pathology, 2006. 168(2): p. 608-620.

148. Schedin, P., Pregnancy-associated breast cancer and metastasis. Nat Rev Cancer, 2006. 6(4): p. 281-91.

149. Schedin, P. and A. Elias, Multistep tumorigenesis and the microenvironment. Breast Cancer Res, 2004. 6(2): p. 93-101.

150. Daling, J.R., et al., The relation of reproductive factors to mortality from breast cancer. Cancer Epidemiol Biomarkers Prev, 2002. 11(3): p. 235-41.

151. Lambe, M., et al., Transient increase in the risk of breast cancer after giving birth. N Engl J Med, 1994. 331(1): p. 5-9.

152. Callihan, E.B., et al., Postpartum diagnosis demonstrates a high risk for metastasis and merits an expanded definition of pregnancy-associated breast cancer. Breast Cancer Res Treat, 2013. 138(2): p. 549-59. 
153. Albrektsen, G., et al., Breast cancer risk by age at birth, time since birth and time intervals between births: exploring interaction effects. Br J Cancer, 2005. 92(1): $p$. 167-75.

154. Rosner, B. and G.A. Colditz, Nurses' health study: log-incidence mathematical model of breast cancer incidence. J Natl Cancer Inst, 1996. 88(6): p. 359-64.

155. Bonnier, P., et al., Influence of pregnancy on the outcome of breast cancer: a case-control study. Societe Francaise de Senologie et de Pathologie Mammaire Study Group. Int J Cancer, 1997. 72(5): p. 720-7.

156. Henderson, B.E. and L. Bernstein, The international variation in breast cancer rates: an epidemiological assessment. Breast Cancer Res Treat, 1991. 18 Suppl 1: p. S11-7.

157. Pathak, D.R., Dual effect of first full term pregnancy on breast cancer risk: empirical evidence and postulated underlying biology. Cancer Causes Control, 2002. 13(4): p. 295-8.

158. Cotroneo, M.S., et al., The Mcs7 quantitative trait locus is associated with an increased susceptibility to mammary cancer in congenic rats and an allelespecific imbalance. Oncogene, 2006. 25(36): p. 5011-7.

159. Haag, J.D., et al., Congenic rats reveal three independent Copenhagen alleles within the Mcs1 quantitative trait locus that confer resistance to mammary cancer. Cancer Res, 2003. 63(18): p. 5808-12.

160. Sharma, D., et al., Quantification of epithelial cell differentiation in mammary glands and carcinomas from DMBA- and MNU-exposed rats. PloS one, 2011. 6(10): p. e26145-e26145.

161. Gould, M.N., The utility of comparative genetics to inform breast cancer prevention strategies. Genetics, 2009. 183(2): p. 409-412.

162. Nandi, S., R.C. Guzman, and J. Yang, Hormones and mammary carcinogenesis in mice, rats, and humans: a unifying hypothesis. Proc Natl Acad Sci U S A, 1995. 92(9): p. 3650-7.

163. Russo, J. and I.H. Russo, Experimentally induced mammary tumors in rats. Breast Cancer Res Treat, 1996. 39(1): p. 7-20.

164. Shepel, L.A., et al., Genetic identification of multiple loci that control breast cancer susceptibility in the rat. Genetics, 1998. 149(1): p. 289-299.

165. Astwood, E.B., C.F. Geschickter, and E.O. Rausch, Development of the mammary gland of the rat: A study of normal, experimental and pathologic changes and their endocrine relationships. American Journal of Anatomy, 1937. 61(3): p. 373405.

166. Lucas, J.N., et al., The rat mammary gland: morphologic changes as an indicator of systemic hormonal perturbations induced by xenobiotics. Toxicol Pathol, 2007. 35(2): p. 199-207.

167. Sternlicht, M.D., Key stages in mammary gland development: the cues that regulate ductal branching morphogenesis. Breast Cancer Res, 2006. 8(1): p. 201.

168. Jin, H.S., et al., Alterations of myoepithelial cells in the rat mammary gland during pregnancy, lactation and involution, and after estradiol treatment. Pathol Int, 2000. 50(5): p. 384-91. 
169. Fernig, D.G., J.A. Smith, and P.S. Rudland, Relationship of growth factors and differentiation in normal and neoplastic development of the mammary gland. Cancer Treat Res, 1991. 53: p. 47-78.

170. Blatchford, D.R., et al., Influence of microenvironment on mammary epithelial cell survival in primary culture. J Cell Physiol, 1999. 181(2): p. 304-11.

171. Filgo, A.J., et al., Mammary Gland Evaluation in Juvenile Toxicity Studies: Temporal Developmental Patterns in the Male and Female Harlan SpragueDawley Rat. Toxicol Pathol, 2016. 44(7): p. 1034-58.

172. Russo, I.H. and J. Russo, Developmental stage of the rat mammary gland as determinant of its susceptibility to 7,12-dimethylbenz[a]anthracene. J Natl Cancer Inst, 1978. 61(6): p. 1439-49.

173. Sinha, Y.N. and H.A. Tucker, Mammary gland growth of rats between 10 and 100 days of age. Am J Physiol, 1966. 210(3): p. 601-5.

174. Ewald, A.J., et al., Collective epithelial migration and cell rearrangements drive mammary branching morphogenesis. Dev Cell, 2008. 14(4): p. 570-81.

175. Russo, J., G. Wilgus, and I.H. Russo, Susceptibility of the mammary gland to carcinogenesis: I Differentiation of the mammary gland as determinant of tumor incidence and type of lesion. Am J Pathol, 1979. 96(3): p. 721-36.

176. Hvid, H., et al., Mammary gland proliferation in female rats: effects of the estrous cycle, pseudo-pregnancy and age. Exp Toxicol Pathol, 2012. 64(4): p. 321-32.

177. Griffith, D.R. and C.W. Turner, Normal growth of rat mammary glands during pregnancy and early lactation. Proc Soc Exp Biol Med, 1961. 106: p. 448-50.

178. Joshi, K., et al., Cellular proliferation in the rat mammary gland during pregnancy and lactation. Lab Invest, 1986. 54(1): p. 52-61.

179. Forsyth, I.A., Variation among species in the endocrine control of mammary growth and function: the roles of prolactin, growth hormone, and placental lactogen. J Dairy Sci, 1986. 69(3): p. 886-903.

180. Munford, R.E., CHANGES IN THE MAMMARY GLANDS OF RATS AND MICE DURING PREGNANCY, LACTATION AND INVOLUTION. 1. HISTOLOGICAL STRUCTURE. J Endocrinol, 1963. 28: p. 1-15.

181. Walker, N.I., R.E. Bennett, and J.F. Kerr, Cell death by apoptosis during involution of the lactating breast in mice and rats. Am J Anat, 1989. 185(1): p. 19-32.

182. Quarrie, L.H., C.V. Addey, and C.J. Wilde, Apoptosis in lactating and involuting mouse mammary tissue demonstrated by nick-end DNA labelling. Cell Tissue Res, 1995. 281(3): p. 413-9.

183. Quarrie, L.H., C.V. Addey, and C.J. Wilde, Programmed cell death during mammary tissue involution induced by weaning, litter removal, and milk stasis. J Cell Physiol, 1996. 168(3): p. 559-69.

184. Zinser, G.M. and J. Welsh, Accelerated mammary gland development during pregnancy and delayed postlactational involution in vitamin D3 receptor null mice. Mol Endocrinol, 2004. 18(9): p. 2208-23.

185. Feng, Z., et al., Glucocorticoid and progesterone inhibit involution and programmed cell death in the mouse mammary gland. The Journal of cell biology, 1995. 131(4): p. 1095-1103. 
186. Li, M., et al., Mammary-derived signals activate programmed cell death during the first stage of mammary gland involution. Proc Natl Acad Sci U S A, 1997. 94(7): p. 3425-30.

187. Andrews, W.W. and S.R. Ojeda, A detailed analysis of the serum luteinizing hormone secretory profile in conscious, free-moving female rats during the time of puberty. Endocrinology, 1981. 109(6): p. 2032-9.

188. Urbanski, H.F. and S.R. Ojeda, The juvenile-peripubertal transition period in the female rat: establishment of a diurnal pattern of pulsatile luteinizing hormone secretion. Endocrinology, 1985. 117(2): p. 644-9.

189. Westwood, F.R., The Female Rat Reproductive Cycle: A Practical Histological Guide to Staging. Toxicologic Pathology, 2008. 36(3): p. 375-384.

190. Astwood, E.B., CHANGES IN THE WEIGHT AND WATER CONTENT OF THE UTERUS OF THE NORMAL ADULT RAT. American Journal of Physiology-Legacy Content, 1939. 126(1): p. 162-170.

191. Blandau, R.J., J.L. Boling, and W.C. Young, The length of heat in the albino rat as determined by the copulatory response. The Anatomical Record, 1941. 79(4): $p$. 453-463.

192. Fata, J.E., V. Chaudhary, and R. Khokha, Cellular turnover in the mammary gland is correlated with systemic levels of progesterone and not 17beta-estradiol during the estrous cycle. Biol Reprod, 2001. 65(3): p. 680-8.

193. Ferguson, D.J. and T.J. Anderson, Morphological evaluation of cell turnover in relation to the menstrual cycle in the "resting" human breast. British journal of cancer, 1981. 44(2): p. 177-181.

194. Olsson, H., et al., Proliferation of the breast epithelium in relation to menstrual cycle phase, hormonal use, and reproductive factors. Breast Cancer Res Treat, 1996. 40(2): p. 187-96.

195. Navarrete, M.A., et al., Assessment of the proliferative, apoptotic and cellular renovation indices of the human mammary epithelium during the follicular and luteal phases of the menstrual cycle. Breast Cancer Res, 2005. 7(3): p. R306-13.

196. Strange, R., et al., Proliferation and apoptosis in mammary epithelium during the rat oestrous cycle. Acta Physiol (Oxf), 2007. 190(2): p. 137-49.

197. Mandl, A.M., The Phases of the Oestrous Cycle in the Adult White Rat. Journal of Experimental Biology, 1951. 28(4): p. 576.

198. Paccola, C.C., et al. The rat estrous cycle revisited: a quantitative and qualitative analysis. 2013.

199. Schedin, P., T. Mitrenga, and M. Kaeck, Estrous Cycle Regulation of Mammary Epithelial Cell Proliferation, Differentiation, and Death in the Sprague-Dawley Rat: A Model for Investigating the Role of Estrous Cycling in Mammary Carcinogenesis. Journal of Mammary Gland Biology and Neoplasia, 2000. 5(2): p. 211-225.

200. Andres, A.C. and R. Strange, Apoptosis in the estrous and menstrual cycles. J Mammary Gland Biol Neoplasia, 1999. 4(2): p. 221-8. 
201. Spornitz, U.M., C.D. Socin, and A.A. Dravid, Estrous stage determination in rats by means of scanning electron microscopic images of uterine surface epithelium. The Anatomical Record, 1999. 254(1): p. 116-126.

202. Smith, M.S., M.E. Freeman, and J.D. Neill, The control of progesterone secretion during the estrous cycle and early pseudopregnancy in the rat: prolactin, gonadotropin and steroid levels associated with rescue of the corpus luteum of pseudopregnancy. Endocrinology, 1975. 96(1): p. 219-26.

203. Dulbecco, R., M. Henahan, and B. Armstrong, Cell types and morphogenesis in the mammary gland. Proceedings of the National Academy of Sciences of the United States of America, 1982. 79(23): p. 7346-7350.

204. Gross, L. and Y. Dreyfuss, Spontaneous tumors in Sprague-Dawley and LongEvans rats and in their F1 hybrids: carcinogenic effect of total-body $x$-irradiation. Proceedings of the National Academy of Sciences of the United States of America, 1979. 76(11): p. 5910-5913.

205. Ratcliffe, H.L., Spontaneous tumors in two colonies of rats of the Wistar Institute of Anatomy and Biology. The American journal of pathology, 1940. 16(3): p. 237254.1.

206. Noble, R.L. and J.H. Cutts, Mammary Tumors of the Rat: \&lt;em\&gt;A Review\&It;/em\&gt. Cancer Research, 1959. 19(11): p. 1125.

207. Russo, J. and I.H. Russo, Biological and molecular bases of mammary carcinogenesis. Lab Invest, 1987. 57(2): p. 112-37.

208. Abba, M.C., et al., DMBA induced mouse mammary tumors display high incidence of activating Pik3caH1047 and loss of function Pten mutations. Oncotarget, 2016. 7(39): p. 64289-64299.

209. Russo, J. and I.H. Russo, Susceptibility of the mammary gland to carcinogenesis. II. Pregnancy interruption as a risk factor in tumor incidence. Am J Pathol, 1980. 100(2): p. 497-512.

210. Maisin, J. and M. Coolen, Au sujet du pouvoir cancerigene du methylcholanthrene. CR Soc. Biol.(Paris), 1936. 123: p. 159-160.

211. Boyland, E. and K.L. Sydnor, The induction of mammary cancer in rats. Br J Cancer, 1962. 16: p. 731-9.

212. Huggins, C., L.C. Grand, and F.P. Brillantes, Mammary Cancer Induced by a Single Feeding of Polynuclear Hydrocarbons, and its Suppression. Nature, 1961. 189(4760): p. 204-207.

213. Wilson, R.H., F. DeEds, and A.J. Cox, The toxicity and carcinogenic activity of 2acetaminofluorene. Cancer Research, 1941. 1(8): p. 595-608.

214. Geyer, R.P., et al., Effect of dose and hormones on tumor production in rats given emulsified 9, 10-dimethyl-1,2-benzanthracene intravenously. Cancer Res, 1953. 13(7:1): p. 503-6.

215. Geyer, R.P., et al., Tumor production in rats injected intravenously with oil emulsions containing 9,10-dimethyl-1,2-benzanthracene. Cancer Res, 1951. 11(6): p. 474-8. 
216. Huggins, C., S. Morii, and L.C. Grand, Mammary cancer induced by a single dose of polynuclear hydrocarbons: routes of administration. Ann Surg, 1961.

154(6)Suppl: p. 315-8.

217. Welsch, C.W., Host factors affecting the growth of carcinogen-induced rat mammary carcinomas: a review and tribute to Charles Brenton Huggins. Cancer Res, 1985. 45(8): p. 3415-43.

218. Bombonati, A. and D.C. Sgroi, The molecular pathology of breast cancer progression. J Pathol, 2011. 223(2): p. 307-17.

219. Murad, T.M. and E. Von Haam, Studies of mammary carcinoma induced by 7,12dimethylbenz(a)anthracene administration. Cancer Res, 1972. 32(7): p. 1404-15.

220. McCormick, G.M. and R.C. Moon, EFFECT OF PREGNANCY AND LACTATION ON GROWTH OF MAMMARY TUMOURS INDUCED BY 7,12-DIMETHLBENZ(A) ANTHRACENE (DMBA). Br J Cancer, 1965. 19: p. 160-6.

221. Russo, J., et al., Pathogenesis of Mammary Carcinomas Induced in Rats by 7, 12Dimethylbenz[a]anthracene2. JNCI: Journal of the National Cancer Institute, 1977. 59(2): p. 435-445.

222. Lin, Y., et al., Role of mammary epithelial and stromal P450 enzymes in the clearance and metabolic activation of 7,12-dimethylbenz(a)anthracene in mice. Toxicol Lett, 2012. 212(2): p. 97-105.

223. Kerdelhue, B., C. Forest, and X. Coumoul, Dimethyl-Benz(a)anthracene: $A$ mammary carcinogen and a neuroendocrine disruptor. Biochim Open, 2016. 3: p. 49-55.

224. Dao, T.L., Mammary cancer induction by 7,12-dimethylbenz(a)anthracene: relation to age. Science, 1969. 165(3895): p. 810-1.

225. Nagasawa, H., R. Yanai, and H. Taniguchi, Importance of mammary gland DNA synthesis on carcinogen-induced mammary tumorigenesis in rats. Cancer Res, 1976. 36(7 pt 1): p. 2223-6.

226. Nagasawa, H. and R. Yanai, Frequency of mammary cell division in relation to age: its significance in the induction of mammary tumors by carcinogen in rats. J Natl Cancer Inst, 1974. 52(2): p. 609-10.

227. Russo, J., et al., Molecular and cellular basis of the mammary gland susceptibility to carcinogenesis. Environ Health Perspect, 1983. 49: p. 185-99.

228. Izzotti, A., et al., Patterns of DNA adduct formation in liver and mammary epithelial cells of rats treated with 7,12-dimethylbenz(a)anthracene, and selective effects of chemopreventive agents. Cancer Res, 1999. 59(17): p. 428590.

229. Kleiner, H.E., et al., Role of cytochrome 4501 family members in the metabolic activation of polycyclic aromatic hydrocarbons in mouse epidermis. Chem Res Toxicol, 2004. 17(12): p. 1667-74.

230. Li, D., et al., Aromatic DNA adducts in adjacent tissues of breast cancer patients: clues to breast cancer etiology. Cancer Res, 1996. 56(2): p. 287-93.

231. Daniel, F.B. and N.J. Joyce, DNA adduct formation by 7,12dimethylbenz[a]anthracene and its noncarcinogenic 2-fluoro analogue in female Sprague-Dawley rats. J Natl Cancer Inst, 1983. 70(1): p. 111-8. 
232. Isaacs, J.T., Genetic control of resistance to chemically induced mammary adenocarcinogenesis in the rat. Cancer Res, 1986. 46(8): p. 3958-63.

233. Moore, C.J., W.A. Tricomi, and M.N. Gould, Comparison of 7,12dimethylbenz[a]anthracene metabolism and DNA binding in mammary epithelial cells from three rat strains with differing susceptibilities to mammary carcinogenesis. Carcinogenesis, 1988. 9(11): p. 2099-102.

234. Lan, H., et al., Genetic loci controlling breast cancer susceptibility in the WistarKyoto rat. Genetics, 2001. 157(1): p. 331-339.

235. Hsu, L.C., et al., Genetic identification of Mcs-1, a rat mammary carcinoma suppressor gene. Cancer Res, 1994. 54(10): p. 2765-70.

236. Gould, K.A., et al., Genetic determination of susceptibility to estrogen-induced mammary cancer in the ACl rat: mapping of Emca1 and Emca2 to chromosomes 5 and 18. Genetics, 2004. 168(4): p. 2113-25.

237. Quan, X., et al., Genetic identification of distinct loci controlling mammary tumor multiplicity, latency, and aggressiveness in the rat. Mammalian Genome, 2006. 17(4): p. 310-321.

238. Schaffer, B.S., et al., Genetic bases of estrogen-induced tumorigenesis in the rat: mapping of loci controlling susceptibility to mammary cancer in a Brown Norway $x$ ACl intercross. Cancer Res, 2006. 66(15): p. 7793-800.

239. Ren, X., et al., Mapping of Mcs30, a new mammary carcinoma susceptibility quantitative trait locus (QTL30) on rat chromosome 12: identification of fry as a candidate Mcs gene. PLoS One, 2013. 8(9): p. e70930.

240. Drinkwater, N.R. and M.N. Gould, The long path from QTL to gene. PLoS genetics, 2012. 8(9): p. e1002975-e1002975.

241. Lan, H., et al., Genetic loci controlling breast cancer susceptibility in the WistarKyoto rat. Genetics, 2001. 157(1): p. 331-9.

242. Kim, H.Y., et al., Gene expression profiles of a mouse congenic strain carrying an obesity susceptibility QTL under obesigenic diets. Genes Nutr, 2010. 5(3): p. 23750.

243. Le, S., Z.C. Martin, and D.J. Samuelson, Physical Confirmation and Comparative Genomics of the Rat Mammary carcinoma susceptibility 3 Quantitative Trait Locus. G3 (Bethesda), 2017. 7(6): p. 1767-1773.

244. Tsuda, H., et al., Correlation between long-term survival in breast cancer patients and amplification of two putative oncogene-coamplification units: hst-1/int-2 and c-erbB-2/ear-1. Cancer Res, 1989. 49(11): p. 3104-8.

245. Easton, D.F., et al., Genome-wide association study identifies novel breast cancer susceptibility loci. Nature, 2007. 447(7148): p. 1087-93.

246. Hunter, D.J., et al., A genome-wide association study identifies alleles in FGFR2 associated with risk of sporadic postmenopausal breast cancer. Nat Genet, 2007. 39(7): p. 870-4.

247. Kibriya, M.G., et al., A pilot genome-wide association study of early-onset breast cancer. Breast Cancer Res Treat, 2009. 114(3): p. 463-77. 
248. denDekker, A.D., et al., Rat Mcs1b is concordant to the genome-wide associationidentified breast cancer risk locus at human $5 q 11.2$ and MIER3 is a candidate cancer susceptibility gene. Cancer research, 2012. 72(22): p. 6002-6012.

249. Antoniou, A.C., et al., Common breast cancer-predisposition alleles are associated with breast cancer risk in BRCA1 and BRCA2 mutation carriers. American journal of human genetics, 2008. 82(4): p. 937-948.

250. Garcia-Closas, M., et al., Heterogeneity of breast cancer associations with five susceptibility loci by clinical and pathological characteristics. PLoS genetics, 2008. 4(4): p. e1000054-e1000054.

251. Broeks, A., et al., Low penetrance breast cancer susceptibility loci are associated with specific breast tumor subtypes: findings from the Breast Cancer Association Consortium. Human molecular genetics, 2011. 20(16): p. 3289-3303.

252. Campa, D., et al., Interactions between genetic variants and breast cancer risk factors in the breast and prostate cancer cohort consortium. Journal of the National Cancer Institute, 2011. 103(16): p. 1252-1263.

253. Broeks, A., et al., Low penetrance breast cancer susceptibility loci are associated with specific breast tumor subtypes: findings from the Breast Cancer Association Consortium. Hum Mol Genet, 2011. 20(16): p. 3289-303.

254. Zheng, Q., et al., Association between Mitogen-Activated Protein Kinase Kinase Kinase 1 Polymorphisms and Breast Cancer Susceptibility: A Meta-Analysis of 20 Case-Control Studies. PLoS ONE, 2014. 9(3): p. e90771.

255. Sawyer, E., et al., Genetic predisposition to in situ and invasive lobular carcinoma of the breast. PLoS Genet, 2014. 10(4): p. e1004285.

256. denDekker, A.D., et al., Rat Mcs1b is concordant to the genome-wide associationidentified breast cancer risk locus at human $5 q 11.2$ and MIER3 is a candidate cancer susceptibility gene. Cancer Res, 2012. 72(22): p. 6002-12.

257. Paterno, G.D., et al., cDNA cloning of a novel, developmentally regulated immediate early gene activated by fibroblast growth factor and encoding a nuclear protein. J Biol Chem, 1997. 272(41): p. 25591-5.

258. Thorne, L.B., et al., Cloning and characterization of the mouse ortholog of mi-er1. DNA Seq, 2005. 16(3): p. 237-40.

259. McCarthy, P.L., G.D. Paterno, and L.L. Gillespie, Protein expression pattern of human MIER1 alpha, a novel estrogen receptor binding protein. Journal of molecular histology, 2013. 44(4): p. 469-479.

260. Paterno, G.D., et al., Genomic organization of the human mi-er1 gene and characterization of alternatively spliced isoforms: regulated use of a facultative intron determines subcellular localization. Gene, 2002. 295(1): p. 79-88.

261. Clements, J.A., et al., Differential splicing alters subcellular localization of the alpha but not beta isoform of the MIER1 transcriptional regulator in breast cancer cells. PloS one, 2012. 7(2): p. e32499-e32499.

262. Li, S., G.D. Paterno, and L.L. Gillespie, Nuclear localization of the transcriptional regulator MIER1a requires interaction with $H D A C 1 / 2$ in breast cancer cells. PloS one, 2013. 8(12): p. e84046-e84046. 
263. Ding, Z., et al., The SANT domain of human MI-ER1 interacts with Sp1 to interfere with GC box recognition and repress transcription from its own promoter. J Biol Chem, 2004. 279(27): p. 28009-16.

264. Derwish, R., G.D. Paterno, and L.L. Gillespie, Differential HDAC1 and 2 Recruitment by Members of the MIER Family. PloS one, 2017. 12(1): p. e0169338-e0169338.

265. Peng, M., et al., MIER3 suppresses colorectal cancer progression by downregulating Sp1, inhibiting epithelial-mesenchymal transition. Sci Rep, 2017. 7(1): p. 11000.

266. Derwish, R., G.D. Paterno, and L.L. Gillespie, Differential HDAC1 and 2 Recruitment by Members of the MIER Family. PLoS One, 2017. 12(1): p. e0169338.

267. Joshi, P., et al., The functional interactome landscape of the human histone deacetylase family. Mol Syst Biol, 2013. 9: p. 672.

268. Li, S., G. Paterno, and L. Gillespie, Nuclear Localization of the Transcriptional Regulator MIER1a Requires Interaction with HDAC1/2 in Breast Cancer Cells. Vol. 8. 2013. e84046.

269. Ding, Z., L.L. Gillespie, and G.D. Paterno, Human MI-ER1 alpha and beta function as transcriptional repressors by recruitment of histone deacetylase 1 to their conserved ELM2 domain. Mol Cell Biol, 2003. 23(1): p. 250-8.

270. Blackmore, T.M., et al., The transcriptional cofactor MIER1-beta negatively regulates histone acetyltransferase activity of the CREB-binding protein. BMC Res Notes, 2008. 1: p. 68.

271. Lakisic, G., et al., Role of the BAHD1 Chromatin-Repressive Complex in Placental Development and Regulation of Steroid Metabolism. PLOS Genetics, 2016. 12(3): p. e1005898.

272. Thorne, L.B., et al., Protein expression of the transcriptional regulator MI-ER1 alpha in adult mouse tissues. J Mol Histol, 2008. 39(1): p. 15-24.

273. Paterno, G.D., et al., Molecular cloning of human er1 CDNA and its differential expression in breast tumours and tumour-derived cell lines. Gene, 1998. 222(1): p. 77-82.

274. McCarthy, P.L., et al., Changes in subcellular localisation of MI-ER1 alpha, a novel oestrogen receptor-alpha interacting protein, is associated with breast cancer progression. Br J Cancer, 2008. 99(4): p. 639-46.

275. Li, S., G.D. Paterno, and L.L. Gillespie, Insulin and IGF-1, but not 178-estradiol, alter the subcellular localization of MIER1 $\alpha$ in MCF7 breast carcinoma cells. BMC Res Notes, 2015. 8: p. 356.

276. Duderstadt, E.L., et al., Chemical carcinogen-induced rat mammary carcinogenesis is a potential model of p21-activated kinase positive female breast cancer. Physiol Genomics, 2021. 53(2): p. 61-68.

277. Letessier, A., et al., Frequency, prognostic impact, and subtype association of $8 p 12,8 q 24,11 q 13,12 p 13,17 q 12$, and 20q13 amplifications in breast cancers. BMC Cancer, 2006. 6: p. 245. 
278. Pereira, B., et al., The somatic mutation profiles of 2,433 breast cancers refines their genomic and transcriptomic landscapes. Nat Commun, 2016. 7: p. 11479.

279. Dawson, S.J., et al., A new genome-driven integrated classification of breast cancer and its implications. Embo j, 2013. 32(5): p. 617-28.

280. Ali, H.R., et al., Genome-driven integrated classification of breast cancer validated in over 7,500 samples. Genome Biol, 2014. 15(8): p. 431.

281. Curtis, C., et al., The genomic and transcriptomic architecture of 2,000 breast tumours reveals novel subgroups. Nature, 2012. 486(7403): p. 346-52.

282. Shrestha, Y., et al., PAK1 is a breast cancer oncogene that coordinately activates MAPK and MET signaling. Oncogene, 2012. 31(29): p. 3397-3408.

283. Holm, C., et al., Association between Pak1 expression and subcellular localization and tamoxifen resistance in breast cancer patients. J Natl Cancer Inst, 2006. 98(10): p. 671-80.

284. Uhlen, M., et al., Proteomics. Tissue-based map of the human proteome. Science, 2015. 347(6220): p. 1260419.

285. Radu, M., et al., PAK signalling during the development and progression of cancer. Nature reviews. Cancer, 2014. 14(1): p. 13-25.

286. Ormandy, C.J., et al., Cyclin D1, EMS1 and 11q13 amplification in breast cancer. Breast Cancer Res Treat, 2003. 78(3): p. 323-35.

287. Fang, F., et al., p21-activated kinase 1 (PAK1) expression correlates with prognosis in solid tumors: A systematic review and meta-analysis. Oncotarget, 2016. 7(19): p. 27422-9.

288. Rayala, S.K., et al., P21-activated kinase 1 regulation of estrogen receptor-alpha activation involves serine 305 activation linked with serine 118 phosphorylation. Cancer Res, 2006. 66(3): p. 1694-701.

289. Adam, L., et al., Regulation of microfilament reorganization and invasiveness of breast cancer cells by kinase dead p21-activated kinase-1. J Biol Chem, 2000. 275(16): p. 12041-50.

290. Kumar, R. and D.Q. Li, PAKs in Human Cancer Progression: From Inception to Cancer Therapeutic to Future Oncobiology. Adv Cancer Res, 2016. 130: p. 137209.

291. Sells, M.A., et al., Human p21-activated kinase (Pak1) regulates actin organization in mammalian cells. Curr Biol, 1997. 7(3): p. 202-10.

292. Yellapu, N.K., M. Pulaganti, and S.B. Pakala, Bioinformatics exploration of PAK1 (P21-activated kinase-1) revealed potential network gene elements in breast invasive carcinoma. J Biomol Struct Dyn, 2017. 35(10): p. 2269-2279.

293. Sells, M.A., J.T. Boyd, and J. Chernoff, p21-activated kinase 1 (Pak1) regulates cell motility in mammalian fibroblasts. J Cell Biol, 1999. 145(4): p. 837-49.

294. Acconcia, F., et al., Phosphorylation-dependent regulation of nuclear localization and functions of integrin-linked kinase. Proc Natl Acad Sci U S A, 2007. 104(16): p. 6782-7.

295. Yang, H.J., et al., Overexpression of ILK1 in breast cancer associates with poor prognosis. Tumour Biol, 2013. 34(6): p. 3933-8. 
296. Gould, M.N., Rodent models for the study of etiology, prevention and treatment of breast cancer. Semin Cancer Biol, 1995. 6(3): p. 147-52.

297. Gibbs, R.A., et al., Genome sequence of the Brown Norway rat yields insights into mammalian evolution. Nature, 2004. 428(6982): p. 493-521.

298. Kent, W.J., et al., The human genome browser at UCSC. Genome Res, 2002. 12(6): p. 996-1006.

299. Application Note: Amplification Efficiency of TaqMan Gene Expression Assays. 2006, Applied Biosystems 127AP05-03.

300. Schindelin, J., et al., Fiji: an open-source platform for biological-image analysis. Nature Methods, 2012. 9(7): p. 676-682.

301. Duderstadt, E., Rat Pak1 sequencing primers (supplemental table 1), in Mendeley Data, V1 doi: 10.17632/s5ywd3525d.1. 2020.

302. Turi, A., et al., UTRdb and UTRsite (RELEASE 2010): a collection of sequences and regulatory motifs of the untranslated regions of eukaryotic $m R N A$ s. Nucleic Acids Research, 2009. 38(suppl_1): p. D75-D80.

303. Kozomara, A., M. Birgaoanu, and S. Griffiths-Jones, miRBase: from microRNA sequences to function. Nucleic Acids Research, 2018. 47(D1): p. D155-D162.

304. Kwek, S.S., et al., Co-amplified genes at $8 p 12$ and $11 q 13$ in breast tumors cooperate with two major pathways in oncogenesis. Oncogene, 2009. 28(17): p. 1892-903.

305. Vadlamudi, R.K., et al., Regulatable expression of p21-activated kinase-1 promotes anchorage-independent growth and abnormal organization of mitotic spindles in human epithelial breast cancer cells. J Biol Chem, 2000. 275(46): p. 36238-44.

306. Wang, Y., et al., P21-Activated Kinase Inhibitors FRAX486 and IPA3: Inhibition of Prostate Stromal Cell Growth and Effects on Smooth Muscle Contraction in the Human Prostate. PLoS One, 2016. 11(4): p. e0153312.

307. Hirokawa, Y., et al., Signal therapy of breast cancers by the HDAC inhibitor FK228 that blocks the activation of PAK1 and abrogates the tamoxifen-resistance. Cancer Biol Ther, 2005. 4(9): p. 956-60.

308. Balasenthil, S., et al., Estrogen receptor activation at serine 305 is sufficient to upregulate cyclin D1 in breast cancer cells. FEBS Lett, 2004. 567(2-3): p. 243-7.

309. Ong, C.C., et al., Targeting p21-activated kinase 1 (PAK1) to induce apoptosis of tumor cells. Proceedings of the National Academy of Sciences, 2011. 108(17): p. 7177.

310. Abba, M.C., et al., Transcriptomic changes in human breast cancer progression as determined by serial analysis of gene expression. Breast cancer research : BCR, 2004. 6(5): p. R499-R513.

311. Li, Q., et al., p21-Activated kinase 1 coordinates aberrant cell survival and pericellular proteolysis in a three-dimensional culture model for premalignant progression of human breast cancer. Neoplasia (New York, N.Y.), 2008. 10(4): p. 314-329. 
312. Cekanova, M. and K. Rathore, Animal models and therapeutic molecular targets of cancer: utility and limitations. Drug design, development and therapy, 2014. 8: p. 1911-1921.

313. Wang, R.A., et al., PAK1 hyperactivation is sufficient for mammary gland tumor formation. Oncogene, 2006. 25(20): p. 2931-6.

314. Shepel, L.A., et al., Genetic identification of multiple loci that control breast cancer susceptibility in the rat. Genetics, 1998. 149(1): p. 289-99.

315. Oeffinger, K.C., et al., Breast Cancer Screening for Women at Average Risk: 2015 Guideline Update From the American Cancer Society. JAMA, 2015. 314(15): p. 1599-1614.

316. DeSantis, C., et al., Breast cancer statistics, 2013. CA Cancer J Clin, 2014. 64(1): p. 52-62.

317. Carroll, J.C., et al., Hereditary breast and ovarian cancers. Can Fam Physician, 2008. 54(12): p. 1691-2.

318. Economopoulou, P., G. Dimitriadis, and A. Psyrri, Beyond BRCA: new hereditary breast cancer susceptibility genes. Cancer Treat Rev, 2015. 41(1): p. 1-8.

319. Mavaddat, N., et al., Genetic susceptibility to breast cancer. Mol Oncol, 2010. 4(3): p. 174-91.

320. Smith, P., et al., A genome wide linkage search for breast cancer susceptibility genes. Genes Chromosomes Cancer, 2006. 45(7): p. 646-55.

321. Decker, B., et al., Targeted Resequencing of the Coding Sequence of 38 Genes Near Breast Cancer GWAS Loci in a Large Case-Control Study. Cancer Epidemiol Biomarkers Prev, 2019. 28(4): p. 822-825.

322. Michailidou, K., et al., Association analysis identifies 65 new breast cancer risk loci. Nature, 2017. 551(7678): p. 92-94.

323. Colletti, J.A., 2nd, et al., Validation of six genetic determinants of susceptibility to estrogen-induced mammary cancer in the rat and assessment of their relevance to breast cancer risk in humans. G3 (Bethesda, Md.), 2014. 4(8): p. 1385-1394.

324. Sanders, J., J.D. Haag, and D.J. Samuelson, Physical confirmation and mapping of overlapping rat mammary carcinoma susceptibility QTLs, Mcs2 and Mcs6. PLoS One, 2011. 6(5): p. e19891.

325. Samuelson, D.J., et al., Physical evidence of Mcs5, a QTL controlling mammary carcinoma susceptibility, in congenic rats. Carcinogenesis, 2003. 24(9): p. 145560.

326. Veillet, A.L., et al., Mcs5c: a mammary carcinoma susceptibility locus located in a gene desert that associates with tenascin $C$ expression. Cancer prevention research (Philadelphia, Pa.), 2011. 4(1): p. 97-106.

327. Chen, C., et al., Varied pathways of stage IA lung adenocarcinomas discovered by integrated gene expression analysis. Int J Biol Sci, 2011. 7(5): p. 551-66.

328. Richmond, J., et al., Interleukin-16 as a marker of Sézary syndrome onset and stage. J Clin Immunol, 2011. 31(1): p. 39-50.

329. Alexandrakis, M.G., et al., Serum level of interleukin-16 in multiple myeloma patients and its relationship to disease activity. Am J Hematol, 2004. 75(2): p. 101-6. 
330. Milke, L., et al., Depletion of tristetraprolin in breast cancer cells increases interleukin-16 expression and promotes tumor infiltration with monocytes/macrophages. Carcinogenesis, 2013. 34(4): p. 850-7.

331. Kovacs, E., The serum levels of IL-12 and IL-16 in cancer patients. Relation to the tumour stage and previous therapy. Biomed Pharmacother, 2001. 55(2): p. 1116.

332. Gao, L.B., et al., The association of interleukin-16 polymorphisms with IL-16 serum levels and risk of colorectal and gastric cancer. Carcinogenesis, 2009. 30(2): p. 295-9.

333. Zhu, J., et al., IL-16 polymorphism and risk of renal cell carcinoma: association in a Chinese population. Int J Urol, 2010. 17(8): p. 700-7.

334. Pérez-Ramírez, C., et al., Interleukins as new prognostic genetic biomarkers in non-small cell lung cancer. Surg Oncol, 2017. 26(3): p. 278-285.

335. Batai, K., et al., Fine-mapping of IL16 gene and prostate cancer risk in African Americans. Cancer Epidemiol Biomarkers Prev, 2012. 21(11): p. 2059-68.

336. Thomas, G., et al., Multiple loci identified in a genome-wide association study of prostate cancer. Nature Genetics, 2008. 40(3): p. 310-315.

337. Bakht, M.K., et al., Neuroendocrine differentiation of prostate cancer leads to PSMA suppression. Endocr Relat Cancer, 2018. 26(2): p. 131-146.

338. Perner, S., et al., Prostate-specific membrane antigen expression as a predictor of prostate cancer progression. Hum Pathol, 2007. 38(5): p. 696-701.

339. Kasoha, M., et al., Prostate-specific membrane antigen (PSMA) expression in breast cancer and its metastases. Clin Exp Metastasis, 2017. 34(8): p. 479-490.

340. Tolkach, Y., et al., Prostate-specific membrane antigen in breast cancer: $a$ comprehensive evaluation of expression and a case report of radionuclide therapy. Breast Cancer Res Treat, 2018. 169(3): p. 447-455.

341. Shono, T., et al., Enhanced expression of NADPH oxidase Nox4 in human gliomas and its roles in cell proliferation and survival. Int J Cancer, 2008. 123(4): p. 78792.

342. Weyemi, U., et al., Intracellular expression of reactive oxygen species-generating NADPH oxidase NOX4 in normal and cancer thyroid tissues. Endocr Relat Cancer, 2010. 17(1): p. 27-37.

343. Yamaura, M., et al., NADPH oxidase 4 contributes to transformation phenotype of melanoma cells by regulating G2-M cell cycle progression. Cancer Res, 2009. 69(6): p. 2647-54.

344. Tang, C.T., et al., NOX4-driven ROS formation regulates proliferation and apoptosis of gastric cancer cells through the GLI1 pathway. Cell Signal, 2018. 46: p. 52-63.

345. Lin, X.L., et al., Overexpression of NOX4 predicts poor prognosis and promotes tumor progression in human colorectal cancer. Oncotarget, 2017. 8(20): p. 33586-33600.

346. Wu, Q.Q., et al., Downregulated NOX4 underlies a novel inhibitory role of microRNA-137 in prostate cancer. J Cell Biochem, 2019. 120(6): p. 10215-10227. 
347. Zhang, C., et al., NOX4 promotes non-small cell lung cancer cell proliferation and metastasis through positive feedback regulation of PI3K/Akt signaling.

Oncotarget, 2014. 5(12): p. 4392-405.

348. Degasper, C., et al., NADPH oxidase 4 expression in the normal endometrium and in endometrial cancer. Tumour Biol, 2019. 41(2): p. 1010428319830002.

349. Yu, B., et al., MiR-140-5p inhibits cell proliferation and metastasis by regulating MUC1 via BCL2A1/MAPK pathway in triple negative breast cancer. Cell Cycle, 2019. 18(20): p. 2641-2650.

350. Kønig, S.M., et al., Alterations of the interactome of $\mathrm{Bcl}-2$ proteins in breast cancer at the transcriptional, mutational and structural level. PLoS Comput Biol, 2019. 15(12): p. e1007485.

351. Kalra, S., et al., Association of CYP2C19*2 and ALDH1A1*1/*2 variants with disease outcome in breast cancer patients: results of a global screening array. Eur J Clin Pharmacol, 2018. 74(10): p. 1291-1298.

352. Tervasmäki, A., et al., Recurrent CYP2C19 deletion allele is associated with triplenegative breast cancer. BMC Cancer, 2014. 14: p. 902.

353. Saito, K., et al., Functional analysis of a novel glioma antigen, EFTUD1. Neuro Oncol, 2014. 16(12): p. 1618-29.

354. Sheng, L., et al., Identification of vitamin D(3) target genes in human breast cancer tissue. J Steroid Biochem Mol Biol, 2016. 164: p. 90-97.

355. Golmohammadzadeh, G., et al., Polymorphisms in Phase I (CYP450) Genes CYP1A1 (rs4646421), CYP1B1 (rs1056836), CYP19A1 (rs749292) and CYP2C8 (rs1058930) and Their Relation to Risk of Breast Cancer: A Case-Control Study in Mazandaran Province in North of Iran. Open Access Maced J Med Sci, 2019. 7(15): p. 2488-2496.

356. Li, C., et al., Identifying hepatocellular carcinoma-related hub genes by bioinformatics analysis and CYP2C8 is a potential prognostic biomarker. Gene, 2019. 698: p. 9-18.

357. Chen, P., et al., Co-expression network analysis identified six hub genes in association with metastasis risk and prognosis in hepatocellular carcinoma. Oncotarget, 2017. 8(30): p. 48948-48958.

358. Eun, H.S., et al., Cytochrome P450 $4 A 11$ expression in tumor cells: A favorable prognostic factor for hepatocellular carcinoma patients. J Gastroenterol Hepatol, 2019. 34(1): p. 224-233.

359. Kim, S., et al., Alteration of CYP4A11 expression in renal cell carcinoma: diagnostic and prognostic implications. J Cancer, 2020. 11(6): p. 1478-1485.

360. Li, W., et al., Downregulation of ARNT2 promotes tumor growth and predicts poor prognosis in human hepatocellular carcinoma. J Gastroenterol Hepatol, 2015. 30(6): p. 1085-93.

361. Kimura, Y., et al., ARNT2 Regulates Tumoral Growth in Oral Squamous Cell Carcinoma. J Cancer, 2016. 7(6): p. 702-10.

362. Martinez, V., et al., Drug metabolism-related genes as potential biomarkers: analysis of expression in normal and tumour breast tissue. Breast Cancer Res Treat, 2008. 110(3): p. 521-30. 
363. Yang, B., et al., ARNT2 is downregulated and serves as a potential tumor suppressor gene in non-small cell lung cancer. Tumour Biol, 2015. 36(3): p. 21119.

364. Jia, Y., et al., Overexpression of ARNT2 is associated with decreased cell proliferation and better prognosis in gastric cancer. Mol Cell Biochem, 2019. 450(1-2): p. 97-103.

365. Lee, H.S., et al., Combined use of CEMIP and CA 19-9 enhances diagnostic accuracy for pancreatic cancer. Sci Rep, 2018. 8(1): p. 3383.

366. Sabates-Bellver, J., et al., Transcriptome profile of human colorectal adenomas. Mol Cancer Res, 2007. 5(12): p. 1263-75.

367. Birkenkamp-Demtroder, K., et al., Repression of KIAA1199 attenuates Wntsignalling and decreases the proliferation of colon cancer cells. Br J Cancer, 2011. 105(4): p. 552-61.

368. Matsuzaki, S., et al., Clinicopathologic significance of KIAA1199 overexpression in human gastric cancer. Ann Surg Oncol, 2009. 16(7): p. 2042-51.

369. Chivu Economescu, M., et al., Identification of potential biomarkers for early and advanced gastric adenocarcinoma detection. Hepatogastroenterology, 2010. 57(104): p. 1453-64.

370. Evensen, N.A., et al., Unraveling the role of KIAA1199, a novel endoplasmic reticulum protein, in cancer cell migration. J Natl Cancer Inst, 2013. 105(18): p. 1402-16.

371. Shen, F., et al., CEMIP promotes ovarian cancer development and progression via the PI3K/AKT signaling pathway. Biomed Pharmacother, 2019. 114: p. 108787.

372. Zhang, P., et al., AMPK/GSK36/B-catenin cascade-triggered overexpression of CEMIP promotes migration and invasion in anoikis-resistant prostate cancer cells by enhancing metabolic reprogramming. Faseb j, 2018. 32(7): p. 3924-3935.

373. Chen, L., et al., Primary Tumor Site Specificity is Preserved in Patient-Derived Tumor Xenograft Models. Front Genet, 2019. 10: p. 738.

374. Wu, S.G., et al., miR-508-5p acts as an anti-oncogene by targeting MESDC1 in hepatocellular carcinoma. Neoplasma, 2017. 64(1): p. 40-47.

375. Huang, L., et al., The RNA-binding Protein MEX3B Mediates Resistance to Cancer Immunotherapy by Downregulating HLA-A Expression. Clin Cancer Res, 2018. 24(14): p. 3366-3376.

376. de Groot, J.S., et al., Validation of DNA promoter hypermethylation biomarkers in breast cancer--a short report. Cell Oncol (Dordr), 2014. 37(4): p. 297-303.

377. Tao, R., et al., Methylation profile of single hepatocytes derived from hepatitis $B$ virus-related hepatocellular carcinoma. PLoS One, 2011. 6(5): p. e19862.

378. Eissa, M.A.L., et al., Promoter methylation of ADAMTS1 and BNC1 as potential biomarkers for early detection of pancreatic cancer in blood. Clin Epigenetics, 2019. 11(1): p. 59.

379. Pangeni, R.P., et al., The GALNT9, BNC1 and CCDC8 genes are frequently epigenetically dysregulated in breast tumours that metastasise to the brain. Clin Epigenetics, 2015. 7(1): p. 57. 
380. Wu, Y., et al., Decreased Expression of BNC1 and BNC2 Is Associated with Genetic or Epigenetic Regulation in Hepatocellular Carcinoma. Int J Mol Sci, 2016. 17(2).

381. Boldrup, L., et al., p63 Transcriptionally regulates BNC1, a Pol I and Pol II transcription factor that regulates ribosomal biogenesis and epithelial differentiation. Eur J Cancer, 2012. 48(9): p. 1401-6.

382. García-Díez, I., et al., Transcriptome and cytogenetic profiling analysis of matched in situ/invasive cutaneous squamous cell carcinomas from immunocompetent patients. Genes Chromosomes Cancer, 2019. 58(3): p. 164174.

383. Delic, S., et al., Identification and functional validation of CDH11, PCSK6 and SH3GL3 as novel glioma invasion-associated candidate genes. Neuropathol Appl Neurobiol, 2012. 38(2): p. 201-12.

384. Fang, W.J., et al., Genome-wide analysis of aberrant DNA methylation for identification of potential biomarkers in colorectal cancer patients. Asian Pac J Cancer Prev, 2012. 13(5): p. 1917-21.

385. Nguyen, S.T., et al., Identification of a predictive gene expression signature of cervical lymph node metastasis in oral squamous cell carcinoma. Cancer Sci, 2007. 98(5): p. 740-6.

386. Koo, B.H., et al., ADAMTSL3/punctin-2, a gene frequently mutated in colorectal tumors, is widely expressed in normal and malignant epithelial cells, vascular endothelial cells and other cell types, and its mRNA is reduced in colon cancer. Int J Cancer, 2007. 121(8): p. 1710-6.

387. Zhou, X., et al., Genome-wide CRISPR knockout screens identify ADAMTSL3 and PTEN genes as suppressors of HCC proliferation and metastasis, respectively. J Cancer Res Clin Oncol, 2020. 146(6): p. 1509-1521.

388. Zhao, J., et al., A Six-LncRNA Expression Signature Associated with Prognosis of Colorectal Cancer Patients. Cell Physiol Biochem, 2018. 50(5): p. 1882-1890.

389. Dieci, M.V., et al., Rare breast cancer subtypes: histological, molecular, and clinical peculiarities. The oncologist, 2014. 19(8): p. 805-813.

390. Bombonati, A. and D.C. Sgroi, The molecular pathology of breast cancer progression. The Journal of pathology, 2011. 223(2): p. 307-317.

391. Makki, J., Diversity of Breast Carcinoma: Histological Subtypes and Clinical Relevance. Clinical medicine insights. Pathology, 2015. 8: p. 23-31.

392. Singh, M., J.N. McGinley, and H.J. Thompson, A comparison of the histopathology of premalignant and malignant mammary gland lesions induced in sexually immature rats with those occurring in the human. Lab Invest, 2000. 80(2): p. 22131.

393. Feng, M., et al., Histopathological alterations during breast carcinogenesis in a rat model induced by 7,12-Dimethylbenz (a) anthracene and estrogenprogestogen combinations. International journal of clinical and experimental medicine, 2015. 8(1): p. 346-357.

394. Russo, J., Significance of rat mammary tumors for human risk assessment. Toxicologic pathology, 2015. 43(2): p. 145-170. 
395. Wei, S., Papillary Lesions of the Breast: An Update. Arch Pathol Lab Med, 2016. 140(7): p. 628-43.

396. Rakha, E.A. and I.O. Ellis, Diagnostic challenges in papillary lesions of the breast. Pathology, 2018. 50(1): p. 100-110.

397. Pal, S.K., et al., Papillary carcinoma of the breast: an overview. Breast Cancer Res Treat, 2010. 122(3): p. 637-45.

398. Kadota, K., et al., The cribriform pattern identifies a subset of acinar predominant tumors with poor prognosis in patients with stage I lung adenocarcinoma: a conceptual proposal to classify cribriform predominant tumors as a distinct histologic subtype. Mod Pathol, 2014. 27(5): p. 690-700.

399. Branca, G., et al., An Updated Review of Cribriform Carcinomas with Emphasis on Histopathological Diagnosis and Prognostic Significance. Oncol Rev, 2017. 11(1): p. 317.

400. Kwan, M.L., et al., Pre-diagnosis body mass index and survival after breast cancer in the After Breast Cancer Pooling Project. Breast Cancer Res Treat, 2012. 132(2): p. 729-39.

401. Chan, D.S., et al., Body mass index and survival in women with breast cancersystematic literature review and meta-analysis of 82 follow-up studies. Ann Oncol, 2014. 25(10): p. 1901-14.

402. Greenlee, H., et al., Association between Body Mass Index and Cancer Survival in a Pooled Analysis of 22 Clinical Trials. Cancer Epidemiol Biomarkers Prev, 2017. 26(1): p. 21-29.

403. Loi, S., et al., Obesity and outcomes in premenopausal and postmenopausal breast cancer. Cancer Epidemiol Biomarkers Prev, 2005. 14(7): p. 1686-91.

404. Copson, E.R., et al., Obesity and the outcome of young breast cancer patients in the UK: the POSH study. Ann Oncol, 2015. 26(1): p. 101-112.

405. Rosenberg, L., K. Czene, and P. Hall, Obesity and poor breast cancer prognosis: an illusion because of hormone replacement therapy? Br J Cancer, 2009. 100(9): p. 1486-91.

406. Li, C.I., K.E. Malone, and J.R. Daling, Interactions between body mass index and hormone therapy and postmenopausal breast cancer risk (United States). Cancer Causes Control, 2006. 17(5): p. 695-703.

407. Neuhouser, M.L., et al., Overweight, Obesity, and Postmenopausal Invasive Breast Cancer Risk: A Secondary Analysis of the Women's Health Initiative Randomized Clinical Trials. JAMA Oncol, 2015. 1(5): p. 611-21.

408. Sebastiani, F., et al., Increased Incidence of Breast Cancer in Postmenopausal Women with High Body Mass Index at the Modena Screening Program. J Breast Cancer, 2016. 19(3): p. 283-291.

409. Picon-Ruiz, M., et al., Obesity and adverse breast cancer risk and outcome: Mechanistic insights and strategies for intervention. CA Cancer J Clin, 2017. 67(5): p. 378-397.

410. Brouckaert, O., et al., Body mass index, age at breast cancer diagnosis, and breast cancer subtype: a cross-sectional study. Breast Cancer Research and Treatment, 2018. 168(1): p. 189-196. 
411. Ooi, B.N.S., et al., The genetic interplay between body mass index, breast size and breast cancer risk: a Mendelian randomization analysis. International journal of epidemiology, 2019. 48(3): p. 781-794.

412. Enger, S.M., et al., Body size, physical activity, and breast cancer hormone receptor status: results from two case-control studies. Cancer Epidemiol Biomarkers Prev, 2000. 9(7): p. 681-7.

413. Suzuki, R., et al., Body weight and postmenopausal breast cancer risk defined by estrogen and progesterone receptor status among Swedish women: A prospective cohort study. Int J Cancer, 2006. 119(7): p. 1683-9.

414. Ahn, J., et al., Adiposity, adult weight change, and postmenopausal breast cancer risk. Arch Intern Med, 2007. 167(19): p. 2091-102.

415. Rosenberg, L.U., et al., Risk factors for hormone receptor-defined breast cancer in postmenopausal women. Cancer Epidemiol Biomarkers Prev, 2006. 15(12): p. 2482-8.

416. Canchola, A.J., et al., Body size and the risk of postmenopausal breast cancer subtypes in the California Teachers Study cohort. Cancer Causes Control, 2012.

417. Michels, K.B., K.L. Terry, and W.C. Willett, Longitudinal study on the role of body size in premenopausal breast cancer. Arch Intern Med, 2006. 166(21): p. 2395402.

418. Berstad, P., et al., A case-control study of body mass index and breast cancer risk in white and African-American women. Cancer Epidemiol Biomarkers Prev, 2010. 19(6): p. 1532-44.

419. Harris, H.R., et al., Body fat distribution and risk of premenopausal breast cancer in the Nurses' Health Study II. J Natl Cancer Inst, 2011. 103(3): p. 273-8.

420. Harris, H.R., et al., Body size across the life course, mammographic density, and risk of breast cancer. Am J Epidemiol, 2011. 174(8): p. 909-18.

421. Boyd, N.F., et al., Body Size, Mammographic Density, and Breast Cancer Risk. Cancer Epidemiology Biomarkers \&amp;amp; Prevention, 2006. 15(11): p. 2086.

422. Engmann, N.J., et al., Combined effect of volumetric breast density and body mass index on breast cancer risk. Breast Cancer Research and Treatment, 2019. 177(1): p. 165-173.

423. Gilbert, C.A. and J.M. Slingerland, Cytokines, obesity, and cancer: new insights on mechanisms linking obesity to cancer risk and progression. Annu Rev Med, 2013. 64: p. 45-57.

424. Picon-Ruiz, M., et al., Interactions between Adipocytes and Breast Cancer Cells Stimulate Cytokine Production and Drive Src/Sox2/miR-302b-Mediated Malignant Progression. Cancer Res, 2016. 76(2): p. 491-504.

425. Dirat, B., et al., Cancer-associated adipocytes exhibit an activated phenotype and contribute to breast cancer invasion. Cancer Res, 2011. 71(7): p. 2455-65.

426. Rekwot, P.I., et al., The role of pheromones and biostimulation in animal reproduction. Anim Reprod Sci, 2001. 65(3-4): p. 157-70.

427. Mora, O.A. and M.M. Cabrera, Pheromonal male-induced diestrus and cyclicity in aging intact and young estrogenized female rats. Biol Reprod, 1994. 50(3): p. 603-6. 
428. Mora, O.A., M.M. Cabrera, and J.E. Sánchez-Criado, Hormonal pattern of the pheromonal restoration of cyclic activity in aging irregularly cycling and persistent-estrus female rats. Biol Reprod, 1994. 51(5): p. 920-5.

429. Tomioka, M., T. Murayama, and M. Kashiwayanagi, Increases in plasma concentration of progesterone by protease-sensitive urinary pheromones in female rats. Biol Pharm Bull, 2005. 28(9): p. 1770-2.

430. Musatov, S., et al., Silencing of estrogen receptor alpha in the ventromedial nucleus of hypothalamus leads to metabolic syndrome. Proc Natl Acad Sci U S A, 2007. 104(7): p. 2501-6.

431. Shi, H. and D.J. Clegg, Sex differences in the regulation of body weight. Physiol Behav, 2009. 97(2): p. 199-204.

432. Asarian, L. and N. Geary, Cyclic estradiol treatment normalizes body weight and restores physiological patterns of spontaneous feeding and sexual receptivity in ovariectomized rats. Horm Behav, 2002. 42(4): p. 461-71.

433. Heine, P.A., et al., Increased adipose tissue in male and female estrogen receptoralpha knockout mice. Proc Natl Acad Sci U S A, 2000. 97(23): p. 12729-34.

434. Clegg, D.J., et al., Gonadal hormones determine sensitivity to central leptin and insulin. Diabetes, 2006. 55(4): p. 978-87.

435. Ainslie, D.A., et al., Estrogen deficiency causes central leptin insensitivity and increased hypothalamic neuropeptide Y. Int J Obes Relat Metab Disord, 2001. 25(11): p. 1680-8.

436. Machinal, F., et al., In vivo and in vitro ob gene expression and leptin secretion in rat adipocytes: evidence for a regional specific regulation by sex steroid hormones. Endocrinology, 1999. 140(4): p. 1567-74.

437. Vogler, M., BCL2A1: the underdog in the BCL2 family. Cell Death Differ, 2012. 19(1): p. 67-74.

438. Capuco, A.V., et al., Concurrent pregnancy retards mammary involution: effects on apoptosis and proliferation of the mammary epithelium after forced weaning of mice. Biol Reprod, 2002. 66(5): p. 1471-6.

439. Edmonds, B.T., et al., Elongation factor-1 alpha is an overexpressed actin binding protein in metastatic rat mammary adenocarcinoma. Journal of Cell Science, 1996. 109(11): p. 2705.

440. Duttaroy, A., et al., Apoptosis rate can be accelerated or decelerated by overexpression or reduction of the level of elongation factor-1 alpha. Exp Cell Res, 1998. 238(1): p. 168-76.

441. Duderstadt, E.L., M.A. Sanders, and D.J. Samuelson, A Method to Pre-Screen Rat Mammary Gland Whole-Mounts Prior To RNAscope. J Mammary Gland Biol Neoplasia, 2021.

442. Burska, A.N., et al., Gene expression analysis in RA: towards personalized medicine. The pharmacogenomics journal, 2014. 14(2): p. 93-106.

443. Patsialou, A., et al., Selective gene-expression profiling of migratory tumor cells in vivo predicts clinical outcome in breast cancer patients. Breast cancer research : BCR, 2012. 14(5): p. R139-R139. 
444. van 't Veer, L.J., et al., Gene expression profiling predicts clinical outcome of breast cancer. Nature, 2002. 415(6871): p. 530-6.

445. Hamburg, M.A. and F.S. Collins, The path to personalized medicine. N Engl J Med, 2010. 363(4): p. 301-4.

446. Wang, F., et al., RNAscope: a novel in situ RNA analysis platform for formalinfixed, paraffin-embedded tissues. The Journal of molecular diagnostics : JMD, 2012. 14(1): p. 22-29.

447. Gall, J.G. and M.L. Pardue, Formation and detection of RNA-DNA hybrid molecules in cytological preparations. Proceedings of the National Academy of Sciences of the United States of America, 1969. 63(2): p. 378-383.

448. Pardue, M.L. and J.G. Gall, Molecular hybridization of radioactive DNA to the DNA of cytological preparations. Proceedings of the National Academy of Sciences of the United States of America, 1969. 64(2): p. 600-604.

449. Tolg, C., M. Cowman, and E.A. Turley, Mouse Mammary Gland Whole Mount Preparation and Analysis. Bio-protocol, 2018. 8(13): p. e2915.

450. McGinley, J.N. and H.J. Thompson, Quantitative Assessment of Mammary Gland Density in Rodents Using Digital Image Analysis. Biological Procedures Online, 2011. 13(1): p. 4.

451. Tucker, D.K., et al., Sectioning Mammary Gland Whole Mounts for Lesion Identification. Journal of visualized experiments : JoVE, 2017(125): p. 55796.

452. Dapson, R.W., The history, chemistry and modes of action of carmine and related dyes. Biotech Histochem, 2007. 82(4-5): p. 173-87.

453. Singh, M., J.N. McGinley, and H.J. Thompson, $A$ Comparison of the Histopathology of Premalignant and Malignant Mammary Gland Lesions Induced in Sexually Immature Rats with those Occurring in the Human. Laboratory Investigation, 2000. 80(2): p. 221-231.

454. Hinck, L. and I. Näthke, Changes in cell and tissue organization in cancer of the breast and colon. Current opinion in cell biology, 2014. 26: p. 87-95.

455. Woditschka, S., et al., A short-term rat mammary carcinogenesis model for the prevention of hormonally responsive and nonresponsive in situ carcinomas. Cancer prevention research (Philadelphia, Pa.), 2009. 2(2): p. 153-160.

456. Lewis, F., et al., Unlocking the archive--gene expression in paraffin-embedded tissue. J Pathol, 2001. 195(1): p. 66-71.

457. Hedegaard, J., et al., Next-generation sequencing of RNA and DNA isolated from paired fresh-frozen and formalin-fixed paraffin-embedded samples of human cancer and normal tissue. PLoS One, 2014. 9(5): p. e98187.

458. Bresters, D., et al., The duration of fixation influences the yield of HCV CDNA-PCR products from formalin-fixed, paraffin-embedded liver tissue. J Virol Methods, 1994. 48(2-3): p. 267-72.

459. Macabeo-Ong, M., et al., Effect of duration of fixation on quantitative reverse transcription polymerase chain reaction analyses. Mod Pathol, 2002. 15(9): p. 979-87. 
460. Baena-Del Valle, J.A., et al., Rapid Loss of RNA Detection by In Situ Hybridization in Stored Tissue Blocks and Preservation by Cold Storage of Unstained Slides. Am J Clin Pathol, 2017. 148(5): p. 398-415.

461. Wang, F., et al., RNAscope: a novel in situ RNA analysis platform for formalinfixed, paraffin-embedded tissues. J Mol Diagn, 2012. 14(1): p. 22-9.

462. Blacher, S., et al., Quantitative Assessment of Mouse Mammary Gland Morphology Using Automated Digital Image Processing and TEB Detection. Endocrinology, 2016. 157(4): p. 1709-1716.

463. Uhlén, M., et al., Tissue-based map of the human proteome. Science, 2015. 347(6220): p. 1260419.

464. Ma, X.J., et al., Gene expression profiles of human breast cancer progression. Proc Natl Acad Sci U S A, 2003. 100(10): p. 5974-9.

465. Arpino, G., R. Laucirica, and R.M. Elledge, Premalignant and In Situ Breast Disease: Biology and Clinical Implications. Annals of Internal Medicine, 2005. 143(6): p. 446-457.

466. Santen, R.J. and R. Mansel, Benign Breast Disorders. New England Journal of Medicine, 2005. 353(3): p. 275-285.

467. Guray, M. and A.A. Sahin, Benign breast diseases: classification, diagnosis, and management. Oncologist, 2006. 11(5): p. 435-49.

468. O'Malley, F.P. and A.L. Bane, The spectrum of apocrine lesions of the breast. Adv Anat Pathol, 2004. 11(1): p. 1-9.

469. Shaaban, A.M., et al., Breast cancer risk in usual ductal hyperplasia is defined by estrogen receptor-alpha and Ki-67 expression. Am J Pathol, 2002. 160(2): p. 597604.

470. Cichon, M.A., et al., Microenvironmental influences that drive progression from benign breast disease to invasive breast cancer. Journal of mammary gland biology and neoplasia, 2010. 15(4): p. 389-397.

471. Allred, D.C., S.K. Mohsin, and S.A. Fuqua, Histological and biological evolution of human premalignant breast disease. Endocr Relat Cancer, 2001. 8(1): p. 47-61.

472. Damiani, S., et al., Myoepithelial cells and basal lamina in poorly differentiated in situ duct carcinoma of the breast. Virchows Archiv, 1999. 434(3): p. 227-234.

473. Sontag, L. and D.E. Axelrod, Evaluation of pathways for progression of heterogeneous breast tumors. J Theor Biol, 2005. 232(2): p. 179-89.

474. Hanahan, D. and R.A. Weinberg, The hallmarks of cancer. Cell, 2000. 100(1): p. 57-70.

475. O'Connell, P., et al., Analysis of loss of heterozygosity in 399 premalignant breast lesions at 15 genetic loci. J Natl Cancer Inst, 1998. 90(9): p. 697-703.

476. Sotiriou, C., et al., Gene Expression Profiling in Breast Cancer: Understanding the Molecular Basis of Histologic Grade To Improve Prognosis. JNCl: Journal of the National Cancer Institute, 2006. 98(4): p. 262-272.

477. Stephens, P.J., et al., The landscape of cancer genes and mutational processes in breast cancer. Nature, 2012. 486(7403): p. 400-4. 
478. Elenbaas, B., et al., Human breast cancer cells generated by oncogenic transformation of primary mammary epithelial cells. Genes Dev, 2001. 15(1): p. 50-65.

479. Williams, C., et al., Gene expression in murine mammary epithelial stem cell-like cells shows similarities to human breast cancer gene expression. Breast Cancer Res, 2009. 11(3): p. R26.

480. Lu, J., et al., Gene expression changes associated with chemically induced rat mammary carcinogenesis. Molecular Carcinogenesis, 1997. 20(2): p. 204-215.

481. Al-Dhaheri, W., I. Hassouna, and S.M. Karam, Genetic polymorphisms and protein expression of $P 53$ and BRCA1 in preneoplastic and neoplastic rat mammary glands. Oncol Rep, 2018. 39(5): p. 2193-2200.

482. Bach, K., et al., Differentiation dynamics of mammary epithelial cells revealed by single-cell RNA sequencing. Nat Commun, 2017. 8(1): p. 2128.

483. Castro, N.P., et al., Evidence that molecular changes in cells occur before morphological alterations during the progression of breast ductal carcinoma. Breast Cancer Res, 2008. 10(5): p. R87.

484. Gregory, K.J., et al., Gene expression signature of atypical breast hyperplasia and regulation by SFRP1. Breast Cancer Res, 2019. 21(1): p. 76.

485. Ma, X.-J., et al., Gene expression profiles of human breast cancer progression. Proceedings of the National Academy of Sciences of the United States of America, 2003. 100(10): p. 5974-5979.

486. Vargas, A.C., et al., Gene expression profiling of tumour epithelial and stromal compartments during breast cancer progression. Breast Cancer Res Treat, 2012. 135(1): p. 153-65.

487. Carter, J.H. and H.W. Carter, Adrenal regulation of mammary tumorigenesis in female Sprague-Dawley rats: histopathology of mammary tumors. Cancer Res, 1988. 48(13): p. 3808-15.

488. Januškevičienè, I. and V. Petrikaitè, Heterogeneity of breast cancer: The importance of interaction between different tumor cell populations. Life Sci, 2019. 239: p. 117009.

489. Perou, C.M., et al., Molecular portraits of human breast tumours. Nature, 2000. 406(6797): p. 747-52.

490. Esparza-López, J., et al., Breast Cancer Intra-Tumor Heterogeneity: One Tumor, Different Entities. Rev Invest Clin, 2017. 69(2): p. 66-76.

491. Gupta, A., M. Mutebi, and A. Bardia, Gene-Expression-Based Predictors for Breast Cancer. Ann Surg Oncol, 2015. 22(11): p. 3418-32.

492. Fu, J., et al., Gene expression profiling leads to discovery of correlation of matrix metalloproteinase 11 and heparanase 2 in breast cancer progression. BMC Cancer, 2015. 15: p. 473.

493. UniProt: a worldwide hub of protein knowledge. Nucleic Acids Res, 2019. 47(D1): p. D506-d515.

494. Gaudet, P., et al., Phylogenetic-based propagation of functional annotations within the Gene Ontology consortium. Brief Bioinform, 2011. 12(5): p. 449-62. 
495. Wiseman, B.S. and Z. Werb, Stromal effects on mammary gland development and breast cancer. Science, 2002. 296(5570): p. 1046-9.

496. Russo, J., H. Lynch, and I.H. Russo, Mammary gland architecture as a determining factor in the susceptibility of the human breast to cancer. Breast J, 2001. 7(5): p. 278-91.

497. Hennighausen, L. and G.W. Robinson, Information networks in the mammary gland. Nat Rev Mol Cell Biol, 2005. 6(9): p. 715-25.

498. Howlin, J., J. McBryan, and F. Martin, Pubertal mammary gland development: insights from mouse models. J Mammary Gland Biol Neoplasia, 2006. 11(3-4): p. 283-97.

499. Darcy, K.M., et al., Mammary fibroblasts stimulate growth, alveolar morphogenesis, and functional differentiation of normal rat mammary epithelial cells. In Vitro Cell Dev Biol Anim, 2000. 36(9): p. 578-92.

500. Cases, S., et al., Development of the mammary gland requires DGAT1 expression in stromal and epithelial tissues. Development, 2004. 131(13): p. 3047.

501. Lukashev, M.E. and Z. Werb, ECM signalling: orchestrating cell behaviour and misbehaviour. Trends Cell Biol, 1998. 8(11): p. 437-41.

502. Lanigan, F., et al., Molecular links between mammary gland development and breast cancer. Cell Mol Life Sci, 2007. 64(24): p. 3159-84.

503. Malhotra, G.K., et al., Shared signaling pathways in normal and breast cancer stem cells. J Carcinog, 2011. 10: p. 38.

504. Hynes, N.E. and C.J. Watson, Mammary gland growth factors: roles in normal development and in cancer. Cold Spring Harb Perspect Biol, 2010. 2(8): p. a003186.

505. Hvid, H., et al., Mammary gland proliferation in female rats: effects of the estrous cycle, pseudo-pregnancy and age. Exp Toxicol Pathol, 2012. 64(4): p. 321-32.

506. Clarke, R.B., Steroid receptors and proliferation in the human breast. Steroids, 2003. 68(10-13): p. 789-794.

507. Mallepell, S., et al., Paracrine signaling through the epithelial estrogen receptor alpha is required for proliferation and morphogenesis in the mammary gland. Proc Natl Acad Sci U S A, 2006. 103(7): p. 2196-201.

508. Russo, J., et al., Pattern of distribution of cells positive for estrogen receptor alpha and progesterone receptor in relation to proliferating cells in the mammary gland. Breast Cancer Res Treat, 1999. 53(3): p. 217-27.

509. Fendrick, J.L., A.M. Raafat, and S.Z. Haslam, Mammary gland growth and development from the postnatal period to postmenopause: ovarian steroid receptor ontogeny and regulation in the mouse. J Mammary Gland Biol Neoplasia, 1998. 3(1): p. 7-22.

510. Korenman, S.G., Oestrogen window hypothesis of the aetiology of breast cancer. Lancet, 1980. 1(8170): p. 700-1.

511. Henderson, B.E., R. Ross, and L. Bernstein, Estrogens as a cause of human cancer: the Richard and Hinda Rosenthal Foundation award lecture. Cancer Res, 1988. 48(2): p. 246-53. 
512. Olsson, H.L. and M.L. Olsson, The Menstrual Cycle and Risk of Breast Cancer: $A$ Review. Frontiers in oncology, 2020. 10: p. 21-21.

513. Vogel, P.M., et al., The correlation of histologic changes in the human breast with the menstrual cycle. The American journal of pathology, 1981. 104(1): p. 23-34.

514. Potten, C.S., et al., The effect of age and menstrual cycle upon proliferative activity of the normal human breast. Br J Cancer, 1988. 58(2): p. 163-70.

515. Korenman, S.G., The endocrinology of breast cancer. Cancer, 1980. 46(4 Suppl): p. 874-8.

516. Pike, M.C., et al., 'Hormonal' risk factors, 'breast tissue age' and the ageincidence of breast cancer. Nature, 1983. 303(5920): p. 767-70.

517. Rodgers, K.M., et al., Environmental chemicals and breast cancer: An updated review of epidemiological literature informed by biological mechanisms. Environ Res, 2018. 160: p. 152-182.

518. Hoover, R.N., et al., Adverse health outcomes in women exposed in utero to diethylstilbestrol. N Engl J Med, 2011. 365(14): p. 1304-14.

519. Land, C.E., et al., Breast Cancer Risk From Low-Dose Exposures to lonizing Radiation: Results of Parallel Analysis of Three Exposed Populations of Women. JNCl: Journal of the National Cancer Institute, 1980. 65(2): p. 353-376.

520. McGregor, D.H., et al., Breast Cancer Incidence Among Atomic Bomb Survivors, Hiroshima and Nagasaki, 1950-69. JNCl: Journal of the National Cancer Institute, 1977. 59(3): p. 799-811.

521. Brown, N.M., et al., Prenatal TCDD and predisposition to mammary cancer in the rat. Carcinogenesis, 1998. 19(9): p. 1623-9.

522. Durando, M., et al., Prenatal bisphenol A exposure induces preneoplastic lesions in the mammary gland in Wistar rats. Environ Health Perspect, 2007. 115(1): p. 80-6.

523. Jenkins, S., et al., Prenatal TCDD exposure predisposes for mammary cancer in rats. Reprod Toxicol, 2007. 23(3): p. 391-6.

524. Fielden, M.R., et al., Normal mammary gland morphology in pubertal female mice following in utero and lactational exposure to genistein at levels comparable to human dietary exposure. Toxicol Lett, 2002. 133(2-3): p. 181-91.

525. Hovey, R.C., et al., Effects of neonatal exposure to diethylstilbestrol, tamoxifen, and toremifene on the BALB/C mouse mammary gland. Biol Reprod, 2005. 72(2): p. 423-35.

526. Betancourt, A.M., et al., In utero exposure to bisphenol A shifts the window of susceptibility for mammary carcinogenesis in the rat. Environ Health Perspect, 2010. 118(11): p. 1614-9.

527. Fachal, L. and A.M. Dunning, From candidate gene studies to GWAS and postGWAS analyses in breast cancer. Curr Opin Genet Dev, 2015. 30: p. 32-41.

528. Dhakal, P. and M.J. Soares, Single-step PCR-based genetic sex determination of rat tissues and cells. Biotechniques, 2017. 62(5): p. 232-233.

529. Young, W.C., J.L. Boling, and R.J. Blandau, The vaginal smear picture, sexual receptivity and time of ovulation in the albino rat. The Anatomical Record, 1941. 80(1): p. 37-45. 
530. Cora, M.C., L. Kooistra, and G. Travlos, Vaginal Cytology of the Laboratory Rat and Mouse: Review and Criteria for the Staging of the Estrous Cycle Using Stained Vaginal Smears. Toxicologic Pathology, 2015. 43(6): p. 776-793.

531. Berryhill, G.E., et al., TRIENNIAL LACTATION SYMPOSIUM/BOLFA: Dietary regulation of allometric ductal growth in the mammary glands. J Anim Sci, 2017. 95(12): p. 5664-5674.

532. Soberon, F. and M.E. Van Amburgh, Effects of preweaning nutrient intake in the developing mammary parenchymal tissue. J Dairy Sci, 2017. 100(6): p. 49965004.

533. Daniel, C.W., G.B. Silberstein, and P. Strickland, Direct action of 17 beta-estradiol on mouse mammary ducts analyzed by sustained release implants and steroid autoradiography. Cancer Res, 1987. 47(22): p. 6052-7.

534. Tucker, H.L., et al., Tamoxifen impairs prepubertal mammary development and alters expression of estrogen receptor $\alpha$ (ESR1) and progesterone receptors (PGR). Domest Anim Endocrinol, 2016. 54: p. 95-105.

535. Hovey, R.C., J.F. Trott, and B.K. Vonderhaar, Establishing a framework for the functional mammary gland: from endocrinology to morphology. J Mammary Gland Biol Neoplasia, 2002. 7(1): p. 17-38.

536. Rezaei, R., et al., Amino acids and mammary gland development: nutritional implications for milk production and neonatal growth. J Anim Sci Biotechnol, 2016. 7: p. 20.

537. Kalirai, H. and R.B. Clarke, Human breast epithelial stem cells and their regulation. J Pathol, 2006. 208(1): p. 7-16.

538. Eden, J.A., Breast cancer, stem cells and sex hormones: part 1. The impact of fetal life and infancy. Maturitas, 2010. 67(2): p. 117-20.

539. Balogh, G.A., et al., Genomic signature induced by pregnancy in the human breast. Int J Oncol, 2006. 28(2): p. 399-410.

540. D'Cruz, C.M., et al., Persistent parity-induced changes in growth factors, TGFbeta3, and differentiation in the rodent mammary gland. Mol Endocrinol, 2002. 16(9): p. 2034-51.

541. Russo, J., et al., Influence of age and parity on the susceptibility of rat mammary gland epithelial cells in primary cultures to 7,12-dimethylbenz(a)anthracene. In Vitro, 1981. 17(10): p. 877-84.

542. Solari, F., A. Bateman, and J. Ahringer, The Caenorhabditis elegans genes egl-27 and egr-1 are similar to MTA1, a member of a chromatin regulatory complex, and are redundantly required for embryonic patterning. Development, 1999. 126(11): p. 2483-94.

543. Ding, Z., L.L. Gillespie, and G.D. Paterno, Human MI-ER1 alpha and beta function as transcriptional repressors by recruitment of histone deacetylase 1 to their conserved ELM2 domain. Molecular and cellular biology, 2003. 23(1): p. 250-258.

544. Manavathi, B., K. Singh, and R. Kumar, MTA family of coregulators in nuclear receptor biology and pathology. Nucl Recept Signal, 2007. 5: p. e010.

545. Boyer, L.A., R.R. Latek, and C.L. Peterson, The SANT domain: a unique histonetail-binding module? Nat Rev Mol Cell Biol, 2004. 5(2): p. 158-63. 
546. Aasland, R., A.F. Stewart, and T. Gibson, The SANT domain: a putative DNAbinding domain in the SWI-SNF and ADA complexes, the transcriptional corepressor N-CoR and TFIIIB. Trends Biochem Sci, 1996. 21(3): p. 87-8.

547. Boyer, L.A., et al., Essential role for the SANT domain in the functioning of multiple chromatin remodeling enzymes. Mol Cell, 2002. 10(4): p. 935-42.

548. Vargova, K., et al., MYB transcriptionally regulates the miR-155 host gene in chronic lymphocytic leukemia. Blood, 2011. 117(14): p. 3816-25.

549. Bantscheff, M., et al., Chemoproteomics profiling of HDAC inhibitors reveals selective targeting of HDAC complexes. Nat Biotechnol, 2011. 29(3): p. 255-65.

550. Mulligan, P., et al., CDYL bridges REST and histone methyltransferases for gene repression and suppression of cellular transformation. Mol Cell, 2008. 32(5): p. 718-26.

551. Escamilla-Del-Arenal, M., et al., Cdyl, a new partner of the inactive $X$ chromosome and potential reader of H3K27me3 and H3K9me2. Mol Cell Biol, 2013. 33(24): p. 5005-20.

552. Derwish, R., Investigating the role of mesoderm induction early response (MIER) family members as transcriptional co-repressors (Unpublished doctoral dissertation). Memorial University of Newfoundland. 2019.

553. Orkin, Stuart H. and K. Hochedlinger, Chromatin Connections to Pluripotency and Cellular Reprogramming. Cell, 2011. 145(6): p. 835-850.

554. Ting, A.H., K.M. McGarvey, and S.B. Baylin, The cancer epigenome--components and functional correlates. Genes Dev, 2006. 20(23): p. 3215-31.

555. Lafon-Hughes, L., et al., Chromatin-remodelling mechanisms in cancer. Mutat Res, 2008. 658(3): p. 191-214.

556. Fog, C.K., K.T. Jensen, and A.H. Lund, Chromatin-modifying proteins in cancer. APMIS, 2007. 115(10): p. 1060-1089.

557. Trojer, P. and D. Reinberg, Facultative heterochromatin: is there a distinctive molecular signature? Mol Cell, 2007. 28(1): p. 1-13.

558. Perissi, V., et al., Deconstructing repression: evolving models of co-repressor action. Nat Rev Genet, 2010. 11(2): p. 109-23.

559. Kelly, Richard D.W. and Shaun M. Cowley, The physiological roles of histone deacetylase (HDAC) 1 and 2: complex co-stars with multiple leading parts. Biochemical Society Transactions, 2013. 41(3): p. 741-749.

560. Laugesen, A. and K. Helin, Chromatin Repressive Complexes in Stem Cells, Development, and Cancer. Cell Stem Cell, 2014. 14(6): p. 735-751.

561. Gibbons, R.J., Histone modifying and chromatin remodelling enzymes in cancer and dysplastic syndromes. Hum Mol Genet, 2005. 14 Spec No 1: p. R85-92.

562. Hon, G.C., et al., Global DNA hypomethylation coupled to repressive chromatin domain formation and gene silencing in breast cancer. Genome Res, 2012. 22(2): p. 246-58.

563. Satheshkumar, P.S., et al., Inhibition of the Ubiquitin-Proteasome System Prevents Vaccinia Virus DNA Replication and Expression of Intermediate and Late Genes. Journal of Virology, 2009. 83(6): p. 2469. 
564. Eng, J.K., A.L. McCormack, and J.R. Yates, An approach to correlate tandem mass spectral data of peptides with amino acid sequences in a protein database. J Am Soc Mass Spectrom, 1994. 5(11): p. 976-89.

565. Mellacheruvu, D., et al., The CRAPome: a contaminant repository for affinity purification-mass spectrometry data. Nature methods, 2013. 10(8): p. 730-736.

566. Ashburner, M., et al., Gene ontology: tool for the unification of biology. The Gene Ontology Consortium. Nature genetics, 2000. 25(1): p. 25-29.

567. Chen, J., et al., ToppGene Suite for gene list enrichment analysis and candidate gene prioritization. Nucleic acids research, 2009. 37(Web Server issue): p. W305W311.

568. Mutarelli, M., et al., Time-course analysis of genome-wide gene expression data from hormone-responsive human breast cancer cells. BMC Bioinformatics, 2008. 9 Suppl 2(Suppl 2): p. S12.

569. Bertucci, F., et al., Gene expression profiling and clinical outcome in breast cancer. Omics, 2006. 10(4): p. 429-43.

570. Thomas, P. and T.G. Smart, HEK293 cell line: a vehicle for the expression of recombinant proteins. J Pharmacol Toxicol Methods, 2005. 51(3): p. 187-200.

571. Ooi, A., et al., A Guide to Transient Expression of Membrane Proteins in HEK-293 Cells for Functional Characterization. Frontiers in Physiology, 2016. 7: p. 300.

572. Rokhlin, O.W., et al., Mechanisms of cell death induced by histone deacetylase inhibitors in androgen receptor-positive prostate cancer cells. Mol Cancer Res, 2006. 4(2): p. 113-23.

573. Dang, L., et al., Proteasome inhibitor MG132 inhibits the proliferation and promotes the cisplatin-induced apoptosis of human esophageal squamous cell carcinoma cells. Int J Mol Med, 2014. 33(5): p. 1083-8.

574. Johmura, Y., et al., Fbxo22-mediated KDM4B degradation determines selective estrogen receptor modulator activity in breast cancer. J Clin Invest, 2018. 128(12): p. 5603-5619.

575. Dong, D.D., et al., Functional analysis of the LXXLL motifs of the human glucocorticoid receptor: association with altered ligand affinity. J Steroid Biochem Mol Biol, 2006. 101(2-3): p. 106-17.

576. Delage-Mourroux, R., et al., Analysis of estrogen receptor interaction with a repressor of estrogen receptor activity (REA) and the regulation of estrogen receptor transcriptional activity by REA. J Biol Chem, 2000. 275(46): p. 35848-56.

577. Kawajiri, K., et al., Role of the LXXLL-motif and activation function 2 domain in subcellular localization of Dax-1 (dosage-sensitive sex reversal-adrenal hypoplasia congenita critical region on the X chromosome, gene 1). Mol Endocrinol, 2003. 17(6): p. 994-1004.

578. Lakisic, G., et al., Role of the BAHD1 Chromatin-Repressive Complex in Placental Development and Regulation of Steroid Metabolism. PLoS Genet, 2016. 12(3): p. e1005898.

579. Jamaladdin, S., et al., Histone deacetylase (HDAC) 1 and 2 are essential for accurate cell division and the pluripotency of embryonic stem cells. Proc Natl Acad Sci U S A, 2014. 111(27): p. 9840-5. 
580. Bierne, H., et al., Human BAHD1 promotes heterochromatic gene silencing. Proc Natl Acad Sci U S A, 2009. 106(33): p. 13826-31.

581. Dechat, T., et al., Nuclear lamins: major factors in the structural organization and function of the nucleus and chromatin. Genes Dev, 2008. 22(7): p. 832-53.

582. Rhodes, D.R., et al., ONCOMINE: a cancer microarray database and integrated data-mining platform. Neoplasia, 2004. 6(1): p. 1-6.

583. Györffy, B., et al., An online survival analysis tool to rapidly assess the effect of 22,277 genes on breast cancer prognosis using microarray data of 1,809 patients. Breast Cancer Res Treat, 2010. 123(3): p. 725-31.

584. Moriya, H., Quantitative nature of overexpression experiments. Mol Biol Cell, 2015. 26(22): p. 3932-9.

585. Li, G.W., et al., Quantifying absolute protein synthesis rates reveals principles underlying allocation of cellular resources. Cell, 2014. 157(3): p. 624-35.

586. Habchi, J., et al., Introducing protein intrinsic disorder. Chem Rev, 2014. 114(13): p. 6561-88.

587. Liu, Z. and Y. Huang, Advantages of proteins being disordered. Protein Sci, 2014. 23(5): p. 539-50.

588. Ishida, T. and K. Kinoshita, PrDOS: prediction of disordered protein regions from amino acid sequence. Nucleic Acids Research, 2007. 35(suppl_2): p. W460-W464.

589. Gsponer, J., et al., Tight regulation of unstructured proteins: from transcript synthesis to protein degradation. Science, 2008. 322(5906): p. 1365-8.

590. Savkur, R.S. and T.P. Burris, The coactivator $L X X L L$ nuclear receptor recognition motif. J Pept Res, 2004. 63(3): p. 207-12.

591. Liu, B., et al., A transcriptional corepressor of Stat1 with an essential LXXLL signature motif. Proc Natl Acad Sci U S A, 2001. 98(6): p. 3203-7.

592. Davis, M.B., et al., Identification of common and cell type specific $L X X L L$ motif ECR cofactors using a bioinformatics refined candidate RNAi screen in Drosophila melanogaster cell lines. BMC Dev Biol, 2011. 11: p. 66.

593. Plevin, M.J., M.M. Mills, and M. Ikura, The LxxLL motif: a multifunctional binding sequence in transcriptional regulation. Trends Biochem Sci, 2005. 30(2): p. 66-9.

594. McInerney, E.M., et al., Determinants of coactivator LXXLL motif specificity in nuclear receptor transcriptional activation. Genes Dev, 1998. 12(21): p. 3357-68.

595. Callebaut, I., J.C. Courvalin, and J.P. Mornon, The BAH (bromo-adjacent homology) domain: a link between DNA methylation, replication and transcriptional regulation. FEBS Lett, 1999. 446(1): p. 189-93.

596. Goodwin, G.H. and R.H. Nicolas, The BAH domain, polybromo and the RSC chromatin remodelling complex. Gene, 2001. 268(1-2): p. 1-7.

597. Yang, N. and R.M. Xu, Structure and function of the BAH domain in chromatin biology. Crit Rev Biochem Mol Biol, 2013. 48(3): p. 211-21.

598. Lebreton, A., et al., A bacterial protein targets the BAHD1 chromatin complex to stimulate type III interferon response. Science, 2011. 331(6022): p. 1319-21.

599. Libertini, E., et al., Overexpression of the Heterochromatinization Factor BAHD1 in HEK293 Cells Differentially Reshapes the DNA Methylome on Autosomes and X Chromosome. Front Genet, 2015. 6: p. 339. 
600. Weimann, M., et al., $A$ Y2H-seq approach defines the human protein methyltransferase interactome. Nat Methods, 2013. 10(4): p. 339-42.

601. Xue, Y., et al., NURD, a novel complex with both ATP-dependent chromatinremodeling and histone deacetylase activities. Mol Cell, 1998. 2(6): p. 851-61.

602. Zhang, Y., et al., Analysis of the NuRD subunits reveals a histone deacetylase core complex and a connection with DNA methylation. Genes Dev, 1999. 13(15): p. 1924-35.

603. Laherty, C.D., et al., Histone deacetylases associated with the mSin3 corepressor mediate mad transcriptional repression. Cell, 1997. 89(3): p. 349-56.

604. Humphrey, G.W., et al., Stable histone deacetylase complexes distinguished by the presence of SANT domain proteins COREST/kiaa0071 and Mta-L1. J Biol Chem, 2001. 276(9): p. 6817-24.

605. Laherty, C.D., et al., SAP30, a component of the mSin3 corepressor complex involved in $\mathrm{N}$-CoR-mediated repression by specific transcription factors. Mol Cell, 1998. 2(1): p. 33-42.

606. Bowen, N.J., et al., Mi-2/NuRD: multiple complexes for many purposes. Biochim Biophys Acta, 2004. 1677(1-3): p. 52-7.

607. Millard, C.J., et al., Class I HDACs share a common mechanism of regulation by inositol phosphates. Mol Cell, 2013. 51(1): p. 57-67.

608. Wang, L., et al., Atrophin recruits HDAC1/2 and G9a to modify histone H3K9 and to determine cell fates. EMBO Rep, 2008. 9(6): p. 555-62.

609. Plaster, N., et al., REREa/Atrophin-2 interacts with histone deacetylase and Fgf8 signaling to regulate multiple processes of zebrafish development. Dev Dyn, 2007. 236(7): p. 1891-904.

610. Feinberg, A.P., Phenotypic plasticity and the epigenetics of human disease. Nature, 2007. 447(7143): p. 433-40.

611. Moss, T.J. and L.L. Wallrath, Connections between epigenetic gene silencing and human disease. Mutat Res, 2007. 618(1-2): p. 163-74.

612. Wang, G.G., C.D. Allis, and P. Chi, Chromatin remodeling and cancer, Part I: Covalent histone modifications. Trends Mol Med, 2007. 13(9): p. 363-72.

613. Dechat, T., S.A. Adam, and R.D. Goldman, Nuclear lamins and chromatin: when structure meets function. Adv Enzyme Regul, 2009. 49(1): p. 157-66.

614. Goldman, R.D., et al., Nuclear lamins: building blocks of nuclear architecture. Genes Dev, 2002. 16(5): p. 533-47.

615. Shumaker, D.K., E.R. Kuczmarski, and R.D. Goldman, The nucleoskeleton: lamins and actin are major players in essential nuclear functions. Curr Opin Cell Biol, 2003. 15(3): p. 358-66.

616. Ludérus, M.E., et al., Binding of matrix attachment regions to lamin B1. Cell, 1992. 70(6): p. 949-59.

617. Zhao, K., et al., Binding of matrix attachment regions to nuclear lamin is mediated by the rod domain and depends on the lamin polymerization state. FEBS Lett, 1996. 380(1-2): p. 161-4. 
618. Taniura, H., C. Glass, and L. Gerace, A chromatin binding site in the tail domain of nuclear lamins that interacts with core histones. J Cell Biol, 1995. 131(1): p. 3344.

619. van Steensel, B. and A.S. Belmont, Lamina-Associated Domains: Links with Chromosome Architecture, Heterochromatin, and Gene Repression. Cell, 2017. 169(5): p. 780-791.

620. Meuleman, W., et al., Constitutive nuclear lamina-genome interactions are highly conserved and associated with A/T-rich sequence. Genome Res, 2013. 23(2): p. 270-80.

621. Graziano, S., et al., Causes and consequences of genomic instability in laminopathies: Replication stress and interferon response. Nucleus, 2018. 9(1): p. 258-275.

622. Gaydos, L.J., W. Wang, and S. Strome, Gene repression. H3K27me and PRC2 transmit a memory of repression across generations and during development. Science, 2014. 345(6203): p. 1515-8.

623. Zhao, D., et al., The BAH domain of BAHD1 is a histone H3K27me3 reader. Protein Cell, 2016. 7(3): p. 222-6.

624. Ma, P. and R.M. Schultz, HDAC1 and HDAC2 in mouse oocytes and preimplantation embryos: Specificity versus compensation. Cell Death \& Differentiation, 2016. 23(7): p. 1119-1127.

625. Khier, H., et al., Molecular cloning and characterization of the mouse histone deacetylase 1 gene: integration of a retrovirus in 129SV mice. Biochim Biophys Acta, 1999. 1489(2-3): p. 365-73.

626. Taplick, J., et al., Homo-oligomerisation and nuclear localisation of mouse histone deacetylase 1. J Mol Biol, 2001. 308(1): p. 27-38.

627. Reichert, N., M.-A. Choukrallah, and P. Matthias, Multiple roles of class I HDACs in proliferation, differentiation, and development. Cellular and Molecular Life Sciences, 2012. 69(13): p. 2173-2187.

628. Brunmeir, R., S. Lagger, and C. Seiser, Histone deacetylase HDAC1/HDAC2controlled embryonic development and cell differentiation. Int J Dev Biol, 2009. 53(2-3): p. 275-89.

629. Levy, D.E. and J.E. Darnell, Jr., Stats: transcriptional control and biological impact. Nat Rev Mol Cell Biol, 2002. 3(9): p. 651-62.

630. Ramana, C.V., et al., Complex roles of Stat1 in regulating gene expression. Oncogene, 2000. 19(21): p. 2619-27.

631. Sadzak, I., et al., Recruitment of Stat1 to chromatin is required for interferoninduced serine phosphorylation of Stat1 transactivation domain. Proc Natl Acad Sci U S A, 2008. 105(26): p. 8944-9.

632. Darnell, J.E., Jr., I.M. Kerr, and G.R. Stark, Jak-STAT pathways and transcriptional activation in response to IFNs and other extracellular signaling proteins. Science, 1994. 264(5164): p. 1415-21.

633. Schindler, C. and J.E. Darnell, Jr., Transcriptional responses to polypeptide ligands: the JAK-STAT pathway. Annu Rev Biochem, 1995. 64: p. 621-51. 
634. Shuai, K., et al., Activation of transcription by IFN-gamma: tyrosine phosphorylation of a 91-kD DNA binding protein. Science, 1992. 258(5089): p. 1808-12.

635. Chatterjee-Kishore, M., F. van den Akker, and G.R. Stark, Association of STATs with relatives and friends. Trends Cell Biol, 2000. 10(3): p. 106-11.

636. Shuai, K., Modulation of STAT signaling by STAT-interacting proteins. Oncogene, 2000. 19(21): p. 2638-2644.

637. de la Serna, I.L., Y. Ohkawa, and A.N. Imbalzano, Chromatin remodelling in mammalian differentiation: lessons from ATP-dependent remodellers. Nat Rev Genet, 2006. 7(6): p. 461-73.

638. Nusinzon, I. and C.M. Horvath, Interferon-stimulated transcription and innate antiviral immunity require deacetylase activity and histone deacetylase 1. Proceedings of the National Academy of Sciences, 2003. 100(25): p. 14742.

639. Kamitani, S., et al., KAP1 regulates type I interferon/STAT1-mediated IRF-1 gene expression. Biochem Biophys Res Commun, 2008. 370(2): p. 366-70.

640. Lebreton, A., et al., A Bacterial Protein Targets the BAHD1 Chromatin Complex to Stimulate Type III Interferon Response. Science, 2011. 331(6022): p. 1319.

641. Lebreton, A., et al., Structural basis for the inhibition of the chromatin repressor BAHD1 by the bacterial nucleomodulin LntA. mBio, 2014. 5(1): p. e00775-13.

642. Meyer, T., K. Gavenis, and U. Vinkemeier, Cell type-specific and tyrosine phosphorylation-independent nuclear presence of STAT1 and STAT3. Exp Cell Res, 2002. 272(1): p. 45-55.

643. Braunstein, J., et al., STATs dimerize in the absence of phosphorylation. J Biol Chem, 2003. 278(36): p. 34133-40.

644. Yang, J. and G.R. Stark, Roles of unphosphorylated STATs in signaling. Cell Res, 2008. 18(4): p. 443-51.

645. Silver-Morse, L. and W.X. Li, JAK-STAT in heterochromatin and genome stability. Jakstat, 2013. 2(3): p. e26090.

646. Hu, X., et al., Unphosphorylated STAT5A stabilizes heterochromatin and suppresses tumor growth. Proceedings of the National Academy of Sciences, 2013. 110(25): p. 10213.

647. Dutta, P., et al., Unphosphorylated STAT3 in heterochromatin formation and tumor suppression in lung cancer. BMC Cancer, 2020. 20(1): p. 145.

648. Jovanovic, J., et al., The epigenetics of breast cancer. Mol Oncol, 2010. 4(3): p. 242-54.

649. Hughes, K. and C.J. Watson, The spectrum of STAT functions in mammary gland development. Jakstat, 2012. 1(3): p. 151-8.

650. Haricharan, S. and Y. Li, STAT signaling in mammary gland differentiation, cell survival and tumorigenesis. Mol Cell Endocrinol, 2014. 382(1): p. 560-569.

651. Santos-Rosa, H. and C. Caldas, Chromatin modifier enzymes, the histone code and cancer. Eur J Cancer, 2005. 41(16): p. 2381-402.

652. Tae, S., et al., Bromodomain protein 7 interacts with PRMT5 and PRC2, and is involved in transcriptional repression of their target genes. Nucleic Acids Res, 2011. 39(13): p. 5424-38. 
653. Holliday, H., et al., Epigenomics of mammary gland development. Breast Cancer Res, 2018. 20(1): p. 100.

654. Zhu, J., et al., Genome-wide Chromatin State Transitions Associated with Developmental and Environmental Cues. Cell, 2013. 152(3): p. 642-654.

655. Meshorer, E., et al., Hyperdynamic Plasticity of Chromatin Proteins in Pluripotent Embryonic Stem Cells. Developmental Cell, 2006. 10(1): p. 105-116.

656. Ho, L. and G.R. Crabtree, Chromatin remodelling during development. Nature, 2010. 463(7280): p. 474-84.

657. Michalak, E.M., et al., Canonical PRC2 function is essential for mammary gland development and affects chromatin compaction in mammary organoids. PLoS Biol, 2018. 16(8): p. e2004986.

658. Pellacani, D., et al., Analysis of Normal Human Mammary Epigenomes Reveals Cell-Specific Active Enhancer States and Associated Transcription Factor Networks. Cell Rep, 2016. 17(8): p. 2060-2074.

659. Chung, C.-Y., et al., Single-Cell Chromatin Analysis of Mammary Gland Development Reveals Cell-State Transcriptional Regulators and Lineage Relationships. Cell Reports, 2019. 29(2): p. 495-510.e6. 


\section{APPENDIX}

\section{PERMISSION TO USE PUBLISHED WORK}

For research article: Duderstadt EL, McQuaide SA, Sanders MA, Samuelson DJ.

Chemical carcinogen-induced rat mammary carcinogenesis is a potential model of p21-activated kinase positive female breast cancer. Physiological Genomics 2021. PMID: 33346690. DOI: 10.1152/physiolgenomics.00112.2020

Permission from The American Physiological Society:

Feb 19, 2021

This Agreement between Emily Duderstadt ("You") and The American Physiological Society ("The American Physiological Society") consists of your license details and the terms and conditions provided by The American Physiological Society and Copyright Clearance Center.

License Number: 5012520903215

License date: Feb 19, 2021

Licensed Content Publisher: The American Physiological Society

Licensed Content Publication: Physiological Genomics

Licensed Content Title: Chemical carcinogen-induced rat mammary carcinogenesis is a potential model of p21-activated kinase positive female breast cancer

Licensed Content Author: Emily L. Duderstadt, et al

Licensed Content Date: Feb 1, 2021

Licensed Content Volume: 53

Licensed Content Issue: 2

Type of Use: Thesis/Dissertation

Requestor type: Author of original work

Requestor: Emily Duderstadt

Institution name: University of Louisville

The APS hereby grants to you a nonexclusive limited license to reuse published material as requested by you, provided you have disclosed complete and accurate details of your proposed reuse of articles, figures, tables, images, and /or data in new or derivative works. Licenses are for a one-time English language use with a maximum distribution equal to the number of copies identified by you in the licensing process, unless additional options for translations or World Rights were included in your request. Any form of print or electronic republication must be completed within three years from the date hereof. Copies prepared before then may be distributed thereafter

(C)The American Physiological Society (APS). All rights reserved. 


\section{PERMISSION TO USE PUBLISHED WORK}

For research article: Duderstadt EL, Sanders MA, Samuelson DJ. A Method to PreScreen Rat Mammary Gland Whole-Mounts Prior To RNAscope. Journal of mammary gland biology and neoplasia 2021. PMID: 33866475. DOI: 10.1007/s10911-021-09484-5

Permission from Springer Nature License:

April 27, 2021

This Agreement between University of Louisville -- Emily Duderstadt ("You") and Springer Nature ("Springer Nature") consists of your license details and the terms and conditions provided by Springer Nature and Copyright Clearance Center.

License Number

License date

Licensed Content Publisher

Licensed Content Publication

Licensed Content Title

Licensed Content Author

Licensed Content Date

Type of Use

Requestor type

Format

Portion

Order reference number
5054801076280

Apr 23, 2021

Springer Nature

Journal of Mammary Gland Biology and Neoplasia A Method to Pre-Screen Rat Mammary Gland WholeMounts Prior To RNAscope

Emily L. Duderstadt et al

Apr 17, 2021

Thesis/Dissertation

academic/university or research institute

print and electronic

full article/chapter

s10911-021-09484-5

The Licensor grants you a personal, non-exclusive, non-transferable, world-wide licence to reproduce the Licensed Material for the purpose specified in your order only. Licences are granted for the specific use requested in the order and for no other use. 


\author{
CURRICULUM VITAE \\ Emily Lauren Duderstadt \\ Department of Biochemistry and Molecular Genetics \\ University of Louisville School of Medicine \\ 580 S. Preston Street, Louisville KY 40202 \\ (513) 259-5040 \\ eldude01@louisville.edu
}

\title{
Education
}

\begin{tabular}{llr}
\hline $2016-$ present & University of Louisville & Louisville, KY \\
& PhD, Biochemistry and Molecular Genetics & \\
& GPA: 3.9/4.0 & \\
& Qualifying Exam Passed: 5/2018, PhD expected spring 2021 \\
$2016-2017$ & University of Louisville & Louisville, KY \\
& M.S., Biochemistry and Molecular Genetics & \\
$2012-2016$ & Wittenberg University & Springfield, OH \\
& B.S., Suma cum laude, Biochemistry and Molecular Biology \\
& GPA 3.9/4.0
\end{tabular}

\section{Research Experience}

2016 - present $\quad$ University of Louisville

Louisville, KY

Doctoral student; Dissertation Advisor: David J. Samuelson

PhD Dissertation: Genetic and Functional Studies of Rat Mammary Cancer Susceptibility

i. Physical mapping of a rat mammary carcinoma susceptibility locus to identify candidate breast cancer variants/genes

ii. Exploration of candidate cancer gene, Mier3, expression during rat mammary cancer progression and windows of susceptibility

iii. Mechanistic analysis of Mier3 protein domain interactions

$2014-2016$ 
i. One pot synthesis of substituted and halogenated derivative dibenzoxanthenes

ii. Structural analysis of compounds by NMR

iii. Testing of potential cytotoxic effects by measuring singlet oxygen production and DPPH antioxidant assays

$2013-2014$

Wittenberg University Springfield, $\mathrm{OH}$

Undergraduate Researcher; Advisor: Kristen K. Cline, PhD

Project: Concentrations of Copper and Chromium Metals in

Treated and Untreated Water Samples from Ohio Counties

i. Chelation ion chromatography of treated and source untreated water samples

ii. Atomic absorbance spectroscopy to determine copper and chromium concentrations with respect to EPA standards, and recommendations to Ohio counties

Teaching Experience

2017

Teaching Assistant, Advanced Biochemistry, University of Louisville

$2014-2016$

Teaching Assistant, Organic Chemistry, Wittenberg University

$2013-2014$

Teaching Assistant, General Chemistry, Wittenberg University

\section{Publications}

Emily L. Duderstadt, Sarah A. McQuaide, Mary Ann Sanders, and David J. Samuelson. Chemical Carcinogen Induced Rat Mammary Carcinogenesis is a Potential Model of p21 activated kinase (PAK1) Positive Breast Cancer. Physiological Genomics 2020, PMID: 33346690

Selected for APSselect, a collection from the American Physiological Society that showcases some of the best recently published articles in physiological research

Emily L. Duderstadt, Mary Ann Sanders, David J. Samuelson.

A method to pre-screen rat mammary gland whole-mounts prior to RNAscope. Journal of Mammary Gland Biology and Neoplasia 2021

\section{Selected Posters and Presentations}

EL Duderstadt. Genetic and Functional Studies of Rat Mammary Cancer Susceptibility. Research Conference Seminars, Department of Biochemistry \& Molecular Genetics, University of Louisville, August 26, 2019 and September 14, 2020

EL Duderstadt, MA Sanders, DJ Samuelson. Expression of Breast Cancer Candidate Gene MIER3 in Rat Mammary Tissue During Development and Disease Progression. Research Louisville, Louisville KY, July 2019

EL Duderstadt, SA McQuiade, DJ Samuelson. Overexpression of p21-activated kinase (Pak1) in Rat Mammary Cancer Tumorigenesis. Research Louisville, Louisville KY, July 2017 


\section{Grant Applications}

NIH F31 Fellowship

Identifier: OGMB190586, Submitted: December 2018

Project title: Functional analysis of mesoderm induction early response, family member 3 Awards and Honors

2020 Doctoral Dissertation Completion Award, University of Louisville Graduate School

$2014 \quad$ Alpha Lambda Delta National Honor Society

$2014 \quad$ Omicron Delta Kappa National Honor Society

$2014 \quad$ Mortar Board National Honor Society

2012 - 2016 Provost Scholarship, Wittenberg University

\section{Work Experience:}

2014 - 2016 Chemical Stockroom Assistant, Wittenberg University, Springfield, OH

2014 Reading Assistant for AP Exams (College Board), Cincinnati OH

Service

2017 - 2019 Volunteer, Kentucky Science Museum, Brain Days Outreach, Louisville, KY

2015 - $2016 \quad$ Volunteer, Boonshoft Museum of Discovery, Springfield, OH

2013 - 2016 Pianist and coordinator, Campus Services, Wittenberg University, Springfield, $\mathrm{OH}$ 